Comparative Effectiveness Review

Number 217

\title{
Management of Infertility
}




\section{Comparative Effectiveness Review}

Number 217

\section{Management of Infertility}

\section{Prepared for:}

Agency for Healthcare Research and Quality

U.S. Department of Health and Human Services

5600 Fishers Lane

Rockville, MD 20857

www.ahrq.gov

Contract No. 290-2015-00004-I

Prepared by:

Duke Evidence-based Practice Center

Durham, NC

\section{Investigators:}

Evan R. Myers, M.D., M.P.H.

Jennifer L. Eaton, M.D., M.S.C.I.

Kara A. McElligott, M.D., M.P.H.

Patricia G. Moorman, Ph.D., M.S.P.H.

Ranee Chatterjee, M.D., M.P.H.

Arthurine K. Zakama, M.D.

Karen Goldstein, M.D., M.S.P.H.

Jennifer Strauss, Ph.D.

Remy R. Coeytaux, M.D., Ph.D.

Adam Goode, D.P.T., Ph.D.

Ethan Borre, B.A.

Geeta K. Swamy, M.D.

Amanda J. McBroom, Ph.D.

Kathryn Lallinger, M.S.L.S.

Robyn Schmidt, B.A.

J. Kelly Davis, B.A.

Victor Hasselblad, Ph.D.

Gillian D. Sanders, Ph.D. 


\section{Key Messages}

\section{Purpose of Review}

Evaluate the comparative effectiveness and safety of treatments for common causes of infertility.

\section{Key Messages}

- The ability to compare the effectiveness of treatments would be enhanced by greater consistency in reporting of outcomes, particularly live birth rates, as well as reporting of diagnosis-specific outcomes for treatments, such as assisted reproductive technology, that are used for multiple diagnoses.

- Letrozole most likely results in more live births with lower multiple births than clomiphene alone in women with polycystic ovary syndrome.

- For women with unexplained infertility, there is most likely shorter time to pregnancy for women with immediate in vitro fertilization (IVF) than for those who undergo other treatments prior to IVF. For the outcomes of live birth, multiple births, ectopic pregnancy, miscarriage, low birthweight, and ovarian hyperstimulation syndrome however, there may be no difference between the two groups.

- Across all diagnoses, elective single-embryo transfer results in slightly lower live birth rates but substantially lower reductions in multiple birth rates than multiple-embryo transfer. 
This report is based on research conducted by the Duke Evidence-based Practice Center (EPC) under contract to the Agency for Healthcare Research and Quality (AHRQ), Rockville, MD (Contract No. 290-2015-00004-I. The findings and conclusions in this document are those of the authors, who are responsible for its contents; the findings and conclusions do not necessarily represent the views of AHRQ. Therefore, no statement in this report should be construed as an official position of AHRQ or of the U.S. Department of Health and Human Services.

\section{None of the investigators have any affiliations or financial involvement that conflicts with the material presented in this report.}

The information in this report is intended to help healthcare decision makers—patients and clinicians, health system leaders, and policymakers, among others-make well-informed decisions and thereby improve the quality of healthcare services. This report is not intended to be a substitute for the application of clinical judgment. Anyone who makes decisions concerning the provision of clinical care should consider this report in the same way as any medical reference and in conjunction with all other pertinent information, i.e., in the context of available resources and circumstances presented by individual patients.

This report is made available to the public under the terms of a licensing agreement between the author and the Agency for Healthcare Research and Quality. This report may be used and reprinted without permission except those copyrighted materials that are clearly noted in the report. Further reproduction of those copyrighted materials is prohibited without the express permission of copyright holders.

AHRQ or U.S. Department of Health and Human Services endorsement of any derivative products that may be developed from this report, such as clinical practice guidelines, other quality enhancement tools, or reimbursement or coverage policies, may not be stated or implied.

This report may periodically be assessed for the currency of conclusions. If an assessment is done, the resulting surveillance report describing the methodology and findings will be found on the Effective Health Care Program website at www.effectivehealthcare.ahrq.gov. Search on the title of the report.

Persons using assistive technology may not be able to fully access information in this report. For assistance contact epc@ahrq.hhs.gov.

Suggested citation: Myers ER, Eaton JL, McElligott KA, Moorman PG, Chatterjee R, Zakama AK, Goldstein K, Strauss J, Coeytaux RR, Goode A, Borre E, Swamy GK, McBroom AJ, Lallinger K, Schmidt R, Davis JK, Hasselblad V, Sanders GD. Management of Infertility. Comparative Effectiveness Review No. 217. (Prepared by the Duke Evidence-based Practice Center under Contract No. 290-2015-00004-I.) AHRQ Publication No. 19-EHC014-EF. Rockville, MD: Agency for Healthcare Research and Quality; May 2019. Posted final reports are located on the Effective Health Care Program search page.

DOI: https://doi.org/10.23970/AHRQEPCCER217. 


\section{Preface}

The Agency for Healthcare Research and Quality (AHRQ), through its Evidence-based Practice Centers (EPCs), sponsors the development of systematic reviews to assist public- and private-sector organizations in their efforts to improve the quality of healthcare in the United States. These reviews provide comprehensive, science-based information on common, costly medical conditions, and new healthcare technologies and strategies.

Systematic reviews are the building blocks underlying evidence-based practice; they focus attention on the strength and limits of evidence from research studies about the effectiveness and safety of a clinical intervention. In the context of developing recommendations for practice, systematic reviews can help clarify whether assertions about the value of the intervention are based on strong evidence from clinical studies. For more information about AHRQ EPC systematic reviews, see www.effectivehealthcare.ahrq.gov/reference/purpose.cfm.

AHRQ expects that these systematic reviews will be helpful to health plans, providers, purchasers, government programs, and the healthcare system as a whole. Transparency and stakeholder input are essential to the Effective Health Care Program. Please visit the website (www.effectivehealthcare.ahrq.gov) to see draft research questions and reports or to join an email list to learn about new program products and opportunities for input.

If you have comments on this systematic review, they may be sent by mail to the Task Order Officer named below at: Agency for Healthcare Research and Quality, 5600 Fishers Lane, Rockville, MD 20857, or by email to epc@ahrq.hhs.gov.

Gopal Khanna, M.B.A.

Director

Agency for Healthcare Research and Quality

Stephanie Chang, M.D., M.P.H.

Director

Evidence-based Practice Center Program

Center for Evidence and Practice

Improvement

Agency for Healthcare Research and Quality
Arlene S. Bierman, M.D., M.S.

Director

Center for Evidence and Practice

Improvement

Agency for Healthcare Research and Quality

Suchitra Iyer, Ph.D.

Task Order Officer

Center for Practice Improvement

Agency for Healthcare Research and Quality 


\section{Acknowledgments}

The authors thank Megan von Isenburg, M.S.L.S., for help with the literature search and retrieval; Samantha Bowen, Ph.D., for project leadership; Megan Chobot, M.S.L.S., for assistance with project coordination; and Liz Wing, M.A., and Rebecca Gray, Ph.D., for editorial assistance.

\section{Key Informants}

In designing the study questions, the EPC consulted several Key Informants who represent the end-users of research. The EPC sought the Key Informant input on the priority areas for research and synthesis. Key Informants are not involved in the analysis of the evidence or the writing of the report. Therefore, in the end, study questions, design, methodological approaches, and/or conclusions do not necessarily represent the views of individual Key Informants.

Key Informants must disclose any financial conflicts of interest greater than \$5,000 and any other relevant business or professional conflicts of interest. Because of their role as end-users, individuals with potential conflicts may be retained. The TOO and the EPC work to balance, manage, or mitigate any conflicts of interest.

The list of Key Informants who provided input to this report follows:

Sheree Boulet, Dr.P.H., M.P.H.

Centers for Disease Control and Prevention

Atlanta, GA

Barbara Collura, M.A.

RESOLVE: The National Fertility

Association

McLean, VA

Esther Eisenberg, M.D.

National Institute of Child Health and

Human Development

National Institutes of Health

Bethesda, MD

Tina Groat, M.D., M.B.A., FACOG

UnitedHealthcare

Minneapolis, MN
Dmitry Kissin, M.D., M.P.H.

Centers for Disease Control and Prevention

Atlanta, GA

Dan I. Lebovic, M.D.

Center for Reproductive Medicine and

Advanced Reproductive Technologies

Minneapolis, MN

Richard S. Legro, M.D.

Pennsylvania State University

State College, PA

Bradley J. Van Voorhis, M.D.

Carver College of Medicine

University of Iowa

Iowa City, IA 


\section{Technical Expert Panel}

In designing the study questions and methodology at the outset of this report, the EPC consulted several technical and content experts. Broad expertise and perspectives were sought. Divergent and conflicting opinions are common and perceived as healthy scientific discourse that results in a thoughtful, relevant systematic review. Therefore, in the end, study questions, design, methodologic approaches, and/or conclusions do not necessarily represent the views of individual technical and content experts.

Technical Experts must disclose any financial conflicts of interest greater than $\$ 5,000$ and any other relevant business or professional conflicts of interest. Because of their unique clinical or content expertise, individuals with potential conflicts may be retained. The TOO and the EPC work to balance, manage, or mitigate any potential conflicts of interest identified.

The list of Technical Experts who provided input to this report follows:

Sheree Boulet, Dr.P.H., M.P.H.*

Centers for Disease Control and Prevention

Atlanta, GA

Barbara Collura, M.A.

RESOLVE: The National Fertility

Association

McLean, VA

Esther Eisenberg, M.D.

National Institute of Child Health and

Human Development

National Institutes of Health

Bethesda, MD

Audrey Gassman, M.D.*

Center for Drug Evaluation and Research

U.S. Food and Drug Administration

Silver Spring, MD

David Grainger, M.D., M.P.H.*

Center for Reproductive Medicine

University of Kansas School of Medicine

Wichita, KS

*Provided input on Draft Report.
Tina Groat, M.D., M.B.A., FACOG

UnitedHealthcare

Minneapolis, $\mathrm{MN}$

Dan I. Lebovic, M.D.*

Center for Reproductive Medicine and

Advanced Reproductive Technologies

Minneapolis, $\mathrm{MN}$

Richard S. Legro, M.D.

Pennsylvania State University

State College, PA

Jay Sandlow, M.D.*

Medical College of Wisconsin

Milwaukee, WI

Bradley J. Van Voorhis, M.D.*

Carver College of Medicine

University of Iowa

Iowa City, IA 


\section{Peer Reviewers}

Prior to publication of the final evidence report, EPCs sought input from independent Peer Reviewers without financial conflicts of interest. However, the conclusions and synthesis of the scientific literature presented in this report do not necessarily represent the views of individual reviewers.

Peer Reviewers must disclose any financial conflicts of interest greater than $\$ 5,000$ and any other relevant business or professional conflicts of interest. Because of their unique clinical or content expertise, individuals with potential nonfinancial conflicts may be retained. The TOO and the EPC work to balance, manage, or mitigate any potential nonfinancial conflicts of interest identified.

The list of Peer Reviewers follows:

Kurt T. Barnhart, M.D., M.S.C.E.

University of Pennsylvania Medical Center Philadelphia, PA

Clarisa Gracia, M.D., M.S.C.E. Perelman School of Medicine University of Pennsylvania Philadelphia, PA
Brooke V. Rossi, M.D.

School of Medicine

Case Western Reserve University

Cleveland, $\mathrm{OH}$

John M. Thorp, Jr., M.D.

University of North Carolina

Chapel Hill, NC 


\section{Management of Infertility}

\section{Structured Abstract}

Objective. Previous studies have demonstrated varying success for treatment of infertility. Much of this literature, however, does not focus on treatment of women with specific diagnoses. This systematic review evaluated the comparative effectiveness and safety of fertility treatment strategies for (a) women of reproductive age (18-44) who are infertile due to polycystic ovary syndrome (PCOS), endometriosis, unknown reasons, or tubal or peritoneal factors or (b) couples with male factor infertility, and evaluated short- and long-term health outcomes of gamete donors in infertility.

Data sources. We searched PubMed ${ }^{\circledR}$, Embase $^{\circledR}$, and the Cochrane Database of Systematic Reviews for English-language studies published from January 1, 2007, to October 3, 2018, that reported live birth rates, pregnancy and neonatal outcomes, time to pregnancy, and short-term and long-term adverse outcomes for mothers and children born after infertility treatment. For male and female donors, we searched for studies reporting short- and long-term adverse effects and quality-of-life outcomes.

Review methods. Two investigators screened each abstract and full-text article for inclusion; abstracted data; and performed quality ratings, applicability ratings, and evidence grading. Where appropriate, random-effects models were used to compute summary estimates of effects.

Results. We identified a total of 151 studies/primary articles that met our inclusion criteria: 56 for PCOS, 7 for endometriosis, 50 for infertility secondary to unknown causes, 8 for tubal/peritoneal factor infertility, 23 for male factor infertility, and 5 for outcomes in male and female gamete donors. There were also 21 studies that adjusted for cause of infertility but whose findings were relevant across all infertility diagnoses. For women with infertility associated with PCOS, there was moderate strength of evidence (SOE) that letrozole results in higher live birth rates than clomiphene while reducing multiple births and with no difference in ectopic pregnancies (moderate SOE). No differences were seen in low birthweight or time to pregnancy (low SOE). There was moderate SOE that there is no difference between clomiphene and metformin as primary therapy. Comparing laparoscopic ovarian drilling with oral agents, live birth rates were not different (moderate SOE). For couples with unexplained infertility, there is no difference between the oral agents of letrozole and anastrozole for the outcome of ectopic pregnancy (low SOE), but evidence is insufficient for other outcomes of interest. There was also no difference between differing adjunct treatments used in combination with oral agents and intrauterine insemination (IUI) for the outcomes of live birth, miscarriage, and ovarian hyperstimulation syndrome (OHSS) (low SOE for all outcomes). Time to pregnancy was shorter with immediate in vitro fertilization (IVF) compared with strategies that started with clomiphene and IUI or gonadotropins and IUI, followed by IVF if necessary (moderate SOE). For couples with male factor infertility, live birth rate (moderate SOE) and miscarriage (low SOE) did not differ between intracytoplasmic sperm injection (ICSI) and intracytoplasmic morphological sperm injection. (The latter is not used in the United States.) For oocyte donors, studies suggested a lower incidence of OHSS with gonadotropin-releasing hormone ( $\mathrm{GnRH})$ agonist trigger than with human chorionic gonadotropin (hCG) trigger (low SOE). However, there was a lack of evidence on any long-term outcomes. Evidence concerning specific comparisons was 
insufficient for couples with tubal factor or endometriosis infertility. Findings applicable across all indications for infertility for couples undergoing assisted reproductive technology (ART) included lower live birth rates for African-Americans compared with other racial/ethnic groups (low SOE); lower live birth rates but significant reductions in multiple birth rates with elective single-embryo transfer compared with multiple embryo transfer (low SOE); no increase in most maternal cancers after ART treatment after adjustment for infertility in general or specific causes (low SOE); and, for children born after ART, a possible increased risk of neurodevelopmental disorders after ICSI compared with IVF (low SOE).

Conclusions. Although there is evidence supporting some strategies for treatment of infertility, both for specific diagnoses and for couples with any diagnosis, consensus on which outcomes to collect and report, and which areas of uncertainty are most important to resolve, is needed in order to design future studies that will improve the ability of patients and clinicians to make optimal decisions. 


\section{Contents}

Evidence Summary ………………................................................................................................. ES-1

Introduction ........................................................................................................................................... 1

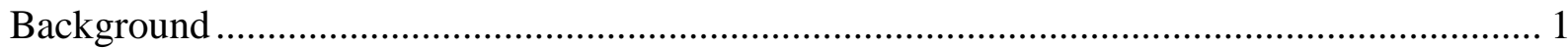

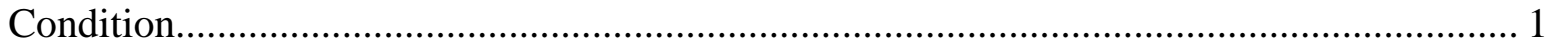

Treatment Strategies ………………………………………………………………….. 1

Complexity of Decision Making for Treatments of Infertility ................................................ 3

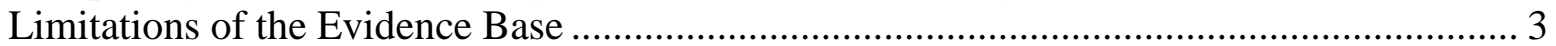

Scope and Key Questions .............................................................................................. 4

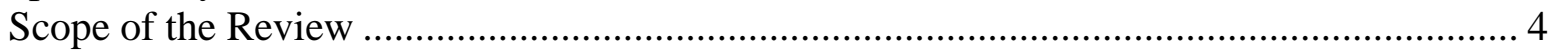

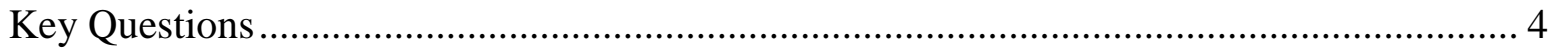

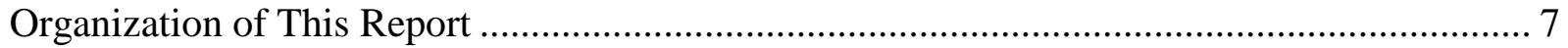

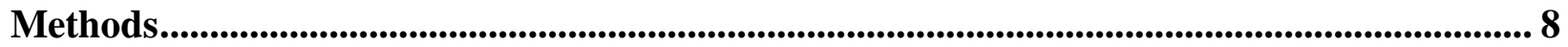

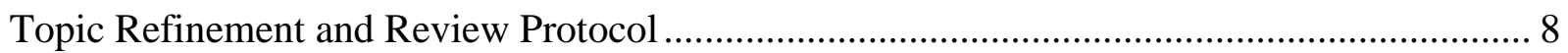

Literature Search Strategy................................................................................................ 8

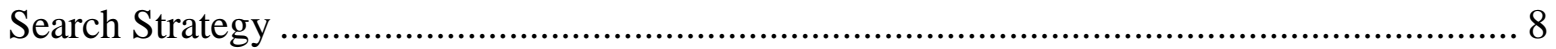

Inclusion and Exclusion Criteria..................................................................................... 9

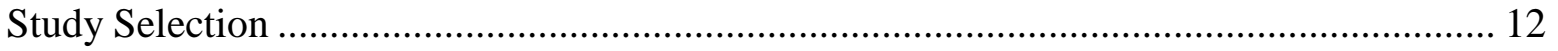

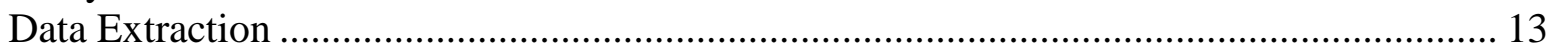

Quality (Risk of Bias) Assessment of Individual Studies.......................................................... 13

Data Synthesis.................................................................................................................. 14

Strength of the Body of Evidence ........................................................................................ 15

Applicability ...................................................................................................................... 16

Peer Review and Public Commentary .................................................................................... 16

Results ................................................................................................................................................... 17

Results of Literature Searches ...................................................................................... 17

Description of Included Studies......................................................................................... 19

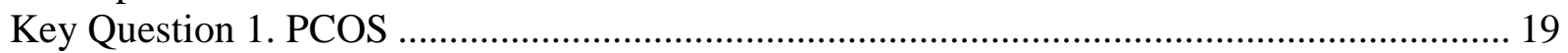

Description of Studies Included for KQ 1 (PCOS) ............................................................. 19

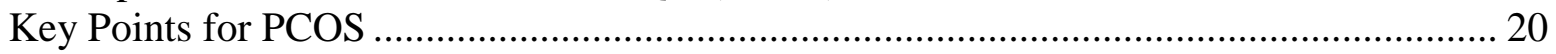

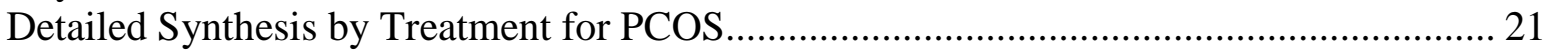

Subgroups of Interest for PCOS .................................................................................... 46

Strength of Evidence for PCOS ...................................................................................... 46

Key Question 2. Endometriosis ............................................................................................. 52

Description of Included Studies for KQ 2 (Endometriosis)................................................. 53

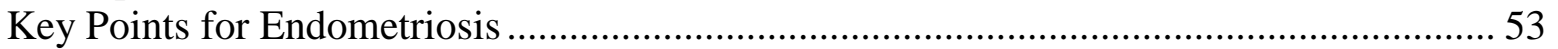

Detailed Synthesis by Treatment for Endometriosis ............................................................... 53

Subpopulations of Interest for Endometriosis....................................................................... 55

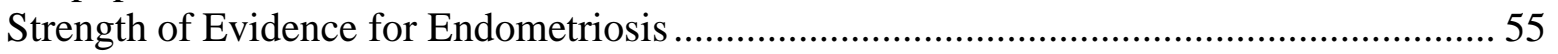

Key Question 3. Unexplained Infertility................................................................................ 57

Description of Included Studies for KQ 3 (Unexplained Infertility) .................................... 57

Key Points for Unexplained Infertility .................................................................................. 57

Detailed Synthesis by Treatment for Unexplained Infertility ................................................. 58

Strength of Evidence for Unexplained Infertility ............................................................. 74 
Key Question 4. Tubal and Peritoneal Factor Infertility......................................................... 77

Description of Included Studies for KQ 4 (Tubal and Peritoneal Factor Infertility)............. 78

Key Points for Tubal or Peritoneal Factor Infertility........................................................... 78

Detailed Synthesis by Treatment for Tubal or Peritoneal Factor Infertility ........................... 78

Strength of Evidence for Tubal and Peritoneal Factor Infertility ........................................... 80

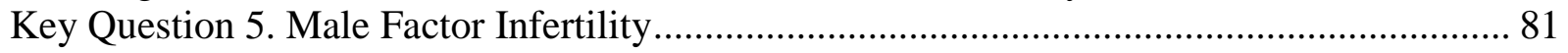

Description of Included Studies for KQ 5 (Male Factor) ……………..................................... 82

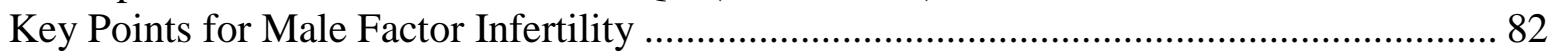

Detailed Synthesis by Treatment for Male Factor Infertility.................................................. 83

Strength of Evidence for Male Factor Infertility .................................................................... 90

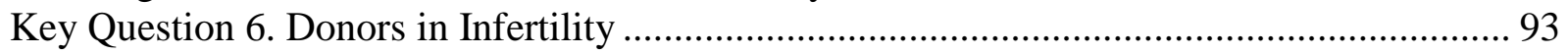

Description of Included Studies for KQ 6 (Donors) ............................................................. 93

Key Points for Donors ............................................................................................................. 93

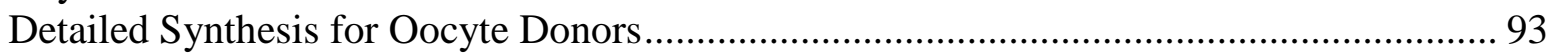

Strength of Evidence for Donors ...................................................................................... 95

Key Findings Across All Infertility Diagnoses........................................................................ 95

Description of Included Studies Across All KQs ………................................................... 95

Key Points for Any Infertility Diagnosis ............................................................................. 96

Subgroups of Interest (With Any Infertility Diagnosis) ......................................................... 99

Strength of Evidence (With Any Infertility Diagnosis) ..................................................... 102

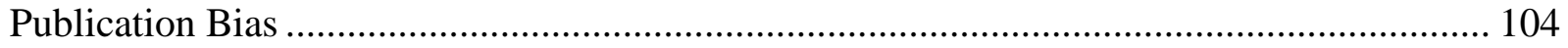

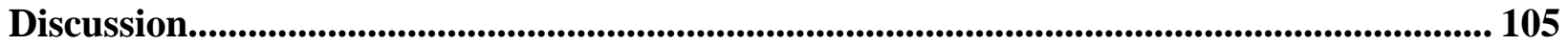

Key Findings and Strength of Evidence ………………..................................................... 105

Findings in Relation to What Is Already Known.................................................................... 106

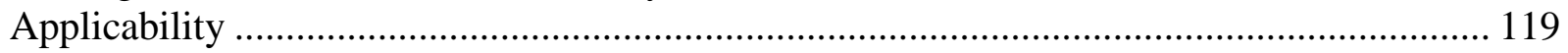

Implications for Clinical and Policy Decision Making............................................................. 121

Limitations of the Systematic Review Process ...................................................................... 122

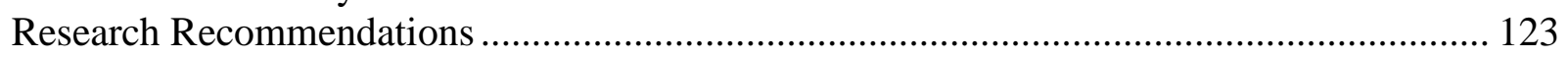

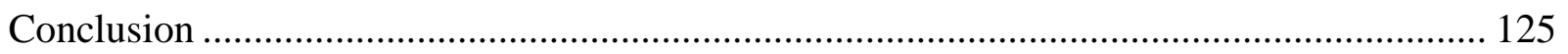

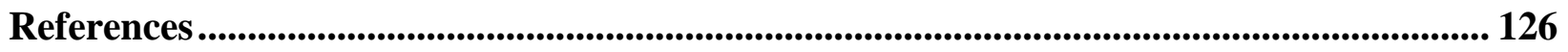

Acronyms and Abbreviations ......................................................................................................... 149

\section{Tables}

Table A. Summary of strength of evidence for major outcomes-KQ 1 (PCOS) .................... ES-9

Table B. Summary of strength of evidence for major outcomes-KQ 2 (endometriosis) ..... ES-12

Table C. Summary of strength of evidence for major outcomes-KQ 3 (unexplained infertility)

Table D. Summary of strength of evidence for major outcomes-KQ 4 (tubal and peritoneal factor infertility)............................................................................................ ES-15

Table E. Summary of strength of evidence for major outcomes-KQ 5 (male factor infertility)

Table F. Summary of strength of evidence for major outcomes-KQ 6 (donors in infertility)

Table G. Summary of strength of evidence for major outcomes - all infertility diagnoses ... ES-19

Table 1. Inclusion and exclusion criteria 


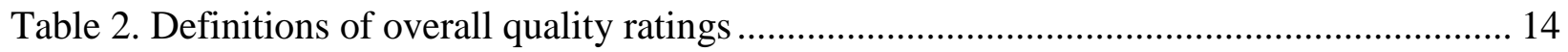

Table 3. Key to primary and companion articles ........................................................................... 17

Table 4. Outcomes for comparisons of oral agents alone in women with PCOS .......................... 24

Table 5. Outcomes for comparisons of oral agents alone versus acupuncture in women with

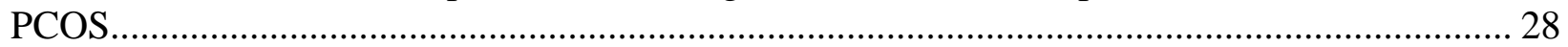

Table 6. Outcomes for oral agents alone versus oral agents with IUI in women with PCOS ...... 29

Table 7. Outcomes for oral agents alone versus surgical management in women with PCOS .... 31

Table 8. Outcomes for oral agents versus gonadotropins in women with PCOS ......................... 34

Table 9. Outcomes for lifestyle intervention versus oral contraceptive pills or no intervention in

women with PCOS............................................................................................................... 36

Table 10. Outcomes for surgical management versus gonadotropins in women with PCOS ...... 37

Table 11. Outcomes for gonadotropins with IUI in women with PCOS ...................................... 38

Table 12. Outcomes for comparisons of IVF treatments in women with PCOS ........................... 41

Table 13. Outcomes for adjuncts to IVF in women with PCOS.............................................. 43

Table 14. Outcomes for fresh versus frozen embryo transfer in IVF in women with PCOS ....... 44

Table 15. Outcomes for comparisons of ICSI treatment in women with PCOS ............................ 45

Table 16. Strength of evidence for major outcomes-KQ 1 (PCOS)............................................. 47

Table 17. Strength of evidence for major outcomes-KQ 2 (endometriosis) ............................... 56

Table 18. Outcomes for comparisons of oral agents without IUI in women with unexplained

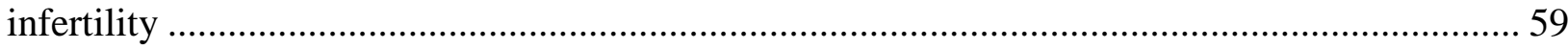

Table 19. Outcomes for comparisons of oral agents versus unstimulated IUI versus expectant management in women with unexplained infertility

Table 20. Outcomes for comparisons of oral agents with IUI in women with unexplained infertility

Table 21. Outcomes for comparisons of oral agents with IUI versus gonadotropins with IUI for

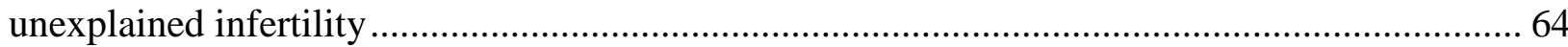

Table 22. Outcomes for comparisons of different treatment strategies for controlled ovarian hyperstimulation with gonadotropins and IUI in women with unexplained infertility ..................66 Table 23. Outcomes for comparisons of immediate IVF versus other treatments prior to IVF in

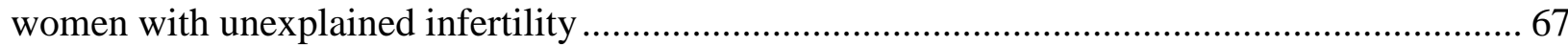

Table 24. Strength of evidence for major outcomes-KQ 3 (unexplained infertility).................. 74 Table 25. Strength of evidence for major outcomes-KQ 4 (tubal and peritoneal factor

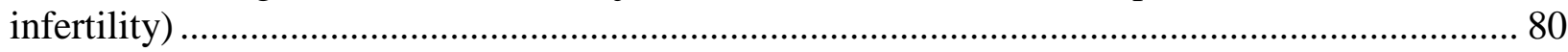

Table 26. Outcomes for comparisons of IVF in couples with male infertility ............................. 84

Table 27. Strength of evidence for major outcomes-KQ 5 (male factor infertility) ................... 90

Table 28. Strength of evidence for major outcomes-KQ 6 (donor) .............................................. 95

Table 29. Cancer risk (95\% CI) for ever use of clomiphene citrate by infertility diagnosis ........ 97

Table 30. Cancer risk (95\% CI) for ever use of gonadotropins by infertility diagnosis................ 97

Table 31. Cancer risk (SIR 95\% CI) among women who underwent assisted reproduction by

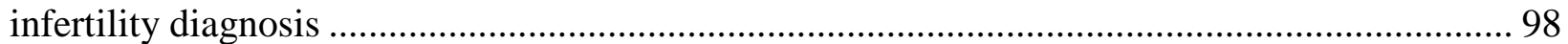

Table 32. Birth rates by number of embryos transferred ............................................................. 100

Table 33. Outcomes with fresh and frozen IVF cycles............................................................... 100

Table 34. Strength of evidence for major outcomes_across all infertility diagnoses ................ 102

Table 35. Report findings and major guidelines/recommendations-KQ 1. PCOS..................... 108

Table 36. Report findings and major guidelines/recommendations-KQ 2, endometriosis ...... 110 
Table 37. Report findings and major guidelines/recommendations-KQ 3, unexplained infertility

Table 38. Report findings and major guidelines/recommendations-KQ 4, tubal and peritoneal factor infertility 112 Table 39. Report findings and major guidelines/recommendations-KQ 5, male factor infertility

Table 40. Report findings and major guidelines/recommendations-KQ 6, donors. 115 Table 41. Report findings and major guidelines/recommendations—all KQs: long-term outcomes of treatments 117

Table 42. Potential issues with applicability of included studies 119

\section{Figures}

Figure A. Analytic framework ES-5

Figure 1. Analytic framework

Figure 2. Literature flow diagram........................................................................................... 18

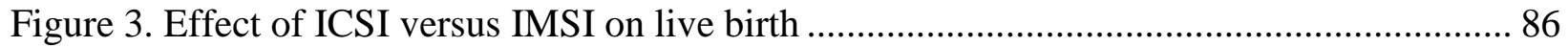

\section{Appendixes}

Appendix A. Exact Search Strings

Appendix B. Data Abstraction Elements

Appendix C. List of Included Studies

Appendix D. List of Excluded Studies

Appendix E. Characteristics of Included Studies

Appendix F. AMSTAR Quality Assessment for Systematic Reviews

Appendix G. Risk of Bias Assessment for Included Studies

Appendix H. Supplemental Project To Assess the Transparency of Reporting for Trials

Evaluating Treatment for Infertility 


\section{Evidence Summary}

\section{Background}

\section{Condition and Therapeutic Strategies}

"Infertility" has traditionally been defined as failure to achieve pregnancy after 12 months of regular unprotected intercourse with the same partner (or after 6 months for women greater than 35 years of age). However, as many as half of such couples will conceive without intervention over the next 12-24 months. Because of this, the term "subfertility" is preferred by many. ${ }^{1}$ From a population perspective, couples who meet the dichotomous criteria for "infertility" include couples who are "normal" but who are in the upper end of the population distribution for "time to pregnancy," and couples who have a physiological or anatomical cause for a prolonged time to pregnancy. However, to be concise, we will use the term "infertility" throughout this report.

Self-reported infertility in the United States, using the 12-month definition, affected approximately 6 percent of married women aged 15-44 in the 2006-2010 National Survey of Family Growth (the most recent available data). ${ }^{2}$ In one population-based study, approximately 10 percent of pregnant women reported receiving infertility treatment, with 29 percent of these women using fertility-enhancing medications; 21 percent using assisted reproductive technology (ART), including in vitro fertilization (IVF); 15 percent using artificial insemination with fertility-enhancing drugs; and 23 percent using other treatments, including surgery. ${ }^{3}$ Other estimates of the prevalence of infertility treatment are similar. ${ }^{4-8}$ Particularly in the United States, where availability of infertility services is variable depending on a number of factors, particularly insurance coverage, utilization of infertility treatments may underestimate the overall burden of infertility.

The most common demographic factor associated with female infertility is "advanced reproductive age," although the probability of pregnancy begins to decline by the mid-20's, the slope of decline sharply increases by age $35 .{ }^{9}$ Other common causes of female infertility include polycystic ovary syndrome (PCOS), endometriosis, occlusion of the fallopian tubes from prior infectious disease, ${ }^{6}$ and infertility secondary to cancer treatment. ${ }^{10-12}$ Isolated male factor infertility affects approximately 17 percent of couples seeking treatment, with 34.6 percent of couples having both male and female diagnoses. ${ }^{13}$

Treatment options are usually dependent on the underlying etiology of infertility. For female causes, options include surgical management of tubal occlusion, surgical treatment of endometriosis, ovarian "drilling" for treatment of PCOS, use of ovulation-induction agents including oral (clomiphene citrate or letrozole) and injected drugs (gonadotropins), artificial insemination with either partner or donor sperm (depending on partner fertility status), and ART, which includes both traditional IVF (fertilization of the egg by the sperm occurs without direct manipulation) and IVF with intra-cytoplasmic sperm injection (ICSI) (fertilization occurs via direct injection of sperm into the egg). ${ }^{14,15}$ Treatment options for male factor infertility include medical treatment of a diagnosed endocrinopathy or other conditions affecting sperm production, empiric treatments with hormonal or other agents, surgical management of varicocele, intrauterine insemination, IVF, ICSI, or use of donor sperm. ${ }^{16}$ Options appropriate for some diagnoses (e.g., ovulation induction in PCOS or unexplained infertility) may not be appropriate for others (e.g., women with documented tubal occlusion). In other cases, the appropriate 
comparisons may involve sequencing or combinations of treatment options-for example, one strategy might consist of several cycles of ovulation induction, followed by ART only if pregnancy does not occur, compared to proceeding directly to ART. Note that throughout this report, we use the term "adjunct treatments" to refer to interventions performed within a major treatment category (for example, comparison of metformin to placebo as pretreatment in women with PCOS undergoing IVF).

Although there has been ongoing debate about the most appropriate outcome for evaluation of infertility treatments, there is a growing consensus that live birth is the most important patientcentered outcome. ${ }^{17,18}$ Trade-offs between outcomes (particularly multiple gestations), time to pregnancy, and out-of-pocket costs might be different between the various treatment strategies even if cumulative live birth rates were identical.

Different treatments also carry different safety risks. There are known short-term risks such as ovarian hyperstimulation syndrome (OHSS) or acute risks associated with any surgery. Surgery may have additional longer-term risks which may affect subsequent fertility (such as scarring or decreased ovarian reserve with procedures such as laparoscopic ovarian drilling (LOD). The literature suggests that observed associations between infertility treatment and female reproductive cancers, particularly ovarian cancer, are likely the result of the underlying infertility rather than treatment itself. There is, however, some uncertainty surrounding some cancer outcomes in subgroups of patients. ${ }^{19-21}$

Some adverse pregnancy outcomes, such as preterm birth, are associated with infertility treatment; however, many of the conditions associated with infertility are also associated with these adverse outcomes, complicating assessment of comparative effectiveness. ${ }^{22-25}$ There may also be direct effects of some treatments that have unclear implications for long-term health in children born after these treatments. ${ }^{26,27}$ Finally, infertility clearly has an emotional impact, ${ }^{12,28,29}$ and the comparative effects of infertility treatments on quality of life are an important consideration for both women and men.

There may be significant variation in outcomes of different treatments in specific subpopulations. For example, age affects the likelihood of conception, and the risk of many pregnancy complications associated with infertility treatments, such as preterm birth or low birthweight, are also increased with higher maternal age. Obesity is common in women with PCOS, and, like older maternal age, is also associated with adverse pregnancy outcomes independent of its association with infertility. The utilization and outcomes of infertility treatment differ among different racial and ethnic groups, even after adjusting for insurance coverage. $^{30-33}$

Finally, a unique subpopulation is women who donate oocytes for use by other couples in ART. There are almost no data on the long-term safety of multiple courses of ovulation induction for the purposes of oocyte donation. ${ }^{34}$ In addition, there are complex ethical and legal considerations, including the balance between fair compensation and inducement, ${ }^{35}$ and sharing information about donors with recipients. ${ }^{36}$

\section{Scope and Key Questions}

This systematic review evaluates the comparative safety and effectiveness of available treatment strategies for women of reproductive age (18-44) who are infertile due to PCOS, endometriosis, unknown reasons, or tubal or peritoneal factors; the comparative safety and effectiveness of available treatment strategies for couples with male factor infertility; and the short- and long-term health outcomes of donors in infertility. 
The specific Key Questions (KQs) addressed in this review are listed below, and Figure A displays the analytic framework that guided our work.

- KQ 1. What are the comparative safety and effectiveness of available treatment strategies for women with polycystic ovary syndrome who are infertile and who wish to become pregnant?

o KQ 1a. Does the optimal treatment strategy vary by patient characteristics such as age, ovarian reserve, race, body mass index (BMI), presence of other potential causes of female infertility, or presence of male factor infertility?

- KQ 2. What are the comparative safety and effectiveness of available treatment strategies for women with endometriosis who are infertile and who wish to become pregnant?

o KQ 2a. Does the optimal treatment strategy vary by patient characteristics such as age, ovarian reserve, race, BMI, stage of endometriosis, presence of other potential causes of female infertility, or presence of male factor infertility?

- KQ 3. What are the comparative safety and effectiveness of available treatment strategies for women who are infertile for unknown reasons and who wish to become pregnant?

o KQ 3a. Does the optimal treatment strategy vary by patient characteristics such as age, ovarian reserve, race, BMI, presence of other potential causes of female infertility, or presence of male factor infertility?

- KQ 4. What are the comparative safety and effectiveness of available treatment strategies for women with tubal or peritoneal factors (e.g., pelvic adhesions) who are infertile and who wish to become pregnant?

$0 \mathrm{KQ} 4 \mathrm{a}$. Does the optimal treatment strategy vary by patient characteristics such as age, ovarian reserve, race, BMI, presence of other potential causes of female infertility, or presence of male factor infertility?

- KQ 5. What are the comparative safety and effectiveness of available treatment strategies for couples with male factor infertility and no evidence of an underlying diagnosis associated with infertility in the female partner?

- KQ 5a. Does the optimal treatment strategy vary by characteristics in either partner such as age, ovarian reserve, race, or BMI?

- KQ 6. What are the short- and long-term health outcomes of donors in infertility? 
o KQ 6a. For female oocyte donors:

1. Do specific aspects of the pre-donation evaluation identify potential donors at greater risk for short- or long-term adverse outcomes (e.g., OHSS, quality-of-life issues)?

2. Do short- and long-term outcomes differ among different stimulation/retrieval protocols?

o KQ 6b. For male semen donors:

- Are there long-term health, quality-of-life, or other adverse outcomes associated with donation? 
Figure A. Analytic framework

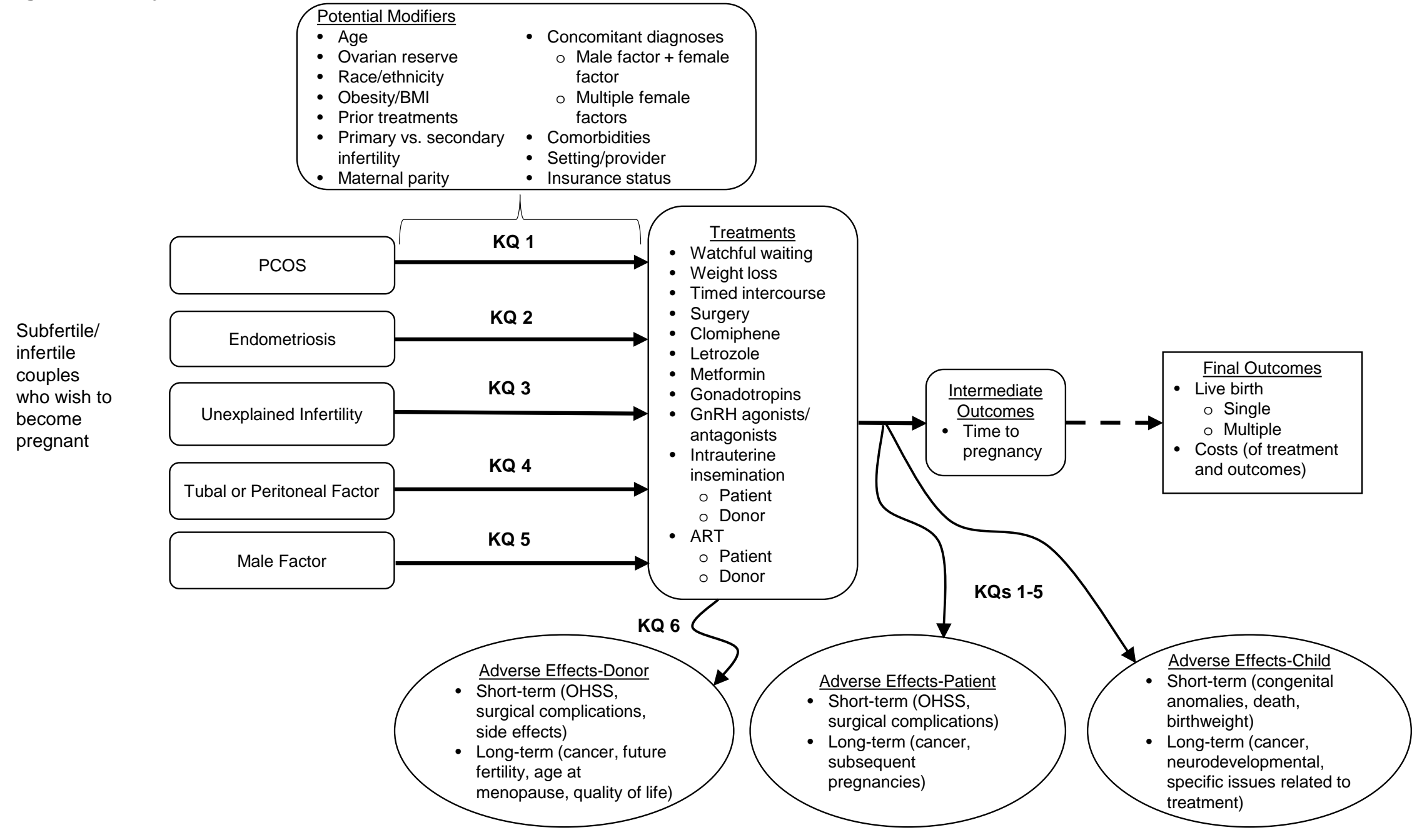

Abbreviations: ART=assisted reproductive technology; BMI=body mass index; GnRH=gonadotropin-releasing hormone; KQ=Key Question; OHSS=ovarian hyperstimulation syndrome; PCOS=polycystic ovary syndrome 


\section{Methods}

Detailed methods are available in the full report and the posted protocol (http://effectivehealthcare.ahrq.gov/index.cfm ).

\section{Literature Search Strategy}

To identify relevant published literature, we searched PubMed ${ }^{\circledR}$, Embase $^{\circledR}$, and the Cochrane Database of Systematic Reviews, limiting the searches to studies conducted in adults and published from January 1, 2007, to October 3, 2018. Selection of the 2007 start date was based on establishing a one-year overlap with the search dates from a previous Agency for Healthcare Research and Quality (AHRQ) evidence report that assessed ART ${ }^{37}$ and input from Key Informants, who felt that the previous AHRQ review and more recent existing Cochrane reviews in this topic area would identify relevant high-quality studies. An experienced search librarian guided all searches. The exact search strings used are given in Appendix A of the full report.

We supplemented the electronic searches with a manual search of citations from a set of key primary and review articles. The reference lists for identified pivotal articles were manually hand-searched and cross-referenced against our database, and additional relevant articles not already under consideration were retrieved for screening. All citations were imported into an electronic bibliographical database (EndNote ${ }^{\circledR}$ Version X7; Thomson Reuters, Philadelphia, PA).

As a mechanism to ascertain publication bias in recent studies, we searched ClinicalTrials.gov to identify completed but unpublished studies (we also explored the possibility of publication bias in any quantitative synthesis of the included literature through meta-analysis techniques).

Approaches to identifying relevant gray literature included notification through the Federal Register to stakeholders, such as drug and device manufacturers, of the opportunity to submit scientific information packets. We also searched the ClinicalTrials.gov study registry and the World Health Organization (WHO) International Clinical Trials Registry Platform (ICTRP) search portal to identify potentially relevant study records, and subsequently searched for relevant articles from completed studies.

We specified our inclusion and exclusion criteria based on the PICOTS (populations, interventions, comparators, outcomes, timing, and settings) identified for each question. For citations retrieved from PubMed, Embase, and the Cochrane Database of Systematic Reviews, two reviewers independently screened each title and abstract for potential relevance to the research questions using prespecified inclusion/exclusion criteria. Articles included by either reviewer underwent full-text screening. Articles meeting eligibility criteria at the full-text stage were included for data abstraction. Based on their clinical and methodological expertise, a pair of researchers were assigned to abstract data from each of the eligible articles. One researcher abstracted the data, and the second over-read the article and the accompanying abstraction to check for accuracy and completeness. Disagreements were resolved by consensus or by obtaining a third reviewer's opinion if consensus could not be reached.

\section{Risk of Bias Assessment of Individual Studies}

We assessed methodological quality, or risk of bias, for each individual study using a components approach, assessing each study for specific aspects of design or conduct (such as allocation concealment for randomized controlled trials (RCTs), or use of methods to address 
potential confounding), as detailed in AHRQ's Methods Guide for Effectiveness and Comparative Effectiveness Reviews. ${ }^{38}$ Briefly, we rated each study as being of good, fair, or poor quality based on its adherence to well-accepted standard methodologies. For each study, one investigator assigned a summary quality rating, which was then reviewed by a second investigator; disagreements were resolved by consensus or by a third investigator if agreement could not be reached.

We also rated quality for identified systematic reviews to provide additional context for the findings of the included studies. Rating was performed using AMSTAR (A Measurement Tool to Assess the Methodological Quality of Systematic Reviews). ${ }^{39}$ For each study, one investigator assigned a summary quality rating, which was then reviewed by a second investigator; disagreements were resolved by consensus or by a third investigator if agreement could not be reached. Reviews were then assigned overall quality scores of good (low risk of bias), fair (moderate risk of bias), or poor (high risk of bias). The consistency of the findings from these systematic reviews were incorporated in to our strength of evidence ratings as described below.

\section{Data Synthesis}

We began by summarizing key features of the included studies for each KQ. To the degree that data are available, we abstracted information on study design; patient characteristics; clinical settings; interventions; and intermediate, final, and adverse event outcomes. If not reported, 95percent confidence intervals for dichotomous outcomes (e.g., live birth rates) were calculated from the numbers provided in the study.

We then determined the feasibility of completing a quantitative synthesis (i.e., meta-analysis, decision analysis, or simulation model). For a meta-analysis, feasibility depends on the volume of relevant literature (requiring at least three relevant studies), conceptual homogeneity of the studies (similar intervention comparisons and outcome definitions), completeness of the reporting of results, and the adequacy and completeness of any existing meta-analyses.

\section{Strength of the Body of Evidence}

We graded the strength of evidence (SOE) for each outcome assessed using the approach described in the AHRQ Methods Guide for Effectiveness and Comparative Effectiveness Reviews. ${ }^{38,40,41}$ We also explored the consistency of our findings with recent systematic reviews and discussed agreement or disagreement, along with possible causes for disagreement and impact on strength of evidence ratings, in the results. A summary rating of high, moderate, or low strength of evidence was assigned for each outcome after discussion by two reviewers. When no evidence was available, or when evidence on the outcome was too weak, sparse, or inconsistent to permit any conclusion to be drawn, a grade of "insufficient" was assigned. This four-level rating scale consisted of the following definitions:

- High Strength of Evidence-We are very confident that the estimate of effect lies close to the true effect for this outcome. The body of evidence has few or no deficiencies. We believe that the findings are stable; i.e., another study would not change the conclusions.

- Moderate Strength of Evidence-We are moderately confident that the estimate of effect lies close to the true effect for this outcome. The body of evidence has some deficiencies. We believe that the findings are likely to be stable, but some doubt remains.

- Low Strength of Evidence-We have limited confidence that the estimate of effect lies close to the true effect for this outcome. The body of evidence has major or numerous 
deficiencies (or both). We believe that additional evidence is needed before concluding either that the findings are stable or that the estimate of effect is close to the true effect.

- Insufficient Strength of Evidence-We have no evidence, we are unable to estimate an effect, or we have no confidence in the estimate of effect for this outcome.

\section{Results}

We briefly summarize the results of our literature searches, description of included studies, key points, and strength of evidence for each KQ. Note we only list here comparisons and outcomes with strength of evidence rated as low, moderate, or high. Full findings are available in the full report.

\section{Summary of Studies}

The literature search yielded 17,391 citations. In total, 1,909 studies were screened in full text, in which 1,748 were excluded for reasons listed in Figure 2 and Appendix D in the full report. We identified 161 articles describing 151 unique studies. The relationship of studies to the review questions is as follows: 56 studies relevant to KQ 1, 7 studies relevant to KQ 2, 50 studies relevant to KQ 3, 8 studies relevant to KQ 4, 23 studies relevant to KQ 5, and 5 studies relevant to KQ 6 (some studies were relevant to more than one KQ). There were also 21 studies relevant to findings across all KQs.

\section{Key Question 1: PCOS}

We identified 61 articles ${ }^{42-102}$ describing 56 studies that addressed the comparative safety and effectiveness of available treatment strategies for infertility in women with PCOS.

Key findings for outcomes in couples where the primary cause of infertility is PCOS include:

- Letrozole has a higher live birth rate than clomiphene citrate alone and lower multiple births, with no difference in ectopic pregnancy, or miscarriage (moderate for all outcomes), low birthweight, or time to pregnancy (low SOE for both these outcomes).

- Clomiphene citrate does not result in higher live birth rates compared with metformin (moderate SOE). Differences are also not found in the rates of multiple birth, ectopic pregnancy, or time to pregnancy (low SOE for all outcomes). There is a higher rate of miscarriage with combination clomiphene and metformin than clomiphene alone (low SOE)

- Letrozole or letrozole and berberine have a higher live birth rate than berberine alone (low SOE) with no difference in multiple births, miscarriage, or low birthweight rates (low SOE)

- There was no difference between clomiphene and tamoxifen for the outcomes of live birth or miscarriage (low SOE)

- There was no difference between laparoscopic ovarian drilling (LOD) and oral agents for live birth (moderate SOE) or miscarriage rates (low SOE). Multiple births were reduced given LOD (moderate SOE).

- Live birth (low SOE) and miscarriage rates (moderate SOE) did not differ between IVF treatment strategies.

- There was no difference in live birth rates for women who underwent lifestyle modification in combination with IVF compared with IVF alone (moderate SOE) 
- There was no difference between type 1 diabetes mellitus diagnoses in children conceived with ART compare to children conceived with no fertility treatment (moderate SOE)

- As with other indications for IVF, use of single-embryo transfer is associated with slightly lower live birth rates but significantly reduced multiple gestation rates (low SOE)

In general, SOE was judged insufficient or low for most outcomes, with the a few exceptions including live births with the use of letrozole versus clomiphene or oral agents versus surgical management, and miscarriage between clomiphene and metformin or oral agents and surgical management which were rated moderate SOE. A common limitation across all comparisons was lack of precision for estimates of rare but important harms such as OHSS or surgical complications (Table A).

Table A. Summary of strength of evidence for major outcomes-KQ 1 (PCOS)

\begin{tabular}{|c|c|c|c|c|}
\hline Comparison & Outcome & $\begin{array}{l}\text { Study Design } \\
\text { (Sample Size) }\end{array}$ & Conclusion & $\begin{array}{l}\text { Strength of } \\
\text { Evidence } \\
\text { (Rationale) }^{\mathrm{a}}\end{array}$ \\
\hline \multirow{4}{*}{$\begin{array}{l}\text { Oral agents } \\
\text { alone: Letrozole } \\
\text { vs. } \\
\text { Berberine vs. } \\
\text { Berberine + } \\
\text { Letrozole }\end{array}$} & $\begin{array}{l}\text { Live birth } \\
\text { (any/patient) }\end{array}$ & $\begin{array}{l}1 \mathrm{RCT}^{81} \\
(644)\end{array}$ & $\begin{array}{l}\text { Improvement: Letrozole or letrozole } \\
\text { and berberine increase live birth rates } \\
\text { compared to berberine alone. }\end{array}$ & $\begin{array}{l}\text { Low } \\
\text { (Imprecise, } 1 \\
\text { study) }\end{array}$ \\
\hline & $\begin{array}{l}\text { Pregnancy } \\
\text { complications: } \\
\text { Multiple births }\end{array}$ & $\begin{array}{l}1 \mathrm{RCT}^{81} \\
(644)\end{array}$ & $\begin{array}{l}\text { No difference: No significant difference } \\
\text { between letrozole, berberine, or } \\
\text { combination therapy }\end{array}$ & $\begin{array}{l}\text { Low } \\
\text { (Imprecise, } 1 \\
\text { study }\end{array}$ \\
\hline & $\begin{array}{l}\text { Pregnancy } \\
\text { complications: } \\
\text { Miscarriage }\end{array}$ & $\begin{array}{l}1 \mathrm{RCT}^{81} \\
(644)\end{array}$ & $\begin{array}{l}\text { No difference: No significant difference } \\
\text { between letrozole, berberine, or } \\
\text { combination therapy }\end{array}$ & $\begin{array}{l}\text { Low } \\
\text { (Imprecise, } 1 \\
\text { study }\end{array}$ \\
\hline & $\begin{array}{l}\text { Neonatal } \\
\text { outcomes: } \\
\text { Birthweight }\end{array}$ & $\begin{array}{l}1 \mathrm{RCT}^{81} \\
(644)\end{array}$ & $\begin{array}{l}\text { No difference: No significant difference } \\
\text { between letrozole, berberine, or } \\
\text { combination therapy }\end{array}$ & $\begin{array}{l}\text { Low } \\
\text { (Imprecise, one } \\
\text { study }\end{array}$ \\
\hline \multirow[t]{5}{*}{$\begin{array}{l}\text { Oral agents } \\
\text { alone: Letrozole } \\
\text { vs. Clomiphene }\end{array}$} & $\begin{array}{l}\text { Live birth } \\
\text { (any/patient) }\end{array}$ & $\begin{array}{l}2 \mathrm{RCTS}^{44,85} \\
(909) \\
1 \text { SR } \\
\text { (9 studies, } 1783 \\
\text { patients) }\end{array}$ & $\begin{array}{l}\text { Improvement: Letrozole has higher } \\
\text { live birth rates than clomiphene. }\end{array}$ & $\begin{array}{l}\text { Moderate } \\
\text { (Imprecise) }\end{array}$ \\
\hline & $\begin{array}{l}\text { Pregnancy } \\
\text { complications: } \\
\text { Multiple births }\end{array}$ & $\begin{array}{l}3 \mathrm{RCTs}^{44,76,85} \\
(886) \\
1 \mathrm{SR} \\
(11 \text { studies, } 2385 \\
\text { patients) }{ }^{103}\end{array}$ & $\begin{array}{l}\text { Improvement: Letrozole has lower } \\
\text { rates of multiple birth compared to } \\
\text { clomiphene }\end{array}$ & $\begin{array}{l}\text { Moderate } \\
\text { (Inconsistent) }\end{array}$ \\
\hline & $\begin{array}{l}\text { Pregnancy } \\
\text { complications: } \\
\text { Ectopic } \\
\text { pregnancy }\end{array}$ & $\begin{array}{l}3 \mathrm{RCTs} \mathrm{s}^{44,76,85} \\
(886)\end{array}$ & $\begin{array}{l}\text { No difference: No difference between } \\
\text { letrozole and clomiphene. }\end{array}$ & $\begin{array}{l}\text { Moderate } \\
\text { (Imprecise) }\end{array}$ \\
\hline & $\begin{array}{l}\text { Pregnancy } \\
\text { complications: } \\
\text { Miscarriage }\end{array}$ & $\begin{array}{l}\text { 3 RCTs } \text { R4,76,85 }^{4} \\
\text { (886) } \\
1 \text { SR } \\
\text { (12 studies, } 2385 \\
\text { patients) }\end{array}$ & $\begin{array}{l}\text { No difference: No statistical difference } \\
\text { between letrozole and clomiphene }\end{array}$ & $\begin{array}{l}\text { Moderate } \\
\text { (Imprecise) }\end{array}$ \\
\hline & $\begin{array}{l}\text { Neonatal } \\
\text { outcomes: } \\
\text { Birthweight }\end{array}$ & $\begin{array}{l}1 \mathrm{RCT}^{44} \\
(750)\end{array}$ & $\begin{array}{l}\text { No difference: No significant difference } \\
\text { in birthweight between letrozole and } \\
\text { clomiphene }\end{array}$ & $\begin{array}{l}\text { Low } \\
\text { (1 study) }\end{array}$ \\
\hline
\end{tabular}




\begin{tabular}{|c|c|c|c|c|}
\hline Comparison & Outcome & $\begin{array}{l}\text { Study Design } \\
\text { (Sample Size) }\end{array}$ & Conclusion & $\begin{array}{l}\text { Strength of } \\
\text { Evidence } \\
\text { (Rationale) }^{\mathrm{a}}\end{array}$ \\
\hline & $\begin{array}{l}\text { Time to } \\
\text { pregnancy }\end{array}$ & $\begin{array}{l}1 \mathrm{RCT}^{44} \\
(750)\end{array}$ & $\begin{array}{l}\text { No difference: No significant difference } \\
\text { in time to pregnancy between } \\
\text { clomiphene vs. letrozole }\end{array}$ & $\begin{array}{l}\text { Low } \\
\text { (1 study) }\end{array}$ \\
\hline \multirow[t]{5}{*}{$\begin{array}{l}\text { Oral agents } \\
\text { alone: } \\
\text { Clomiphene vs. } \\
\text { Metformin vs. } \\
\text { Metformin + } \\
\text { Clomiphene }\end{array}$} & $\begin{array}{l}\text { Live birth } \\
\text { (any/patient) }\end{array}$ & 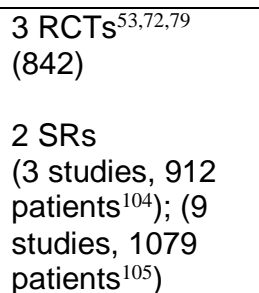 & $\begin{array}{l}\text { No difference: No statistical difference } \\
\text { between clomiphene and metformin or } \\
\text { between clomiphene and combination } \\
\text { therapy of metformin and clomiphene }\end{array}$ & $\begin{array}{l}\text { Moderate } \\
\text { (Suspected } \\
\text { reporting bias) }\end{array}$ \\
\hline & $\begin{array}{l}\text { Pregnancy } \\
\text { complications: } \\
\text { Multiple births }\end{array}$ & $\begin{array}{l}3 \mathrm{RCTS}^{63,70,72} \\
(921) \\
1 \mathrm{SR}^{105} \\
\text { (9 studies, } 1079 \\
\text { patients) }\end{array}$ & $\begin{array}{l}\text { No difference: No differences in } \\
\text { multiple birth rates between clomiphene } \\
\text { alone, metformin alone, and } \\
\text { clomiphene plus metformin }\end{array}$ & $\begin{array}{l}\text { Low } \\
\text { (Imprecise, } \\
\text { suspected } \\
\text { reporting bias) }\end{array}$ \\
\hline & $\begin{array}{l}\text { Pregnancy } \\
\text { complications: } \\
\text { Ectopic } \\
\text { pregnancy }\end{array}$ & $\begin{array}{l}3 \mathrm{RCTs}^{70,72,79} \\
(1,005)\end{array}$ & $\begin{array}{l}\text { No difference: No difference between } \\
\text { studied oral agents. Very few ectopic } \\
\text { pregnancies overall. }\end{array}$ & $\begin{array}{l}\text { Low } \\
\text { (Imprecise } \\
\text { findings with } \\
\text { moderate study } \\
\text { limitations) }\end{array}$ \\
\hline & $\begin{array}{l}\text { Pregnancy } \\
\text { complications: } \\
\text { Miscarriage }\end{array}$ & $\begin{array}{l}3 \mathrm{RCTS}^{63,70,72,79} \\
(817) \\
1 \mathrm{SR}^{105} \\
\text { (9 studies, } 1079 \\
\text { patients) }\end{array}$ & $\begin{array}{l}\text { Increase: Higher rate of miscarriage in } \\
\text { the combined therapy group } \\
\text { (clomiphene and metformin) compared } \\
\text { to clomiphene alone }\end{array}$ & $\begin{array}{l}\text { Low } \\
\text { (Suspected } \\
\text { reporting bias, } \\
\text { imprecise) }\end{array}$ \\
\hline & $\begin{array}{l}\text { Time to } \\
\text { Pregnancy }\end{array}$ & $\begin{array}{l}1 \mathrm{RCT}^{53} \\
(343)\end{array}$ & $\begin{array}{l}\text { No difference: No significant difference } \\
\text { in time to pregnancy between } \\
\text { clomiphene vs. metformin }\end{array}$ & $\begin{array}{l}\text { Low } \\
\text { (1 study) }\end{array}$ \\
\hline \multirow[t]{2}{*}{$\begin{array}{l}\text { Oral agents } \\
\text { alone: } \\
\text { Clomiphene vs. } \\
\text { Tamoxifen }\end{array}$} & $\begin{array}{l}\text { Live birth } \\
\text { (any/patient) }\end{array}$ & $\begin{array}{l}1 \mathrm{RCT}^{99} \\
(88) \\
1 \mathrm{SR}^{106} \\
\text { (2 studies, } 195 \\
\text { women) }\end{array}$ & $\begin{array}{l}\text { No difference: No significant difference } \\
\text { in live birth rates between tamoxifen } \\
\text { and clomiphene }\end{array}$ & $\begin{array}{l}\text { Low } \\
\text { (Imprecise) }\end{array}$ \\
\hline & $\begin{array}{l}\text { Pregnancy } \\
\text { complications: } \\
\text { Miscarriage }\end{array}$ & $\begin{array}{l}1 \mathrm{RCT}^{99} \\
(88) \\
1 \mathrm{SR}^{106} \\
\text { (2 studies, } 195 \\
\text { women) }\end{array}$ & $\begin{array}{l}\text { No difference: No significant difference } \\
\text { in miscarriage rates between tamoxifen } \\
\text { and clomiphene }\end{array}$ & $\begin{array}{l}\text { Low } \\
\text { (limprecise) }\end{array}$ \\
\hline \multirow{3}{*}{$\begin{array}{l}\text { Active } \\
\text { Acupuncture + } \\
\text { Clomiphene vs. } \\
\text { Control } \\
\text { Acupuncture + } \\
\text { Clomiphene vs. } \\
\text { Active } \\
\text { Acupuncture + } \\
\text { Placebo vs. } \\
\text { Control } \\
\text { Acupuncture + } \\
\text { Placebo }\end{array}$} & Live birth & $\begin{array}{l}1 \mathrm{RCT}^{96} \\
(1000)\end{array}$ & $\begin{array}{l}\text { Improvement: Live birth rates } \\
\text { significantly higher for clomiphene vs. } \\
\text { placebo; not significantly different for } \\
\text { active vs. control } \\
\text { Acupuncture }\end{array}$ & $\begin{array}{l}\text { Low } \\
(1 \text { study with } \\
\text { potential risk of } \\
\text { bias) }\end{array}$ \\
\hline & $\begin{array}{l}\text { Pregnancy } \\
\text { complications: } \\
\text { Ectopic } \\
\text { pregnancy }\end{array}$ & $\begin{array}{l}1 \mathrm{RCT}^{96} \\
(1000)\end{array}$ & $\begin{array}{l}\text { No difference: no significant difference } \\
\text { in ectopic pregnancy rates between oral } \\
\text { agents and acupuncture strategies. }\end{array}$ & $\begin{array}{l}\text { Low } \\
\text { (1 study with } \\
\text { potential risk of } \\
\text { bias) }\end{array}$ \\
\hline & $\begin{array}{l}\text { Pregnancy } \\
\text { complications: } \\
\text { Miscarriage }\end{array}$ & $\begin{array}{l}1 \mathrm{RCT}^{96} \\
(1000)\end{array}$ & $\begin{array}{l}\text { No difference: no significant difference } \\
\text { in miscarriage rates between oral } \\
\text { agents and acupuncture strategies. }\end{array}$ & $\begin{array}{l}\text { Low } \\
\text { (1 study with } \\
\text { potential risk of } \\
\text { bias) }\end{array}$ \\
\hline
\end{tabular}




\begin{tabular}{|c|c|c|c|c|}
\hline Comparison & Outcome & $\begin{array}{l}\text { Study Design } \\
\text { (Sample Size) }\end{array}$ & Conclusion & $\begin{array}{l}\text { Strength of } \\
\text { Evidence } \\
\text { (Rationale) }^{\mathrm{a}}\end{array}$ \\
\hline & $\begin{array}{l}\text { Neonatal } \\
\text { outcomes: } \\
\text { Congenital } \\
\text { Abnormalities }\end{array}$ & $\begin{array}{c}1 \mathrm{RCT}^{96} \\
(1000)\end{array}$ & $\begin{array}{l}\text { No difference: no significant difference } \\
\text { in congenital abnormality rates between } \\
\text { oral agents and acupuncture strategies. }\end{array}$ & $\begin{array}{l}\text { Low } \\
\text { ( } 1 \text { study with } \\
\text { potential risk of } \\
\text { bias) }\end{array}$ \\
\hline & $\begin{array}{l}\text { Neonatal } \\
\text { Death }\end{array}$ & $\begin{array}{l}1 \text { RCT }^{96} \\
(1000)\end{array}$ & $\begin{array}{l}\text { No difference: no significant difference } \\
\text { in neonatal death rates between oral } \\
\text { agents and acupuncture strategies. }\end{array}$ & $\begin{array}{l}\text { Low } \\
\text { (1 study with } \\
\text { potential risk of } \\
\text { bias) } \\
\end{array}$ \\
\hline \multirow[t]{3}{*}{$\begin{array}{l}\text { Oral agents } \\
\text { alone vs. LOD }\end{array}$} & $\begin{array}{l}\text { Live birth } \\
\text { (any/patient) }\end{array}$ & $\begin{array}{l}1 \mathrm{SR}^{107} \\
\text { (8 studies, } 1,034 \\
\text { women) }\end{array}$ & $\begin{array}{l}\text { No difference: No statistically } \\
\text { significant differences between LOD } \\
\text { and oral agents }\end{array}$ & $\begin{array}{l}\text { Moderate } \\
\text { (Suspected } \\
\text { reporting bias) }\end{array}$ \\
\hline & $\begin{array}{l}\text { Pregnancy } \\
\text { complications: } \\
\text { Multiple births } \\
\end{array}$ & $\begin{array}{l}1 \mathrm{SR}^{107} \\
\text { (15 studies, } 1,129 \\
\text { women) }\end{array}$ & $\begin{array}{l}\text { Reduction: There was a reduction in } \\
\text { multiple births given LOD as compared } \\
\text { to oral agents }\end{array}$ & $\begin{array}{l}\text { Moderate } \\
\text { (Suspected } \\
\text { reporting bias) }\end{array}$ \\
\hline & $\begin{array}{l}\text { Pregnancy } \\
\text { complications: } \\
\text { Miscarriage }\end{array}$ & $\begin{array}{l}1 \mathrm{RCT}^{97} \\
(80) \\
1 \mathrm{SR}^{107} \\
\text { (15 studies, 1,592 } \\
\text { women) }\end{array}$ & $\begin{array}{l}\text { No difference: No significant } \\
\text { differences in miscarriage between } \\
\text { LOD and oral agents }\end{array}$ & $\begin{array}{l}\text { Low } \\
\text { (Imprecise, } \\
\text { suspected } \\
\text { reporting bias) }\end{array}$ \\
\hline $\begin{array}{l}\text { Clomiphene } \\
\text { citrate vs. low- } \\
\text { dose FSH }\end{array}$ & $\begin{array}{l}\text { Pregnancy } \\
\text { complications: } \\
\text { Ectopic } \\
\text { pregnancy }\end{array}$ & $\begin{array}{l}\text { 3 RCTs } 54,82,95 \\
(1072)\end{array}$ & $\begin{array}{l}\text { No difference: Ectopic pregnancy rate } \\
\text { did not differ between FSH and } \\
\text { clomiphene strategies. }\end{array}$ & $\begin{array}{l}\text { Low } \\
\text { (Imprecise) }\end{array}$ \\
\hline $\begin{array}{l}\text { Lifestyle } \\
\text { modifications + } \\
\text { IVF vs. IVF } \\
\text { alone } \\
\end{array}$ & Live birth & $\begin{array}{l}3 \mathrm{RCTs}^{75,78,87} \\
(1688)\end{array}$ & $\begin{array}{l}\text { No difference: No difference in live } \\
\text { birth rates for women who underwent } \\
\text { lifestyle modification in combination } \\
\text { with IVF compared with IVF alone }\end{array}$ & $\begin{array}{l}\text { Moderate } \\
\text { (Heterogeneity } \\
\text { in interventions) }\end{array}$ \\
\hline \multirow[t]{2}{*}{$\begin{array}{l}\text { ART IVF: GnRH } \\
\text { agonist +/- IVF } \\
\text { vs. GnRH } \\
\text { antagonist +/- } \\
\text { IVF }\end{array}$} & $\begin{array}{l}\text { Live birth } \\
\text { (cycle) }\end{array}$ & $\begin{array}{l}4 \mathrm{RCTs} \mathrm{s}^{48,52,68,71} \\
(408)\end{array}$ & $\begin{array}{l}\text { No difference: No significant difference } \\
\text { in included studies but varying } \\
\text { interventions and comparators with low } \\
\text { numbers of live birth }\end{array}$ & $\begin{array}{l}\text { Low } \\
\text { (Imprecise } \\
\text { findings with } \\
\text { moderate study } \\
\text { limitations) } \\
\end{array}$ \\
\hline & $\begin{array}{l}\text { Pregnancy } \\
\text { complications: } \\
\text { Miscarriage }\end{array}$ & $\begin{array}{l}\text { 3 RCTs }{ }^{68,71,77} \\
(279)\end{array}$ & $\begin{array}{l}\text { No difference: No differences in } \\
\text { miscarriage rates for GnRH agonist vs. } \\
\text { antagonist, or hCG medium, hCG-free } \\
\text { medium with transfer, and hCG-free } \\
\text { medium without transfer. }\end{array}$ & $\begin{array}{l}\text { Moderate } \\
\text { (Imprecise } \\
\text { findings with } \\
\text { moderate study } \\
\text { limitations) } \\
\end{array}$ \\
\hline \multirow{6}{*}{$\begin{array}{l}\text { ART IVF: Fresh } \\
\text { vs. Frozen } \\
\text { Embryos in IVF } \\
\text { for PCOS }\end{array}$} & $\begin{array}{l}\text { Live birth } \\
\text { (any/cycle) }\end{array}$ & $\begin{array}{l}1 \mathrm{RCT}^{80} \\
(1508)\end{array}$ & $\begin{array}{l}\text { Improvement: Live birth rates were } \\
\text { significantly higher with frozen embryo } \\
\text { transfer compared to fresh embryos }\end{array}$ & $\begin{array}{l}\text { Low } \\
\text { (1 study) }\end{array}$ \\
\hline & $\begin{array}{l}\text { Pregnancy } \\
\text { complications: } \\
\text { Multiple births } \\
\end{array}$ & $\begin{array}{l}1 \mathrm{RCT}^{80} \\
(1508)\end{array}$ & $\begin{array}{l}\text { No difference: No difference in multiple } \\
\text { births with fresh versus frozen embryo } \\
\text { transfer }\end{array}$ & $\begin{array}{l}\text { Low } \\
\text { (1 study) }\end{array}$ \\
\hline & $\begin{array}{l}\text { Pregnancy } \\
\text { complications: } \\
\text { Ectopic } \\
\text { pregnancy }\end{array}$ & $\begin{array}{l}1 \mathrm{RCT}^{80} \\
(1508)\end{array}$ & $\begin{array}{l}\text { Reduction: Ectopic pregnancies were } \\
\text { reduced with frozen embryo transfer }\end{array}$ & $\begin{array}{l}\text { Low } \\
\text { (1 study) }\end{array}$ \\
\hline & $\begin{array}{l}\text { Pregnancy } \\
\text { complications: } \\
\text { Miscarriage }\end{array}$ & $\begin{array}{l}1 \mathrm{RCT}^{80} \\
(1508)\end{array}$ & $\begin{array}{l}\text { Reduction: Miscarriages were reduced } \\
\text { with frozen embryo transfer }\end{array}$ & $\begin{array}{l}\text { Low } \\
\text { (1 study) }\end{array}$ \\
\hline & $\begin{array}{l}\text { Neonatal } \\
\text { Outcomes: } \\
\text { Congenital } \\
\text { abnormalities }\end{array}$ & $\begin{array}{l}1 \mathrm{RCT}^{80} \\
(1508)\end{array}$ & $\begin{array}{l}\text { No difference: No difference } \\
\text { congenital abnormalities with fresh } \\
\text { versus frozen embryo transfer }\end{array}$ & $\begin{array}{l}\text { Low } \\
\text { (1 study) }\end{array}$ \\
\hline & $\begin{array}{l}\text { Neonatal } \\
\text { Death }\end{array}$ & $\begin{array}{l}1 \mathrm{RCT}^{80} \\
(1508)\end{array}$ & $\begin{array}{l}\text { No difference: No difference neonatal } \\
\text { deaths with fresh versus frozen embryo } \\
\text { transfer }\end{array}$ & $\begin{array}{l}\text { Low } \\
\text { (1 study) }\end{array}$ \\
\hline
\end{tabular}




\begin{tabular}{|l|l|l|l|l|}
\hline \multicolumn{1}{|c|}{ Comparison } & \multicolumn{1}{|c|}{ Outcome } & \multicolumn{1}{|c|}{$\begin{array}{c}\text { Study Design } \\
\text { (Sample Size) }\end{array}$} & \multicolumn{1}{c|}{$\begin{array}{c}\text { Conclusion } \\
\text { Evidence } \\
\text { (Rationale) }\end{array}$} \\
\hline $\begin{array}{l}\text { ART vs. no } \\
\text { infertility } \\
\text { treatment }\end{array}$ & $\begin{array}{l}\text { Long-term } \\
\text { outcomes: } \\
\text { Child (type 1 } \\
\text { diabetes } \\
\text { mellitus) }\end{array}$ & $\begin{array}{l}1 \text { Obs }^{90} \\
(565,116 \\
\text { pregnancies) }\end{array}$ & $\begin{array}{l}\text { No difference: No significant difference } \\
\text { found between type 1 diabetes mellitus } \\
\text { diagnoses in children born to patients } \\
\text { with PCOS infertility conceived with } \\
\text { ART compared to children conceived } \\
\text { with no fertility treatment }\end{array}$ & $\begin{array}{l}\text { Moderate } \\
\text { (Imprecise) }\end{array}$ \\
\hline
\end{tabular}

${ }^{a}$ Criteria for downgrading strength of evidence is described as Rationale; when these criteria are insufficient for understanding the final strength of evidence, additional explanation is provided.

Abbreviations: ART=assisted reproductive technology; GnRH=gonadotropin-releasing hormone; hCG=human chorionic gonadotropin; IVF=in vitro fertilization; KQ=Key Question; LOD=laparoscopic ovarian drilling/diathermy; Obs=observational study; PCOS=polycystic ovary syndrome; RCT=randomized controlled trial; SR=systematic review

\section{Key Question 2. Endometriosis}

We identified seven individual studies that addressed infertility treatment for women with endometriosis. ${ }^{91,92,108-112}$

Key findings for couples where the primary cause of infertility is endometriosis in the female partner included:

- As with other indications for IVF, use of single-embryo transfer is associated with slightly lower live birth rates but significantly reduced multiple gestation rates (low SOE)

- The live birth rate per cycle was higher in couples who underwent ART than those who used intrauterine insemination (IUI) (low SOE)

- SOE was rated insufficient for all other comparisons/outcomes.

In general, the SOE across all outcomes was judged to be insufficient or low, primarily due to imprecision and small numbers of studies, especially for both short-term harms (such as OHSS) (Table B).

Table B. Summary of strength of evidence for major outcomes-KQ 2 (endometriosis)

\begin{tabular}{|c|c|c|c|c|}
\hline Comparison & Outcome & $\begin{array}{l}\text { Study Design } \\
\text { (Sample Size) }\end{array}$ & Conclusion & $\begin{array}{l}\text { Strength of } \\
\text { Evidence } \\
\text { (Rationale) }^{\mathrm{a}}\end{array}$ \\
\hline $\begin{array}{l}\text { ART: IVF/ICSI } \\
\text { vs. no } \\
\text { treatment }\end{array}$ & Live birth & $\begin{array}{l}1 \text { Obs }^{111} \\
(69,028 \text { cycles })\end{array}$ & $\begin{array}{l}\text { Improvement: For women with } \\
\text { endometriosis, the live birth rate per } \\
\text { cycle was higher in couples who } \\
\text { underwent } 2 \text { embryo transfer } \\
(51.5 \%) \text { as compared with single } \\
\text { embryo transfer }(46.6 \%) \\
(p<0.0001) \text {. }\end{array}$ & $\begin{array}{l}\text { Low } \\
\text { (Imprecise) }\end{array}$ \\
\hline IUI vs. ART & Live birth & $\begin{array}{l}1 \text { Obs }^{92} \\
(19,884)\end{array}$ & $\begin{array}{l}\text { Improvement: For women with } \\
\text { endometriosis, the live birth rate per } \\
\text { cycle was higher in couples who } \\
\text { underwent ART than those who } \\
\text { used IUI }\end{array}$ & $\begin{array}{l}\text { Low } \\
\text { (1 study) }\end{array}$ \\
\hline
\end{tabular}

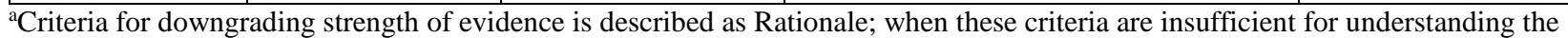
final strength of evidence, additional explanation is provided.

Abbreviations: ART=assisted reproductive technology; ICSI=intra-cytoplasmic sperm injection; IUI=intrauterine insemination; IVF=in vitro fertilization; KQ=Key Question; Obs=observational study 


\section{Key Question 3. Unexplained Infertility}

We identified 50 individual studies that met inclusion criteria for KQ 3 and had unexplained infertility (infertility with no other documented female or male diagnosis). ${ }^{75,91,92,111,113-158}$

Key findings for couples with unexplained infertility included:

- There is no difference between the oral agents of letrozole and anastrozole for the outcome of ectopic pregnancy (low SOE) but evidence is insufficient for other outcomes of interest.

- There is no difference between letrozole and clomiphene for outcomes of multiple births or miscarriage (moderate SOE).

- There is no difference between differing adjunct treatments used in combination with oral agents and IUI for the outcomes of live birth, miscarriage, and OHSS (low SOE for all outcomes).

- There are no differences between immediate IVF versus other treatments prior to IVF for the outcomes of live birth, multiple births, ectopic pregnancy, miscarriage, low birthweight, and OHSS (low SOE for all outcomes). There is however shorter time to pregnancy with immediate IVF (moderate SOE).

- As with other indications for IVF, use of single-embryo transfer is associated with slightly lower live birth rates but significantly reduced multiple gestation rates (low SOE)

SOE for most outcomes was judged to be insufficient or low, primarily because of imprecision or small numbers of studies of fair quality. Two exceptions were multiple births and miscarriages for oral agents without IUI where an existing systematic review existed, and time to pregnancy between different strategies for sequencing treatment, where precision was reasonable. In both cases SOE for these outcomes was judged to be moderate (Table C).

Table C. Summary of strength of evidence for major outcomes-KQ 3 (unexplained infertility)

\begin{tabular}{|c|c|c|c|c|}
\hline Comparison & Outcome & $\begin{array}{l}\text { Study Design } \\
\text { (Sample Size) }\end{array}$ & Conclusion & $\begin{array}{l}\text { Strength of } \\
\text { Evidence } \\
\text { (Rationale) }^{\mathrm{a}}\end{array}$ \\
\hline \multirow[t]{3}{*}{$\begin{array}{l}\text { Oral Agents } \\
\text { Without IUI }\end{array}$} & $\begin{array}{l}\text { Pregnancy } \\
\text { complications: } \\
\text { Ectopic } \\
\text { pregnancy }\end{array}$ & $\begin{array}{l}2 \mathrm{RCTs}^{132,157} \\
(1,168)\end{array}$ & $\begin{array}{l}\text { No difference: No difference } \\
\text { between letrozole and anastrozole: }\end{array}$ & $\begin{array}{l}\text { Low } \\
\text { (Moderate } \\
\text { study } \\
\text { limitations) }\end{array}$ \\
\hline & $\begin{array}{l}\text { Pregnancy } \\
\text { complications: } \\
\text { Multiple births }\end{array}$ & $\begin{array}{l}1 \mathrm{SR}^{159} \\
\text { (5 studies, } 395 \\
\text { patients) }\end{array}$ & $\begin{array}{l}\text { No difference: No difference } \\
\text { between letrozole and clomiphene } \\
\text { citrate }\end{array}$ & Moderate \\
\hline & $\begin{array}{l}\text { Pregnancy } \\
\text { complications: } \\
\text { Miscarriage }\end{array}$ & $\begin{array}{l}3 \text { RCTs }^{113,132,157} \\
(1,248) \\
1 \text { SR }^{159} \\
\text { (5 studies, } 395 \\
\text { patients) }\end{array}$ & $\begin{array}{l}\text { No difference: No difference } \\
\text { between letrozole and clomiphene } \\
\text { citrate }\end{array}$ & Moderate \\
\hline \multirow[t]{2}{*}{$\begin{array}{l}\text { Clomiphene } \\
\text { Citrate vs. } \\
\text { Expectant } \\
\text { Management }\end{array}$} & $\begin{array}{l}\text { Pregnancy } \\
\text { complications: } \\
\text { Ectopic } \\
\text { Pregnancy }\end{array}$ & $\begin{array}{l}2 \mathrm{RCTs}^{136,149} \\
(781)\end{array}$ & $\begin{array}{l}\text { No difference: No significant } \\
\text { difference in ectopic pregnancy rates } \\
\text { between clomiphene and expectant } \\
\text { management }\end{array}$ & $\begin{array}{l}\text { Low } \\
\text { (Imprecise, } \\
\text { heterogeneous } \\
\text { interventions) }\end{array}$ \\
\hline & $\begin{array}{l}\text { Pregnancy } \\
\text { complications: } \\
\text { Miscarriage }\end{array}$ & $\begin{array}{l}2 \mathrm{RCTs}^{136,149} \\
(781)\end{array}$ & $\begin{array}{l}\text { No difference: No significant } \\
\text { difference in ectopic pregnancy rates } \\
\text { between clomiphene and expectant } \\
\text { management }\end{array}$ & $\begin{array}{l}\text { Low } \\
\text { (Imprecise, } \\
\text { heterogeneous } \\
\text { interventions) }\end{array}$ \\
\hline
\end{tabular}




\begin{tabular}{|c|c|c|c|c|}
\hline Comparison & Outcome & $\begin{array}{l}\text { Study Design } \\
\text { (Sample Size) }\end{array}$ & Conclusion & $\begin{array}{l}\text { Strength of } \\
\text { Evidence } \\
\text { (Rationale) }\end{array}$ \\
\hline $\begin{array}{l}\text { Oral Agents vs. } \\
\text { Unstimulated } \\
\text { IUI vs. } \\
\text { Expectant } \\
\text { Management }\end{array}$ & Live birth & $\begin{array}{l}\text { 1 SR }{ }^{160} \\
\text { (3 studies, 370) }\end{array}$ & $\begin{array}{l}\text { Improvement: A significant increase } \\
\text { in live births was found for women } \\
\text { treated with IUI and ovarian } \\
\text { hyperstimulation compared to women } \\
\text { treated with IUI only }\end{array}$ & $\begin{array}{l}\text { Low } \\
\text { (Inconsistent) }\end{array}$ \\
\hline \multirow[t]{3}{*}{$\begin{array}{l}\text { Adjunct } \\
\text { Treatments with } \\
\text { Oral Agents } \\
\text { and IUI }\end{array}$} & Live birth & $\begin{array}{l}5 \\
\text { RCTs } 124,130,140,153,156 \\
(1859)\end{array}$ & $\begin{array}{l}\text { No difference: No difference } \\
\text { between adjunct treatments with oral } \\
\text { agents and IUI }\end{array}$ & $\begin{array}{l}\text { Low } \\
\text { (Moderate } \\
\text { study } \\
\text { limitations) } \\
\end{array}$ \\
\hline & $\begin{array}{l}\text { Pregnancy } \\
\text { complications: } \\
\text { Miscarriage }\end{array}$ & $\begin{array}{l}5 \\
\text { RCTs } 130,138,142,143,156 \\
(1859)\end{array}$ & $\begin{array}{l}\text { No difference: No difference } \\
\text { between adjunct treatments with oral } \\
\text { agents and IUI }\end{array}$ & $\begin{array}{l}\text { Low } \\
\text { (Moderate } \\
\text { study } \\
\text { limitations) } \\
\end{array}$ \\
\hline & $\begin{array}{l}\text { Short term } \\
\text { adverse effects } \\
\text { of treatment: } \\
\text { OHSS }\end{array}$ & $\begin{array}{l}\text { 3 RCTs } 124,138,156 \\
(1189)\end{array}$ & $\begin{array}{l}\text { No difference: No difference } \\
\text { between adjunct treatments with oral } \\
\text { agents and IUI }\end{array}$ & $\begin{array}{l}\text { Low } \\
\text { (Moderate } \\
\text { study } \\
\text { limitations) } \\
\end{array}$ \\
\hline \multirow{2}{*}{$\begin{array}{l}\text { Oral Agents } \\
\text { With IUI vs. } \\
\text { Gonadotropins } \\
\text { With IUI }\end{array}$} & $\begin{array}{l}\text { Pregnancy } \\
\text { complications: } \\
\text { Miscarriage }\end{array}$ & $\begin{array}{l}3 \text { RCTs }^{144,152,155} \\
(1,654)\end{array}$ & $\begin{array}{l}\text { No difference: No difference } \\
\text { between oral agents with IUI versus } \\
\text { gonadotropins with IUI }\end{array}$ & $\begin{array}{l}\text { Low } \\
\text { (Imprecise) }\end{array}$ \\
\hline & $\begin{array}{l}\text { Pregnancy } \\
\text { complications: } \\
\text { Multiple births }\end{array}$ & $\begin{array}{l}1 \mathrm{RCT}^{144} \\
(742)\end{array}$ & $\begin{array}{l}\text { Increased risk: Greater multiple } \\
\text { gestations with gonadotropins } \\
\text { compared to either clomiphene or } \\
\text { letrozole }\end{array}$ & $\begin{array}{l}\text { Low } \\
\text { (one study) }\end{array}$ \\
\hline \multirow{7}{*}{$\begin{array}{l}\text { Immediate IVF } \\
\text { vs. Other } \\
\text { Treatments } \\
\text { Prior to IVF }\end{array}$} & Live birth & $\begin{array}{l}3 \mathrm{RCTS}^{118,120,131,151} \\
(812)\end{array}$ & $\begin{array}{l}\text { No difference: Live birth does not } \\
\text { differ between differing strategies of } \\
\text { other treatments prior to IVF }\end{array}$ & $\begin{array}{l}\text { Low } \\
\text { (Imprecise) }\end{array}$ \\
\hline & $\begin{array}{l}\text { Pregnancy } \\
\text { complications: } \\
\text { Multiple births }\end{array}$ & $\begin{array}{l}2 \mathrm{RCTs}^{118,131} \\
(657)\end{array}$ & $\begin{array}{l}\text { No difference: No significant } \\
\text { difference between other treatments } \\
\text { prior to IVF and immediate IVF. }\end{array}$ & $\begin{array}{l}\text { Low } \\
\text { (Imprecise) }\end{array}$ \\
\hline & $\begin{array}{l}\text { Pregnancy } \\
\text { complications: } \\
\text { Ectopic } \\
\text { pregnancy }\end{array}$ & $\begin{array}{l}3 \mathrm{RCTS}^{118,120,131,151} \\
(812)\end{array}$ & $\begin{array}{l}\text { No difference: No significant } \\
\text { difference between other treatments } \\
\text { prior to IVF and immediate IVF. }\end{array}$ & $\begin{array}{l}\text { Low } \\
\text { (Imprecise) }\end{array}$ \\
\hline & $\begin{array}{l}\text { Pregnancy } \\
\text { complications: } \\
\text { Miscarriage }\end{array}$ & $\begin{array}{l}3 \mathrm{RCTS}^{118,120,131,151} \\
(812)\end{array}$ & $\begin{array}{l}\text { No difference: No significant } \\
\text { difference between other treatments } \\
\text { prior to IVF and immediate IVF. }\end{array}$ & $\begin{array}{l}\text { Low } \\
\text { (Imprecise) }\end{array}$ \\
\hline & $\begin{array}{l}\text { Neonatal } \\
\text { outcomes: } \\
\text { Birthweight }\end{array}$ & $\begin{array}{l}2 \mathrm{RCTS}^{118,131} \\
(657)\end{array}$ & $\begin{array}{l}\text { No difference: No significant } \\
\text { difference between other treatments } \\
\text { prior to IVF and immediate IVF. }\end{array}$ & $\begin{array}{l}\text { Low } \\
\text { (Imprecise) }\end{array}$ \\
\hline & $\begin{array}{l}\text { Time to } \\
\text { pregnancy }\end{array}$ & $\begin{array}{l}\text { 2 RCTs } 118,131 \\
(657)\end{array}$ & $\begin{array}{l}\text { Reduction: Shorter time to } \\
\text { pregnancy with immediate IVF } \\
\text { compared with other treatments prior } \\
\text { to IVF }\end{array}$ & Moderate \\
\hline & $\begin{array}{l}\text { Short term } \\
\text { adverse effects } \\
\text { of treatment: } \\
\text { OHSS }\end{array}$ & $\begin{array}{l}2 \mathrm{RCTs}^{118,131} \\
(657)\end{array}$ & $\begin{array}{l}\text { No difference: No significant } \\
\text { difference between other treatments } \\
\text { prior to IVF and immediate IVF. }\end{array}$ & $\begin{array}{l}\text { Low } \\
\text { (Imprecise) }\end{array}$ \\
\hline $\begin{array}{l}\text { ART: IVF vs. } \\
\text { ICSI }\end{array}$ & $\begin{array}{l}\text { Neonatal } \\
\text { outcomes: Birth } \\
\text { weight }\end{array}$ & $\begin{array}{l}1 \text { Obs }^{91} \\
(90,401 \text { cycles })\end{array}$ & $\begin{array}{l}\text { No difference: No significant } \\
\text { differences in rates of } r \text { low birth } \\
\text { weight between ICSI versus } \\
\text { conventional-IVF cycles }\end{array}$ & $\begin{array}{l}\text { Low } \\
\text { (1 study with } \\
\text { moderate } \\
\text { study } \\
\text { limitations) } \\
\end{array}$ \\
\hline $\begin{array}{l}\text { ART: } \\
\text { Unspecified }\end{array}$ & $\begin{array}{l}\text { Long-term } \\
\text { outcomes: Child } \\
\text { (cancer) }\end{array}$ & $\begin{array}{l}1 \mathrm{Obs}^{121} \\
(33,840)\end{array}$ & $\begin{array}{l}\text { No difference: The overall cancer } \\
\text { incidence was not elevated in } \\
\text { children born after assisted } \\
\text { conception for unexplained infertility. }\end{array}$ & $\begin{array}{l}\text { Low } \\
\text { (Moderate } \\
\text { study } \\
\text { limitations) } \\
\end{array}$ \\
\hline
\end{tabular}


${ }^{\mathrm{a}}$ Criteria for downgrading strength of evidence is described as Rationale; when these criteria are insufficient for understanding the final strength of evidence, additional explanation is provided.

Abbreviations: ART=assisted reproductive technology; ICSI=intra-cytoplasmic sperm injection; IUI=intrauterine insemination; IVF=in vitro fertilization; KQ=Key Question; Obs=observational study; RCT=randomized controlled trial

\section{Key Question 4. Tubal and Peritoneal Factor Infertility}

We identified eight individual studies ${ }^{90,91,111,161-165}$ that addressed outcomes after treatment for tubal or peritoneal factor infertility.

Key findings for patients with tubal or peritoneal factor infertility included:

- As with other indications for IVF, use of single-embryo transfer is associated with slightly lower live birth rates but significantly reduced multiple gestation rates (low SOE)

- The live birth rate was lower in women undergoing ICSI as compared to conventional IVF (low SOE)

- There was no difference between type 1 diabetes mellitus diagnoses in children born to patients with tubal factor infertility conceived with ART compared to children conceived with no fertility treatment (moderate SOE)

- SOE was rated insufficient for all other comparisons/outcomes.

The SOE was judged to be insufficient for most outcomes primarily due to imprecision based on few studies meeting our inclusion criteria (Table D).

Table D. Summary of strength of evidence for major outcomes-KQ 4 (tubal and peritoneal factor infertility)

\begin{tabular}{|c|c|c|c|c|}
\hline Comparison & Outcome & $\begin{array}{c}\text { Study Design } \\
\text { and Sample Size }\end{array}$ & Conclusion & $\begin{array}{l}\text { Strength of } \\
\text { Evidence } \\
\text { (Rationale) }^{\mathrm{a}} \\
\end{array}$ \\
\hline $\begin{array}{l}\text { ART: 2-embryo } \\
\text { transfer vs. 1- } \\
\text { embryo transfer }\end{array}$ & Live birth (patient) & $\begin{array}{l}1 \text { Obs }^{111} \\
(69,028 \text { cycles })\end{array}$ & $\begin{array}{l}\text { Improvement. The live birth } \\
\text { rate per cycle was higher in } \\
\text { couples who underwent } 2 \\
\text { embryo transfer as compared } \\
\text { with single embryo transfer }\end{array}$ & $\begin{array}{l}\text { Low } \\
\text { (Imprecise) }\end{array}$ \\
\hline $\begin{array}{l}\text { ART: IVF+ICSI } \\
\text { vs. IVF }\end{array}$ & $\begin{array}{l}\text { Neonatal } \\
\text { outcomes: Birth } \\
\text { weight }\end{array}$ & $\begin{array}{l}1 \text { Obs }^{91} \\
(90,401 \text { cycles })\end{array}$ & $\begin{array}{l}\text { No difference: No significant } \\
\text { differences in rates of } r \text { low } \\
\text { birth weight between ICSI } \\
\text { versus conventional-IVF } \\
\text { cycles }\end{array}$ & $\begin{array}{l}\text { Low } \\
\text { (1 study with } \\
\text { moderate study } \\
\text { limitations) }\end{array}$ \\
\hline $\begin{array}{l}\text { ART vs. no } \\
\text { fertility treatment }\end{array}$ & $\begin{array}{l}\text { Long-term } \\
\text { outcomes: Child } \\
\text { (type } 1 \text { diabetes } \\
\text { mellitus) }\end{array}$ & $\begin{array}{l}1 \text { Obs }^{90} \\
(565,116 \\
\text { pregnancies) }\end{array}$ & $\begin{array}{l}\text { No difference: No significant } \\
\text { difference found between } \\
\text { type } 1 \text { diabetes mellitus } \\
\text { diagnoses in children born to } \\
\text { patients with tubal factor } \\
\text { infertility conceived with ART } \\
\text { compared to children } \\
\text { conceived with no fertility } \\
\text { treatment }\end{array}$ & $\begin{array}{l}\text { Moderate } \\
\text { (Imprecise) }\end{array}$ \\
\hline
\end{tabular}

${ }^{a}$ Criteria for downgrading strength of evidence is described as Rationale; when these criteria are insufficient for understanding the final strength of evidence, additional explanation is provided.

Abbreviations: ART=assisted reproductive technology; ICSI=intra-cytoplasmic sperm injection; IVF=in vitro fertilization; $\mathrm{KQ}=$ Key Question; Obs=observational study 


\section{Key Question 5. Male Factor Infertility}

We identified 23 individual studies ${ }^{75,90-92,111,115,121,130,147,153,166-179}$ that addressed the comparative effectiveness or safety of interventions applied to patients with male factor infertility.

Key findings for patients with male factor infertility included:

- Live birth rate (moderate SOE) and miscarriage (low SOE) did not differ between intracytoplasmic sperm injection (ICSI) and intracytoplasmic morphological sperm injection (IMSI). Of note, IMSI is not used in the United States.

- There was no difference in live birth rates or any adverse pregnancy events between couples using frozen embryo versus fresh embryo transfer (low SOE)

- The overall cancer incidence was not elevated in children born after assisted conception for male factor infertility (low SOE).

- There was no difference between type 1 diabetes mellitus diagnoses in children born to patients with male factor infertility conceived with ART compared to children conceived with no fertility treatment (moderate SOE)

- Live birth rate (low SOE) improved with vitamin E or zinc supplementation relative to placebo or no supplementation.

- As with other indications for IVF, use of single-embryo transfer is associated with slightly lower live birth rates but significantly reduced multiple gestation rates (low SOE)

The SOE was judged to be insufficient or low for all outcomes except for the comparison of IVF versus ICSI for live birth and long term outcomes related to diabetes (Table E).

Table E. Summary of strength of evidence for major outcomes-KQ 5 (male factor infertility)

\begin{tabular}{|c|c|c|c|c|}
\hline Comparison & Outcome & $\begin{array}{c}\text { Study Design } \\
\text { and Sample Size }\end{array}$ & Conclusion & $\begin{array}{l}\text { Strength of } \\
\text { Evidence } \\
\text { (Rationale) }^{\mathrm{a}}\end{array}$ \\
\hline $\begin{array}{l}\text { ART IVF: ICSI or } \\
\text { assisted hatching } \\
\text { (1 embryo } \\
\text { transferred) vs. } \\
\text { ICSI or assisted } \\
\text { hatching (multiple } \\
\text { embryos } \\
\text { transferred) } \\
\text { TESE vs. } \\
\text { ejaculated OAT }\end{array}$ & Live birth & $\begin{array}{l}2 \text { Obs }^{111,171} \\
\text { (272,717 cycles) }\end{array}$ & $\begin{array}{l}\text { Improvement. Greater live births } \\
\text { with multiple embryos transferred } \\
\text { compared to } 1 \text { embryo transferred }\end{array}$ & $\begin{array}{l}\text { Low } \\
\text { (Imprecise) }\end{array}$ \\
\hline \multirow[t]{2}{*}{$\begin{array}{l}\text { ART IVF: Frozen } \\
\text { vs. fresh embryo } \\
\text { transfer }\end{array}$} & Live birth & $\begin{array}{l}\text { 1 } \mathrm{RCT}^{177} \\
(2,157 \text { patients })\end{array}$ & $\begin{array}{l}\text { No difference: no difference in live } \\
\text { birth rates between couples using } \\
\text { frozen embryo versus fresh embryo } \\
\text { transfer }\end{array}$ & $\begin{array}{l}\text { Low } \\
\text { (1 study, } \\
\text { heterogeneous } \\
\text { infertility } \\
\text { indication) }\end{array}$ \\
\hline & $\begin{array}{l}\text { Pregnancy } \\
\text { complications: } \\
\text { Ectopic } \\
\text { pregnancy }\end{array}$ & $\begin{array}{l}\text { 1 } \mathrm{RCT}^{177} \\
(2,157 \text { patients) }\end{array}$ & $\begin{array}{l}\text { No difference: no difference in } \\
\text { ectopic pregnancy rates between } \\
\text { couples using frozen embryo } \\
\text { versus fresh embryo transfer }\end{array}$ & $\begin{array}{l}\text { Low } \\
\text { (1 study, } \\
\text { heterogeneous } \\
\text { infertility } \\
\text { indication) }\end{array}$ \\
\hline
\end{tabular}




\begin{tabular}{|c|c|c|c|c|}
\hline Comparison & Outcome & $\begin{array}{c}\text { Study Design } \\
\text { and Sample Size }\end{array}$ & Conclusion & $\begin{array}{l}\text { Strength of } \\
\text { Evidence } \\
\text { (Rationale) }^{\mathrm{a}}\end{array}$ \\
\hline & $\begin{array}{l}\text { Pregnancy } \\
\text { complications: } \\
\text { Multiple births }\end{array}$ & $\begin{array}{l}\text { 1 } \mathrm{RCT}^{177} \\
(2,157 \text { patients })\end{array}$ & $\begin{array}{l}\text { No difference: no difference in } \\
\text { multiple birth rates between } \\
\text { couples using frozen embryo } \\
\text { versus fresh embryo transfer }\end{array}$ & $\begin{array}{l}\text { Low } \\
\text { (1 study, } \\
\text { heterogeneous } \\
\text { infertility } \\
\text { indication) }\end{array}$ \\
\hline & $\begin{array}{l}\text { Pregnancy } \\
\text { complications: } \\
\text { Miscarriage }\end{array}$ & $\begin{array}{l}\text { 1 } \mathrm{RCT}^{177} \\
(2,157 \text { patients) }\end{array}$ & $\begin{array}{l}\text { No difference: no difference in } \\
\text { miscarriage rates between couples } \\
\text { using frozen embryo versus fresh } \\
\text { embryo transfer }\end{array}$ & $\begin{array}{l}\text { Low } \\
\text { (1 study, } \\
\text { heterogeneous } \\
\text { infertility } \\
\text { indication) }\end{array}$ \\
\hline & $\begin{array}{l}\text { Neonatal } \\
\text { outcomes: } \\
\text { Birthweight }\end{array}$ & $\begin{array}{l}\text { 1 } \mathrm{RCT}^{177} \\
(2,157 \text { patients })\end{array}$ & $\begin{array}{l}\text { No difference: no difference in low } \\
\text { birthweight rates between couples } \\
\text { using frozen embryo versus fresh } \\
\text { embryo transfer }\end{array}$ & $\begin{array}{l}\text { Low } \\
\text { (1 study, } \\
\text { heterogeneous } \\
\text { infertility } \\
\text { indication) }\end{array}$ \\
\hline & $\begin{array}{l}\text { Neonatal } \\
\text { outcomes: } \\
\text { Congenital } \\
\text { anomalies }\end{array}$ & $\begin{array}{l}\text { 1 } \mathrm{RCT}^{177} \\
(2,157 \text { patients })\end{array}$ & $\begin{array}{l}\text { No difference: no difference in } \\
\text { congenital anomalies rates } \\
\text { between couples using frozen } \\
\text { embryo versus fresh embryo } \\
\text { transfer }\end{array}$ & $\begin{array}{l}\text { Low } \\
\text { (1 study, } \\
\text { heterogeneous } \\
\text { infertility } \\
\text { indication) }\end{array}$ \\
\hline \multirow[t]{3}{*}{ IVF vS. ICSI } & Live birth & $\begin{array}{l}3 \mathrm{RCTs}^{166,170,173} \\
\text { (497 patients) } \\
2 \text { Obs }^{168,172} \\
\text { (771,661 cycles) }\end{array}$ & $\begin{array}{l}\text { No difference. Meta-analysis of } 3 \\
\text { RCTs does not demonstrate a } \\
\text { difference between ICSI and IMSI. }\end{array}$ & $\begin{array}{l}\text { Moderate } \\
\text { (Moderate study } \\
\text { limitations) }\end{array}$ \\
\hline & $\begin{array}{l}\text { Pregnancy } \\
\text { complications: } \\
\text { Miscarriage }\end{array}$ & $\begin{array}{l}1 \mathrm{RCT}^{166} \\
\text { (121 patients) } \\
1 \text { Obs }^{168} \\
(499,135 \text { cycles) } \\
1 \mathrm{SR}^{180} \\
\text { (6 studies, } 552 \\
\text { women) }\end{array}$ & $\begin{array}{l}\text { No difference. Both included } \\
\text { studies and an existing systematic } \\
\text { review supported no difference in } \\
\text { miscarriage. SOE was reduced } \\
\text { because of quality of included } \\
\text { studies and imprecision of findings. }\end{array}$ & $\begin{array}{l}\text { Low } \\
\text { (High study } \\
\text { limitations, } \\
\text { imprecise) }\end{array}$ \\
\hline & $\begin{array}{l}\text { Neonatal } \\
\text { outcomes: } \\
\text { Birthweight }\end{array}$ & $\begin{array}{l}1 \mathrm{RCT}^{166} \\
\text { (121 patients) } \\
3 \text { Obs }^{91,168,172} \\
\text { (862,062 cycles) }\end{array}$ & $\begin{array}{l}\text { No difference: No significant } \\
\text { differences in rates of low birth } \\
\text { weight between ICSI versus } \\
\text { conventional-IVF cycles }\end{array}$ & $\begin{array}{l}\text { Low } \\
\text { (Moderate study } \\
\text { limitations) }\end{array}$ \\
\hline \multirow[t]{2}{*}{ ART: Unspecified } & $\begin{array}{l}\text { Long-term } \\
\text { outcomes: } \\
\text { Child (cancer) }\end{array}$ & $\begin{array}{l}1 \text { Obs }^{121} \\
\text { (924,427 patients) }\end{array}$ & $\begin{array}{l}\text { No difference: The overall cancer } \\
\text { incidence was not elevated in } \\
\text { children born after assisted } \\
\text { conception for male factor infertility. }\end{array}$ & $\begin{array}{l}\text { Low } \\
\text { (Moderate study } \\
\text { limitations) }\end{array}$ \\
\hline & $\begin{array}{l}\text { Long-term } \\
\text { outcomes: } \\
\text { Child (type } 1 \\
\text { diabetes } \\
\text { mellitus) }\end{array}$ & $\begin{array}{l}1 \mathrm{Obs}^{90} \\
(565,116 \\
\text { pregnancies) }\end{array}$ & $\begin{array}{l}\text { No difference: No significant } \\
\text { difference found between type } 1 \\
\text { diabetes mellitus diagnoses in } \\
\text { children born to patients with male } \\
\text { factor infertility conceived with ART } \\
\text { compared to children conceived } \\
\text { with no fertility treatment }\end{array}$ & $\begin{array}{l}\text { Moderate } \\
\text { (Imprecise) }\end{array}$ \\
\hline $\begin{array}{l}\text { Other strategies: } \\
\text { Antioxidant use } \\
\text { for Male Infertility }\end{array}$ & Live birth & $\begin{array}{l}1 \mathrm{SR}^{181} \\
\text { (4 studies of } 277 \\
\text { couples) }\end{array}$ & $\begin{array}{l}\text { Improvement: Increase in live birth } \\
\text { rate associated with vitamin E or } \\
\text { zinc supplementation relative to } \\
\text { placebo or no supplementation }\end{array}$ & $\begin{array}{l}\text { Low } \\
\text { (Imprecise, } \\
\text { small studies) }\end{array}$ \\
\hline
\end{tabular}

${ }^{\mathrm{a}}$ Criteria for downgrading strength of evidence is described as Rationale; when these criteria are insufficient for understanding the final strength of evidence, additional explanation is provided. 
Abbreviations: ART=assisted reproductive technology; ICSI=intra-cytoplasmic sperm injection; IVF=in vitro fertilization; $\mathrm{KQ}=$ Key Question; OAT=oligo-astheno-teratozoospermia; Obs=observational study; $\mathrm{RCT}=$ randomized controlled trial; TESE=extracted testicular sperm

\section{Key Question 6. Donors in Infertility}

We identified one fair-quality RCT ${ }^{182}$ and four retrospective observational studies, three fairquality, ${ }^{183-185}$ and one poor-quality, ${ }^{186}$ that addressed short- or long-term health outcomes of donors in infertility.

Key findings for outcomes of sperm and oocyte donors included:

- For oocyte donors, observational studies suggest a lower incidence of OHSS with GnRH agonist trigger than with human chorionic gonadotropin (hCG) trigger (low SOE). However, there was a lack of evidence on any long-term outcomes.

- There was a lack of evidence on any short or long-term outcomes for sperm donors

Table F summarizes the SOE for KQ 6 and specifically for the incidence of OHSS with GnRH agonist trigger versus hCG trigger. All other short- and long-term outcomes had insufficient SOE or were not evaluated in the limited set of included studies.

Table F. Summary of strength of evidence for major outcomes-KQ 6 (donors in infertility)

\begin{tabular}{|l|l|l|l|l|}
\hline \multicolumn{1}{|c|}{ Comparison } & \multicolumn{1}{|c|}{ Outcome } & \multicolumn{1}{|c|}{$\begin{array}{l}\text { Study Design } \\
\text { (Sample Size) }\end{array}$} & \multicolumn{1}{c|}{ Conclusion } & $\begin{array}{l}\text { Strength of } \\
\text { Evidence } \\
\text { (Rationale) }\end{array}$ \\
\hline $\begin{array}{l}\text { GnRH agonist } \\
\text { (leuprolide acetate) vs. } \\
\text { hCG trigger }\end{array}$ & $\begin{array}{l}\text { Short term adverse } \\
\text { effects of treatment: } \\
\text { OHSS }\end{array}$ & $\begin{array}{l}\text { 2 Obs }{ }^{183,184} \\
(3824)\end{array}$ & $\begin{array}{l}\text { Improvement: Lower } \\
\text { incidence of OHSS with GnRH } \\
\text { agonist trigger than with hCG } \\
\text { trigger. }\end{array}$ & $\begin{array}{l}\text { Low } \\
\text { (Moderate } \\
\text { Study } \\
\text { limitations, } \\
\text { imprecise) }\end{array}$ \\
\hline
\end{tabular}

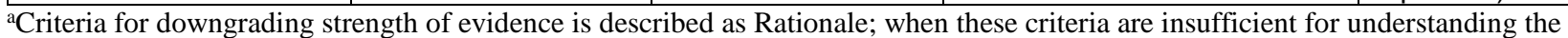
final strength of evidence, additional explanation is provided.

Abbreviations: GnRH=gonadotropin-releasing hormone; hCG= human chorionic gonadotropin; KQ=Key Question; Obs=observational study; OHSS=ovarian hyperstimulation syndrome

\section{Findings Applicable Across All Infertility Diagnoses}

We identified 26 articles ${ }^{21,167,187-210}$ described in 21 studies that addressed outcomes after treatment for infertility and adjusted for cause of infertility and therefore were considered relevant across all infertility diagnoses.

Findings applicable across all KQs for patients who undergo IVF/ICSI include:

- Clomiphene or gonadotropins ever use was not associated with increased risk of maternal cancer (low SOE).

- Women who undergo IVF demonstrated an increased risk of ovarian neoplasms and colorectal malignancies (low SOE) compared to women who do not undergo IVF. There is no evidence of a difference in invasive ovarian cancers (low SOE).

- For children born after ART, ICSI may be associated with an increased risk of autism compared to IVF (low SOE).

- In the United States, live birth rates after IVF/ICSI are lower for African-Americans than for other racial/ethnic groups after adjusting for other prognostic factors (low SOE). 
- Elective single-embryo transfer is associated with lower live birth rates but a significant reduction in multiple birth rates compared to multiple-embryo transfer (low SOE for both outcomes).

- There was no difference in the odds of low birth weight between ICSI versus conventional IVF cycles (low SOE). However, among couples undergoing ART with a singleton pregnancy, frozen embryo transfers result in a higher average birthweight, with a subsequent reduction in the incidence of low birthweight and an increase in the incidence of macrosomia (low SOE).

Table G summarizes the SOE for findings that are applicable across all infertility diagnoses.

Table G. Summary of strength of evidence for major outcomes-all infertility diagnoses

\begin{tabular}{|c|c|c|c|c|}
\hline Comparison & Outcome & $\begin{array}{l}\text { Study Design } \\
\text { (Sample Size) }\end{array}$ & Conclusion & $\begin{array}{l}\text { Strength of } \\
\text { Evidence } \\
\text { (Rationale) }^{\mathrm{a}}\end{array}$ \\
\hline $\begin{array}{l}\text { Clomiphene } \\
\text { citrate and } \\
\text { gonadotropin }\end{array}$ & $\begin{array}{l}\text { Long-term } \\
\text { outcomes: } \\
\text { Maternal cancer }\end{array}$ & $\begin{array}{l}1 \text { Obs }^{187} \\
\text { (9892 patients) }\end{array}$ & $\begin{array}{l}\text { No difference. Ever use of } \\
\text { clomiphene citrate was not } \\
\text { statistically significantly } \\
\text { associated with maternal } \\
\text { ovarian, breast, endometrial, } \\
\text { lung, thyroid, colon, or } \\
\text { melanoma cancer. } \\
\text { Gonadotropin use was not } \\
\text { associated with increased risk } \\
\text { for breast or endometrial } \\
\text { cancer }\end{array}$ & $\begin{array}{l}\text { Low } \\
\text { (Size of cohort not } \\
\text { sufficient to detect } \\
\text { modest increases } \\
\text { in risk) }\end{array}$ \\
\hline \multirow[t]{6}{*}{ ART: IVF } & Live birth (by race) & $\begin{array}{l}1 \text { Obs }^{211} \\
(13,473 \text { cycles })\end{array}$ & $\begin{array}{l}\text { Greater disparity. Lower live } \\
\text { birth rate for blacks as } \\
\text { compared to white }(p<0.001)\end{array}$ & $\begin{array}{l}\text { Low } \\
\text { (Imprecise, } 1 \\
\text { study) }\end{array}$ \\
\hline & $\begin{array}{l}\text { Live birth (by } \\
\text { number of embryos } \\
\text { transferred) }\end{array}$ & $\begin{array}{l}1 \text { Obs }^{111} \\
(69,028 \text { cycles })\end{array}$ & $\begin{array}{l}\text { Improvement. Increased live } \\
\text { birth rate per cycle with } 2 \\
\text { embryo transfer as compared } \\
\text { to single embryo transfer }\end{array}$ & $\begin{array}{l}\text { Low } \\
\text { (Imprecise, } \\
\text { findings with } \\
\text { moderate study } \\
\text { limitations) }\end{array}$ \\
\hline & $\begin{array}{l}\text { Pregnancy } \\
\text { complications: } \\
\text { Multiple births (by } \\
\text { number of embryos } \\
\text { transferred) }\end{array}$ & $\begin{array}{l}1 \text { Obs }^{111} \\
(69,028 \text { cycles })\end{array}$ & $\begin{array}{l}\text { Greater risk. Multiple live } \\
\text { birth rates are significantly } \\
\text { higher with a 2-embryo } \\
\text { transfer than a single embryo } \\
\text { transfer, but do not increase } \\
\text { further with 3- or 4-embryo } \\
\text { transfers }\end{array}$ & $\begin{array}{l}\text { Low } \\
\text { (Imprecise, } \\
\text { findings with } \\
\text { moderate study } \\
\text { limitations) }\end{array}$ \\
\hline & $\begin{array}{l}\text { Neonatal } \\
\text { outcomes: } \\
\text { Birthweight }\end{array}$ & $\begin{array}{l}1 \text { Obs }^{193} \\
(8,948)\end{array}$ & $\begin{array}{l}\text { No difference: No significant } \\
\text { difference in rates of low } \\
\text { birthweight using ART by } \\
\text { assisted hatching, source of } \\
\text { oocytes/semen, number of } \\
\text { embryos or ICSI }\end{array}$ & $\begin{array}{l}\text { Low } \\
\text { (Imprecise) }\end{array}$ \\
\hline & $\begin{array}{l}\text { Neonatal } \\
\text { outcomes: } \\
\text { Congenital } \\
\text { Anomalies }\end{array}$ & $\begin{array}{l}1 \text { Obs }^{197} \\
(64,861)\end{array}$ & $\begin{array}{l}\text { Greater risk. Risk of birth } \\
\text { defects was greater in infants } \\
\text { conceived using ART }\end{array}$ & $\begin{array}{l}\text { Low } \\
\text { (1 study) }\end{array}$ \\
\hline & $\begin{array}{l}\text { Long-term } \\
\text { outcomes: Child } \\
\text { (Autism) }\end{array}$ & $\begin{array}{l}1 \text { Obs }^{188} \\
(42,383)\end{array}$ & $\begin{array}{l}\text { Greater risk. Risk of autism } \\
\text { was greater in children } \\
\text { conceived with ART with ICSI } \\
\text { as compared to ART without } \\
\text { ICSI }\end{array}$ & $\begin{array}{l}\text { Low } \\
\text { (Imprecise) }\end{array}$ \\
\hline
\end{tabular}




\begin{tabular}{|c|c|c|c|c|}
\hline Comparison & Outcome & $\begin{array}{l}\text { Study Design } \\
\text { (Sample Size) }\end{array}$ & Conclusion & $\begin{array}{l}\text { Strength of } \\
\text { Evidence } \\
\text { (Rationale) }^{\mathrm{a}}\end{array}$ \\
\hline & $\begin{array}{l}\text { Long-term } \\
\text { outcomes: } \\
\text { Maternal (cancer) }\end{array}$ & $\begin{array}{l}2 \text { Obs }^{167,209} \\
(280,950)\end{array}$ & $\begin{array}{l}\text { Greater risk. IVF was } \\
\text { associated with a statistically } \\
\text { significant increased risk of all } \\
\text { ovarian neoplasms and } \\
\text { borderline ovarian tumors, } \\
\text { and colorectal cancer } \\
\text { No difference: IVF however } \\
\text { was not associated with an } \\
\text { increased risk of invasive } \\
\text { ovarian cancer, or melanoma }\end{array}$ & $\begin{array}{l}\text { Low } \\
\text { (Imprecise, older } \\
\text { study) }\end{array}$ \\
\hline $\begin{array}{l}\text { IVF+ICSI vs. } \\
\text { IVF }\end{array}$ & $\begin{array}{l}\text { Neonatal } \\
\text { outcomes: Birth } \\
\text { weight }\end{array}$ & $\begin{array}{l}1 \text { Obs }^{91} \\
(90,401 \text { cycles })\end{array}$ & $\begin{array}{l}\text { No difference: No significant } \\
\text { difference in the odds of low } \\
\text { birth weight between ICSI } \\
\text { versus conventional-IVF } \\
\text { cycles }\end{array}$ & $\begin{array}{l}\text { Low } \\
\text { (1 study with } \\
\text { moderate study } \\
\text { limitations) }\end{array}$ \\
\hline
\end{tabular}

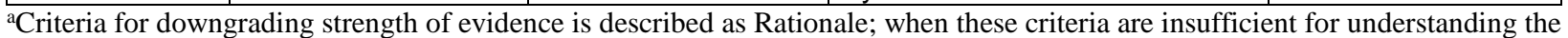
final strength of evidence, additional explanation is provided.

Abbreviations: ART=assisted reproductive technology; ICSI=intra-cytoplasmic sperm injection; IUI=intrauterine insemination; IVF=in vitro fertilization; KQ=Key Question; Obs=observational study

\section{Discussion}

In this Comparative Effectiveness Review, we reviewed 151 studies described in 161 publications that directly compared infertility management strategies in couples with infertility due to PCOS (KQ 1) or endometriosis (KQ 2); unexplained infertility (KQ 3); tubal and peritoneal factor infertility (KQ 4); and male factor infertility (KQ 5). We also explored the comparative safety and effectiveness of management strategies for donors in infertility (KQ 6). Although the ultimate goal with any infertility management strategy is to improve live birth rates of healthy infants to a healthy couple, many studies initially identified in our review only reported on pregnancy rates or focused on other short-term outcomes and did not differentiate by the underlying causes of infertility. Our findings are based on those 151 studies which evaluated the comparative effectiveness of infertility management strategies in couples with a known cause of infertility (including unexplained infertility) and which evaluated the outcome of live birth or another long-term outcome.

\section{Findings in Relation to What Is Already Known}

The 2008 AHRQ Evidence Report on "Effectiveness of ART"37 found that approximately 80 percent of the 478 included studies were performed outside the United States, and that the majority of RCTs did not report delivery rates and obstetric outcomes. In that review, most studies did not have sufficient power to detect clinically meaningful differences in live birth rates, and had still lower power to detect differences in less frequent outcomes such as multiple births and complications. In addition, the previous report focused on outcomes of specific treatments (ovulation induction, superovulation, and IVF/ICSI) rather than a wider range of potential treatments, and infertility diagnosis was considered as subgroup analyses, rather than the primary basis for comparing treatments.

Methods for evidence synthesis, in particular for rating strength of evidence, have also been revised since that report. Although an increasing number of studies are using live birth rate as the 
primary outcome, the majority of the literature, particularly randomized trials, is still based on pregnancy or ongoing pregnancy. Lack of precision for comparative estimates of rates for less common but important outcomes, such as complications, continues to be a major limitation.

We compared our findings to evidence-based guidelines from the National Institute for Health and Care Excellence (NICE) in the United Kingdom (UK), and the American Society for Reproductive Medicine (ASRM). In general, findings of our review were concordant with the guidelines, with differences primarily attributable to differences in inclusion/exclusion criteria.

For women with PCOS, both NICE and ASRM support use of clomiphene citrate alone as first-line therapy, with the NICE guidance recommending ultrasound monitoring for dose adjustment to minimize risk of multiple pregnancy, followed by combination therapy with metformin or gonadotropins for women who do not conceive after a 3-6 month course of clomiphene alone. Both our review and NICE suggest letrozole may be superior to clomiphene as first line therapy, and that pretreatment with metformin may improve outcomes in women with PCOS being treated with gonadotropins.

For women with endometriosis, ASRM concluded that evidence for surgical treatment of women with mild to moderate endometriosis was insufficient to recommend treatment, while the NICE guidance suggests some benefit, and our review was inconclusive. For those patients going directly to ART, surgical treatment of endometriosis, including endometrioma, prior to ART does not improve outcomes.

For women with unexplained infertility, NICE recommends against use of oral agents entirely, while ASRM suggests clomiphene plus IUI may improve cycle fecundity compared to expectant management; our review found insufficient evidence. Based on our review, immediate IVF results in higher live birth rates and shorter time to pregnancy in women aged 38-42 compared with a trial of clomiphene and IUI or gonadotropins and IUI, with most live births ultimately resulting from IVF.

For women with suspected tubal factor infertility, both NICE and ASRM recommend imaging for diagnosis (which is outside the scope of our review), although, when ART is readily available and affordable, proceeding directly to ART without a definitive diagnosis of tubal disease may be more efficient.

For male factor infertility, our review found no relevant findings compared to the recommendations, primarily because of limited data on live birth outcomes.

For both male and female donors, both NICE and ASRM recommend psychological evaluation and counseling, including, for females, the short term risks of ovarian stimulation and oocyte collection; our review found evidence on outcomes was limited only to the known shortterm risks of these procedures, with no evidence on potential longer term risks.

For long-term outcomes in women and children after infertility treatment, our review found limited or inconsistent evidence. Risks of adverse longer term maternal cancer outcomes were generally not increased after adjustment for the risk associated with infertility itself. ICSI however may be associated with an increased risk of neurodevelopmental disorders in children compared to those conceived through IVF. The NICE guidance was generally consistent with this assessment, and recommended that patients should be informed that any absolute risk was low, while there was still uncertainty about longer-term outcomes.

In general, our current review's findings are consistent with the NICE and ASRM guidelines - there is a general consensus that the overall body of evidence for many aspects of infertility treatment across all patient groups is limited. One consistent limitation is the relative paucity of studies utilizing live birth per couple as the primary outcome. 


\section{Applicability}

Two broad issues relate to the overall applicability of the available evidence to clinical practice in the United States — one geographic and one temporal. Many of the RCTs meeting our criteria were performed outside of the United States. Leaving aside any issues related to differences in study oversight or reporting, the populations of these studies may differ from U.S. infertility patients in two potentially important ways.

The first issue is that there may be clinically relevant differences between populations in terms of non-clinical factors affecting outcomes. For example, live birth rates for AfricanAmerican women undergoing ART in the US are lower than for white women ${ }^{211}$, which may reflect issues related to socioeconomic status, insurance coverage, or other factors (such as wellestablished racial differences in the risk of many adverse pregnancy outcomes). Differences in access to infertility services between countries may lead to differences in the likelihood of treatment success. Although the estimate of any relative difference between two interventions derived from an unbiased RCT should in theory be independent of the probability of specific outcomes, the more clinically relevant absolute difference may be substantially different (e.g., the risk of preterm birth in African-American compared to white women is consistently elevated). To the extent that the probability of specific outcomes of interest may differ between populations because of differences in genetic risk, exposures to other factors affecting risk, or non-biological factors such as access to care, there may be substantial differences in estimates of absolute risk differences. For relatively uncommon but important outcomes, these differences might also affect precision of estimates - confidence intervals for any treatment effect will be wider in populations where the outcome is less common.

In addition to the potential impact of race/ethnicity, there may be important differences in the distribution of socioeconomic status between populations. Access to infertility diagnosis and treatment varies across countries, and certainly within the United States. ${ }^{212}$ Differences in socioeconomic status could affect applicability in several ways. Differences in access to care may lead to differences in the spectrum of severity of "disease" for U.S. patients who given the financial burden of treatment options they may wait longer to undergo evaluations. Although summary statistics of baseline characteristics may allow some judgment of comparability, there may be potentially important differences in the distribution that are obscured by the typical reporting of means and standard deviations (particularly if the underlying characteristic is not normally distributed), or by differences within a given stage. Socioeconomic status may also potentially affect some important outcomes independently of any specific treatment-for example, neurodevelopmental outcomes such as specific learning skills may be strongly correlated with parental socioeconomic status.

The second issue is that changes in practice over time have a major impact on applicability, particularly for long-term outcomes. The long lag time between exposure to infertility treatment and the potential development of longer term outcomes such as cancer means that data available today necessarily reflect women exposed to treatments at least 10 years in the past; even if the specific exposure is similar, there may be differences between past and current practice in potentially important attributes such as dosage, timing, patient selection criteria, use of adjunctive treatments, etc. For example, evidence that immediate use of IVF leads to shorter time to pregnancy than strategies where IVF is used only after a trial of agents such as clomiphene or gonadotropins has led to a change in guidelines. ${ }^{213}$ which now suggest that the cumulative exposure to gonadotropins during the course of treatment is likely to decrease compared to earlier cohorts of women, reducing any long-term risks. 
In addition, there may be cohort effects in terms of other exposures that may affect the absolute risk of some outcomes (e.g., changes in the use of postmenopausal hormone replacement therapy or ages of mammography screening affecting breast cancer risk), which in turn would impact any additional absolute risk due to exposure to infertility treatments. Because of this phenomenon, there is likely to always be some unresolvable uncertainty about long-term outcomes for both parents undergoing current infertility treatments and their children..

\section{Research Recommendations}

In an era of constrained resources, future clinical research, especially comparative effectiveness research — which helps resolve current uncertainties regarding clinical or policy decisions - should receive priority. For most of the KQs, there are multiple areas of remaining uncertainty based on the existing evidence. In part because of the diversity of causes and treatment options, it is difficult to make specific recommendations for specific topics.

Before setting a specific agenda for future research in infertility, we believe a more general approach to identifying priorities would be helpful. Achieving consensus on the relative priority of specific outcomes, incorporating the perspective of multiple stakeholders (similar to the approach used for developing a research agenda for comparative effectiveness research for uterine fibroids. $^{214,215}$ Ideally, these outcome priorities would be used for subsequent evidence syntheses and guideline development.

As part of this consensus process, additional areas of discussion include:

- Formal consideration of the limits of acceptability for specific quantitative harms (e.g., preterm birth) and clinically meaningful differences in benefits (e.g., live birth).

- Formal discussion of the potential role of cost-effectiveness in decision making, including issues of willingness-to-pay and appropriate choice of outcome. This is particularly important because there are significant methodological challenges to the use of "standard" measures such as quality-adjusted life expectancy in the setting of infertility treatment.

- Issues related to study design, particularly from the patient stakeholder perspective. For example, in settings where patients and/or clinicians may have strong preferences for specific treatments, recruitment into RCTs may be difficult. ${ }^{216}$ In the uterine fibroid consensus process, patient stakeholders strongly preferred observational designs to randomized treatment assignment. ${ }^{214}$ Discussion of potential trade-offs between risk of bias, efficiency, ability to measure all relevant potential confounders and effect modifiers, appropriateness of alternative approaches such as Zelen randomization (where subjects are randomized prior to consent, then allowed to either receive the assigned treatment or choose the alternative ${ }^{217}$ ), and the likelihood that a specific study design would resolve a specific area of uncertainty should all be included.

- Issues related to data reporting. Particularly for ART and other treatments which are used for multiple indications, reporting of results separately by indication in both randomized trials and large observational studies would be extremely useful. Although these subgroup results may have insufficient power to detect clinically relevant differences within the context of individual studies (particularly RCTs), their routine publication would eventually allow synthesis of results using methods such as meta-analysis (including individual-level meta-analysis.) 
The Society for Assisted Reproductive Technology Clinic Outcome Reporting System (SART CORS) and the National ART Surveillance System (NASS), which includes data submitted through SART CORS (the majority of clinics providing ART as well as a smaller number of non-SART participating clinics who report directly to the Centers for Disease Control and Prevention (CDC), are outstanding examples of what a large-scale, population-based registry can achieve in terms of providing data on treatment outcomes. However, the major limitation of the database in the past has been that data are only published on a per-cycle, rather than percouple, basis. Recently the database methods have changed and now they are publicly reporting the cumulative success rate per patient. Results, however, are still reported at the clinic level, so patients who receive care at more than one clinic do not have the full range of outcomes captured, and there is no mechanism for prospectively collecting long-term outcomes of patients or children. Facilitating reporting of results so that outcomes are reported on a per-couple basis will substantially improve the ability to generate estimates of the likely outcome of specific ART-related decisions.

Based on input from key informants and our technical expert panel (TEP), we structured the review based on infertility diagnosis, and required studies to report outcomes specifically by diagnosis, or to adjust for diagnosis in multivariable analyses. As noted above, this led to exclusion of a number of papers, particularly those related to ART methods. There is clear evidence that the probability of some outcomes of interest, both short-term (e.g., OHSS) and long-term (certain cancers) differs based on underlying diagnosis. Although this may not be the case for all outcomes, we believe it would be helpful for future studies of interventions performed in patients with different underlying diagnoses to report results separately by diagnosis. Within an individual study powered on the basis of the total patients, estimates of diagnosis-specific outcomes may be too imprecise to confidently rule out clinically relevant differences - consistency of reporting would allow formal synthesis of estimates across studies.

We found very limited evidence on outcomes among sperm or oocyte donors. Oocyte donors, who undergo controlled ovarian hyperstimulation and oocyte retrieval in the same manner as patients undergoing IVF using their own eggs, have, in theory, at least the same risk of shortterm adverse events as patients. The frequency with which oocyte donors are used is increasing, and evidence from the SART CORS database suggests that the risk of certain pregnancy complications is lower when donor oocytes are used. ${ }^{34,218}$ If demand for donor oocytes continues to increase, much more evidence on the specific short- and long-term outcomes of donation (especially if a donor undergoes multiple cycles) is needed.

\section{Conclusions}

There is evidence supporting some strategies for treatment of infertility, both for specific diagnoses and for couples with any diagnosis, in part because of recent adaptation of more rigorous methods for evaluating treatments for infertility, particularly regarding treatments for PCOS and approaches to timing of interventions in patients undergoing ART. In addition, ongoing refinements to the SART CORS database continue to make it a valuable resource, particularly for data on short-term outcomes. However, given the diversity of infertility causes and treatments, there is considerable residual uncertainty about the optimal treatment options for specific patients. Consensus on which outcomes to report (such as encouraging reporting of live birth rates on a per couple basis as well as per cycle, and, for studies of treatment such as ART, reporting of both overall and diagnosis-specific outcomes) and which areas of uncertainty are 
most important to resolve (in order to prioritize research) is needed to improve the ability of patients and clinicians to make decisions about the most appropriate treatment. 


\section{References}

1. Gnoth C, Godehardt E, Frank-Herrmann P, et al. Definition and prevalence of subfertility and infertility. Hum Reprod. 2005 May;20(5):1144-7. doi: 10.1093/humrep/deh870. PMID: 15802321.

2. Chandra A, Copen CE, Stephen EH. Infertility and impaired fecundity in the United States, 1982-2010: data from the National Survey of Family Growth. Natl Health Stat Report. 2013 Aug 14(67):1-18. PMID: 24988820.

3. Simonsen SE, Baksh L, Stanford JB. Infertility treatment in a population-based sample: 2004-2005. Matern Child Health J. 2012 May;16(4):877-86. doi: 10.1007/s10995-011-0809-6. PMID: 21559776.

4. Greil AL, McQuillan J, Shreffler KM, et al. Race-ethnicity and medical services for infertility: stratified reproduction in a population-based sample of U.S. women. J Health Soc Behav. 2011 Dec;52(4):493-509. doi: http://dx.doi.org/10.1177/002214651141823 6. PMID: 22031500.

5. Hammoud AO, Gibson M, Stanford J, et al. In vitro fertilization availability and utilization in the United States: a study of demographic, social, and economic factors. Fertil Steril. 2009 May;91(5):1630-5. doi: 10.1016/j.fertnstert.2007.10.038. PMID: 18539275.

6. Macaluso M, Wright-Schnapp TJ, Chandra A, et al. A public health focus on infertility prevention, detection, and management. Fertil Steril. 2010 Jan;93(1):16.e1-0. doi: 10.1016/j.fertnstert.2008.09.046. PMID: 18992879.

7. Sunderam S, Kissin DM, Flowers L, et al. Assisted reproductive technology surveillance-United States, 2009. MMWR Surveill Summ. 2012 Nov 2;61(7):1-23. PMID: 23114281.

8. Louis JF, Thoma ME, Sorensen DN, et al. The prevalence of couple infertility in the United States from a male perspective: evidence from a nationally representative sample. Andrology. 2013 Sep;1(5):741-8. doi: 10.1111/j.2047-2927.2013.00110.x. PMID: 23843214.
9. Eijkemans MJ, van Poppel F, Habbema DF, et al. Too old to have children? Lessons from natural fertility populations. Hum Reprod. 2014 Jun;29(6):1304-12. doi: 10.1093/humrep/deu056. PMID: 24676403.

10. Barton SE, Najita JS, Ginsburg ES, et al. Infertility, infertility treatment, and achievement of pregnancy in female survivors of childhood cancer: a report from the Childhood Cancer Survivor Study cohort. Lancet Oncol. 2013 Aug;14(9):87381. doi: 10.1016/s1470-2045(13)70251-1. PMID: 23856401.

11. Howard-Anderson J, Ganz PA, Bower JE, et al. Quality of life, fertility concerns, and behavioral health outcomes in younger breast cancer survivors: a systematic review. J Natl Cancer Inst. 2012 Mar 7;104(5):386405. doi: 10.1093/jnci/djr541. PMID: 22271773.

12. Letourneau JM, Ebbel EE, Katz PP, et al. Pretreatment fertility counseling and fertility preservation improve quality of life in reproductive age women with cancer. Cancer. 2012 Mar 15;118(6):1710-7. doi: 10.1002/cncr.26459. PMID: 21887678.

13. Odisho AY, Nangia AK, Katz PP, et al. Temporal and geospatial trends in male factor infertility with assisted reproductive technology in the United States from 19992010. Fertil Steril. 2014 Aug;102(2):469-75. doi: 10.1016/j.fertnstert.2014.05.006. PMID: 24931206.

14. Practice Committee of the American Society for Reproductive Medicine. Diagnostic evaluation of the infertile female: a committee opinion. Fertil Steril. 2012 Aug;98(2):302-7. doi: 10.1016/j.fertnstert.2012.05.032. PMID: 22698637.

15. National Collaborating Centre for Women's and Children's Health (UK). Fertility: Assessment and Treatment for People with Fertility Problems. London (UK): RCOG Press; 2013 Feb. (NICE Clinical Guidelines, No. 156.) Available at:

http://www.ncbi.nlm.nih.gov/pubmedhealth/ PMH0068976/. PMID: 25340218. 
16. Hwang K, Walters RC, Lipshultz LI. Contemporary concepts in the evaluation and management of male infertility. Nat Rev Urol. 2011 Feb;8(2):86-94. doi: 10.1038/nrurol.2010.230. PMID: 21243017.

17. Barnhart KT. Live birth is the correct outcome for clinical trials evaluating therapy for the infertile couple. Fertil Steril. 2014 May;101(5):1205-8. doi: 10.1016/j.fertnstert.2014.03.026. PMID: 24786740.

18. Legro RS, Wu X, Barnhart KT, et al. Improving the reporting of clinical trials of infertility treatments (IMPRINT): modifying the CONSORT statement. Hum Reprod. 2014 Oct 10;29(10):2075-82. doi: 10.1093/humrep/deu218. PMID: 25217611.

19. Kurta ML, Moysich KB, Weissfeld JL, et al. Use of fertility drugs and risk of ovarian cancer: results from a U.S.-based casecontrol study. Cancer Epidemiol Biomarkers Prev. 2012 Aug;21(8):1282-92. doi: 10.1158/1055-9965.epi-12-0426. PMID: 22707710.

20. Rizzuto I, Behrens RF, Smith LA. Risk of ovarian cancer in women treated with ovarian stimulating drugs for infertility. Cochrane Database of Systematic Reviews. 2013;8:CD008215. doi: http://dx.doi.org/10.1002/14651858.CD0082 15.pub2. PMID: 23943232.

21. Trabert B, Lamb EJ, Scoccia B, et al. Ovulation-inducing drugs and ovarian cancer risk: results from an extended followup of a large United States infertility cohort. Fertil Steril. 2013 Dec;100(6):1660-6. doi: 10.1016/j.fertnstert.2013.08.008. PMID: 24011610.

22. Beck S, Wojdyla D, Say L, et al. The worldwide incidence of preterm birth: a systematic review of maternal mortality and morbidity. Bull World Health Organ. 2010 Jan;88(1):31-8. doi: 10.2471/blt.08.062554. PMID: 20428351.

23. Messerlian C, Maclagan L, Basso O. Infertility and the risk of adverse pregnancy outcomes: a systematic review and metaanalysis. Hum Reprod. 2013 Jan;28(1):12537. doi: 10.1093/humrep/des347. PMID: 23042798.
24. Kawwass JF, Crawford S, Kissin DM, et al. Tubal factor infertility and perinatal risk after assisted reproductive technology. Obstet Gynecol. 2013 Jun;121(6):1263-71. doi: 10.1097/AOG.0b013e31829006d9. PMID: 23812461.

25. Stern JE, Luke B, Tobias M, et al. Adverse pregnancy and birth outcomes associated with underlying diagnosis with and without assisted reproductive technology treatment. Fertil Steril. 2015 Jun;103(6):1438-45. doi: 10.1016/j.fertnstert.2015.02.027. PMID: 25813277.

26. Manipalviratn S, DeCherney A, Segars J. Imprinting disorders and assisted reproductive technology. Fertil Steril. 2009 Feb;91(2):305-15. doi: 10.1016/j.fertnstert.2009.01.002. PMID: 19201275.

27. Batcheller A, Cardozo E, Maguire M, et al. Are there subtle genome-wide epigenetic alterations in normal offspring conceived by assisted reproductive technologies? Fertil Steril. 2011 Dec;96(6):1306-11. doi: 10.1016/j.fertnstert.2011.09.037. PMID: 22035969.

28. Greil AL, McQuillan J, Lowry M, et al. Infertility treatment and fertility-specific distress: A longitudinal analysis of a population-based sample of U.S. women. Soc Sci Med. 2011 Jul;73(1):87-94. doi: 10.1016/j.socscimed.2011.04.023. PMID: 21645954.

29. Wilkins KM, Warnock JK, Serrano E. Depressive symptoms related to infertility and infertility treatments. Psychiatr Clin North Am. 2010 Jun;33(2):309-21. doi: 10.1016/j.psc.2010.01.009. PMID: 20385339.

30. Feinberg EC, Larsen FW, Wah RM, et al. Economics may not explain Hispanic underutilization of assisted reproductive technology services. Fertil Steril. 2007 Nov;88(5):1439-41. doi: 10.1016/j.fertnstert.2007.01.031. PMID: 17561005. 
31. Fujimoto VY, Luke B, Brown MB, et al. Racial and ethnic disparities in assisted reproductive technology outcomes in the United States. Fertil Steril. 2010

Feb;93(2):382-90. doi: 10.1016/j.fertnstert.2008.10.061. PMID: 19081561.

32. McCarthy-Keith DM, Schisterman EF, Robinson RD, et al. Will decreasing assisted reproduction technology costs improve utilization and outcomes among minority women? Fertil Steril. 2010 Dec;94(7):25879. doi: 10.1016/j.fertnstert.2010.02.021. PMID: 20356585.

33. Wellons MF, Fujimoto VY, Baker VL, et al. Race matters: a systematic review of racial/ethnic disparity in Society for Assisted Reproductive Technology reported outcomes. Fertil Steril. 2012 Aug;98(2):4069. doi: 10.1016/j.fertnstert.2012.05.012. PMID: 22698638.

34. Myers ER. Outcomes of donor oocyte cycles in assisted reproduction. JAMA. 2013 Dec 11;310(22):2403-4. doi: 10.1001/jama.2013.280925. PMID: 24135802.

35. Klitzman RL, Sauer MV. Kamakahi vs ASRM and the future of compensation for human eggs. Am J Obstet Gynecol. 2015 Mar 26doi: 10.1016/j.ajog.2015.03.046. PMID: 25816784.

36. De Melo-Martin I. The ethics of anonymous gamete donation: is there a right to know one's genetic origins? Hastings Cent Rep. 2014 Mar-Apr;44(2):28-35. doi: 10.1002/hast.285. PMID: 24532424.

37. Myers ER, McCrory DC, Mills AA, et al. Effectiveness of Assisted Reproductive Technology. Evidence Report/Technology Assessment No. 167 (Prepared by the Duke University Evidence-based Practice Center under Contract No. 290-02-0025.) AHRQ Publication No. 08-E012. Rockville, MD: Agency for Healthcare Research and Quality. May 2008. Available at: http://www.ncbi.nlm.nih.gov/books/NBK38 549/. Accessed November 13, 2018.
38. Agency for Healthcare Research and Quality (AHRQ). Methods Guide for Effectiveness and Comparative Effectiveness Reviews. AHRQ Publication No. 10(14)-EHC063-EF. Rockville, MD: Agency for Healthcare Research and Quality. January 2014. Available at: https://www.effectivehealthcare.ahrq.gov/to pics/cer-methods-guide/overview. Accessed January 18, 2018.

39. Shea BJ, Grimshaw JM, Wells GA, et al. Development of AMSTAR: a measurement tool to assess the methodological quality of systematic reviews. BMC Med Res Methodol. 2007 Feb 15;7:10. doi: 10.1186/1471-2288-7-10. PMID: 17302989.

40. Owens DK, Lohr KN, Atkins D, et al. AHRQ series paper 5: grading the strength of a body of evidence when comparing medical interventions-Agency for Healthcare Research and Quality and the Effective Health Care Program. J Clin Epidemiol. 2010 May;63(5):513-23. PMID: 19595577.

41. Berkman ND, Lohr KN, Ansari M, et al. Grading the Strength of a Body of Evidence When Assessing Health Care Interventions for the Effective Health Care Program of the Agency for Healthcare Research and Quality: An Update. Methods Guide for Comparative Effectiveness Reviews (Prepared by the RTI-UNC Evidence-based Practice Center under Contract No. 2902007-10056-I). AHRQ Publication No. 13(14)-EHC130-EF. Rockville, MD: Agency for Healthcare Research and Quality. November 2013. Available at: https://effectivehealthcare.ahrq.gov/sites/def ault/files/pdf/methods-guidance-gradingevidence_methods.pdf. Accessed November 13, 2018.

42. Rashidi M, Najmi Z, Mobasseri A. Advantages of Recombinant FollicleStimulating Hormone over Human Menopausal Gonadotropin in Intrauterine Insemination: A Randomized Clinical Trial in Polycystic Ovary Syndrome-Associated Infertility. Gynecol Obstet Invest. 2015 Jul 23;81(2):118-23. doi: 10.1159/000435773. PMID: 26228499. 
43. Polotsky AJ, Allshouse AA, Casson PR, et al. Impact of Male and Female Weight, Smoking, and Intercourse Frequency on Live Birth in Women With Polycystic Ovary Syndrome. J Clin Endocrinol Metab. 2015 Jun;100(6):2405-12. doi: 10.1210/jc.20151178. PMID: 25856211.

44. Legro RS, Brzyski RG, Diamond MP, et al. Letrozole versus clomiphene for infertility in the polycystic ovary syndrome. N Engl J Med. 2014 Jul 10;371(2):119-29. doi: 10.1056/NEJMoa1313517. PMID: 25006718.

45. Yazici G, Savas A, Tasdelen B, et al. Role of luteal phase support on gonadotropin ovulation induction cycles in patients with polycystic ovary syndrome. J Reprod Med. 2014 Jan-Feb;59(1-2):25-30. PMID: 24597283.

46. Ghanem ME, Elboghdady LA, Hassan M, et al. Clomiphene citrate co-treatment with low dose urinary FSH versus urinary FSH for clomiphene resistant PCOS: randomized controlled trial. J Assist Reprod Genet. 2013 Nov;30(11):1477-85. doi: 10.1007/s10815013-0090-2. PMID: 24014214.

47. An Y, Sun Z, Zhang Y, et al. The use of berberine for women with polycystic ovary syndrome undergoing IVF treatment. Clin Endocrinol (Oxf). 2014 Mar;80(3):425-31. doi: 10.1111/cen.12294. PMID: 23869585.

48. Choi MH, Lee SH, Kim HO, et al. Comparison of assisted reproductive technology outcomes in infertile women with polycystic ovary syndrome: In vitro maturation, GnRH agonist, and GnRH antagonist cycles. Clin Exp Reprod Med. 2012 Dec;39(4):166-71. doi: 10.5653/cerm.2012.39.4.166. PMID: 23346527.

49. Nahuis MJ, Oude Lohuis E, Kose N, et al. Long-term follow-up of laparoscopic electrocautery of the ovaries versus ovulation induction with recombinant FSH in clomiphene citrate-resistant women with polycystic ovary syndrome: an economic evaluation. Hum Reprod. 2012

Dec;27(12):3577-82. doi: 10.1093/humrep/des336. PMID: 23001778.
50. Mehrabian F, Eessaei F. The laparoscopic ovarian electrocautery versus gonadotropin therapy in infertile women with clomiphene citrate-resistant polycystic ovary syndrome; a randomized controlled trial. J Pak Med Assoc. 2012 Mar;62(3 Suppl 2):S42-4. PMID: 22768457.

51. Zheng X, Wang L, Zhen X, et al. Effect of hCG priming on embryonic development of immature oocytes collected from unstimulated women with polycystic ovarian syndrome. Reprod Biol Endocrinol. 2012;10:40. doi: 10.1186/1477-7827-10-40. PMID: 22621829.

52. Kim CH, Moon JW, Kang HJ, et al. Effectiveness of GnRH antagonist multiple dose protocol applied during early and late follicular phase compared with GnRH agonist long protocol in non-obese and obese patients with polycystic ovary syndrome undergoing IVF/ICSI. Clin Exp Reprod Med. 2012 Mar;39(1):22-7. doi: 10.5653/cerm.2012.39.1.22. PMID: 22563547.

53. Morin-Papunen L, Rantala AS, UnkilaKallio L, et al. Metformin improves pregnancy and live-birth rates in women with polycystic ovary syndrome (PCOS): a multicenter, double-blind, placebocontrolled randomized trial. J Clin Endocrinol Metab. 2012 May;97(5):1492500. doi: 10.1210/jc.2011-3061. PMID: 22419702.

54. Homburg R, Hendriks ML, Konig TE, et al. Clomifene citrate or low-dose FSH for the first-line treatment of infertile women with anovulation associated with polycystic ovary syndrome: a prospective randomized multinational study. Hum Reprod. 2012 Feb;27(2):468-73. doi: 10.1093/humrep/der401. PMID: 22128296.

55. Palomba S, Falbo A, Carrillo L, et al. Metformin reduces risk of ovarian hyperstimulation syndrome in patients with polycystic ovary syndrome during gonadotropin-stimulated in vitro fertilization cycles: a randomized, controlled trial. Fertil Steril. 2011 Dec;96(6):1384-90.e4. doi: 10.1016/j.fertnstert.2011.09.020. PMID: 21982727. 
56. Abu Hashim H, Foda O, Ghayaty E, et al. Laparoscopic ovarian diathermy after clomiphene failure in polycystic ovary syndrome: is it worthwhile? A randomized controlled trial. Arch Gynecol Obstet. 2011 Nov;284(5):1303-9. doi: 10.1007/s00404011-1983-x. PMID: 21755338.

57. Kjotrod SB, Carlsen SM, Rasmussen PE, et al. Use of metformin before and during assisted reproductive technology in nonobese young infertile women with polycystic ovary syndrome: a prospective, randomized, double-blind, multi-centre study. Hum Reprod. 2011 Aug;26(8):204553. doi: 10.1093/humrep/der154. PMID: 21606131.

58. Nahuis MJ, Kose N, Bayram N, et al. Longterm outcomes in women with polycystic ovary syndrome initially randomized to receive laparoscopic electrocautery of the ovaries or ovulation induction with gonadotrophins. Hum Reprod. 2011 Jul;26(7):1899-904. doi: 10.1093/humrep/der141. PMID: 21576081.

59. Abdellah MS. Reproductive outcome after letrozole versus laparoscopic ovarian drilling for clomiphene-resistant polycystic ovary syndrome. Int J Gynaecol Obstet. 2011 Jun;113(3):218-21. doi: 10.1016/j.ijgo.2010.11.026. PMID: 21457973.

60. Abu Hashim H, Ombar O, Abd Elaal I. Intrauterine insemination versus timed intercourse with clomiphene citrate in polycystic ovary syndrome: a randomized controlled trial. Acta Obstet Gynecol Scand. 2011 Apr;90(4):344-50. doi: 10.1111/j.1600-0412.2010.01063.x. PMID: 21306326.

61. Hosseini MA, Aleyasin A, Saeedi H, et al. Comparison of gonadotropin-releasing hormone agonists and antagonists in assisted reproduction cycles of polycystic ovarian syndrome patients. J Obstet Gynaecol Res. 2010 Jun;36(3):605-10. doi: 10.1111/j.14470756.2010.01247.x. PMID: 20598044.
62. Stadtmauer LA, Sarhan A, Duran EH, et al. The impact of a gonadotropin-releasing hormone antagonist on gonadotropin ovulation induction cycles in women with polycystic ovary syndrome: a prospective randomized study. Fertil Steril. 2011 Jan;95(1):216-20. doi: 10.1016/j.fertnstert.2010.05.023. PMID: 20594551.

63. Johnson NP, Stewart AW, Falkiner J, et al. PCOSMIC: a multi-centre randomized trial in women with PolyCystic Ovary Syndrome evaluating Metformin for Infertility with Clomiphene. Hum Reprod. 2010 Jul;25(7):1675-83. doi: 10.1093/humrep/deq100. PMID: 20435692.

64. Tehraninejad ES, Nasiri R, Rashidi B, et al. Comparison of GnRH antagonist with long GnRH agonist protocol after OCP pretreatment in PCOs patients. Arch Gynecol Obstet. 2010 Sep;282(3):319-25. doi: 10.1007/s00404-010-1429-x. PMID: 20379731.

65. Aboulghar M, Saber W, Amin Y, et al. Prospective, randomized study comparing highly purified urinary follicle-stimulating hormone (FSH) and recombinant FSH for in vitro fertilization/intracytoplasmic sperm injection in patients with polycystic ovary syndrome. Fertil Steril. 2010

Nov;94(6):2332-4. doi: 10.1016/j.fertnstert.2010.01.051. PMID: 20188364.

66. Palomba S, Falbo A, Battista L, et al. Laparoscopic ovarian diathermy vs clomiphene citrate plus metformin as second-line strategy for infertile anovulatory patients with polycystic ovary syndrome: a randomized controlled trial. Am J Obstet Gynecol. 2010 Jun;202(6):577.e1-8. doi: 10.1016/j.ajog.2009.11.042. PMID: 20096821.

67. Rausch ME, Legro RS, Barnhart HX, et al. Predictors of pregnancy in women with polycystic ovary syndrome. J Clin Endocrinol Metab. 2009 Sep;94(9):3458-66. doi: 10.1210/jc.2009-0545. PMID: 19509098. 
68. Kurzawa R, Ciepiela P, Baczkowski T, et al. Comparison of embryological and clinical outcome in GnRH antagonist vs. GnRH agonist protocols for in vitro fertilization in PCOS non-obese patients. A prospective randomized study. J Assist Reprod Genet. 2008 Aug;25(8):365-74. doi: 10.1007/s10815-008-9249-7. PMID: 18802744.

69. Amer SA, Li TC, Metwally M, et al. Randomized controlled trial comparing laparoscopic ovarian diathermy with clomiphene citrate as a first-line method of ovulation induction in women with polycystic ovary syndrome. Hum Reprod. 2009 Jan;24(1):219-25. doi: 10.1093/humrep/den325. PMID: 18794162.

70. Zain MM, Jamaluddin R, Ibrahim A, et al. Comparison of clomiphene citrate, metformin, or the combination of both for first-line ovulation induction, achievement of pregnancy, and live birth in Asian women with polycystic ovary syndrome: a randomized controlled trial. Fertil Steril. 2009 Feb;91(2):514-21. doi: 10.1016/j.fertnstert.2007.12.002. PMID: 18321486 .

71. Ge HS, Huang XF, Zhang W, et al. Exposure to human chorionic gonadotropin during in vitro maturation does not improve the maturation rate and developmental potential of immature oocytes from patients with polycystic ovary syndrome. Fertil Steril. 2008 Jan;89(1):98-103. doi: 10.1016/j.fertnstert.2007.02.021. PMID: 17524398.

72. Legro RS, Barnhart HX, Schlaff WD, et al. Clomiphene, metformin, or both for infertility in the polycystic ovary syndrome. N Engl J Med. 2007 Feb 8;356(6):551-66. doi: 10.1056/NEJMoa063971. PMID: 17287476.

73. Abu Hashim H, Mashaly AM, Badawy A. Letrozole versus laparoscopic ovarian diathermy for ovulation induction in clomiphene-resistant women with polycystic ovary syndrome: a randomized controlled trial. Arch Gynecol Obstet. 2010 Nov;282(5):567-71. doi: 10.1007/s00404010-1566-2. PMID: 20577748.
74. Zakherah MS, Nasr A, El Saman AM, et al. Clomiphene citrate plus tamoxifen versus laparoscopic ovarian drilling in women with clomiphene-resistant polycystic ovary syndrome. Int J Gynaecol Obstet. 2010 Mar;108(3):240-3. doi: 10.1016/j.ijgo.2009.10.004. PMID: 19944418.

75. Mutsaerts MA, van Oers AM, Groen H, et al. Randomized Trial of a Lifestyle Program in Obese Infertile Women. N Engl J Med. 2016 May 19;374(20):1942-53. doi: 10.1056/NEJMoa1505297. PMID: 27192672.

76. Ghahiri A, Mogharehabed N, Mamourian $\mathrm{M}$. Letrozole as the first-line treatment of infertile women with poly cystic ovarian syndrome (PCOS) compared with clomiphene citrate: A clinical trial. Adv Biomed Res. 2016;5:6. doi: 10.4103/22779175.175237. PMID: 26962508.

77. Wang Y, Chen Q, Wang N, et al. Controlled Ovarian Stimulation Using

Medroxyprogesterone Acetate and hMG in Patients With Polycystic Ovary Syndrome Treated for IVF: A Double-Blind Randomized Crossover Clinical Trial. Medicine (Baltimore). 2016

Mar;95(9):e2939. doi: 10.1097/md.0000000000002939. PMID: 26945402.

78. Legro RS, Dodson WC, Kris-Etherton PM, et al. Randomized Controlled Trial of Preconception Interventions in Infertile Women With Polycystic Ovary Syndrome. J Clin Endocrinol Metab. 2015 Nov;100(11):4048-58. doi: 10.1210/jc.20152778. PMID: 26401593.

79. Kar S, Sanchita S. Clomiphene citrate, metformin or a combination of both as the first line ovulation induction drug for Asian Indian women with polycystic ovarian syndrome: A randomized controlled trial. J Hum Reprod Sci. 2015;8(4):197-201.

80. Chen ZJ, Shi Y, Sun Y, et al. Fresh versus Frozen Embryos for Infertility in the Polycystic Ovary Syndrome. N Engl J Med. 2016 Aug 11;375(6):523-33. doi: 10.1056/NEJMoa1513873. PMID: 27509101. 
81. Wu XK, Wang YY, Liu JP, et al. Randomized controlled trial of letrozole, berberine, or a combination for infertility in the polycystic ovary syndrome. Fertil Steril. 2016 Sep 1;106(3):757-65.e1. doi: 10.1016/j.fertnstert.2016.05.022. PMID: 27336209.

82. Hossein-Rashidi B, Khandzad B, ShahrokhTehraninejad E, et al. Recombinant FSH Compared to Clomiphene Citrate as the First-Line in Ovulation Induction in Polycystic Ovary Syndrome Using Newly Designed Pens: A Randomized Controlled Trial. J Family Reprod Health. 2016 Mar;10(1):42-8. PMID: 27385973.

83. Jacob SL, Brewer C, Tang T, et al. A short course of metformin does not reduce OHSS in a GnRH antagonist cycle for women with PCOS undergoing IVF: a randomised placebo-controlled trial. Hum Reprod. 2016 Dec;31(12):2756-64. doi: 10.1093/humrep/dew268. PMID: 27816925.

84. Aghahosseini M, Aleyasin A, Chegini V, et al. Low-dose hCG as trigger day and $35 \mathrm{hr}$ later have different ovarian hyperstimulation syndrome occurrence in females undergoing In vitro fertilization: An RCT. Int J Reprod Biomed (Yazd). 2017 Nov;15(11):735-40. PMID: 29404536.

85. Amer SA, Smith J, Mahran A, et al. Doubleblind randomized controlled trial of letrozole versus clomiphene citrate in subfertile women with polycystic ovarian syndrome. Hum Reprod. 2017 Aug 1;32(8):1631-8. doi: 10.1093/humrep/dex227. PMID: 28854590.

86. de Wilde MA, Lamain-de Ruiter M, Veltman-Verhulst SM, et al. Increased rates of complications in singleton pregnancies of women previously diagnosed with polycystic ovary syndrome predominantly in the hyperandrogenic phenotype. Fertil Steril. 2017 Aug;108(2):333-40. doi: 10.1016/j.fertnstert.2017.06.015. PMID: 28778282.

87. Einarsson S, Bergh C, Friberg B, et al. Weight reduction intervention for obese infertile women prior to IVF: a randomized controlled trial. Hum Reprod. 2017 Aug 1;32(8):1621-30. doi:

10.1093/humrep/dex235. PMID: 28854592.
88. Emekci Ozay O, Ozay AC, Cagliyan E, et al. Myo-inositol administration positively effects ovulation induction and intrauterine insemination in patients with polycystic ovary syndrome: a prospective, controlled, randomized trial. Gynecol Endocrinol. 2017 Jul;33(7):524-8. doi: 10.1080/09513590.2017.1296127. PMID: 28277112.

89. Hassan A, Shehata N, Wahba A. Cost effectiveness of letrozole and purified urinary FSH in treating women with clomiphene citrate-resistant polycystic ovarian syndrome: a randomized controlled trial. Hum Fertil (Camb). 2017

Apr;20(1):37-42. doi: 10.1080/14647273.2016.1242783. PMID: 27825272.

90. Kettner LO, Matthiesen NB, RamlauHansen $\mathrm{CH}$, et al. Fertility treatment and childhood type 1 diabetes mellitus: a nationwide cohort study of 565,116 live births. Fertil Steril. 2016 Dec;106(7):17516. doi: 10.1016/j.fertnstert.2016.09.009. PMID: 27773424.

91. Keyhan S, Truong T, Li YJ, et al. Preterm Delivery and Low Birth Weight Among Neonates Conceived With Intracytoplasmic Sperm Injection Compared With Conventional In Vitro Fertilization. Obstet Gynecol. 2018 Feb;131(2):262-8. doi: 10.1097/aog.0000000000002423. PMID: 29324596.

92. Malchau SS, Henningsen AA, Loft A, et al. The long-term prognosis for live birth in couples initiating fertility treatments. Hum Reprod. 2017 Jul 1;32(7):1439-49. doi: 10.1093/humrep/dex096. PMID: 28472455.

93. van Oers AM, Groen H, Mutsaerts MA, et al. Effectiveness of lifestyle intervention in subgroups of obese infertile women: a subgroup analysis of a RCT. Hum Reprod. 2016 Dec;31(12):2704-13. doi: 10.1093/humrep/dew252. PMID: 27798042.

94. van Oers AM, Mutsaerts MAQ, Burggraaff JM, et al. Association between periconceptional weight loss and maternal and neonatal outcomes in obese infertile women. PLoS One. 2018;13(3):e0192670. doi: 10.1371/journal.pone.0192670. PMID: 29590118. 
95. Weiss NS, Nahuis MJ, Bordewijk E, et al. Gonadotrophins versus clomifene citrate with or without intrauterine insemination in women with normogonadotropic anovulation and clomifene failure (MOVIN): a randomised, two-by-two factorial trial. Lancet. 2018 Feb 24;391(10122):75865. doi: 10.1016/s0140-6736(17)33308-1. PMID: 29273245.

96. Wu XK, Stener-Victorin E, Kuang HY, et al. Effect of Acupuncture and Clomiphene in Chinese Women With Polycystic Ovary Syndrome: A Randomized Clinical Trial. JAMA. 2017 Jun 27;317(24):2502-14. doi: 10.1001/jama.2017.7217. PMID: 28655015.

97. Ibrahim MH, Tawfic M, Hassan MM, et al. Letrozole versus laparoscopic ovarian drilling in infertile women with PCOS resistant to clomiphene citrate. Middle East Fertility Society Journal. 2017;22(4):251-4. doi: 10.1016/j.mefs.2017.02.003.

98. Mohammadi Yeganeh L, Moini A, Shiva M, et al. Methylprednisolone for prevention of ovarian hyperstimulation syndrome in patients with polycystic ovarian syndrome undergoing in-vitro fertilisation: a randomised controlled trial. J Obstet Gynaecol. 2018;38(2):241-6. doi: 10.1080/01443615.2017.1346593.

99. Topçu HO, Batioğlu AS, İslimye M. Tamoxifen versus clomiphene citrate for ovulation induction in women with polycystic ovary syndrome: A prospective randomized trial. J Reprod Med. 2017;62(5):507-12.

100. Zahran KM, Mostafa WA, Abbas AM, et al. Clomiphene citrate plus cabergoline versus clomiphene citrate for induction of ovulation in infertile euprolactinemic patients with polycystic ovary syndrome: A randomized clinical trial. Middle East Fertility Society Journal. 2018doi: 10.1016/j.mefs.2017.12.008.

101. Badawy A, Allam A, Abulatta M. Extending clomiphene treatment in clomipheneresistant women with PCOS: a randomized controlled trial. Reprod Biomed Online. 2008 Jun;16(6):825-9. PMID: 18549692.
102. Elsedeek M, Elgindy E. Comparison between two clomiphene citrate protocols for induction of ovulation in clomiphene resistant polycystic ovary syndrome. Middle East Fertility Society Journal. 2014;19(4):243-7.

103. Franik S, Kremer JA, Nelen WL, et al. Aromatase inhibitors for subfertile women with polycystic ovary syndrome. Cochrane Database Syst Rev. 2014;2:Cd010287. doi: 10.1002/14651858.CD010287.pub2. PMID: 24563180 .

104. Sun X, Zhang D, Zhang W. Effect of metformin on ovulation and reproductive outcomes in women with polycystic ovary syndrome: a meta-analysis of randomized controlled trials. Arch Gynecol Obstet. 2013 Aug;288(2):423-30. doi: 10.1007/s00404013-2756-5. PMID: 23430028.

105. Morley LC, Tang T, Yasmin E, et al. Insulin-sensitising drugs (metformin, rosiglitazone, pioglitazone, D-chiro-inositol) for women with polycystic ovary syndrome, oligo amenorrhoea and subfertility. Cochrane Database Syst Rev. 2017 Nov 29;11:Cd003053. doi: 10.1002/14651858.CD003053.pub6. PMID: 29183107.

106. Brown J, Farquhar C. Clomiphene and other antioestrogens for ovulation induction in polycystic ovarian syndrome. Cochrane Database Syst Rev. 2016 Dec 15;12:Cd002249. doi: 10.1002/14651858.CD002249.pub5. PMID: 27976369.

107. Farquhar C, Brown J, Marjoribanks J. Laparoscopic drilling by diathermy or laser for ovulation induction in anovulatory polycystic ovary syndrome. Cochrane Database Syst Rev. 2012;6:Cd001122. doi: http://dx.doi.org/10.1002/14651858.CD0011 22.pub4. PMID: 22696324.

108. Zhu S, Liu D, Huang W, et al. Postlaparoscopic oral contraceptive combined with Chinese herbal mixture in treatment of infertility and pain associated with minimal or mild endometriosis: a randomized controlled trial. BMC Complement Altern Med. 2014;14:222. doi: 10.1186/1472-688214-222. PMID: 24996447. 
109. Stewart LM, Holman CD, Aboagye-Sarfo P, et al. In vitro fertilization, endometriosis, nulliparity and ovarian cancer risk. Gynecol Oncol. 2013 Feb;128(2):260-4. doi: 10.1016/j.ygyno.2012.10.023. PMID: 23116937.

110. Abu Hashim H, El Rakhawy M, Abd Elaal I. Randomized comparison of superovulation with letrozole vs. clomiphene citrate in an IUI program for women with recently surgically treated minimal to mild endometriosis. Acta Obstet Gynecol Scand. 2012 Mar;91(3):338-45. doi: 10.1111/j.1600-0412.2011.01346.x. PMID: 22181973.

111. Luke B, Brown MB, Grainger DA, et al. Practice patterns and outcomes with the use of single embryo transfer in the United States. Fertil Steril. 2010 Feb;93(2):490-8. doi: 10.1016/j.fertnstert.2009.02.077. PMID: 19376512.

112. Muller V, Kogan I, Yarmolinskaya M, et al. Dienogest treatment after ovarian endometrioma removal in infertile women prior to IVF. Gynecol Endocrinol. 2017;33(sup1):18-21. doi: 10.1080/09513590.2017.1415676. PMID: 29264985.

113. Yapca OE, Delibas IB, Karaca I, et al. Timelimited hydrotubation combined with clomiphene citrate treatment for unexplained infertility. Clin Exp Obstet Gynecol. 2015;42(3):311-4. PMID: 26152000.

114. Tartagni M, Cicinelli MV, Baldini D, et al. Dehydroepiandrosterone decreases the agerelated decline of the in vitro fertilization outcome in women younger than 40 years old. Reprod Biol Endocrinol. 2015;13:18. doi: 10.1186/s12958-015-0014-3. PMID: 25884390 .

115. Erdem M, Abay S, Erdem A, et al. Recombinant FSH increases live birth rates as compared to clomiphene citrate in intrauterine insemination cycles in couples with subfertility: a prospective randomized study. Eur J Obstet Gynecol Reprod Biol. 2015 Jun;189:33-7. doi: 10.1016/j.ejogrb.2015.03.023. PMID: 25855325.
116. Butts SF, Owen C, Mainigi M, et al. Assisted hatching and intracytoplasmic sperm injection are not associated with improved outcomes in assisted reproduction cycles for diminished ovarian reserve: an analysis of cycles in the United States from 2004 to 2011. Fertil Steril. 2014

Oct;102(4):1041-7.e1. doi: 10.1016/j.fertnstert.2014.06.043. PMID: 25086790.

117. Seckin B, Turkcapar F, Yildiz Y, et al. Effect of luteal phase support with vaginal progesterone in intrauterine insemination cycles with regard to follicular response: a prospective randomized study. J Reprod Med. 2014 May-Jun;59(5-6):260-6. PMID: 24937967.

118. Goldman MB, Thornton KL, Ryley D, et al. A randomized clinical trial to determine optimal infertility treatment in older couples: the Forty and Over Treatment Trial (FORT-T). Fertil Steril. 2014

Jun;101(6):1574-81.e1-2. doi: 10.1016/j.fertnstert.2014.03.012. PMID: 24796764.

119. Yildiz F, Bozkurt N, Erdem A, et al. Effect of Pertubation on Pregnancy Rates before Intrauterine Insemination Treatment in Patients with Unexplained Infertility. Int J Fertil Steril. 2014 Apr;8(1):77-84. PMID: 24695882.

120. van Rumste MM, Custers IM, van Wely M, et al. IVF with planned single-embryo transfer versus IUI with ovarian stimulation in couples with unexplained subfertility: an economic analysis. Reprod Biomed Online. 2014 Mar;28(3):336-42. doi: 10.1016/j.rbmo.2013.10.021. PMID: 24456703.

121. Williams CL, Bunch KJ, Stiller CA, et al. Cancer risk among children born after assisted conception. N Engl J Med. 2013 Nov 7;369(19):1819-27. doi: 10.1056/NEJMoa1301675. PMID: 24195549 . 
122. Majumdar G, Majumdar A. A prospective randomized study to evaluate the effect of hyaluronic acid sperm selection on the intracytoplasmic sperm injection outcome of patients with unexplained infertility having normal semen parameters. J Assist Reprod Genet. 2013 Nov;30(11):1471-5. doi: 10.1007/s10815-013-0108-9. PMID: 24085466.

123. Vitek WS, Galarraga O, Klatsky PC, et al. Management of the first in vitro fertilization cycle for unexplained infertility: a costeffectiveness analysis of split in vitro fertilization-intracytoplasmic sperm injection. Fertil Steril. 2013

Nov;100(5):1381-8. doi: 10.1016/j.fertnstert.2013.06.035. PMID: 23876534.

124. Rashidi M, Aaleyasin A, Aghahosseini M, et al. Advantages of recombinant folliclestimulating hormone over human menopausal gonadotropin for ovarian stimulation in intrauterine insemination: a randomized clinical trial in unexplained infertility. Eur J Obstet Gynecol Reprod Biol. 2013 Jul;169(2):244-7. doi: 10.1016/j.ejogrb.2013.03.002. PMID: 23541417.

125. Rubio C, Bellver J, Rodrigo L, et al. Preimplantation genetic screening using fluorescence in situ hybridization in patients with repetitive implantation failure and advanced maternal age: two randomized trials. Fertil Steril. 2013 Apr;99(5):1400-7. doi: 10.1016/j.fertnstert.2012.11.041. PMID: 23260857.

126. Ragni G, Levi-Setti PE, Fadini R, et al. Clomiphene citrate versus high doses of gonadotropins for in vitro fertilisation in women with compromised ovarian reserve: a randomised controlled non-inferiority trial. Reprod Biol Endocrinol. 2012;10:114. doi: 10.1186/1477-7827-10-114. PMID: 23249758.

127. Custers IM, van Rumste MM, van der Steeg JW, et al. Long-term outcome in couples with unexplained subfertility and an intermediate prognosis initially randomized between expectant management and immediate treatment. Hum Reprod. 2012 Feb;27(2):444-50. doi: 10.1093/humrep/der389. PMID: 22114108.
128. Kim CH, Howles CM, Lee HA. The effect of transdermal testosterone gel pretreatment on controlled ovarian stimulation and IVF outcome in low responders. Fertil Steril. 2011 Feb;95(2):679-83. doi: 10.1016/j.fertnstert.2010.07.1077. PMID: 20801436.

129. Wiser A, Gonen O, Ghetler Y, et al. Addition of dehydroepiandrosterone (DHEA) for poor-responder patients before and during IVF treatment improves the pregnancy rate: a randomized prospective study. Hum Reprod. 2010 Oct;25(10):2496500. doi: 10.1093/humrep/deq220. PMID: 20729538.

130. Bagis T, Haydardedeoglu B, Kilicdag EB, et al. Single versus double intrauterine insemination in multi-follicular ovarian hyperstimulation cycles: a randomized trial. Hum Reprod. 2010 Jul;25(7):1684-90. doi: 10.1093/humrep/deq112. PMID: 20457669.

131. Reindollar RH, Regan MM, Neumann PJ, et al. A randomized clinical trial to evaluate optimal treatment for unexplained infertility: the fast track and standard treatment (FASTT) trial. Fertil Steril. 2010 Aug;94(3):888-99. doi: 10.1016/j.fertnstert.2009.04.022. PMID: 19531445.

132. Badawy A, Shokeir T, Allam AF, et al. Pregnancy outcome after ovulation induction with aromatase inhibitors or clomiphene citrate in unexplained infertility. Acta Obstet Gynecol Scand. 2009;88(2):187-91. doi: 10.1080/00016340802638199. PMID: 19089782.

133. Kansal Kalra S, Ratcliffe S, Gracia CR, et al. Randomized controlled pilot trial of luteal phase recombinant FSH stimulation in poor responders. Reprod Biomed Online. 2008 Dec;17(6):745-50. PMID: 19079956.

134. Oyesanya OA, Olufowobi O, Ross W, et al. Prognosis of oocyte donation cycles: a prospective comparison of the in vitro fertilization-embryo transfer cycles of recipients who used shared oocytes versus those who used altruistic donors. Fertil Steril. 2009 Sep;92(3):930-6. doi: 10.1016/j.fertnstert.2008.07.1769. PMID: 18829002. 
135. Erdem A, Erdem M, Atmaca S, et al. Impact of luteal phase support on pregnancy rates in intrauterine insemination cycles: a prospective randomized study. Fertil Steril. 2009 Jun;91(6):2508-13. doi: 10.1016/j.fertnstert.2008.04.029. PMID: 18692788.

136. Bhattacharya S, Harrild K, Mollison J, et al. Clomifene citrate or unstimulated intrauterine insemination compared with expectant management for unexplained infertility: pragmatic randomised controlled trial. BMJ. 2008;337:a716. doi: 10.1136/bmj.a716. PMID: 18687718.

137. Gregoriou O, Vlahos NF, Konidaris S, et al. Randomized controlled trial comparing superovulation with letrozole versus recombinant follicle-stimulating hormone combined with intrauterine insemination for couples with unexplained infertility who had failed clomiphene citrate stimulation and intrauterine insemination. Fertil Steril. 2008 Sep;90(3):678-83. doi: 10.1016/j.fertnstert.2007.06.099. PMID: 17961561.

138. Morad AWA, Abdelhamid AA. Prospective randomized study for hydrotubation with or without lidocaine before intrauterine insemination in unexplained infertility. Middle East Fertility Society Journal. 2012;17(4):250-5.

139. Gibreel A, Badawy A, El-Refai W, et al. Endometrial scratching to improve pregnancy rate in couples with unexplained subfertility: A randomized controlled trial. J Obstet Gynaecol Res. 2013;39(3):680-4.

140. Ebrahimi M, Asbagh FA, Darvish S. The effect of luteal phase support on pregnancy rates of the stimulated intrauterine insemination cycles in couples with unexplained infertility. International Journal of Fertility and Sterility. 2010;4(2):51-6.

141. Demirol A, Gurgan T. Comparison of different gonadotrophin preparations in intrauterine insemination cycles for the treatment of unexplained infertility: a prospective, randomized study. Hum Reprod. 2007 Jan;22(1):97-100. doi: 10.1093/humrep/del335. PMID: 16954409.
142. Zarei A, Mahboubi M, Parsanezhad ME, et al. Effects of piroxicam administration on pregnancy outcome in intrauterine insemination (IUI) cycles: a randomized clinical trial. Clin Exp Obstet Gynecol. 2016;43(2):225-9. PMID: 27132415.

143. Khosravi D, Taheripanah R, Taheripanah A, et al. Comparison of oral dydrogesterone with vaginal progesteronefor luteal support in IUI cycles: a randomized clinical trial. Iran J Reprod Med. 2015 Jul;13(7):433-8. PMID: 26494991.

144. Diamond MP, Legro RS, Coutifaris C, et al. Letrozole, Gonadotropin, or Clomiphene for Unexplained Infertility. Acta Obstet Gynecol Scand. 2015 Sep 24;373(13):123040. doi: 10.1111/aogs.12781; 10.1056/NEJMoa1414827. PMID: 26398071.

145. Nada AM, ElSetohy KA, Banat MM, et al. Antagonist protocol versus clomiphene in unexplained infertility: A randomized controlled study. Taiwan J Obstet Gynecol. 2016 Jun;55(3):326-30. doi: 10.1016/j.tjog.2016.04.006. PMID: 27343309.

146. Youssef MA, van Wely M, Al-Inany H, et al. A mild ovarian stimulation strategy in women with poor ovarian reserve undergoing IVF: a multicenter randomized non-inferiority trial. Hum Reprod. 2016 Nov 11doi: 10.1093/humrep/dew282. PMID: 27836979.

147. Dhalwani NN, Boulet SL, Kissin DM, et al. Assisted reproductive technology and perinatal outcomes: conventional versus discordant-sibling design. Fertil Steril. 2016;106(3):710-6.e2.

148. Selman H, Rinaldi L. Effectiveness of corifollitropin alfa used for ovarian stimulation of poor responder patients. International Journal of Women's Health. 2016;8:609-15.

149. Farquhar CM, Liu E, Armstrong S, et al. Intrauterine insemination with ovarian stimulation versus expectant management for unexplained infertility (TUI): a pragmatic, open-label, randomised, controlled, two-centre trial. Lancet. 2018 Feb 3;391(10119):441-50. doi: 10.1016/s0140-6736(17)32406-6. PMID: 29174128. 
150. Levi Dunietz G, Holzman C, Zhang Y, et al. Assisted Reproductive Technology and Newborn Size in Singletons Resulting from Fresh and Cryopreserved Embryos Transfer. PLoS One. 2017;12(1):e0169869. doi: 10.1371/journal.pone.0169869. PMID: 28114395.

151. Nandi A, Bhide P, Hooper R, et al. Intrauterine insemination with gonadotropin stimulation or in vitro fertilization for the treatment of unexplained subfertility: a randomized controlled trial. Fertil Steril. 2017 Jun;107(6):1329-35.e2. doi: 10.1016/j.fertnstert.2017.03.028. PMID: 28501361.

152. Pourali L, Ayati S, Tavakolizadeh S, et al. Clomiphene citrate versus letrozole with gonadotropins in intrauterine insemination cycles: A randomized trial. Int J Reprod Biomed (Yazd). 2017 Jan;15(1):49-54. PMID: 28280800.

153. van Rijswijk J, Caanen MR, Mijatovic V, et al. Immobilization or mobilization after IUI: an RCT. Hum Reprod. 2017 Nov 1;32(11):2218-24. doi: 10.1093/humrep/dex302. PMID: 29040538.

154. Jahromi BN, Sadeghi S, Alipour S, et al. Effect of melatonin on the outcome of assisted reproductive technique cycles in women with diminished ovarian reserve: A double-blinded randomized clinical trial. Iranian Journal of Medical Sciences. 2017;42(1):73-8.

155. Danhof NA, van Wely M, Repping S, et al. Follicle stimulating hormone versus clomiphene citrate in intrauterine insemination for unexplained subfertility: a randomized controlled trial. Hum Reprod. 2018 Oct 1;33(10):1866-74. doi: 10.1093/humrep/dey268. PMID: 30137325.

156. Maher MA, Sayyed TM, Elkhouly N. Cervical mucus removal prior to intrauterine insemination: a randomized trial. BJOG. 2018 Jun;125(7):841-7. doi: 10.1111/14710528.15003. PMID: 29078018.

157. Harira M. Use of Letrozole versus clomiphene-estradiol for treating infertile women with unexplained infertility not responding well to clomiphene alone, comparative study. Middle East Fertility Society Journal. 2018doi: 10.1016/j.mefs.2018.05.008.
158. Yu R, Jin H, Huang X, et al. Comparison of modified agonist, mild-stimulation and antagonist protocols for in vitro fertilization in patients with diminished ovarian reserve. J Int Med Res. 2018;46(6):2327-37. doi: 10.1177/0300060518770346.

159. Liu A, Zheng C, Lang J, et al. Letrozole versus clomiphene citrate for unexplained infertility: a systematic review and metaanalysis. J Obstet Gynaecol Res. 2014 May;40(5):1205-16. doi: 10.1111/jog.12393. PMID: 24754848.

160. Veltman-Verhulst SM, Cohlen BJ, Hughes $\mathrm{E}$, et al. Intra-uterine insemination for unexplained subfertility. Cochrane Database Syst Rev. 2012;9:Cd001838. doi: 10.1002/14651858.CD001838.pub4. PMID: 22972053.

161. Kuzmin A, Linde V. Diagnostic and remedial capability of transcervical falloposcopy in conjunction with laparoscopy. Gynecol Endocrinol. 2014 Oct;30 Suppl 1:17-9. doi: 10.3109/09513590.2014.945771. PMID: 25200821.

162. Verhoeve HR, Moolenaar LM, Hompes P, et al. Cost-effectiveness of tubal patency tests. BJOG. 2013 Apr;120(5):583-93. doi: 10.1111/1471-0528.12121. PMID: 23331951.

163. Dreyer K, Lier MC, Emanuel MH, et al. Hysteroscopic proximal tubal occlusion versus laparoscopic salpingectomy as a treatment for hydrosalpinges prior to IVF or ICSI: an RCT. Hum Reprod. 2016 Sep;31(9):2005-16. doi: 10.1093/humrep/dew050. PMID: 27209341.

164. Grimstad FW, Nangia AK, Luke B, et al. Use of ICSI in IVF cycles in women with tubal ligation does not improve pregnancy or live birth rates. Hum Reprod. 2016 Dec;31(12):2750-5. doi: 10.1093/humrep/dew247. PMID: 27738114.

165. Qu F, Wang FF, Wu Y, et al.

Transcutaneous Electrical Acupoint Stimulation Improves the Outcomes of In Vitro Fertilization: A Prospective, Randomized and Controlled Study. Explore (NY). 2017 Sep - Oct;13(5):306-12. doi: 10.1016/j.explore.2017.06.004. PMID: 28915981. 
166. La Sala GB, Nicoli A, Fornaciari E, et al. Intracytoplasmic morphologically selected sperm injection versus conventional intracytoplasmic sperm injection: a randomized controlled trial. Reprod Biol Endocrinol. 2015;13(1):97. doi: 10.1186/s12958-015-0096-y. PMID: 26307050.

167. Spaan M, van den Belt-Dusebout AW, Schaapveld M, et al. Melanoma risk after ovarian stimulation for in vitro fertilization. Hum Reprod. 2015 May;30(5):1216-28. doi: 10.1093/humrep/dev023. PMID: 25743782.

168. Boulet SL, Mehta A, Kissin DM, et al. Trends in use of and reproductive outcomes associated with intracytoplasmic sperm injection. JAMA. 2015 Jan 20;313(3):25563. doi: 10.1001/jama.2014.17985. PMID: 25602996.

169. Razi MH, Halvaei I, Razi Y. Laser assisted zona hatching does not improve live birth rate in patients undergoing their first ICSI cycles. Iran J Reprod Med. 2013 Dec;11(12):1021-6. PMID: 24639729.

170. Leandri RD, Gachet A, Pfeffer J, et al. Is intracytoplasmic morphologically selected sperm injection (IMSI) beneficial in the first ART cycle? a multicentric randomized controlled trial. Andrology. 2013 Sep;1(5):692-7. doi: 10.1111/j.20472927.2013.00104.x. PMID: 23788532.

171. Tsai CC, Huang FJ, Wang LJ, et al. Clinical outcomes and development of children born after intracytoplasmic sperm injection (ICSI) using extracted testicular sperm or ejaculated extreme severe oligo-asthenoteratozoospermia sperm: a comparative study. Fertil Steril. 2011 Sep;96(3):567-71. doi: 10.1016/j.fertnstert.2011.06.080. PMID: 21880275.

172. Nangia AK, Luke B, Smith JF, et al. National study of factors influencing assisted reproductive technology outcomes with male factor infertility. Fertil Steril. 2011 Sep;96(3):609-14. doi: 10.1016/j.fertnstert.2011.06.026. PMID: 21733503.
173. Balaban B, Yakin K, Alatas C, et al. Clinical outcome of intracytoplasmic injection of spermatozoa morphologically selected under high magnification: a prospective randomized study. Reprod Biomed Online. 2011 May;22(5):472-6. doi: 10.1016/j.rbmo.2010.11.003. PMID: 21324747.

174. Belva F, Bonduelle M, Schiettecatte J, et al. Salivary testosterone concentrations in pubertal ICSI boys compared with spontaneously conceived boys. Hum Reprod. 2011 Feb;26(2):438-41. doi: 10.1093/humrep/deq345. PMID: 21138905.

175. Hershko-Klement A, Sukenik-Halevy R, Biron Shental T, et al. Intracytoplasmic morphologically selected sperm injection and congenital birth defects: a retrospective cohort study. Andrology. 2016 Sep;4(5):887-93. doi: 10.1111/andr.12221. PMID: 27317040.

176. Hajizadeh Maleki B, Tartibian B. Moderate aerobic exercise training for improving reproductive function in infertile patients: A randomized controlled trial. Cytokine. 2017 Apr;92:55-67. doi: 10.1016/j.cyto.2017.01.007. PMID: 28092795.

177. Shi Y, Sun Y, Hao C, et al. Transfer of Fresh versus Frozen Embryos in Ovulatory Women. N Engl J Med. 2018 Jan 11;378(2):126-36. doi: 10.1056/NEJMoa1705334. PMID: 29320646.

178. Xiong X, Dickey RP, Buekens P, et al. Use of Intracytoplasmic Sperm Injection and Birth Outcomes in Women Conceiving through In Vitro Fertilization. Paediatr Perinat Epidemiol. 2017 Mar;31(2):108-15. doi: 10.1111/ppe.12339. PMID: 28140471.

179. Rahman A, Francomano D, Sagnella F, et al. The effect on clinical results of adding recombinant LH in late phase of ovarian stimulation of patients with repeated implantation failure: A pilot study. Eur Rev Med Pharmacol Sci. 2017;21(23):5485-90. 
180. Teixeira DM, Barbosa MA, Ferriani RA, et al. Regular (ICSI) versus ultra-high magnification (IMSI) sperm selection for assisted reproduction. Cochrane Database Syst Rev. 2013;7:Cd010167. doi: http://dx.doi.org/10.1002/14651858.CD0101 67.pub2. PMID: 23884963.

181. Showell MG, Mackenzie-Proctor R, Brown $\mathrm{J}$, et al. Antioxidants for male subfertility. Cochrane Database Syst Rev. 2014;12:Cd007411. doi: 10.1002/14651858.CD007411.pub3. PMID: 25504418.

182. Sismanoglu A, Tekin HI, Erden HF, et al. Ovulation triggering with GnRH agonist vs. hCG in the same egg donor population undergoing donor oocyte cycles with $\mathrm{GnRH}$ antagonist: a prospective randomized crossover trial. J Assist Reprod Genet. 2009 May;26(5):251-6. doi: 10.1007/s10815-0099326-6. PMID: 19629674.

183. Bodri D, Guillen JJ, Polo A, et al. Complications related to ovarian stimulation and oocyte retrieval in 4052 oocyte donor cycles. Reprod Biomed Online. 2008 Aug;17(2):237-43. PMID: 18681998.

184. Bodri D, Guillen JJ, Galindo A, et al. Triggering with human chorionic gonadotropin or a gonadotropin-releasing hormone agonist in gonadotropin-releasing hormone antagonist-treated oocyte donor cycles: findings of a large retrospective cohort study. Fertil Steril. 2009

Feb;91(2):365-71. doi: 10.1016/j.fertnstert.2007.11.049. PMID: 18367175.

185. Maxwell KN, Cholst IN, Rosenwaks Z. The incidence of both serious and minor complications in young women undergoing oocyte donation. Fertil Steril. 2008 Dec;90(6):2165-71. doi: 10.1016/j.fertnstert.2007.10.065. PMID: 18249368.

186. Kramer W, Schneider J, Schultz N. US oocyte donors: a retrospective study of medical and psychosocial issues. Hum Reprod. 2009 Dec;24(12):3144-9. doi: 10.1093/humrep/dep309. PMID: 19729378.
187. Brinton LA, Moghissi KS, Scoccia B, et al. Effects of fertility drugs on cancers other than breast and gynecologic malignancies. Fertil Steril. 2015 Jul 29;104(4):980-8. doi: 10.1016/j.fertnstert.2015.06.045. PMID: 26232746.

188. Kissin DM, Zhang Y, Boulet SL, et al. Association of assisted reproductive technology (ART) treatment and parental infertility diagnosis with autism in ARTconceived children. Hum Reprod. 2015 Feb;30(2):454-65. doi: 10.1093/humrep/deu338. PMID: 25518976.

189. Brinton LA, Scoccia B, Moghissi KS, et al. Long-term relationship of ovulationstimulating drugs to breast cancer risk. Cancer Epidemiol Biomarkers Prev. 2014 Apr;23(4):584-93. doi: 10.1158/10559965.epi-13-0996. PMID: 24700523.

190. Brinton LA, Westhoff CL, Scoccia B, et al. Fertility drugs and endometrial cancer risk: results from an extended follow-up of a large infertility cohort. Hum Reprod. 2013 Oct;28(10):2813-21. doi: 10.1093/humrep/det323. PMID: 23943795.

191. van Leeuwen FE, Klip H, Mooij TM, et al. Risk of borderline and invasive ovarian tumours after ovarian stimulation for in vitro fertilization in a large Dutch cohort. Hum Reprod. 2011 Dec;26(12):3456-65. doi: 10.1093/humrep/der322. PMID: 22031719.

192. Schendelaar P, Middelburg KJ, Bos AF, et al. The Groningen ART cohort study: the effects of ovarian hyperstimulation and the IVF laboratory procedures on neurological condition at 2 years. Hum Reprod. 2011 Mar;26(3):703-12. doi: 10.1093/humrep/deq377. PMID: 21227942.

193. Luke B, Stern JE, Kotelchuck M, et al. Birth Outcomes by Infertility Treatment: Analyses of the Population-Based Cohort: Massachusetts Outcomes Study of Assisted Reproductive Technologies (MOSART). J Reprod Med. 2016 Mar-Apr;61(3-4):114-27. PMID: 27172633. 
194. Toftager M, Bogstad J, Bryndorf T, et al. Risk of severe ovarian hyperstimulation syndrome in GnRH antagonist versus GnRH agonist protocol: RCT including 1050 first IVF/ICSI cycles. Hum Reprod. 2016 Jun;31(6):1253-64. doi: 10.1016/j.fertnstert.2016.03.037. PMID: 27060174.

195. Spaan M, van den Belt-Dusebout AW, Burger CW, et al. Risk of Colorectal Cancer After Ovarian Stimulation for In Vitro Fertilization. Clin Gastroenterol Hepatol. 2016 May;14(5):729-37.e5. doi: 10.1016/j.cgh.2015.12.018. PMID: 26687912.

196. Chang J, Boulet SL, Jeng G, et al. Outcomes of in vitro fertilization with preimplantation genetic diagnosis: an analysis of the United States Assisted Reproductive Technology Surveillance Data, 2011-2012. Fertil Steril. 2016 Feb;105(2):394-400. doi: 10.1016/j.fertnstert.2015.10.018. PMID: 26551441.

197. Boulet SL, Kirby RS, Reefhuis J, et al. Assisted reproductive technology and birth defects among liveborn infants in Florida, Massachusetts, and Michigan, 2000-2010. JAMA Pediatrics. 2016;170(6).

198. Londra L, Moreau C, Strobino D, et al. Is the type of gonadotropin-releasing hormone suppression protocol for ovarian hyperstimulation associated with ectopic pregnancy in fresh autologous cycles for in vitro fertilization? Fertil Steril. 2016;106(3):666-72.

199. Provost MP, Thomas SM, Yeh JS, et al. State Insurance Mandates and Multiple Birth Rates After In Vitro Fertilization. Obstet Gynecol. 2016.

200. Barad DH, Darmon SK, Kushnir VA, et al. Impact of preimplantation genetic screening on donor oocyte-recipient cycles in the United States. Am J Obstet Gynecol. 2017 Nov;217(5):576.e1-.e8. doi: 10.1016/j.ajog.2017.07.023. PMID: 28735705.
201. Crawford S, Boulet SL, Kawwass JF, et al. Cryopreserved oocyte versus fresh oocyte assisted reproductive technology cycles, United States, 2013. Fertil Steril. 2017 Jan;107(1):110-8. doi: 10.1016/j.fertnstert.2016.10.002. PMID: 27842997.

202. Knudtson JF, Failor CM, Gelfond JA, et al. Assisted hatching and live births in firstcycle frozen embryo transfers. Fertil Steril. 2017 Oct;108(4):628-34. doi: 10.1016/j.fertnstert.2017.07.011. PMID: 28863938.

203. Mancuso AC, Boulet SL, Duran E, et al. Elective single embryo transfer in women less than age 38 years reduces multiple birth rates, but not live birth rates, in United States fertility clinics. Fertil Steril. 2016 Oct;106(5):1107-14. doi: 10.1016/j.fertnstert.2016.06.017. PMID: 27376458.

204. Litzky JF, Boulet SL, Esfandiari N, et al. Effect of frozen/thawed embryo transfer on birthweight, macrosomia, and low birthweight rates in US singleton infants. Am J Obstet Gynecol. 2018;218(4):433.e1.e10. doi: 10.1016/j.ajog.2017.12.223.

205. Toftager M, Bogstad J, Lossl K, et al. Cumulative live birth rates after one ART cycle including all subsequent frozen-thaw cycles in 1050 women: secondary outcome of an RCT comparing GnRH-antagonist and GnRH-agonist protocols. Hum Reprod. 2017 Mar 1;32(3):556-67. doi: 10.1093/humrep/dew358. PMID: 28130435.

206. Wang ET, Kathiresan ASQ, Bresee C, et al. Abnormal implantation after fresh and frozen in vitro fertilization cycles. Fertil Steril. 2017 May;107(5):1153-8. doi: 10.1016/j.fertnstert.2017.03.012. PMID: 28433367.

207. Magnusson A, Wennerholm UB, Kallen K, et al. The association between the number of oocytes retrieved for IVF, perinatal outcome and obstetric complications. Hum Reprod. 2018 Oct 1;33(10):1939-47. doi: 10.1093/humrep/dey266. PMID: 30124838. 
208. Peeraer K, Couck I, Debrock S, et al. Frozen-thawed embryo transfer in a natural or mildly hormonally stimulated cycle in women with regular ovulatory cycles: a RCT. Hum Reprod. 2015 Nov;30(11):255262. doi: 10.1093/humrep/dev224. PMID: 26364081.

209. Williams CL, Jones ME, Swerdlow AJ, et al. Risks of ovarian, breast, and corpus uteri cancer in women treated with assisted reproductive technology in Great Britain, 1991-2010: data linkage study including 2.2 million person years of observation. BMJ. 2018 Jul 11;362:k2644. doi: 10.1136/bmj.k2644. PMID: 29997145.

210. Litzky JF, Boulet SL, Esfandiari N, et al. Birthweight in infants conceived through in vitro fertilization following blastocyst or cleavage-stage embryo transfer: a national registry study. J Assist Reprod Genet. 2018;35(6):1027-37. doi: 10.1007/s10815018-1168-7.

211. Dhillon RK, Smith PP, Malhas R, et al. Investigating the effect of ethnicity on IVF outcome. Reprod Biomed Online. 2015 Jun 3;31(3):356-63. doi:

10.1016/j.rbmo.2015.05.015. PMID: 26208448.

212. Adashi EY, Dean LA. Access to and use of infertility services in the United States: framing the challenges. Fertil Steril. 2016 May;105(5):1113-8. doi: 10.1016/j.fertnstert.2016.01.017. PMID: 26826275.

213. Practice Committee of the American Society for Reproductive Medicine. Effectiveness and treatment for unexplained infertility. Fertil Steril. 2006 Nov;86(5 Suppl 1):S1114. doi: 10.1016/j.fertnstert.2006.07.1475. PMID: 17055802.
214. Gliklich RE, Leavy MB, Velentgas P, et al. Identification of Future Research Needs in the Comparative Management of Uterine Fibroid Disease. A Report on the PrioritySetting Process, Preliminary Data Analysis, and Research Plan. Effective Healthcare Research Report No. 31. (Prepared by the Outcome DEcIDE Center, under Contract No. HHSA 290-2005-0035-I, TO5). AHRQ Publication No. 11-EHC023-EF. Rockville, MD: Agency for Healthcare Research and Quality. March 2011. Available at: https://effectivehealthcare.ahrq.gov/topics/ut erine-fibroids-2010/research. Accessed November 13, 2014.

215. Gliklich RE, Leavy MB, Velentgas P, et al. Incorporating stakeholder perspectives in developing a translation table framework for comparative effectiveness research. J Comp Eff Res. 2012 May;1(3):281-92. doi: 10.2217/cer.12.25. PMID: 24237409.

216. Vercellini P, Somigliana E, Cortinovis I, et al. "You can't always get what you want": from doctrine to practicability of study designs for clinical investigation in endometriosis. BMC Womens Health. 2015;15:89. doi: 10.1186/s12905-015-02484. PMID: 26490454.

217. Adamson J, Cockayne S, Puffer S, et al. Review of randomised trials using the postrandomised consent (Zelen's) design. Contemp Clin Trials. 2006 Aug;27(4):30519. doi: 10.1016/j.cct.2005.11.003. PMID: 16455306.

218. Kawwass JF, Monsour M, Crawford S, et al. Trends and outcomes for donor oocyte cycles in the United States, 2000-2010. JAMA. 2013 Dec 11;310(22):2426-34. doi: 10.1001/jama.2013.280924. PMID: 24135860. 


\section{Introduction}

\section{Background}

\section{Condition}

"Infertility" has traditionally been defined as failure to achieve pregnancy after 12 months of regular unprotected intercourse with the same partner (or after 6 months for women greater than 35 years of age). However, as many as half of such couples will conceive without intervention over the next 12-24 months. Because of this, the term "subfertility" is preferred by many. ${ }^{1}$ From a population perspective, couples who meet the dichotomous criteria for "infertility" include couples who are "normal" but who are in the upper end of the population distribution for "time to pregnancy", and couples who have a physiological or anatomical cause for a prolonged time to pregnancy. However, to be concise, we use the term "infertility" throughout this report.

Self-reported infertility in the United States, using the 12-month definition, affected approximately 6 percent of married women aged 15-44 in the 2006-2010 National Survey of Family Growth (the most recent available data). ${ }^{2}$ In one population-based study, approximately 10 percent of pregnant women reported receiving infertility treatment, with 29 percent of these women using fertility-enhancing medications; 21 percent using assisted reproductive technology (ART), including in vitro fertilization (IVF); 15 percent using artificial insemination with fertility-enhancing drugs; and 23 percent using other treatments, including surgery. ${ }^{3}$ Other estimates of the prevalence of infertility treatment are similar. ${ }^{4-8}$ Particularly in the US, where availability of infertility services is variable depending on a number of factors, particularly insurance coverage, utilization of infertility treatments may underestimate the overall burden of infertility.

The most common demographic factor associated with female infertility is "advanced reproductive age"; although the probability of pregnancy begins to decline by the mid-20's, the slope of decline sharply increases by age $35 .{ }^{9}$ For example, the prevalence of "unexplained infertility" (infertility with no other documented female or male diagnosis) is substantially higher in older women, ${ }^{10}$ and "diminished ovarian reserve," which is most commonly associated with increased age, is the single most common diagnosis among women undergoing ART, accounting for 27.5 percent of cycles. ${ }^{11}$ Other common causes of female infertility include polycystic ovary syndrome (PCOS), endometriosis, and occlusion of the fallopian tubes from prior infectious disease. ${ }^{6}$ A growing number of women also experience infertility secondary to cancer treatment. ${ }^{12-14}$. Although there are other potentially treatable causes of infertility (conditions other than PCOS which affect ovulatory function, congenital uterine anomalies, uterine fibroids), this review is based on the most common conditions, based on input from our Technical Expert Panel.

Based on estimates of patients attending ART clinics, isolated male factor infertility affects approximately 17 percent of couples seeking treatment, with 34.6 percent of couples having both male and female diagnoses. ${ }^{15}$

\section{Treatment Strategies}

Treatment options are usually dependent on the underlying etiology of infertility. For female causes, options include surgical management of tubal occlusion, surgical treatment of endometriosis, ovarian “drilling” for treatment of PCOS, use of ovulation-induction agents 
including oral (clomiphene citrate or letrozole) and injected drugs (gonadotropins), artificial insemination with either partner or donor sperm (depending on partner fertility status), and ART, which includes both traditional IVF (fertilization of the egg by the sperm occurs without direct manipulation) and IVF with intra-cytoplasmic sperm injection (ICSI) (fertilization occurs via direct injection of sperm into the egg). ${ }^{16,17}$ Treatment options for male factor infertility include medical treatment of a diagnosed endocrinopathy or other conditions affecting sperm production, empiric treatments with hormonal or other agents, surgical management of varicocele, intrauterine insemination, IVF, ICSI, or use of donor sperm. ${ }^{18}$ Options appropriate for some diagnoses (e.g., ovulation induction in PCOS or unexplained infertility) may not be appropriate for others (e.g., women with documented tubal occlusion). In other cases, the appropriate comparisons may involve sequencing or combinations of treatment options-for example, one strategy might consist of several cycles of ovulation induction, followed by ART only if pregnancy does not occur, compared to proceeding directly to ART. Note that throughout this report, we use the term "adjunct treatments" to refer to interventions performed within a major treatment category (for example, comparison of metformin to placebo as pretreatment in women with PCOS undergoing IVF).

\section{Benefits}

There has been ongoing debate about the most appropriate outcome for evaluation of infertility treatments - ovulation (in anovulatory women such as PCOS patients), pregnancy, live birth, or term live birth. ${ }^{19-23}$ However, there is a growing consensus that live birth is the most important patient-centered outcome. ${ }^{22,23}$ Trade-offs in outcomes (particularly multiple gestations), time to pregnancy, and out-of-pocket costs might be different among the various treatment strategies even if cumulative live birth rates are identical.

\section{Harms}

Different treatments also carry different safety risks. There are known short-term risks such as ovarian hyperstimulation syndrome (OHSS) or acute risks associated with any surgery. Surgery may have additional longer-term risks which may affect subsequent fertility (such as scarring or decreased ovarian reserve with procedures such as laparoscopic ovarian drilling (LOD). The literature suggests that observed associations between infertility treatment and female reproductive cancers, particularly ovarian cancer, are likely the result of the underlying infertility rather than treatment itself. There is, however, some uncertainty surrounding some cancer outcomes in subgroups of patients. ${ }^{24-26}$

Some adverse pregnancy outcomes, such as preterm birth, are associated with infertility treatment; however, many of the conditions associated with infertility are also associated with these adverse outcomes, complicating assessment of comparative effectiveness. ${ }^{19,21,27,28}$ There may also be treatments that have unclear effects on the long-term health in children born following these treatments For example, there is the possibility that epigenetic changes from treatments such as IVF/ICSI may lead to increased risk of some disorders (e.g., BeckwithWiedemann syndrome) later in life-or that an increase in multiple births or other causes of prematurity or fetal growth restriction/low birthweight from treatments may result in poor neurodevelopmental outcomes. ${ }^{29,30}$ Finally, infertility clearly has an emotional impact, ${ }^{14,31,32}$ and the comparative effects of infertility treatments on quality of life are an important consideration for both women and men. 
There may be significant variation in outcomes of different treatments in specific subpopulations. For example, age affects the likelihood of conception, and the risk of many pregnancy complications associated with infertility treatments, such as preterm birth or low birthweight, are also increased with higher maternal age. Obesity is common in women with PCOS, and, like older maternal age, is also associated with adverse pregnancy outcomes independent of its association with infertility. The utilization and outcomes of infertility treatment differ among different racial and ethnic groups, even after adjusting for insurance coverage. $^{33-36}$

Finally, a unique subpopulation is women who donate oocytes for use by other couples in ART. An increasing number of women undergoing ART are receiving donor oocytes, ${ }^{37}$ and there are almost no data on the long-term safety of multiple courses of ovulation induction for the purposes of oocyte donation. ${ }^{38}$ In addition, there are complex ethical and legal considerations, including the balance between fair compensation and inducement, ${ }^{39}$ and sharing information about donors with recipients. ${ }^{40}$

\section{Complexity of Decision Making for Treatments of Infertility}

Infertility treatment is a topic where decision making is particularly complex for patients, clinicians, and policymakers. Decision making involves both partners (although the intensity and risks of treatment are quite different), consideration of outcomes for both parents and infants over short- and long-term time frames, trade-offs between short-term success and long-term adverse outcomes, and in some cases preferences for process as well as outcome. In addition, time is an important consideration, particularly for women aged 35 and older. There is clear variation in patient preferences for different treatments and outcomes, and there has been relatively little empirical work focused on the decision-making aspects of infertility treatment. There are large differences in the costs of different infertility treatments and variation in the degree of coverage for infertility diagnosis and treatment, and many patients face significant outof-pocket costs. ${ }^{41}$ There is substantial evidence that the availability of coverage affects access to treatment and treatment choices. ${ }^{42-45}$ Time lost from work may also be a consideration (particularly in the context of the need to make out-of-pocket payments).

There are a number of areas where controversy or uncertainty about the evidence adds to the difficulty of decision making. For example, the optimal trade-off between ART success and the risk of preterm birth and long-term health outcomes (such as neurodevelopmental problems) in infants associated with the number of embryos transferred is unclear. All things being equal, transfer of more embryos results in both a greater chance of success in a given ART cycle and a greater chance of multiple pregnancies - single-embryo transfer greatly reduces the chance of multiple gestation, but may require more cycles to achieve a pregnancy. ${ }^{46,47}$ Other areas of uncertainty include optimal timing of embryo transfer ${ }^{48}$ and use of fresh versus frozen embryos, ${ }^{49,50}$ in terms of both achieving pregnancy and outcomes of those pregnancies, as well as timing of ART relative to other options, especially since the risk of higher order multiples (triplets or higher) is greater with ovulation induction, although ART is more invasive and expensive on a per-cycle basis. ${ }^{51-53}$

\section{Limitations of the Evidence Base}

Methodological limitations of the literature contribute to the uncertainty. For example, the National ART Surveillance System (NASS) is an excellent resource for observational data on U.S. population-based outcomes for ART. However, it has been limited by (a) use of the ART 
cycle (rather than the individual patient) as the unit of analysis; (b) lack of long-term follow-up data for individual patients $\mathrm{s}^{38}$; and (c) some concern about underreporting of some adverse outcomes. ${ }^{54}$ NASS 2.0, introduced at the end of 2016, now includes both unique patient and cycle identifiers, meaning that cumulative success rates per patient should be available in future years. ${ }^{55}$ In addition, mechanisms for capturing outcomes from patients who receive care at multiple clinics have been put into place. On the other hand, randomized controlled trials (RCTs) may not provide data on important long-term outcomes, or may be underpowered to detect clinically relevant differences in complications of treatment.

\section{Scope and Key Questions}

\section{Scope of the Review}

The present review evaluates the comparative safety and effectiveness of available treatment strategies for women of reproductive age (18-44) who are infertile due to PCOS, endometriosis, unknown reasons, or tubal or peritoneal factors; the comparative safety and effectiveness of available treatment strategies for couples with male factor infertility; and the short- and longterm health outcomes of both oocyte and sperm donors. For all questions, we consider only treatment options begun after completion of a diagnostic evaluation.

\section{Key Questions}

The specific Key Questions (KQs) addressed in this review are listed below, and Figure 1 displays the analytic framework that guided our work.

- KQ 1. What are the comparative safety and effectiveness of available treatment strategies for women with polycystic ovary syndrome (PCOS) who are infertile and who wish to become pregnant?

o KQ 1a. Does the optimal treatment strategy vary by patient characteristics such as age, ovarian reserve, race, body mass index (BMI), presence of other potential causes of female infertility, or presence of male factor infertility?

- KQ 2. What are the comparative safety and effectiveness of available treatment strategies for women with endometriosis who are infertile and who wish to become pregnant?

o KQ 2a. Does the optimal treatment strategy vary by patient characteristics such as age, ovarian reserve, race, BMI, stage of endometriosis, presence of other potential causes of female infertility, or presence of male factor infertility?

- KQ 3. What are the comparative safety and effectiveness of available treatment strategies for women who are infertile for unknown reasons and who wish to become pregnant?

o KQ 3a. Does the optimal treatment strategy vary by patient characteristics such as age, ovarian reserve, race, BMI, 
presence of other potential causes of female infertility, or

presence of male factor infertility?

- KQ 4. What are the comparative safety and effectiveness of available treatment strategies for women with tubal or peritoneal factors

(e.g., pelvic adhesions) who are infertile and who wish to become pregnant?

o KQ 4a. Does the optimal treatment strategy vary by patient characteristics such as age, ovarian reserve, race, BMI, presence of other potential causes of female infertility, or presence of male factor infertility?

- KQ 5. What are the comparative safety and effectiveness of available treatment strategies for couples with male factor infertility and no evidence of an underlying diagnosis associated with infertility in the female partner?

- KQ 5a. Does the optimal treatment strategy vary by characteristics in either partner such as age, ovarian reserve, race, or BMI?

- KQ 6. What are the short- and long-term health outcomes of donors in infertility?

o KQ 6a. For female oocyte donors:

3. Do specific aspects of the pre-donation evaluation identify potential donors at greater risk for short- or long-term adverse outcomes (e.g., OHSS, quality-of-life issues)?

4. Do short- and long-term outcomes differ among different stimulation/retrieval protocols?

o KQ 6b. For male semen donors:

1. Are there long-term health, quality-of-life, or other adverse outcomes associated with donation? 
Figure 1. Analytic framework

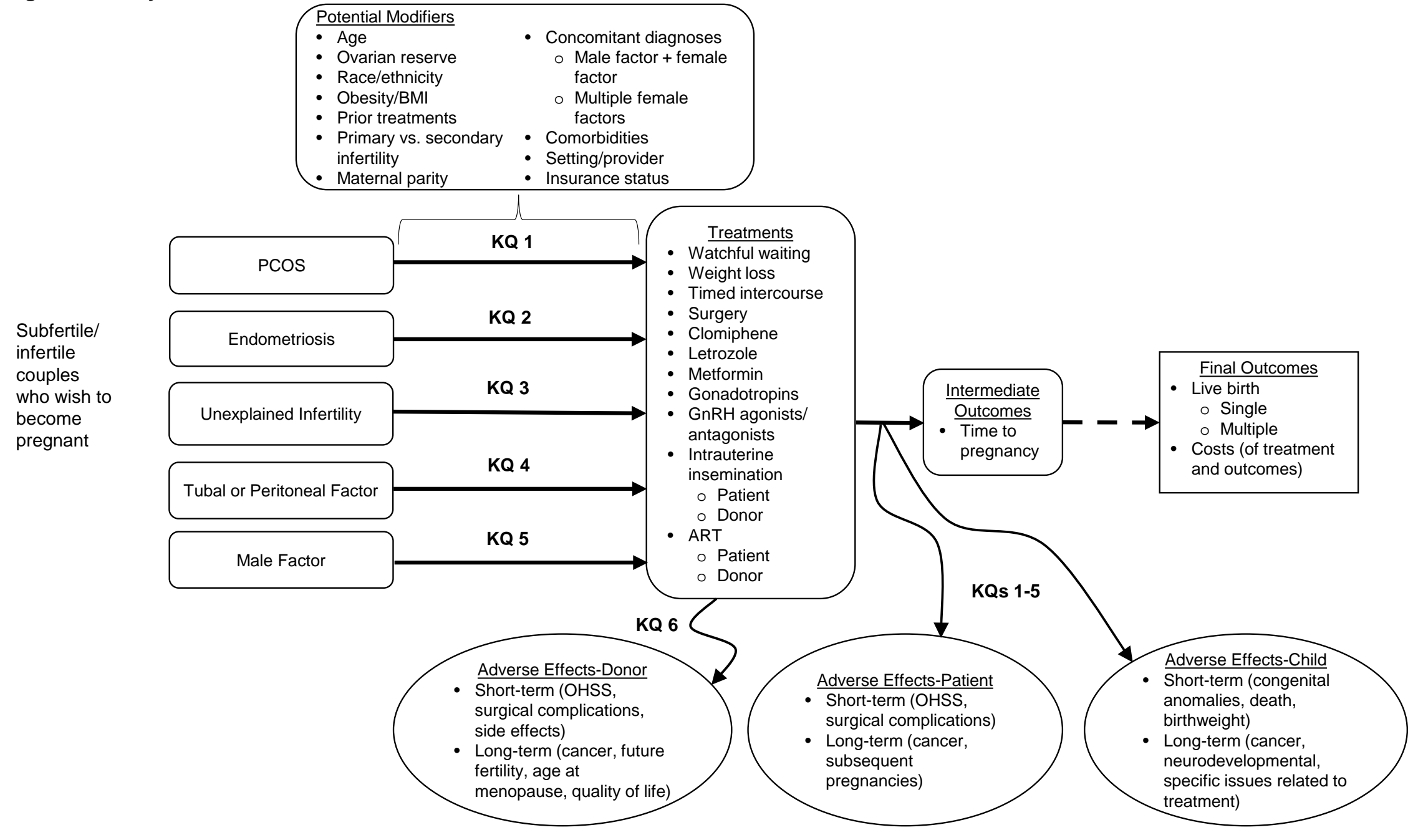

Abbreviations: ART=assisted reproductive technology; BMI=body mass index; GnRH=gonadotropin-releasing hormone; KQ=Key Question; OHSS=ovarian hyperstimulation syndrome; PCOS=polycystic ovary syndrome 
Figure 1 depicts the KQs within the context of the populations, interventions, comparators, outcomes, timings, and settings (PICOTS) considered in this review. The figure illustrates how a wide range of treatments for infertility may result in intermediate outcomes such as time to pregnancy and/or final outcomes such as live birth (single or multiple) or costs in couples with different underlying causes of infertility. A separate KQ focuses on outcomes in female and male donors in infertility. Short- and long-term adverse effects may occur at any point during treatment and may affect donors, patients, and/or children. Optimal treatment strategies may vary by important patient characteristics and/or by setting/provider.

\section{Organization of This Report}

The remainder of the report details our methodology and presents the results of our literature synthesis, with summary tables and strength of evidence grading for major comparisons and outcomes. In the discussion section, we offer our conclusions, summarized findings, and other information that may be relevant to translating this work for clinical practice and future research.

Appendixes provide further details on our methods and the studies we assessed, as follows:

- Appendix A. Exact Search Strings

- Appendix B. Data Abstraction Elements

- Appendix C. List of Included Studies

- Appendix D. List of Excluded Studies

- Appendix E. Characteristics of Included Studies

- Appendix F. AMSTAR Quality Assessment for Systematic Reviews

- Appendix G. Risk of Bias Assessment for Included Studies

- Appendix H. Supplemental Project To Assess the Transparency of Reporting for Trials Evaluating Treatment for Infertility

A list of abbreviations and acronyms is provided at the end of the report. 


\section{Methods}

Methods for this systematic review follow the Agency for Healthcare Research and Quality (AHRQ) Methods Guide for Effectiveness and Comparative Effectiveness Reviews ${ }^{56}$ and the Preferred Reporting Items for Systematic Reviews and Meta-Analyses (PRISMA) checklist. ${ }^{57}$ See the review protocol (http://effectivehealthcare.ahrq.gov/index.cfm/search-for-guidesreviews-and-reports/?productid=2131\&pageaction=displayproduct) for full details.

\section{Topic Refinement and Review Protocol}

The topic of this report and preliminary Key Questions (KQs) arose through a public nomination and initial development by the Scientific Resource Center (SRC) for AHRQ's Effective Health Care (EHC) program. During the subsequent topic refinement phase, a panel of key informants gave input to the Evidence-based Practice Center (EPC) on the KQs to be examined; these KQs were posted on AHRQ's EHC website for public comment in June 2015 for 3 weeks and revised in response to comments. We then drafted a protocol for the systematic review and recruited a Technical Expert Panel (TEP) to provide high-level content and methodological expertise throughout the development of the review. The Key Informants and TEP represented members of medical professional societies and clinician/researchers in the areas of obstetrics and gynecology, assisted reproductive technology, and reproductive medicine; scientific experts; payers; Federal agencies; and patients/consumers. The finalized protocol is posted on the EHC website. ${ }^{58}$ The PROSPERO registration is CRD42016025750.

\section{Literature Search Strategy}

\section{Search Strategy}

To identify relevant published literature, we searched PubMed ${ }^{\circledR}$, Embase $^{\circledR}$, and the Cochrane Database of Systematic Reviews, limiting the searches to studies conducted in adults and published from January 1, 2007, to October 3, 2018. We selected the 2007 start date to establish a one-year overlap with the search dates from a 2008 AHRQ evidence report that assessed assisted reproductive technology (ART).$^{59}$ Also, the key informants felt that existing Cochrane reviews would identify older relevant high-quality studies, particularly evidence from randomized controlled trials (RCTs), while primary studies and other systematic reviews published after 2008 would identify studies most relevant to current practice in infertility. An experienced search librarian guided all searches. The exact search strings used are given in Appendix A.

We supplemented the electronic searches with a manual search. The reference lists for identified pivotal articles ${ }^{60-112}$ were manually searched and cross-referenced against our database, and additional relevant articles not already under consideration were retrieved for screening. All citations were imported into an electronic bibliographical database (EndNote ${ }^{\circledR}$ Version X8; Thomson Reuters, Philadelphia, PA).

To ascertain publication bias in recent studies, we searched ClinicalTrials.gov to identify completed but unpublished studies. We also explored publication bias in any quantitative synthesis of the included literature through meta-analysis techniques. In Appendix $\mathrm{H}$, we use this report to explore in more detail the utility of ClinicalTrials.gov for detecting selective reporting, and the impact of selective reporting on the estimates of treatment effect. Note that these evaluations of publication bias require studies to have been registered on ClinicalTrials.gov or 
published in the peer-reviewed literature and therefore are not able to reflect studies which found no difference or negative results but did not reach one of these outlets.

Approaches to identifying relevant gray literature included notification to stakeholders of the opportunity to submit scientific information packets of material relevant to the KQs. This notification was coordinated by the SRC. We also searched the ClinicalTrials.gov study registry and the World Health Organization (WHO) International Clinical Trials Registry Platform (ICTRP) search portal to identify potentially relevant study records, and subsequently searched for relevant articles from completed studies.

\section{Inclusion and Exclusion Criteria}

We specified our inclusion and exclusion criteria based on the PICOTS (populations, interventions, comparators, outcomes, timing, and settings) identified for each question. Note that the outcomes of interest are ordered in approximate relative importance to patients, based on input from topical experts and Key Informants, rather than temporal occurrence in the clinical pathway. Table 1 lists inclusion and exclusion criteria.

Table 1. Inclusion and exclusion criteria

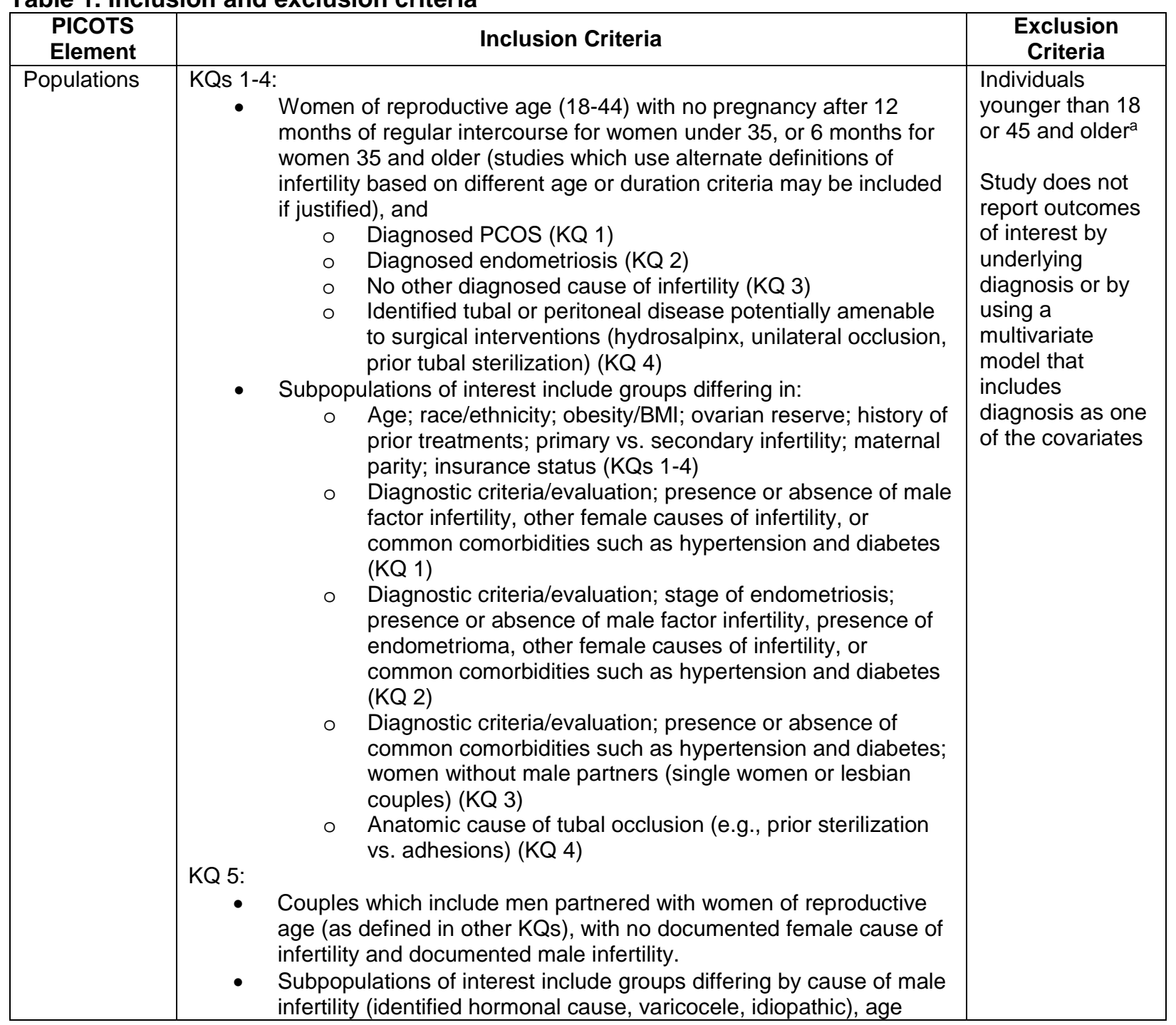




\begin{tabular}{|c|c|c|}
\hline $\begin{array}{l}\text { PICOTS } \\
\text { Element }\end{array}$ & Inclusion Criteria & $\begin{array}{c}\text { Exclusion } \\
\text { Criteria }\end{array}$ \\
\hline & $\begin{array}{ll}\text { (male and female), race/ethnicity, obesity/BMI, history of prior } \\
\text { treatments, primary vs. secondary infertility, diagnostic criteria used } \\
\text { for male infertility, insurance status, and presence or absence of } \\
\text { common comorbidities such as hypertension and diabetes. } \\
\text { KQ 6: } \\
\text { - Women of reproductive age (18-44) who are potential donors of } \\
\text { oocytes for ART, and males donating semen for intrauterine } \\
\text { insemination or ART }\end{array}$ & \\
\hline Interventions & $\begin{array}{l}\text { KQ 1: Clomiphene citrate, letrozole, diet/exercise/other weight loss } \\
\text { strategies, timed intercourse using various technologies in conjunction with } \\
\text { oral ovulation induction, metformin, combination oral medications, ovulation } \\
\text { induction with gonadotropins with or without intrauterine insemination (IUI), } \\
\text { surgery (ovarian drilling), ART (IVF and ICSI) with patient and donor oocytes } \\
\text { KQ 2: Surgical excision of endometriotic implants, alternative surgical } \\
\text { approaches to destruction of lesions (e.g., laser vaporization), gonadotropin- } \\
\text { releasing hormone agonists or antagonists, timed intercourse with various } \\
\text { technologies, ovulation induction with gonadotropins with or without IUI, ART } \\
\text { (IVF and ICSI) with patient and donor oocytes } \\
\text { KQ 3: Timed intercourse with various technologies, oral ovulation induction } \\
\text { agents (e.g., clomiphene citrate), superovulation with gonadotropins with and } \\
\text { without IUI, ART (IVF and ICSI) with patient and donor oocytes, watchful } \\
\text { waiting } \\
\text { KQ 4: Surgical repair, ART (IVF and ICSI) with patient and donor oocytes } \\
\text { KQ 5: ICSI (note that interventions and comparators may vary depending on } \\
\text { underlying cause of male factor infertility), testicular sperm extraction, } \\
\text { vasectomy reversal, surgical repair of varicocele, IUI, donor insemination, } \\
\text { ART, treatment of underlying endocrinopathy } \\
\text { KQ 6: Pre-donation testing strategies; ovulation induction with gonadotropins } \\
\text { using different induction/retrieval protocols; semen donation (men) }\end{array}$ & \\
\hline Comparators & $\begin{array}{l}\text { KQ 1: Any other active intervention (e.g., clomiphene vs. metformin), or } \\
\text { timing/sequence of interventions (e.g., ovulation induction/IUl followed by } \\
\text { ART if unsuccessful vs. proceeding directly to ART, or timed intercourse with } \\
\text { oral medications or injectable gonadotropins) } \\
\text { 2: Either direct between two alternatives (e.g., surgery vs. GnRH } \\
\text { agonists/antagonists), or timing/sequence of interventions (e.g., ovulation } \\
\text { induction/IUI followed by ART if unsuccessful vs. proceeding directly to ART) } \\
\text { KQ 3: Any other active intervention, or timing/sequencing of timing/sequence } \\
\text { of interventions (e.g., ovulation induction/IUI followed by ART if unsuccessful } \\
\text { vs. proceeding directly to ART) } \\
\text { KQ 4: Other active interventions (including combinations of therapy such as } \\
\text { surgical removal of hydrosalpinx followed by ART) } \\
\text { KQ 5: Other active interventions } \\
\text { KQ } 6 \text { (women): Pre-donation testing strategies; controlled ovarian } \\
\text { hyperstimulation with gonadotropins using different induction/retrieval } \\
\text { protocols; non-donors (women and men) }\end{array}$ & \\
\hline Outcomes & $\begin{array}{cc}\text { KQ 1-5: } & \\
\text { - } & \text { Live birth (both cumulative and per cycle) } \\
0 \quad \text { Live singleton birth } \\
0 \quad \text { Live multiple birth } \\
\begin{array}{cc}\text { Pregnancy complications } \\
0 \quad \text { Multiple births (and associated complications) }\end{array} \\
\end{array}$ & \\
\hline
\end{tabular}




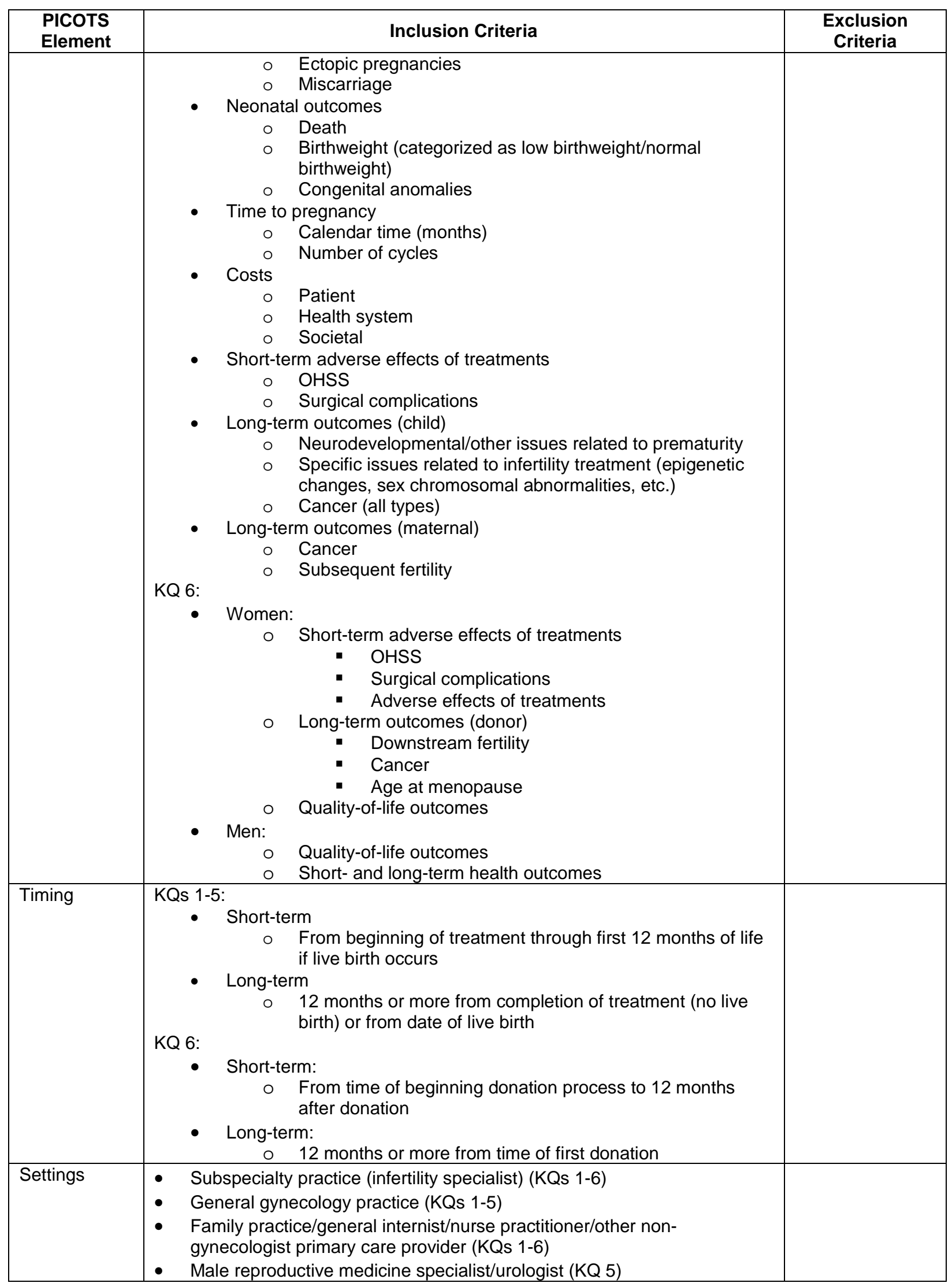




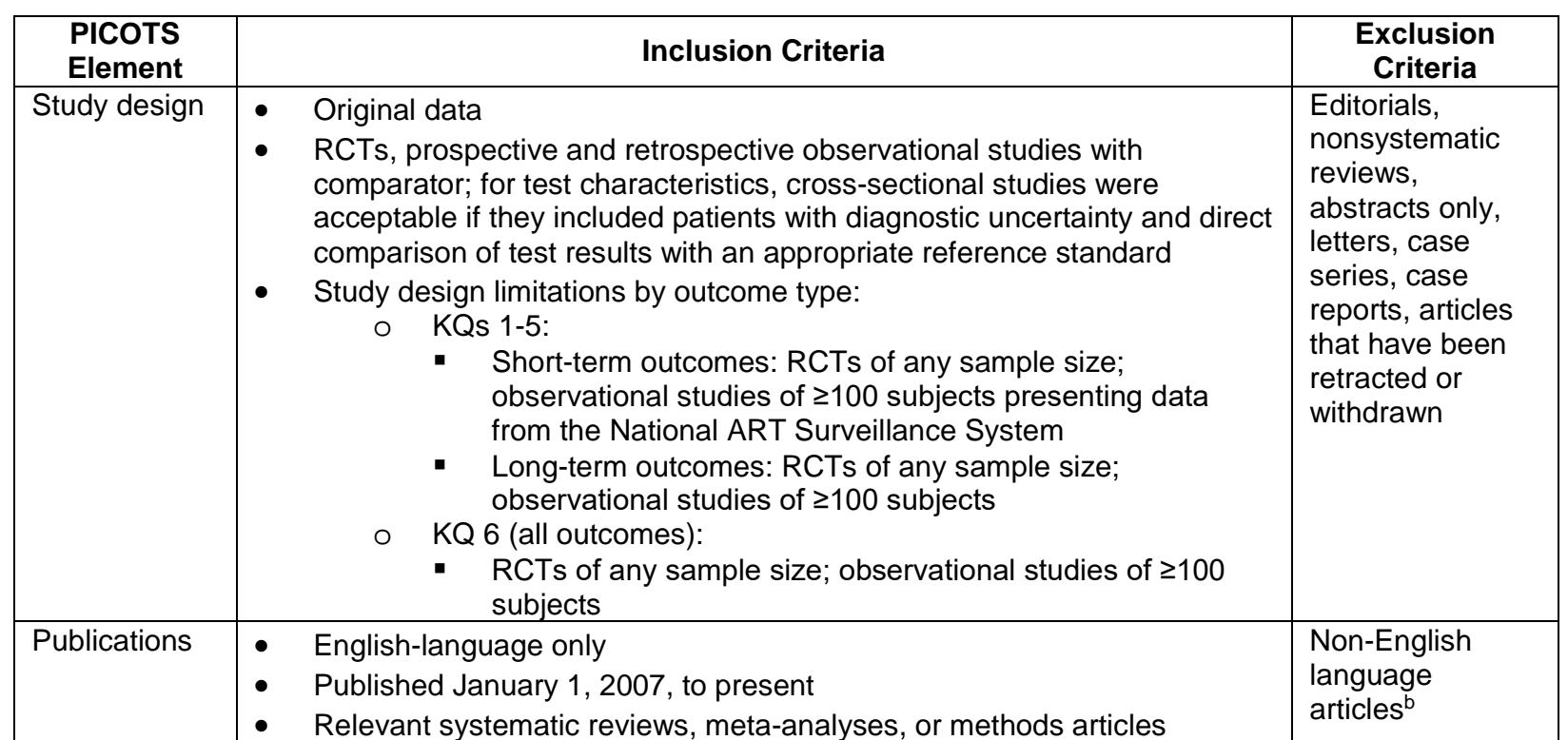

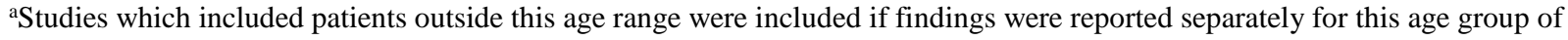
interest or if at least $80 \%$ of women were within this age range. bNon-English articles were excluded due to: (1) the high volume of literature available in English-language publications (including the majority of known important studies); and (2) concerns about the applicability of non-English publication studies to populations in the United States.

Abbreviations: ART=assisted reproductive technology; BMI=body mass index; GnRH=gonadotropin-releasing hormone; hCG=human chorionic gonadotropin; ICSI=intra-cytoplasmic sperm injection; IUI=intrauterine insemination; IVF=in vitro fertilization; KQ=Key Question; OHSS=ovarian hyperstimulation syndrome; PCOS=polycystic ovary syndrome; PICOTS=Populations, Interventions, Comparators, Outcomes, Timing, Settings; RCTs=randomized controlled trials

\section{Study Selection}

For citations retrieved from PubMed, Embase, and the Cochrane Database of Systematic Reviews, two reviewers independently screened each title and abstract for potential relevance to the research questions using prespecified inclusion/exclusion criteria described in Table 1. Articles included by either reviewer underwent full-text screening.

At the full-text screening stage, two reviewers independently reviewed the full text of each article and indicated a decision to include or exclude the article for data abstraction. When paired reviewers arrived at different decisions about whether to include or exclude an article, or about the reason for exclusion, we reconciled the difference through review and discussion among investigators. Articles meeting eligibility criteria were included for data abstraction. All screening results were tracked using the DistillerSR data synthesis software program (Evidence Partners Inc., Manotick, ON, Canada).

Appendix C provides a list of all articles included for data abstraction. Appendix D provides a list of articles excluded at the full-text screening stage, with reasons for exclusion.

While systematic reviews and meta-analyses were not study designs qualifying for inclusion and abstraction under our screening criteria, we did flag relevant articles of these types as part of the screening process. Component references from these systematic reviews were reviewed and when studies met our inclusion criteria, they were included in our report. For systematic reviews which were identified as relevant to the individual KQs but included mostly studies prior to 2007, we summarize the findings from these existing reviews and the consistency of their findings with those from our included studies in the appropriate results sections. 


\section{Data Extraction}

The investigative team created data abstraction forms that were programmed in the DistillerSR software for collection of data from included studies. The abstraction forms were pilot-tested with a sample of included articles to ensure that all relevant data elements were captured and that there was consistency and reproducibility between abstractors. Based on their clinical and methodological expertise, a pair of researchers were assigned to abstract data from each of the eligible articles. One researcher abstracted the data, and the second over-read the article and the accompanying abstraction to check for accuracy and completeness. Disagreements were resolved by consensus or by obtaining a third reviewer's opinion if consensus could not be reached.

We designed the data abstraction forms to collect the data required to evaluate the specified eligibility criteria for inclusion in this review, as well as demographic and other data needed for determining outcomes (intermediate, final, and adverse events outcomes). We paid particular attention to describing the details of the treatment (e.g., for comparisons of in vitro fertilization [IVF] to other therapies, the specific IVF protocol used), patient characteristics (e.g., age of female partners, presence or absence of male factor infertility), setting (e.g., U.S.- vs. non-U.S.based studies), and study design (e.g., RCT vs. observational) that may be related to outcomes. In addition, we described comparators carefully, as treatment standards may have changed during the period covered by the review. The safety outcomes were framed to help identify adverse events, including those from medical therapies (e.g., ovarian hyperstimulation syndrome) and those resulting from procedural complications. Data necessary for assessing quality and applicability, as described in AHRQ's Methods Guide for Effectiveness and Comparative Effectiveness Reviews, ${ }^{56}$ were also abstracted. A complete list of data abstraction elements is provided in Appendix B.

\section{Quality (Risk of Bias) Assessment of Individual Studies}

We assessed methodological quality, or risk of bias, for each individual study using a components approach, assessing each study for specific aspects of design or conduct (such as allocation concealment for RCTs, or use of methods to address potential confounding), as detailed in AHRQ's Methods Guide for Effectiveness and Comparative Effectiveness Reviews. ${ }^{56}$ Briefly, we rated each study as being of good, fair, or poor quality based on its adherence to well-accepted standard methodologies. For each study, one investigator assigned a summary quality rating, which was then reviewed by a second investigator; disagreements were resolved by consensus or by a third investigator if agreement could not be reached. Table 2 describes the overall study quality assessment ratings. Appendix $\mathrm{G}$ presents the risk of bias assessment components for the individual included studies. 
Table 2. Definitions of overall quality ratings

\begin{tabular}{|l|l|}
\hline \multicolumn{1}{|c|}{ Quality Rating } & \multicolumn{1}{c|}{ Description } \\
\hline Good (low risk of bias) & $\begin{array}{l}\text { These studies had the least bias, and the results were considered valid. } \\
\text { These studies adhered to the commonly held concepts of high quality, } \\
\text { including the following: a clear description of the population, setting, } \\
\text { approaches, and comparison groups; appropriate measurement of } \\
\text { outcomes; appropriate statistical and analytical methods and reporting; no } \\
\text { reporting errors; a low dropout rate; and clear reporting of dropouts. }\end{array}$ \\
\hline $\begin{array}{l}\text { Fair (moderate risk of } \\
\text { bias) }\end{array}$ & $\begin{array}{l}\text { These studies were susceptible to some bias, but not enough to invalidate } \\
\text { the results. They did not meet all the criteria required for a rating of good } \\
\text { quality because they had some deficiencies, but no flaw was likely to } \\
\text { cause major bias. The study may have been missing information, making it } \\
\text { difficult to assess limitations and potential problems. }\end{array}$ \\
\hline Poor (high risk of bias) & $\begin{array}{l}\text { These studies had significant flaws that might have invalidated the results. } \\
\text { They had serious errors in design, analysis, or reporting; large amounts of } \\
\text { missing information; or discrepancies in reporting. }\end{array}$ \\
\hline
\end{tabular}

The grading was outcome-specific such that a given study that analyzed its primary outcome well but did an incomplete analysis of a secondary outcome could be assigned a different quality grade for each of the two outcomes. Studies of different designs were graded within the context of their respective designs as good, fair, or poor (Appendix G).

We also rated the quality of systematic reviews that were identified and discussed in the report using AMSTAR (A Measurement Tool to Assess the Methodological Quality of Systematic Reviews). ${ }^{113}$ For each study, one investigator assigned a summary quality rating, a second investigator reviewed the rating; disagreements were resolved by consensus or by a third investigator. Reviews were then assigned overall quality scores according to the following categories $^{114}$ :

- Good (low risk of bias)—Systematic reviews that have few or no methodological shortcomings and a low risk of bias.

- Fair (moderate risk of bias) — Systematic reviews that have some methodological flaws but the investigators conclude that the flaws will not seriously bias or invalidate the results.

- Poor (high risk of bias)_-Systematic reviews that contain a serious flaw or flaws that, in the judgment of the investigators, are highly likely to bias or invalidate the results.

The AMSTAR quality assessment components for the individual systematic reviews are detailed in Appendix F.

\section{Data Synthesis}

We began by summarizing key features of the included studies for each KQ. To the degree that data are available, we abstracted information on study design; patient characteristics; clinical settings; interventions; and intermediate, final, and adverse event outcomes. If not reported, 95percent confidence intervals for dichotomous outcomes (e.g., live birth rates) were calculated from the numbers provided in the study, in order to characterize the degree of precision of a particular estimate. This helped inform grading of the strength of evidence, as well as provided insight about the degree to which lack of statistical power may have affected study conclusions about lack of a treatment effect- 
We then determined the feasibility of completing a quantitative synthesis (i.e., meta-analysis, decision analysis, or simulation model). For a meta-analysis, feasibility depends on the volume of relevant literature (requiring at least three relevant studies), conceptual homogeneity of the studies (similar intervention comparisons and outcome definitions), completeness of the reporting of results, and the adequacy and completeness of any existing meta-analyses.

When the above criteria were met and a meta-analysis was considered appropriate, we used random-effects models within the Comprehensive Meta-Analysis software to synthesize the available evidence quantitatively. We tested for heterogeneity using graphical displays and test statistics (Q and $\mathrm{I}^{2}$ statistics), while recognizing that the ability of statistical methods to detect heterogeneity may be limited. For comparison, we also performed fixed-effect meta-analyses. We present summary estimates, standard errors, and confidence intervals. We anticipated that intervention effects may be heterogeneous. We hypothesized that the methodological quality of individual studies, study type, the characteristics of the comparator, and patients' underlying clinical presentation would be associated with the intervention effects. If there were sufficient studies, we performed subgroup analyses and/or meta-regression analyses to examine these hypotheses. We performed quantitative and qualitative syntheses separately by study type and discuss their consistency qualitatively.

\section{Strength of the Body of Evidence}

We rated strength of evidence using the approach described in the AHRQ Methods Guide for Effectiveness and Comparative Effectiveness Reviews. ${ }^{56,115,116}$ We graded the strength of evidence separately for each outcome; thus, the strength of evidence for two separate outcomes in a given study may be graded differently. The grades are presented in the strength of evidence tables throughout the report.

Briefly, the approach requires assessment of five domains: study quality (previously named risk of bias, and described above), consistency, directness, precision, and reporting bias (which includes publication bias, outcome reporting, and analysis reporting biases). When the body of evidence for a particular outcome included both RCTs and observational studies, we graded each study type separately using design-specific criteria. In considering the overall strength of the entire body of evidence, we considered the extent to which the observational evidence was consistent with RCT data, particularly with regard to direction and magnitude of effect. We also explored the consistency of our findings with recent systematic reviews and discussed agreement or disagreement, along with possible causes for disagreement and impact on strength of evidence ratings, in the text. Because of the risk of unmeasured confounding, observational studies generally would not contribute to estimates of the magnitude or precision of effect, when RCT data were available. If there were other issues (such as differences in when and where RCTs were performed compared to observational studies, and how these differences might affect applicability), this would generally increase uncertainty about the magnitude and precision of any treatment effect. ${ }^{117}$ The five domains were considered qualitatively, and a summary rating of high, moderate, or low strength of evidence was assigned after discussion by two reviewers. When no evidence was available or when evidence on the outcome was too weak, sparse, or inconsistent to permit any conclusion to be drawn, a grade of "insufficient" was assigned. This four-level rating scale consisted of the following definitions:

- High Strength of Evidence-We are very confident that the estimate of effect lies close to the true effect for this outcome. The body of evidence has few or no deficiencies. We believe that the findings are stable; i.e., another study would not change the conclusions. 
- Moderate Strength of Evidence-We are moderately confident that the estimate of effect lies close to the true effect for this outcome. The body of evidence has some deficiencies. We believe that the findings are likely to be stable, but some doubt remains.

- Low Strength of Evidence-We have limited confidence that the estimate of effect lies close to the true effect for this outcome. The body of evidence has major or numerous deficiencies (or both). We believe that additional evidence is needed before concluding either that the findings are stable or that the estimate of effect is close to the true effect.

- Insufficient Strength of Evidence-We have no evidence, we are unable to estimate an effect, or we have no confidence in the estimate of effect for this outcome.

\section{Applicability}

We assessed applicability across our KQs using the method described in AHRQ's EPC Methods Guide for Effectiveness and Comparative Effectiveness Reviews ${ }^{56,118}$ In brief, this method uses the PICOTS format as a way to organize information relevant to applicability. The most important issue with respect to applicability is whether the outcomes were different across studies that recruit different populations (e.g., age groups, U.S. vs. non-U.S. settings) or used different methods to implement the interventions of interest; that is, important characteristics are those that affect baseline (control group) rates of events, intervention group rates of events, or both. We used a checklist applied to each abstracted study to guide the assessment of applicability (Appendix B). For each study, one investigator assigned a summary quality rating, which was then reviewed by a second investigator; disagreements were resolved by consensus or by a third investigator if agreement could not be reached. We summarize issues of applicability qualitatively.

\section{Peer Review and Public Commentary}

Experts in the fields of reproductive endocrinology, reproductive epidemiology, urology, and women's reproductive health, and individuals representing stakeholder and user communities were invited to provide external peer review of the draft report. AHRQ, an associate editor, and members of the TEP were also were invited to provide comments. In addition, the draft report was posted on the AHRQ EHC website for public comment from April 3, 2018, through May 1, 2018. We have addressed all reviewer comments, revising the text as appropriate, and documented everything in a disposition of comments report that will be made available 3 months after the Agency posts the final report on the EHC website. A list of peer reviewers submitting comments on the draft report is provided in the front matter of this report. 


\section{Results}

We begin by describing the results of our literature searches. We then provide an overall description of the included studies. The remainder of the chapter is organized by Key Question (KQ). Under each of the six KQs, we begin with a brief description of the included studies, followed by a bulleted list of the key points of the findings and a detailed synthesis of the evidence. Within each KQ the detailed syntheses are organized first by treatment comparison and then by outcome. The outcomes of interest are ordered in approximate relative importance to patients, based on input from topical experts and Key Informants, rather than temporal occurrence in the clinical pathway: live birth, pregnancy complications, neonatal outcomes, time to pregnancy, costs, short term adverse effects of treatment, and long term outcomes. We conducted quantitative syntheses where possible, as described in the Methods chapter. Although not considered as formal included articles, we discuss findings from relevant systematic reviews - and whether these findings are consistent or not with the evidence from our included articles. We end each treatment section by highlighting any evidence for specific subgroups of interest. Each KQ results section concludes with a summary of the strength of evidence for the main findings. For findings applicable across all KQs, please refer to "Key Findings Across All Infertility Diagnoses," which is presented at the end of the results section. For a list of abbreviations, please refer to the end of the report.

\section{Results of Literature Searches}

Searches of PubMed, Embase, and the Cochrane Database of Systematic Reviews yielded 21,467 citations, 17,263 of which were unique. Manual searching of gray literature databases and bibliographies of key articles or referral by investigators identified 128 additional citations, for a total of 17,391 citations. We received no responses from manufacturers to our requests for scientific information packets. After applying inclusion/exclusion criteria the title-and-abstract level, 1,909 full-text articles were retrieved and screened. Of these, 1,748 were excluded at the full-text screening stage, leaving 161 articles for data abstraction. These 161 articles described 151 unique studies. Studies with more than one article are listed in Table 3. Note that although four studies used the National ART Surveillance System (NASS), they were not considered overlapping studies in terms of the underlying patient population and so are reported separately.

Table 3. Key to primary and companion articles

\begin{tabular}{|c|c|c|}
\hline Study Designation & Primary Abstracted Article & Companion Articles \\
\hline \multirow[t]{2}{*}{ LIFEStyle } & \multirow[t]{2}{*}{ Mutsaerts, $2016^{119}$} & van Oers, $2016^{120}$ \\
\hline & & van Oers, $2018^{121}$ \\
\hline \multirow{4}{*}{$\begin{array}{l}\text { NASS (National Assisted } \\
\text { Reproductive Technology } \\
\text { Surveillance System) }\end{array}$} & Butts, $2014^{122}$ & None \\
\hline & Kawwass, $2013^{27}$ & None \\
\hline & Luke, $2010^{123}$ & None \\
\hline & Nangia, $2011^{124}$ & None \\
\hline \multirow[t]{2}{*}{ OMEGA Project } & \multirow[t]{2}{*}{ Spaan, $2015^{125}$} & van Leeuwen, $2011^{126}$ \\
\hline & & Spaan, $2016^{127}$ \\
\hline \multirow{2}{*}{$\begin{array}{l}\text { PPCOS (Pregnancy in Polycystic } \\
\text { Ovary Syndrome) }\end{array}$} & \multirow[t]{2}{*}{ Legro, $2007^{128}$} & Rausch, $2009^{129}$ \\
\hline & & Legro, 2006 $130 a$ \\
\hline \multirow{3}{*}{$\begin{array}{l}\text { PPCOS (Pregnancy in Polycystic } \\
\text { Ovary Syndrome) } 2\end{array}$} & \multirow[t]{3}{*}{ Legro, $2014^{131}$} & Polotsky, $2015^{132}$ \\
\hline & & Legro, $2014^{133 a}$ \\
\hline & & Legro, $2012^{134 a}$ \\
\hline $\begin{array}{l}\text { SUIT (Scottish Unexplained } \\
\text { Infertility Trial) }\end{array}$ & Bhattacharya, $2008^{135}$ & None \\
\hline
\end{tabular}




\begin{tabular}{|c|c|c|}
\hline Study Designation & Primary Abstracted Article & Companion Articles \\
\hline \multirow[t]{3}{*}{ None } & \multirow[t]{3}{*}{ Brinton, $2015^{136}$} & Brinton, $2014^{137}$ \\
\hline & & Brinton, $2013^{138}$ \\
\hline & & Trabert, $2013^{26}$ \\
\hline None & Custers, $2012^{139}$ & Steures, 2006 $140 a$ \\
\hline None & Nahuis, $2011^{141}$ & Nahuis, $2012^{142}$ \\
\hline
\end{tabular}

aUsed for background information only.

Figure 2 depicts the flow of articles through the literature search and screening process.

\section{Figure 2. Literature flow diagram}

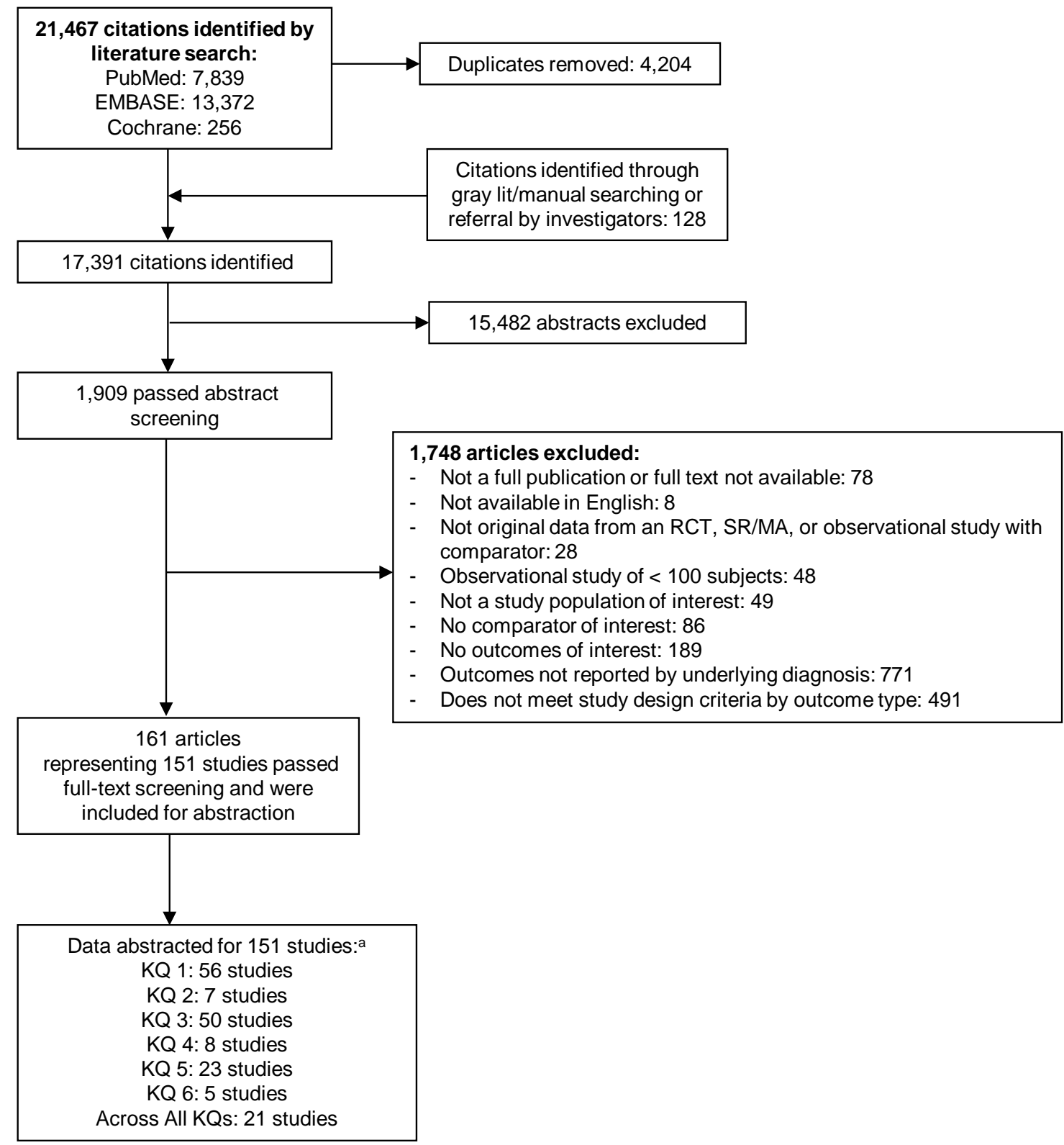

aSome studies are relevant to more than one KQ.

Abbreviations: KQ=Key Question; RCT=randomized controlled trial; SR/MA=systematic review/meta-analysis 


\section{Description of Included Studies}

Overall, we included 151 studies described in 161 publications: 56 studies were relevant to KQ 1, 7 studies to KQ 2, 50 studies to KQ 3, 8 studies to KQ 4, 23 studies to KQ 5, and 5 studies to KQ 6 (some studies were relevant to more than one KQ). Of the 151 included studies, 21 studies had adjusted their results for cause of infertility, but did not report their findings for specific causes of infertility and are discussed at the end of the results section. Globally the evidence supporting findings varying by patient characteristics such as age, ovarian reserve, race, $\mathrm{BMI}$, and presence of other potential causes was minimal. We highlight in the report those cases where findings in these specific subgroups was possible.

Studies were conducted wholly or partly in continental Europe or the United Kingdom (52 studies, 34\%), the United States or Canada (34 studies, 23\%), the Middle East (32 studies, 21\%), Asia (19 studies, 12\%), Africa (10 studies, 7\%), and other locations (Latin America [1 study; this study also had sites in the UK/Europe] and Australia/New Zealand [3 studies], total 2\%). Appendix C provides a detailed listing of included articles. Appendix D provides a complete list of articles excluded at the full-text screening stage, with reasons for exclusion. Further details on the studies included for each KQ are provided in the relevant results sections, below, and in Appendix E. Detailed risk of bias information for each included study is reported in Appendix G.

\section{Key Question 1. PCOS}

KQ 1. What are the comparative safety and effectiveness of available treatment strategies for women with polycystic ovary syndrome (PCOS) who are infertile and who wish to become pregnant?
KQ 1a. Does the optimal treatment strategy vary by patient
characteristics such as age, ovarian reserve, race, body mass index (BMI), presence of other potential causes of female infertility, or presence of male factor infertility?

\section{Description of Studies Included for KQ 1 (PCOS)}

We identified 61 articles ${ }^{119-121,128,129,131,132,141-194}$ describing 56 studies that addressed the comparative safety and effectiveness of available treatment strategies for infertility in women with PCOS. Four studies were described in nine publications, as follows:

- PCOS Study: Legro, 2007: Primary report ${ }^{128}$ and a companion paper ${ }^{129}$

- PCOS 2 Study: Legro, 2014: Primary report ${ }^{131}$ and a companion paper $^{132}$

- Mutsaerts, 2016: Primary report ${ }^{119}$ and two companion papers ${ }^{120,121}$

- Nahuis, 2011: Primary report ${ }^{141}$ and a companion paper ${ }^{142}$

Of the 56 included studies, 52 were randomized controlled trials (RCTs). Twenty five of these were good quality, ${ }^{119,128,131,145,151-153,156,157,160,162,163,167-169,172,174,175,177-179,181-183,187} 25$ were fair quality, ${ }^{141,143,146,148-150,154,155,158,159,161,164-166,170,171,173,176,188-194}$ and 2 were poor quality. ${ }^{144,147}$ In addition, we identified three good-quality ${ }^{180,184,186}$ observational studies and one fair-quality observational study. ${ }^{185}$

Much of the research was done abroad, in subspecialty clinics and funded by an unclear or unknown sources. The breakdown of geographic location was: 5 studies in Africa, ${ }^{154,156,162,168,169}$ 11 studies in Asia, ${ }^{146,147,149,150,166,167,171,173-175,188} 1$ study in Australia/NZ, ${ }^{160} 17$ studies in the 
Middle East, 143,145,148,157,158,161,170,176,178,182,183,189-194 5 studies in the United States, 128,131,159,172,185 16 studies in the United Kingdom or continental Europe, 119,141,144,151,153,155,163-165,177,179-181,184,186,187 and 1 study in the UK/Europe and Latin America. ${ }^{152}$ Settings included 2 studies conducted in general gynecology practices, ${ }^{173,182} 3$ studies conducted in a hospital, ${ }^{180,183,191} 2$ studies conducted in a combination of gynecological or subspecialty practices, ${ }^{153,193} 6$ studies where the setting was unclear or not reported, ${ }^{131,141,144,147,177,184}$ while the remaining 42 studies were conducted in subspecialty practices. Last, 8 studies reported government funding, ${ }^{119,131,141,149,164,171,172,187} 3$ studies reported industry funding, ${ }^{152,159,186} 7$ studies reported non-government, non-industry funding, ${ }^{148,157,165,178,181,183,184} 6$ studies reported a combination of funding from a variety of sources, ${ }^{128,146,160,174,179,188}$ while the remaining 33 studies did not report a funding source or it was unclear.

Further details on the characteristics of studies included for this KQ are provided in the following sections and Appendix E.

In addition to the above studies, seven systematic reviews; six good quality, ${ }^{66,81,90,195-197}$ one fair quality, ${ }^{78}$ addressed the comparative effectiveness of various treatments for infertility in women with PCOS are also discussed below and the consistency of their findings with our included studies are incorporated in to our strength of evidence ratings. In general, the randomized trials used standardized diagnostic criteria for PCOS, while the nonrandomized observational studies may have included other ovulatory disorders.

\section{Key Points for PCOS}

Key findings for outcomes in couples where the primary cause of infertility is PCOS include:

- Letrozole has a higher live birth rate than clomiphene citrate alone and lower multiple births, with no difference in ectopic pregnancy, or miscarriage (moderate for all outcomes), low birthweight, or time to pregnancy (low strength of evidence [SOE] for both these outcomes).

- Clomiphene citrate does not result in higher live birth rates compared with metformin (moderate SOE). Differences are also not found in the rates of multiple birth, ectopic pregnancy, or time to pregnancy (low SOE for all outcomes). There is a higher rate of miscarriage with combination clomiphene and metformin than clomiphene alone (low SOE)

- Letrozole or letrozole and berberine have a higher live birth rate than berberine alone (low SOE) with no difference in multiple births, miscarriage, or low birthweight rates (low SOE)

- There was no difference between clomiphene and tamoxifen for the outcomes of live birth or miscarriage (low SOE)

- There was no difference between laparoscopic ovarian drilling (LOD) and oral agents for live birth (moderate SOE) or miscarriage rates (low SOE). Multiple births were reduced given LOD (moderate SOE).

- Live birth (low SOE) and miscarriage rates (moderate SOE) did not differ between in vitro fertilization (IVF) treatment strategies.

- There was no difference in live birth rates for women who underwent lifestyle modification in combination with IVF compared with IVF alone (moderate SOE)

- There was no difference between type 1 diabetes mellitus diagnoses in children conceived with assisted reproductive technology (ART) compared to children conceived with no fertility treatment (moderate SOE) 
- As with other indications for IVF, use of single-embryo transfer is associated with slightly lower live birth rates but significantly reduced multiple gestation rates (low SOE)

\section{Detailed Synthesis by Treatment for PCOS}

Included studies and their findings for the following treatments for PCOS are detailed in this section:

1. Oral Agents Alone

2. Oral Agents Alone Versus Acupuncture

3. Oral Agents Alone Versus Oral Agents With Intrauterine Insemination (IUI)

4. Oral Agents Alone Versus Surgical Management

5. Oral Agents Versus Gonadotropins

6. Lifestyle Interventions

7. Surgical Management Versus Gonadotropins

8. Gonadotropins With IUI

9. Assisted Reproductive Technology (ART)
a. IVF
b. Adjuncts to IVF
c. Fresh Versus Frozen in IVF
d. Intra-cytoplasmic sperm injection (ICSI)

\section{Oral Agents Alone for PCOS}

Oral agents used to induce ovulation in women with PCOS include selective estrogen receptor modulators (e.g., clomiphene citrate, tamoxifen), aromatase inhibitors (e.g., letrozole), dopamine agonists (e.g., cabergoline) and herbal medicines (e.g., berberine). We identified 12 randomized controlled trials (RCTs) 128,131,151,160,166,170,173,175,179,191,192,194 (6 good quality, 6 fair quality) that addressed outcomes between different oral agents for ovulation induction without intrauterine insemination (IUI). These studies varied in the medication type used for oral ovulation as well as in adjunct treatments. These 12 studies included 3217 patients combined.

We also identified two systematic reviews that explored metformin versus clomiphene, ${ }^{78,90}$ one that focused on letrozole versus clomiphene, ${ }^{66}$ and one that examined clomiphene and other elective estrogen receptor modulators. ${ }^{198}$

Findings for our specific outcomes of interest are summarized in Table 4.

\section{Letrozole Versus Clomiphene}

Letrozole resulted in significantly more live births than clomiphene (moderate SOE). This finding was based on a good-quality meta-analysis by Franik et al. comparing clomiphene to letrozole ${ }^{66}$ and which included the RCT by Legro et al. ${ }^{131}$ This meta-analysis of 9 RCTs (1783 subjects) reported a significant increase in live birth rate with letrozole (pooled odds ratio [OR] 1.64; 95\% confidence interval [CI], 1.32 to 2.04). One additional recent good-quality RCT by Amer et al. ${ }^{179}$ reported live birth rates that were non-significantly higher in the letrozole group compared to the clomiphene group ( $48.8 \%$ versus $35.4 \%$, $\mathrm{p}=0.089$ ).

Multiple births was explored in the meta-analysis demonstrating a lower rate of multiple pregnancies with letrozole compared to clomiphene (OR 0.38, 95\% CI, 0.17 to 0.84 ) based on 11 studies with 2385 subjects ${ }^{66}$ (moderate SOE). Our 3 included RCTs showed no difference in multiple births with very low rates of the outcome across the studies. ${ }^{131,170,179}$ 
The meta-analysis did not report on ectopic pregnancies. The three individual RCTs reported no significant difference in ectopic pregnancy between the clomiphene and letrozole arms and a meta analysis of these three studies representing 886 women showed no difference $(\mathrm{OR}=0.72$, 95\% CI 0.24 to 2.17) (SOE moderate). ${ }^{131,170,179}$

Miscarriage was reported in all 3 studies and in the meta-analysis by Franik and colleagues, with several definitions of miscarriage utilized. From the meta analysis, 2385 patients from 12 trials demonstrated no difference between letrozole and clomiphene in miscarriage rates (pooled OR 1.32, 95\% CI 0.92 to 1.88) (moderate SOE). ${ }^{66}$ The two additional individual RCTs not included in the meta analysis comprised an additional 136 women and also reported no significant differences in miscarriage between the treatment arms. ${ }^{170,179}$

Neonatal death was reported in one good-quality RCT, ${ }^{131}$ which compared clomiphene to letrozole. There was not a significant difference between clomiphene and letrozole for cases of neonatal death although given the rarity of the outcome, much larger data sets are needed (insufficient SOE).

Finally, one good-quality RCT reported time to pregnancy for clomiphene versus letrozole. ${ }^{131}$ It did not show a significant difference in mean days to pregnancy between study arms (low SOE).

\section{Metformin Versus Clomiphene}

Five studies compared clomiphene to metformin (or metformin in combination with clomiphene). ${ }^{128,151,160,166,173}$ Three evaluated live birth as an outcome. One RCT ${ }^{151}$ demonstrated a significantly greater live birth rate with the combination of metformin and clomiphene compared to clomiphene alone, while another $\mathrm{RCT}^{173}$ reported non-significantly higher live birth rates with the combination of metformin and clomiphene than either metformin alone or clomiphene alone. The third $\mathrm{RCT}^{128}$ resulted in greater live births with clomiphene compared to metformin but no difference between clomiphene and combination therapy (Table 4).

Two meta-analyses, ${ }^{78,199}$ one good quality ${ }^{199}$ and one fair quality, ${ }^{78}$ also compared metformin and clomiphene. Both meta-analyses reported no significant difference in live birth rates between women treated with metformin and clomiphene. The fair-quality meta-analysis by Sun et al., ${ }^{78}$ reported an OR of 0.89; 95\% CI, 0.71 to 1.13 in 4 RCTs (1012 patients) and the good-quality meta-analysis ${ }^{199}$ reported an OR of $0.71,95 \%$ CI, 0.49 to 1.01 based on five studies. The latter meta-analysis reported evidence of heterogeneity by BMI, with live birth rates lower in the metformin group among obese women (OR 0.30, 95\% CI, 0.17 to 0.52 ) but higher in the nonobese group (OR 1.71, 95\% CI, 1.00 to 2.94 ).

The 2017 meta-analysis included four of our identified RCTs. ${ }^{128,151,166,173}$ Both meta-analyses also reported no difference in live birth rates comparing metformin plus clomiphene to clomiphene alone. ${ }^{78,199}$ Sun reported an OR of 0.99; 95\% CI, 0.84 to 1.17) based on 3 studies with 912 patients $^{78}$ and Morley et al. reported an OR of 1.21, 95\% CI, 0.92 to 1.59 based on 9 studies with 1079 women. ${ }^{199}$ Together we rated the SOE as moderate for no difference in live birth rates.

Miscarriages were reported as an outcome in four of the RCTs ${ }^{128,160,166,173}$ and synthesized in the 2017 meta-analysis. ${ }^{199}$ The meta-analysis (which included three ${ }^{128,166,173}$ of the four individual included RCTs) reported no differences in miscarriage rates between metformin and clomiphene, whereas one RCT reported reduced miscarriages in the clomiphene group. ${ }^{160}$ The meta-analysis reported an increased risk for miscarriage in women taking clomiphene plus metformin compared to clomiphene alone (OR 1.59, 95\% CI, 1.03 to 2.46) based on 9 studies 
with 1096 women. ${ }^{199}$ None of the individual studies reported a statistically significant difference between the groups, although in each of the studies the miscarriage rate was higher in the clomiphene plus metformin group than the clomiphene group. ${ }^{128,160,166,173}$ Together these studies supported a low SOE of a higher rate of miscarriage in the combined therapy group.

The 2017 meta-analysis ${ }^{199}$ and our included studies did not support a difference in multiple births between clomiphene and metformin. Given the imprecision in these findings and suspected reporting bias of the included studies the strength of evidence was rated as low. Our included studies also did not support a difference in ectopic pregnancy (low SOE for both outcomes).

For the outcomes of congenital anomalies, the anomalies reported in these studies varied in severity and type and given the rarity of outcomes and imprecise evidence the SOE was rated as insufficient. None of the studies however found significant differences between intervention groups.

One good-quality RCT reported time to pregnancy for clomiphene versus metformin or placebo in combination with clomiphene. ${ }^{151}$ It did not show a significant difference in mean days to pregnancy between study arms (low SOE).

\section{Tamoxifen Versus Clomiphene}

One fair-quality RCT compared tamoxifen with clomiphene. ${ }^{191}$ Live births and miscarriages were not significantly different between the treatment arms. A meta-analysis ${ }^{198}$ also reported on tamoxifen versus clomiphene and found no significant differences in live births (OR 1.24, 95\% CI 0.59-2.62) in two studies with a total of 195 women or in miscarriages (OR 1.81, 95\% CI 0.80-4.12) in four studies with a total of 653 women. Within that meta analysis, both outcomes were judged to have a low grade of evidence. We rated the strength of evidence for both outcomes as low for no difference between tamoxifen and clomiphene.

\section{Cabergoline Versus Clomiphene}

One fair-quality RCT compared clomiphene alone to clomiphene plus cabergoline. ${ }^{192}$ Live birth rates were not reported, and no significant differences in miscarriages or multiple pregnancies were reported. SOE was rated as insufficient given findings from one small study with potential limitations.

\section{Letrozole Versus Berberine}

One good-quality RCT compared letrozole, berberine and a combination of the two. ${ }^{175}$ Live births were similar between the letrozole and combination arms (36.3\% and $34.4 \%, \mathrm{p}=0.69)$, and both of these arms had significantly higher live birth rates than the berberine arm $(22.0 \%$, $\mathrm{p}=0.001$ ) (low SOE). No significant differences between treatment arms were reported for multiple births, miscarriage or birthweight (low SOE for all three outcomes).

There was no evidence regarding costs, short-term adverse effects, and long-term child or maternal outcomes. 
Table 4. Outcomes for comparisons of oral agents alone in women with PCOS

\begin{tabular}{|c|c|c|c|c|c|c|c|}
\hline Outcome & $\begin{array}{c}\text { Study } \\
\text { Design } \\
\text { (N } \\
\text { Patients) }\end{array}$ & Intervention & Comparator & $\begin{array}{c}\text { Results } \\
\text { Intervention } \\
N \\
(\%) \\
(95 \% \mathrm{Cl})\end{array}$ & $\begin{array}{c}\text { Results } \\
\text { Comparator } \\
N \\
(\%) \\
(95 \% \mathrm{Cl})\end{array}$ & $\begin{array}{c}\mathbf{P} \\
\text { Value }\end{array}$ & $\begin{array}{l}\text { Summary } \\
\text { of Study } \\
\text { Findings }\end{array}$ \\
\hline \multirow[t]{10}{*}{$\begin{array}{l}\text { Live birth: } \\
\text { Any/patient }\end{array}$} & $\begin{array}{l}\text { Legro, } \\
2014^{131} \\
\text { RCT } \\
(750) \\
\end{array}$ & $\mathrm{CC}$ & Letrozole & $\begin{array}{c}72 / 376 \\
(19.1) \\
(15.3 \text { to } 23.3)\end{array}$ & $\begin{array}{c}103 / 374 \\
(27.5) \\
(23.1 \text { to } 32.2)\end{array}$ & 0.007 & $\begin{array}{l}\text { Greater live } \\
\text { births with } \\
\text { letrozole } \\
\text { compared to } \\
\text { clomiphene }\end{array}$ \\
\hline & $\begin{array}{l}\text { Amer, } \\
2017^{179} \\
\\
\text { RCT } \\
(159)\end{array}$ & $\mathrm{CC}$ & Letrozole & $\begin{array}{l}28 / 79 \\
(35.4)\end{array}$ & $\begin{array}{l}39 / 80 \\
(48.8)\end{array}$ & 0.089 & $\begin{array}{l}\text { No } \\
\text { difference }\end{array}$ \\
\hline & $\begin{array}{l}\text { Morin- } \\
\text { Papunen, } \\
2012^{151} \\
\text { RCT } \\
(320)\end{array}$ & $\mathrm{CC}$ & $\begin{array}{l}\text { Metformin+ } \\
\text { CC }\end{array}$ & $\begin{array}{c}46 / 160 \\
(28.9) \\
\text { (22.0 to } 36.0)\end{array}$ & $\begin{array}{c}66 / 160 \\
(41.1) \\
\text { (33.8 to } 38.9)\end{array}$ & 0.03 & $\begin{array}{l}\text { Greater live } \\
\text { births with } \\
\text { combination } \\
\text { of metformin } \\
\text { and } \\
\text { clomiphene } \\
\text { compared to } \\
\text { clomiphene } \\
\text { alone }\end{array}$ \\
\hline & \multirow[t]{2}{*}{$\begin{array}{l}\text { Legro, } \\
2007^{128} \\
\text { RCT } \\
(626)\end{array}$} & \multirow[t]{2}{*}{$\mathrm{CC}$} & Metformin & $\begin{array}{c}47 / 209 \\
(22.5) \\
\text { (17.1 to } 28.4)\end{array}$ & $\begin{array}{c}15 / 208 \\
(7.2) \\
\text { (4.1 to } 11.1)\end{array}$ & $<0.001$ & $\begin{array}{l}\text { Greater live } \\
\text { births with } \\
\text { clomiphene } \\
\text { compared to } \\
\text { metformin } \\
\end{array}$ \\
\hline & & & $\begin{array}{l}\text { Metformin+ } \\
\text { CC }\end{array}$ & $\begin{array}{c}47 / 209 \\
(22.5) \\
(17.1 \text { to } 28.4) \\
\end{array}$ & $\begin{array}{c}56 / 209 \\
(26.8) \\
\text { (21.0 to } 33.0) \\
\end{array}$ & 0.31 & $\begin{array}{l}\text { No } \\
\text { difference }\end{array}$ \\
\hline & \multirow{2}{*}{$\begin{array}{l}\text { Kar, } \\
2015^{173} \\
\\
\text { RCT } \\
(105)\end{array}$} & \multirow[t]{2}{*}{$\mathrm{CC}$} & Metformin & $\begin{array}{c}9 / 32 \\
(28.1) \\
\end{array}$ & $\begin{array}{c}9 / 24 \\
(37.5) \\
\end{array}$ & 0.46 & $\begin{array}{l}\text { No } \\
\text { difference }\end{array}$ \\
\hline & & & $\begin{array}{l}\text { Metformin+ } \\
\text { CC }\end{array}$ & $\begin{array}{c}9 / 32 \\
(28.1)\end{array}$ & $\begin{array}{l}10 / 24 \\
(41.6)\end{array}$ & 0.29 & $\begin{array}{l}\text { No } \\
\text { difference }\end{array}$ \\
\hline & $\begin{array}{l}\text { Topçu, } \\
2017^{191} \\
\text { RCT } \\
\text { (88) }\end{array}$ & $\mathrm{CC}$ & Tamoxifen & $\begin{array}{c}6 / 46 \\
(13.0)\end{array}$ & $\begin{array}{c}8 / 42 \\
(19.0)\end{array}$ & 0.617 & $\begin{array}{l}\text { No } \\
\text { difference }\end{array}$ \\
\hline & \multirow{2}{*}{$\begin{array}{l}\text { Wu, } \\
2016^{175} \\
\\
\text { RCT } \\
(644)\end{array}$} & \multirow[t]{2}{*}{ Letrozole } & Berberine & $\begin{array}{l}78 / 215 \\
(36.3)\end{array}$ & $\begin{array}{c}47 / 214 \\
(22.0)\end{array}$ & 0.001 & \multirow[b]{2}{*}{$\begin{array}{l}\text { Greater live } \\
\text { births for } \\
\text { letrozole or } \\
\text { letrozole + } \\
\text { berberine } \\
\text { compared } \\
\text { with } \\
\text { berberine } \\
\text { alone. No } \\
\text { difference } \\
\text { between } \\
\text { letrozole } \\
\text { and } \\
\text { letrozole + } \\
\text { berberine } \\
\end{array}$} \\
\hline & & & $\begin{array}{l}\text { Berberine + } \\
\text { Letrozole }\end{array}$ & $\begin{array}{c}78 / 215 \\
(36.3)\end{array}$ & $\begin{array}{l}74 / 215 \\
(34.4)\end{array}$ & 0.687 & \\
\hline
\end{tabular}




\begin{tabular}{|c|c|c|c|c|c|c|c|}
\hline Outcome & $\begin{array}{c}\text { Study } \\
\text { Design } \\
\text { (N } \\
\text { Patients) }\end{array}$ & Intervention & Comparator & $\begin{array}{c}\text { Results } \\
\text { Intervention } \\
\mathbf{N} \\
(\%) \\
(95 \% \mathrm{Cl})\end{array}$ & $\begin{array}{c}\text { Results } \\
\text { Comparator } \\
N \\
(\%) \\
(95 \% \mathrm{Cl})\end{array}$ & $\begin{array}{c}\mathbf{P} \\
\text { Value }\end{array}$ & $\begin{array}{l}\text { Summary } \\
\text { of Study } \\
\text { Findings }\end{array}$ \\
\hline \multirow[t]{12}{*}{$\begin{array}{l}\text { Pregnancy } \\
\text { complications: } \\
\text { Multiple births }\end{array}$} & $\begin{array}{l}\text { Legro, } \\
2014^{131} \\
\\
\text { RCT } \\
(750)\end{array}$ & $\mathrm{CC}$ & Letrozole & $\begin{array}{c}5 / 376 \\
(1.3) \\
\text { (0.4 to } 2.7)\end{array}$ & $\begin{array}{c}4 / 374 \\
(1.1) \\
\text { (0.3 to } 2.3)\end{array}$ & 0.175 & $\begin{array}{l}\text { No } \\
\text { difference }\end{array}$ \\
\hline & $\begin{array}{l}\text { Ghahiri, } \\
20166^{170} \\
\text { RCT } \\
(100)\end{array}$ & $\mathrm{CC}$ & Letrozole & $\begin{array}{c}0 / 24 \\
(0)\end{array}$ & $\begin{array}{c}0 / 24 \\
(0)\end{array}$ & NS & $\begin{array}{l}\text { No } \\
\text { difference }\end{array}$ \\
\hline & $\begin{array}{l}\text { Amer, } \\
2017^{179} \\
\text { RCT } \\
(159)\end{array}$ & $\mathrm{CC}$ & Letrozole & $\begin{array}{l}0 / 34 \\
(0.0)\end{array}$ & $\begin{array}{l}3 / 49 \\
(6.1)\end{array}$ & 0.201 & $\begin{array}{l}\text { No } \\
\text { difference }\end{array}$ \\
\hline & \multirow{2}{*}{$\begin{array}{l}\text { Johnson, } \\
2010^{160} \\
\text { RCT } \\
(171)\end{array}$} & \multirow[t]{2}{*}{$\mathrm{CC}$} & Metformin & $\begin{array}{c}1 / 36 \\
(2.8) \\
(0.1 \text { to } 10.0)\end{array}$ & $\begin{array}{c}1 / 35 \\
(2.9) \\
\text { (0.1 to 10.3) }\end{array}$ & 0.98 & $\begin{array}{l}\text { No } \\
\text { difference }\end{array}$ \\
\hline & & & $\begin{array}{l}\text { Metformin+ } \\
\text { CC }\end{array}$ & $\begin{array}{c}1 / 36 \\
(2.8) \\
(0.1 \text { to } 10.0) \\
\end{array}$ & $\begin{array}{c}1 / 35 \\
(2.9) \\
(0.1 \text { to } 10.3) \\
\end{array}$ & 0.98 & $\begin{array}{l}\text { No } \\
\text { difference }\end{array}$ \\
\hline & \multirow[t]{2}{*}{$\begin{array}{l}\text { Zain, } \\
2009^{166}\end{array}$} & \multirow[t]{2}{*}{$\mathrm{CC}$} & Metformin & $\begin{array}{c}0 / 39 \\
(0)\end{array}$ & $\begin{array}{c}0 / 38 \\
(0)\end{array}$ & NS & $\begin{array}{l}\text { No } \\
\text { difference }\end{array}$ \\
\hline & & & $\begin{array}{l}\text { Metformin+ } \\
\text { CC }\end{array}$ & $\begin{array}{c}0 / 39 \\
(0)\end{array}$ & $\begin{array}{c}0 / 38 \\
(0)\end{array}$ & NS & $\begin{array}{l}\text { No } \\
\text { difference }\end{array}$ \\
\hline & \multirow{2}{*}{$\begin{array}{l}\text { Legro, } \\
2007^{128} \\
\\
\text { RCT } \\
(626)\end{array}$} & \multirow[t]{2}{*}{$\mathrm{CC}$} & Metformin & $\begin{array}{c}3 / 50 \\
(6.0) \\
(1.3 \text { to } 14.0)\end{array}$ & $\begin{array}{c}0 / 18 \\
(0)\end{array}$ & 0.29 & $\begin{array}{l}\text { No } \\
\text { difference }\end{array}$ \\
\hline & & & $\begin{array}{l}\text { Metformin+ } \\
\text { CC }\end{array}$ & $\begin{array}{c}3 / 50 \\
(6.0) \\
(1.3 \text { to } 14.0) \\
\end{array}$ & $\begin{array}{c}3 / 65 \\
(4.6) \\
(1.0 \text { to } 10.8) \\
\end{array}$ & 0.74 & $\begin{array}{l}\text { No } \\
\text { difference }\end{array}$ \\
\hline & $\begin{array}{l}\text { Zahran, } \\
2018^{192} \\
\\
\text { RCT } \\
(130) \\
\end{array}$ & $\mathrm{CC}$ & $\begin{array}{l}\mathrm{CC}+ \\
\text { Cabergoline }\end{array}$ & $\begin{array}{c}1 / 8 \\
(12.5)\end{array}$ & $\begin{array}{c}2 / 19 \\
(10.5)\end{array}$ & 0.83 & $\begin{array}{l}\text { No } \\
\text { difference }\end{array}$ \\
\hline & \multirow{2}{*}{$\begin{array}{l}\text { Wu, } \\
2016^{175} \\
\\
\text { RCT } \\
(644)\end{array}$} & \multirow[t]{2}{*}{ Letrozole } & Berberine & $\begin{array}{l}1 / 78 \\
(1.2)\end{array}$ & $\begin{array}{l}0 / 47 \\
(0.0)\end{array}$ & NS & \multirow[t]{2}{*}{$\begin{array}{l}\text { No } \\
\text { difference }\end{array}$} \\
\hline & & & $\begin{array}{l}\text { Berberine + } \\
\text { Letrozole }\end{array}$ & $\begin{array}{l}1 / 78 \\
(1.2)\end{array}$ & $\begin{array}{l}3 / 74 \\
(4.1)\end{array}$ & 0.357 & \\
\hline \multirow[t]{2}{*}{$\begin{array}{l}\text { Pregnancy } \\
\text { complications: } \\
\text { Ectopic } \\
\text { pregnancy }\end{array}$} & $\begin{array}{l}\text { Legro, } \\
2014^{131} \\
\text { RCT } \\
(750)\end{array}$ & $\mathrm{CC}$ & Letrozole & $\begin{array}{c}3 / 376 \\
(0.8) \\
(0.2 \text { to } 1.9\end{array}$ & $\begin{array}{c}4 / 374 \\
(1.1) \\
\text { (0.3 to } 2.3)\end{array}$ & 0.67 & $\begin{array}{l}\text { No } \\
\text { difference }\end{array}$ \\
\hline & $\begin{array}{l}\text { Ghahiri, } \\
2016^{170} \\
\text { RCT } \\
(100)\end{array}$ & $\mathrm{CC}$ & Letrozole & $\begin{array}{l}2 / 24 \\
(8.3)\end{array}$ & $\begin{array}{c}3 / 29 \\
(10.3)\end{array}$ & 0.80 & $\begin{array}{l}\text { No } \\
\text { difference }\end{array}$ \\
\hline
\end{tabular}




\begin{tabular}{|c|c|c|c|c|c|c|c|}
\hline Outcome & $\begin{array}{c}\text { Study } \\
\text { Design } \\
\text { (N } \\
\text { Patients) }\end{array}$ & Intervention & Comparator & $\begin{array}{c}\text { Results } \\
\text { Intervention } \\
\mathbf{N} \\
(\%) \\
(95 \% \mathrm{Cl})\end{array}$ & $\begin{array}{c}\text { Results } \\
\text { Comparator } \\
\mathbf{N} \\
(\%) \\
(95 \% \mathrm{Cl})\end{array}$ & $\begin{array}{c}\mathbf{P} \\
\text { Value }\end{array}$ & $\begin{array}{l}\text { Summary } \\
\text { of Study } \\
\text { Findings }\end{array}$ \\
\hline & $\begin{array}{l}\text { Amer, } \\
2017^{179} \\
\\
\text { RCT } \\
(159)\end{array}$ & $\mathrm{CC}$ & Letrozole & $\begin{array}{l}0 / 34 \\
(0.0)\end{array}$ & $\begin{array}{l}1 / 49 \\
(2.0)\end{array}$ & NS & $\begin{array}{l}\text { No } \\
\text { difference }\end{array}$ \\
\hline & $\begin{array}{l}\text { Zain, } \\
2009^{166}\end{array}$ & $\mathrm{CC}$ & Metformin & $\begin{array}{c}0 / 39 \\
(0)\end{array}$ & $\begin{array}{c}0 / 38 \\
(0)\end{array}$ & NS & $\begin{array}{l}\text { No } \\
\text { difference }\end{array}$ \\
\hline & $\begin{array}{l}\mathrm{RCT} \\
(124)\end{array}$ & & $\begin{array}{l}\text { Metformin+ } \\
\text { CC }\end{array}$ & $\begin{array}{c}0 / 39 \\
(0)\end{array}$ & $\begin{array}{c}0 / 38 \\
(0)\end{array}$ & NS & $\begin{array}{l}\text { No } \\
\text { difference }\end{array}$ \\
\hline & $\begin{array}{l}\text { Legro, } \\
2007^{128}\end{array}$ & $\mathrm{CC}$ & Metformin & $\begin{array}{c}2 / 209 \\
(1.0) \\
\text { (0.1 to } 2.6)\end{array}$ & $\begin{array}{c}0 / 208 \\
(0)\end{array}$ & 0.16 & $\begin{array}{l}\text { No } \\
\text { difference }\end{array}$ \\
\hline & $\begin{array}{l}\text { RCT } \\
(626)\end{array}$ & & $\begin{array}{l}\text { Metformin+ } \\
\text { CC }\end{array}$ & $\begin{array}{c}2 / 209 \\
(1.0) \\
\text { (0.1 to } 2.6)\end{array}$ & $\begin{array}{c}2 / 209 \\
(1.0) \\
\text { (0.1 to } 2.6)\end{array}$ & NS & $\begin{array}{l}\text { No } \\
\text { difference }\end{array}$ \\
\hline & $\mathrm{Kar}_{173} 2015$ & $\mathrm{CC}$ & Metformin & $\begin{array}{l}1 / 10 \\
(10)\end{array}$ & $\begin{array}{l}0 / 13 \\
(0.0)\end{array}$ & NS & $\begin{array}{l}\text { No } \\
\text { difference }\end{array}$ \\
\hline & $\begin{array}{l}\text { RCT } \\
(105)\end{array}$ & & $\begin{array}{l}\text { Metformin+ } \\
\text { CC }\end{array}$ & $\begin{array}{l}1 / 10 \\
(10)\end{array}$ & $\begin{array}{l}0 / 12 \\
(0.0)\end{array}$ & NS & $\begin{array}{l}\text { No } \\
\text { difference }\end{array}$ \\
\hline \multirow[t]{9}{*}{$\begin{array}{l}\text { Pregnancy } \\
\text { complications: } \\
\text { Miscarriage }\end{array}$} & $\begin{array}{l}\text { Legro, } \\
2014^{131} \\
\\
\text { RCT } \\
(750)\end{array}$ & CC & Letrozole & $\begin{array}{c}30 / 103 \\
(29.1) \\
(20.8 \text { to } 38.2)\end{array}$ & $\begin{array}{c}49 / 154 \\
(31.8) \\
\text { (24.7 to } 39.4)\end{array}$ & 0.65 & $\begin{array}{l}\text { No } \\
\text { difference }\end{array}$ \\
\hline & $\begin{array}{l}\text { Ghahiri, } \\
20166^{170} \\
\text { RCT } \\
(100) \\
\end{array}$ & $\mathrm{CC}$ & Letrozole & $\begin{array}{c}6 / 24 \\
(25.0)\end{array}$ & $\begin{array}{c}5 / 29 \\
(17.2)\end{array}$ & 0.38 & $\begin{array}{l}\text { No } \\
\text { difference }\end{array}$ \\
\hline & $\begin{array}{l}\text { Amer, } \\
2017^{179} \\
\text { RCT } \\
(159)\end{array}$ & CC & Letrozole & $\begin{array}{c}6 / 34 \\
(17.6)\end{array}$ & $\begin{array}{c}9 / 49 \\
(18.4)\end{array}$ & 0.93 & $\begin{array}{l}\text { No } \\
\text { difference }\end{array}$ \\
\hline & \multirow{2}{*}{$\begin{array}{l}\text { Legro, } \\
2007^{128} \\
\\
\text { RCT } \\
(626)\end{array}$} & \multirow[t]{2}{*}{$\mathrm{CC}$} & Metformin & $\begin{array}{c}16 / 62 \\
(25.8) \\
(15.8 \text { to } 37.3) \\
\end{array}$ & $\begin{array}{c}10 / 25 \\
(40.0) \\
\text { (22.1 to } 59.4) \\
\end{array}$ & 0.19 & $\begin{array}{l}\text { No } \\
\text { difference }\end{array}$ \\
\hline & & & $\begin{array}{l}\text { Metformin+ } \\
\text { CC }\end{array}$ & $\begin{array}{c}16 / 62 \\
(25.8) \\
(15.8 \text { to } 37.3)\end{array}$ & $\begin{array}{c}24 / 80 \\
(30.0) \\
(20.5 \text { to } 40.4)\end{array}$ & 0.58 & $\begin{array}{l}\text { No } \\
\text { difference }\end{array}$ \\
\hline & \multirow{2}{*}{$\begin{array}{l}\text { Zain, } \\
\text { 2009166 } \\
\\
\text { RCT } \\
(124) \\
\end{array}$} & \multirow[t]{2}{*}{$\mathrm{CC}$} & Metformin & $\begin{array}{c}0 / 39 \\
(0)\end{array}$ & $\begin{array}{c}0 / 38 \\
(0)\end{array}$ & NS & $\begin{array}{l}\text { No } \\
\text { difference }\end{array}$ \\
\hline & & & $\begin{array}{l}\text { Metformin+ } \\
\text { CC }\end{array}$ & $\begin{array}{c}0 / 39 \\
(0)\end{array}$ & $\begin{array}{l}1 / 38 \\
(2.6)\end{array}$ & 0.31 & $\begin{array}{l}\text { No } \\
\text { difference }\end{array}$ \\
\hline & \multirow[t]{2}{*}{$\begin{array}{l}\text { Johnson, } \\
2010^{160} \\
\text { RCT } \\
(171)\end{array}$} & \multirow[t]{2}{*}{ CC } & Metformin & $\begin{array}{c}0 / 36 \\
(0)\end{array}$ & $\begin{array}{c}4 / 35 \\
(11.4)\end{array}$ & 0.037 & $\begin{array}{l}\text { Reduced } \\
\text { miscarriage } \\
\text { with } \\
\text { clomiphene } \\
\text { compared to } \\
\text { metformin }\end{array}$ \\
\hline & & & $\begin{array}{l}\text { Metformin+ } \\
\text { CC }\end{array}$ & $\begin{array}{c}0 / 36 \\
(0)\end{array}$ & $\begin{array}{l}3 / 35 \\
(8 / 6)\end{array}$ & 0.073 & $\begin{array}{l}\text { No } \\
\text { difference }\end{array}$ \\
\hline
\end{tabular}




\begin{tabular}{|c|c|c|c|c|c|c|c|}
\hline Outcome & $\begin{array}{c}\text { Study } \\
\text { Design } \\
\text { (N } \\
\text { Patients) }\end{array}$ & Intervention & Comparator & $\begin{array}{c}\text { Results } \\
\text { Intervention } \\
\mathrm{N} \\
(\%) \\
(95 \% \mathrm{Cl}) \\
\end{array}$ & $\begin{array}{c}\text { Results } \\
\text { Comparator } \\
N \\
(\%) \\
(95 \% \mathrm{Cl}) \\
\end{array}$ & $\begin{array}{c}P \\
\text { Value }\end{array}$ & $\begin{array}{l}\text { Summary } \\
\text { of Study } \\
\text { Findings }\end{array}$ \\
\hline & \multirow[t]{2}{*}{$\begin{array}{l}\text { Kar, } 2015 \\
173\end{array}$} & \multirow[t]{2}{*}{$\mathrm{CC}$} & Metformin & $\begin{array}{l}0 / 10 \\
(0.0)\end{array}$ & $\begin{array}{c}4 / 13 \\
(30.7) \\
\end{array}$ & NS & $\begin{array}{l}\text { No } \\
\text { difference }\end{array}$ \\
\hline & & & $\begin{array}{l}\text { Metformin+ } \\
\text { CC }\end{array}$ & $\begin{array}{l}0 / 10 \\
(0.0)\end{array}$ & $\begin{array}{c}2 / 12 \\
(16.6)\end{array}$ & NS & $\begin{array}{l}\text { No } \\
\text { difference }\end{array}$ \\
\hline & $\begin{array}{l}\text { Topçu, } \\
2017191 \\
\text { RCT } \\
(88)\end{array}$ & $\mathrm{CC}$ & Tamoxifen & $\begin{array}{c}3 / 9 \\
(33.3)\end{array}$ & $\begin{array}{c}2 / 10 \\
(20.0)\end{array}$ & NS & $\begin{array}{l}\text { No } \\
\text { difference }\end{array}$ \\
\hline & $\begin{array}{l}\text { Zahran, } \\
2018^{192} \\
\text { RCT } \\
(130)\end{array}$ & $\mathrm{CC}$ & $\begin{array}{l}\mathrm{CC}+ \\
\text { Cabergoline }\end{array}$ & $\begin{array}{c}2 / 8 \\
(25.0)\end{array}$ & $\begin{array}{c}3 / 19 \\
(15.8)\end{array}$ & 0.74 & $\begin{array}{l}\text { No } \\
\text { difference }\end{array}$ \\
\hline & \multirow{2}{*}{$\begin{array}{l}\text { Wu, } \\
2016^{175} \\
\text { RCT } \\
(644)\end{array}$} & \multirow[t]{2}{*}{ Letrozole } & Berberine & $\begin{array}{l}17 / 98 \\
(17.4)\end{array}$ & $\begin{array}{l}14 / 61 \\
(23.0)\end{array}$ & 0.386 & \multirow[t]{2}{*}{$\begin{array}{l}\text { No } \\
\text { difference }\end{array}$} \\
\hline & & & $\begin{array}{l}\text { Berberine + } \\
\text { Letrozole }\end{array}$ & $\begin{array}{l}17 / 98 \\
(17.4)\end{array}$ & $\begin{array}{c}27 / 105 \\
(25.7)\end{array}$ & 0.148 & \\
\hline \multirow[t]{3}{*}{$\begin{array}{l}\text { Neonatal } \\
\text { outcomes: } \\
\text { Birthweight } \\
(\mathrm{kg})\end{array}$} & $\begin{array}{l}\text { Legro, } \\
2014^{131} \\
\text { RCT } \\
(750)\end{array}$ & $\mathrm{CC}$ & Letrozole & $\begin{array}{c}\text { Mean }=3.23 \\
(+/-0.7153)\end{array}$ & $\begin{array}{c}\text { Mean }=3.23 \\
(+/-0.6574)\end{array}$ & 0.83 & $\begin{array}{l}\text { No } \\
\text { difference }\end{array}$ \\
\hline & $\begin{array}{l}\text { Wu, } \\
2016^{175}\end{array}$ & Letrozole & Berberine & $\begin{array}{l}\text { Mean = 3.463 } \\
(+/-0.575 \text { SD })\end{array}$ & $\begin{array}{c}\text { Mean }=3.542 \\
(+/-0.399 \\
\text { SD })\end{array}$ & 0.216 & \multirow[t]{2}{*}{$\begin{array}{l}\text { No } \\
\text { difference }\end{array}$} \\
\hline & $\begin{array}{l}\text { RCT } \\
(644)\end{array}$ & & $\begin{array}{l}\text { Berberine + } \\
\text { Letrozole }\end{array}$ & $\begin{array}{c}\text { Mean }=3.463 \\
(+/-0.575 \\
\text { SD })\end{array}$ & $\begin{array}{c}\text { Mean }=3.484 \\
(+/-0.504 \\
\text { SD })\end{array}$ & 0.246 & \\
\hline \multirow[t]{4}{*}{$\begin{array}{l}\text { Neonatal } \\
\text { outcomes: } \\
\text { Congenital } \\
\text { anomalies }\end{array}$} & $\begin{array}{l}\text { Legro, } \\
2014^{131} \\
\text { RCT } \\
(750)\end{array}$ & $\mathrm{CC}$ & Letrozole & $\begin{array}{c}1 / 66 \\
(1.5) \\
(0.0 \text { to } 5.5)\end{array}$ & $\begin{array}{c}4 / 102 \\
(3.9) \\
(1.1 \text { to } 8.4)\end{array}$ & 0.37 & $\begin{array}{l}\text { No } \\
\text { difference }\end{array}$ \\
\hline & $\begin{array}{l}\text { Amer, } \\
2017^{179} \\
\text { RCT } \\
(159)\end{array}$ & $\mathrm{CC}$ & Letrozole & 0 & 0 & NS & $\begin{array}{l}\text { No } \\
\text { difference }\end{array}$ \\
\hline & \multirow[t]{2}{*}{$\begin{array}{l}\text { Legro, } \\
2007^{128}\end{array}$} & \multirow[t]{2}{*}{$\mathrm{CC}$} & Metformin & $\begin{array}{c}0 / 209 \\
(0)\end{array}$ & $\begin{array}{c}0 / 208 \\
(0)\end{array}$ & NS & $\begin{array}{l}\text { No } \\
\text { difference }\end{array}$ \\
\hline & & & $\begin{array}{l}\text { Metformin+ } \\
\text { CC }\end{array}$ & $\begin{array}{c}0 / 209 \\
(0)\end{array}$ & $\begin{array}{c}2 / 209 \\
(1.0) \\
(0.1 \text { to } 2.6)\end{array}$ & 0.15 & $\begin{array}{l}\text { No } \\
\text { difference }\end{array}$ \\
\hline $\begin{array}{l}\text { Time to } \\
\text { pregnancy } \\
\text { (days) }\end{array}$ & $\begin{array}{l}\text { Legro, } \\
2014^{131} \\
\text { RCT } \\
(750)\end{array}$ & $\mathrm{CC}$ & Letrozole & 85.9 & 90.4 & 0.27 & $\begin{array}{l}\text { No } \\
\text { difference }\end{array}$ \\
\hline
\end{tabular}




\begin{tabular}{|l|l|l|l|c|c|c|c|}
\hline Outcome & $\begin{array}{c}\text { Study } \\
\text { Design } \\
\mathbf{( N} \\
\text { Patients) }\end{array}$ & Intervention & Comparator & $\begin{array}{c}\text { Results } \\
\text { Intervention } \\
\mathbf{N} \\
\mathbf{( \% )} \\
(\mathbf{9 5 \%} \mathbf{C l})\end{array}$ & $\begin{array}{c}\text { Results } \\
\text { Comparator } \\
\mathbf{N} \\
\mathbf{( \% )} \\
\mathbf{( 9 5 \% ~ C l )}\end{array}$ & $\begin{array}{c}\mathbf{P} \\
\text { Value }\end{array}$ & $\begin{array}{c}\text { Summary } \\
\text { of Study } \\
\text { Findings }\end{array}$ \\
\hline & $\begin{array}{l}\text { Morin- } \\
\text { Papunen, } \\
2012^{151}\end{array}$ & CC & $\begin{array}{l}\text { Metformin+ } \\
\text { CC }\end{array}$ & 172 & 171 & 0.9 & $\begin{array}{l}\text { No } \\
\text { difference }\end{array}$ \\
& & & & & & \\
\\
RCT & & & & & & & \\
\hline
\end{tabular}

Abbreviations: $\mathrm{CC}=$ clomiphene citrate; $\mathrm{CI}=$ confidence interval; $\mathrm{N}=$ number of patients; $\mathrm{NS}=$ not statistically significant; PCOS=polycystic ovary syndrome; RCT=randomized control trial; SD=standard deviation.

\section{Oral Agents Alone Versus Acupuncture for PCOS}

One fair-quality factorial RCT with 1000 women ${ }^{188}$ compared clomiphene and acupuncture alone and combined. Results for live births, miscarriage, ectopic pregnancy and congenital abnormality are summarized in Table 5 . When analyzing the main effects of acupuncture and clomiphene, the live birth rate was significantly higher in the clomiphene group as compared to placebo whereas it was not significantly different for the active and control acupuncture. SOE was rated as low for all outcomes given findings from one study with potential risk of bias.

Table 5. Outcomes for comparisons of oral agents alone versus acupuncture in women with PCOS

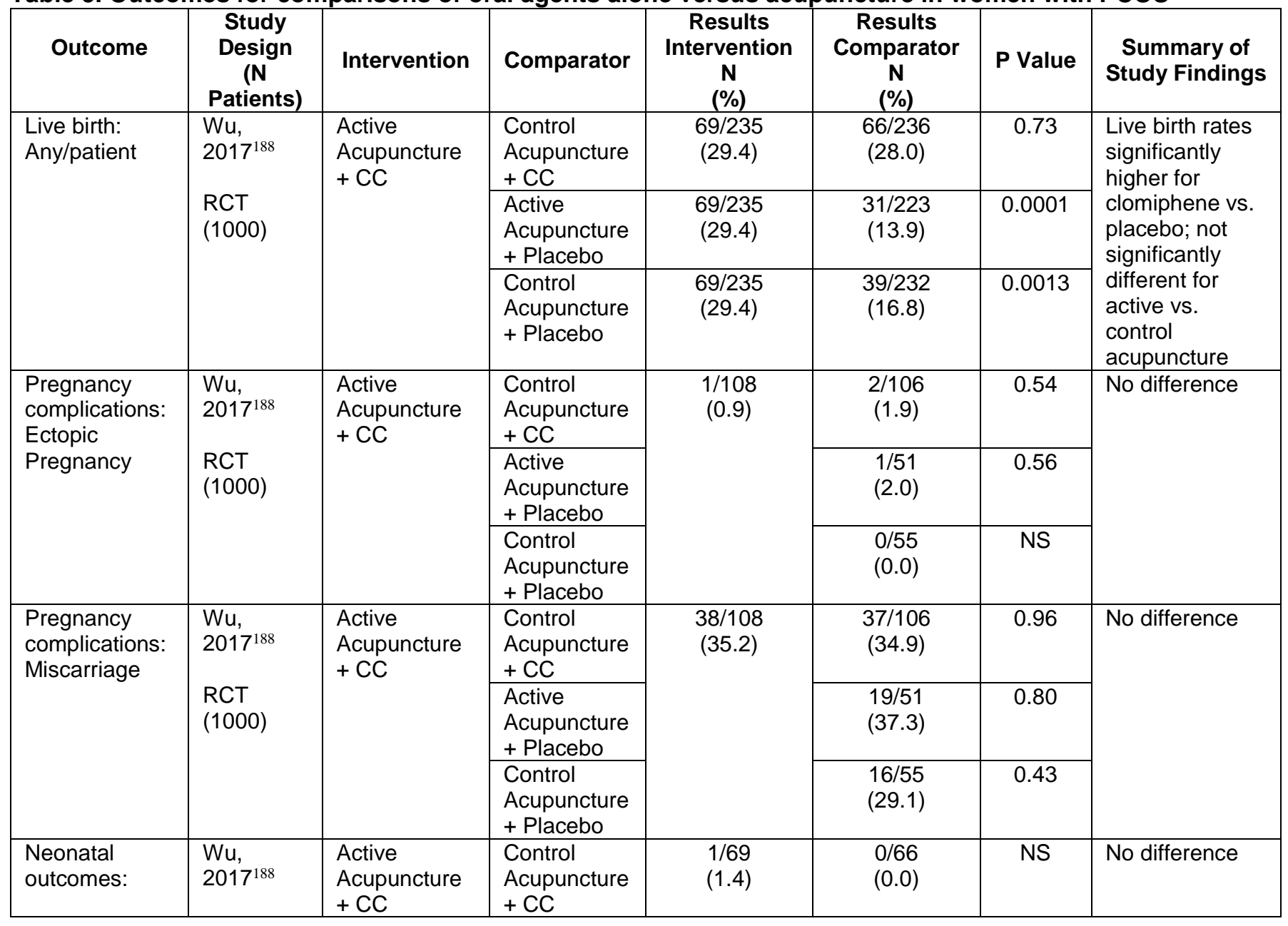




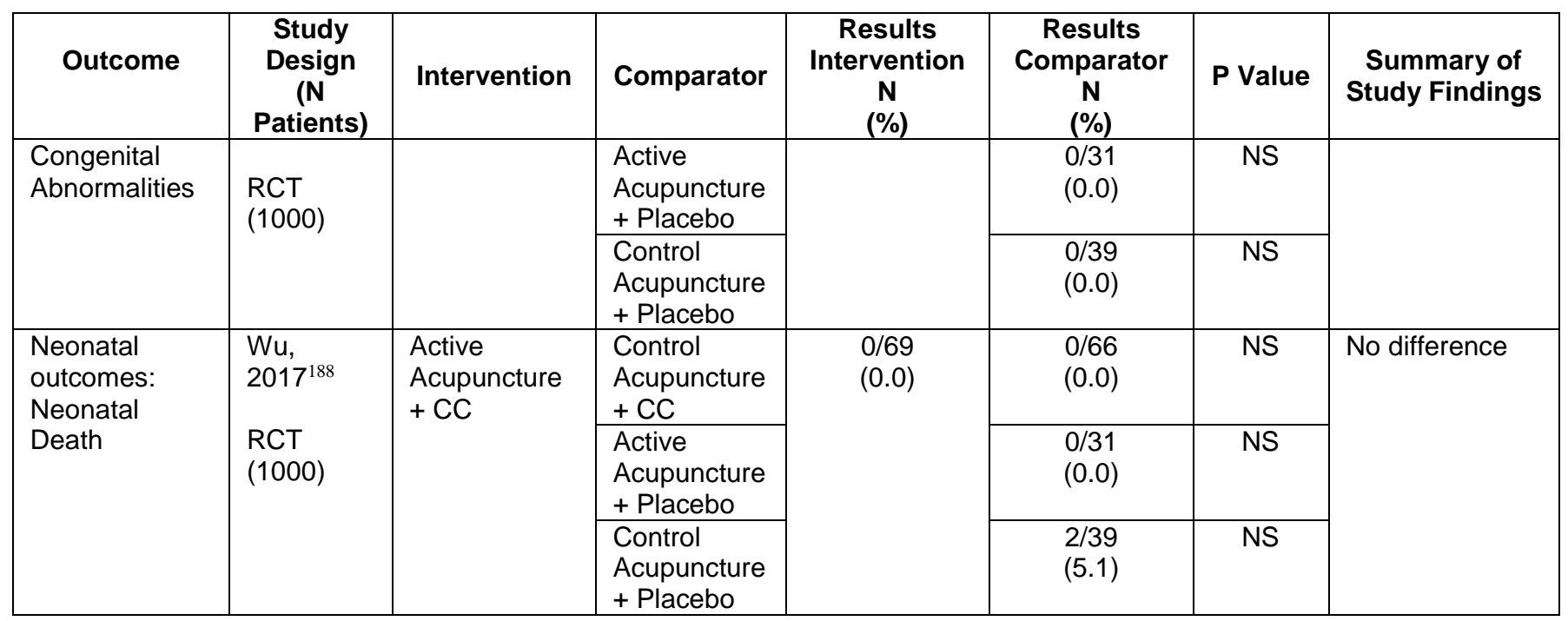

Abbreviations: $\mathrm{CC}=$ clomiphene citrate; $\mathrm{CI}=$ confidence interval; $\mathrm{N}=$ number of patients; $\mathrm{NS}=$ not statistically significant; PCOS=polycystic ovary syndrome; RCT=randomized control trial; SD=standard deviation.

\section{Oral Agents Alone Versus Oral Agents With IUI for PCOS}

One good-quality RCT $\mathrm{T}^{157}$ compared clomiphene citrate without IUI to clomiphene citrate with IUI in women with PCOS. The results for live birth (reported as any live birth per patient), pregnancy complications (multiple births, ectopic, miscarriage) were reported. Results for live births and pregnancy complications are summarized in Table 6 (insufficient SOE). There was no evidence for this treatment regarding neonatal outcomes, time to pregnancy, costs, short-term adverse effects, and long-term child or maternal outcomes.

A second good-quality RCT ${ }^{182}$ compared Myo-inositol before and during ovulation induction followed by IUI compared to ovulation induction and IUI alone in women with PCOS. Frequency of OHSS and multiple births were reported and are summarized in Table 6 (insufficient SOE).

Table 6. Outcomes for oral agents alone versus oral agents with IUI in women with PCOS

\begin{tabular}{|c|c|c|c|c|c|c|c|}
\hline Outcome & $\begin{array}{c}\text { Study } \\
\text { Design } \\
\text { (N } \\
\text { Patients) }\end{array}$ & Intervention & Comparator & $\begin{array}{c}\text { Results } \\
\text { Intervention } \\
\mathbf{N} \\
(\%) \\
(95 \% \mathrm{Cl})\end{array}$ & $\begin{array}{c}\text { Results } \\
\text { Comparator } \\
N \\
(\%) \\
(95 \% \mathrm{Cl})\end{array}$ & P Value & $\begin{array}{l}\text { Summary } \\
\text { of Study } \\
\text { Findings }\end{array}$ \\
\hline $\begin{array}{l}\text { Live birth: } \\
\text { Any/patient }\end{array}$ & $\begin{array}{l}\text { Abu } \\
\text { Hashim, } \\
2011^{157} \\
\text { RCT } \\
(188)\end{array}$ & $\mathrm{CC}+\mathrm{IUI}$ & $\begin{array}{l}\mathrm{CC}+\text { timed } \\
\text { Intercourse }\end{array}$ & $\begin{array}{c}18 / 93 \\
(19.4 \%) \\
(12.0 \% \text { to } \\
27.9 \%)\end{array}$ & $\begin{array}{c}17 / 95 \\
(17.9 \%) \\
(10.9 \% \text { to } \\
26.2 \%)\end{array}$ & 0.33 & $\begin{array}{l}\text { No } \\
\text { difference }\end{array}$ \\
\hline $\begin{array}{l}\text { Pregnancy } \\
\text { complications: } \\
\text { Multiple births }\end{array}$ & $\begin{array}{l}\text { Abu } \\
\text { Hashim, } \\
2011^{157} \\
\text { RCT } \\
(188)\end{array}$ & $\mathrm{CC}+\mathrm{IUI}$ & $\begin{array}{l}\mathrm{CC}+\text { timed } \\
\text { Intercourse }\end{array}$ & $\begin{array}{c}2 / 22 \\
(9.1 \%) \\
(1.2 \% \text { to } \\
23.8 \%)\end{array}$ & $\begin{array}{c}2 / 21 \\
(9.5 \%) \\
(1.2 \% \text { to } \\
24.9 \%)\end{array}$ & 0.46 & $\begin{array}{l}\text { No } \\
\text { difference }\end{array}$ \\
\hline
\end{tabular}




\begin{tabular}{|c|c|c|c|c|c|c|c|}
\hline Outcome & $\begin{array}{c}\text { Study } \\
\text { Design } \\
\text { (N } \\
\text { Patients) }\end{array}$ & Intervention & Comparator & $\begin{array}{c}\text { Results } \\
\text { Intervention } \\
N \\
(\%) \\
(95 \% \mathrm{Cl})\end{array}$ & $\begin{array}{c}\text { Results } \\
\text { Comparator } \\
N \\
(\%) \\
(95 \% \mathrm{Cl})\end{array}$ & $P$ Value & $\begin{array}{l}\text { Summary } \\
\text { of Study } \\
\text { Findings }\end{array}$ \\
\hline & $\begin{array}{l}\text { Emekci } \\
\text { Ozay } \\
2017^{182} \\
\\
\text { RCT } \\
(196) \\
\end{array}$ & $\begin{array}{l}\mathrm{MYO} \\
+\mathrm{COH} / \mathrm{IUI}\end{array}$ & $\mathrm{COH} / \mathrm{IUI}$ & $\begin{array}{c}2 / 16 \\
(12.5 \%)\end{array}$ & $\begin{array}{c}1 / 11 \\
(9.1 \%)\end{array}$ & 0.78 & $\begin{array}{l}\text { No } \\
\text { difference }\end{array}$ \\
\hline $\begin{array}{l}\text { Pregnancy } \\
\text { complications: } \\
\text { Ectopic } \\
\text { pregnancy }\end{array}$ & $\begin{array}{l}\text { Abu } \\
\text { Hashim, } \\
2011^{157} \\
\text { RCT } \\
(188)\end{array}$ & $\mathrm{CC}+\mathrm{IUI}$ & $\begin{array}{l}\mathrm{CC}+\text { timed } \\
\text { Intercourse }\end{array}$ & $\begin{array}{l}0 / 93 \\
(0 \%)\end{array}$ & $\begin{array}{l}0 / 95 \\
(0 \%)\end{array}$ & NS & $\begin{array}{l}\text { No } \\
\text { difference }\end{array}$ \\
\hline $\begin{array}{l}\text { Pregnancy } \\
\text { complications: } \\
\text { Miscarriage }\end{array}$ & $\begin{array}{l}\text { Abu } \\
\text { Hashim, } \\
2011^{157} \\
\text { RCT } \\
(188)\end{array}$ & $C C+I U I$ & $\begin{array}{l}\mathrm{CC}+\text { timed } \\
\text { Intercourse }\end{array}$ & $\begin{array}{c}4 / 22 \\
(18.2 \%) \\
(5.4 \% \text { to } \\
36.3 \%)\end{array}$ & $\begin{array}{c}4 / 21 \\
(19.0 \%) \\
(5.7 \% \text { to } \\
37.9 \%)\end{array}$ & 0.31 & $\begin{array}{l}\text { No } \\
\text { difference }\end{array}$ \\
\hline $\begin{array}{l}\text { Short-term } \\
\text { adverse } \\
\text { effects: OHSS }\end{array}$ & $\begin{array}{l}\text { Emekci } \\
\text { Ozay } \\
2017^{182} \\
\\
\text { RCT } \\
(196)\end{array}$ & MYO +IUI & IUI alone & $\begin{array}{c}1 / 86 \\
(1.2 \%)\end{array}$ & $\begin{array}{c}3 / 90 \\
(3.3 \%)\end{array}$ & 0.35 & $\begin{array}{l}\text { No } \\
\text { difference }\end{array}$ \\
\hline
\end{tabular}

Abbreviations: $\mathrm{CC}=$ clomiphene citrate; $\mathrm{CI}=$ confidence interval; $\mathrm{COH}=$ controlled ovarian hyperstimulation; IUI=intrauterine insemination; $\mathrm{MYO}=\mathrm{Myo}$-inositol; $\mathrm{N}=$ number of patients; $\mathrm{NR}=$ not reported; $\mathrm{NS}=$ not statistically significant; OHSS=0varian hyperstimulation syndrome; PCOS=polycystic ovary syndrome; RCT=randomized control trial; SD=standard deviation

\section{Oral Agents Alone Versus Surgical Management for PCOS}

Seven RCTs ${ }^{154,156,163,165,168,169,189}$ (four good quality, three fair quality) compared the use of oral agents without IUI to surgical management in women with PCOS. These studies varied in the medication used as well as in the specific surgical methods. These 7 studies included 918 patients. One good-quality systematic review of 25 studies also explored laparoscopic ovarian drilling versus oral agents alone. ${ }^{81}$ All but one ${ }^{189}$ of the seven included studies were included in this systematic review and therefore the findings of this systematic review are highlighted when they assessed outcomes of interest.

Outcomes are summarized in Table 7 and demonstrate that there were no significant differences in treatment outcomes between pharmacologic and surgical approaches for live birth within the individual studies. The systematic review of 25 studies by Farquhar et al. ${ }^{81}$ included 8 studies (1034 women) where live birth was evaluated also found no significant differences in live birth rates comparing LOD to oral agents (OR $=0.77,95 \%$ CI 0.59 to 1.01 ) (moderate SOE). When exploring specific oral agents, this review found that LOD compared to clomiphene plus tamoxifen (OR 0.81; 95\% CI, 0.42 to 1.53 based on 1 trial, $\mathrm{n}=150$ ), or to letrozole (OR 0.97; $95 \%$ CI, 0.59 to 1.59 based on 3 trials, $n=318$ ) did not support a difference in live birth dates, but it did find a significantly lower birth rate with LOD compared to clomiphene plus metformin (OR 0.44; 95\% CI, 0.24 to 0.82 based on 2 trials, $\mathrm{n}=159$ ).

Our included studies did not demonstrate a difference in miscarriage. The systematic review synthesized findings from 15 studies (1592 women) and found no difference between LOD and 
other treatments (OR 1.1, 95\% CI 0.74 to 1.61) (low SOE). The evidence was downgraded since there were inadequate explanations of randomization (in 3 trials), allocation concealment (8 trials) and inadequate or no blinding reported in 8 trials. The one study not included in the systematic review $^{189}$ also showed no significant difference in miscarriage (18\% vs. $\left.7 \%, \mathrm{p}=0.41\right)$.

The multiple pregnancy rate was evaluated in 12 studies (1129 women) and found to be lower in women undergoing LOD (OR 0.21, 95\% CI 0.08 to 0.58) (moderate SOE). ${ }^{81}$

The evidence did not show any differences between treatments for the outcomes of OHSS, and surgical complications but was considered insufficient SOE given the small numbers of events and the imprecision in the included studies.

There was no evidence for this treatment regarding neonatal outcomes, time to pregnancy, costs, and long-term child or maternal outcomes.

Table 7. Outcomes for oral agents alone versus surgical management in women with PCOS

\begin{tabular}{|c|c|c|c|c|c|c|c|}
\hline Outcome & $\begin{array}{c}\text { Study } \\
\text { Design } \\
\text { (N } \\
\text { Patients) }\end{array}$ & Intervention & Comparator & $\begin{array}{c}\text { Results } \\
\text { Intervention } \\
\mathbf{N} \\
(\%) \\
(95 \% \mathrm{Cl}) \\
\end{array}$ & $\begin{array}{c}\text { Results } \\
\text { Comparator } \\
\mathrm{N} \\
(\%) \\
(95 \% \mathrm{Cl}) \\
\end{array}$ & $\begin{array}{c}\mathbf{P} \\
\text { Value }\end{array}$ & $\begin{array}{c}\text { Summary of } \\
\text { Study } \\
\text { Findings }\end{array}$ \\
\hline \multirow[t]{5}{*}{$\begin{array}{l}\text { Live birth: } \\
\text { Any/patient }\end{array}$} & $\begin{array}{l}\text { Abu } \\
\text { Hashim, } \\
2011^{154} \\
\text { RCT } \\
(176)\end{array}$ & $\mathrm{CC}$ & LOD & $\begin{array}{c}25 / 89 \\
(28.1) \\
(19.3 \text { to } 37.8)\end{array}$ & $\begin{array}{c}28 / 87 \\
(32.2) \\
(22.8 \text { to } 42.3)\end{array}$ & 0.55 & No difference \\
\hline & $\begin{array}{l}\text { Palomba, } \\
2010^{163} \\
\text { RCT } \\
(47)\end{array}$ & $\mathrm{CC}$ & LOD & $\begin{array}{c}12 / 23 \\
(52.2) \\
(32.2 \text { to } 71.8)\end{array}$ & $\begin{array}{c}13 / 24 \\
(54.2) \\
\text { (34.5 to } 73.2)\end{array}$ & 1 & No difference \\
\hline & $\begin{array}{l}\text { Amer, } \\
2009^{165} \\
\text { RCT } \\
(65)\end{array}$ & $\mathrm{CC}$ & LOD & $\begin{array}{c}18 / 32 \\
(56.3) \\
(39.1 \text { to } 72.7)\end{array}$ & $\begin{array}{c}15 / 33 \\
(45.5) \\
(29.1 \text { to } 62.3)\end{array}$ & 0.27 & No difference \\
\hline & $\begin{array}{l}\text { Abu } \\
\text { Hashim, } \\
2010^{168} \\
\text { RCT } \\
(260)\end{array}$ & Letrozole & LOD & $\begin{array}{c}32 / 128 \\
(25.0) \\
(17.9 \text { to } 32.8)\end{array}$ & $\begin{array}{c}33 / 132 \\
(25.0) \\
(18.0 \text { to } 32.7)\end{array}$ & 1 & No difference \\
\hline & $\begin{array}{l}\text { Zakherah } \\
, 2010^{169} \\
\text { RCT } \\
(150)\end{array}$ & $\begin{array}{l}\mathrm{CC}+ \\
\text { tamoxifen }\end{array}$ & LOD & $\begin{array}{c}37 / 75 \\
(49.3) \\
(38.1 \text { to } 60.6)\end{array}$ & $\begin{array}{c}33 / 75 \\
(44.0) \\
(33.0 \text { to } 55.3)\end{array}$ & 0.35 & No difference \\
\hline $\begin{array}{l}\text { Pregnancy } \\
\text { complications: } \\
\text { Multiple births }\end{array}$ & $\begin{array}{l}\text { Abdellah, } \\
2011^{156} \\
\text { RCT } \\
(140)\end{array}$ & Letrozole & LOD & $\begin{array}{c}0 / 23 \\
(0)\end{array}$ & $\begin{array}{c}0 / 16 \\
(0)\end{array}$ & NS & No difference \\
\hline
\end{tabular}




\begin{tabular}{|c|c|c|c|c|c|c|c|}
\hline Outcome & $\begin{array}{c}\text { Study } \\
\text { Design } \\
\text { (N } \\
\text { Patients) }\end{array}$ & Intervention & Comparator & $\begin{array}{c}\text { Results } \\
\text { Intervention } \\
N \\
(\%) \\
(95 \% \mathrm{Cl}) \\
\end{array}$ & $\begin{array}{c}\text { Results } \\
\text { Comparator } \\
N \\
(\%) \\
(95 \% \mathrm{Cl}) \\
\end{array}$ & $\begin{array}{c}P \\
\text { Value }\end{array}$ & $\begin{array}{c}\text { Summary of } \\
\text { Study } \\
\text { Findings }\end{array}$ \\
\hline & $\begin{array}{l}\text { Abu } \\
\text { Hashim, } \\
2010^{168} \\
\text { RCT } \\
(260)\end{array}$ & Letrozole & LOD & $\begin{array}{c}0 / 128 \\
(0)\end{array}$ & $\begin{array}{c}0 / 132 \\
(0)\end{array}$ & NS & No difference \\
\hline & $\begin{array}{l}\text { Amer, } \\
2009^{165} \\
\\
\text { RCT } \\
(65)\end{array}$ & $\mathrm{CC}$ & LOD & $\begin{array}{c}0 / 20 \\
(0)\end{array}$ & $\begin{array}{c}0 / 17 \\
(0)\end{array}$ & NS & No difference \\
\hline \multirow[t]{6}{*}{$\begin{array}{l}\text { Pregnancy } \\
\text { complications: } \\
\text { Miscarriage }\end{array}$} & $\begin{array}{l}\text { Abu } \\
\text { Hashim, } \\
2011^{154} \\
\text { RCT } \\
(176)\end{array}$ & $\mathrm{CC}$ & LOD & $\begin{array}{c}5 / 30 \\
(16.7) \\
\text { (5.8 to } 31.7 \text { ) }\end{array}$ & $\begin{array}{c}6 / 34 \\
(17.6) \\
\text { (7.0 to } 31.6)\end{array}$ & 0.92 & No difference \\
\hline & $\begin{array}{l}\text { Abdellah, } \\
2011^{156} \\
\text { RCT } \\
(140)\end{array}$ & Letrozole & LOD & $\begin{array}{c}2 / 25 \\
(8.0) \\
\text { (1.0 to } 2.1)\end{array}$ & $\begin{array}{c}4 / 20 \\
(20.0) \\
\text { (6.1 to } 39.6)\end{array}$ & 0.231 & No difference \\
\hline & $\begin{array}{l}\text { Amer, } \\
2009^{165} \\
\\
\text { RCT } \\
(65)\end{array}$ & $\mathrm{CC}$ & LOD & $\begin{array}{c}2 / 20 \\
(10.0) \\
\text { (1.3 to } 26.0)\end{array}$ & $\begin{array}{c}2 / 17 \\
(11.8) \\
\text { (1.6 to } 30.2)\end{array}$ & 0.62 & No difference \\
\hline & $\begin{array}{l}\text { Abu } \\
\text { Hashim, } \\
2010^{168} \\
\text { RCT } \\
(260)\end{array}$ & Letrozole & LOD & $\begin{array}{c}4 / 128 \\
(3.1) \\
\text { (0.9 to } 6.7)\end{array}$ & $\begin{array}{c}4 / 132 \\
(3.0) \\
(0.8 \text { to } 6.5)\end{array}$ & 0.92 & No difference \\
\hline & $\begin{array}{l}\text { Zakherah } \\
, 2010^{169} \\
\text { RCT } \\
(150)\end{array}$ & $\begin{array}{l}\mathrm{CC}+ \\
\text { tamoxifen }\end{array}$ & LOD & $\begin{array}{c}3 / 40 \\
(7.5) \\
(1.6 \text { to } 17.3)\end{array}$ & $\begin{array}{c}5 / 38 \\
(13.2) \\
(4.5 \text { to } 25.4)\end{array}$ & 0.06 & No difference \\
\hline & $\begin{array}{l}\text { Ibrahim, } \\
2017^{189} \\
\text { RCT } \\
(80)\end{array}$ & Letrozole & LOD & $\begin{array}{c}1 / 14 \\
(7)\end{array}$ & $\begin{array}{l}2 / 11 \\
(18)\end{array}$ & 0.41 & No difference \\
\hline $\begin{array}{l}\text { Short-term } \\
\text { adverse } \\
\text { effects: OHSS }\end{array}$ & $\begin{array}{l}\text { Amer, } \\
2009^{165} \\
\\
\text { RCT } \\
(65)\end{array}$ & $\mathrm{CC}$ & LOD & $\begin{array}{c}1 / 32 \\
(3.1) \\
(0.1 \text { to } 11.2)\end{array}$ & $\begin{array}{c}0 / 33 \\
(0)\end{array}$ & 0.31 & No difference \\
\hline
\end{tabular}




\begin{tabular}{|c|c|c|c|c|c|c|c|}
\hline Outcome & $\begin{array}{c}\text { Study } \\
\text { Design } \\
\text { (N } \\
\text { Patients) }\end{array}$ & Intervention & Comparator & $\begin{array}{c}\text { Results } \\
\text { Intervention } \\
N \\
(\%) \\
(95 \% \mathrm{Cl})\end{array}$ & $\begin{array}{c}\text { Results } \\
\text { Comparator } \\
N \\
(\%) \\
(95 \% \mathrm{Cl})\end{array}$ & $\begin{array}{c}P \\
\text { Value }\end{array}$ & $\begin{array}{c}\text { Summary of } \\
\text { Study } \\
\text { Findings }\end{array}$ \\
\hline & $\begin{array}{l}\text { Abu } \\
\text { Hashim, } \\
2010^{168} \\
\text { RCT } \\
(260)\end{array}$ & Letrozole & LOD & $\begin{array}{c}0 / 128 \\
(0)\end{array}$ & $\begin{array}{c}0 / 132 \\
(0)\end{array}$ & NS & No difference \\
\hline \multirow[t]{2}{*}{$\begin{array}{l}\text { Surgical } \\
\text { complications }\end{array}$} & $\begin{array}{l}\text { Palomba, } \\
2010^{163} \\
\text { RCT } \\
(47)\end{array}$ & $\mathrm{CC}$ & LOD & NA & $\begin{array}{c}0 / 24 \\
(0)\end{array}$ & $\mathrm{NR}$ & No difference \\
\hline & $\begin{array}{l}\text { Amer, } \\
2009165 \\
\\
\text { RCT } \\
(65)\end{array}$ & $\mathrm{CC}$ & LOD & NA & $\begin{array}{c}1 / 33 \\
(3.0) \\
(0.1 \text { to } 10.9)\end{array}$ & $\mathrm{NR}$ & No difference \\
\hline
\end{tabular}

Abbreviations: $\mathrm{CC}=$ clomiphene citrate; $\mathrm{CI}=$ confidence interval; $\mathrm{LOD}=$ laparoscopic ovarian drilling; $\mathrm{N}=$ number of patients; $\mathrm{NA}=$ not applicable; $\mathrm{NR}=$ not reported; NS=not statistically significant; OHSS=ovarian hyperstimulation syndrome; PCOS=polycystic ovary syndrome; RCT=randomized control trial

\section{Oral Agents Versus Gonadotropins for PCOS}

Five RCTs, three good-quality and two fair-quality, compared clomiphene to folliclestimulating hormone (FSH) in women with PCOS with variation across the studies in the treatment protocols. ${ }^{145,152,176,187,193}$ Treatment with clomiphene citrate was compared to FSH only in two studies. ${ }^{152,176}$ Another study with a factorial design compared FSH with or without IUI to CC with or without IUI. ${ }^{187}$ The fourth study compared clomiphene plus urinary FSH to FSH alone $^{145}$ and a fifth study compared extended clomiphene treatment to human menopausal gonadotropin. ${ }^{193}$ Outcomes for the studies are summarized in Table 8.

Higher birth rates and higher miscarriage rates were reported with FSH treatment compared to CC in one good-quality RCT ${ }^{187}$, whereas no differences between FSH and CC were observed in two other studies, one good-quality ${ }^{152}$ and one fair quality. ${ }^{176}$ SOE was rated as insufficient for both outcomes given inconsistent evidence from the included studies. Ectopic pregnancies and birthweight did not significantly differ between groups (SOE low).

A good-quality RCT ${ }^{145}$ compared clomiphene plus urinary FSH to FSH alone. No statistically significant difference in live birth or pregnancy complications were reported. Given the limited evidence and imprecise findings these outcomes were rated insufficient for strength of evidence.

There was no evidence for these treatment comparisons regarding time to pregnancy, costs, short-term adverse effects, and long-term child outcomes.

One RCT compared letrozole to FSH among women with clomiphene resistant PCOS ${ }^{183}$. Outcomes of interested that were reported include miscarriage rate and OHSS development with no difference seen between groups.

We also identified one meta-analysis of five studies with 264 women that compared ovulation induction using gonadotropins with and without metformin. ${ }^{197}$ Live births were significantly higher in the metformin group 457/1000 versus 267/1000 (OR 2.31, 95\% CI 1.24 to 4.33) based on two studies including 180 women. Miscarriage rates were not significantly 
different, based on three studies including 84 women (OR 0.62, 95\% CI 0.19 to 2.01). OHSS also was not significantly different based on two studies including180 women (OR 0.32, 95\% CI 0.01 to 8.23). The authors of the meta-analysis judged the overall SOE to be low.

Table 8. Outcomes for oral agents versus gonadotropins in women with PCOS

\begin{tabular}{|c|c|c|c|c|c|c|c|}
\hline Outcome & $\begin{array}{c}\text { Study } \\
\text { Design } \\
\text { (N } \\
\text { Patients) }\end{array}$ & Intervention & Comparator & $\begin{array}{c}\text { Results } \\
\text { Intervention } \\
N \\
(\%) \\
(95 \% \mathrm{Cl}) \\
\end{array}$ & $\begin{array}{c}\text { Results } \\
\text { Comparator } \\
N \\
(\%) \\
(95 \% \mathrm{Cl}) \\
\end{array}$ & P Value & $\begin{array}{l}\text { Summary } \\
\text { of Study } \\
\text { Findings }\end{array}$ \\
\hline \multirow[t]{6}{*}{$\begin{array}{l}\text { Live birth: } \\
\text { Any/patient }\end{array}$} & $\begin{array}{l}\text { Homburg, } \\
2012^{152} \\
\text { RCT } \\
(302)\end{array}$ & CC & FSH & $\begin{array}{c}53 / 143 \\
(37.1) \\
(29.4 \text { to } 45.1)\end{array}$ & $\begin{array}{c}72 / 159 \\
(45.3) \\
(37.6 \text { to } 53.0)\end{array}$ & 0.12 & $\begin{array}{l}\text { No } \\
\text { difference }\end{array}$ \\
\hline & $\begin{array}{l}\text { Hossein- } \\
\text { Rashidi, } \\
2016^{176} \\
\text { RCT } \\
(104)\end{array}$ & CC & $\mathrm{FSH}$ & $\begin{array}{l}5 / 52 \\
(9.6)\end{array}$ & $\begin{array}{c}5 / 44 \\
(11.4)\end{array}$ & 0.78 & $\begin{array}{l}\text { No } \\
\text { difference }\end{array}$ \\
\hline & \multirow{3}{*}{$\begin{array}{l}\text { Weiss, } \\
20188^{187} \\
\text { RCT } \\
(666)\end{array}$} & \multirow[t]{3}{*}{ CC } & $\mathrm{CC}+\mathrm{IUI}$ & \multirow[t]{3}{*}{$\begin{array}{c}66 / 171 \\
(38.6)\end{array}$} & $\begin{array}{c}72 / 163 \\
(44.2)\end{array}$ & \multirow{3}{*}{$\begin{array}{l}\text { FSH vs. } \\
\text { CC: } \\
0.0124\end{array}$} & \multirow{3}{*}{$\begin{array}{l}\text { Greater } \\
\text { live births } \\
\text { for FSH } \\
\text { than CC; } \\
\text { no } \\
\text { significant } \\
\text { difference } \\
\text { between } \\
\text { IUI and } \\
\text { intercours } \\
\text { e. }\end{array}$} \\
\hline & & & $\mathrm{FSH}$ & & $\begin{array}{l}78 / 163 \\
(47.9) \\
\end{array}$ & & \\
\hline & & & $\mathrm{FSH}+\mathrm{IUI}$ & & $\begin{array}{l}89 / 164 \\
(54.3)\end{array}$ & & \\
\hline & $\begin{array}{l}\text { Ghanem, } \\
2013^{145} \\
\text { RCT } \\
(159) \\
\end{array}$ & $\mathrm{CC}+\mathrm{FSH}$ & $\mathrm{FSH}$ & $\begin{array}{c}22 / 87 \\
(25.3) \\
(16.8 \text { to } 34.9)\end{array}$ & $\begin{array}{c}19 / 87 \\
(21.8) \\
(13.9 \text { to } 31.0)\end{array}$ & 0.85 & $\begin{array}{l}\text { No } \\
\text { difference }\end{array}$ \\
\hline $\begin{array}{l}\text { Pregnancy } \\
\text { complications: } \\
\text { Multiple births }\end{array}$ & $\begin{array}{l}\text { Homburg, } \\
2012^{152} \\
\text { RCT } \\
(302)\end{array}$ & $\mathrm{CC}$ & $\mathrm{FSH}$ & $\begin{array}{c}0 / 143 \\
(0)\end{array}$ & $\begin{array}{c}2 / 159 \\
(1.3) \\
(0.2 \text { to } 3.5)\end{array}$ & 0.17 & $\begin{array}{l}\text { No } \\
\text { difference }\end{array}$ \\
\hline \multirow[t]{4}{*}{$\begin{array}{l}\text { Pregnancy } \\
\text { complications: } \\
\text { Ectopic } \\
\text { pregnancy }\end{array}$} & $\begin{array}{l}\text { Homburg, } \\
2012^{152} \\
\text { RCT } \\
(302)\end{array}$ & $\mathrm{CC}$ & $\mathrm{FSH}$ & $\begin{array}{c}1 / 143 \\
(0.7) \\
(0.0 \text { to } 2.6)\end{array}$ & $\begin{array}{c}1 / 159 \\
(0.6) \\
(0.0 \text { to } 2.3)\end{array}$ & 0.91 & $\begin{array}{l}\text { No } \\
\text { difference }\end{array}$ \\
\hline & \multirow{3}{*}{$\begin{array}{l}\text { Weiss, } \\
2018^{187} \\
\\
\text { RCT } \\
(666)\end{array}$} & \multirow[t]{3}{*}{ CC } & $\mathrm{CC}+\mathrm{IUI}$ & \multirow[t]{3}{*}{$\begin{array}{c}1 / 171 \\
(0.6)\end{array}$} & $\begin{array}{c}3 / 163 \\
(1.8)\end{array}$ & \multirow{3}{*}{$\begin{array}{l}0.31 \\
\text { NS }\end{array}$} & \multirow[t]{3}{*}{$\begin{array}{l}\text { No } \\
\text { difference }\end{array}$} \\
\hline & & & $\mathrm{FSH}$ & & $\begin{array}{c}1 / 163 \\
(0.6) \\
\end{array}$ & & \\
\hline & & & $\mathrm{FSH}+\mathrm{IUI}$ & & $\begin{array}{c}1 / 164 \\
(0.6) \\
\end{array}$ & & \\
\hline $\begin{array}{l}\text { Pregnancy } \\
\text { complications: } \\
\text { Miscarriage }\end{array}$ & $\begin{array}{l}\text { Homburg, } \\
2012^{152} \\
\text { RCT } \\
(302)\end{array}$ & $\mathrm{CC}$ & $\mathrm{FSH}$ & $\begin{array}{c}5 / 143 \\
(3.5) \\
\text { (1.2 to } 7.1)\end{array}$ & $\begin{array}{c}7 / 159 \\
(4.4) \\
(1.8 \text { to } 8.1)\end{array}$ & 0.68 & $\begin{array}{l}\text { No } \\
\text { difference }\end{array}$ \\
\hline
\end{tabular}




\begin{tabular}{|c|c|c|c|c|c|c|c|}
\hline Outcome & $\begin{array}{c}\text { Study } \\
\text { Design } \\
\text { (N } \\
\text { Patients) }\end{array}$ & Intervention & Comparator & $\begin{array}{c}\text { Results } \\
\text { Intervention } \\
\mathbf{N} \\
(\%) \\
(95 \% \mathrm{Cl}) \\
\end{array}$ & $\begin{array}{c}\text { Results } \\
\text { Comparator } \\
N \\
(\%) \\
(95 \% \mathrm{Cl}) \\
\end{array}$ & P Value & $\begin{array}{c}\text { Summary } \\
\text { of Study } \\
\text { Findings }\end{array}$ \\
\hline & $\begin{array}{l}\text { Hossein- } \\
\text { Rashidi, } \\
2016^{176} \\
\text { RCT } \\
(104)\end{array}$ & $\mathrm{CC}$ & $\mathrm{FSH}$ & $\begin{array}{l}0 / 52 \\
(0.0)\end{array}$ & $\begin{array}{l}1 / 44 \\
(2.3)\end{array}$ & 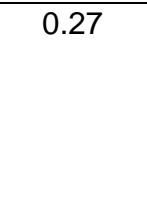 & $\begin{array}{l}\text { No } \\
\text { difference }\end{array}$ \\
\hline & $\begin{array}{l}\text { Weiss, } \\
2018^{187}\end{array}$ & \multirow[t]{3}{*}{ CC } & $\mathrm{CC}+\mathrm{IUI}$ & \multirow[t]{3}{*}{$\begin{array}{c}3 / 171 \\
(1.8)\end{array}$} & $\begin{array}{c}8 / 163 \\
(4.9)\end{array}$ & \multirow{3}{*}{$\begin{array}{c}\text { FSH vS. } \\
\text { CC: } 0.02 \text {; } \\
\text { IUI vs. } \\
\text { inter- } \\
\text { course } \\
0.05\end{array}$} & \multirow{3}{*}{$\begin{array}{l}\text { Higher } \\
\text { miscarriag } \\
\text { e rate with } \\
\text { FSH and } \\
\text { with IUI }\end{array}$} \\
\hline & \multirow{2}{*}{$\begin{array}{l}\text { RCT } \\
(666)\end{array}$} & & $\mathrm{FSH}$ & & $\begin{array}{c}9 / 163 \\
(5.5) \\
\end{array}$ & & \\
\hline & & & $\mathrm{FSH}+\mathrm{IUI}$ & & $\begin{array}{c}15 / 164 \\
(9.1)\end{array}$ & & \\
\hline & $\begin{array}{l}\text { Hassan } \\
2017^{183}\end{array}$ & Letrozole & $\mathrm{FSH}$ & $2 / 21$ & $3 / 24$ & 0.999 & $\begin{array}{l}\text { No } \\
\text { difference }\end{array}$ \\
\hline & $\begin{array}{l}\text { Badawy } \\
2008^{193}\end{array}$ & $\begin{array}{l}\text { Extended } \\
\text { CC }\end{array}$ & $\mathrm{FSH}$ & $5 / 160$ & $4 / 158$ & NS & $\begin{array}{l}\text { No } \\
\text { difference }\end{array}$ \\
\hline \multirow{3}{*}{$\begin{array}{l}\text { Neonatal } \\
\text { outcomes: } \\
\text { Birthweight } \\
(\mathrm{kg})\end{array}$} & \multirow[t]{3}{*}{$\begin{array}{l}\text { Weiss, } \\
2018^{187}\end{array}$} & \multirow[t]{3}{*}{$\mathrm{CC}$} & CC+IUI & \multirow[t]{3}{*}{$\begin{array}{c}3.408 \\
(0.491 \text { SD) }\end{array}$} & $\begin{array}{c}3.178 \\
(0.714 \mathrm{SD})\end{array}$ & \multirow{3}{*}{$\begin{array}{c}\text { FSH vs. } \\
\text { CC: } 0.96 \text {; } \\
\text { IUI vs. } \\
\text { inter- } \\
\text { course } \\
0.14\end{array}$} & \multirow[t]{3}{*}{$\begin{array}{l}\text { No } \\
\text { difference }\end{array}$} \\
\hline & & & $\mathrm{FSH}$ & & $\begin{array}{c}3.302 \\
(0.769 \mathrm{SD})\end{array}$ & & \\
\hline & & & FSH+IUI & & $\begin{array}{c}3.279 \\
(0.695 \text { SD) }\end{array}$ & & \\
\hline
\end{tabular}

Abbreviations: $\mathrm{CC}=$ clomiphene citrate; $\mathrm{CI}=$ confidence interval; $\mathrm{FSH}=$ follicle-stimulating hormone; IUI=intrauterine insemination; N=number of patients; NR=not reported; NS=not statistically significant; PCOS=polycystic ovary syndrome; $\mathrm{RCT}=$ randomized control trial; $\mathrm{SD}=$ standard deviation 


\section{Lifestyle Interventions for PCOS}

Three studies looked at methods of lifestyle modifications for women with PCOS and their impact on outcomes of interest. One good-quality 3-arm study ${ }^{172}$ compared preconception continuous oral contraceptives (OCPs) to lifestyle modification with carloric restriction, weight loss medication and increased physical activity to a combined treatment of OCPs and lifestyle modifications. After the preconception intervention, all women started standard ovulation induction for four cycles with clomiphene citrate. The primary outcome was live birth rate, and relevant secondary outcomes included fecundity per ovulated patient, and adverse outcomes (ectopic pregnancy). Results are summarized in Table 9. There was no evidence for difference in live birth rate between arms, though fecundity per patient who ovulated was higher in women randomized to lifestyle intervention compared to OCP alone.

One good-quality multicenter RCT (LIFEstyle study) ${ }^{119}$ conducted a predetermined subgroup analyses based on ovulatory status (anovulatory vs. ovulatory). The intervention consisted of a 6-month program aimed at loss of 5-10\% of original body weight. Of those women who were anovulatory, $76 \%$ of women in the intervention group (pre-treatment lifestyle) and $74 \%$ in the control group (prompt treatment) met criteria for PCOS. Outcomes reported include overall and healthy live birth $<24$ months. There were no significant differences between lifestyle intervention and control on healthy live birth rate or overall live birth rate between ovulatory and anovulatory women. In addition, the effect of lifestyle intervention on overall and healthy birth rate was not altered by ovulatory status.

Finally, a third good-quality multi-center RCT ${ }^{181}$ compared a pre-IVF treatment 12 week strict low calorie liquid formula diet (LCD) to IVF treatment alone. 23\% of intervention participants and $18.3 \%$ of IVF only participants had PCOS, and a subgroup analysis was conducted for this group of 81 women (Table 9). No difference was seen between arms in the live birth rates. Combined, these three studies supported a moderate SOE for no difference in live birth rates from lifestyle modification interventions

Table 9. Outcomes for lifestyle intervention versus oral contraceptive pills or no intervention in women with PCOS

\begin{tabular}{|c|c|c|c|c|c|c|c|}
\hline Outcome & $\begin{array}{c}\text { Study } \\
\text { Design } \\
\text { (N } \\
\text { Patients) }\end{array}$ & Intervention & Comparator & $\begin{array}{c}\text { Results } \\
\text { Intervention } \\
N \\
(\%) \\
(95 \% \mathrm{Cl})\end{array}$ & $\begin{array}{c}\text { Results } \\
\text { Comparator } \\
N \\
(\%) \\
(95 \% \mathrm{Cl})\end{array}$ & $\begin{array}{c}P \\
\text { Value }\end{array}$ & $\begin{array}{l}\text { Summary } \\
\text { of Study } \\
\text { Findings }\end{array}$ \\
\hline \multirow[t]{4}{*}{$\begin{array}{l}\text { Live birth: } \\
\text { Any/patient }\end{array}$} & $\begin{array}{l}\text { Legro } \\
2015^{172}\end{array}$ & $\begin{array}{l}\text { Lifestyle + } \\
\text { CC }\end{array}$ & $\mathrm{OCP}+\mathrm{CC}$ & \multirow[t]{2}{*}{$\begin{array}{l}13 / 50 \\
(26.0)\end{array}$} & $\begin{array}{c}5 / 49 \\
(10.2)\end{array}$ & 0.06 & \multirow[t]{2}{*}{$\begin{array}{l}\text { No } \\
\text { difference }\end{array}$} \\
\hline & $\begin{array}{l}\text { RCT } \\
(132)\end{array}$ & & $\begin{array}{l}\text { OCP } \\
+ \text { Lifestyle + } \\
\text { CC }\end{array}$ & & $\begin{array}{l}12 / 50 \\
(24.0)\end{array}$ & 0.08 & \\
\hline & $\begin{array}{l}\text { Mutsaerts, } \\
2016^{119} \\
\text { RCT } \\
(564)\end{array}$ & Lifestyle & No Lifestyle & $\begin{array}{l}72 / 123 \\
(58.5)\end{array}$ & $\begin{array}{l}83 / 140 \\
(59.3)\end{array}$ & 0.90 & $\begin{array}{l}\text { No } \\
\text { difference }\end{array}$ \\
\hline & $\begin{array}{l}\text { Einarsson, } \\
2017^{181} \\
\text { RCT } \\
(317)\end{array}$ & LCD +IVF & IVF alone & $\begin{array}{l}11 / 40 \\
(27.5)\end{array}$ & $\begin{array}{c}9 / 41 \\
(22.0)\end{array}$ & 0.75 & $\begin{array}{l}\text { No } \\
\text { difference }\end{array}$ \\
\hline
\end{tabular}




\begin{tabular}{|c|c|c|c|c|c|c|c|}
\hline Outcome & $\begin{array}{c}\text { Study } \\
\text { Design } \\
\text { (N } \\
\text { Patients) }\end{array}$ & Intervention & Comparator & $\begin{array}{c}\text { Results } \\
\text { Intervention } \\
N \\
(\%) \\
(95 \% \mathrm{Cl})\end{array}$ & $\begin{array}{c}\text { Results } \\
\text { Comparator } \\
N \\
(\%) \\
(95 \% \mathrm{Cl})\end{array}$ & $\begin{array}{c}\mathbf{P} \\
\text { Value }\end{array}$ & $\begin{array}{l}\text { Summary } \\
\text { of Study } \\
\text { Findings }\end{array}$ \\
\hline \multirow[t]{2}{*}{$\begin{array}{l}\text { Live birth: Per- } \\
\text { ovulated } \\
\text { patient }\end{array}$} & \multirow[t]{2}{*}{$\begin{array}{l}\text { Legro } \\
2015^{172} \\
\text { RCT } \\
(132)\end{array}$} & \multirow[t]{2}{*}{$\begin{array}{l}\text { Lifestyle + } \\
\text { CC }\end{array}$} & $\mathrm{OCP}+\mathrm{CC}$ & \multirow[t]{2}{*}{$\begin{array}{l}13 / 36 \\
(36.1)\end{array}$} & $\begin{array}{c}5 / 36 \\
(13.9)\end{array}$ & 0.04 & $\begin{array}{l}\text { Increased } \\
\text { live birth } \\
\text { rate for } \\
\text { Lifestyle } \\
\text { intervention } \\
\text { than OCPs } \\
\text { alone. }\end{array}$ \\
\hline & & & $\begin{array}{l}\text { OCP } \\
+ \text { Lifestyle + } \\
\text { CC }\end{array}$ & & $\begin{array}{l}12 / 39 \\
(30.8)\end{array}$ & 0.10 & $\begin{array}{l}\text { No } \\
\text { difference }\end{array}$ \\
\hline \multirow{2}{*}{$\begin{array}{l}\text { Pregnancy } \\
\text { complications: } \\
\text { Ectopic } \\
\text { pregnancy }\end{array}$} & \multirow[t]{2}{*}{$\begin{array}{l}\text { Legro } \\
2015^{172}\end{array}$} & \multirow[t]{2}{*}{$\begin{array}{l}\text { Lifestyle + } \\
\text { CC }\end{array}$} & $\mathrm{OCP}+\mathrm{CC}$ & \multirow[t]{2}{*}{$\begin{array}{l}1 / 16 \\
(6.3)\end{array}$} & $\begin{array}{l}0 / 8 \\
(0)\end{array}$ & \multirow[t]{2}{*}{ NS } & \multirow[t]{2}{*}{$\begin{array}{l}\text { No } \\
\text { difference }\end{array}$} \\
\hline & & & $\begin{array}{l}\text { OCP+ } \\
\text { Lifestyle + } \\
\text { CC }\end{array}$ & & $\begin{array}{l}0 / 14 \\
(0)\end{array}$ & & \\
\hline
\end{tabular}

Abbreviations: $\mathrm{CC}$ = clomiphene citrate; $\mathrm{CI}=$ confidence interval; $\mathrm{hMG}=$ human menopausal gonadotropin; LCD=low calorie liquid formula diet; $\mathrm{N}=$ number of patients; $\mathrm{NR}=$ not reported; $\mathrm{NS}=$ not statistically significant; $\mathrm{OCP}=$ oral contraceptives; OHSS=ovarian hyperstimulation syndrome; PCOS=polycystic ovary syndrome; RCT=randomized control trial; $\mathrm{rFSH}=$ recombinant follicle-stimulating hormone

\section{Surgical Management Versus Gonadotropins for PCOS}

Three articles reporting two fair-quality RCTs ${ }^{141,142,148}$ compared surgical management to gonadotropins with IUI for PCOS. These studies varied in the medication type/protocol used for oral ovulation induction as well as in the specific methods used for laparoscopic ovarian electrocauterization (a form of laparoscopic ovarian drilling) and the protocol following surgery. The two studies included 272 patients.

Results are summarized in Table 10. In the study reporting live birth, ${ }^{141}$ rates were not significantly different between treatments. Multiple births and costs were also not different between strategies. ${ }^{142}$ In a study comparing adverse outcomes, OHSS was reported in $3.8 \%$ of subjects receiving hMG, and no cases in the laparoscopic electrocauterization arm. ${ }^{148}$ Given the evidence from one small fair-quality study for each of these outcomes it was graded as insufficient. There was no evidence for this treatment regarding neonatal outcomes, time to pregnancy, and long-term child or maternal outcomes.

Table 10. Outcomes for surgical management versus gonadotropins in women with PCOS

\begin{tabular}{|c|c|c|c|c|c|c|c|}
\hline Outcome & $\begin{array}{c}\text { Study } \\
\text { Design } \\
\text { (N Patients) }\end{array}$ & Intervention & Comparator & $\begin{array}{c}\text { Results } \\
\text { Intervention } \\
\mathbf{N} \\
(\%) \\
(95 \% \mathrm{Cl})\end{array}$ & $\begin{array}{c}\text { Results } \\
\text { Comparator } \\
N \\
(\%) \\
(95 \% \mathrm{Cl})\end{array}$ & $\begin{array}{c}P \\
\text { Value }\end{array}$ & $\begin{array}{c}\text { Summary of } \\
\text { Study } \\
\text { Findings }\end{array}$ \\
\hline $\begin{array}{l}\text { Live birth: } \\
\text { Any/patient }\end{array}$ & $\begin{array}{l}\text { Nahuis, } \\
2011^{141} \\
\\
\text { RCT } \\
(168)\end{array}$ & LEC & $\mathrm{rFSH}$ & $\begin{array}{c}71 / 83 \\
(85.5) \\
\text { (77.3 to } 92.2)\end{array}$ & $\begin{array}{c}69 / 85 \\
(81.2) \\
(72.3 \text { to } \\
88.7)\end{array}$ & 0.63 & $\begin{array}{l}\text { No } \\
\text { difference }\end{array}$ \\
\hline $\begin{array}{l}\text { Pregnancy } \\
\text { complications: } \\
\text { Multiple births }\end{array}$ & $\begin{array}{l}\text { Nahuis, } \\
2011^{141} \\
\\
\text { RCT } \\
(168)\end{array}$ & LEC & $\mathrm{rFSH}$ & $\begin{array}{c}7 / 134 \\
(5.2) \\
\text { (2.1 to } 9.6)\end{array}$ & $\begin{array}{c}10 / 124 \\
(8.1) \\
(4.0 \text { to } 13.4)\end{array}$ & 0.35 & $\begin{array}{l}\text { No } \\
\text { difference }\end{array}$ \\
\hline
\end{tabular}




\begin{tabular}{|c|c|c|c|c|c|c|c|}
\hline Outcome & $\begin{array}{c}\text { Study } \\
\text { Design } \\
\text { (N Patients) }\end{array}$ & Intervention & Comparator & $\begin{array}{c}\text { Results } \\
\text { Intervention } \\
N \\
(\%) \\
(95 \% \mathrm{Cl})\end{array}$ & $\begin{array}{c}\text { Results } \\
\text { Comparator } \\
N \\
(\%) \\
(95 \% \mathrm{Cl})\end{array}$ & $\begin{array}{c}\mathbf{P} \\
\text { Value }\end{array}$ & $\begin{array}{c}\text { Summary of } \\
\text { Study } \\
\text { Findings }\end{array}$ \\
\hline $\begin{array}{l}\text { Mean direct } \\
\text { medical costs } \\
\text { in euros }\end{array}$ & $\begin{array}{l}\text { Nahuis, } \\
2011^{142} \\
\\
\text { RCT } \\
(168)\end{array}$ & LEC & $\mathrm{rFSH}$ & $\begin{array}{c}9,560 \text { euros } \\
(8,212 \text { to } \\
10,907)\end{array}$ & $\begin{array}{c}11,708 \\
\text { euros } \\
(9,845 \text { to } \\
13,561)\end{array}$ & NS & $\begin{array}{l}\text { No } \\
\text { difference }\end{array}$ \\
\hline $\begin{array}{l}\text { Short-term } \\
\text { adverse } \\
\text { effects: OHSS }\end{array}$ & $\begin{array}{l}\text { Mehrabian, } \\
2012^{148} \\
\text { RCT } \\
(104)\end{array}$ & LEC & hMG & $\begin{array}{c}0 / 52 \\
(0)\end{array}$ & $\begin{array}{c}2 / 52 \\
(3.8) \\
\text { (0.5 to } 10.4)\end{array}$ & 0.16 & $\begin{array}{l}\text { No } \\
\text { difference }\end{array}$ \\
\hline
\end{tabular}

Abbreviations: $\mathrm{CI}=$ confidence interval; LEC=laparoscopic electrocauterization; $\mathrm{hMG}=$ human menopausal gonadotropin; $\mathrm{N}=$ number of patients; $\mathrm{NR}=$ not reported; NS=not statistically significant; OHSS=ovarian hyperstimulation syndrome;

PCOS=polycystic ovary syndrome; RCT=randomized control trial; rFSH=recombinant follicle-stimulating hormone

\section{Gonadotropins With IUI for PCOS}

Two fair-quality RCTs ${ }^{143,159}$ examined the effects of gonadotropins with IUI. These studies varied in the medication type used for oral ovulation as well as in adjunct treatments. These two studies included 374 patients. Table 11 summarizes the findings for live birth, pregnancy complications, and short-tern adverse effects. Live birth was investigated by both studies but used varying outcomes for measuring live birth. The strength of evidence was rated as insufficient given evidence from one fair-quality trial for either live birth measure. These same two studies examined pregnancy complications, reporting on miscarriage and multiple births. Neither study found significant differences between their intervention groups for either outcome. The imprecision of the findings and the quality of the included studies resulted in insufficient strength of evidence.

Finally, one fair-quality RCTs ${ }^{143}$ reported on short-term adverse effects of treatment, specifically OHSS (insufficient SOE). There was no evidence regarding neonatal outcomes, time to pregnancy, costs, and long-term child or maternal outcomes.

Table 11. Outcomes for gonadotropins with IUI in women with PCOS

\begin{tabular}{|c|c|c|c|c|c|c|c|}
\hline Outcome & $\begin{array}{c}\text { Study } \\
\text { Design } \\
\text { (N Patients) }\end{array}$ & Intervention & Comparator & $\begin{array}{c}\text { Results } \\
\text { Intervention } \\
N \\
(\%) \\
(95 \% \mathrm{Cl})\end{array}$ & $\begin{array}{c}\text { Results } \\
\text { Comparator } \\
N \\
(\%) \\
(95 \% \mathrm{Cl})\end{array}$ & $\begin{array}{c}P \\
\text { Value }\end{array}$ & $\begin{array}{l}\text { Summary of } \\
\text { Study Findings }\end{array}$ \\
\hline \multirow[t]{2}{*}{$\begin{array}{l}\text { Live birth: } \\
\text { Any/cycle }\end{array}$} & \multirow{2}{*}{$\begin{array}{l}\text { Stadtmauer, } \\
\text { 2011 }{ }^{159} \\
\text { RCT } \\
(98)\end{array}$} & \multirow[t]{2}{*}{$\mathrm{rFSH}$} & $\begin{array}{l}\text { rFSH + } \\
\text { GnRH } \\
\text { antagonist } \\
\text { (flexible) }\end{array}$ & $\begin{array}{l}10 / 53 \\
(18.9)\end{array}$ & $\begin{array}{l}18 / 54 \\
(33.3)\end{array}$ & 0.09 & No difference \\
\hline & & & $\begin{array}{l}\text { rFSH + } \\
\text { GnRH } \\
\text { antagonist } \\
\text { (start day) }\end{array}$ & $\begin{array}{l}10 / 53 \\
(18.9)\end{array}$ & $\begin{array}{c}7 / 47 \\
(12.3)\end{array}$ & 0.37 & No difference \\
\hline $\begin{array}{l}\text { Live birth: } \\
\text { Single/cycle }\end{array}$ & $\begin{array}{l}\text { Rashidi, } \\
2015^{143} \\
\text { RCT } \\
(276) \\
\end{array}$ & rFSH & hMG & $\begin{array}{c}21 / 132 \\
(15.9)\end{array}$ & $\begin{array}{c}14 / 144 \\
(9.7)\end{array}$ & 0.14 & No difference \\
\hline
\end{tabular}




\begin{tabular}{|c|c|c|c|c|c|c|c|}
\hline Outcome & $\begin{array}{c}\text { Study } \\
\text { Design } \\
\text { (N Patients) }\end{array}$ & Intervention & Comparator & $\begin{array}{c}\text { Results } \\
\text { Intervention } \\
N \\
(\%) \\
(95 \% \mathrm{Cl}) \\
\end{array}$ & $\begin{array}{c}\text { Results } \\
\text { Comparator } \\
\mathrm{N} \\
(\%) \\
(95 \% \mathrm{Cl}) \\
\end{array}$ & $\begin{array}{c}P \\
\text { Value }\end{array}$ & $\begin{array}{l}\text { Summary of } \\
\text { Study Findings }\end{array}$ \\
\hline \multirow[t]{3}{*}{$\begin{array}{l}\text { Pregnancy } \\
\text { complications: } \\
\text { Multiple births }\end{array}$} & $\begin{array}{l}\text { Rashidi, } \\
2015^{143} \\
\text { RCT } \\
(276)\end{array}$ & $\mathrm{rFSH}$ & hMG & $\begin{array}{c}1 / 132 \\
(0.8 \%) \\
(0.0 \text { to } 2.8)\end{array}$ & $\begin{array}{c}2 / 144 \\
(1.4) \\
(0.2 \text { to } 3.8)\end{array}$ & 0.62 & No difference \\
\hline & \multirow{2}{*}{$\begin{array}{l}\text { Stadtmauer, } \\
2011^{159} \\
\text { RCT } \\
(98)\end{array}$} & \multirow[t]{2}{*}{$\mathrm{rFSH}$} & $\begin{array}{l}\mathrm{rFSH}+ \\
\text { GnRH } \\
\text { antagonist } \\
\text { (flexible) }\end{array}$ & $\begin{array}{c}0 / 10 \\
(0)\end{array}$ & $\begin{array}{c}2 / 18 \\
(11.1) \\
(1.5 \text { to } 28.7\end{array}$ & 0.27 & No difference \\
\hline & & & $\begin{array}{l}\mathrm{rFSH}+ \\
\text { GnRH } \\
\text { antagonist } \\
\text { (start day) }\end{array}$ & $\begin{array}{c}0 / 10 \\
(0)\end{array}$ & $\begin{array}{c}1 / 7 \\
(14.3) \\
\text { (0.4 to } 45.9)\end{array}$ & 0.22 & No difference \\
\hline \multirow[t]{3}{*}{$\begin{array}{l}\text { Pregnancy } \\
\text { complications: } \\
\text { Miscarriage }\end{array}$} & $\begin{array}{l}\text { Rashidi, } \\
2015^{143} \\
\text { RCT } \\
(276) \\
\end{array}$ & $\mathrm{rFSH}$ & hMG & $\begin{array}{c}3 / 25 \\
(12.0) \\
\text { (2.7 to } 27.0)\end{array}$ & $\begin{array}{c}3 / 18 \\
(16.7) \\
\text { (3.8 to } 36.4)\end{array}$ & 0.63 & No difference \\
\hline & \multirow{2}{*}{$\begin{array}{l}\text { Stadtmauer, } \\
\text { 2011 }^{159} \\
\text { RCT } \\
(98)\end{array}$} & \multirow[t]{2}{*}{$\mathrm{rFSH}$} & $\begin{array}{l}\text { rFSH + } \\
\text { GnRH } \\
\text { antagonist } \\
\text { (flexible) }\end{array}$ & $\begin{array}{c}2 / 12 \\
(16.7) \\
\text { (2.3 to } 41.3)\end{array}$ & $\begin{array}{c}1 / 19 \\
(5.3) \\
(0.1 \text { to } 18.5)\end{array}$ & 0.30 & No difference \\
\hline & & & $\begin{array}{l}\text { rFSH + } \\
\text { GnRH } \\
\text { antagonist } \\
\text { (start day) }\end{array}$ & $\begin{array}{c}2 / 12 \\
(16.7) \\
\text { (2.3 to } 41.3)\end{array}$ & $\begin{array}{c}2 / 9 \\
(22.2) \\
(3.2 \text { to } 52.7)\end{array}$ & 0.75 & No difference \\
\hline $\begin{array}{l}\text { Short-term } \\
\text { adverse } \\
\text { effects: OHSS }\end{array}$ & $\begin{array}{l}\begin{array}{l}\text { Rashidi, } \\
2015^{143}\end{array} \\
\text { RCT } \\
(276)\end{array}$ & $\mathrm{rFSH}$ & $\mathrm{hMG}$ & $\begin{array}{c}1 / 132 \\
(0.8) \\
(0.0 \text { to } 2.8)\end{array}$ & $\begin{array}{c}2 / 144 \\
(1.4) \\
(0.2 \text { to } 3.8)\end{array}$ & 0.61 & No difference \\
\hline
\end{tabular}

Abbreviations: $\mathrm{CI}=$ confidence interval; $\mathrm{GnRH}=$ gonadotropin-releasing hormone; $\mathrm{hMG}=$ human menopausal gonadotropin; $\mathrm{N}=$ number of patients; $\mathrm{NR}=$ not reported; $\mathrm{NS}=$ not statistically significant; OHSS=ovarian hyperstimulation syndrome; PCOS=polycystic ovary syndrome; RCT=randomized control trial; rFSH=recombinant follicle-stimulating hormone

\section{Assisted Reproductive Technology (ART) for PCOS}

\section{IVF for PCOS}

Ten RCTs 146,147,150,153,161,162,164,167,171,178 (4 good, 5 fair, 1 poor-quality) compared outcomes for patients with PCOS undergoing IVF. These studies varied in the medication type used for ovulation stimulation, adjunct treatments, and laboratory phase methods. One study explored in vitro maturation (IVM) which involves immature oocyte retrieval with subsequent oocyte IVM. ${ }^{147}$ These 10 studies included 2,176 patients combined. There was no evidence regarding neonatal outcomes, time to pregnancy, costs, and long-term child or maternal outcomes.

Table 12 summarizes the findings from these studies related to live birth, pregnancy complications, and short-term adverse effects. Live birth was investigated in five studies with one $^{146}$ reporting it as any live birth per patient and four ${ }^{147,150,164,167}$ reporting any live birth per cycle . One of the studies reporting any live birth/patient compared berberine (an alkaloid extracted from Chinese medicinal herbs), metformin, and placebo as adjunct therapies to IVF. The results were significant for berberine, resulting in more live births than metformin and 
placebo; metformin resulted in significantly more live births than placebo. ${ }^{146}$ This evidence however was rated as insufficient strength of evidence given findings form one fair quality trial.

Of the four studies that reported live birth according to any live birth per cycle, three incorporated gonadotropin use as adjunct therapy. The results of any live birth per cycle were not significant across intervention groups (low SOE).

Three studies examined pregnancy complications, ${ }^{164,167,171}$ with all three reporting on miscarriage, one ${ }^{164}$ reporting on multiple births, and one ${ }^{171}$ reporting on ectopic pregnancies. None of the studies reported significant differences between intervention groups for miscarriages (moderate SOE) but given the heterogeneity in intervention protocols we did not perform a metaanalysis of these findings synthesis. Multiple births were reported in one study ${ }^{164}$ without significant differences measured but the strength of evidence was rated as insufficient given findings from one fair-quality trial. Ectopic pregnancies were not significantly different in the one fair-quality study that reported this outcome (insufficient SOE). ${ }^{171}$

Five studies examined short-term adverse effects of ART ${ }^{153,161,162,164,178}$ with all 6 reporting on OHSS. One study ${ }^{153}$ compared metformin with placebo as oral ovulation induction in addition to IVF. Two studies ${ }^{161,178}$ reported outcomes by severity of OHSS. Heterogeneity in comparisons and findings as well as the studies being underpowered to detect differences in OHSS led to an insufficient strength of evidence rating.

Three observational studies explored the comparative effectiveness of ART with other infertility treatments in women with PCOS. ${ }^{180,184,186}$

One nationwide birth cohort study ${ }^{184}$ identified all pregnancies with a live-single born child over an 8 year period in Denmark and compared the incidence of type I diabetes among those conceived with fertility treatment to those conceived naturally. There was no association between PCOS infertility as an indicator for fertility treatment and the subsequent development of Type I diabetes in offspring (adjusted HR 0.98, 95\% CI 0.32 to 3.05) (moderate SOE).

A second Danish national cohort study from registry data examined success rates across complete fertility treatment courses including insemination, ART, and natural conception among couples treated using homologous gametes and no previous live births due to fertility treatment. ${ }^{186} 13.7 \%$ (95\% CI 13.1 to 14.4 ) of women with first treatment by IUI and $4.2 \%$ (95\%CI 3.7 to 4.6) of women with first treatment with ART were diagnosed with anovulation as the specified cause of female infertility. Across all women, anovulatory infertility was predictor of high live birthrate. The adjusted OR for live birth within 2 years from first IUI treatment was 1.31 (95\% CI 1.15 to 1.50 ; $\mathrm{p}<0.0001$ ) and 1.57 for ART (95\% CI 1.18 to 2.11; $\mathrm{p}=0.002$ ). Live birthrate differed by maternal age for women with anovulatory infertility less than 35 years 45.3 (95\% CI 42.8 to 47.9) with IUI and 14.8 (95\% CI 13.1 to 16.7) for ART. This compares to 31.7 (95\% CI 25.2 to 38.9) for IUI among women over 35 years and 6.9 (95\% CI 3.7 to 11.5) for ART.

Finally, a prospective cohort study ${ }^{180}$ of women with PCOS compared maternal and neonatal outcomes with a reference population. Of the 188 included women with PCOS, 14 percent had conceived spontaneously, 68 percent had undergone ovulation induction, and 16 percent underwent IVF/ICSI. A subgroup analysis found no differences in maternal or neonatal (including small for gestational age and neonatal death) complications across presence/absence and type of fertility treatment. 
Table 12. Outcomes for comparisons of IVF treatments in women with PCOS

\begin{tabular}{|c|c|c|c|c|c|c|c|}
\hline Outcome & $\begin{array}{c}\text { Study } \\
\text { Design } \\
\text { (N Patients) }\end{array}$ & Intervention & Comparator & $\begin{array}{c}\text { Results } \\
\text { Intervention } \\
N \\
(\%) \\
(95 \% \mathrm{Cl})\end{array}$ & $\begin{array}{c}\text { Results } \\
\text { Comparator } \\
N \\
(\%) \\
(95 \% \mathrm{Cl})\end{array}$ & $P$ Value & $\begin{array}{l}\text { Summary } \\
\text { of Study } \\
\text { Findings }\end{array}$ \\
\hline \multirow[t]{2}{*}{$\begin{array}{l}\text { Live birth: } \\
\text { Any/patient }\end{array}$} & \multirow[t]{2}{*}{$\begin{array}{l}\text { An, 2014 }{ }^{146} \\
\text { RCT } \\
(109)\end{array}$} & \multirow[t]{2}{*}{$\begin{array}{l}\text { Berberine } \\
+ \text { IVF }\end{array}$} & $\begin{array}{l}\text { Metformin } \\
+ \text { IVF }\end{array}$ & $\begin{array}{c}18 / 37 \\
(48.6) \\
(32.9 \text { to } 64.5)\end{array}$ & $\begin{array}{c}14 / 38 \\
(36.8) \\
(22.5 \text { to } \\
52.5) \\
\end{array}$ & 0.30 & $\begin{array}{l}\text { No } \\
\text { difference }\end{array}$ \\
\hline & & & $\begin{array}{l}\text { Placebo } \\
+ \text { IVF }\end{array}$ & $\begin{array}{c}18 / 37 \\
(48.6) \\
(32.9 \text { to } 64.5)\end{array}$ & $\begin{array}{c}7 / 34 \\
(20.6) \\
\text { (9.0 to } 35.5)\end{array}$ & 0.013 & $\begin{array}{l}\text { Greater live } \\
\text { birth with } \\
\text { berberine } \\
\text { compared } \\
\text { to placebo }\end{array}$ \\
\hline \multirow[t]{6}{*}{$\begin{array}{l}\text { Live birth: } \\
\text { Any/cycle }\end{array}$} & \multirow[t]{2}{*}{$\begin{array}{l}\text { Choi, } \\
2012^{147} \\
\text { RCT } \\
(61)\end{array}$} & \multirow[t]{2}{*}{ IVM + hCG } & $\begin{array}{l}\text { GnRH } \\
\text { agonist }\end{array}$ & \multirow[t]{2}{*}{$\begin{array}{c}5 / 14 \\
(35.7) \\
(13.9 \text { to } 61.4)\end{array}$} & $\begin{array}{c}5 / 14 \\
(35.7) \\
(13.9 \text { to } \\
61.4) \\
\end{array}$ & NS & $\begin{array}{l}\text { No } \\
\text { difference }\end{array}$ \\
\hline & & & $\begin{array}{l}\text { GnRH } \\
\text { antagonist }\end{array}$ & & $\begin{array}{c}10 / 39 \\
(25.6) \\
(13.4 \text { to } \\
40.2) \\
\end{array}$ & 0.47 & $\begin{array}{l}\text { No } \\
\text { difference }\end{array}$ \\
\hline & $\begin{array}{l}\text { Kim, } 2012^{150} \\
\text { RCT } \\
(208)\end{array}$ & $\begin{array}{l}\text { GnRH } \\
\text { agonist }\end{array}$ & $\begin{array}{l}\text { GnRH } \\
\text { antagonist }\end{array}$ & $\begin{array}{c}36 / 103 \\
(35.0) \\
(26.1 \text { to } 44.4)\end{array}$ & $\begin{array}{c}36 / 105 \\
(34.3) \\
(25.6 \text { to } \\
43.6) \\
\end{array}$ & 0.92 & $\begin{array}{l}\text { No } \\
\text { difference }\end{array}$ \\
\hline & $\begin{array}{l}\text { Kurzawa, } \\
2008^{164} \\
\text { RCT } \\
(70)\end{array}$ & $\begin{array}{l}\text { GnRH } \\
\text { agonist }\end{array}$ & $\begin{array}{l}\text { GnRH } \\
\text { antagonist }\end{array}$ & $\begin{array}{c}18 / 37 \\
(48.6) \\
(32.9 \text { to } 64.5)\end{array}$ & $\begin{array}{c}14 / 33 \\
(42.4) \\
(26.4 \text { to } \\
59.4)\end{array}$ & 0.481 & $\begin{array}{l}\text { No } \\
\text { difference }\end{array}$ \\
\hline & \multirow[t]{2}{*}{$\begin{array}{l}\text { Ge } 2008^{167} \\
\text { RCT } \\
(62)\end{array}$} & \multirow[t]{2}{*}{$\begin{array}{l}\mathrm{hCG} \\
\text { medium }\end{array}$} & $\begin{array}{l}\text { hCG free } \\
\text { medium + } \\
\text { transfer }\end{array}$ & \multirow[t]{2}{*}{$\begin{array}{c}9 / 29 \\
(31.0) \\
(15.9 \text { to } 48.7)\end{array}$} & $\begin{array}{c}10 / 30 \\
(33.3) \\
(17.9 \text { to } \\
50.8) \\
\end{array}$ & 0.85 & $\begin{array}{l}\text { No } \\
\text { difference }\end{array}$ \\
\hline & & & $\begin{array}{l}\text { hCG free } \\
\text { medium }\end{array}$ & & $\begin{array}{c}10 / 30 \\
(33.3) \\
(17.9 \text { to } \\
50.8) \\
\end{array}$ & 0.85 & $\begin{array}{l}\text { No } \\
\text { difference }\end{array}$ \\
\hline $\begin{array}{l}\text { Pregnancy } \\
\text { complications: } \\
\text { Multiple births }\end{array}$ & $\begin{array}{l}\text { Kurzawa, } \\
2008^{164} \\
\text { RCT } \\
(70)\end{array}$ & $\begin{array}{l}\text { GnRH } \\
\text { agonist } \\
+ \text { IVF }\end{array}$ & $\begin{array}{l}\text { GnRH } \\
\text { antagonist } \\
+ \text { IVF }\end{array}$ & $\begin{array}{c}5 / 37 \\
(13.5) \\
\text { (4.7 to } 26.1)\end{array}$ & $\begin{array}{c}3 / 33 \\
(9.1) \\
\text { (2.0 to } 20.8)\end{array}$ & 0.5 & $\begin{array}{l}\text { No } \\
\text { difference }\end{array}$ \\
\hline \multirow[t]{3}{*}{$\begin{array}{l}\text { Pregnancy } \\
\text { complications: } \\
\text { Miscarriage }\end{array}$} & $\begin{array}{l}\text { Kurzawa, } \\
2008^{164} \\
\text { RCT } \\
(70) \\
\end{array}$ & $\begin{array}{l}\text { GnRH } \\
\text { agonist } \\
+ \text { IVF }\end{array}$ & $\begin{array}{l}\text { GnRH } \\
\text { antagonist }\end{array}$ & $\begin{array}{c}2 / 37 \\
(5.4) \\
\text { (0.7 to } 14.5)\end{array}$ & $\begin{array}{c}6 / 33 \\
(18.2) \\
\text { (7.2 to } 32.8)\end{array}$ & 0.154 & $\begin{array}{l}\text { No } \\
\text { difference }\end{array}$ \\
\hline & \multirow[t]{2}{*}{$\begin{array}{l}{\text { Ge } 2008^{167}} \\
\text { RCT } \\
(62)\end{array}$} & \multirow[t]{2}{*}{$\begin{array}{l}\text { hCG } \\
\text { medium }\end{array}$} & $\begin{array}{l}\text { hCG-free } \\
\text { medium + } \\
\text { transfer }\end{array}$ & $\begin{array}{c}3 / 29 \\
(10.3) \\
(2.3 \text { to } 23.5)\end{array}$ & $\begin{array}{c}3 / 30 \\
(10.0) \\
(17.9 \text { to } \\
50.8) \\
\end{array}$ & 0.97 & $\begin{array}{l}\text { No } \\
\text { difference }\end{array}$ \\
\hline & & & $\begin{array}{l}\text { hCG-free } \\
\text { medium }\end{array}$ & $\begin{array}{c}3 / 29 \\
(10.3) \\
\text { (2.3 to } 23.5) \\
\end{array}$ & $\begin{array}{c}2 / 30 \\
(6.7) \\
\text { (0.8 to } 17.8) \\
\end{array}$ & 0.62 & $\begin{array}{l}\text { No } \\
\text { difference }\end{array}$ \\
\hline
\end{tabular}




\begin{tabular}{|c|c|c|c|c|c|c|c|}
\hline Outcome & $\begin{array}{c}\text { Study } \\
\text { Design } \\
\text { (N Patients) }\end{array}$ & Intervention & Comparator & $\begin{array}{c}\text { Results } \\
\text { Intervention } \\
\mathrm{N} \\
(\%) \\
(95 \% \mathrm{Cl})\end{array}$ & $\begin{array}{c}\text { Results } \\
\text { Comparator } \\
N \\
(\%) \\
(95 \% \mathrm{Cl})\end{array}$ & $P$ Value & $\begin{array}{l}\text { Summary } \\
\text { of Study } \\
\text { Findings }\end{array}$ \\
\hline & $\begin{array}{l}\text { Wang, } \\
2016^{171} \\
\\
\text { RCT } \\
(120)\end{array}$ & $\begin{array}{l}\text { MPA + } \\
\text { hMG+IVF }\end{array}$ & $\begin{array}{l}\text { Short } \\
\text { protocol } \\
\text { (Decapeptyl, } \\
\text { hMG, hCG) }\end{array}$ & $\begin{array}{c}4 / 75 \\
(5.33)\end{array}$ & $\begin{array}{c}7 / 84 \\
(8.33)\end{array}$ & 0.457 & $\begin{array}{l}\text { No } \\
\text { difference }\end{array}$ \\
\hline $\begin{array}{l}\text { Pregnancy } \\
\text { complications: } \\
\text { Ectopic } \\
\text { pregnancy }\end{array}$ & $\begin{array}{l}\text { Wang, } \\
2016^{171} \\
\\
\text { RCT } \\
(120)\end{array}$ & $\begin{array}{l}\text { MPA + } \\
\text { hMG+IVF }\end{array}$ & $\begin{array}{l}\text { Short } \\
\text { protocol } \\
\text { (Decapeptyl, } \\
\text { hMG, hCG) }\end{array}$ & $\begin{array}{c}1 / 49 \\
(2.04)\end{array}$ & $\begin{array}{c}1 / 45 \\
(2.22)\end{array}$ & 0.952 & $\begin{array}{l}\text { No } \\
\text { difference }\end{array}$ \\
\hline \multirow[t]{2}{*}{$\begin{array}{l}\text { Short-term } \\
\text { adverse } \\
\text { effects: OHSS, } \\
\text { any }\end{array}$} & $\begin{array}{l}\text { Palomba, } \\
2011^{153} \\
\text { RCT } \\
(120)\end{array}$ & $\begin{array}{l}\text { Metformin+ } \\
\text { IVF }\end{array}$ & Placebo+IVF & $\begin{array}{c}5 / 60 \\
(8.3) \\
(2.8 \text { to } 16.5)\end{array}$ & $\begin{array}{c}18 / 60 \\
(30.0) \\
(19.2 \text { to } \\
42.1)\end{array}$ & 0.003 & $\begin{array}{l}\text { Reduced } \\
\text { OHSS with } \\
\text { metformin } \\
\text { compared } \\
\text { to placebo }\end{array}$ \\
\hline & $\begin{array}{l}\text { Aboulghar, } \\
2010^{162} \\
\text { RCT } \\
(84)\end{array}$ & $\mathrm{rFSH}$ & uFSH & $\begin{array}{l}0 / 42 \\
(0)\end{array}$ & $\begin{array}{c}1 / 42 \\
(2.4) \\
\text { (0.1 to } 8.6)\end{array}$ & 0.31 & $\begin{array}{l}\text { No } \\
\text { difference }\end{array}$ \\
\hline $\begin{array}{l}\text { Short-term } \\
\text { adverse } \\
\text { effects: OHSS, } \\
\text { moderate }\end{array}$ & $\begin{array}{l}\text { Tehranineja } \\
\text { d, 2010161 } \\
\text { RCT } \\
\text { (90) }\end{array}$ & $\begin{array}{l}\text { GnRH } \\
\text { agonist }\end{array}$ & $\begin{array}{l}\text { GnRH } \\
\text { antagonist }\end{array}$ & $\begin{array}{c}10 / 45 \\
(22.2) \\
(11.5 \text { to } 35.3)\end{array}$ & $\begin{array}{c}0 / 45 \\
(0)\end{array}$ & 0.001 & $\begin{array}{l}\text { Reduced } \\
\text { OHSS with } \\
\text { GnRH } \\
\text { antagonist } \\
\text { compared } \\
\text { to GnRH } \\
\text { agonist }\end{array}$ \\
\hline \multirow[t]{2}{*}{$\begin{array}{l}\text { Short-term } \\
\text { adverse } \\
\text { effects: OHSS, } \\
\text { severe }\end{array}$} & $\begin{array}{l}\text { Tehranineja } \\
\text { d, } 2010^{161}\end{array}$ & $\begin{array}{l}\text { GnRH } \\
\text { agonist }\end{array}$ & $\begin{array}{l}\text { GnRH } \\
\text { antagonist }\end{array}$ & $\begin{array}{c}5 / 45 \\
(11.1) \\
\text { (3.8 to } 21.7)\end{array}$ & $\begin{array}{c}0 / 45 \\
(0)\end{array}$ & 0.02 & $\begin{array}{l}\text { Reduced } \\
\text { OHSS with } \\
\text { GnRH } \\
\text { antagonist } \\
\text { compared } \\
\text { to GnRH } \\
\text { agonist }\end{array}$ \\
\hline & $\begin{array}{l}\text { Aghahossein } \\
\text { i, 2017 }{ }^{178} \\
\text { RCT } \\
(100)\end{array}$ & $\begin{array}{l}\text { Low-dose } \\
\text { hCG at time } \\
\text { of GnRH } \\
\text { agonist }\end{array}$ & $\begin{array}{l}\text { Low-dose } \\
\text { hCG } 35 \text { hrs } \\
\text { after GnRH } \\
\text { agonist }\end{array}$ & $\begin{array}{l}13 / 40 \\
(32.5)\end{array}$ & $\begin{array}{c}5 / 40 \\
(12.5)\end{array}$ & 0.03 & $\begin{array}{l}\text { Reduced } \\
\text { OHSS with } \\
\text { hCG given } \\
35 \text { hours } \\
\text { after GnRH } \\
\text { agonist }\end{array}$ \\
\hline
\end{tabular}

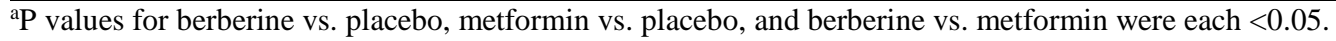

Abbreviations: $\mathrm{CI}=$ confidence interval; $\mathrm{GnRH}=$ gonadotropin-releasing hormone; $\mathrm{hCG}=$ human chorionic gonadotropin; $\mathrm{hMG=}$ human menopausal gonadotropin; IVF=in vitro fertilization; IVM=in vitro maturation; MPA=medroxyprogesterone acetate; $\mathrm{N}=$ number of patients; NR=not reported; NS=not statistically significant; OHSS=0varian hyperstimulation syndrome; PCOS=polycystic ovary syndrome; RCT=randomized control trial; $\mathrm{rFSH}=$ recombinant follicle-stimulating hormone; uFSH=urinary follicle-stimulating hormone

\section{Adjuncts to IVF for PCOS}

Three studies looked at adjuncts to IVF in PCOS women. ${ }^{155,177,190}$ Specifically, two RCTs $^{155,177}$ (one fair quality and one good quality) compared metformin with placebo as pretreatment prior to IVF in PCOS patients. One study ${ }^{155}$ presented any live birth per patient for 3 study populations: intention-to-treat (all women randomized to treatment), spontaneous pregnancy (all women with a positive urinary pregnancy test prior to controlled ovarian 
stimulation), and those women randomized to the study who started ovarian stimulation (ART population). Note that we judge only the intention-to-treat results as fair quality, with the others poor (due to increased risk of bias). The other study of pretreatment with metformin reported significantly lower birth rates in the metformin group. The live birth results are presented in Table 13 (insufficient SOE). There was no difference in OHSS in one study ${ }^{177}$ (Table 13) and no evidence regarding pregnancy complications, neonatal outcomes, time to pregnancy, costs, other short-term adverse effects, and long-term child or maternal outcomes. The third study ${ }^{190}$ reported on OHSS in women treated with methylprednisolone as an adjunct to IVF. No differences overall or by severity of OHSS were reported. (Table 13). SOE was rated as insufficient for all outcomes given the small studies with varying adjuncts.

Table 13. Outcomes for adjuncts to IVF in women with PCOS

\begin{tabular}{|c|c|c|c|c|c|c|c|}
\hline Outcome & $\begin{array}{c}\text { Study } \\
\text { Design } \\
\text { (N Patients) }\end{array}$ & $\begin{array}{l}\text { Intervention } \\
\text { (population) }\end{array}$ & $\begin{array}{l}\text { Comparator } \\
\text { (population) }\end{array}$ & $\begin{array}{c}\text { Results } \\
\text { Intervention } \\
\mathbf{N} \\
(\%)\end{array}$ & $\begin{array}{c}\text { Results } \\
\text { Comparator } \\
\mathbf{N} \\
(\%)\end{array}$ & $\begin{array}{c}\mathbf{P} \\
\text { Value }\end{array}$ & $\begin{array}{c}\text { Summary of } \\
\text { Study } \\
\text { Findings }\end{array}$ \\
\hline \multirow[t]{2}{*}{$\begin{array}{l}\text { Live birth: } \\
\text { Any/patient }\end{array}$} & \multirow[t]{2}{*}{$\begin{array}{l}\text { Kjotrod, } \\
2011^{155} \\
\text { RCT } \\
(149)\end{array}$} & $\begin{array}{l}\text { Metformin } \\
\text { (Intention to } \\
\text { treat) }\end{array}$ & $\begin{array}{l}\text { Placebo } \\
\text { (Intention to } \\
\text { treat) }\end{array}$ & $\begin{array}{l}36 / 74 \\
(48.6)\end{array}$ & $\begin{array}{l}24 / 75 \\
(32.0)\end{array}$ & 0.038 & $\begin{array}{l}\text { Greater live } \\
\text { births with } \\
\text { metformin } \\
\text { compared to } \\
\text { placebo } \\
\text { within the } \\
\text { intention to } \\
\text { treat } \\
\text { analysis }\end{array}$ \\
\hline & & $\begin{array}{l}\text { Metformin } \\
(\mathrm{ART})\end{array}$ & $\begin{array}{l}\text { Placebo } \\
\text { (ART) }\end{array}$ & $\begin{array}{l}21 / 56 \\
(37.5)\end{array}$ & $\begin{array}{l}16 / 56 \\
(28.6)\end{array}$ & 0.32 & $\begin{array}{l}\text { No } \\
\text { difference }\end{array}$ \\
\hline $\begin{array}{l}\text { Live birth: } \\
\text { Any/embryo } \\
\text { transfer }\end{array}$ & $\begin{array}{l}\text { Jacob, } \\
2016^{177} \\
\text { RCT } \\
(153)\end{array}$ & Metformin & Placebo & $\begin{array}{l}16 / 58 \\
(27.6)\end{array}$ & $\begin{array}{l}33 / 64 \\
(51.6)\end{array}$ & 0.01 & $\begin{array}{l}\text { Lower birth } \\
\text { rates in } \\
\text { metformin } \\
\text { group. }\end{array}$ \\
\hline \multirow[t]{2}{*}{$\begin{array}{l}\text { Short-term } \\
\text { adverse } \\
\text { effects: } \\
\text { OHSS, any }\end{array}$} & $\begin{array}{l}\text { Jacob, } \\
2016^{177} \\
\text { RCT } \\
(153)\end{array}$ & Metformin & Placebo & $\begin{array}{l}21 / 75 \\
(28.0)\end{array}$ & $\begin{array}{l}16 / 74 \\
(21.6)\end{array}$ & 0.726 & $\begin{array}{l}\text { No } \\
\text { difference }\end{array}$ \\
\hline & $\begin{array}{l}\text { Yeganeh, } \\
2018^{190} \\
\text { RCT } \\
(219) \\
\end{array}$ & $\begin{array}{l}\text { Methy- } \\
\text { prednisolone }\end{array}$ & Placebo & $\begin{array}{l}18 / 93 \\
(19.4)\end{array}$ & $\begin{array}{l}15 / 91 \\
(16.5)\end{array}$ & 0.61 & $\begin{array}{l}\text { No } \\
\text { difference }\end{array}$ \\
\hline $\begin{array}{l}\text { Short-term } \\
\text { adverse } \\
\text { effects: } \\
\text { OHSS, mild }\end{array}$ & $\begin{array}{l}\text { Yeganeh, } \\
2018^{190} \\
\text { RCT } \\
(219) \\
\end{array}$ & $\begin{array}{l}\text { Methy- } \\
\text { prednisolone }\end{array}$ & Placebo & $\begin{array}{l}10 / 93 \\
(10.8)\end{array}$ & $\begin{array}{l}5 / 91 \\
(5.5)\end{array}$ & NS & $\begin{array}{l}\text { No } \\
\text { difference }\end{array}$ \\
\hline $\begin{array}{l}\text { Short-term } \\
\text { adverse } \\
\text { effects: } \\
\text { OHSS, } \\
\text { moderate/ } \\
\text { severe }\end{array}$ & $\begin{array}{l}\text { Jacob, } \\
2016^{177} \\
\text { RCT } \\
(153)\end{array}$ & Metformin & Placebo & $\begin{array}{c}12 / 75 \\
(16)\end{array}$ & $\begin{array}{c}9 / 74 \\
(12.2)\end{array}$ & 0.66 & $\begin{array}{l}\text { No } \\
\text { difference }\end{array}$ \\
\hline
\end{tabular}




\begin{tabular}{|c|c|c|c|c|c|c|c|}
\hline Outcome & $\begin{array}{c}\text { Study } \\
\text { Design } \\
\text { (N Patients) }\end{array}$ & $\begin{array}{l}\text { Intervention } \\
\text { (population) }\end{array}$ & $\begin{array}{l}\text { Comparator } \\
\text { (population) }\end{array}$ & $\begin{array}{c}\text { Results } \\
\text { Intervention } \\
\mathbf{N} \\
(\%)\end{array}$ & $\begin{array}{c}\text { Results } \\
\text { Comparator } \\
\mathrm{N} \\
(\%)\end{array}$ & $\begin{array}{c}\mathbf{P} \\
\text { Value }\end{array}$ & $\begin{array}{c}\text { Summary of } \\
\text { Study } \\
\text { Findings }\end{array}$ \\
\hline $\begin{array}{l}\text { OHSS, } \\
\text { moderate }\end{array}$ & $\begin{array}{l}\text { Yeganeh, } \\
2018^{190} \\
\text { RCT } \\
(219)\end{array}$ & $\begin{array}{l}\text { Methy- } \\
\text { prednisolone }\end{array}$ & Placebo & $\begin{array}{l}4 / 93 \\
(4.3)\end{array}$ & $\begin{array}{l}5 / 91 \\
(5.5)\end{array}$ & NS & $\begin{array}{l}\text { No } \\
\text { difference }\end{array}$ \\
\hline $\begin{array}{l}\text { OHSS, } \\
\text { severe }\end{array}$ & $\begin{array}{l}\text { Yeganeh, } \\
2018^{190} \\
\text { RCT } \\
(219)\end{array}$ & $\begin{array}{l}\text { Methy- } \\
\text { prednisolone }\end{array}$ & Placebo & $\begin{array}{l}4 / 93 \\
(4.3)\end{array}$ & $\begin{array}{l}5 / 91 \\
(5.5)\end{array}$ & NS & $\begin{array}{l}\text { No } \\
\text { difference }\end{array}$ \\
\hline
\end{tabular}

Abbreviations: ART=assisted reproductive technology; CI=confidence interval, N=number of patients; NS=not statistically significant; OHSS=ovarian hyperstimulation Syndrome; PCOS=polycystic ovary syndrome; RCT=randomized control trial

\section{Fresh Versus Frozen Embryos in IVF for PCOS}

One good-quality RCT ${ }^{174}$ compared outcomes in women with PCOS undergoing IVF with transfer of frozen versus fresh embryos. Outcomes presented in Table 14 include live births, multiple births, and parental and neonatal outcomes. Live birth rates were significantly higher in the frozen embryo transfer group, with no significant difference in multiple births. Miscarriages and ectopic pregnancies were higher in the fresh embryo transfer group. No significant differences were observed for neonatal deaths or congenital abnormalities. SOE was low for all outcomes given findings from one study.

Table 14. Outcomes for fresh versus frozen embryo transfer in IVF in women with PCOS

\begin{tabular}{|c|c|c|c|c|c|c|c|}
\hline Outcome & $\begin{array}{c}\text { Study } \\
\text { Design } \\
\text { (N Patients) }\end{array}$ & Intervention & Comparator & $\begin{array}{c}\text { Results } \\
\text { Intervention } \\
\mathrm{N} \\
(\%) \\
(95 \% \mathrm{Cl}) \\
\end{array}$ & $\begin{array}{c}\text { Results } \\
\text { Comparator } \\
N \\
(\%) \\
(95 \% \mathrm{Cl}) \\
\end{array}$ & $\begin{array}{c}P \\
\text { Value }\end{array}$ & $\begin{array}{c}\text { Summary of } \\
\text { Study } \\
\text { Findings }\end{array}$ \\
\hline $\begin{array}{l}\text { Live birth: } \\
\text { Any/patient }\end{array}$ & $\begin{array}{l}\text { Chen, } \\
2016^{174} \\
\text { RCT } \\
(1508)\end{array}$ & $\begin{array}{l}\text { Frozen } \\
\text { Embryo } \\
\text { Transfer in } \\
\text { IVF }\end{array}$ & $\begin{array}{l}\text { Fresh } \\
\text { Embryo } \\
\text { Transfer in } \\
\text { IVF }\end{array}$ & $\begin{array}{c}368 / 746 \\
(49.3)\end{array}$ & $\begin{array}{c}320 / 762 \\
(42.0)\end{array}$ & 0.004 & $\begin{array}{l}\text { Greater live } \\
\text { births with } \\
\text { frozen } \\
\text { embryo } \\
\text { transfer }\end{array}$ \\
\hline $\begin{array}{l}\text { Pregnancy } \\
\text { complications: } \\
\text { Multiple births }\end{array}$ & $\begin{array}{l}\text { Chen, } \\
2016^{174} \\
\text { RCT } \\
(1508)\end{array}$ & $\begin{array}{l}\text { Frozen } \\
\text { Embryo } \\
\text { Transfer in } \\
\text { IVF }\end{array}$ & $\begin{array}{l}\text { Fresh } \\
\text { Embryo } \\
\text { Transfer in } \\
\text { IVF }\end{array}$ & $\begin{array}{c}118 / 746 \\
(15.8)\end{array}$ & $\begin{array}{c}108 / 762 \\
(14.2)\end{array}$ & 0.41 & $\begin{array}{l}\text { No } \\
\text { difference }\end{array}$ \\
\hline $\begin{array}{l}\text { Pregnancy } \\
\text { complications: } \\
\text { Miscarriage }\end{array}$ & $\begin{array}{l}\text { Chen, } \\
2016^{174} \\
\text { RCT } \\
(1508) \\
\end{array}$ & $\begin{array}{l}\text { Frozen } \\
\text { Embryo } \\
\text { Transfer in } \\
\text { IVF }\end{array}$ & $\begin{array}{l}\text { Fresh } \\
\text { Embryo } \\
\text { Transfer in } \\
\text { IVF }\end{array}$ & $\begin{array}{c}108 / 492 \\
(22.0)\end{array}$ & $\begin{array}{c}161 / 492 \\
(32.7)\end{array}$ & $\begin{array}{c}<0.00 \\
1\end{array}$ & $\begin{array}{l}\text { Lower } \\
\text { miscarriages } \\
\text { with frozen } \\
\text { embryo } \\
\text { transfer }\end{array}$ \\
\hline $\begin{array}{l}\text { Pregnancy } \\
\text { complications: } \\
\text { Ectopic } \\
\text { pregnancy }\end{array}$ & $\begin{array}{l}\text { Chen, } \\
2016^{174} \\
\text { RCT } \\
(1508)\end{array}$ & $\begin{array}{l}\text { Frozen } \\
\text { Embryo } \\
\text { Transfer in } \\
\text { IVF }\end{array}$ & $\begin{array}{l}\text { Fresh } \\
\text { Embryo } \\
\text { Transfer in } \\
\text { IVF }\end{array}$ & $\begin{array}{c}10 / 492 \\
(2.0)\end{array}$ & $\begin{array}{c}54 / 762 \\
(7.1)\end{array}$ & $\begin{array}{c}<0.00 \\
1\end{array}$ & $\begin{array}{l}\text { Lower } \\
\text { ectopic } \\
\text { pregnancies } \\
\text { with frozen } \\
\text { embryo } \\
\text { transfer }\end{array}$ \\
\hline
\end{tabular}




\begin{tabular}{|c|c|c|c|c|c|c|c|}
\hline Outcome & $\begin{array}{c}\text { Study } \\
\text { Design } \\
\text { (N Patients) }\end{array}$ & Intervention & Comparator & $\begin{array}{c}\text { Results } \\
\text { Intervention } \\
N \\
(\%) \\
(95 \% \mathrm{Cl})\end{array}$ & $\begin{array}{c}\text { Results } \\
\text { Comparator } \\
N \\
(\%) \\
(95 \% \mathrm{Cl})\end{array}$ & $\begin{array}{c}\mathbf{P} \\
\text { Value }\end{array}$ & $\begin{array}{c}\text { Summary of } \\
\text { Study } \\
\text { Findings }\end{array}$ \\
\hline $\begin{array}{l}\text { Neonatal } \\
\text { outcomes: } \\
\text { Stillbirth }\end{array}$ & $\begin{array}{l}\text { Chen, } \\
2016^{174} \\
\text { RCT } \\
(1508)\end{array}$ & $\begin{array}{l}\text { Frozen } \\
\text { Embryo } \\
\text { Transfer in } \\
\text { IVF }\end{array}$ & $\begin{array}{l}\text { Fresh } \\
\text { Embryo } \\
\text { Transfer in } \\
\text { IVF }\end{array}$ & $\begin{array}{c}2 / 370 \\
(0.5)\end{array}$ & $\begin{array}{c}0 \\
(0)\end{array}$ & 0.50 & $\begin{array}{l}\text { No } \\
\text { difference }\end{array}$ \\
\hline $\begin{array}{l}\text { Neonatal } \\
\text { outcomes: } \\
\text { Congenital } \\
\text { abnormalities }\end{array}$ & $\begin{array}{l}\text { Chen, } \\
2016^{174} \\
\text { RCT } \\
(1508)\end{array}$ & $\begin{array}{l}\text { Frozen } \\
\text { Embryo } \\
\text { Transfer in } \\
\text { IVF }\end{array}$ & $\begin{array}{l}\text { Fresh } \\
\text { Embryo } \\
\text { Transfer in } \\
\text { IVF }\end{array}$ & $\begin{array}{c}24 / 491 \\
(4.9)\end{array}$ & $\begin{array}{c}17 / 432 \\
(3.9)\end{array}$ & 0.52 & $\begin{array}{l}\text { No } \\
\text { difference }\end{array}$ \\
\hline
\end{tabular}

Abbreviations: $\mathrm{CI}=$ confidence interval, $\mathrm{IVF}=$ in vitro fertilization; $\mathrm{N}=$ number of patients; $\mathrm{PCOS}=$ polycystic ovary syndrome; RCT=randomized control trial

\section{ICSI for PCOS}

Two fair-quality RCTs ${ }^{149,158}$ compared different methods for ICSI. These studies varied in the medication type used for ovulation stimulation and adjunct treatments. These 2 studies included 186 patients combined. Outcomes are summarized in Table 15. Live birth was investigated in one study ${ }^{149}$ reporting it as any live birth per patient. The pregnancy complication of miscarriage was investigated in the other study. ${ }^{158}$ Neither outcome was significantly different between treatments but the strength of evidence was rated as insufficient given findings from one small fair-quality study. Only the second study evaluated the short-term adverse effect of OHSS. The use of GnRH antagonist reduced the incidence of OHSS significantly, primarily through its effect on moderate/severe OHSS although again the evidence from one fair-quality study results in an insufficient strength of evidence rating.

There was no evidence regarding neonatal outcomes, time to pregnancy, costs, and long-term child or maternal outcomes.

We also identified one good-quality meta-analysis that examined HCG priming for fertility treatments with in vitro maturation. ${ }^{196}$ For women with PCOS, outcomes of interest were reported in only one study ${ }^{149}$ which we have described in our results of individual studies.

Table 15. Outcomes for comparisons of ICSI treatment in women with PCOS

\begin{tabular}{|c|c|c|c|c|c|c|c|}
\hline Outcome & $\begin{array}{c}\text { Study } \\
\text { Design } \\
\text { (N } \\
\text { Patients) }\end{array}$ & Intervention & Comparator & $\begin{array}{c}\text { Results } \\
\text { Intervention } \\
\mathbf{N} \\
(\%) \\
(95 \% \mathrm{Cl})\end{array}$ & $\begin{array}{c}\text { Results } \\
\text { Comparator } \\
\mathrm{N} \\
(\%) \\
(95 \% \mathrm{Cl})\end{array}$ & $\begin{array}{c}P \\
\text { Value }\end{array}$ & $\begin{array}{l}\text { Summary } \\
\text { of Study } \\
\text { Findings }\end{array}$ \\
\hline $\begin{array}{l}\text { Live birth: } \\
\text { Any/patient }\end{array}$ & $\begin{array}{l}\text { Zheng, } \\
2012^{149} \\
\text { RCT } \\
(82)\end{array}$ & hCG Priming & $\begin{array}{l}\text { No hCG } \\
\text { Priming }\end{array}$ & $\begin{array}{c}9 / 40 \\
(22.5) \\
(11.1 \text { to } \\
36.5)\end{array}$ & $\begin{array}{c}13 / 42 \\
(31.0) \\
(18.1 \text { to } 45.5)\end{array}$ & 0.39 & $\begin{array}{l}\text { No } \\
\text { difference }\end{array}$ \\
\hline $\begin{array}{l}\text { Pregnancy } \\
\text { complications: } \\
\text { Miscarriage }\end{array}$ & $\begin{array}{l}\text { Hosseini, } \\
2010^{158} \\
\text { RCT } \\
(112)\end{array}$ & $\begin{array}{l}\text { GnRH } \\
\text { agonist }\end{array}$ & $\begin{array}{l}\text { GnRH } \\
\text { antagonist }\end{array}$ & $\begin{array}{c}4 / 55 \\
(7.3) \\
(2.1 \text { to } 15.4)\end{array}$ & $\begin{array}{c}8 / 57 \\
(17.6) \\
(6.9 \text { to } 25.8)\end{array}$ & 0.219 & $\begin{array}{l}\text { No } \\
\text { difference }\end{array}$ \\
\hline
\end{tabular}




\begin{tabular}{|c|c|c|c|c|c|c|c|}
\hline Outcome & $\begin{array}{c}\text { Study } \\
\text { Design } \\
\text { (N } \\
\text { Patients) }\end{array}$ & Intervention & Comparator & $\begin{array}{c}\text { Results } \\
\text { Intervention } \\
N \\
(\%) \\
(95 \% \mathrm{Cl})\end{array}$ & $\begin{array}{c}\text { Results } \\
\text { Comparator } \\
\mathrm{N} \\
(\%) \\
(95 \% \mathrm{Cl})\end{array}$ & $\begin{array}{c}P \\
\text { Value }\end{array}$ & $\begin{array}{l}\text { Summary } \\
\text { of Study } \\
\text { Findings }\end{array}$ \\
\hline $\begin{array}{l}\text { Short-term } \\
\text { adverse } \\
\text { effects: OHSS }\end{array}$ & $\begin{array}{l}\text { Hosseini, } \\
2010^{158} \\
\text { RCT } \\
(112)\end{array}$ & $\begin{array}{l}\text { GnRH } \\
\text { agonist }\end{array}$ & $\begin{array}{l}\text { GnRH } \\
\text { antagonist }\end{array}$ & $\begin{array}{c}35 / 55 \\
(63.6) \\
(50.6 \text { to } \\
75.7)\end{array}$ & $\begin{array}{c}25 / 57 \\
(43.9) \\
(34.0 \text { to } 60.5)\end{array}$ & 0.036 & $\begin{array}{l}\text { Reduced } \\
\text { OHSS with } \\
\text { GnRH } \\
\text { antagonist } \\
\text { compared } \\
\text { to GnRH } \\
\text { agonist }\end{array}$ \\
\hline $\begin{array}{l}\text { Short-term } \\
\text { adverse } \\
\text { effects: OHSS, } \\
\text { mild }\end{array}$ & $\begin{array}{l}\text { Hosseini, } \\
2010^{158} \\
\text { RCT } \\
(112)\end{array}$ & $\begin{array}{l}\text { GnRH } \\
\text { agonist }\end{array}$ & $\begin{array}{l}\text { GnRH } \\
\text { antagonist }\end{array}$ & $\begin{array}{c}18 / 55 \\
(32.7) \\
(21.1 \text { to } \\
45.6)\end{array}$ & $\begin{array}{c}16 / 57 \\
(29) \\
(18.7 \text { to } 43.1)\end{array}$ & 0.78 & $\begin{array}{l}\text { No } \\
\text { difference }\end{array}$ \\
\hline $\begin{array}{l}\text { Short-term } \\
\text { adverse } \\
\text { effects: OHSS, } \\
\text { moderate/ } \\
\text { severe }\end{array}$ & $\begin{array}{l}\text { Hosseini, } \\
2010^{158} \\
\text { RCT } \\
(112)\end{array}$ & $\begin{array}{l}\text { GnRH } \\
\text { agonist }\end{array}$ & $\begin{array}{l}\text { GnRH } \\
\text { antagonist }\end{array}$ & $\begin{array}{c}17 / 55 \\
(30.9) \\
(19.5 \text { to } \\
43.6)\end{array}$ & $\begin{array}{c}9 / 57 \\
(15.7) \\
\text { (8.2 to } 28.1)\end{array}$ & 0.09 & $\begin{array}{l}\text { No } \\
\text { difference }\end{array}$ \\
\hline
\end{tabular}

Abbreviations: $\mathrm{CI}=$ confidence interval; $\mathrm{GnRH}=$ gonadotropin-releasing hormone; $\mathrm{hCG}=$ human chorionic gonadotropin; $\mathrm{NR}=$ not reported; OHSS=ovarian hyperstimulation syndrome; PCOS=polycystic ovary syndrome; RCT=randomized control trial

\section{Subgroups of Interest for PCOS}

Three good-quality RCTs ${ }^{128,151,160}$ reported live birth with BMI analyzed as a subgroup in women with PCOS. Interventions and BMI parameters varied between studies. One study ${ }^{151}$ reported BMI as less than 27 or $\geq 27$. This study found no significant differences in live births between metformin and placebo as adjunct therapies to clomiphene by BMI subgroups (BMI <27 $45.5 \%$ vs. $34.2 \%$; $=0.16 \mid \mathrm{BMI} \geq 2735.9 \%$ vs. $22.6 \%$; $=0.1)$.

Another study ${ }^{160}$ reported findings for women with BMI $\leq 32$ or $>32$. This study compared clomiphene, metformin, and clomiphene plus metformin. Pregnancy and live birth rates were low in women with BMI > 32 whatever treatment was used, with no evidence of benefit of metformin over placebo. For women with $\mathrm{BMI} \leq 32$ there was no evidence of significant differences in outcomes whether treated with metformin, clomiphene or both.

The third study ${ }^{128,129}$ compared clomiphene and clomiphene plus metformin (combination therapy) in 626 women. Metformin was used as a reference in the analysis. Patients were divided into BMI subgroups $(<30,30-34, \geq 35)$. When the treatment arms were stratified by BMI, both clomiphene and combination therapy were more successful regarding the outcome of live birth compared to metformin with greatest benefits seen in the low and high BMI categories.

These findings suggest that the addition of metformin to clomiphene does not significantly improve live birth weight in obese women with PCOS. Studies however were not powered to detect differences within subgroups, and different categorization definitions for BMI limit the ability to pool study results.

\section{Strength of Evidence for PCOS}

Table 16 summarizes the SOE for the findings described above. In general, SOE was judged insufficient or low for most outcomes with a few exceptions: There was moderate SOE live births with the use of letrozole versus clomiphene, and for oral agents versus surgical management. There was moderate SOE for miscarriage rates between clomiphene versus 
metformin and for oral agents versus surgical management. A common limitation across all comparisons was lack of precision for estimates of rare but important harms such as OHSS or surgical complications

Table 16. Strength of evidence for major outcomes-KQ 1 (PCOS)

\begin{tabular}{|c|c|c|c|c|}
\hline Comparison & Outcome & $\begin{array}{l}\text { Study Design } \\
\text { (Sample Size) }\end{array}$ & Conclusion & $\begin{array}{c}\text { SOE } \\
\text { (Rationale) }^{\mathrm{a}}\end{array}$ \\
\hline \multirow{4}{*}{$\begin{array}{l}\text { Oral agents } \\
\text { alone: Letrozole } \\
\text { vs. } \\
\text { Berberine vs. } \\
\text { Berberine + } \\
\text { Letrozole }\end{array}$} & $\begin{array}{l}\text { Live birth } \\
\text { (any/patient) }\end{array}$ & $\begin{array}{l}1 \mathrm{RCT}^{175} \\
(644)\end{array}$ & $\begin{array}{l}\text { Improvement: Letrozole or letrozole } \\
\text { and berberine increase live birth rates } \\
\text { compared to berberine alone. }\end{array}$ & $\begin{array}{l}\text { Low } \\
\text { (Imprecise, } 1 \\
\text { study) }\end{array}$ \\
\hline & $\begin{array}{l}\text { Pregnancy } \\
\text { complications: } \\
\text { Multiple births }\end{array}$ & $\begin{array}{l}1 \mathrm{RCT}^{175} \\
(644)\end{array}$ & $\begin{array}{l}\text { No difference: No significant difference } \\
\text { between letrozole, berberine, or } \\
\text { combination therapy }\end{array}$ & $\begin{array}{l}\text { Low } \\
\text { (Imprecise, } 1 \\
\text { study }\end{array}$ \\
\hline & $\begin{array}{l}\text { Pregnancy } \\
\text { complications: } \\
\text { Miscarriage }\end{array}$ & $\begin{array}{l}1 \mathrm{RCT}^{175} \\
(644)\end{array}$ & $\begin{array}{l}\text { No difference: No significant difference } \\
\text { between letrozole, berberine, or } \\
\text { combination therapy }\end{array}$ & $\begin{array}{l}\text { Low } \\
\text { (Imprecise, } 1 \\
\text { study }\end{array}$ \\
\hline & $\begin{array}{l}\text { Neonatal } \\
\text { outcomes: } \\
\text { Birthweight }\end{array}$ & $\begin{array}{l}1 \mathrm{RCT}^{175} \\
(644)\end{array}$ & $\begin{array}{l}\text { No difference: No significant difference } \\
\text { between letrozole, berberine, or } \\
\text { combination therapy }\end{array}$ & $\begin{array}{l}\text { Low } \\
\text { (Imprecise, } 1 \\
\text { study }\end{array}$ \\
\hline \multirow[t]{8}{*}{$\begin{array}{l}\text { Oral agents } \\
\text { alone: Letrozole } \\
\text { vs. Clomiphene }\end{array}$} & $\begin{array}{l}\text { Live birth } \\
\text { (any/patient) }\end{array}$ & 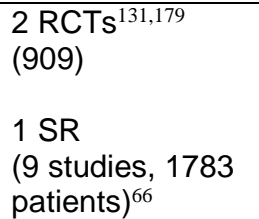 & $\begin{array}{l}\text { Improvement: Letrozole has higher } \\
\text { live birth rates than clomiphene (pooled } \\
\text { OR } 1.64 ; 95 \% \mathrm{Cl}, 1.32 \text { to } 2.04 \text { ). }\end{array}$ & $\begin{array}{l}\text { Moderate } \\
\text { (Imprecise) }\end{array}$ \\
\hline & $\begin{array}{l}\text { Pregnancy } \\
\text { complications: } \\
\text { Multiple births }\end{array}$ & 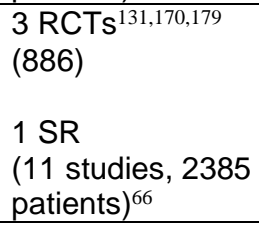 & $\begin{array}{l}\text { Improvement: Letrozole has lower } \\
\text { rates of multiple birth compared to } \\
\text { clomiphene (OR } 0.38 ; 95 \% \mathrm{Cl}, 0.17 \text { to } \\
0.84 \text { ) }\end{array}$ & $\begin{array}{l}\text { Moderate } \\
\text { (Inconsistent) }\end{array}$ \\
\hline & $\begin{array}{l}\text { Pregnancy } \\
\text { complications: } \\
\text { Ectopic } \\
\text { pregnancy }\end{array}$ & $\begin{array}{l}3 \text { RCTs }{ }^{131,170,179} \\
(886)\end{array}$ & $\begin{array}{l}\text { No difference: No difference between } \\
\text { letrozole and clomiphene (OR } 0.72 \text {, } \\
95 \% \mathrm{Cl} 0.24 \text { to } 2.17 \text { ). }\end{array}$ & $\begin{array}{l}\text { Moderate } \\
\text { (Imprecise) }\end{array}$ \\
\hline & $\begin{array}{l}\text { Pregnancy } \\
\text { complications: } \\
\text { Miscarriage }\end{array}$ & 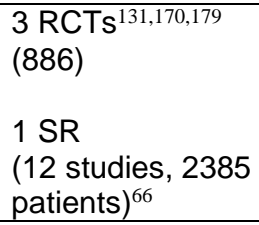 & $\begin{array}{l}\text { No difference: No statistical difference } \\
\text { between letrozole and clomiphene } \\
\text { (pooled OR } 1.32,95 \% \mathrm{Cl} 0.92 \text { to } 1.88 \text { ) }\end{array}$ & $\begin{array}{l}\text { Moderate } \\
\text { (Imprecise) }\end{array}$ \\
\hline & $\begin{array}{l}\text { Neonatal } \\
\text { outcomes: } \\
\text { Death }\end{array}$ & $\begin{array}{l}1 \mathrm{RCT}^{131} \\
(750)\end{array}$ & $\begin{array}{l}\text { Inconclusive: Given the rarity of the } \\
\text { outcome, much larger data sets are } \\
\text { needed. }\end{array}$ & $\begin{array}{l}\text { Insufficient } \\
\text { (Imprecise, } \\
\text { findings from } \\
\text { only } 1 \text { study, } \\
\text { small number of } \\
\text { events) }\end{array}$ \\
\hline & $\begin{array}{l}\text { Neonatal } \\
\text { outcomes: } \\
\text { Birthweight }\end{array}$ & $\begin{array}{l}1 \mathrm{RCT}^{131} \\
(750)\end{array}$ & $\begin{array}{l}\text { No difference: No significant difference } \\
\text { in birthweight between letrozole and } \\
\text { clomiphene }\end{array}$ & $\begin{array}{l}\text { Low } \\
\text { (Findings from } \\
\text { only } 1 \text { study) }\end{array}$ \\
\hline & $\begin{array}{l}\text { Neonatal } \\
\text { outcomes: } \\
\text { Congenital } \\
\text { anomalies }\end{array}$ & $\begin{array}{l}2 \mathrm{RCTs}^{131,179} \\
(909)\end{array}$ & $\begin{array}{l}\text { Inconclusive: No significant difference } \\
\text { in congenital anomaly rates between } \\
\text { letrozole and clomiphene However, } \\
\text { given the rarity of the outcomes, much } \\
\text { larger data sets are needed. }\end{array}$ & $\begin{array}{l}\text { Insufficient } \\
\text { (Imprecise, } \\
\text { small number of } \\
\text { events) }\end{array}$ \\
\hline & $\begin{array}{l}\text { Time to } \\
\text { pregnancy }\end{array}$ & $\begin{array}{l}1 \mathrm{RCT}^{131} \\
(750)\end{array}$ & $\begin{array}{l}\text { No difference: No significant difference } \\
\text { in time to pregnancy between } \\
\text { clomiphene vs. letrozole }\end{array}$ & $\begin{array}{l}\text { Low } \\
\text { (Findings from } \\
\text { only } 1 \text { study) }\end{array}$ \\
\hline
\end{tabular}




\begin{tabular}{|c|c|c|c|c|}
\hline Comparison & Outcome & $\begin{array}{l}\text { Study Design } \\
\text { (Sample Size) }\end{array}$ & Conclusion & $\begin{array}{c}\text { SOE } \\
\text { (Rationale) }^{\mathrm{a}}\end{array}$ \\
\hline \multirow[t]{6}{*}{$\begin{array}{l}\text { Oral agents } \\
\text { alone: } \\
\text { Clomiphene vs. } \\
\text { Metformin vs. } \\
\text { Metformin + } \\
\text { Clomiphene }\end{array}$} & $\begin{array}{l}\text { Live birth } \\
\text { (any/patient) }\end{array}$ & 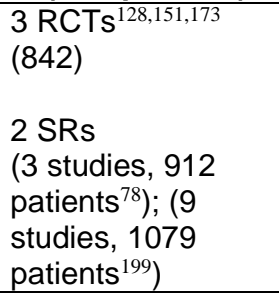 & $\begin{array}{l}\text { No difference: No statistical difference } \\
\text { between clomiphene and metformin or } \\
\text { between clomiphene and combination } \\
\text { therapy of metformin and clomiphene } \\
\text { (OR } 1.21,95 \% \mathrm{Cl} 0.92 \text { to } 1.59 \text { ) }\end{array}$ & $\begin{array}{l}\text { Moderate } \\
\text { (Suspected } \\
\text { reporting bias) }\end{array}$ \\
\hline & $\begin{array}{l}\text { Pregnancy } \\
\text { complications: } \\
\text { Multiple births }\end{array}$ & $\begin{array}{l}3 \mathrm{RCTS}^{128,160,166} \\
(921) \\
1 \mathrm{SR}^{199} \\
\text { (9 studies, } 1079 \\
\text { patients) }\end{array}$ & $\begin{array}{l}\text { No difference: No differences in } \\
\text { multiple birth rates between clomiphene } \\
\text { alone, metformin alone, and } \\
\text { clomiphene plus metformin }\end{array}$ & $\begin{array}{l}\text { Low } \\
\text { (Imprecise, } \\
\text { suspected } \\
\text { reporting bias) }\end{array}$ \\
\hline & $\begin{array}{l}\text { Pregnancy } \\
\text { complications: } \\
\text { Ectopic } \\
\text { pregnancy }\end{array}$ & $\begin{array}{l}3 \text { RCTS }^{128,166,173} \\
(1,005)\end{array}$ & $\begin{array}{l}\text { No difference: No difference between } \\
\text { studied oral agents. Very few ectopic } \\
\text { pregnancies overall. }\end{array}$ & $\begin{array}{l}\text { Low } \\
\text { (Imprecise } \\
\text { findings with } \\
\text { moderate study } \\
\text { limitations) } \\
\end{array}$ \\
\hline & $\begin{array}{l}\text { Pregnancy } \\
\text { complications: } \\
\text { Miscarriage }\end{array}$ & $\begin{array}{l}\text { 3 RCTs }{ }^{128,160,166,173} \\
(817) \\
1 \mathrm{SR}^{199} \text { (9 studies, } \\
1079 \text { patients) }\end{array}$ & $\begin{array}{l}\text { Increase: Higher rate of miscarriage in } \\
\text { the combined therapy group } \\
\text { (clomiphene and metformin) compared } \\
\text { to clomiphene alone (OR } 1.59,95 \% \mathrm{Cl} \text {, } \\
1.03 \text { to } 2.46 \text { ) }\end{array}$ & $\begin{array}{l}\text { Low } \\
\text { (Suspected } \\
\text { reporting bias, } \\
\text { imprecise) }\end{array}$ \\
\hline & $\begin{array}{l}\text { Neonatal } \\
\text { outcomes: } \\
\text { Congenital } \\
\text { anomalies }\end{array}$ & $\begin{array}{l}3 \text { RCTs }{ }^{128,166,173} \\
(1,005)\end{array}$ & $\begin{array}{l}\text { Inconclusive: SOE was insufficient } \\
\text { given the imprecise evidence from } \\
\text { identified studies. Given the rarity of the } \\
\text { outcomes, much larger data sets are } \\
\text { needed. }\end{array}$ & $\begin{array}{l}\text { Insufficient } \\
\text { (Imprecise, } \\
\text { suspected } \\
\text { reporting bias) }\end{array}$ \\
\hline & $\begin{array}{l}\text { Time to } \\
\text { Pregnancy }\end{array}$ & $\begin{array}{l}\text { 1 RCT }^{151} \\
(343)\end{array}$ & $\begin{array}{l}\text { No difference: No significant difference } \\
\text { in time to pregnancy between } \\
\text { clomiphene vs. metformin }\end{array}$ & $\begin{array}{l}\text { Low (Findings } \\
\text { from } 1 \text { study) }\end{array}$ \\
\hline \multirow{2}{*}{$\begin{array}{l}\text { Oral agents } \\
\text { alone: } \\
\text { Clomiphene vs. } \\
\text { Clomiphene + } \\
\text { Cabergoline }\end{array}$} & $\begin{array}{l}\text { Pregnancy } \\
\text { complications: } \\
\text { Multiple births }\end{array}$ & $\begin{array}{l}\text { 1 RCT }^{192} \\
(130)\end{array}$ & $\begin{array}{l}\text { Inconclusive: SOE was insufficient } \\
\text { given the findings from } 1 \text { small study } \\
\text { with potential limitations }\end{array}$ & $\begin{array}{l}\text { Insufficient } \\
\text { ( } 1 \text { study, } \\
\text { moderate } \\
\text { limitations) } \\
\end{array}$ \\
\hline & $\begin{array}{l}\text { Pregnancy } \\
\text { complications: } \\
\text { Miscarriage }\end{array}$ & $\begin{array}{l}\text { 1 RCT }^{192} \\
(130)\end{array}$ & $\begin{array}{l}\text { Inconclusive: SOE was insufficient } \\
\text { given the findings from } 1 \text { small study } \\
\text { with potential limitations }\end{array}$ & $\begin{array}{l}\text { Insufficient } \\
\text { ( } 1 \text { study, } \\
\text { moderate } \\
\text { limitations) } \\
\end{array}$ \\
\hline \multirow[t]{2}{*}{$\begin{array}{l}\text { Oral agents } \\
\text { alone: } \\
\text { Clomiphene vs. } \\
\text { Tamoxifen }\end{array}$} & $\begin{array}{l}\text { Live birth } \\
\text { (any/patient) }\end{array}$ & $\begin{array}{l}1 \mathrm{RCT}^{191} \\
(88) \\
1 \mathrm{SR}^{198} \\
\text { (2 studies, } 195 \\
\text { women) } \\
\end{array}$ & $\begin{array}{l}\text { No difference: No significant difference } \\
\text { in live birth rates between tamoxifen } \\
\text { and clomiphene (OR } 1.24,95 \% \mathrm{Cl} \\
0.59-2.62 \text { ) }\end{array}$ & $\begin{array}{l}\text { Low } \\
\text { (Imprecise) }\end{array}$ \\
\hline & $\begin{array}{l}\text { Pregnancy } \\
\text { complications: } \\
\text { Miscarriage }\end{array}$ & $\begin{array}{l}1 \mathrm{RCT}^{191} \\
\text { (88) } \\
1 \mathrm{SR}^{198} \\
\text { (2 studies, } 195 \\
\text { women) } \\
\end{array}$ & $\begin{array}{l}\text { No difference: No significant difference } \\
\text { in miscarriage rates between tamoxifen } \\
\text { and clomiphene (OR } 1.81,95 \% \mathrm{Cl} \\
0.80-4.12 \text { ) }\end{array}$ & $\begin{array}{l}\text { Low } \\
\text { (Imprecise) }\end{array}$ \\
\hline $\begin{array}{l}\text { Oral agents } \\
\text { alone vs. } \\
\text { acupuncture: } \\
\text { Active }\end{array}$ & Live birth & $\begin{array}{l}1 \mathrm{RCT}^{188} \\
(1000)\end{array}$ & $\begin{array}{l}\text { Improvement: Live birth rates } \\
\text { significantly higher for clomiphene vs. } \\
\text { placebo; not significantly different for } \\
\text { active vs. control acupuncture }\end{array}$ & $\begin{array}{l}\text { Low } \\
\text { (1 study with } \\
\text { potential risk of } \\
\text { bias) }\end{array}$ \\
\hline
\end{tabular}




\begin{tabular}{|c|c|c|c|c|}
\hline Comparison & Outcome & $\begin{array}{l}\text { Study Design } \\
\text { (Sample Size) }\end{array}$ & Conclusion & $\begin{array}{c}\text { SOE } \\
\text { (Rationale) }\end{array}$ \\
\hline \multirow{4}{*}{$\begin{array}{l}\text { Acupuncture + } \\
\text { Clomiphene vs. } \\
\text { Control } \\
\text { Acupuncture + } \\
\text { Clomiphene vs. } \\
\text { Active } \\
\text { Acupuncture + } \\
\text { Placebo vs. } \\
\text { Control } \\
\text { Acupuncture + } \\
\text { Placebo }\end{array}$} & $\begin{array}{l}\text { Pregnancy } \\
\text { complications: } \\
\text { Ectopic } \\
\text { pregnancy }\end{array}$ & $\begin{array}{l}1 \mathrm{RCT}^{188} \\
(1000)\end{array}$ & $\begin{array}{l}\text { No difference: no significant difference } \\
\text { in ectopic pregnancy rates between oral } \\
\text { agents and acupuncture strategies. }\end{array}$ & $\begin{array}{l}\text { Low } \\
(1 \text { study with } \\
\text { potential risk of } \\
\text { bias) }\end{array}$ \\
\hline & $\begin{array}{l}\text { Pregnancy } \\
\text { complications: } \\
\text { Miscarriage }\end{array}$ & $\begin{array}{l}1 \mathrm{RCT}^{188} \\
(1000)\end{array}$ & $\begin{array}{l}\text { No difference: no significant difference } \\
\text { in miscarriage rates between oral } \\
\text { agents and acupuncture strategies. }\end{array}$ & $\begin{array}{l}\text { Low } \\
(1 \text { study with } \\
\text { potential risk of } \\
\text { bias) }\end{array}$ \\
\hline & $\begin{array}{l}\text { Neonatal } \\
\text { outcomes: } \\
\text { Congenital } \\
\text { Abnormalities }\end{array}$ & $\begin{array}{l}1 \mathrm{RCT}^{188} \\
(1000)\end{array}$ & $\begin{array}{l}\text { No difference: no significant difference } \\
\text { in congenital abnormality rates between } \\
\text { oral agents and acupuncture strategies. }\end{array}$ & $\begin{array}{l}\text { Low } \\
(1 \text { study with } \\
\text { potential risk of } \\
\text { bias) }\end{array}$ \\
\hline & $\begin{array}{l}\text { Neonatal } \\
\text { Death }\end{array}$ & $\begin{array}{l}\text { 1 RCT } 188 \\
(1000)\end{array}$ & $\begin{array}{l}\text { No difference: no significant difference } \\
\text { in neonatal death rates between oral } \\
\text { agents and acupuncture strategies. }\end{array}$ & $\begin{array}{l}\text { Low (1 study } \\
\text { with potential } \\
\text { risk of bias) }\end{array}$ \\
\hline \multirow{4}{*}{$\begin{array}{l}\text { Oral agents } \\
\text { alone vs. oral } \\
\text { agents with IUI: } \\
\text { Clomiphene } \\
\text { without IUI vs. } \\
\text { Clomiphene } \\
\text { with IUI }\end{array}$} & Live birth & $\begin{array}{l}1 \mathrm{RCT}^{157} \\
(188)\end{array}$ & $\begin{array}{l}\text { Inconclusive: SOE was insufficient } \\
\text { given evidence from } 1 \text { small trial. }\end{array}$ & $\begin{array}{l}\text { Insufficient } \\
\text { (Imprecise, } 1 \\
\text { small trial) }\end{array}$ \\
\hline & $\begin{array}{l}\text { Pregnancy } \\
\text { complications: } \\
\text { Multiple births }\end{array}$ & $\begin{array}{l}1 \mathrm{RCT}^{157} \\
(188)\end{array}$ & $\begin{array}{l}\text { Inconclusive: SOE was insufficient } \\
\text { given evidence from } 1 \text { small trial. }\end{array}$ & $\begin{array}{l}\text { Insufficient } \\
\text { (Imprecise, } 1 \\
\text { small trial) }\end{array}$ \\
\hline & $\begin{array}{l}\text { Pregnancy } \\
\text { complications: } \\
\text { Ectopic } \\
\text { pregnancy }\end{array}$ & $\begin{array}{l}1 \mathrm{RCT}^{157} \\
(188)\end{array}$ & $\begin{array}{l}\text { Inconclusive: SOE was insufficient } \\
\text { given evidence from } 1 \text { small trial. }\end{array}$ & $\begin{array}{l}\text { Insufficient } \\
\text { (Imprecise, } 1 \\
\text { small trial) }\end{array}$ \\
\hline & $\begin{array}{l}\text { Pregnancy } \\
\text { complications: } \\
\text { Miscarriage }\end{array}$ & $\begin{array}{l}\text { 1 RCT } \\
\text { (188) }\end{array}$ & $\begin{array}{l}\text { Inconclusive: SOE was insufficient } \\
\text { given evidence from } 1 \text { small trial. }\end{array}$ & $\begin{array}{l}\text { Insufficient } \\
\text { (Imprecise, } 1 \\
\text { small trial) }\end{array}$ \\
\hline \multirow{2}{*}{$\begin{array}{l}\text { Oral agents } \\
\text { alone vs. oral } \\
\text { agents with IUI: } \\
\text { Myo-inositol + } \\
\text { Ovulation } \\
\text { induction + IUI } \\
\text { vs. Ovulation } \\
\text { induction and } \\
\text { IUI alone }\end{array}$} & $\begin{array}{l}\text { Pregnancy } \\
\text { complications: } \\
\text { Multiple births }\end{array}$ & $\begin{array}{l}\text { 1 RCT } \\
\text { (196) }\end{array}$ & $\begin{array}{l}\text { Inconclusive: SOE was insufficient } \\
\text { given evidence from } 1 \text { small trial. }\end{array}$ & $\begin{array}{l}\text { Insufficient } \\
\text { (Imprecise, } 1 \\
\text { small trial) }\end{array}$ \\
\hline & $\begin{array}{l}\text { Adverse } \\
\text { events: } \\
\text { OHSS }\end{array}$ & $\begin{array}{l}1 \mathrm{RCT}^{182} \\
(196)\end{array}$ & $\begin{array}{l}\text { Inconclusive: SOE was insufficient } \\
\text { given evidence from } 1 \text { small trial. }\end{array}$ & $\begin{array}{l}\text { Insufficient } \\
\text { (Imprecise, } 1 \\
\text { small trial) }\end{array}$ \\
\hline \multirow[t]{4}{*}{$\begin{array}{l}\text { Oral agents vs. } \\
\text { LOD }\end{array}$} & $\begin{array}{l}\text { Live birth } \\
\text { (any/patient) }\end{array}$ & $\begin{array}{l}1 \mathrm{SR}^{81} \\
\text { (8 studies, } 1,034 \\
\text { women) }\end{array}$ & $\begin{array}{l}\text { No difference: No statistically } \\
\text { significant differences between LOD } \\
\text { and oral agents (OR }=0.77,95 \% \mathrm{Cl} \\
0.59 \text { to } 1.01)\end{array}$ & $\begin{array}{l}\text { Moderate } \\
\text { (Suspected } \\
\text { reporting bias) }\end{array}$ \\
\hline & $\begin{array}{l}\text { Pregnancy } \\
\text { complications: } \\
\text { Multiple births }\end{array}$ & $\begin{array}{l}1 \mathrm{SR}^{81} \\
\text { (15 studies, } 1,129 \\
\text { women) }\end{array}$ & $\begin{array}{l}\text { Reduction: There was a reduction in } \\
\text { multiple births given LOD as compared } \\
\text { to oral agents (OR } 0.21,95 \% \mathrm{Cl} 0.08 \text { to } \\
0.58 \text { ) }\end{array}$ & $\begin{array}{l}\text { Moderate } \\
\text { (Suspected } \\
\text { reporting bias) }\end{array}$ \\
\hline & $\begin{array}{l}\text { Pregnancy } \\
\text { complications: } \\
\text { Miscarriage }\end{array}$ & $\begin{array}{l}1 \mathrm{RCT}^{189} \\
(80) \\
1 \mathrm{SR}^{81} \\
\text { (15 studies, 1,592 } \\
\text { women) }\end{array}$ & $\begin{array}{l}\text { No difference: No significant } \\
\text { differences in miscarriage between } \\
\text { LOD and oral agents (OR 1.1, } 95 \% \mathrm{Cl} \\
0.74 \text { to } 1.61 \text { ) }\end{array}$ & $\begin{array}{l}\text { Low } \\
\text { (Imprecise, } \\
\text { suspected } \\
\text { reporting bias) }\end{array}$ \\
\hline & $\begin{array}{l}\text { Short term } \\
\text { adverse } \\
\text { effects of } \\
\text { treatment: } \\
\text { OHSS }\end{array}$ & $\begin{array}{l}2 \mathrm{RCTs}^{165,168} \\
(325)\end{array}$ & $\begin{array}{l}\text { Inconclusive: SOE was insufficient } \\
\text { given the imprecision and small } \\
\text { numbers of events in the identified } \\
\text { studies. }\end{array}$ & $\begin{array}{l}\text { Insufficient } \\
\text { (Imprecise, } \\
\text { suspected } \\
\text { reporting bias, } 1 \\
\text { small study) }\end{array}$ \\
\hline
\end{tabular}




\begin{tabular}{|c|c|c|c|c|}
\hline Comparison & Outcome & $\begin{array}{l}\text { Study Design } \\
\text { (Sample Size) }\end{array}$ & Conclusion & $\begin{array}{c}\text { SOE } \\
\text { (Rationale) }^{\mathrm{a}}\end{array}$ \\
\hline & $\begin{array}{l}\text { Short term } \\
\text { adverse } \\
\text { effects of } \\
\text { treatment: } \\
\text { Surgical } \\
\text { complications }\end{array}$ & $\begin{array}{l}2 \mathrm{RCTs}^{163,165} \\
(89)\end{array}$ & $\begin{array}{l}\text { Inconclusive: SOE was insufficient } \\
\text { given the imprecision and small } \\
\text { numbers of events in the identified } \\
\text { studies. }\end{array}$ & $\begin{array}{l}\text { Insufficient } \\
\text { (Imprecise, } \\
\text { suspected } \\
\text { reporting bias, } \\
\text { small sample } \\
\text { size) }\end{array}$ \\
\hline \multirow{4}{*}{$\begin{array}{l}\text { Oral agents } \\
\text { alone vs. } \\
\text { gonadotropins: } \\
\text { Clomiphene } \\
\text { citrate vs. low- } \\
\text { dose FSH }\end{array}$} & Live birth & $\begin{array}{l}3 \mathrm{RCTs}^{152,176,187} \\
(1072)\end{array}$ & $\begin{array}{l}\text { Inconclusive: SOE was insufficient } \\
\text { given the inconsistent evidence from } \\
\text { included studies }\end{array}$ & $\begin{array}{l}\text { Insufficient } \\
\text { (Imprecise, } \\
\text { inconsistent) }\end{array}$ \\
\hline & $\begin{array}{l}\text { Pregnancy } \\
\text { complications: } \\
\text { Multiple births }\end{array}$ & $\begin{array}{l}\text { 1 RCT } 152 \\
(302)\end{array}$ & $\begin{array}{l}\text { Inconclusive: SOE was insufficient } \\
\text { given only } 1 \text { identified trial. }\end{array}$ & $\begin{array}{l}\text { Insufficient } \\
\text { (Imprecise, } 1 \\
\text { study) }\end{array}$ \\
\hline & $\begin{array}{l}\text { Pregnancy } \\
\text { complications: } \\
\text { Ectopic } \\
\text { pregnancy }\end{array}$ & $\begin{array}{l}3 \operatorname{RCTs}^{152,176,187} \\
(1072)\end{array}$ & $\begin{array}{l}\text { No difference: Ectopic pregnancy rate } \\
\text { did not differ between FSH and } \\
\text { clomiphene strategies. }\end{array}$ & $\begin{array}{l}\text { Low } \\
\text { (Imprecise) }\end{array}$ \\
\hline & $\begin{array}{l}\text { Pregnancy } \\
\text { complications: } \\
\text { Miscarriage }\end{array}$ & $\begin{array}{l}3 \text { RCTs }{ }^{152,176,187} \\
(1072)\end{array}$ & $\begin{array}{l}\text { Inconclusive: SOE was insufficient } \\
\text { given the inconsistent evidence from } \\
\text { included studies }\end{array}$ & $\begin{array}{l}\text { Insufficient } \\
\text { (Imprecise, } \\
\text { inconsistent) }\end{array}$ \\
\hline $\begin{array}{l}\text { Oral agents } \\
\text { alone vs. } \\
\text { gonadotropins } \\
\text { Clomiphene } \\
\text { plus urinary } \\
\text { FSH vs. FSH } \\
\text { alone }\end{array}$ & Live birth & $\begin{array}{l}\text { 1 RCT }^{145} \\
(174)\end{array}$ & $\begin{array}{l}\text { Inconclusive SOE was insufficient } \\
\text { given only } 1 \text { identified trial }\end{array}$ & $\begin{array}{l}\text { Insufficient } \\
\text { (Imprecise, } 1 \\
\text { small study) }\end{array}$ \\
\hline $\begin{array}{l}\text { Lifestyle } \\
\text { modifications + } \\
\text { IVF vs. IVF } \\
\text { alone }\end{array}$ & Live birth & $\begin{array}{l}\text { 3 RCTs } \\
\text { (1688) }\end{array}$ & $\begin{array}{l}\text { No difference: No difference in live } \\
\text { birth rates for women who underwent } \\
\text { lifestyle modification in combination } \\
\text { with IVF compared with IVF alone }\end{array}$ & $\begin{array}{l}\text { Moderate } \\
\text { (Heterogeneity } \\
\text { in interventions) }\end{array}$ \\
\hline \multirow[t]{4}{*}{$\begin{array}{l}\text { LEC vs. rFSH } \\
\text { LEC vs. hMG }\end{array}$} & Live birth & $\begin{array}{l}\text { 1 RCT }{ }^{141} \\
\text { (168) }\end{array}$ & $\begin{array}{l}\text { Inconclusive: SOE was insufficient } \\
\text { given only } 1 \text { study with moderate risk of } \\
\text { bias. }\end{array}$ & $\begin{array}{l}\text { Insufficient } \\
\text { (Imprecise, } 1 \\
\text { study with } \\
\text { limitations) } \\
\end{array}$ \\
\hline & $\begin{array}{l}\text { Pregnancy } \\
\text { complications: } \\
\text { Multiple births }\end{array}$ & $\begin{array}{l}\text { 1 RCT }^{141} \\
(168)\end{array}$ & $\begin{array}{l}\text { Inconclusive: SOE was insufficient } \\
\text { given only } 1 \text { study with moderate risk of } \\
\text { bias. }\end{array}$ & $\begin{array}{l}\text { Insufficient } \\
\text { (Imprecise, } 1 \\
\text { study with } \\
\text { limitations) } \\
\end{array}$ \\
\hline & Costs & $\begin{array}{l}1 \mathrm{RCT}^{141} \\
(168)\end{array}$ & $\begin{array}{l}\text { Inconclusive: SOE was insufficient } \\
\text { given only } 1 \text { study with moderate risk of } \\
\text { bias. }\end{array}$ & $\begin{array}{l}\text { Insufficient } \\
\text { (Imprecise, } 1 \\
\text { study with } \\
\text { limitations) } \\
\end{array}$ \\
\hline & $\begin{array}{l}\text { Short term } \\
\text { adverse } \\
\text { effects of } \\
\text { treatment: } \\
\text { OHSS }\end{array}$ & $\begin{array}{l}1 \mathrm{RCT}^{148} \\
(104)\end{array}$ & $\begin{array}{l}\text { Inconclusive: SOE was insufficient } \\
\text { given only } 1 \text { study with moderate risk of } \\
\text { bias. }\end{array}$ & $\begin{array}{l}\text { Insufficient } \\
\text { (Imprecise, } 1 \\
\text { study with } \\
\text { limitations) }\end{array}$ \\
\hline \multirow[t]{2}{*}{$\begin{array}{l}\text { rFSH vs. rFSH } \\
+ \text { GnRH } \\
\text { antagonist or } \\
\text { hMG }\end{array}$} & $\begin{array}{l}\text { Live birth } \\
\text { (any/cycle) }\end{array}$ & $\begin{array}{l}2 \mathrm{RCTs}^{143,159} \\
(374)\end{array}$ & $\begin{array}{l}\text { Inconclusive: SOE was insufficient } \\
\text { given studies with moderate risk of bias } \\
\text { and varying definitions of live birth }\end{array}$ & $\begin{array}{l}\text { Insufficient } \\
\text { (Moderate } \\
\text { study } \\
\text { limitations) } \\
\end{array}$ \\
\hline & $\begin{array}{l}\text { Pregnancy } \\
\text { complications: } \\
\text { Multiple births }\end{array}$ & $\begin{array}{l}\text { 2 RCTs } \mathrm{R}^{143,159} \\
(374)\end{array}$ & $\begin{array}{l}\text { Inconclusive: SOE was insufficient } \\
\text { given imprecise findings from studies of } \\
\text { moderate risk of bias. }\end{array}$ & $\begin{array}{l}\text { Insufficient } \\
\text { (Imprecise } \\
\text { findings with } \\
\text { moderate study } \\
\text { limitations) } \\
\end{array}$ \\
\hline
\end{tabular}




\begin{tabular}{|c|c|c|c|c|}
\hline Comparison & Outcome & $\begin{array}{l}\text { Study Design } \\
\text { (Sample Size) }\end{array}$ & Conclusion & $\begin{array}{c}\text { SOE } \\
\text { (Rationale) }^{\mathrm{a}}\end{array}$ \\
\hline & $\begin{array}{l}\text { Pregnancy } \\
\text { complications: } \\
\text { Miscarriage }\end{array}$ & $\begin{array}{l}2 \mathrm{RCTs}^{143,159} \\
(374)\end{array}$ & $\begin{array}{l}\text { Inconclusive: SOE was insufficient } \\
\text { given imprecise findings from studies of } \\
\text { moderate risk of bias. }\end{array}$ & $\begin{array}{l}\text { Insufficient } \\
\text { (Imprecise } \\
\text { findings with } \\
\text { moderate study } \\
\text { limitations) }\end{array}$ \\
\hline & $\begin{array}{l}\text { Short term } \\
\text { adverse } \\
\text { effects of } \\
\text { treatment: } \\
\text { OHSS }\end{array}$ & $\begin{array}{l}1 \mathrm{RCT}^{143} \\
(276)\end{array}$ & $\begin{array}{l}\text { Inconclusive: SOE was insufficient } \\
\text { given only } 1 \text { study with moderate risk of } \\
\text { bias. }\end{array}$ & $\begin{array}{l}\text { Insufficient } \\
\text { (Imprecise } \\
\text { findings with } \\
\text { moderate study } \\
\text { limitations) }\end{array}$ \\
\hline \multirow[t]{6}{*}{$\begin{array}{l}\text { ART IVF: GnRH } \\
\text { agonist +/- IVF } \\
\text { Vs. GnRH } \\
\text { antagonist +/- } \\
\text { IVF }\end{array}$} & $\begin{array}{l}\text { Live birth } \\
\text { (patient) }\end{array}$ & $\begin{array}{l}1 \mathrm{RCT}^{146} \\
(109)\end{array}$ & $\begin{array}{l}\text { Inconclusive: SOE was insufficient } \\
\text { given only } 1 \text { study with moderate risk of } \\
\text { bias and with imprecise findings }\end{array}$ & $\begin{array}{l}\text { Insufficient } \\
\text { (Imprecise } \\
\text { findings with } \\
\text { moderate study } \\
\text { limitations) }\end{array}$ \\
\hline & $\begin{array}{l}\text { Live birth } \\
\text { (cycle) }\end{array}$ & $\begin{array}{l}4 \mathrm{RCTS}^{147,150,164,167} \\
(408)\end{array}$ & $\begin{array}{l}\text { No difference: No significant difference } \\
\text { in included studies but varying } \\
\text { interventions and comparators with low } \\
\text { numbers of live birth }\end{array}$ & $\begin{array}{l}\text { Low } \\
\text { (Imprecise } \\
\text { findings with } \\
\text { moderate study } \\
\text { limitations) }\end{array}$ \\
\hline & $\begin{array}{l}\text { Pregnancy } \\
\text { complications: } \\
\text { Multiple births }\end{array}$ & $\begin{array}{l}1 \mathrm{RCT}^{164} \\
(70)\end{array}$ & $\begin{array}{l}\text { Inconclusive: SOE was insufficient } \\
\text { given only } 1 \text { small study with moderate } \\
\text { risk of bias. }\end{array}$ & $\begin{array}{l}\text { Insufficient } \\
\text { (Moderate } \\
\text { study } \\
\text { limitations) }\end{array}$ \\
\hline & $\begin{array}{l}\text { Pregnancy } \\
\text { complications: } \\
\text { Ectopic } \\
\text { pregnancy }\end{array}$ & $\begin{array}{l}1 \mathrm{RCT}^{171} \\
(120)\end{array}$ & $\begin{array}{l}\text { Inconclusive: SOE was insufficient } \\
\text { given only } 1 \text { small study with moderate } \\
\text { risk of bias. }\end{array}$ & $\begin{array}{l}\text { Insufficient } \\
\text { (Moderate } \\
\text { study } \\
\text { limitations) }\end{array}$ \\
\hline & $\begin{array}{l}\text { Pregnancy } \\
\text { complications: } \\
\text { Miscarriage }\end{array}$ & $\begin{array}{l}3 \text { RCTs }^{164,167,171} \\
(279)\end{array}$ & $\begin{array}{l}\text { No difference: No differences in } \\
\text { miscarriage rates for GnRH agonist vs. } \\
\text { antagonist, or hCG medium, hCG-free } \\
\text { medium with transfer, and hCG-free } \\
\text { medium without transfer. }\end{array}$ & $\begin{array}{l}\text { Moderate } \\
\text { (Imprecise } \\
\text { findings with } \\
\text { moderate study } \\
\text { limitations) }\end{array}$ \\
\hline & $\begin{array}{l}\text { Short term } \\
\text { adverse } \\
\text { effects of } \\
\text { treatment: } \\
\text { OHSS }\end{array}$ & $\begin{array}{l}5 \\
\text { RCTs }^{153,161,162,164,178} \\
(468)\end{array}$ & $\begin{array}{l}\text { Inconclusive: SOE was insufficient } \\
\text { given that all identified studies were } \\
\text { underpowered to detect differences in } \\
\text { OHSS. }\end{array}$ & $\begin{array}{l}\text { Insufficient } \\
\text { (Imprecise, } \\
\text { underpowered } \\
\text { studies) }\end{array}$ \\
\hline \multirow[t]{2}{*}{ Adjunct to IVF } & $\begin{array}{l}\text { Live birth } \\
\text { (patient) }\end{array}$ & $\begin{array}{l}1 \mathrm{RCT}^{155} \\
(149)\end{array}$ & $\begin{array}{l}\text { Inconclusive: SOE was insufficient } \\
\text { given only } 1 \text { small study with moderate } \\
\text { risk of bias. }\end{array}$ & $\begin{array}{l}\text { Insufficient } \\
\text { (Moderate } \\
\text { study } \\
\text { limitations) }\end{array}$ \\
\hline & $\begin{array}{l}\text { Short term } \\
\text { adverse } \\
\text { effects of } \\
\text { treatment: } \\
\text { OHSS }\end{array}$ & $\begin{array}{l}2 \mathrm{RCTs}^{177,190} \\
(372)\end{array}$ & $\begin{array}{l}\text { Inconclusive: SOE was insufficient } \\
\text { given only small studies with moderate } \\
\text { risk of bias and varying adjuncts. }\end{array}$ & $\begin{array}{l}\text { Insufficient } \\
\text { (Moderate } \\
\text { study } \\
\text { limitations) }\end{array}$ \\
\hline \multirow{3}{*}{$\begin{array}{l}\text { Fresh vS. } \\
\text { Frozen } \\
\text { Embryos in IVF } \\
\text { for PCOS }\end{array}$} & $\begin{array}{l}\text { Live birth } \\
\text { (any/cycle) }\end{array}$ & $\begin{array}{l}1 \mathrm{RCT}^{174} \\
(1508)\end{array}$ & $\begin{array}{l}\text { Improvement: Live birth rates were } \\
\text { significantly higher with frozen embryo } \\
\text { transfer compared to fresh embryos }\end{array}$ & $\begin{array}{l}\text { Low } \\
\text { (1 study) }\end{array}$ \\
\hline & $\begin{array}{l}\text { Pregnancy } \\
\text { complications: } \\
\text { Multiple births }\end{array}$ & $\begin{array}{l}1 \mathrm{RCT}^{174} \\
(1508)\end{array}$ & $\begin{array}{l}\text { No difference: No difference in multiple } \\
\text { births with fresh versus frozen embryo } \\
\text { transfer }\end{array}$ & $\begin{array}{l}\text { Low } \\
\text { (1 study) }\end{array}$ \\
\hline & $\begin{array}{l}\text { Pregnancy } \\
\text { complications: } \\
\text { Ectopic } \\
\text { pregnancy }\end{array}$ & $\begin{array}{l}1 \mathrm{RCT}^{174} \\
(1508)\end{array}$ & $\begin{array}{l}\text { Reduction: Ectopic pregnancies were } \\
\text { reduced with frozen embryo transfer }\end{array}$ & $\begin{array}{l}\text { Low } \\
\text { (1 study) }\end{array}$ \\
\hline
\end{tabular}




\begin{tabular}{|c|c|c|c|c|}
\hline Comparison & Outcome & $\begin{array}{l}\text { Study Design } \\
\text { (Sample Size) }\end{array}$ & Conclusion & $\begin{array}{c}\text { SOE } \\
\text { (Rationale) }^{\mathrm{a}}\end{array}$ \\
\hline & $\begin{array}{l}\text { Pregnancy } \\
\text { complications: } \\
\text { Miscarriage }\end{array}$ & $\begin{array}{l}1 \mathrm{RCT}^{174} \\
(1508)\end{array}$ & $\begin{array}{l}\text { Reduction: Miscarriages were reduced } \\
\text { with frozen embryo transfer }\end{array}$ & $\begin{array}{l}\text { Low } \\
\text { (1 study) }\end{array}$ \\
\hline & $\begin{array}{l}\text { Neonatal } \\
\text { Outcomes: } \\
\text { Congenital } \\
\text { abnormalities }\end{array}$ & $\begin{array}{l}1 \mathrm{RCT}^{174} \\
(1508)\end{array}$ & $\begin{array}{l}\text { No difference: No difference } \\
\text { congenital abnormalities with fresh } \\
\text { versus frozen embryo transfer }\end{array}$ & $\begin{array}{l}\text { Low } \\
\text { (1 study) }\end{array}$ \\
\hline & $\begin{array}{l}\text { Neonatal } \\
\text { Death }\end{array}$ & $\begin{array}{l}1 \mathrm{RCT}^{174} \\
(1508)\end{array}$ & $\begin{array}{l}\text { No difference: No difference neonatal } \\
\text { deaths with fresh versus frozen embryo } \\
\text { transfer }\end{array}$ & $\begin{array}{l}\text { Low } \\
\text { (1 study) }\end{array}$ \\
\hline $\begin{array}{l}\text { ART vs. no } \\
\text { infertility } \\
\text { treatment }\end{array}$ & $\begin{array}{l}\text { Long-term } \\
\text { outcomes: } \\
\text { Child (type } 1 \\
\text { diabetes } \\
\text { mellitus) }\end{array}$ & $\begin{array}{l}1 \text { Obs }^{184} \\
(565,116 \\
\text { pregnancies) }\end{array}$ & $\begin{array}{l}\text { No difference: No significant difference } \\
\text { found between type } 1 \text { diabetes mellitus } \\
\text { diagnoses in children born to patients } \\
\text { with PCOS infertility conceived with } \\
\text { ART compared to children conceived } \\
\text { with no fertility treatment }\end{array}$ & $\begin{array}{l}\text { Moderate } \\
\text { (Imprecise) }\end{array}$ \\
\hline \multirow[t]{3}{*}{$\begin{array}{l}\text { ART ICSI: } \\
\text { GnRH agonist } \\
\text { vs. GnRH } \\
\text { antagonist }\end{array}$} & Live birth & $\begin{array}{l}1 \mathrm{RCT}^{149} \\
(82)\end{array}$ & $\begin{array}{l}\text { Inconclusive: SOE was insufficient } \\
\text { given only } 1 \text { small study with moderate } \\
\text { risk of bias. }\end{array}$ & $\begin{array}{l}\text { Insufficient } \\
\text { (Imprecise } \\
\text { findings with } \\
\text { moderate study } \\
\text { limitations, } 1 \\
\text { small study) } \\
\end{array}$ \\
\hline & $\begin{array}{l}\text { Pregnancy } \\
\text { complications: } \\
\text { Miscarriage }\end{array}$ & $\begin{array}{l}1 \mathrm{RCT}^{158} \\
(112)\end{array}$ & $\begin{array}{l}\text { Inconclusive: SOE was insufficient } \\
\text { given only } 1 \text { small study with moderate } \\
\text { risk of bias. }\end{array}$ & $\begin{array}{l}\text { Insufficient } \\
\text { (Imprecise } \\
\text { findings with } \\
\text { moderate study } \\
\text { limitations, } 1 \\
\text { small study) }\end{array}$ \\
\hline & $\begin{array}{l}\text { Short term } \\
\text { adverse } \\
\text { effects of } \\
\text { treatment: } \\
\text { OHSS }\end{array}$ & $\begin{array}{l}1 \mathrm{RCT}^{158} \\
(112)\end{array}$ & $\begin{array}{l}\text { Inconclusive: SOE was insufficient } \\
\text { given only } 1 \text { small study with moderate } \\
\text { risk of bias. }\end{array}$ & $\begin{array}{l}\text { Insufficient } \\
\text { (Imprecise } \\
\text { findings with } \\
\text { moderate study } \\
\text { limitations, } 1 \\
\text { small study) }\end{array}$ \\
\hline
\end{tabular}

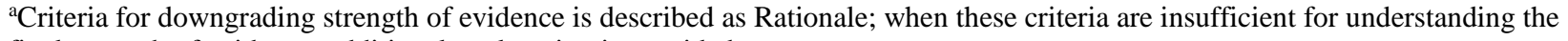
final strength of evidence, additional explanation is provided.

Abbreviations: ART=assisted reproductive technology; BMI=body mass index; CI=confidence interval; FSH=follicle-stimulating hormone; GnRH=gonadotropin-releasing hormone; hCG=human chorionic gonadotropin; hMG=human menopausal gonadotropin; IUI=intrauterine insemination; IVF=in vitro fertilization; IVM=in vitro maturation; KQ=Key Question; LEC=laparoscopic electrocauterization; LOD=laparoscopic ovarian drilling/diathermy; N=number of patients/participants; NA=not applicable; NR=not reported; Obs=observational study; OHSS=ovarian hyperstimulation syndrome; OR=odds ratio; PCOS=polycystic ovary syndrome; RCT=randomized controlled trial; $\mathrm{rFSH}=$ recombinant follicle-stimulating hormone; $\mathrm{SR}=$ systematic review; uFSH=urinary follicle-stimulating hormone

\section{Key Question 2. Endometriosis}

KQ 2. What are the comparative safety and effectiveness of available treatment strategies for women with endometriosis who are infertile and who wish to become pregnant?
KQ 2a. Does the optimal treatment strategy vary by patient characteristics such as age, ovarian reserve, race, BMI, stage of endometriosis, presence of other potential causes of female infertility, or presence of male factor infertility? 
Endometriosis is a condition defined by the presence of endometrial tissue outside the uterus, most commonly on the ovary and peritoneum. It is observed in approximately $6-10$ percent of women of fertile age, and in up to 35-50 percent of women with infertility, pelvic pain, or both. ${ }^{200}$ Endometriosis severity is defined in a system of stages that are based on a weighted point system. The severity of the disease is classified as minimal (Stage I), mild (Stage II), moderate (Stage III), or severe (Stage IV) based on surgical findings. ${ }^{201}$

\section{Description of Included Studies for KQ 2 (Endometriosis)}

We identified 7 individual studies that addressed infertility treatment for women with endometriosis. ${ }^{123,185,186,202-205}$

Three studies were RCTs; one was good quality, ${ }^{204}$ and two were fair quality. ${ }^{202,205}$ The remaining four were observational cohorts; two were good quality, ${ }^{185,186}$ and two were fair quality. ${ }^{123,203}$ Geographical locations varied; one study was located in Africa, ${ }^{204}$ one was located in Asia, ${ }^{202}$ one in Australia/NZ, ${ }^{203}$ two in the United States, ${ }^{123,185}$ and the remaining two were located in the United Kingdom or continental Europe. ${ }^{186,205}$ Five studies were conducted in subspecialty practices. ${ }^{185,186,202,204,205}$ The setting was unclear for the remaining two studies. ${ }^{123,203}$ One study reported funding from government sources, ${ }^{203}$ one study reported funding from industry, ${ }^{186}$ one study reported non-government, non-industry funding, ${ }^{123}$ and the remaining four studies did not report a funding source or the source was unclear. ${ }^{185,202,204,205}$

The main classes of treatment examined were: oral ovulation/superovulation with or without IUI, gonadotropins with or without IUI, ART (IVF or ICSI), surgical treatment, and surgery with hormonal adjunctive therapy. We did not perform meta-analysis because of the lack of studies reporting results for similar outcomes and treatment comparisons.

In addition to the above studies, we discuss two good-quality systematic reviews ${ }^{60,64}$ that addressed the comparative effectiveness of various treatments for infertility in women with endometriosis, and incorporate the consistency of their findings with included studies into our strength of evidence (SOE) ratings.

\section{Key Points for Endometriosis}

Key findings for couples where the primary cause of infertility is endometriosis in the female partner included:

- As with other indications for IVF, use of single-embryo transfer is associated with slightly lower live birth rates but significantly reduced multiple gestation rates (low SOE)

- The live birth rate per cycle was higher in couples who underwent ART than those who used IUI (low SOE)

- SOE was rated insufficient for all other comparisons/outcomes.

\section{Detailed Synthesis by Treatment for Endometriosis}

Included studies and their findings for the following treatments for endometriosis are detailed in this section:

1. Oral Agents With IUI

2. Adjunctive Hormonal Therapy After Surgical Treatment

3. ART 


\section{Oral Agents With IUI After Laparoscopic Treatment for Endometriosis}

One good-quality RCT examined pregnancy outcomes after ovulation induction with IUI in 136 infertile women with minimal/mild endometriosis who sought infertility treatment 6 to 12 months after laparoscopic treatment. ${ }^{204}$ Women were randomized to treatment with clomiphene or letrozole for 5 days starting on day 3 of the menstrual cycle. Human chorionic gonadotropin (hCG; 10,000 IU) was given intramuscularly when one follicle measuring at least $18 \mathrm{~mm}$ was identified. IUI was performed 32-36 hours after HCG injection. There was no significant difference in live birth rate among women treated with letrozole and IUI (44.9\%) as compared with clomiphene citrate and IUI (40.3\%; RR 0.89; 95\% CI, 0.43 to 1.58) (low SOE). There was also no significant difference in miscarriage rate among women treated with letrozole and IUI (12.4\%) as compared with clomiphene citrate and IUI (12.9\%; RR 1.13; 95\% CI, 0.76 to 1.68). Both outcomes were rated as insufficient SOE given findings from one small study. Other pregnancy complication outcomes, neonatal outcomes, time to pregnancy, costs, short term adverse effects of treatments, or long term effects were not evaluated.

\section{Adjunctive Hormonal Therapy After Surgical Treatment for Endometriosis}

Two fair-quality RCTs evaluated the use of hormonal therapy after surgery for IVF. A fairquality RCT examined the efficacy of hormonal therapy as an adjunctive treatment with surgical correction of endometriosis. ${ }^{202}$ This trial studied short-term oral contraceptives, with and without a "Dan'e" Chinese herbal mixture, compared with no treatment, immediately after surgery in 156 women with endometriosis. After the surgical treatment, participants were randomly allocated to three groups: (a) a combined oral contraceptive for 63 days, (b) combined oral contraceptive for 63 days + Dan'e Chinese herbal mixture for the last 30 days, or (c) no medical treatment. The mean duration of follow-up was $22.17 \pm 3.39$ months.

A total of 73 pregnancies occurred. The total pregnancy rate was $46.80 \%$. There was no difference in live birth rate (70\% vs. $81.2 \%$ vs. $79.2 \%$ ), ectopic pregnancy rate (5\% vs. 0 vs. $8.3 \%$ ), or miscarriage rate ( $20 \%$ vs. $18.7 \%$ vs. $12.5 \%$ ) in any of the three arms of the study (p $>0.05$ ). One stillbirth occurred in this study, in the group that was treated with oral contraceptive alone. Other outcomes of interest were not evaluated.

A second fair-quality RCT, performed in Russia, examined 144 women who had laparoscopic surgery for removal of ovarian endometriomas and tested the effects of 3 agents for 6 months prior to IVF. The 3 treatments tested were 1) Progestin, dienogest 2mg orally daily; 2) GnRH agonist, triptorelin 3.75 mg intramuscularly monthly; 3) no hormonal therapy prior to IVF. ${ }^{205}$ The live birth rates for participants who received dienogest were significantly higher compared to those on no therapy with live birth rates of $36.8 \%$ versus $11.1 \%$ respectively, $\mathrm{P}=0.013$. The live birth rates for those receiving the GnRH agonist compared to no therapy were not statistically significantly different and were $28.6 \%$ versus $11.1 \%$ respectively, $\mathrm{P}=0.234 .{ }^{205}$

The imprecise evidence from fair-quality studies performed in Russia and China resulted in insufficient strength of evidence across the outcomes assessed.

\section{ART for Endometriosis}

Two observational studies ( 1 fair, ${ }^{123} 1$ good quality ${ }^{186}$ ) looked at the use of ART interventions in women with endometriosis-related infertility. 
A good-quality cohort study of the national Danish ART registry and Medical Birth registry compared live birth rates after treatment with ART versus IUI for women with endometriosis, stratified by age. ${ }^{186}$ Compared to IUI and natural conception, live birth rates were higher among women who received ART. For women $\geq 35$ years of age live birthrate $(95 \% \mathrm{CI})$ for women undergoing ART was 39.0 (24.2, 55.5); undergoing IUI 0 (0.0 to 8.6); and natural conception 4.9 (0.6 to 16.5). ${ }^{186}$ For women $<35$ years of age, live birthrate $(95 \% \mathrm{CI})$ for women undergoing ART was 51.3 (44.1 to 58.4); undergoing IUI 0.5 (0.0 to 2.8); and natural conception 9.6 (5.9 to 14.7) (low SOE).

One fair-quality observational study ${ }^{123}$ examined the effectiveness of ART using data from the Society for Assisted Reproductive Technology Clinic Outcome Reporting System (SART CORS) database, a collective cohort of 305,774 pregnancies in the United States. The goodquality retrospective analysis examined live birth rates, maternal complications, and neonatal complications based on the number of embryos transferred in 69,028 ART cycles. ${ }^{123}$ In this study, 7,104 cycles of IVF or ICSI were performed in couples with the diagnosis of endometriosis. In this endometriosis subgroup, the live birth rate per cycle after treatment with either IVF or ICSI was $48.7 \%$. For women with endometriosis, the live birth rate per cycle was higher in couples who underwent two embryo transfer ( $n=3808$ cycles, live birth rate=51.5\%) as compared with single-embryo transfer $(n=319$ cycles, live birth rate $=46.6 \%)(p<0.0001)$ (low SOE).

\section{Subpopulations of Interest for Endometriosis}

A 2014 good-quality systematic review compared IVF outcomes in 20,167 women (78 publications) with endometriosis (2227 women with stage I-II endometriosis, 1703 women with stage III-IV endometriosis) to 121,931 women without endometriosis. ${ }^{64}$ This publication included randomized and nonrandomized controlled trials. They concluded that live birth rates were similar regardless of whether surgical correction of endometrioma was performed. Women with more severe endometriosis had similar outcomes as compared with women with less severe endometriosis. Specifically, for the comparison of women with Stage-III/IV versus Stage-I/II endometriosis, results were as follows: live birth, RR 0.94 (95\% CI, 0.80 to 1.11); clinical pregnancy, RR 0.90 (95\% CI, 0.82 to 1.00); miscarriage, RR 0.99 (95\% CI, 0.73 to 1.36).

In 2015, Hamdan and colleagues published a good-quality systematic review ${ }^{60}$ investigating the association between endometriosis and reproductive outcomes in women undergoing ART. This meta-analysis included 33 studies (30 retrospective observational, 3 RCTs). Fourteen of these studies overlapped with systematic review by Barbosa and colleagues discussed above. ${ }^{64}$ In women with endometrioma, those who had surgical treatment before IVF/ICSI had a similar live birth rate (OR 0.90; 95\% CI, 0.63 to 1.28), a similar clinical pregnancy rate (OR 0.97; 95\% CI, 0.78 to 1.20 ), and a similar miscarriage rate (OR 1.32; 95\% CI, 0.66 to 2.65) compared to those with untreated endometrioma.

In summary, the severity of endometriosis, surgical treatment of endometriosis prior to ART, and adjunctive hormonal therapy do not appear to influence fertility outcomes. ${ }^{60,64}$

\section{Strength of Evidence for Endometriosis}

Table 17 summarizes the SOE for the findings described above. In general, the SOE across all outcomes was judged to be insufficient or low, primarily due to imprecision and small numbers of studies, especially for both short-term harms (such as OHSS) and long-term harms. 
Table 17. Strength of evidence for major outcomes-KQ 2 (endometriosis)

\begin{tabular}{|c|c|c|c|c|}
\hline Comparison & Outcome & $\begin{array}{l}\text { Study Design } \\
\text { (Sample Size) }\end{array}$ & Conclusion & $\begin{array}{l}\text { Strength of } \\
\text { Evidence } \\
\text { (Rationale) }^{\mathrm{a}}\end{array}$ \\
\hline \multirow[t]{2}{*}{$\begin{array}{l}\text { Letrozole vs. } \\
\text { Clomiphene } \\
\text { with IUI }\end{array}$} & $\begin{array}{l}\text { Live birth } \\
\text { (any/patient) }\end{array}$ & $\begin{array}{l}1 \mathrm{RCT}^{204} \\
(136)\end{array}$ & $\begin{array}{l}\text { Inconclusive: SOE was insufficient } \\
\text { given imprecise evidence from } 1 \\
\text { study with moderate risk of bias }\end{array}$ & $\begin{array}{l}\text { Insufficient } \\
\text { (Imprecise } \\
\text { findings from one } \\
\text { small study with } \\
\text { limitations) }\end{array}$ \\
\hline & $\begin{array}{l}\text { Pregnancy } \\
\text { complications: } \\
\text { Miscarriage }\end{array}$ & $\begin{array}{l}1 \mathrm{RCT}^{204} \\
(136)\end{array}$ & $\begin{array}{l}\text { Inconclusive: SOE was insufficient } \\
\text { given imprecise evidence from } 1 \\
\text { study with moderate risk of bias }\end{array}$ & $\begin{array}{l}\text { Insufficient } \\
\text { (Imprecise } \\
\text { findings from one } \\
\text { small study with } \\
\text { limitations) }\end{array}$ \\
\hline \multirow[t]{4}{*}{$\begin{array}{l}\text { Hormonal } \\
\text { Therapy After } \\
\text { Surgical } \\
\text { Treatment vs. } \\
\text { no treatment }\end{array}$} & Live birth & $\begin{array}{l}2 \mathrm{RCTS}^{202,205} \\
(300)\end{array}$ & $\begin{array}{l}\text { Inconclusive: SOE was insufficient } \\
\text { given imprecise evidence from } 2 \\
\text { studies from non-US settings with } \\
\text { moderate risk of bias }\end{array}$ & $\begin{array}{l}\text { Insufficient } \\
\text { (Imprecise } \\
\text { findings with } \\
\text { moderate study } \\
\text { limitations) }\end{array}$ \\
\hline & $\begin{array}{l}\text { Pregnancy } \\
\text { complications: } \\
\text { Miscarriage }\end{array}$ & $\begin{array}{l}1 \mathrm{RCT}^{202} \\
(156)\end{array}$ & $\begin{array}{l}\text { Inconclusive: SOE was insufficient } \\
\text { given imprecise evidence from } 1 \\
\text { study with moderate risk of bias }\end{array}$ & $\begin{array}{l}\text { Insufficient } \\
\text { (Imprecise } \\
\text { findings with } \\
\text { moderate study } \\
\text { limitations) } \\
\end{array}$ \\
\hline & $\begin{array}{l}\text { Pregnancy } \\
\text { complications: } \\
\text { Ectopic } \\
\text { pregnancy }\end{array}$ & $\begin{array}{l}1 \mathrm{RCT}^{202} \\
(156)\end{array}$ & $\begin{array}{l}\text { Inconclusive: SOE was insufficient } \\
\text { given imprecise evidence from } 1 \\
\text { study with moderate risk of bias }\end{array}$ & $\begin{array}{l}\text { Insufficient } \\
\text { (Imprecise } \\
\text { findings with } \\
\text { moderate study } \\
\text { limitations) }\end{array}$ \\
\hline & $\begin{array}{l}\text { Neonatal } \\
\text { outcomes: } \\
\text { Death }\end{array}$ & $\begin{array}{l}1 \mathrm{RCT}^{202} \\
(156)\end{array}$ & $\begin{array}{l}\text { Inconclusive: SOE was insufficient } \\
\text { given imprecise evidence from } 1 \\
\text { study with moderate risk of bias }\end{array}$ & $\begin{array}{l}\text { Insufficient } \\
\text { (Imprecise } \\
\text { findings with } \\
\text { moderate study } \\
\text { limitations) }\end{array}$ \\
\hline $\begin{array}{l}\text { ART: IVF/ICSI } \\
\text { vs. no } \\
\text { treatment }\end{array}$ & Live birth & $\begin{array}{l}1 \text { Obs }^{123} \\
(69,028 \text { cycles })\end{array}$ & $\begin{array}{l}\text { Improvement: For women with } \\
\text { endometriosis, the live birth rate per } \\
\text { cycle was higher in couples who } \\
\text { underwent } 2 \text { embryo transfer } \\
(51.5 \%) \text { as compared with single- } \\
\text { embryo transfer }(46.6 \%) \\
(p<0.0001) .\end{array}$ & $\begin{array}{l}\text { Low } \\
\text { (Imprecise) }\end{array}$ \\
\hline IUI vs. ART & Live birth & $\begin{array}{l}1 \text { Obs }^{186} \\
(19,884)\end{array}$ & $\begin{array}{l}\text { Improvement: For women with } \\
\text { endometriosis, the live birth rate per } \\
\text { cycle was higher in couples who } \\
\text { underwent ART than those who } \\
\text { used IUI }\end{array}$ & Low (one study) \\
\hline
\end{tabular}

${ }^{a}$ Criteria for downgrading strength of evidence is described as Rationale; when these criteria are insufficient for understanding the final strength of evidence, additional explanation is provided.

Abbreviations: ART=assisted reproductive technology; $\mathrm{CI}=$ confidence interval; HR=hazard ratio; ICSI=intra-cytoplasmic sperm injection; IUI=intrauterine insemination; IVF=in vitro fertilization; KQ=Key Question; $\mathrm{N}=$ number of patients/participants; $\mathrm{NA}=$ not applicable; Obs=observational study; $\mathrm{RCT}=$ randomized controlled trial; $\mathrm{RR}=$ relative risk 


\title{
Key Question 3. Unexplained Infertility
}

KQ 3. What are the comparative safety and effectiveness of available treatment strategies for women who are infertile for unknown reasons and who wish to become pregnant?

\author{
KQ 3a. Does the optimal treatment strategy vary by patient \\ characteristics such as age, ovarian reserve, race, BMI, presence of \\ other potential causes of female infertility, or presence of male factor \\ infertility?
}

Unexplained infertility is defined as infertility with no other documented female or male diagnosis.

\section{Description of Included Studies for KQ 3 (Unexplained Infertility)}

We identified 50 individual studies that met inclusion criteria for KQ 3. 52,119,122,123,135,139,185,186,206-247

Twenty-one studies were conducted in the United Kingdom or continental Europe, ${ }^{119,135,139,186,206,207,209,211-213,217,218,221,224-226,230,237,240,242,244 ~} 10$ in the United States, ${ }^{52,122,123,185,210,215,223,233,236,239 ~} 11$ in the Middle East, ${ }^{208,216,220,222,229,231,232,241,243,245,246}$ three in Asia, ${ }^{214,219,247}$ four in Africa, ${ }^{227,228,234,235}$ and one in Australia/NZ. ${ }^{238}$ Forty-two studies were RCTs; 18 were good quality, ${ }^{52,119,135,139,208-210,226-230,233-235,237,238,246} 22$ were fair quality, ${ }^{206,207,212,214,216-225,231,232,240,242-245,247}$ and 2 were poor quality. ${ }^{211,241}$ Eight studies were observational studies; four were good quality, ${ }^{186,215,236,239}$ and four were fair quality. ${ }^{122,123,185,213}$ Eleven studies were solely government-funded, ${ }^{52,119,122,135,139,210,213,218,233,239,243}$ seven studies received non-government, non-industry funding, ${ }^{123,211,215,235,241,244,247}$ four were solely funded by industry, ${ }^{186,212,220,223}$ and one study reported a combination of funding sources. ${ }^{236}$ The remaining 27 studies did not report a funding source or the funding source was unclear. The type of practice was not specified in six studies, ${ }^{123,212-214,232,239}$ while one study was performed in a general gynecology practice, ${ }^{231}$ two studies were performed in a hospital setting, ${ }^{246,247}$ and the remaining 42 studies were performed at a subspecialty practice.

In addition to the above studies, 6 systematic reviews; $5 \operatorname{good}^{70,84,87,92,95}, 1$ fair quality ${ }^{94}$ addressed the comparative effectiveness of various treatments in women with unexplained infertility are discussed below and the consistency of their findings with our included studies are incorporated in to our strength of evidence ratings.

\section{Key Points for Unexplained Infertility}

Key findings for couples with unexplained infertility included:

- There is no difference between the oral agents of letrozole and anastrozole for the outcome of ectopic pregnancy (low SOE) but evidence is insufficient for other outcomes of interest.

- There is no difference between letrozole and clomiphene for outcomes of multiple births or miscarriage (moderate SOE).

- There is no difference between differing adjunct treatments used in combination with oral agents and IUI for the outcomes of live birth, miscarriage, and OHSS (low SOE for all outcomes). 
- There are no differences between immediate IVF versus other treatments prior to IVF for the outcomes of live birth, multiple births, ectopic pregnancy, miscarriage, low birthweight, and OHSS (low SOE for all outcomes). There is however shorter time to pregnancy with immediate IVF (moderate SOE).

- As with other indications for IVF, use of single-embryo transfer is associated with slightly lower live birth rates but significantly reduced multiple gestation rates (low SOE)

\section{Detailed Synthesis by Treatment for Unexplained Infertility}

Included studies and their findings for the following treatments for unexplained infertility are detailed in this section:

1. Oral Agents Without IUI

2. Oral Agents Versus Unstimulated IUI Versus Expectant Management

3. Different Adjunct Treatments Combined With Oral Agents and IUI

4. Oral Agents With IUI Versus Gonadotropins With IUI

5. Different Treatment Strategies for Controlled Ovarian Hyperstimulation with Gonadotropins and IUI

6. Immediate IVF Versus Other Treatments Prior to IVF

7. Expectant Management Versus Other Interventions

8. ART
a. IVF
b. ICSI
c. IVF Versus ICSI
d. Unspecified ART

\section{Oral Agents Without IUI for Unexplained Infertility}

Three RCTs, provided evidence on outcomes among women with unexplained infertility treated with oral agents alone. One fair-quality study ${ }^{206}$ compared clomiphene citrate alone to clomiphene citrate plus hydrotubation, another fair-quality study ${ }^{22}$ compared clomiphene to letrozole and anastrozole, a third good-quality trial compared use of letrozole compare with clomiphene with estradiol in women with unexplained fertility who did not respond initially to clomiphene alone. ${ }^{246}$ Results are summarized in Table 18 . The studies did not identify significant differences in outcomes between clomiphene alone and comparators, but sample sizes were small, with wide confidence intervals for rates of all outcomes. Outcomes of multiple births, costs, short term adverse effects, and long term outcomes were not evaluated in the included studies.

A good-quality systematic review and meta-analysis compared outcomes between clomiphene citrate and letrozole. ${ }^{70}$ Six studies were included representing 1776 patients, of which one ${ }^{222}$ was included in the present systematic review. The meta-analysis did not assess live birth rates. Miscarriage however was examined in 5 studies (395 patients), including the study by Badawy and colleagues. ${ }^{222}$ Within these studies, the miscarriage rate was 24/195 $(12.3 \%)$ in the letrozole group versus 36/200 (18.0\%) in the clomiphene citrate group (RR 0.65; 95\% CI, 0.33 to 1.29 , $\mathrm{p}=0.22$ ) (moderate SOE). Multiple pregnancy rates were also evaluated in these same 5 trials and again demonstrated no difference between letrozole and clomiphene citrate (RR 0.57, 95\% CI 0.25 to 1.27 ) (moderate SOE). ${ }^{70}$ 
Table 18. Outcomes for comparisons of oral agents without IUI in women with unexplained infertility

\begin{tabular}{|c|c|c|c|c|c|c|c|}
\hline Outcome & $\begin{array}{l}\text { Study } \\
\text { Design } \\
\text { (N } \\
\text { Patients) }\end{array}$ & Intervention & Comparator & $\begin{array}{c}\text { Results } \\
\text { Intervention } \\
\mathbf{N} \\
(\%) \\
(95 \% \mathrm{Cl})\end{array}$ & $\begin{array}{c}\text { Results } \\
\text { Comparator } \\
N \\
(\%) \\
(95 \% \mathrm{Cl})\end{array}$ & $\begin{array}{c}P \\
\text { Value }\end{array}$ & $\begin{array}{c}\text { Summary of } \\
\text { Study } \\
\text { Findings }\end{array}$ \\
\hline $\begin{array}{l}\text { Live birth: } \\
\text { Any/patient }\end{array}$ & $\begin{array}{l}\text { Yapca, } \\
2015^{206} \\
\text { RCT } \\
(80)\end{array}$ & CC & $\begin{array}{l}\mathrm{CC}+ \\
\text { hydrotubation }\end{array}$ & $\begin{array}{c}2 / 40 \\
\text { (5) } \\
\text { (0.6 to 13.5) }\end{array}$ & $\begin{array}{c}8 / 40 \\
(20) \\
(9.3 \text { to } 33.5)\end{array}$ & 0.043 & $\begin{array}{l}\text { Greater live } \\
\text { births with } \\
\text { clomiphene } \\
\text { and } \\
\text { hydrotubatio } \\
\text { n compared } \\
\text { to } \\
\text { clomiphene } \\
\text { alone } \\
\text { alo }\end{array}$ \\
\hline \multirow{3}{*}{$\begin{array}{l}\text { Pregnancy } \\
\text { complications: } \\
\text { Ectopic } \\
\text { pregnancy }\end{array}$} & \multirow[t]{2}{*}{$\begin{array}{l}\text { Badawy, } \\
2009^{222}\end{array}$} & \multirow[t]{2}{*}{$\mathrm{CC}$} & Letrozole & \multirow[t]{2}{*}{$\begin{array}{c}1 / 420 \\
(0.2) \\
(0.06 \text { to } 0.9)\end{array}$} & $\begin{array}{c}0 / 269 \\
(0) \\
(0 \text { to } 0.9)\end{array}$ & 0.43 & $\begin{array}{l}\text { No } \\
\text { difference }\end{array}$ \\
\hline & & & Anastrozole & & $\begin{array}{c}0 / 107 \\
(0) \\
(0 \text { to } 2.3)\end{array}$ & 0.62 & $\begin{array}{l}\text { No } \\
\text { difference }\end{array}$ \\
\hline & $\begin{array}{l}\text { Harira } \\
2018^{246} \\
\\
\text { RCT } \\
(172)\end{array}$ & $\begin{array}{l}\text { CC+ } \\
\text { estradiol }\end{array}$ & Letrozole & $\begin{array}{l}0 / 86 \\
(0)\end{array}$ & $\begin{array}{c}0 / 86 \\
(0)\end{array}$ & NS & $\begin{array}{l}\text { No } \\
\text { difference }\end{array}$ \\
\hline \multirow[t]{4}{*}{$\begin{array}{l}\text { Pregnancy } \\
\text { complications: } \\
\text { Miscarriage }\end{array}$} & $\begin{array}{l}\text { Yapca, } \\
2015^{206} \\
\text { RCT } \\
(80)\end{array}$ & $\mathrm{CC}$ & $\begin{array}{l}\mathrm{CC}+ \\
\text { hydrotubation }\end{array}$ & $\begin{array}{c}1 / 40 \\
(2.5) \\
(0.06 \text { to } 9.0)\end{array}$ & $\begin{array}{c}0 / 40 \\
(0) \\
\text { (0 to } 6.1 \text { ) }\end{array}$ & 0.31 & $\begin{array}{l}\text { No } \\
\text { difference }\end{array}$ \\
\hline & \multirow{2}{*}{$\begin{array}{l}\text { Badawy, } \\
2009^{222} \\
\text { RCT } \\
(996)\end{array}$} & \multirow[t]{2}{*}{$\mathrm{CC}$} & Letrozole & \multirow[t]{2}{*}{$\begin{array}{c}8 / 77 \\
(10.4) \\
\text { (4.7 to } 18.1)\end{array}$} & $\begin{array}{c}4 / 36 \\
(11.1) \\
\text { (3.2 to } 23.1)\end{array}$ & 0.91 & $\begin{array}{l}\text { No } \\
\text { difference }\end{array}$ \\
\hline & & & Anastrozole & & $\begin{array}{c}2 / 15 \\
(13.3) \\
(1.8 \text { to } 33.9)\end{array}$ & 0.74 & $\begin{array}{l}\text { No } \\
\text { difference }\end{array}$ \\
\hline & $\begin{array}{l}\text { Harira } \\
2018^{246} \\
\\
\text { RCT } \\
(172)\end{array}$ & $\begin{array}{l}\text { CC } \\
\text { +estradiol }\end{array}$ & Letrozole & $4 / 86(4.6)$ & $3 / 86(3.4)$ & 0.67 & $\begin{array}{l}\text { No } \\
\text { difference }\end{array}$ \\
\hline $\begin{array}{l}\text { Short-term } \\
\text { adverse effects: } \\
\text { OHSS }\end{array}$ & $\begin{array}{l}\text { Harira } \\
2018^{246} \\
\text { RCT } \\
(172)\end{array}$ & $\begin{array}{l}\text { CC } \\
\text { +estradiol }\end{array}$ & Letrozole & $0 / 86$ & $0 / 86$ & NS & $\begin{array}{l}\text { No } \\
\text { difference }\end{array}$ \\
\hline \multirow{2}{*}{$\begin{array}{l}\text { Neonatal } \\
\text { outcomes: } \\
\text { Birthweight } \\
\text { (small for } \\
\text { gestational age) }\end{array}$} & \multirow{2}{*}{$\begin{array}{l}\text { Badawy, } \\
2009^{222} \\
\text { RCT } \\
(996)\end{array}$} & \multirow[t]{2}{*}{$\mathrm{CC}$} & Letrozole & \multirow[t]{2}{*}{$\begin{array}{c}3 / 65 \\
(4.6) \\
(1.0 \text { to } 10.8)\end{array}$} & $\begin{array}{c}2 / 30 \\
(6.7) \\
\text { (0.8 to } 17.8) \\
\end{array}$ & 0.68 & $\begin{array}{l}\text { No } \\
\text { difference }\end{array}$ \\
\hline & & & Anastrozole & & $\begin{array}{c}1 / 11 \\
(9.1) \\
(950.3 \text { to } \\
30.8) \\
\end{array}$ & 0.54 & $\begin{array}{l}\text { No } \\
\text { difference }\end{array}$ \\
\hline Neonatal death & $\begin{array}{l}\text { Badawy, } \\
2009^{222}\end{array}$ & $\mathrm{CC}$ & Letrozole & $\begin{array}{c}0 / 65 \\
(0) \\
\text { (0 to } 3.8)\end{array}$ & $\begin{array}{c}1 / 30 \\
(3.3) \\
(0.09 \text { to } \\
11.9)\end{array}$ & 0.14 & $\begin{array}{l}\text { No } \\
\text { difference }\end{array}$ \\
\hline
\end{tabular}




\begin{tabular}{|l|l|l|l|c|c|c|c|}
\hline Outcome & $\begin{array}{c}\text { Study } \\
\text { Design } \\
\mathbf{( N} \\
\text { Patients) }\end{array}$ & Intervention & Comparator & $\begin{array}{c}\text { Results } \\
\text { Intervention } \\
\mathbf{N} \\
(\%) \\
(\mathbf{9 5 \%} \mathbf{C l})\end{array}$ & $\begin{array}{c}\text { Results } \\
\text { Comparator } \\
\mathbf{N} \\
(\mathbf{\%}) \\
(\mathbf{9 5 \%} \mathbf{C l})\end{array}$ & $\begin{array}{c}\mathbf{P} \\
\text { Value }\end{array}$ & $\begin{array}{c}\text { Summary of } \\
\text { Study } \\
\text { Findings }\end{array}$ \\
\hline & $\begin{array}{l}\mathrm{RCT} \\
(996)\end{array}$ & & Anastrozole & & $\begin{array}{c}0 / 11 \\
(0) \\
(0 \text { to 20.8) }\end{array}$ & NS & $\begin{array}{l}\text { No } \\
\text { difference }\end{array}$ \\
\hline
\end{tabular}

Abbreviations: $\mathrm{CC}=$ clomiphene citrate; $\mathrm{CI}=$ confidence interval; $\mathrm{N}=$ number of patients; $\mathrm{NR}=$ not reported; NS= not statistically significant; OHSS=ovarian hyperstimulation syndrome; RCT=randomized control trial

\section{Oral Agents Versus Unstimulated IUI Versus Expectant Management for Unexplained Infertility}

One good-quality RCT ${ }^{135}$ compared outcomes between oral agents, unstimulated IUI, and expectant management. A second good-quality RCT compared IUI with either clomiphene citrate or letrozole to expectant management. ${ }^{238}$ Results of both studies are summarized in Table 19 for live birth, pregnancy complications, and time to pregnancy. Other outcomes of interest were not reported.

Table 19. Outcomes for comparisons of oral agents versus unstimulated IUI versus expectant management in women with unexplained infertility

\begin{tabular}{|c|c|c|c|c|c|c|c|}
\hline Outcome & $\begin{array}{l}\text { Study } \\
\text { Design } \\
\text { (N } \\
\text { Patients) }\end{array}$ & Intervention & Comparator & $\begin{array}{c}\text { Results } \\
\text { Intervention } \\
N \\
(\%) \\
(95 \% \mathrm{Cl}) \\
\end{array}$ & $\begin{array}{c}\text { Results } \\
\text { Comparator } \\
N \\
(\%) \\
(95 \% \mathrm{Cl}) \\
\end{array}$ & $\begin{array}{c}\mathbf{P} \\
\text { Value }\end{array}$ & $\begin{array}{l}\text { Summary } \\
\text { of Study } \\
\text { Findings }\end{array}$ \\
\hline \multirow[t]{3}{*}{$\begin{array}{l}\text { Live birth: } \\
\text { Any/patient }\end{array}$} & \multirow[t]{2}{*}{$\begin{array}{l}\text { Bhattachar } \\
\text { ya, 2008 } \\
\text { RCT } \\
\text { (576) }\end{array}$} & \multirow[t]{2}{*}{ CC } & IUI & \multirow[t]{2}{*}{$\begin{array}{c}26 / 192 \\
(13.5) \\
\text { (9.1 to } 18.7)\end{array}$} & $\begin{array}{c}43 / 191 \\
(22.5) \\
(16.9 \text { to } \\
28.7)\end{array}$ & 0.022 & $\begin{array}{l}\text { Greater live } \\
\text { births with } \\
\text { IUI } \\
\text { compared } \\
\text { to } \\
\text { clomiphene }\end{array}$ \\
\hline & & & $\begin{array}{l}\text { Expectant } \\
\text { management }\end{array}$ & & $\begin{array}{c}32 / 193 \\
(16.5) \\
(11.7 \text { to } \\
22.1) \\
\end{array}$ & 0.40 & $\begin{array}{l}\text { No } \\
\text { difference }\end{array}$ \\
\hline & $\begin{array}{l}\text { Farquhar, } \\
2018^{238} \\
\text { RCT } \\
(201)\end{array}$ & $\begin{array}{l}\text { IUI } \\
\text { CC or } \\
\text { letrozole }\end{array}$ & $\begin{array}{l}\text { Expectant } \\
\text { management }\end{array}$ & $\begin{array}{c}31 / 101 \\
(31)\end{array}$ & $\begin{array}{c}9 / 100 \\
(9)\end{array}$ & $\begin{array}{c}0.000 \\
1\end{array}$ & $\begin{array}{l}\text { Greater live } \\
\text { births with } \\
\text { IUI and oral } \\
\text { agents than } \\
\text { with } \\
\text { expectant } \\
\text { manageme } \\
\text { nt }\end{array}$ \\
\hline \multirow{3}{*}{$\begin{array}{l}\text { Pregnancy } \\
\text { complications: } \\
\text { Ectopic } \\
\text { pregnancy }\end{array}$} & \multirow[t]{2}{*}{$\begin{array}{l}\text { Bhattachar } \\
\text { ya, 2008 }\end{array}$} & \multirow[t]{2}{*}{$\mathrm{CC}$} & IUI & \multirow[t]{2}{*}{$\begin{array}{c}0 / 192 \\
(0) \\
(0 \text { to } 1.3)\end{array}$} & $\begin{array}{c}2 / 191 \\
(1.0) \\
(0.1 \text { to } 2.9)\end{array}$ & 0.24 & $\begin{array}{l}\text { No } \\
\text { difference }\end{array}$ \\
\hline & & & $\begin{array}{l}\text { Expectant } \\
\text { management }\end{array}$ & & $\begin{array}{c}1 / 193 \\
(0.5) \\
(0.01 \text { to } 1.9)\end{array}$ & 0.68 & $\begin{array}{l}\text { No } \\
\text { difference }\end{array}$ \\
\hline & $\begin{array}{l}\text { Farquhar, } \\
2018^{238} \\
\text { RCT } \\
(201)\end{array}$ & $\begin{array}{l}\text { IUI } \\
\text { CC or } \\
\text { letrozole }\end{array}$ & $\begin{array}{l}\text { Expectant } \\
\text { management }\end{array}$ & $\begin{array}{l}4 / 101 \\
(3.9)\end{array}$ & $\begin{array}{c}0 / 100 \\
(0)\end{array}$ & $\begin{array}{c}0.097 \\
4\end{array}$ & $\begin{array}{l}\text { No } \\
\text { difference }\end{array}$ \\
\hline
\end{tabular}




\begin{tabular}{|c|c|c|c|c|c|c|c|}
\hline Outcome & $\begin{array}{c}\text { Study } \\
\text { Design } \\
\text { (N } \\
\text { Patients) }\end{array}$ & Intervention & Comparator & $\begin{array}{c}\text { Results } \\
\text { Intervention } \\
N \\
(\%) \\
(95 \% \mathrm{Cl})\end{array}$ & $\begin{array}{c}\text { Results } \\
\text { Comparator } \\
N \\
(\%) \\
(95 \% \mathrm{Cl})\end{array}$ & $\begin{array}{c}P \\
\text { Value }\end{array}$ & $\begin{array}{l}\text { Summary } \\
\text { of Study } \\
\text { Findings }\end{array}$ \\
\hline \multirow[t]{3}{*}{$\begin{array}{l}\text { Pregnancy } \\
\text { complications: } \\
\text { Miscarriage }\end{array}$} & $\begin{array}{l}\text { Bhattachar } \\
\text { ya, } 2008^{135}\end{array}$ & $\mathrm{CC}$ & IUI & \multirow[t]{2}{*}{$\begin{array}{c}10 / 38 \\
(26.3) \\
(13.8 \text { to } \\
41.2)\end{array}$} & $\begin{array}{c}9 / 55 \\
(16.3) \\
\text { (7.9 to } 27.1)\end{array}$ & 0.24 & $\begin{array}{l}\text { No } \\
\text { difference }\end{array}$ \\
\hline & (576) & & $\begin{array}{l}\text { Expectant } \\
\text { management }\end{array}$ & & $\begin{array}{c}14 / 46 \\
(30.4) \\
(18.2 \text { to } \\
44.3) \\
\end{array}$ & 0.68 & $\begin{array}{l}\text { No } \\
\text { difference }\end{array}$ \\
\hline & $\begin{array}{l}\text { Farquhar, } \\
2018^{238} \\
\text { RCT } \\
(201)\end{array}$ & $\begin{array}{l}\text { IUI } \\
\text { CC or } \\
\text { letrozole }\end{array}$ & $\begin{array}{l}\text { Expectant } \\
\text { management }\end{array}$ & $\begin{array}{c}6 / 37 \\
(16.2)\end{array}$ & $\begin{array}{l}1 / 11 \\
(9.1)\end{array}$ & 0.153 & $\begin{array}{l}\text { No } \\
\text { difference }\end{array}$ \\
\hline $\begin{array}{l}\text { Time to } \\
\text { pregnancy }\end{array}$ & $\begin{array}{l}\text { Bhattachar } \\
\text { ya, 2008 } \\
\text { RCT } \\
\text { (576) }\end{array}$ & $\begin{array}{l}\mathrm{CC} \\
\mathrm{IUI}\end{array}$ & $\begin{array}{l}\text { Expectant } \\
\text { management }\end{array}$ & $\begin{array}{c}\mathrm{HR} \text { of CC } \\
\text { compared to } \\
\text { expectant } \\
\text { managemen } \\
\text { t } 0.83 \\
(0.42 \text { to } \\
1.63)\end{array}$ & $\begin{array}{c}\text { HR of IUI } \\
\text { compared to } \\
\text { expectant } \\
\text { managemen } \\
\text { t } 1.40 \\
(0.77 \text { to } \\
2.56)\end{array}$ & NS & $\begin{array}{l}\text { No } \\
\text { difference }\end{array}$ \\
\hline
\end{tabular}

Abbreviations: $\mathrm{CC}=$ clomiphene citrate; $\mathrm{CI}=$ confidence interval; $\mathrm{HR}=$ hazard ratio; $\mathrm{IUI}=$ intrauterine insemination; $\mathrm{N}=$ number of patients; NS=not statistically significant; RCT=randomized control trial

This comparison was also evaluated in two good-quality systematic reviews. ${ }^{87,92}$ Both of these systematic reviews included our included study by Bhattacharya and colleagues. ${ }^{135}$

One systematic review examined outcomes with clomiphene citrate for unexplained infertility as compared to expectant management. ${ }^{92}$ Seven RCTs were included (1,159 patients), of which only the one study by Bhattacharya ${ }^{135}$ reported on outcomes of interest. In this study, there was no benefit of clomiphene citrate over placebo for live birth (OR 0.79; 95\% CI, 0.44 to 1.38 , $\mathrm{p}=0.41$ ) or miscarriage (OR $=0.71,95 \% \mathrm{CI} 0.31$ to 1.61 ). The SOE was rated as insufficient given imprecise findings from one study.

Another good-quality systematic review of 14 studies examined outcomes following ovarian stimulation, IUI, or both in 2,033 patients with unexplained infertility. ${ }^{87}$ Comparisons were made between IUI and timed intercourse (TI) with and without ovarian hyperstimulation. Clomiphene citrate, gonadotropins, or a combination of clomiphene citrate and gonadotropins were utilized for ovarian hyperstimulation. Fourteen RCTs were included, of which one ${ }^{135}$ was included in our systematic review. Overall for the 14 included studies, they noted that the risk of bias was "substantial" due to failure to report allocation concealment and details of randomization.

When comparing IUI versus expectant management, only one good-quality, low risk of bias study was included, also included in the present systematic review. ${ }^{135}$ Live birth rates were not significantly differently between groups (23\% with IUI, 16\% with expectant management; OR 1.60; 95\% CI, 0.92 to 2.78). Miscarriage rates were similar between groups (OR 0.77; 95\% CI, 0.28 to 2.11). There were 2 ectopic pregnancies in the IUI group (OR 5.06; 95\% CI, 0.24 to 106.21) (insufficient SOE).

For the comparison of IUI versus TI with ovarian hyperstimulation, only 2 studies examined live birth rates. Live birth rates were similar between groups (OR 1.59; 95\% CI, 0.88 to 2.88).

For the comparison of IUI in a natural cycle versus IUI in a stimulated cycle, 3 studies examined live birth rates, all excluded from the present review due to publication prior to 2007. 
Live birth rates were significantly higher with ovarian hyperstimulation/IUI compared to IUI alone (OR 2.07; 95\% CI, 1.22 to 3.50) (low SOE).

Finally, only one study was included for in the meta analysis which compared IUI in a natural cycle versus TI in a stimulated cycle (also included in the present study). ${ }^{135}$ Odds of live birth were higher with IUI than with ovarian hyperstimulation/TI (OR 1.95; 95\% CI, 1.10 to 3.44) (insufficient SOE).

Overall this systematic review suggested that there is evidence that IUI with ovarian hyperstimulation increases the live birth rate compared to IUI alone. Other comparisons had insufficient SOE.

\section{Different Adjunct Treatments Combined With Oral Agents or Gonadotropins and IUI for Unexplained infertility}

Two good-quality RCTs ${ }^{227,229}$ and six fair-quality RCTs ${ }^{216,221,231,232,242,245}$ compared outcomes between different adjunct treatment strategies for use of oral agents (usually CC) and/or gonadotropins with IUI. Interventions included luteal progesterone using a vaginal pessary, ${ }^{229}$ hydrotubation with saline compared to lidocaine, ${ }^{227}$ double versus single insemination, ${ }^{221}$, piroxicam $^{231}$, vaginal progesterone versus oral dygesterone ${ }^{232}$, supine immobilization, ${ }^{242}$ cervical mucus removal, ${ }^{245}$ and adjunct stimulation with either rFSH or HMG. ${ }^{216}$ Results are summarized in Table 20. Other than an increase in live birth for women in the cervical mucus removal group (RR 1.60, 95\% CI 1.12 to 2.28), none of the studies identified significant differences in outcomes between interventions for the outcomes of live birth, miscarriage, or OHSS (low SOE for all outcomes). No other outcomes of interest were evaluated.

Table 20. Outcomes for comparisons of oral agents with IUI in women with unexplained infertility

\begin{tabular}{|c|c|c|c|c|c|c|c|}
\hline Outcome & $\begin{array}{l}\text { Study } \\
\text { Design } \\
\text { (N } \\
\text { Patients) }\end{array}$ & Intervention & Comparator & $\begin{array}{c}\text { Results } \\
\text { Intervention } \\
\mathrm{N} \\
(\%) \\
(95 \% \mathrm{Cl}) \\
\end{array}$ & $\begin{array}{c}\text { Results } \\
\text { Comparator } \\
\mathrm{N} \\
(\%) \\
(95 \% \mathrm{Cl})\end{array}$ & $\begin{array}{c}P \\
\text { Value }\end{array}$ & $\begin{array}{c}\text { Summary } \\
\text { of Study } \\
\text { Findings }\end{array}$ \\
\hline \multirow[t]{4}{*}{$\begin{array}{l}\text { Live birth: } \\
\text { Any/patient }\end{array}$} & $\begin{array}{l}\text { Ebrahimi, } \\
2010^{229} \\
\text { RCT } \\
(200)\end{array}$ & $\begin{array}{l}\mathrm{CC} / \mathrm{hMG}+ \\
\text { Progesteron } \\
\mathrm{e}\end{array}$ & $\begin{array}{l}\text { CC/hmG+No } \\
\text { support }\end{array}$ & $\begin{array}{c}19 / 98 \\
(19.4) \\
(12.2 \text { to } 27.7)\end{array}$ & $\begin{array}{c}15 / 102 \\
(14.7) \\
(85.6 \text { to } \\
22.2)\end{array}$ & 0.38 & $\begin{array}{l}\text { No } \\
\text { difference }\end{array}$ \\
\hline & $\begin{array}{l}\text { Bagis, } \\
2010^{221} \\
\text { RCT } \\
(228)\end{array}$ & $\begin{array}{l}\text { CC+Double } \\
\text { insemination }\end{array}$ & $\begin{array}{l}\mathrm{CC}+\text { Single } \\
\text { insemination }\end{array}$ & $\begin{array}{c}1 / 19 \\
(5.3) \\
(0.1 \text { to } 18.5)\end{array}$ & $\begin{array}{c}2 / 17 \\
(11.7) \\
(1.5 \text { to } 30.2)\end{array}$ & 0.48 & $\begin{array}{l}\text { No } \\
\text { difference }\end{array}$ \\
\hline & $\begin{array}{l}\text { Rashidi, } \\
2013^{216} \\
\text { RCT } \\
(259)\end{array}$ & $\mathrm{CC}+\mathrm{rFSH}$ & $\mathrm{CC}+\mathrm{hMG}$ & $\begin{array}{c}19 / 132 \\
(14.5) \\
(9.0 \text { to } 21.0)\end{array}$ & $\begin{array}{c}16 / 127 \\
(12.6) \\
(7.4 \text { to } 18.9)\end{array}$ & 0.12 & $\begin{array}{l}\text { No } \\
\text { difference }\end{array}$ \\
\hline & $\begin{array}{l}\text { van } \\
\text { Rijswijk, } \\
2017^{242} \\
\text { RCT } \\
(481)\end{array}$ & $\begin{array}{l}\text { Supine } \\
\text { immobilizatio } \\
n+I U I\end{array}$ & $\begin{array}{l}\text { Immediate } \\
\text { mobilization + } \\
\text { IUI }\end{array}$ & $\begin{array}{c}73 / 226 \\
(32)\end{array}$ & $\begin{array}{c}92 / 245 \\
(37)\end{array}$ & 0.13 & $\begin{array}{l}\text { No } \\
\text { difference }\end{array}$ \\
\hline
\end{tabular}




\begin{tabular}{|c|c|c|c|c|c|c|c|}
\hline Outcome & $\begin{array}{c}\text { Study } \\
\text { Design } \\
\text { (N } \\
\text { Patients) }\end{array}$ & Intervention & Comparator & $\begin{array}{c}\text { Results } \\
\text { Intervention } \\
N \\
(\%) \\
(95 \% \mathrm{Cl})\end{array}$ & $\begin{array}{c}\text { Results } \\
\text { Comparator } \\
\mathrm{N} \\
(\%) \\
(95 \% \mathrm{Cl})\end{array}$ & $\begin{array}{c}\mathbf{P} \\
\text { Value }\end{array}$ & $\begin{array}{l}\text { Summary } \\
\text { of Study } \\
\text { Findings }\end{array}$ \\
\hline & $\begin{array}{l}\text { Maher, } \\
2018^{245} \\
\text { RCT } \\
(714)\end{array}$ & $\begin{array}{l}\text { Cervical } \\
\text { mucus } \\
\text { removal }\end{array}$ & $\begin{array}{l}\text { No mucus } \\
\text { removal }\end{array}$ & $\begin{array}{l}97 / 361 \\
(26.9)\end{array}$ & $\begin{array}{l}66 / 353 \\
(18.7)\end{array}$ & 0.009 & $\begin{array}{l}\text { Greater } \\
\text { live birth } \\
\text { rate in the } \\
\text { cervical } \\
\text { mucus } \\
\text { removal } \\
\text { group }\end{array}$ \\
\hline $\begin{array}{l}\text { Pregnancy } \\
\text { complications: } \\
\text { Ectopic } \\
\text { pregnancy }\end{array}$ & $\begin{array}{l}\text { Morad, } \\
2012^{227} \\
\text { RCT } \\
(216)\end{array}$ & $\begin{array}{l}\text { Lidocaine } \\
\text { hydrotubatio } \\
\mathrm{n} \text { prior to CC } \\
+\mathrm{IUI}\end{array}$ & $\begin{array}{l}\text { Saline } \\
\text { hydrotubation } \\
\text { prior to CC + } \\
\text { IUI }\end{array}$ & $\begin{array}{c}1 / 109 \\
(0.9) \\
(0.02 \text { to } 3.4)\end{array}$ & $\begin{array}{c}1 / 107 \\
(0.9) \\
(0.02 \text { to } 3.4)\end{array}$ & 0.99 & $\begin{array}{l}\text { No } \\
\text { difference }\end{array}$ \\
\hline \multirow[t]{5}{*}{$\begin{array}{l}\text { Pregnancy } \\
\text { complications: } \\
\text { Miscarriage }\end{array}$} & $\begin{array}{l}\text { Bagis, } \\
2010^{221} \\
\\
\text { RCT } \\
(228)\end{array}$ & $\begin{array}{l}\text { CC+Double } \\
\text { insemination }\end{array}$ & $\begin{array}{l}\text { CC+Single } \\
\text { insemination }\end{array}$ & $\begin{array}{c}6 / 20 \\
(30) \\
(12.6 \text { to } 51)\end{array}$ & $\begin{array}{c}4 / 16 \\
(25) \\
\text { (7.8 to } 48.1)\end{array}$ & 0.74 & $\begin{array}{l}\text { No } \\
\text { difference }\end{array}$ \\
\hline & $\begin{array}{l}\text { Morad, } \\
2012^{227} \\
\\
\text { RCT } \\
(216)\end{array}$ & $\begin{array}{l}\text { Lidocaine } \\
\text { hydrotubatio } \\
\text { n prior to CC } \\
+ \text { IUI }\end{array}$ & $\begin{array}{l}\text { Saline } \\
\text { hydrotubation } \\
\text { prior to CC + } \\
\text { IUI }\end{array}$ & $\begin{array}{c}1 / 109 \\
(0.9) \\
(0.02 \text { to } 3.4)\end{array}$ & $\begin{array}{c}1 / 107 \\
(0.9) \\
(0.02 \text { to } 3.4)\end{array}$ & 0.99 & $\begin{array}{l}\text { No } \\
\text { difference }\end{array}$ \\
\hline & $\begin{array}{l}\text { Zarei, } \\
2016^{231} \\
\text { RCT } \\
(260)\end{array}$ & $\begin{array}{l}\text { Piroxicam+ } \\
\mathrm{CC}+\mathrm{rFSH}+ \\
\mathrm{hCG}+\mathrm{IUI}\end{array}$ & $\begin{array}{l}\text { Placebo+ CC + } \\
\text { rFSH + hCG + } \\
\text { IUI }\end{array}$ & $\begin{array}{c}5 / 130 \\
(4)\end{array}$ & $\begin{array}{c}5 / 130 \\
(4)\end{array}$ & 0.82 & $\begin{array}{l}\text { No } \\
\text { difference }\end{array}$ \\
\hline & $\begin{array}{l}\text { Khosravi, } \\
2015^{232} \\
\text { RCT } \\
(150)\end{array}$ & $\begin{array}{l}\text { Oral } \\
\text { dygesterone } \\
+\mid \mathrm{IUI}\end{array}$ & $\begin{array}{l}\text { Vaginal } \\
\text { progesterone + } \\
\text { IUI }\end{array}$ & $\begin{array}{l}2 / 75 \\
(9.1)\end{array}$ & $\begin{array}{c}3 / 75 \\
(15.8)\end{array}$ & 0.056 & $\begin{array}{l}\text { No } \\
\text { difference }\end{array}$ \\
\hline & $\begin{array}{l}\text { Maher, } \\
2018^{245} \\
\text { RCT } \\
(714)\end{array}$ & $\begin{array}{l}\text { Cervical } \\
\text { mucus } \\
\text { removal }\end{array}$ & $\begin{array}{l}\text { No mucus } \\
\text { removal }\end{array}$ & $\begin{array}{l}14 / 361 \\
(12.5)\end{array}$ & $\begin{array}{l}11 / 353 \\
(14.3)\end{array}$ & 0.72 & $\begin{array}{l}\text { No } \\
\text { difference }\end{array}$ \\
\hline \multirow[t]{3}{*}{$\begin{array}{l}\text { Short-term } \\
\text { adverse } \\
\text { effects: OHSS }\end{array}$} & $\begin{array}{l}\text { Morad, } \\
2012^{227} \\
\text { RCT } \\
(216)\end{array}$ & $\begin{array}{l}\text { Lidocaine } \\
\text { hydrotubatio } \\
\text { n prior to CC } \\
+ \text { IUI }\end{array}$ & $\begin{array}{l}\text { Saline } \\
\text { hydrotubation } \\
\text { prior to CC + } \\
\text { IUI }\end{array}$ & $\begin{array}{c}1 / 109 \\
(0.9) \\
(0.02 \text { to } 3.4)\end{array}$ & $\begin{array}{c}1 / 107 \\
(0.9) \\
(0.02 \text { to } 3.4)\end{array}$ & 0.99 & $\begin{array}{l}\text { No } \\
\text { difference }\end{array}$ \\
\hline & $\begin{array}{l}\text { Rashidi, } \\
2013^{216} \\
\text { RCT } \\
\text { (259) }\end{array}$ & $\mathrm{CC}+\mathrm{rFSH}$ & $\mathrm{CC}+\mathrm{hMG}$ & $\begin{array}{c}6 / 132 \\
(4.5) \\
(1.7 \text { to } 8.7)\end{array}$ & $\begin{array}{c}5 / 127 \\
(3.9) \\
(1.3 \text { to } 7.9)\end{array}$ & 0.81 & $\begin{array}{l}\text { No } \\
\text { difference }\end{array}$ \\
\hline & $\begin{array}{l}\text { Maher, } \\
2018^{245} \\
\\
\text { RCT } \\
(714)\end{array}$ & $\begin{array}{l}\text { Cervical } \\
\text { mucus } \\
\text { removal }\end{array}$ & $\begin{array}{l}\text { No mucus } \\
\text { removal }\end{array}$ & $\begin{array}{c}18 / 361 \\
(5)\end{array}$ & $\begin{array}{c}15 / 353 \\
(4.2)\end{array}$ & 0.61 & $\begin{array}{l}\text { No } \\
\text { difference }\end{array}$ \\
\hline
\end{tabular}

Abbreviations: $\mathrm{CC}=$ clomiphene citrate; $\mathrm{CI}=$ confidence interval; $\mathrm{hMG}=$ human menopausal gonadotropin; IUI=intrauterine insemination; $\mathrm{N}=$ number of patients; $\mathrm{NR}=$ not reported; $\mathrm{NS}=$ not statistically significant; $\mathrm{OHSS}=$ ovarian hyperstimulation syndrome; rFSH=recombinant follicle-stimulating hormone; RCT=randomized control trial 


\section{Oral Agents With IUI Versus Gonadotropins With IUI for Unexplained Infertility}

Six RCTs (4 good, ${ }^{208,226,233,234}$ one fair, ${ }^{244}$ and one poor quality ${ }^{241}$ ) compared outcomes between oral agents/IUI and gonadotropins/IUI. Table 21 summarizes the findings from these studies. Evidence supported no difference in miscarriage rates between strategies (low SOE). All other outcomes had inconsistent and imprecise findings resulting in insufficient SOE.

Table 21. Outcomes for comparisons of oral agents with IUI versus gonadotropins with IUI for unexplained infertility

\begin{tabular}{|c|c|c|c|c|c|c|c|}
\hline Outcome & $\begin{array}{l}\text { Study } \\
\text { Design } \\
\text { (N } \\
\text { Patients) }\end{array}$ & Intervention & Comparator & $\begin{array}{c}\text { Results } \\
\text { Intervention } \\
\mathbf{N} \\
(\%) \\
\end{array}$ & $\begin{array}{c}\text { Results } \\
\text { Comparator } \\
\mathrm{N} \\
(\%) \\
\end{array}$ & $\begin{array}{c}P \\
\text { Value }\end{array}$ & $\begin{array}{l}\text { Summary } \\
\text { of Study } \\
\text { Findings }\end{array}$ \\
\hline \multirow[t]{5}{*}{$\begin{array}{l}\text { Live birth: } \\
\text { Any/patient }\end{array}$} & $\begin{array}{l}\text { Erdem, } \\
2015^{208} \\
\text { RCT } \\
(174)\end{array}$ & $\mathrm{CC}$ & rFSH with IUI & $\begin{array}{l}42 / 94 \\
(44.7)\end{array}$ & $\begin{array}{l}25 / 87 \\
(28.7)\end{array}$ & 0.026 & $\begin{array}{l}\text { Greater } \\
\text { live births } \\
\text { with CC }\end{array}$ \\
\hline & $\begin{array}{l}\text { Gregoriou, } \\
2008^{226} \\
\text { RCT } \\
(50)\end{array}$ & Letrozole & rFSH with IUI & $\begin{array}{l}7 / 25 \\
(28)\end{array}$ & $\begin{array}{l}5 / 25 \\
(20)\end{array}$ & 0.51 & $\begin{array}{l}\text { No } \\
\text { difference }\end{array}$ \\
\hline & \multirow[t]{2}{*}{$\begin{array}{l}\text { Diamond, } \\
2015^{233} \\
\text { RCT } \\
(900)\end{array}$} & CC with IUI & \multirow[t]{2}{*}{$\begin{array}{l}\text { Subcutaneous } \\
\text { gonadotropin } \\
\text { with IUI }\end{array}$} & $\begin{array}{l}70 / 300 \\
(23.3)\end{array}$ & \multirow[t]{2}{*}{$\begin{array}{c}97 / 301 \\
(32.2)\end{array}$} & 0.02 & $\begin{array}{l}\text { Greater } \\
\text { live birth } \\
\text { with } \\
\text { gonadotro } \\
\text { pins } \\
\text { compared } \\
\text { to CC }\end{array}$ \\
\hline & & $\begin{array}{l}\text { Letrozole } \\
\text { with IUI }\end{array}$ & & $\begin{array}{c}56 / 299 \\
(18.7)\end{array}$ & & $\begin{array}{c}0.000 \\
1\end{array}$ & $\begin{array}{l}\text { Greater } \\
\text { live birth } \\
\text { with } \\
\text { gonadotro } \\
\text { pins } \\
\text { compared } \\
\text { to } \\
\text { letrozole }\end{array}$ \\
\hline & $\begin{array}{l}\text { Danhof, } \\
2018^{244} \\
\text { RCT } \\
(738)\end{array}$ & CC with IUI & FSH with IUI & $\begin{array}{c}92 / 369 \\
(25)\end{array}$ & $\begin{array}{l}105 / 369 \\
(28)\end{array}$ & 0.36 & $\begin{array}{l}\text { No } \\
\text { significant } \\
\text { difference }\end{array}$ \\
\hline \multirow[t]{4}{*}{$\begin{array}{l}\text { Pregnancy } \\
\text { complications: } \\
\text { Miscarriage }\end{array}$} & $\begin{array}{l}\text { Pourali, } \\
2017^{241} \\
\text { RCT } \\
(180)\end{array}$ & $\begin{array}{l}\mathrm{CC}+\mathrm{HMG}+ \\
\mathrm{IUI}\end{array}$ & $\begin{array}{l}\text { Letrozole + hMG } \\
+ \text { IUI }\end{array}$ & $\begin{array}{l}5 / 87 \\
(5.7)\end{array}$ & $\begin{array}{l}4 / 83 \\
(4.8)\end{array}$ & 0.80 & $\begin{array}{l}\text { No } \\
\text { difference }\end{array}$ \\
\hline & \multirow[t]{2}{*}{$\begin{array}{l}\text { Diamond, } \\
2015^{233}\end{array}$} & CC with IUI & \multirow{2}{*}{$\begin{array}{l}\text { Subcutaneous } \\
\text { gonadotropin } \\
\text { with IUI }\end{array}$} & $\begin{array}{c}31 / 106 \\
(29.3)\end{array}$ & $\begin{array}{c}51 / 140 \\
(36.4)\end{array}$ & 0.24 & $\begin{array}{l}\text { No } \\
\text { difference }\end{array}$ \\
\hline & & $\begin{array}{l}\text { Letrozole } \\
\text { with IUI }\end{array}$ & & $\begin{array}{l}26 / 85 \\
(30.6)\end{array}$ & & 0.37 & $\begin{array}{l}\text { No } \\
\text { difference }\end{array}$ \\
\hline & $\begin{array}{l}\text { Danhof, } \\
2018^{244} \\
\text { RCT } \\
(738)\end{array}$ & CC with IUI & FSH with IUI & $\begin{array}{c}31 / 369 \\
(8)\end{array}$ & $\begin{array}{c}32 / 369 \\
(9)\end{array}$ & 0.63 & $\begin{array}{l}\text { No } \\
\text { difference }\end{array}$ \\
\hline
\end{tabular}




\begin{tabular}{|c|c|c|c|c|c|c|c|}
\hline Outcome & $\begin{array}{c}\text { Study } \\
\text { Design } \\
\text { (N } \\
\text { Patients) }\end{array}$ & Intervention & Comparator & $\begin{array}{c}\text { Results } \\
\text { Intervention } \\
\mathrm{N} \\
(\%) \\
\end{array}$ & $\begin{array}{c}\text { Results } \\
\text { Comparator } \\
N \\
(\%) \\
\end{array}$ & $\begin{array}{c}\mathbf{P} \\
\text { Value }\end{array}$ & $\begin{array}{c}\text { Summary } \\
\text { of Study } \\
\text { Findings }\end{array}$ \\
\hline \multirow[t]{2}{*}{$\begin{array}{l}\text { Pregnancy } \\
\text { complications: } \\
\text { Multiple births }\end{array}$} & \multirow[t]{2}{*}{$\begin{array}{l}\text { Diamond, } \\
2015^{233} \\
\text { RCT } \\
(900)\end{array}$} & CC with IUI & \multirow[t]{2}{*}{$\begin{array}{l}\text { Subcutaneous } \\
\text { gonadotropin } \\
\text { with IUI }\end{array}$} & $\begin{array}{l}4 / 70 \\
(5.7)\end{array}$ & $\begin{array}{l}31 / 97 \\
(32.0)\end{array}$ & $\begin{array}{c}<0.00 \\
01\end{array}$ & $\begin{array}{l}\text { Greater } \\
\text { multiple } \\
\text { gestations } \\
\text { with } \\
\text { gonadotro } \\
\text { pins } \\
\text { compared } \\
\text { to CC }\end{array}$ \\
\hline & & $\begin{array}{l}\text { Letrozole } \\
\text { with IUI }\end{array}$ & & $\begin{array}{c}8 / 56 \\
(14.3)\end{array}$ & & 0.015 & $\begin{array}{l}\text { Greater } \\
\text { multiple } \\
\text { gestations } \\
\text { with } \\
\text { gonadotro } \\
\text { pins } \\
\text { compared } \\
\text { to } \\
\text { letrozole }\end{array}$ \\
\hline \multirow[t]{2}{*}{$\begin{array}{l}\text { Short-term } \\
\text { adverse } \\
\text { effects: OHSS }\end{array}$} & $\begin{array}{l}\text { Nada, } \\
2016^{234} \\
\\
\text { RCT } \\
(595)\end{array}$ & CC with IUI & $\begin{array}{l}\text { GnRH antagonist } \\
\text { with IUI }\end{array}$ & $\begin{array}{l}6 / 297 \\
(2)\end{array}$ & $\begin{array}{c}30 / 298 \\
(10)\end{array}$ & $\begin{array}{c}<0.00 \\
01\end{array}$ & $\begin{array}{l}\text { Higher } \\
\text { rate of } \\
\text { mild } \\
\text { OHSS } \\
\text { among } \\
\text { those in } \\
\text { GnRH } \\
\text { antagonist } \\
\text { group }\end{array}$ \\
\hline & $\begin{array}{l}\text { Pourali, } \\
2017^{241} \\
\\
\text { RCT } \\
(170)\end{array}$ & $\begin{array}{l}\text { CC + HMG + } \\
\text { IUI }\end{array}$ & $\begin{array}{l}\text { Letrozole + hMG } \\
+ \text { IUI }\end{array}$ & $\begin{array}{l}5 / 87 \\
\text { (5.7) }\end{array}$ & $\begin{array}{c}0 / 83 \\
(0)\end{array}$ & 0.027 & $\begin{array}{l}\text { Rate of } \\
\text { cancelled } \\
\text { cycles for } \\
\text { OHSS } \\
\text { higher in } \\
\text { CC group }\end{array}$ \\
\hline $\begin{array}{l}\text { Ectopic } \\
\text { pregnancy }\end{array}$ & $\begin{array}{l}\text { Danhof, } \\
2018^{244} \\
\text { RCT } \\
(738)\end{array}$ & CC with IUI & FSH with IUI & $\begin{array}{l}3 / 369 \\
(1)\end{array}$ & $\begin{array}{l}2 / 369 \\
(1)\end{array}$ & 1 & $\begin{array}{l}\text { No } \\
\text { difference }\end{array}$ \\
\hline
\end{tabular}

Abbreviations: $\mathrm{CC}=$ clomiphene citrate; $\mathrm{CI}=$ confidence interval; FSH=follicle-stimulating hormone; hMG=human menopausal gonadotropin; IUI=intrauterine insemination; N=number of patients; NS=not statistically significant; OHSS=ovarian hyperstimulation syndrome; RCT=randomized control trial; rFSH=recombinant follicle-stimulating hormone; uFSH=urinary follicle-stimulating hormone

\section{Different Treatment Strategies for Controlled Ovarian Hyperstimulation With Gonadotropins and IUI for Unexplained Infertility}

Two good-quality RCTs, ${ }^{209,230}$ two fair-quality RCTs ${ }^{221,225}$ and one poor-quality RCT ${ }^{211}$ compared outcomes between different treatment strategies of gonadotropins/IUI. Interventions included luteal progesterone compared to no luteal support, ${ }^{209,225}$ uterine cavity perturbation versus no additional therapy, ${ }^{211}$ and type of gonadotropin (rFSH, highly purified urinary FSH, or HMG). ${ }^{230}$ Results are summarized in Table 22. The studies were consistent in terms of the direction of benefit (greater live births with progesterone compared to no support) but inconsistent in terms of their statistical significance and with imprecise findings (insufficient SOE). No difference was reported between uterine perturbation versus no intervention 
(insufficient SOE). The imprecise findings from heterogeneous interventions for miscarriage resulted in an insufficient strength of evidence rating. Finally, evidence from one study with no events provided insufficient evidence to support statements about the impact on OHSS. No other outcomes of interest were reported.

Table 22. Outcomes for comparisons of different treatment strategies for controlled ovarian hyperstimulation with gonadotropins and IUI in women with unexplained infertility

\begin{tabular}{|c|c|c|c|c|c|c|c|}
\hline Outcome & $\begin{array}{c}\text { Study } \\
\text { Design } \\
\text { (N } \\
\text { Patients) }\end{array}$ & Intervention & Comparator & $\begin{array}{c}\text { Results } \\
\text { Intervention } \\
\mathbf{N} \\
(\%) \\
(95 \% \mathrm{Cl}) \\
\end{array}$ & $\begin{array}{c}\text { Results } \\
\text { Comparator } \\
\mathbf{N} \\
(\%) \\
(95 \% \mathrm{Cl}) \\
\end{array}$ & $\begin{array}{c}P \\
\text { Value }\end{array}$ & $\begin{array}{c}\text { Summary } \\
\text { of Study } \\
\text { Findings }\end{array}$ \\
\hline \multirow[t]{3}{*}{$\begin{array}{l}\text { Live birth: } \\
\text { Any/patient }\end{array}$} & $\begin{array}{l}\text { Seckin, } \\
2014^{209} \\
\text { RCT } \\
(149)\end{array}$ & Progesterone & No support & $\begin{array}{l}14 / 71 \\
(19.7)\end{array}$ & $\begin{array}{l}11 / 78 \\
(14.1)\end{array}$ & 0.36 & $\begin{array}{l}\text { No } \\
\text { difference }\end{array}$ \\
\hline & $\begin{array}{l}\text { Erdem, } \\
2009^{225} \\
\text { RCT } \\
(214)\end{array}$ & Progesterone & No support & $\begin{array}{c}\text { 39/223 (per } \\
\text { cycle) } \\
(17.5)\end{array}$ & $\begin{array}{c}19 / 204 \text { (per } \\
\text { cycle) } \\
(9.3)\end{array}$ & 0.016 & $\begin{array}{l}\text { Greater } \\
\text { live births } \\
\text { with } \\
\text { progestero } \\
\text { ne } \\
\text { compared } \\
\text { to no } \\
\text { support }\end{array}$ \\
\hline & $\begin{array}{l}\text { Yildiz, } \\
2014^{211} \\
\text { RCT } \\
(180) \\
\end{array}$ & $\begin{array}{l}\text { Uterine } \\
\text { perturbation }\end{array}$ & $\begin{array}{l}\text { No } \\
\text { intervention }\end{array}$ & $\begin{array}{c}10 / 79 \\
(12.7) \\
(6.3 \text { to } 20.8)\end{array}$ & $\begin{array}{c}20 / 101 \\
(19.8) \\
(12.6 \text { to } 28.1)\end{array}$ & 0.20 & $\begin{array}{l}\text { No } \\
\text { difference }\end{array}$ \\
\hline \multirow[t]{4}{*}{$\begin{array}{l}\text { Pregnancy } \\
\text { complications: } \\
\text { Miscarriage }\end{array}$} & $\begin{array}{l}\text { Bagis, } \\
2010^{221} \\
\text { RCT } \\
(228)\end{array}$ & $\begin{array}{l}\text { Double } \\
\text { insemination }\end{array}$ & $\begin{array}{l}\text { Single } \\
\text { insemination }\end{array}$ & $\begin{array}{c}6 / 20 \\
(30) \\
(12.6 \text { to } 51)\end{array}$ & $\begin{array}{c}4 / 16 \\
(25) \\
(7.8 \text { to } 48.1)\end{array}$ & 0.74 & $\begin{array}{l}\text { No } \\
\text { difference }\end{array}$ \\
\hline & $\begin{array}{l}\text { Yildiz, } \\
2014^{211} \\
\text { RCT } \\
(180)\end{array}$ & $\begin{array}{l}\text { Uterine } \\
\text { perturbation }\end{array}$ & $\begin{array}{l}\text { No } \\
\text { intervention }\end{array}$ & $\begin{array}{c}1 / 79 \\
(1.3) \\
(0.03 \text { to } 4.5)\end{array}$ & $\begin{array}{c}3 / 101 \\
(3.0) \\
(0.6 \text { to } 7.0)\end{array}$ & 0.44 & $\begin{array}{l}\text { No } \\
\text { difference }\end{array}$ \\
\hline & \multirow[t]{2}{*}{$\begin{array}{l}\text { Demirol, } \\
2007^{230}\end{array}$} & \multirow[t]{2}{*}{ rFSH } & hMG & \multirow[t]{2}{*}{$\begin{array}{c}2 / 81 \\
(2.5) \\
(0.3 \text { to } 6.8)\end{array}$} & $\begin{array}{c}\text { hMG: } \\
2 / 80 \\
(2.5) \\
\text { (0.3 to } 6.9)\end{array}$ & 0.99 & $\begin{array}{l}\text { No } \\
\text { difference }\end{array}$ \\
\hline & & & uFSH & & $\begin{array}{c}\text { uFHS: } \\
1 / 80 \\
(1.2) \\
(0.03 \text { to } 4.5)\end{array}$ & 0.57 & $\begin{array}{l}\text { No } \\
\text { difference }\end{array}$ \\
\hline \multirow[t]{2}{*}{$\begin{array}{l}\text { Short-term } \\
\text { adverse } \\
\text { effects: OHSS }\end{array}$} & \multirow[t]{2}{*}{$\begin{array}{l}\text { Demirol, } \\
2007^{230}\end{array}$} & \multirow[t]{2}{*}{ rFSH } & hMG & \multirow[t]{2}{*}{$\begin{array}{c}0 / 81 \\
(0) \\
(0 \text { to } 3.1)\end{array}$} & $\begin{array}{c}\text { hMG: } \\
\text { 0/80 } \\
(0) \\
(0 \text { to } 3.1)\end{array}$ & NS & $\begin{array}{l}\text { No } \\
\text { difference }\end{array}$ \\
\hline & & & uFSH & & $\begin{array}{c}\text { uFSH: } \\
0 / 80 \\
(0) \\
\text { (0 to } 3.1 \text { ) }\end{array}$ & NS & $\begin{array}{l}\text { No } \\
\text { difference }\end{array}$ \\
\hline
\end{tabular}

Abbreviations: $\mathrm{CI}=$ confidence interval; $\mathrm{FSH}=$ follicle-stimulating hormone; $\mathrm{hMG}=$ human menopausal gonadotropin;

IUI=intrauterine insemination; $\mathrm{N}=$ number of patients; NS=not statistically significant; OHSS=0varian hyperstimulation 
syndrome; RCT=randomized control trial; rFSH=recombinant follicle-stimulating hormone; uFSH=urinary follicle-stimulating hormone

\section{Immediate IVF Versus Other Treatments Prior to IVF for Unexplained Infertility}

Three RCTs ( 2 good, ${ }^{52,210} 1$ fair quality ${ }^{240}$ ) compared different broad strategies for women with unexplained infertility. In the Fast Track and Standard Treatment (FASTT) trial, ${ }^{52}$ women aged 21-39 were randomized to (a) up to 3 cycles of clomiphene citrate/IUI followed by up to 3 cycles of gonadotropins/IUI followed by up to 6 cycles of IVF ("conventional strategy") or (b) up to 3 cycles of clomiphene citrate/IUI followed by 6 cycles of IVF ("fast track"). In the Forty and Over Treatment Trial (FORT-T), ${ }^{210}$ women aged 38-42 with unexplained infertility were randomized to (a) clomiphene citrate/IUI for up to 2 cycles followed by up to 6 cycles IVF, (b) FSH/IUI for up to 2 cycles followed by 6 cycles IVF, or (c) immediate IVF (up to 6 cycles). A final fair-quality RCT randomized 207 couples with unexplained infertility to 3 cycles of IUI plus controlled ovarian hyperstimulation with injectable gonadotropins or to 1 cycle of IVF. ${ }^{240}$

Table 23 presents outcomes of interest. Consistent findings between the three studies found no difference in outcomes between immediate IVF compared with other treatments prior to IVF (low SOE for live birth, ectopic, miscarriage, multiple births, low birthweight, OHSS) while decreasing the time to pregnancy (moderate SOE).

Table 23. Outcomes for comparisons of immediate IVF versus other treatments prior to IVF in women with unexplained infertility ${ }^{\mathrm{a}}$

\begin{tabular}{|c|c|c|c|c|c|c|c|}
\hline Outcome & $\begin{array}{c}\text { Study } \\
\text { Design } \\
\text { (N } \\
\text { Patients) }\end{array}$ & Intervention & Comparator & $\begin{array}{c}\text { Results } \\
\text { Intervention } \\
\mathrm{N} \\
(\%) \\
(95 \% \mathrm{Cl}) \\
\end{array}$ & $\begin{array}{c}\text { Results } \\
\text { Comparator } \\
\mathrm{N} \\
(\%) \\
(95 \% \mathrm{Cl}) \\
\end{array}$ & $\begin{array}{c}\mathbf{P} \\
\text { Value }\end{array}$ & $\begin{array}{l}\text { Summary } \\
\text { of Study } \\
\text { Findings }\end{array}$ \\
\hline \multirow[t]{4}{*}{$\begin{array}{l}\text { Live birth—by } \\
\text { strategy }\end{array}$} & $\begin{array}{l}\text { Reindollar, } \\
2010^{52} \\
\text { RCT } \\
(503)\end{array}$ & $\begin{array}{l}\mathrm{CC} \rightarrow \mathrm{IVF} \text { (fast } \\
\text { track) }\end{array}$ & $\begin{array}{l}\text { CC } \rightarrow \\
\text { gonadotropins } \\
\rightarrow \text { IVF } \\
\text { (conventional) }\end{array}$ & $\begin{array}{c}171 / 256 \\
(66.8) \\
(60.5 \text { to } \\
72.1)\end{array}$ & $\begin{array}{c}150 / 247 \\
(60.7) \\
\text { (56.6 to } 68.9)\end{array}$ & 0.15 & $\begin{array}{l}\text { No } \\
\text { difference }\end{array}$ \\
\hline & \multirow{2}{*}{$\begin{array}{l}\text { Goldman, } \\
2014^{210} \\
\text { RCT } \\
(154)\end{array}$} & \multirow[t]{2}{*}{ IVF } & $\mathrm{CC} / \mathrm{IUI} \rightarrow \mathrm{IVF}$ & \multirow[t]{2}{*}{$\begin{array}{c}24 / 51 \\
(47.1) \\
(31.2 \text { to } \\
63.4)\end{array}$} & $\begin{array}{c}\text { CC/IUI } \rightarrow \text { IVF: } \\
25 / 51 \\
(49.0) \\
\text { (32.9 to } 65.2) \\
\end{array}$ & 0.84 & $\begin{array}{l}\text { No } \\
\text { difference }\end{array}$ \\
\hline & & & $\begin{array}{l}\text { Gonadotropins } \\
\text { / IUI } \rightarrow \text { IVF }\end{array}$ & & $\begin{array}{c}\text { Gonadotropins } \\
\text { / IUI } \rightarrow \text { IVF: } \\
22 / 52 \\
(42.3) \\
(97.5 \mathrm{Cl}, 27.1 \\
\text { to } 58.1)\end{array}$ & 0.63 & $\begin{array}{l}\text { No } \\
\text { difference }\end{array}$ \\
\hline & $\begin{array}{l}\text { Nandi, } \\
2017^{240} \\
\text { RCT } \\
(207)\end{array}$ & $\begin{array}{l}3 \text { cycles IUI + } \\
\text { ovarian } \\
\text { hyperstimulati } \\
\text { on with } \\
\text { gonadotropin } \\
\text { s }\end{array}$ & IVF & $\begin{array}{l}29 / 101 \\
(28.7)\end{array}$ & $\begin{array}{c}36 / 106 \\
(33.9)\end{array}$ & 0.42 & $\begin{array}{l}\text { No } \\
\text { difference }\end{array}$ \\
\hline $\begin{array}{l}\text { Pregnancy } \\
\text { complications: } \\
\text { Ectopic } \\
\text { pregnancy-by } \\
\text { strategy }\end{array}$ & $\begin{array}{l}\text { Reindollar, } \\
2010^{52} \\
\text { RCT } \\
\text { (503) }\end{array}$ & $\begin{array}{l}C C \rightarrow \text { IVF (fast } \\
\text { track) }\end{array}$ & $\begin{array}{l}\mathrm{CC} \rightarrow \\
\text { gonadotropins } \\
\rightarrow \text { IVF } \\
\text { (conventional) }\end{array}$ & $\begin{array}{c}10 / 256 \\
(3.9) \\
(1.9 \text { to } 6.6)\end{array}$ & $\begin{array}{c}8 / 247 \\
(3.2) \\
(1.4 \text { to } 5.8)\end{array}$ & 0.69 & $\begin{array}{l}\text { No } \\
\text { difference }\end{array}$ \\
\hline
\end{tabular}




\begin{tabular}{|c|c|c|c|c|c|c|c|}
\hline Outcome & $\begin{array}{c}\text { Study } \\
\text { Design } \\
\text { (N } \\
\text { Patients) }\end{array}$ & Intervention & Comparator & $\begin{array}{c}\text { Results } \\
\text { Intervention } \\
N \\
(\%) \\
(95 \% \mathrm{Cl})\end{array}$ & $\begin{array}{c}\text { Results } \\
\text { Comparator } \\
N \\
(\%) \\
(95 \% \mathrm{Cl})\end{array}$ & $\begin{array}{c}\mathbf{P} \\
\text { Value }\end{array}$ & $\begin{array}{l}\text { Summary } \\
\text { of Study } \\
\text { Findings }\end{array}$ \\
\hline & $\begin{array}{l}\text { Goldman, } \\
2014^{210} \\
\text { RCT }\end{array}$ & IVF & CC/IUI $\rightarrow I V F$ & \multirow[t]{2}{*}{$\begin{array}{c}0 / 51 \\
(0) \\
(95 \mathrm{Cl} 0 \text { to } \\
4.8)\end{array}$} & $\begin{array}{c}\text { CC/IUI } \rightarrow \text { IVF: } \\
1 / 51 \\
(2.0) \\
(0.04 \text { to } 7.1) \\
\end{array}$ & 0.32 & $\begin{array}{l}\text { No } \\
\text { difference }\end{array}$ \\
\hline & (154) & & $\begin{array}{l}\text { Gonadotropins } \\
\text { / IUI } \rightarrow \text { IVF }\end{array}$ & & $\begin{array}{c}\text { Gonadotropins } \\
\text { / IUI } \rightarrow \text { IVF: } \\
3 / 52 \\
(5.8) \\
\text { (1.2 to } 13.5)\end{array}$ & 0.08 & $\begin{array}{l}\text { No } \\
\text { difference }\end{array}$ \\
\hline & $\begin{array}{l}\text { Nandi, } \\
2017^{240} \\
\text { RCT } \\
(207)\end{array}$ & $\begin{array}{l}3 \text { cycles IUI + } \\
\text { ovarian } \\
\text { hyperstimulati } \\
\text { on with } \\
\text { gonadotropin } \\
\text { s }\end{array}$ & IVF & $\begin{array}{l}2 / 101 \\
(1.98)\end{array}$ & $\begin{array}{c}0 / 106 \\
(0)\end{array}$ & NS & $\begin{array}{l}\text { No } \\
\text { difference }\end{array}$ \\
\hline \multirow[t]{4}{*}{$\begin{array}{l}\text { Pregnancy } \\
\text { complications: } \\
\text { Miscarriage }\end{array}$} & $\begin{array}{l}\text { Reindollar, } \\
2010^{52} \\
\text { RCT } \\
(503) \\
\end{array}$ & $\begin{array}{l}\mathrm{CC} \rightarrow \mathrm{IVF} \text { (fast } \\
\text { track) }\end{array}$ & $\begin{array}{l}\mathrm{CC} \rightarrow \\
\text { gonadotropins } \\
\rightarrow \text { IVF } \\
\text { (conventional) }\end{array}$ & $\begin{array}{c}38 / 256 \\
(14.8) \\
(10.8 \text { to } \\
19.4)\end{array}$ & $\begin{array}{c}32 / 247 \\
(13.0) \\
(9.1 \text { to } 17.4)\end{array}$ & 0.54 & $\begin{array}{l}\text { No } \\
\text { difference }\end{array}$ \\
\hline & \multirow{2}{*}{$\begin{array}{l}\text { Goldman, } \\
2014^{210} \\
\text { RCT } \\
(154)\end{array}$} & \multirow[t]{2}{*}{ IVF } & $\mathrm{CC} / \mathrm{IUI} \rightarrow \mathrm{IVF}$ & \multirow[t]{2}{*}{$\begin{array}{c}11 / 51 \\
(21.6) \\
(11.5 \text { to } \\
33.7)\end{array}$} & $\begin{array}{c}\text { CC/IUI } \rightarrow \text { IVF: } \\
8 / 51 \\
(15.7) \\
(7.2 \text { to } 26.7)\end{array}$ & 0.45 & $\begin{array}{l}\text { No } \\
\text { difference }\end{array}$ \\
\hline & & & $\begin{array}{l}\text { Gonadotropins } \\
\text { / IUI } \rightarrow \text { IVF }\end{array}$ & & $\begin{array}{c}\text { Gonadotropins } \\
\text { / IUI } \rightarrow \text { IVF: } \\
10 / 52 \\
(19.2) \\
\text { (9.8 to } 30.9)\end{array}$ & 0.77 & $\begin{array}{l}\text { No } \\
\text { difference }\end{array}$ \\
\hline & $\begin{array}{l}\text { Nandi, } \\
2017^{240} \\
\text { RCT } \\
(207)\end{array}$ & $\begin{array}{l}3 \text { cycles IUI + } \\
\text { ovarian } \\
\text { hyper- } \\
\text { stimulation } \\
\text { with } \\
\text { gonadotropin } \\
\text { s }\end{array}$ & IVF & $\begin{array}{l}3 / 34 \\
(12)\end{array}$ & $\begin{array}{l}13 / 49 \\
(26.5)\end{array}$ & 0.11 & $\begin{array}{l}\text { No } \\
\text { difference }\end{array}$ \\
\hline \multirow[t]{3}{*}{$\begin{array}{l}\text { Pregnancy } \\
\text { complications: } \\
\text { Multiple births }\end{array}$} & $\begin{array}{l}\text { Reindollar, } \\
2010^{52} \\
\text { RCT } \\
(503)\end{array}$ & $\begin{array}{l}C C \rightarrow I V F \text { (fast } \\
\text { track) }\end{array}$ & $\begin{array}{l}\mathrm{CC} \rightarrow \\
\text { gonadotropins } \\
\rightarrow \text { IVF } \\
\text { (conventional) }\end{array}$ & $\begin{array}{c}34 / 171 \\
\text { (twins) } \\
(19.9) \\
(14.3 \text { to } \\
26.2) \\
\end{array}$ & $\begin{array}{c}\text { 30/150 (twins) } \\
\text { (20) } \\
\text { (14.0 to } 26.7)\end{array}$ & 0.98 & $\begin{array}{l}\text { No } \\
\text { difference }\end{array}$ \\
\hline & \multirow{2}{*}{$\begin{array}{l}\text { Goldman, } \\
2014^{210} \\
\text { RCT } \\
(154)\end{array}$} & \multirow[t]{2}{*}{ IVF } & CC/IUI $\rightarrow I V F$ & \multirow[t]{2}{*}{$\begin{array}{l}3 / 24 \text { (twins) } \\
(12.5) \\
\text { (2.8 to } 28.0 \text { ) }\end{array}$} & $\begin{array}{c}\text { CC/IUI } \rightarrow \text { IVF: } \\
6 / 25 \text { (twins) } \\
(25) \\
\text { (9.8 to } 42.2 \text { ) }\end{array}$ & 0.30 & $\begin{array}{l}\text { No } \\
\text { difference }\end{array}$ \\
\hline & & & $\begin{array}{l}\text { Gonadotropins } \\
\text { / IUI } \rightarrow \text { IVF }\end{array}$ & & $\begin{array}{c}\text { Gonadotropins } \\
\text { / IUI } \rightarrow \text { IVF: } \\
4 / 22 \text { (3 twins, } 1 \\
\text { triplet) } \\
(18.2) \\
\text { (5.4 to } 36.3) \\
\end{array}$ & 0.59 & $\begin{array}{l}\text { No } \\
\text { difference }\end{array}$ \\
\hline $\begin{array}{l}\text { Neonatal } \\
\text { outcomes: Low } \\
\text { birthweight }\end{array}$ & $\begin{array}{l}\text { Reindollar, } \\
2010^{52} \\
\text { RCT } \\
(503)\end{array}$ & $\begin{array}{l}C C \rightarrow I V F \text { (fast } \\
\text { track) }\end{array}$ & $\begin{array}{l}\mathrm{CC} \rightarrow \\
\text { gonadotropins } \\
\rightarrow \text { IVF } \\
\text { (conventional) }\end{array}$ & $\begin{array}{c}30 / 171 \\
(17.5) \\
(95 \mathrm{Cl} \\
12.2 \text { to } 23.6)\end{array}$ & $\begin{array}{c}23 / 150 \\
(15.3) \\
(10.0 \text { to } 21.5)\end{array}$ & 0.59 & $\begin{array}{l}\text { No } \\
\text { difference }\end{array}$ \\
\hline
\end{tabular}




\begin{tabular}{|c|c|c|c|c|c|c|c|}
\hline Outcome & $\begin{array}{c}\text { Study } \\
\text { Design } \\
\text { (N } \\
\text { Patients) }\end{array}$ & Intervention & Comparator & $\begin{array}{c}\text { Results } \\
\text { Intervention } \\
N \\
(\%) \\
(95 \% \mathrm{Cl})\end{array}$ & $\begin{array}{c}\text { Results } \\
\text { Comparator } \\
N \\
(\%) \\
(95 \% \mathrm{Cl})\end{array}$ & $\begin{array}{c}P \\
\text { Value }\end{array}$ & $\begin{array}{l}\text { Summary } \\
\text { of Study } \\
\text { Findings }\end{array}$ \\
\hline & $\begin{array}{l}\text { Goldman, } \\
2014^{210} \\
\text { RCT }\end{array}$ & IVF & $\mathrm{CC} / \mathrm{IUI} \rightarrow \mathrm{IVF}$ & \multirow[t]{2}{*}{$\begin{array}{c}2 / 24 \\
(8.3) \\
\text { (1.1 to } 21.9)\end{array}$} & $\begin{array}{c}\text { CC/IUI } \rightarrow \text { IVF: } \\
2 / 25 \\
(2.8) \\
(1.0 \text { to } 21.1)\end{array}$ & 0.97 & $\begin{array}{l}\text { No } \\
\text { difference }\end{array}$ \\
\hline & (154) & & $\begin{array}{l}\text { Gonadotropins } \\
\text { / IUI } \rightarrow \text { IVF }\end{array}$ & & $\begin{array}{c}\text { Gonadotropins } \\
\text { / IUI } \rightarrow \text { IVF: } \\
2 / 22 \\
(9.1) \\
\text { (1.2 to } 23.8)\end{array}$ & 0.93 & $\begin{array}{l}\text { No } \\
\text { difference }\end{array}$ \\
\hline \multirow[t]{3}{*}{$\begin{array}{l}\text { Neonatal } \\
\text { outcomes: } \\
\text { Neonatal } \\
\text { deaths }\end{array}$} & $\begin{array}{l}\text { Reindollar, } \\
2010^{52} \\
\text { RCT } \\
\text { (503) }\end{array}$ & $\begin{array}{l}\mathrm{CC} \rightarrow \mathrm{IVF} \text { (fast } \\
\text { track) }\end{array}$ & $\begin{array}{l}\mathrm{CC} \rightarrow \\
\text { gonadotropins } \\
\rightarrow \text { IVF } \\
\text { (conventional) }\end{array}$ & $\begin{array}{c}0 / 171 \\
(0) \\
(0 \text { to } 1.5)\end{array}$ & $\begin{array}{c}0 / 150 \\
(0) \\
(0 \text { to } 1.7)\end{array}$ & NS & $\begin{array}{l}\text { No } \\
\text { difference }\end{array}$ \\
\hline & \multirow{2}{*}{$\begin{array}{l}\text { Goldman, } \\
2014^{210} \\
\text { RCT } \\
(154)\end{array}$} & \multirow[t]{2}{*}{ IVF } & $\mathrm{CC} / \mathrm{IUI} \rightarrow \mathrm{IVF}$ & \multirow[t]{2}{*}{$\begin{array}{c}0 / 24 \\
(0) \\
(0 \text { to } 10.0)\end{array}$} & $\begin{array}{c}\text { CC/IUI } \rightarrow \text { IVF: } \\
0 / 25 \\
(0) \\
(0 \text { to } 9.6)\end{array}$ & NS & $\begin{array}{l}\text { No } \\
\text { difference }\end{array}$ \\
\hline & & & $\begin{array}{l}\text { Gonadotropins } \\
\text { / IUI } \rightarrow \text { IVF }\end{array}$ & & $\begin{array}{c}\text { Gonadotropins } \\
\text { / IUI } \rightarrow \text { IVF: } \\
\text { 0/22 } \\
(0) \\
\text { (0 to 10.9) }\end{array}$ & NS & $\begin{array}{l}\text { No } \\
\text { difference }\end{array}$ \\
\hline \multirow[t]{3}{*}{$\begin{array}{l}\text { Time to } \\
\text { pregnancy }\end{array}$} & $\begin{array}{l}\text { Reindollar, } \\
2010^{52} \\
\text { RCT } \\
(503)\end{array}$ & $\begin{array}{l}\mathrm{CC} \rightarrow \mathrm{IVF} \text { (fast } \\
\text { track) }\end{array}$ & $\begin{array}{l}\mathrm{CC} \rightarrow \\
\text { gonadotropins } \\
\rightarrow \text { IVF } \\
\text { (conventional) }\end{array}$ & 8 months & 11 months & 0.045 & $\begin{array}{l}\text { Shorter } \\
\text { time to } \\
\text { pregnancy } \\
\text { with } \\
\text { immediate } \\
\text { IVF } \\
\text { compared } \\
\text { to } \\
\text { gonadotro } \\
\text { pins prior } \\
\text { to IVF }\end{array}$ \\
\hline & \multirow[t]{2}{*}{$\begin{array}{l}\text { Goldman, } \\
2014^{210} \\
\text { RCT } \\
(154)\end{array}$} & \multirow[t]{2}{*}{ IVF } & $\mathrm{CC} / \mathrm{IUI} \rightarrow \mathrm{IVF}$ & \multirow[t]{2}{*}{$\begin{array}{c}8.7 \pm 0.5 \\
\text { months }\end{array}$} & $\begin{array}{c}\text { CC/IUI } \rightarrow \text { IVF: } \\
9.1 \pm 0.6\end{array}$ & $<0.05$ & $\begin{array}{l}\text { Shorter } \\
\text { time to } \\
\text { pregnancy } \\
\text { with } \\
\text { immediate } \\
\text { IVF } \\
\text { compared } \\
\text { to IUI prior } \\
\text { to IVF }\end{array}$ \\
\hline & & & $\begin{array}{l}\text { Gonadotropins } \\
\text { / IUI } \rightarrow \text { IVF }\end{array}$ & & $\begin{array}{c}\text { Gonadotropins } \\
\text { / IUI } \rightarrow \text { IVF: } \\
12.2 \pm 0.7\end{array}$ & $<0.05$ & $\begin{array}{l}\text { Shorter } \\
\text { time to } \\
\text { pregnancy } \\
\text { with } \\
\text { immediate } \\
\text { IVF } \\
\text { compared } \\
\text { to IUI and } \\
\text { gonadotro } \\
\text { pins prior } \\
\text { to IVF }\end{array}$ \\
\hline
\end{tabular}




\begin{tabular}{|c|c|c|c|c|c|c|c|}
\hline Outcome & $\begin{array}{l}\text { Study } \\
\text { Design } \\
\text { (N } \\
\text { Patients) }\end{array}$ & Intervention & Comparator & $\begin{array}{c}\text { Results } \\
\text { Intervention } \\
\mathrm{N} \\
(\%) \\
(95 \% \mathrm{Cl})\end{array}$ & $\begin{array}{c}\text { Results } \\
\text { Comparator } \\
N \\
(\%) \\
(95 \% \mathrm{Cl})\end{array}$ & $\begin{array}{c}\mathbf{P} \\
\text { Value }\end{array}$ & $\begin{array}{l}\text { Summary } \\
\text { of Study } \\
\text { Findings }\end{array}$ \\
\hline \multirow[t]{3}{*}{ OHSS } & $\begin{array}{l}\text { Reindollar, } \\
2010^{52} \\
\text { RCT } \\
(503)\end{array}$ & $\begin{array}{l}\mathrm{CC} \rightarrow \mathrm{IVF} \text { (fast } \\
\text { track) }\end{array}$ & $\begin{array}{l}\mathrm{CC} \rightarrow \\
\text { gonadotropins } \\
\rightarrow \text { IVF } \\
\text { (conventional) }\end{array}$ & $\begin{array}{c}18 / 256 \\
(7.0) \\
\text { (4.2 to } 10.5)\end{array}$ & $\begin{array}{c}18 / 247 \\
\text { ( } 7.3) \\
\text { (4.4 to } 10.8)\end{array}$ & 0.93 & $\begin{array}{l}\text { No } \\
\text { difference }\end{array}$ \\
\hline & \multirow{2}{*}{$\begin{array}{l}\text { Goldman, } \\
2014^{210} \\
\text { RCT } \\
(154)\end{array}$} & \multirow[t]{2}{*}{ IVF } & CC/IUI $\rightarrow I V F$ & \multirow[t]{2}{*}{$\begin{array}{c}3 / 51 \\
(5.9) \\
\text { (1.3 to 13.7) }\end{array}$} & $\begin{array}{c}\text { CC/IUI } \rightarrow \text { IVF: } \\
2 / 51 \\
(3.9) \\
(0.5 \text { to } 10.6)\end{array}$ & 0.65 & $\begin{array}{l}\text { No } \\
\text { difference }\end{array}$ \\
\hline & & & $\begin{array}{l}\text { Gonadotropins } \\
\text { / IUI } \rightarrow \text { IVF }\end{array}$ & & $\begin{array}{c}\text { Gonadotropins } \\
\text { / IUI } \rightarrow \text { IVF: } \\
3 / 52 \\
(5.8) \\
\text { (1.2 to } 13.5) \\
\end{array}$ & 0.98 & $\begin{array}{l}\text { No } \\
\text { difference }\end{array}$ \\
\hline
\end{tabular}

${ }^{a}$ Use of an arrow in the table indicates a second treatment that followed the first treatment.

Abbreviations: $\mathrm{CC}=$ clomiphene citrate; $\mathrm{CI}=$ confidence interval; eSET=elective single-embryo transfer; HR=hazard ratio; IUI=intrauterine insemination; IVF=in vitro fertilization; $\mathrm{N}=$ =number of patients; $\mathrm{NS}=$ =not statistically significant; $\mathrm{OHSS}=\mathrm{ovarian}$ hyperstimulation syndrome; RCT=randomized control trial

A cost-effectiveness analysis of the U.S.-based FASTT trial ${ }^{52}$ reported charges per delivery were \$9,846 lower (95\% CI, \$25,099 lower to \$3,869 higher; $\mathrm{p}=0.084$ ) for the fast track arm than the conventional arm. The difference in the infertility treatment-related charges per delivery was $\$ 5,802$ (95\% CI, $-\$ 14,388$ to $\$ 2,299$; p=0.08) (insufficient SOE). Note that multiple birth rates did not differ significantly between the two arms.

\section{Expectant Management Versus Other Interventions for Unexplained Infertility}

We identified three studies that included expectant management as a strategy and provided evidence on at least one outcome of interest.

One good-quality RCT examined outcomes following luteal phase scratching compared to expectant management in women with unexplained infertility. ${ }^{228}$ The miscarriage rate was 2/16 $(12.5 \%)$ in the endometrial scratch group versus $1 / 6(16.6 \%)$ in the control group $(p=0.79)$. Multiple births were also similar in the two groups (1/54 in the endometrial scratch group versus 0/51 in the control group, $\mathrm{p}=1$ ) (insufficient SOE for both outcomes).

Another good-quality RCT trial of 253 couples compared costs of controlled ovarian stimulation with FSH and IUI to 6 months of expectant management followed by usual treatment. ${ }^{139}$ Three-year outcomes were evaluated. The mean estimated costs per couple in the expectant management group were 3424 euros (95\% CI, 880 to 5968 euros) and in the immediate treatment group 6040 euros (95\% CI, 4055-8121 euros), with an estimated saving of 2616 euros per couple (95\% CI, 385 to 4847 euros). The likelihood of achieving pregnancy and the time to pregnancy did not differ between the groups, suggesting that expectant management was a reasonable cost-savings option. Applicability to U.S. populations is limited because the study was conducted in the Netherlands (insufficient SOE).

A third study was a good-quality RCT from the Netherlands which randomized women with different types of infertility, including unexplained infertility, to a 6-month structured lifestyle intervention for weight loss or to prompt treatment for infertility as per Dutch infertility 
guidelines. ${ }^{119}$ Of the 161 women with unexplained infertility, 86 were assigned to the intervention and 77 to the control groups. Within this group, there were no significant differences in the outcomes of vaginal birth of healthy singleton at term or of live births. Compared to the control group, the RR (95\% CI) of vaginal birth of health singleton at term for the intervention group was 0.73 (0.47 to 1.1); compared to the control group, the RR (95\% CI) of live births for the intervention group was 0.85 (0.64 to 1.1$){ }^{119}$

Finally, a good-quality systematic review ${ }^{84}$ examined outcomes following IVF for unexplained infertility. Within this analysis, the live birth rate was higher with IVF than expectant management (45.8\% vs. 3.7\%, OR 22.00; 95\% CI, 2.56 to 189.37) based on 1 RCT with 51 women. Given the inconsistent findings we rated the SOE as insufficient.

\section{ART for Unexplained Infertility}

\section{IVF for Unexplained Infertility}

Two observational studies ${ }^{236,239}$ and one $\mathrm{RCT}^{247}$ evaluated different IVF strategies in women with unexplained infertility.

The fair quality RCT compared 3 strategies for ovarian stimulation in women with diminished ovarian reserve undergoing IVF or ICSI. ${ }^{247}$ There were 116 participants randomized to one of the 3 protocols: a modified GnRH agonist (triptorelin) protocol; a mild stimulation protocol with letrozole; or an antagonist protocol with triptorelin. There was no significant difference in live birth rate between the 3 groups, with rates of $20.37 \%$ (11/54), $15.38 \%(8 / 52)$ and $13.33 \%(8 / 60)$ respectively. ${ }^{247}$

One good-quality observational study utilizing linked data from SMART Collaborative examined rates of small for gestational age with fresh embryo transfer, frozen embryo transfer, and natural conception. ${ }^{239}$ Among women with unexplained infertility, IVF with fresh embryo transfer was associated with increased odds of small for gestational age (defined as $<10 \%$ ) compared to natural conception (adjusted OR 1.24, 95\% CI 1.10 to 1.38). Results were similar when small for gestational age was defined as $<5 \%$ (adjusted OR 1.24, 95\% CI 1.06 to 1.45 ). When IVF was performed with frozen embryo transfer, however, there was no significant difference in small for gestational age compared to natural conception (adjusted OR 0.71, 95\% CI 0.47 to 1.06 when defined as $<10 \%$; adjusted OR 0.78 , 95\% CI 0.45 to 1.36 when defined as $<5 \%)$.

The second good-quality observational study evaluated low birth weight and demonstrated that among women with unexplained infertility, infants conceived with ART had an increased risk of low birth weight (OR 1.45, 95\% CI 1.29 to 1.63). ${ }^{236}$ However, a discordant sibling-pair analysis in the same study demonstrated no association (OR 1.27, 95\% CI 0.95 to 1.68)

A fair-quality systematic review and meta-analysis examined outcomes following ART with and without preimplantation genetic screening (PGS) in women of advanced maternal age (35+). ${ }^{94}$ Six RCTs were included (1,136 patients), none of the studies within this systematic review were included in the present systematic review. Reasons for exclusion included either being published before 2007, not presenting outcomes of interest by underlying diagnosis, or presenting findings by embryo rather than by cycle or patient. Live birth rate per woman was examined in only one study. In this study the odds of live birth were decreased with ART plus PGS compared with ART without PGS (OR 0.48; 95\% CI, 0.26 to 0.88). Miscarriage was examined in 3 studies. There was no significant difference in miscarriage rate between groups (OR 2.02; 95\% CI, 0.57 to 7.41). The systematic review was limited by inclusion of studies that 
utilized PGS technologies that are no longer current. Therefore, the review is not applicable to today's clinical practice and strength of evidence for all outcomes was rated as insufficient.

\section{ICSI for Unexplained Infertility}

One fair-quality RCT ${ }^{214}$ compared outcomes between different treatment strategies with ICSI. The study randomized patients undergoing ICSI to hyaluronic acid sperm selection (physiological ICSI [PICSI]) or no selection (ICSI). ${ }^{214}$ The live birth rate per patient was 22/71 (31\%) with PICSI versus 21/80 (26.3\%) with ICSI ( $p=0.520)$ (insufficient SOE). The miscarriage rate per patient was 3/25 (12\%) with PICSI versus 7/28 (25\%) with ICSI ( $p=0.227)$ (insufficient SOE).

\section{IVF Versus Combined IVF and ICSI for Unexplained Infertility}

Two observational studies evaluated outcomes for women undergoing IVF as compared to combined IVF and ICSI for unexplained infertility. ${ }^{185,215}$

In one good-quality observational study ${ }^{215}$ the authors performed an adaptive decision analysis to determine whether combined IVF and split-ICSI (randomly assigning sibling oocytes to conventional IVF or combined IVF and ICSI) is cost-effective for patients with unexplained infertility undergoing their first IVF cycle. For patients undergoing one cycle, conventional IVF was preferred, as the incremental cost effectiveness ratio (ICER) of split-ICSI or all ICSI (\$58,766 per additional live birth) did not justify the increase in live birth rate (3\%). For patients undergoing two cycles, split IVF/ICSI was preferred, as the 3.3\% increased cumulative live birth rate was gained at an ICER of \$29,666 per additional live birth (insufficient SOE).

The second observational study utilizing data from SART CORS compared rates of preterm birth and low birth weight among women undergoing conventional IVF versus ICSI. ${ }^{185}$ In this study, 2,922 live births were to women with unexplained infertility. In a matched dataset, among those women with unexplained infertility, the proportion of births with low birth weight was $123 / 1,464$ (8.4\%) in the ICSI group compared to 124/1,458 in the conventional group (8.5\%) (OR 1.03, 95\% CI 0.73 to $1.47, \mathrm{P}=0.86$ ) demonstrating no evidence of a difference between treatment groups (low SOE).

\section{Unspecified ART for Unexplained Infertility}

\section{Long-Term Outcomes After ART for Unexplained Infertility: Child}

Risk for cancer in children conceived after by either IVF or ICSI was evaluated in a cohort of 106,013 children born in Britain between 1992 and 2008 ${ }^{213}$ For 33,840 of these children, the cause of infertility was unexplained. The average duration of follow-up was 6.6 years, with a maximum of 15 years. Cancer diagnoses were identified through the National Registry of Childhood Tumors.

Cancer was diagnosed in 32 children born after assisted conception for unexplained infertility. The standardized incidence ratio (SIR) for all cancers in children conceived with assisted conception for unexplained infertility compared to the general population of the same age was 0.84 (95\% CI, 0.57 to 1.18).

Despite the large number of children in the cohort, the overall number of cancers was small. Conclusions about the risk are tenuous due to the inability to examine risk for individual cancer types by underlying cause of infertility (low SOE). 


\section{Subgroups of Interest for Unexplained Infertility}

Five total studies, one fair-quality observational study, ${ }^{122}$ two fair-quality RCTs, ${ }^{217,243}$ and two good-quality RCTs, ${ }^{235,237}$ reported data on outcomes with IVF in subgroups of interest for women with infertility of unknown etiology.

\section{Ovarian Reserve Status}

A fair-quality observational SART CORS study assessed the impact of assisted hatching IVF (where an embryologist uses micromanipulation under a microscope to create a small hole in the zona pellucidaon) outcomes in patients undergoing an initial IVF cycle with diminished ovarian reserve (DOR). ${ }^{122}$ DOR was diagnosed in one of 2 ways: (1) only elevated FSH and (2) DOR diagnosis in SART CORS database. Among women with elevated FSH only, the live birth rate per cycle was 562/2682 (21\%) with no assisted hatching compared to 571/3470 (21.6\%) with assisted hatching (RR 1.03; 95\% CI, 0.94 to 1.14). After adjustment for possible confounders, the RR was 0.92 (95\% CI, 0.79 to 1.07). Among women with a DOR diagnosis in SART CORS, the live birth rate per cycle was 3190/14,106 (22.6\%) with no assisted hatching compared to 3123/16,033 (19.5\%) with assisted hatching (RR 0.86; 95\% CI, 0.82 to 0.9). After adjustment for possible confounders, the RR was 0.84 (95\% CI, 0.77 to 0.92 ), suggesting worse outcomes with assisted hatching although the limitations of the observational design remain (insufficient SOE).

A good-quality RCT examined the utility of corifollitropin alpha for ovarian stimulation for poor responders undergoing IVF. ${ }^{237}$ Patients were randomized to clomiphene citrate and corifollitropin alpha for the first 7 days of stimulation, followed by recombinant FSH, or to clomiphene citrate with daily FSH. There was no evidence of a difference in live birth rate per transfer was (OR 0.76, 95\% CI 0.19 to 3.04, $\mathrm{P}=0.73$ ) nor in miscarriage rates (OR 1.14, 95\% CI 0.06 to $21.87, \mathrm{P}=1.00)$.

A good-quality RCT evaluated women $\geq 35$ years old and with a low antral follicular count of $<5$ follicles or poor ovarian response or cycle cancellation during a previous IVF irrespective of age and compared the effects of a mild ovarian stimulation protocol compared to a regular ovarian stimulation protocol. ${ }^{235}$ There was a similar risk of pregnancy loss in the 2 groups; compared to the conventional strategy, those randomized to the mild stimulation protocol had an RR (95\% CI) of early pregnancy loss of 1.20 (0.36 to 4.17$){ }^{235}$

Finally, one fair-quality RCT examined the effects of melatonin versus placebo on outcomes with IVF in women receiving ART for the first time and with diminished ovarian reserve. ${ }^{243}$.Women randomized to the melatonin arm received $3 \mathrm{mg}$ at bedtime beginning on day 5 of their cycle prior to the cycle planned for gonadotropin stimulation; women in the placebo group received matching placebo capsules taken similarly. IVF was performed using the same protocol in both groups. Of 32 women in the melatonin arm, 6.2\% had a miscarriage; of 34 women in the placebo arm $2.9 \%$ had a miscarriage demonstrating no evidence of a difference between treatment groups.

\section{Advanced Maternal Age}

The study by Rubio and colleagues compared PGS prior to blastocyst transfer on day 3 to no screening in women with advanced maternal age. ${ }^{217}$ In the advanced maternal age group, the live birth rate per patient was 30/93 (32.5\%) in the PGS group versus 14/90 (15.5\%) in the no-PGS group (OR 2.59; 95\% CI, 1.26 to 5.30) demonstrating an increase in live birth with PGS (insufficient SOE). 
Miscarriage rates and multiple birth rates were similar. In the advanced maternal age population, the miscarriage rate was $16.7 \%$ in the PGS group versus $22.2 \%$ in the no-PGS group ( $>0.05$ ). The twin birth rate was $25 \%$ in the PGS group versus $21.4 \%$ in the no-PGS group ( $>0.05$ ) (insufficient SOE for all outcomes).

\section{Strength of Evidence for Unexplained Infertility}

Table 24 summarizes the SOE for the findings described above. SOE for most outcomes was judged to be insufficient or low, primarily because of imprecision or small numbers of studies of fair quality. One exception was time to pregnancy between different strategies for sequencing treatment, where precision was reasonable, and where SOE was judged to be moderate.

Table 24. Strength of evidence for major outcomes-KQ 3 (unexplained infertility)

\begin{tabular}{|c|c|c|c|c|}
\hline Comparison & Outcome & $\begin{array}{l}\text { Study Design } \\
\text { (Sample Size) }\end{array}$ & Conclusion & $\begin{array}{l}\text { Strength of } \\
\text { Evidence } \\
\text { (Rationale) }^{\mathrm{a}}\end{array}$ \\
\hline \multirow[t]{7}{*}{$\begin{array}{l}\text { Oral Agents } \\
\text { Without IUI }\end{array}$} & $\begin{array}{l}\text { Live birth } \\
\text { (any/patient) }\end{array}$ & $\begin{array}{l}1 \mathrm{RCT}^{206} \\
(80)\end{array}$ & $\begin{array}{l}\text { Inconclusive: SOE was } \\
\text { insufficient given imprecise } \\
\text { evidence from } 1 \text { small study with } \\
\text { moderate risk of bias. }\end{array}$ & $\begin{array}{l}\text { Insufficient } \\
\text { (Imprecise findings, } \\
\text { one small study } \\
\text { with moderate } \\
\text { study limitations) }\end{array}$ \\
\hline & $\begin{array}{l}\text { Pregnancy } \\
\text { complications: } \\
\text { Ectopic } \\
\text { pregnancy }\end{array}$ & $\begin{array}{l}2 \mathrm{RCTs}^{222,246} \\
(1,168)\end{array}$ & $\begin{array}{l}\text { No difference: No difference } \\
\text { between letrozole and } \\
\text { anastrozole: }\end{array}$ & $\begin{array}{l}\text { Low } \\
\text { (Moderate study } \\
\text { limitations) }\end{array}$ \\
\hline & $\begin{array}{l}\text { Pregnancy } \\
\text { complications: } \\
\text { Multiple births }\end{array}$ & $\begin{array}{l}1 \mathrm{SR}^{70} \text { (5 studies, } \\
395 \text { patients) }\end{array}$ & $\begin{array}{l}\text { No difference: No difference } \\
\text { between letrozole and } \\
\text { clomiphene citrate }\end{array}$ & Moderate \\
\hline & $\begin{array}{l}\text { Pregnancy } \\
\text { complications: } \\
\text { Miscarriage }\end{array}$ & $\begin{array}{l}\text { 3 RCTs }{ }^{206,222,246} \\
(1,248) \\
1 \mathrm{SR}^{70} \text { (5 studies, } \\
395 \text { patients) }\end{array}$ & $\begin{array}{l}\text { No difference: No difference } \\
\text { between letrozole and } \\
\text { clomiphene citrate }\end{array}$ & Moderate \\
\hline & $\begin{array}{l}\text { Neonatal } \\
\text { outcomes: } \\
\text { Death }\end{array}$ & $\begin{array}{l}1 \mathrm{RCT}^{222} \\
(996)\end{array}$ & $\begin{array}{l}\text { Inconclusive: SOE was } \\
\text { insufficient given imprecise } \\
\text { evidence from } 1 \text { study with } \\
\text { moderate risk of bias }\end{array}$ & $\begin{array}{l}\text { Insufficient } \\
\text { (Imprecise findings } \\
\text { with moderate } \\
\text { study limitations)) } \\
\end{array}$ \\
\hline & $\begin{array}{l}\text { Neonatal } \\
\text { outcomes: } \\
\text { Birthweight }\end{array}$ & $\begin{array}{l}1 \mathrm{RCT}^{222} \\
(996)\end{array}$ & $\begin{array}{l}\text { Inconclusive: SOE was } \\
\text { insufficient given imprecise } \\
\text { evidence from } 1 \text { study with } \\
\text { moderate risk of bias }\end{array}$ & $\begin{array}{l}\text { Insufficient } \\
\text { (Imprecise findings } \\
\text { with moderate } \\
\text { study limitations)) } \\
\end{array}$ \\
\hline & $\begin{array}{l}\text { Short term } \\
\text { adverse effects } \\
\text { of treatment: } \\
\text { OHSS }\end{array}$ & $\begin{array}{l}1 \mathrm{RCT}^{246} \\
(172)\end{array}$ & $\begin{array}{l}\text { Inconclusive: SOE was } \\
\text { insufficient given imprecise } \\
\text { evidence from } 1 \text { small study }\end{array}$ & $\begin{array}{l}\text { Insufficient } \\
\text { (Imprecise, one } \\
\text { small study) }\end{array}$ \\
\hline \multirow[t]{3}{*}{$\begin{array}{l}\text { Clomiphene } \\
\text { Citrate vs. } \\
\text { Expectant } \\
\text { Management }\end{array}$} & Live birth & $\begin{array}{l}2 \mathrm{RCTs}^{135,238} \\
(781)\end{array}$ & $\begin{array}{l}\text { Inconclusive: SOE was } \\
\text { insufficient given inconsistent } \\
\text { evidence from studies with } \\
\text { heterogeneous interventions. }\end{array}$ & $\begin{array}{l}\text { Insufficient } \\
\text { (Inconsistent, } \\
\text { heterogeneous } \\
\text { interventions) }\end{array}$ \\
\hline & $\begin{array}{l}\text { Pregnancy } \\
\text { complications: } \\
\text { Ectopic } \\
\text { Pregnancy }\end{array}$ & $\begin{array}{l}2 \mathrm{RCTs}^{135,238} \\
(781)\end{array}$ & $\begin{array}{l}\text { No difference: No significant } \\
\text { difference in ectopic pregnancy } \\
\text { rates between clomiphene and } \\
\text { expectant management }\end{array}$ & $\begin{array}{l}\text { Low } \\
\text { (Imprecise, } \\
\text { heterogeneous } \\
\text { interventions) }\end{array}$ \\
\hline & $\begin{array}{l}\text { Pregnancy } \\
\text { complications: } \\
\text { Miscarriage }\end{array}$ & $\begin{array}{l}2 \mathrm{RCTs}^{135,238} \\
(781)\end{array}$ & $\begin{array}{l}\text { No difference: No significant } \\
\text { difference in ectopic pregnancy } \\
\text { rates between clomiphene and } \\
\text { expectant management }\end{array}$ & $\begin{array}{l}\text { Low } \\
\text { (Imprecise, } \\
\text { heterogeneous } \\
\text { interventions) }\end{array}$ \\
\hline
\end{tabular}




\begin{tabular}{|c|c|c|c|c|}
\hline Comparison & Outcome & $\begin{array}{l}\text { Study Design } \\
\text { (Sample Size) }\end{array}$ & Conclusion & $\begin{array}{l}\text { Strength of } \\
\text { Evidence } \\
\text { (Rationale) }^{\mathrm{a}}\end{array}$ \\
\hline \multirow[t]{4}{*}{$\begin{array}{l}\text { Oral Agents vs. } \\
\text { Unstimulated } \\
\text { IUI vs. } \\
\text { Expectant } \\
\text { Management }\end{array}$} & Live birth & $\begin{array}{l}1 \mathrm{SR}^{87} \text { (3 studies, } \\
370 \text { ) }\end{array}$ & $\begin{array}{l}\text { Improvement: A significant } \\
\text { increase in live births was found } \\
\text { for women treated with IUI and } \\
\text { ovarian hyperstimulation } \\
\text { compared to women treated with } \\
\text { IUI only }\end{array}$ & Low (Inconsistent) \\
\hline & $\begin{array}{l}\text { Pregnancy } \\
\text { complications: } \\
\text { Ectopic } \\
\text { pregnancy }\end{array}$ & $\begin{array}{l}\text { 1 RCT }^{135} \\
(580)\end{array}$ & $\begin{array}{l}\text { Inconclusive: SOE was } \\
\text { insufficient given imprecise } \\
\text { evidence from } 1 \text { trial. }\end{array}$ & $\begin{array}{l}\text { Insufficient } \\
\text { (Imprecise, one } \\
\text { study) }\end{array}$ \\
\hline & $\begin{array}{l}\text { Pregnancy } \\
\text { complications: } \\
\text { Miscarriage }\end{array}$ & $\begin{array}{l}1 \mathrm{RCT}^{135} \\
(580)\end{array}$ & $\begin{array}{l}\text { Inconclusive: SOE was } \\
\text { insufficient given imprecise } \\
\text { evidence from } 1 \text { trial. }\end{array}$ & $\begin{array}{l}\text { Insufficient } \\
\text { (Imprecise, one } \\
\text { study) }\end{array}$ \\
\hline & $\begin{array}{l}\text { Time to } \\
\text { pregnancy }\end{array}$ & $\begin{array}{l}1 \mathrm{RCT}^{135} \\
(580)\end{array}$ & $\begin{array}{l}\text { Inconclusive: SOE was } \\
\text { insufficient given imprecise } \\
\text { evidence from } 1 \text { trial. }\end{array}$ & $\begin{array}{l}\text { Insufficient } \\
\text { (Imprecise, one } \\
\text { study) }\end{array}$ \\
\hline \multirow{4}{*}{$\begin{array}{l}\text { Adjunct } \\
\text { Treatments with } \\
\text { Oral Agents } \\
\text { and IUI }\end{array}$} & Live birth & $\begin{array}{l}5 \\
\text { RCTs'216,221,229,242,245 } \\
(1859)\end{array}$ & $\begin{array}{l}\text { No difference: No difference } \\
\text { between adjunct treatments with } \\
\text { oral agents and IUI }\end{array}$ & $\begin{array}{l}\text { Low } \\
\text { (Moderate study } \\
\text { limitations) }\end{array}$ \\
\hline & $\begin{array}{l}\text { Pregnancy } \\
\text { complications: } \\
\text { Ectopic } \\
\text { pregnancy }\end{array}$ & $\begin{array}{l}1 \mathrm{RCT}^{227} \\
(216)\end{array}$ & $\begin{array}{l}\text { Inconclusive: SOE was } \\
\text { insufficient given imprecise } \\
\text { evidence from } 1 \text { trial. }\end{array}$ & $\begin{array}{l}\text { Insufficient } \\
\text { (Imprecise, one } \\
\text { study) }\end{array}$ \\
\hline & $\begin{array}{l}\text { Pregnancy } \\
\text { complications: } \\
\text { Miscarriage }\end{array}$ & $\begin{array}{l}5 \\
\mathrm{RCTS}^{221,227,231,232,245} \\
(1859)\end{array}$ & $\begin{array}{l}\text { No difference: No difference } \\
\text { between adjunct treatments with } \\
\text { oral agents and IUI }\end{array}$ & $\begin{array}{l}\text { Low } \\
\text { (Moderate study } \\
\text { limitations) }\end{array}$ \\
\hline & $\begin{array}{l}\text { Short term } \\
\text { adverse effects } \\
\text { of treatment: } \\
\text { OHSS }\end{array}$ & $\begin{array}{l}3 \mathrm{RCTs}^{216,227,245} \\
(1189)\end{array}$ & $\begin{array}{l}\text { No difference: No difference } \\
\text { between adjunct treatments with } \\
\text { oral agents and IUI }\end{array}$ & $\begin{array}{l}\text { Low } \\
\text { (Moderate study } \\
\text { limitations) }\end{array}$ \\
\hline \multirow{5}{*}{$\begin{array}{l}\text { Oral Agents } \\
\text { With IUI vs. } \\
\text { Gonadotropins } \\
\text { With IUI }\end{array}$} & Live birth & $\begin{array}{l}4 \mathrm{RCTS}^{208,226,233,244} \\
(1708)\end{array}$ & $\begin{array}{l}\text { Inconclusive: Conflicting } \\
\text { findings from RCTs resulted in } \\
\text { insufficient SOE }\end{array}$ & $\begin{array}{l}\text { Insufficient } \\
\text { (Imprecise, } \\
\text { inconsistent) }\end{array}$ \\
\hline & $\begin{array}{l}\text { Pregnancy } \\
\text { complications: } \\
\text { Ectopic } \\
\text { pregnancy }\end{array}$ & $\begin{array}{l}1 \mathrm{RCT}^{244} \\
(738)\end{array}$ & $\begin{array}{l}\text { Inconclusive: SOE was } \\
\text { insufficient given evidence from } \\
1 \text { trial with moderate study } \\
\text { limitations. }\end{array}$ & $\begin{array}{l}\text { Insufficient } \\
\text { (one study, } \\
\text { moderate study } \\
\text { limitations) }\end{array}$ \\
\hline & $\begin{array}{l}\text { Pregnancy } \\
\text { complications: } \\
\text { Miscarriage }\end{array}$ & $\begin{array}{l}\text { 3 RCTs } 233,241,244 \\
(1,654)\end{array}$ & $\begin{array}{l}\text { No difference: No difference } \\
\text { between oral agents with IUI } \\
\text { versus gonadotropins with IUI }\end{array}$ & $\begin{array}{l}\text { Low } \\
\text { (Imprecise) }\end{array}$ \\
\hline & $\begin{array}{l}\text { Pregnancy } \\
\text { complications: } \\
\text { Multiple births }\end{array}$ & $\begin{array}{l}1 \mathrm{RCT}^{233} \\
(742)\end{array}$ & $\begin{array}{l}\text { Increased risk: Greater multiple } \\
\text { gestations with gonadotropins } \\
\text { compared to either clomiphene } \\
\text { or letrozole }\end{array}$ & $\begin{array}{l}\text { Low } \\
\text { (one study) }\end{array}$ \\
\hline & $\begin{array}{l}\text { Short term } \\
\text { adverse effects } \\
\text { of treatment: } \\
\text { OHSS }\end{array}$ & $\begin{array}{l}2 \mathrm{RCTs}^{234,241} \\
(765)\end{array}$ & $\begin{array}{l}\text { Inconclusive: SOE was } \\
\text { insufficient given inconsistent } \\
\text { and imprecise findings. }\end{array}$ & $\begin{array}{l}\text { Insufficient } \\
\text { (Inconsistent and } \\
\text { Imprecise findings } \\
\text { with moderate } \\
\text { study limitations) }\end{array}$ \\
\hline $\begin{array}{l}\text { Different } \\
\text { Treatment } \\
\text { Strategies for } \\
\text { Controlled } \\
\text { Ovarian Hyper }\end{array}$ & Live birth & $\begin{array}{l}3 \mathrm{RCTs}^{209,211,225} \\
(837)\end{array}$ & $\begin{array}{l}\text { Inconclusive: SOE was } \\
\text { insufficient given inconsistent } \\
\text { and imprecise findings. }\end{array}$ & $\begin{array}{l}\text { Insufficient } \\
\text { (Inconsistent and } \\
\text { Imprecise findings } \\
\text { with moderate } \\
\text { study limitations) }\end{array}$ \\
\hline
\end{tabular}




\begin{tabular}{|c|c|c|c|c|}
\hline Comparison & Outcome & $\begin{array}{l}\text { Study Design } \\
\text { (Sample Size) }\end{array}$ & Conclusion & $\begin{array}{l}\text { Strength of } \\
\text { Evidence } \\
\text { (Rationale) }^{\mathrm{a}}\end{array}$ \\
\hline \multirow[t]{2}{*}{$\begin{array}{l}\text { stimulation with } \\
\text { Gonadotropins } \\
\text { \& IUI }\end{array}$} & $\begin{array}{l}\text { Pregnancy } \\
\text { complications: } \\
\text { Miscarriage }\end{array}$ & $\begin{array}{l}3 \text { RCTs } 211,221,230 \\
(929)\end{array}$ & $\begin{array}{l}\text { Inconclusive: SOE was } \\
\text { insufficient given imprecise } \\
\text { evidence about the outcome } \\
\text { from } 3 \text { studies targeting each an } \\
\text { individual intervention. }\end{array}$ & $\begin{array}{l}\text { Insufficient } \\
\text { (Imprecise findings } \\
\text { with moderate } \\
\text { study limitations)) }\end{array}$ \\
\hline & $\begin{array}{l}\text { Short term } \\
\text { adverse effects } \\
\text { of treatment: } \\
\text { OHSS }\end{array}$ & $\begin{array}{l}\text { 1 RCT }^{230} \\
(161)\end{array}$ & $\begin{array}{l}\text { Inconclusive: SOE was } \\
\text { insufficient given imprecise } \\
\text { evidence from } 1 \text { trial with no } \\
\text { events. }\end{array}$ & $\begin{array}{l}\text { Insufficient } \\
\text { (Imprecise, one } \\
\text { small study) }\end{array}$ \\
\hline \multirow[t]{9}{*}{$\begin{array}{l}\text { Immediate IVF } \\
\text { vs. Other } \\
\text { Treatments } \\
\text { Prior to IVF }\end{array}$} & Live birth & $\begin{array}{l}\text { 3 RCTs } 52,210,212,240 \\
(812)\end{array}$ & $\begin{array}{l}\text { No difference: Live birth does } \\
\text { not differ between differing } \\
\text { strategies of other treatments } \\
\text { prior to IVF }\end{array}$ & $\begin{array}{l}\text { Low } \\
\text { (Imprecise) }\end{array}$ \\
\hline & $\begin{array}{l}\text { Pregnancy } \\
\text { complications: } \\
\text { Multiple births }\end{array}$ & $\begin{array}{l}2 \mathrm{RCTs}^{52,210} \\
(657)\end{array}$ & $\begin{array}{l}\text { No difference: No significant } \\
\text { difference between other } \\
\text { treatments prior to IVF and } \\
\text { immediate IVF. }\end{array}$ & $\begin{array}{l}\text { Low } \\
\text { (Imprecise) }\end{array}$ \\
\hline & $\begin{array}{l}\text { Pregnancy } \\
\text { complications: } \\
\text { Ectopic } \\
\text { pregnancy }\end{array}$ & $\begin{array}{l}3 \text { RCTs } 52,210,212,240 \\
(812)\end{array}$ & $\begin{array}{l}\text { No difference: No significant } \\
\text { difference between other } \\
\text { treatments prior to IVF and } \\
\text { immediate IVF. }\end{array}$ & $\begin{array}{l}\text { Low } \\
\text { (Imprecise) }\end{array}$ \\
\hline & $\begin{array}{l}\text { Pregnancy } \\
\text { complications: } \\
\text { Miscarriage }\end{array}$ & $\begin{array}{l}\text { 3 RCTs } 52,210,212,240 \\
(812)\end{array}$ & $\begin{array}{l}\text { No difference: No significant } \\
\text { difference between other } \\
\text { treatments prior to IVF and } \\
\text { immediate IVF. }\end{array}$ & $\begin{array}{l}\text { Low } \\
\text { (Imprecise) }\end{array}$ \\
\hline & $\begin{array}{l}\text { Neonatal } \\
\text { outcomes: } \\
\text { Death }\end{array}$ & $\begin{array}{l}2 \mathrm{RCTS}^{52,210} \\
(657)\end{array}$ & $\begin{array}{l}\text { Inconclusive: SOE was } \\
\text { insufficient given imprecise } \\
\text { evidence for a rare event which } \\
\text { requires a larger data set to draw } \\
\text { inferences }\end{array}$ & $\begin{array}{l}\text { Insufficient } \\
\text { (Imprecise, rare } \\
\text { events) }\end{array}$ \\
\hline & $\begin{array}{l}\text { Neonatal } \\
\text { outcomes: } \\
\text { Birthweight }\end{array}$ & $\begin{array}{l}2 \mathrm{RCTs}^{52,210} \\
(657)\end{array}$ & $\begin{array}{l}\text { No difference: No significant } \\
\text { difference between other } \\
\text { treatments prior to IVF and } \\
\text { immediate IVF. }\end{array}$ & $\begin{array}{l}\text { Low } \\
\text { (Imprecise) }\end{array}$ \\
\hline & $\begin{array}{l}\text { Time to } \\
\text { pregnancy }\end{array}$ & $\begin{array}{l}2 \mathrm{RCTS}^{52,210} \\
(657)\end{array}$ & $\begin{array}{l}\text { Reduction: Shorter time to } \\
\text { pregnancy with immediate IVF } \\
\text { compared with other treatments } \\
\text { prior to IVF }\end{array}$ & Moderate \\
\hline & Costs & $\begin{array}{l}1 \mathrm{RCT}^{52} \\
(619)\end{array}$ & $\begin{array}{l}\text { Inconclusive: Insufficient SOE } \\
\text { given one study with imprecise } \\
\text { and overlapping findings }\end{array}$ & $\begin{array}{l}\text { Insufficient } \\
\text { (Imprecise, one } \\
\text { study) }\end{array}$ \\
\hline & $\begin{array}{l}\text { Short term } \\
\text { adverse effects } \\
\text { of treatment: } \\
\text { OHSS }\end{array}$ & $\begin{array}{l}2 \mathrm{RCTs}^{52,210} \\
(657)\end{array}$ & $\begin{array}{l}\text { No difference: No significant } \\
\text { difference between other } \\
\text { treatments prior to IVF and } \\
\text { immediate IVF. }\end{array}$ & $\begin{array}{l}\text { Low } \\
\text { (Imprecise) }\end{array}$ \\
\hline \multirow[t]{3}{*}{$\begin{array}{l}\text { Expectancy } \\
\text { Management } \\
\text { vs. Other } \\
\text { Interventions }\end{array}$} & Live birth & $\begin{array}{l}1 \mathrm{RCT}^{119} \\
(161) \\
\mathrm{SR}^{84}(1 \text { study, } 51 \\
\text { patients) }\end{array}$ & $\begin{array}{l}\text { Inconclusive: Insufficient SOE } \\
\text { given inconsistent findings from } \\
\text { small studies }\end{array}$ & $\begin{array}{l}\text { Insufficient } \\
\text { (Inconsistent; small } \\
\text { studies) }\end{array}$ \\
\hline & $\begin{array}{l}\text { Pregnancy } \\
\text { complications: } \\
\text { Multiple births }\end{array}$ & $1 \mathrm{RCT}^{228}(105)$ & $\begin{array}{l}\text { Inconclusive: Insufficient SOE } \\
\text { given imprecise findings from } \\
\text { one small study }\end{array}$ & $\begin{array}{l}\text { Insufficient } \\
\text { (Imprecise findings } \\
\text { from small study) }\end{array}$ \\
\hline & $\begin{array}{l}\text { Pregnancy } \\
\text { complications: } \\
\text { Miscarriage }\end{array}$ & $1 \mathrm{RCT}^{228}(105)$ & $\begin{array}{l}\text { Inconclusive: Insufficient SOE } \\
\text { given imprecise findings from } \\
\text { one small study }\end{array}$ & $\begin{array}{l}\text { Insufficient } \\
\text { (Imprecise findings } \\
\text { from small study) }\end{array}$ \\
\hline
\end{tabular}




\begin{tabular}{|c|c|c|c|c|}
\hline Comparison & Outcome & $\begin{array}{l}\text { Study Design } \\
\text { (Sample Size) }\end{array}$ & Conclusion & $\begin{array}{l}\text { Strength of } \\
\text { Evidence } \\
\text { (Rationale) }^{\mathrm{a}}\end{array}$ \\
\hline & Costs & $1 \mathrm{RCT}^{139}(253)$ & $\begin{array}{l}\text { Inconclusive: Insufficient SOE } \\
\text { given imprecise findings from } \\
\text { one small study published } \\
\text { outside US }\end{array}$ & $\begin{array}{l}\text { Insufficient } \\
\text { (Imprecise findings } \\
\text { from small study) }\end{array}$ \\
\hline \multirow[t]{2}{*}{$\begin{array}{l}\text { ART: } \\
\text { ICSI }\end{array}$} & Live birth & $\begin{array}{l}1 \mathrm{RCT}^{214} \\
(156)\end{array}$ & $\begin{array}{l}\text { Inconclusive: Insufficient SOE } \\
\text { given imprecise findings from } \\
\text { one study with moderate risk of } \\
\text { bias }\end{array}$ & $\begin{array}{l}\text { Insufficient } \\
\text { (Imprecise findings } \\
\text { with moderate } \\
\text { study limitations) }\end{array}$ \\
\hline & $\begin{array}{l}\text { Pregnancy } \\
\text { complications: } \\
\text { Miscarriage }\end{array}$ & $\begin{array}{l}1 \mathrm{RCT}^{214} \\
(156)\end{array}$ & $\begin{array}{l}\text { Inconclusive: Insufficient SOE } \\
\text { given imprecise findings from } \\
\text { one study with moderate risk of } \\
\text { bias }\end{array}$ & $\begin{array}{l}\text { Insufficient } \\
\text { (Imprecise findings } \\
\text { with moderate } \\
\text { study limitations) }\end{array}$ \\
\hline \multirow[t]{2}{*}{$\begin{array}{l}\text { ART: } \\
\text { IVF vs. ICSI }\end{array}$} & Costs & $\begin{array}{l}1 \mathrm{Obs}^{215} \\
(154)\end{array}$ & $\begin{array}{l}\text { Inconclusive: SOE was } \\
\text { insufficient given imprecise } \\
\text { evidence from } 1 \text { small } \\
\text { observational trial. }\end{array}$ & $\begin{array}{l}\text { Insufficient } \\
\text { (Imprecise, one } \\
\text { small study) }\end{array}$ \\
\hline & $\begin{array}{l}\text { Neonatal } \\
\text { outcomes: Birth } \\
\text { weight }\end{array}$ & $\begin{array}{l}1 \text { Obs }^{185} \\
(90,401 \text { cycles })\end{array}$ & $\begin{array}{l}\text { No difference: No significant } \\
\text { differences in rates of } r \text { low birth } \\
\text { weight (OR } 1.03,95 \% \mathrm{CI} 0.73 \text { to } \\
\text { 1.47) between ICSI versus } \\
\text { conventional-IVF cycles }\end{array}$ & $\begin{array}{l}\text { Low (one study } \\
\text { with moderate } \\
\text { study limitations) }\end{array}$ \\
\hline $\begin{array}{l}\text { ART: } \\
\text { Unspecified }\end{array}$ & $\begin{array}{l}\text { Long-term } \\
\text { outcomes: Child } \\
\text { (cancer) }\end{array}$ & $\begin{array}{l}1 \mathrm{Obs}^{213} \\
(33,840)\end{array}$ & $\begin{array}{l}\text { No difference: The overall } \\
\text { cancer incidence was not } \\
\text { elevated in children born after } \\
\text { assisted conception for } \\
\text { unexplained infertility. The SIR } \\
\text { for all cancers in children } \\
\text { conceived with assisted } \\
\text { conception for unexplained } \\
\text { infertility compared to the } \\
\text { general population of the same } \\
\text { age was } 0.84(95 \% \mathrm{Cl}, 0.57 \text { to } \\
\text { 1.18). }\end{array}$ & $\begin{array}{l}\text { Low } \\
\text { (Moderate study } \\
\text { limitations) }\end{array}$ \\
\hline
\end{tabular}

${ }^{\mathrm{a} C r i t e r i a}$ for downgrading strength of evidence is described as Rationale; when these criteria are insufficient for understanding the final strength of evidence, additional explanation is provided.

Abbreviations: AH=assisted hatching; AMA=advanced maternal age; ART=assisted reproductive technology; CC=clomiphene citrate; $\mathrm{CI}=$ confidence interval; DHEA=dehydroepiandrosterone; FASTT=Fast Track and Standard Treatment; FSH=folliclestimulating hormone; hMG=human menopausal gonadotropin; HR=hazard ratio; ICER=incremental cost-effectiveness ratio; ICSI=intra-cytoplasmic sperm injection; IUI=intrauterine insemination; IVF=in vitro fertilization; KQ=Key Question; N=number of patients/participants; NA=not applicable; Obs=observational study; OHSS=ovarian hyperstimulation syndrome; OR=odds ratio; PGS=preimplantation genetic screening; $\mathrm{RCT}=$ =randomized controlled trial; $\mathrm{rFSH}=$ recombinant follicle-stimulating hormone; RIF=recurrent implantation failure; SIR=standardized incidence ratio; uFSH=urinary follicle-stimulating hormone

\title{
Key Question 4. Tubal and Peritoneal Factor Infertility
}

KQ 4 . What are the comparative safety and effectiveness of available treatment strategies for women with tubal or peritoneal factors (e.g., pelvic adhesions) who are infertile and who wish to become pregnant?

\author{
KQ 4a. Does the optimal treatment strategy vary by patient \\ characteristics such as age, ovarian reserve, race, BMI, presence of \\ other potential causes of female infertility, or presence of male factor \\ infertility?
}




\section{Description of Included Studies for KQ 4 (Tubal and Peritoneal Factor Infertility)}

We identified eight individual studies ${ }^{123,184,185,248-252}$ that addressed outcomes after treatment for tubal or peritoneal factor infertility.

Among the eight studies that addressed outcomes after treatment for tubal or peritoneal factor infertility, three were RCTs. One was rated as good quality, ${ }^{250}$ one was rated as fair quality, ${ }^{252}$ and one was rated as poor quality. ${ }^{248}$ The remaining five studies were observational; three were good quality, ${ }^{184,249,251}$ and two were fair quality. ${ }^{123,185}$ Geographically, three studies were conducted in the U.K. or continental Europe ${ }^{184,249,250}$, three were conducted in the U.S. ${ }^{123,185,251}$, and two were conducted in Asia. ${ }^{248,252}$ Four studies were conducted in, or used data from, fertility subspecialty clinics, ${ }^{185,250-252}$ whereas setting was not specified for the remaining four. ${ }^{123,184,248,249}$ With regard to funding source, one study was government funded, ${ }^{252}$, one was

industry funded, ${ }^{250}$ two reported non-government, non-industry funding, ${ }^{123,184}$ and, for the remaining four, funding source was not specified or was unclear. ${ }^{185,248,249,251}$

Outcomes and complications of pregnancy were described in three RCTs ${ }^{248,250,252}$ and five observational studies. ${ }^{27,123,124,185,251}$ One observational study reported on long-term outcomes in the child. ${ }^{184}$ One observational study reported on treatment costs. ${ }^{249}$

The main classes of treatment investigated were oral ovulation induction with or without IUI, gonadotropins with or without IUI, surgical treatment and surgery with hormonal adjunctive therapy, and ART (IVF or ICSI) alone or with adjunctive acupressure treatment. We did not perform meta-analysis because of the lack of studies reporting results for similar outcomes and treatment comparisons.

In addition to the above studies, one good-quality systematic review that addressed the comparative effectiveness of various treatments for infertility in women with tubal factor infertility (5 studies, 646 patients) is discussed below and the consistency of its findings with our included studies is incorporated in to our strength of evidence ratings. ${ }^{93}$

\section{Key Points for Tubal or Peritoneal Factor Infertility}

Key findings for patients with tubal or peritoneal factor infertility included:

- As with other indications for IVF, use of single-embryo transfer is associated with slightly lower live birth rates but significantly reduced multiple gestation rates (low SOE)

- The live birth rate was lower in women undergoing ICSI as compared to conventional IVF (low SOE)

- There was no difference between type 1 diabetes mellitus diagnoses in children born to patients with tubal factor infertility conceived with ART compared to children conceived with no fertility treatment (moderate SOE)

- SOE was rated insufficient for all other comparisons/outcomes.

\section{Detailed Synthesis by Treatment for Tubal or Peritoneal Factor Infertility}

Included studies and their findings for the following treatments for tubal or peritoneal infertility are detailed in this section:

1. Surgical Management

2. ART 


\section{Surgical Management for Tubal or Peritoneal Factor Infertility}

Three studies and one systematic review explored the use of surgical management strategies in women with tubal or peritoneal factor infertility.

We identified one RCT of a surgical intervention that examined live birth outcomes. A goodquality $\mathrm{RCT}^{250}$ compared hysteroscopic proximal tubal occlusion via Essure ${ }^{\circledR}$ to laparoscopic salpingectomy prior to IVF/ICSI in 85 women. They found that there was no difference in pregnancy complications of miscarriage or ectopic pregnancy between groups, but that women who underwent laparoscopic salpingectomy had a significantly higher live birth rate than women who underwent Essure ${ }^{\circledR}$ prior to IVF/ICSI in the ITT analysis (21.4\% vs. 46.5\%, respectively). However, this difference was no longer significant in the per protocol analyses $(p=0.143)$. Given imprecise findings from just one small study the SOE was rated as insufficient.

The risk of ectopic pregnancy was examined in one poor-quality RCT of women who underwent transcervical falloposcopy tubal dilatation as an adjunctive therapy during surgical treatment of tubal fertility. ${ }^{248}$ The study examined 468 infertile women with evidence of fallopian tube disease who underwent laparoscopic salpingolysis and/or salpingostomy. Patients were included if they had tubal factor infertility on hysterosalpingography, and were excluded if they had evidence of uterine fibroids, endometriosis, male factor infertility, genital malformations, unilateral salpingectomy, or endocrine pathology. A total of 256 patients were randomized to the intervention, transcervical falloposcopy tubal dilatation, and 212 patients did not receive the additional procedure (control group). The ectopic pregnancy rate in women who underwent transcervical falloposcopy tubal dilatation was $2 \%$ compared with $5.4 \%$ in the control group ( $\mathrm{p}=0.647$ ) (insufficient $\mathrm{SOE}$ ).

One good-quality study evaluated costs of different diagnostic and treatment scenarios for women with tubal infertility. ${ }^{249}$ Six different scenarios involving different diagnostic approaches (no diagnostics, hysterosalpingogram, laparoscopy) and delayed or immediate IVF treatment were examined using a computer-generated Markov model. Costs were based on those in the Netherlands. Costs per live birth of the various diagnostic and treatment scenarios differed by more than 3000 euros. The costliest intervention was no diagnostics with immediate IVF treatment (8927 euros), and the least expensive was no diagnostics with 12 cycles of expectant management followed by 3 cycles of IVF (6459 euros) (insufficient SOE).

Finally, one good-quality Cochrane systematic review involving 5 RCTs with a total of 646 women explored surgical treatment for tubal disease in women undergoing IVF. None of the included trials reported on the primary outcome of live birth, and therefore they were excluded from our systematic review. They did, however, report an increase in ongoing pregnancy and clinical pregnancy with laparoscopic salpingectomy for hydrosalpinges prior to IVF versus nonsurgical management. No significant differences were seen in any of the adverse effects of surgical treatments. ${ }^{93}$

\section{ART for Tubal or Peritoneal Factor Infertility}

One RCT ${ }^{252}$ and four observational studies ${ }^{123,184,185,251}$ examined treatment outcomes in women with tubal factor infertility undergoing IVF/ICSI.

One fair quality RCT ${ }^{252}$ compared three different frequencies of transcutaneous electrical acupoint stimulation (TEAS) and a non-TEAS control group prior to IVF among 481 women with bilateral tubal blockage. ${ }^{252}$ Those assigned to one of the three TEAS arms received 30minutes of TEAS 24 hours before transvaginal oocyte retrieval and 2 hours after embryo transfer. TEAS was administered at low frequency $(2 \mathrm{~Hz})$, high frequency $(100 \mathrm{~Hz})$, or 
alternating low and high frequencies $(2 / 100 \mathrm{~Hz})$. The women receiving TEAS at alternating low and high frequencies $2 / 100 \mathrm{~Hz}$ had a higher live birth rate (55/144, 48.25\%) when compared to each of the other study arms $(\mathrm{p}<0.05)$.

Live birth rates was also evaluated in one observational study. The good-quality study ${ }^{251}$ used the Society for Assisted Reproductive Technology Clinic Outcome Reporting System (SART CORS) database to compare outcomes for conventional IVF and use of ICSI in women with tubal ligation. In adjusted analyses, use of ICSI was associated with lower odds of live birth (AOR 0.77, 0.69-0.85) (low SOE). There were no significant differences in plurality, mean length of gestation, or birth weight. ${ }^{251}$

An analysis of the SART CORS database compared success and complications associated with treatment patterns for women undergoing IVF/ICSI: live birth rates and maternal and neonatal complications based on the number of embryos transferred in 69,028 ART cycles. ${ }^{123}$ Among women with tubal infertility, the live birth rate after treatment with IVF or ICSI was 44.4 percent. In women with tubal fertility, the live birth rate was higher in couples who underwent two embryo transfer (47.5\%) as compared with single-embryo transfer $(41.1 \%)(p=0.05)$ (low SOE). ${ }^{123}$

Other outcomes of interest were evaluated in three observational studies. One fair-quality study ${ }^{185}$ used the SART CORS database to examine prevalence of preterm delivery and low birth weight among singletons conceived with ICSI compared to conventional IVF. In secondary analyses conducted on the subset of patients who used autologous sperm, had a favorable fertility prognosis based on female age $<35$, and $>$ three oocytes retrieved, no significant differences in rates of preterm delivery or low birth weight was observed among those with tubal factor only infertility (preterm delivery OR 1.14, 95\% CI -.79 to 1.65, $\mathrm{p}=.49$; low birth weight OR 0.88, 95\% CI 0.56 to $1.37, \mathrm{p}=.56$ ) (low SOE). ${ }^{185}$

One nationwide birth cohort study identified all pregnancies with a live-single born child over an 8-year period in Denmark and compared the incidence of type I diabetes among those conceived with fertility treatment to those conceived naturally. ${ }^{184}$ There was no association between tubal factor infertility as an indicator for fertility treatment and the subsequent development of Type I diabetes in offspring (HR 1.08, 95\% to 0.61 to 1.91) (moderate SOE).

\section{Strength of Evidence for Tubal and Peritoneal Factor Infertility}

Table 25 summarizes the SOE for the findings described above. The SOE was judged to be insufficient for all outcomes primarily due to imprecision based on few studies meeting our inclusion criteria.

Table 25. Strength of evidence for major outcomes-KQ 4 (tubal and peritoneal factor infertility)

\begin{tabular}{|l|l|l|l|l|}
\hline \multicolumn{1}{|c|}{ Comparison } & \multicolumn{1}{|c|}{ Outcome } & $\begin{array}{l}\text { Study Design } \\
\text { (Sample Size) }\end{array}$ & \multicolumn{1}{c|}{$\begin{array}{c}\text { Conclusion } \\
\text { Strength of } \\
\text { Evidence } \\
\text { (Rationale) }\end{array}$} \\
\hline $\begin{array}{l}\text { Hysteroscopic } \\
\text { proximal } \\
\text { occlusion vs. } \\
\text { laparoscopic } \\
\text { salpingectomy }\end{array}$ & Live birth (patient) & $\begin{array}{l}1 \mathrm{RCTS}{ }^{250} \\
(85)\end{array}$ & $\begin{array}{l}\text { Inconclusive. SOE was } \\
\text { insufficient given imprecise } \\
\text { findings from 1 small study }\end{array}$ & $\begin{array}{l}\text { Insufficient } \\
\text { (Imprecise, 1 } \\
\text { small study) }\end{array}$ \\
\hline $\begin{array}{l}\text { Transcervical } \\
\text { falloposcopy } \\
\text { tubal dilatation } \\
\text { vs. no } \\
\text { intervention }\end{array}$ & $\begin{array}{l}\text { Pregnancy } \\
\text { complications: } \\
\text { Ectopic pregnancy }\end{array}$ & $\begin{array}{l}1 \mathrm{RCT}^{248} \\
(468)\end{array}$ & $\begin{array}{l}\text { Inconclusive. SOE was } \\
\text { insufficient given imprecise } \\
\text { evidence from 1 trial with high } \\
\text { potential limitations }\end{array}$ & $\begin{array}{l}\text { Insufficient } \\
\text { (Imprecise } \\
\text { findings with } \\
\text { high study } \\
\text { limitations, } 1 \\
\text { study) }\end{array}$ \\
\hline
\end{tabular}




\begin{tabular}{|c|c|c|c|c|}
\hline Comparison & Outcome & $\begin{array}{l}\text { Study Design } \\
\text { (Sample Size) }\end{array}$ & Conclusion & $\begin{array}{l}\text { Strength of } \\
\text { Evidence } \\
\text { (Rationale) }^{\mathrm{a}}\end{array}$ \\
\hline $\begin{array}{l}\text { Hysterosal- } \\
\text { pingogram, } \\
\text { laparoscopy, no } \\
\text { intervention vs. } \\
\text { IVF }\end{array}$ & Costs & $\begin{array}{l}1 \mathrm{Obs}^{249} \\
\text { (NA) }\end{array}$ & $\begin{array}{l}\text { Inconclusive. SOE was } \\
\text { insufficient given imprecise } \\
\text { evidence from } 1 \text { modeling } \\
\text { study }\end{array}$ & $\begin{array}{l}\text { Insufficient } \\
\text { (Imprecise; } \\
\text { indirect findings, } \\
1 \text { study) }\end{array}$ \\
\hline $\begin{array}{l}\text { ART: 2-embryo } \\
\text { transfer vs. 1- } \\
\text { embryo transfer }\end{array}$ & Live birth (patient) & $\begin{array}{l}1 \text { Obs }^{123} \\
(69,028 \text { cycles })\end{array}$ & $\begin{array}{l}\text { Improvement. The live birth } \\
\text { rate per cycle was higher in } \\
\text { couples who underwent } 2 \\
\text { embryo transfer as compared } \\
\text { with single-embryo transfer }\end{array}$ & $\begin{array}{l}\text { Low } \\
\text { (Imprecise) }\end{array}$ \\
\hline $\begin{array}{l}\text { ART: } \\
\text { Frequencies of } \\
\text { transcutaneous } \\
\text { electrical } \\
\text { acupoint } \\
\text { stimulation prior } \\
\text { to IVF }\end{array}$ & Live birth (patient) & $1 \mathrm{RCT}^{252}(481)$ & $\begin{array}{l}\text { Inconclusive. SOE was } \\
\text { insufficient given imprecise } \\
\text { findings from } 1 \text { fair-quality } \\
\text { study }\end{array}$ & $\begin{array}{l}\text { Insufficient } \\
\text { (Imprecise, } 1 \\
\text { fair-quality } \\
\text { study) }\end{array}$ \\
\hline $\begin{array}{l}\text { ART: IVF vs. } \\
\text { ICSI }\end{array}$ & Live birth (patient) & $\begin{array}{l}1 \mathrm{Obs}^{251} \\
(7145)\end{array}$ & $\begin{array}{l}\text { Reduction: Lower odds of } \\
\text { live birth with ICSI }\end{array}$ & $\begin{array}{l}\text { Low } \\
\text { (1 study) }\end{array}$ \\
\hline $\begin{array}{l}\text { ART: IVF+ICSI } \\
\text { vs. IVF }\end{array}$ & $\begin{array}{l}\text { Neonatal } \\
\text { outcomes: Birth } \\
\text { weight }\end{array}$ & $\begin{array}{l}1 \text { Obs }^{185} \\
(90,401 \text { cycles })\end{array}$ & $\begin{array}{l}\text { No difference: No significant } \\
\text { differences in rates of low } \\
\text { birth weight (OR } 0.88,95 \% \mathrm{Cl} \\
0.56 \text { to } 1.37 \text { ) between ICSI } \\
\text { versus conventional-IVF } \\
\text { cycles }\end{array}$ & $\begin{array}{l}\text { Low } \\
\text { (1 study with } \\
\text { moderate study } \\
\text { limitations) }\end{array}$ \\
\hline $\begin{array}{l}\text { ART vs. no } \\
\text { fertility treatment }\end{array}$ & $\begin{array}{l}\text { Long-term } \\
\text { outcomes: Child } \\
\text { (type } 1 \text { diabetes } \\
\text { mellitus) }\end{array}$ & $\begin{array}{l}1 \mathrm{Obs}^{184} \\
(565,116 \\
\text { pregnancies) }\end{array}$ & $\begin{array}{l}\text { No difference: No significant } \\
\text { difference found between } \\
\text { type } 1 \text { diabetes mellitus } \\
\text { diagnoses in children born to } \\
\text { patients with tubal factor } \\
\text { infertility conceived with ART } \\
\text { compared to children } \\
\text { conceived with no fertility } \\
\text { treatment }\end{array}$ & $\begin{array}{l}\text { Moderate } \\
\text { (Imprecise) }\end{array}$ \\
\hline
\end{tabular}

${ }^{a}$ Criteria for downgrading strength of evidence is described as Rationale; when these criteria are insufficient for understanding the final strength of evidence, additional explanation is provided.

Abbreviations: ART=assisted reproductive technology; $\mathrm{CI}=$ confidence interval; HR=hazard ratio; ICSI=intra-cytoplasmic sperm injection; IUI=intrauterine insemination; IVF=in vitro fertilization; $\mathrm{KQ}=$ Key Question; $\mathrm{N}=$ number of patients/participants; NA=not applicable; Obs=observational study; RCT=randomized controlled trial; RR=relative risk; SIR=standardized incidence ratio

\title{
Key Question 5. Male Factor Infertility
}

KQ 5. What are the comparative safety and effectiveness of available treatment strategies for couples with male factor infertility and no evidence of an underlying diagnosis associated with infertility in the female partner?

\author{
KQ 5a. Does the optimal treatment strategy vary by characteristics in \\ either partner such as age, ovarian reserve, race, or BMI?
}




\section{Description of Included Studies for KQ 5 (Male Factor)}

We identified 23 individual studies ${ }^{119,123-125,184-186,208,213,221,236,242,253-264}$ that addressed the comparative effectiveness or safety of interventions applied to patients with male factor infertility.

Eleven studies were RCTs $119,208,221,242,253,255,256,258,261,262,264$ and 12 were observational studies. ${ }^{123,124,184-186,213,236,254,257,259,260,263}$ Of the 11 RCTs, four studies were rated good quality, ${ }^{119,208,261,262}$ five were rated fair quality, ${ }^{221,242,256,258,264}$ and two were rated poor quality. ${ }^{253,255}$ Of the 12 observational studies, three were rated good quality, ${ }^{184,186,236}$ seven were rated fair quality, ${ }^{123,124,185,213,254,260,263}$ and two were rated poor quality. ${ }^{257,259}$

Of the 23 studies, six were conducted in the United States, ${ }^{123,124,185,236,254,263} 10$ in the UK or continental Europe, ${ }^{119,184,186,213,221,242,253,256,259,264}$ five in the Middle East, ${ }^{208,255,258,260,261}$ and two in Asia. ${ }^{257,262}$ All but three studies were conducted in subspecialty practices; the remaining three did not report the setting or the setting was unclear. ${ }^{123,184,213}$ Finally, six studies reported government funding, ${ }^{119,213,254,256,259,262}$ one reported industry funding, ${ }^{186}$ three studies reported non-government, non-industry funding, ${ }^{123,184,257}$ two studies reported a combination of funding sources, ${ }^{124,236}$ and the remaining eleven studies did not report funding source or the funding source was unclear. ${ }^{185,208,221,242,253,255,258,260,261,263,264}$ The interventions and comparisons evaluated in the included studies are summarized in Appendix E.

In addition to the above studies, four good-quality systematic reviews ${ }^{72,79,265,266}$ that addressed the comparative effectiveness of various treatments in men with male factor infertility are discussed below and the consistency of their findings with our included studies are incorporated in to our strength of evidence ratings.

\section{Key Points for Male Factor Infertility}

Key findings for patients with male factor infertility included:

- Live birth rate (moderate SOE) and miscarriage (low SOE) did not differ between intracytoplasmic sperm injection (ICSI) and intracytoplasmic morphological sperm injection (IMSI). Of note, IMSI is not used in the United States.

- There was no difference in live birth rates or any adverse pregnancy events between couples using frozen embryo versus fresh embryo transfer (low SOE)

- The overall cancer incidence was not elevated in children born after assisted conception for male factor infertility (low SOE).

- There was no difference between type 1 diabetes mellitus diagnoses in children born to patients with male factor infertility conceived with ART compared to children conceived with no fertility treatment (moderate SOE)

- Live birth rate (low SOE) improved with vitamin E or zinc supplementation relative to placebo or no supplementation.

- As with other indications for IVF, use of single-embryo transfer is associated with slightly lower live birth rates but significantly reduced multiple gestation rates (low SOE). 


\section{Detailed Synthesis by Treatment for Male Factor Infertility}

Included studies and their findings for the following treatments for unexplained infertility are detailed in this section:

1. IUI With Adjunct Treatment for Female Partners

2. ART
a. IVF
b. ICSI
c. IVF Versus ICSI
d. ART Unspecified

3. Other Strategies

\section{IUI With Adjunct Treatments for Female Partners With Male Factor Infertility}

A single fair-quality $\mathrm{RCT}^{221}$ that compared single versus double IUI in multifollicular ovarian hyperstimulation cycles reported live birth rates and pregnancy complications. Clomiphene citrate or recombinant FSH or both were used for ovarian hyperstimulation in both study arms. Of the 228 women in the trial, male factor was the cause of infertility for 67 couples in the single IUI arm and 65 couples in the double IUI arm. Among the couples with male factor infertility, double IUI was associated with a crude OR of 0.71 (95\% CI, 0.21 to 2.37; p=0.764) relative to single IUI for live birth rate. This trial was conducted in a single subspecialty clinic in Turkey and given the imprecise findings was rated as insufficient strength of evidence.

A single good-quality RCT ${ }^{208}$ compared rFSH with clomiphene citrate in IUI cycles in couples with infertility. Of the 219 couples in the trial, male factor was the cause of infertility for 15 couples in rFSH group and 22 couples in the clomiphene citrate group. Among the couples with male factor infertility, the live birth rate per patient was 20 percent in the rFSH group and 13.6 percent in the clomiphene citrate group (p value reported as "not significant”). This trial was conducted in a single subspecialty clinic in Turkey (insufficient SOE).

Related to these included studies, two good-quality systematic reviews published in $2007^{265}$ and $2017^{266}$ evaluated outcomes associated with IUI for male-factor infertility. The study by Bensdorp, 2007 compared ovarian hyperstimulation with IUI with IUI alone among couples with male factor infertility did not demonstrate a significant difference in live birth rates (OR 0.87; 95\% CI, 0.28 to 2.70). The more recent systematic review by Cissen, 2016 also compared ovarian hyperstimulation with IUI with IUI alone among couples with male factor infertility summarizing 3 RCTs of a total of 346 couples (OR 1.34, 95\% CI 0.77, 2.33; $\mathrm{I}^{2}=0 \%$ ) findings no evidence of a difference with live births. They noted very low quality of evidence for this outcome given potential high risk of bias in the included studies, inconsistencies in the data, and imprecise findings. This review also identified two RCTs (1 low quality and 1 very low quality) examining IVF versus IUI in natural cycles or cycles with ovarian hyperstimulation. This review found no evidence of a difference in live birth rates between IVF versus IUI in natural cycles (1 RCT, 53 couples; OR 0.77, 95\% CI 0.25, 2.53) or IVF versus IUI in cycles with ovarian hyperstimulation ( 2 RCTs, 86 couples; OR 1.03 95\% CI 0.43, 2.45; $\mathrm{I}^{2}=0 \%$ ) and again with very low quality evidence. 


\section{ART for Male Factor Infertility}

\section{IVF for Male Factor Infertility}

Three studies reported findings from comparisons of varying IVF procedures for patients with male factor infertility. ${ }^{13,257,262}$ Findings for these studies are summarized in Table 26. We identified one good-quality RCT that investigated fresh versus frozen embryo transfer on multiple outcomes including live birth rates, multiples, birth weight, ectopic pregnancy, and congenital anomalies. ${ }^{262}$ Patients within this RCT had either tubal or male factor infertility and the results were not stratified by type. Approximately 40 percent of the population had male factor or both male and tubal factor infertility. The strength of evidence for the findings from this trial was reduced given the inclusion of couples with tubal factor infertility in the cohort.

Two additional studies reported live birth rate per cycle, ${ }^{123,257}$, one of which also reports neonatal outcomes and congenital anomalies. ${ }^{257}$

Table 26. Outcomes for comparisons of IVF in couples with male infertility

\begin{tabular}{|c|c|c|c|c|c|c|c|}
\hline Outcome & $\begin{array}{c}\text { Study } \\
\text { Design } \\
\text { (N Patients) }\end{array}$ & Intervention & Comparator & $\begin{array}{c}\text { Results } \\
\text { Intervention } \\
\mathbf{N} \\
(\%) \\
(95 \% \mathrm{Cl}) \\
\end{array}$ & $\begin{array}{c}\text { Results } \\
\text { Comparator } \\
\mathrm{N} \\
(\%) \\
(95 \% \mathrm{Cl}) \\
\end{array}$ & $\begin{array}{c}P \\
\text { value }\end{array}$ & $\begin{array}{l}\text { Summary } \\
\text { of Study } \\
\text { Findings }\end{array}$ \\
\hline \multirow[t]{3}{*}{$\begin{array}{l}\text { Live birth: } \\
\text { Any/patient }\end{array}$} & $\begin{array}{c}\text { Tsai, } 2011^{257} \\
\text { RCT } \\
\text { (191 cycles) }\end{array}$ & TESE & $\begin{array}{l}\text { Ejaculated } \\
\text { OAT }\end{array}$ & $40.7 \%$ & $30.2 \%$ & 0.197 & $\begin{array}{c}\text { No } \\
\text { difference }\end{array}$ \\
\hline & $\begin{array}{c}\text { Luke, } \\
2010^{123} \\
\\
\text { RCT } \\
\text { (69,028 ART } \\
\text { cycles) }\end{array}$ & $\begin{array}{c}\text { ICSI or } \\
\text { assisted } \\
\text { hatching (1 } \\
\text { embryo } \\
\text { transferred) }\end{array}$ & $\begin{array}{c}\text { ICSI or } \\
\text { assisted } \\
\text { hatching } \\
\text { (multiple } \\
\text { embryos } \\
\text { transferred) }\end{array}$ & $44.7 \%$ & $\begin{array}{c}52.1 \%(2 \\
\text { embryos) } \\
46.9 \%(3 \\
\text { embryos) } \\
40.4 \%(4 \\
\text { embryos) } \\
\text { (number } \\
\text { transferred) }\end{array}$ & $\begin{array}{c}<0.00 \\
1\end{array}$ & $\begin{array}{c}\text { Greater } \\
\text { live births } \\
\text { with } \\
\text { multiple } \\
\text { embryos } \\
\text { transferre } \\
\text { d } \\
\text { compared } \\
\text { to } 1 \\
\text { embryo } \\
\text { transferre } \\
\text { d } \\
\end{array}$ \\
\hline & $\begin{array}{c}\text { Shi, } 2018^{262} \\
\text { RCT } \\
(2,157 \\
\text { patients }) \\
\end{array}$ & $\begin{array}{l}\text { Frozen } \\
\text { embryo } \\
\text { transfer }\end{array}$ & $\begin{array}{l}\text { Fresh embryo } \\
\text { transfer }\end{array}$ & $48.7 \%$ & $50.2 \%$ & 0.50 & $\begin{array}{c}\text { No } \\
\text { difference }\end{array}$ \\
\hline $\begin{array}{l}\text { Pregnancy } \\
\text { complications: } \\
\text { Ectopic } \\
\text { pregnancy }\end{array}$ & $\begin{array}{c}\text { Shi, } 2018^{262} \\
\text { RCT } \\
(2,157 \\
\text { patients }) \\
\end{array}$ & $\begin{array}{l}\text { Frozen } \\
\text { embryo } \\
\text { transfer }\end{array}$ & $\begin{array}{l}\text { Fresh embryo } \\
\text { transfer }\end{array}$ & $2.7 \%$ & $1.7 \%$ & 0.23 & $\begin{array}{c}\text { No } \\
\text { difference }\end{array}$ \\
\hline $\begin{array}{l}\text { Pregnancy } \\
\text { complications: } \\
\text { Miscarriage }\end{array}$ & $\begin{array}{c}\text { Shi, } 2018^{262} \\
\text { RCT } \\
(2,157 \\
\text { patients) } \\
\end{array}$ & $\begin{array}{l}\text { Frozen } \\
\text { embryo } \\
\text { transfer }\end{array}$ & $\begin{array}{l}\text { Fresh embryo } \\
\text { transfer }\end{array}$ & $\begin{array}{c}\text { Among clinical } \\
\text { pregnancies } \\
9.4 \%\end{array}$ & $\begin{array}{c}\text { Among } \\
\text { clinical } \\
\text { pregnancies } \\
11.5 \%\end{array}$ & 0.22 & $\begin{array}{c}\text { No } \\
\text { difference }\end{array}$ \\
\hline $\begin{array}{l}\text { Pregnancy } \\
\text { complications: } \\
\text { Multiple births }\end{array}$ & $\begin{array}{c}\text { Shi, } 2018^{262} \\
\text { RCT } \\
(2,157 \\
\text { patients }) \\
\end{array}$ & $\begin{array}{l}\text { Frozen } \\
\text { embryo } \\
\text { transfer }\end{array}$ & $\begin{array}{l}\text { Fresh embryo } \\
\text { transfer }\end{array}$ & $\begin{array}{c}\text { Singleton } 31.3 \% \\
\text { Twin } 17.4 \% \\
\text { Triplet } 0.1 \%\end{array}$ & $\begin{array}{c}\text { Singleton } \\
34.0 \% \\
\text { Twin } 16.0 \% \\
\text { Triplet } 0.2 \%\end{array}$ & $\begin{array}{l}0.18 \\
0.40 \\
1.00\end{array}$ & $\begin{array}{c}\text { No } \\
\text { difference }\end{array}$ \\
\hline
\end{tabular}




\begin{tabular}{|c|c|c|c|c|c|c|c|}
\hline Outcome & $\begin{array}{c}\text { Study } \\
\text { Design } \\
\text { (N Patients) }\end{array}$ & Intervention & Comparator & $\begin{array}{c}\text { Results } \\
\text { Intervention } \\
N \\
(\%) \\
(95 \% \mathrm{Cl})\end{array}$ & $\begin{array}{c}\text { Results } \\
\text { Comparator } \\
N \\
(\%) \\
(95 \% \mathrm{Cl})\end{array}$ & $\begin{array}{c}P \\
\text { value }\end{array}$ & $\begin{array}{l}\text { Summary } \\
\text { of Study } \\
\text { Findings }\end{array}$ \\
\hline \multirow[t]{2}{*}{$\begin{array}{l}\text { Neonatal } \\
\text { outcomes: } \\
\text { Birthweight }\end{array}$} & $\begin{array}{c}\text { Tsai, } 2011^{257} \\
\text { RCT } \\
\text { (191 cycles) } \\
\end{array}$ & TESE & $\begin{array}{l}\text { Ejaculated } \\
\text { extreme } \\
\text { severe OAT } \\
\text { sperm } \\
\end{array}$ & $38.3 \%$ & $23.8 \%$ & 0.292 & $\begin{array}{c}\text { No } \\
\text { difference }\end{array}$ \\
\hline & $\begin{array}{c}\text { Shi, } 2018^{262} \\
\text { RCT } \\
(2,157 \\
\text { patients })\end{array}$ & $\begin{array}{l}\text { Frozen } \\
\text { embryo } \\
\text { transfer }\end{array}$ & $\begin{array}{c}\text { Fresh embryo } \\
\text { transfer }\end{array}$ & $3373 g(+/-515)$ & $\begin{array}{c}3380 \mathrm{~g}(+/- \\
502)\end{array}$ & 0.85 & $\begin{array}{c}\text { No } \\
\text { difference }\end{array}$ \\
\hline \multirow[t]{2}{*}{$\begin{array}{l}\text { Neonatal } \\
\text { outcomes: } \\
\text { Congenital } \\
\text { anomalies }\end{array}$} & $\begin{array}{c}\text { Tsai, } 2011^{257} \\
\text { RCT } \\
\text { (191 cycles) } \\
\end{array}$ & TESE & $\begin{array}{l}\text { Ejaculated } \\
\text { extreme } \\
\text { sever OAT } \\
\text { sperm } \\
\end{array}$ & $\begin{array}{l}1.7 \% \text { (major) } \\
8.3 \% \text { (minor) }\end{array}$ & $\begin{array}{l}4.7 \% \text { (major) } \\
4.8 \% \text { (minor) }\end{array}$ & $\begin{array}{l}0.454 \\
0.591\end{array}$ & $\begin{array}{c}\text { No } \\
\text { difference }\end{array}$ \\
\hline & $\begin{array}{c}\text { Shi, } 2018^{262} \\
\text { RCT } \\
(2,157 \\
\text { patients })\end{array}$ & $\begin{array}{l}\text { Frozen } \\
\text { embryo } \\
\text { transfer }\end{array}$ & $\begin{array}{c}\text { Fresh embryo } \\
\text { transfer }\end{array}$ & $2.2 \%$ & $3.6 \%$ & 0.12 & $\begin{array}{c}\text { No } \\
\text { difference }\end{array}$ \\
\hline
\end{tabular}

Abbreviations: $\mathrm{CI}=$ confidence interval; $\mathrm{HR}=$ hazard ratio; ICSI=intra-cytoplasmic sperm injection; N=number of patients; NS=not statistically significant; OAT=oligo-astheno-teratozoospermia; RCT=randomized control trial; SOE = strength of evidence; TESE=extracted testicular sperm

\section{ICSI for Male Factor Infertility}

Two RCTs were identified for analyzing outcomes of male factor infertility while undergoing variations of ICSI. ${ }^{255,264}$ One fair-quality RCT investigated the effects of rFSH and rLH versus rFSH alone on miscarriage in couples with repeated implantation failures. The study found a significantly lower miscarriage rate in the rFSH and rLH group (21\%) as compared to the rFSH alone group (37.5\%; $<.01){ }^{264}$ Strength of evidence was rated as insufficient given findings from one small study with potential limitations.

The second RCT was rated as poor-quality. ${ }^{255}$ This study was conducted in Iran and had randomly allocated 182 couples undergoing ICSI for male factor infertility to laser assisted hatching $(n=90)$ or intact embryo transfer without laser assisted hatching $(n=92)$. The live birth rate per patient was $11.1 \%$ in the laser assisted hatching arm and $8.6 \%$ in the control arm $(p=0.06)$. This study reported 2 sets of twins among the 10 live births in the laser assisted hatching arm and 2 sets of twins among the 8 live births in the conventional ICSI arm (OR 0.6; 95\% CI, 0.08 to 6.9). In addition, one congenital anomaly was reported among the 10 live births in the laser assisted hatching arm and zero congenital anomalies among the 8 livebirths in the conventional ICSI arm (OR 0.9; 95\% CI, 0.07 to 1.1) (insufficient SOE for all outcomes). ${ }^{255}$

\section{IVF Versus ICSI for Male Factor Infertility}

\section{Live Birth}

Three RCTs ( 2 fair quality ${ }^{256,258}$ and 1 poor quality ${ }^{253}$ ) and 2 fair-quality observational studies ${ }^{124,254}$ reported live birth rates for comparisons between IVF and ICSI for couples with male factor infertility.

We conducted a meta-analysis of the live birth rates per cycle reported by the 3 RCTs summarized above that compared ICSI with IMSI (Figure 3). The summary estimate of the OR for live birth per cycle associated with ICSI was 1.218 (95\% CI, 0.779 to 1.903) using a fixed 
effect model (with identical results using a random effects model). The Q-value was 0.2913 with 2 degrees of freedom, with an $\mathrm{I}^{2}$ of $0 \%$, suggesting no significant heterogeneity. This metaanalysis corroborates the findings of each of the three primary studies, which is that there is no significant difference in live birth rates per cycle between ICSI and IMSI procedures for male factor infertility. The systematic review published in $2013^{79}$ included 9 trials with 2,014 couples however only the study by Balaban and colleagues ${ }^{258}$ reported on live birth. We rated the strength of evidence for no difference in live birth as moderate based on the findings of our meta-analysis.

Figure 3. Effect of ICSI versus IMSI on live birth

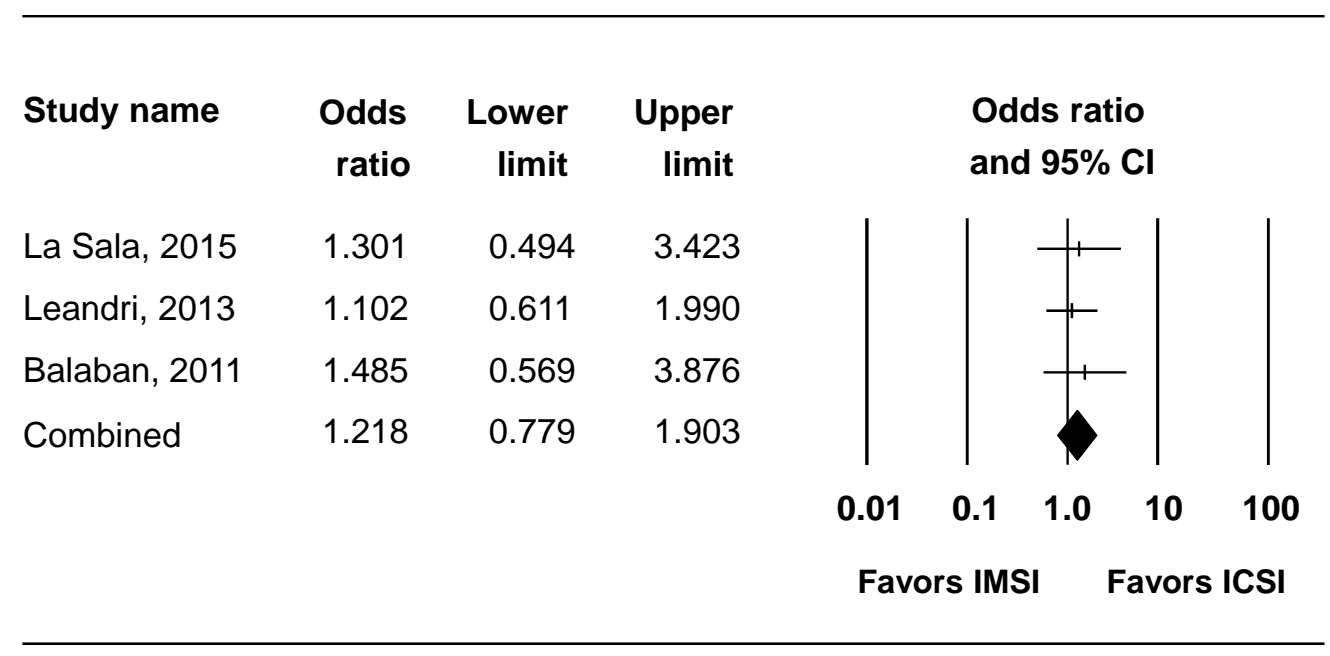

Abbreviations: $\mathrm{CI}=$ confidence interval; ICSI=intra-cytoplasmic sperm injection; IMSI=intra-cytoplasmic morphologically selected sperm injection

We also identified two fair-quality observational studies ${ }^{124,254}$ that analyzed data from the SART CORS database to compare ICSI with IVF for male factor infertility. These two studies may report data from the same patients. One of the studies compared live birth rates of both couples with male factor infertility who received conventional IVF ( $\mathrm{N}=4973$ cycles) or combined IVF with ICSI ( $\mathrm{N}=72,459$ cycles). ${ }^{124}$ Among couples with male factor infertility, the live birth rate for the 1989 cycles that resulted in a pregnancy after treatment with conventional IVF was 84.9\%, compared with $85.2 \%$ live birth rate among the approximately 32,896 cycles that resulted in a pregnancy after treatment with ICSI. This corresponds to an OR of 0.98 (95\% CI, 0.90 to 1.06) for live birth rate given a pregnancy associated with IVF relative to ICSI among couples with male factor infertility and supports the findings from our meta-analysis. The second study compared outcomes associated with 10,933 cycles of conventional IVF with 153,968 ICSI cycles for male factor infertility reported to the SART registry during 1996-2012. ${ }^{254}$ The adjusted RR for live birth per cycle among transfers associated with conventional IVF relative to ICSI was 0.98 (95\% CI, 0.95 to 1.02; $>$ >0.99). The multivariate analysis was adjusted for maternal age, number of prior live births, number of prior miscarriages, number of prior assisted reproductive technology cycles, number of oocytes retrieved, number of embryos cryopreserved, donor egg/embryo, donor sperm, use of preimplantation genetic testing, and infertility diagnosis (tubal factor, endometriosis, uterine factor, ovulatory disorder, and diminished ovarian reserve). Again 
this observational study supports the findings from our meta analysis of no difference between IVF and ICSI for the outcome of live birth (moderate SOE).

\section{Miscarriage}

We identified two studies (one poor-quality $\mathrm{RCT}^{253}$ and one fair-quality observational study $^{254}$ ) that compared ICSI to IMSI for male factor infertility in relation to miscarriage rates. The RCT ${ }^{253}$ reported miscarriage rates of 20.5\% (15/73) for ICSI and 22.9\% (11/48) for IMSI $(\mathrm{p}=0.823)$ while the observational study ${ }^{254}$ calculated an adjusted RR of 0.97 (95\% CI, 0.91 to 1.04) for miscarriage among pregnancies for ICSI relative to IVF among couples with male factor infertility.

Our findings are consistent with the good-quality systematic review by Teixeira and colleagues which consisted of 6 RCTs (552 women) $)^{79}$ that concluded that there is no evidence of effect on miscarriage between ICSI and IMSI. Although all of these studies support there being no difference in miscarriage rates, the SOE was rated as low given large imprecision in the findings and the high risk of bias in the included studies.

\section{Multiple Births}

In addition to miscarriage rates, the two studies above ${ }^{253,254}$ reviewed multiple birth events when comparing ICSI to IMSI for male factor infertility. The poor-quality RCT reported that $22.2 \%(2 / 9)$ and $36.4 \%(4 / 11)$ of births were twin deliveries in the ICSI and IMSI arms, respectively $(\mathrm{p}=0.496){ }^{253}$ The fair-quality observational study by Boulet and associates ${ }^{254}$ compared outcomes associated with conventional IVF with ICSI calculated an adjusted RR of 0.87 (95\% CI, 0.83 to 0.91 ) for multiple live births among pregnancies for ICSI relative to IVF among couples with male factor infertility. Given the quality of these two studies and the imprecision of the findings the SOE was rated as insufficient.

\section{Birthweight}

One poor-quality RCT ${ }^{253}$ and three fair-quality observational studies compared birthweight for ICSI versus conventional IVF. ${ }^{124,185,254}$ Boulet's observational study ${ }^{254}$ compared birthweight for ICSI (4230 live births) versus conventional IVF (60,273 live births). Among couples with male factor infertility, they calculated an adjusted RR of low birthweight of 0.93 (95\% CI, 0.88 to 0.98 ) for ICSI relative to IVF. The second observational study ${ }^{124}$ evaluated outcomes associated with conventional IVF versus ICSI among both couples with male factor infertility reported singleton birth weights of 3270 grams (standard deviation [SD] 580) among conventional IVF recipients versus 3266 grams (SD 637) among ICSI recipients ( $p=0.67)$. The third fair-quality observational study ${ }^{185}$ examined neonatal outcomes for fresh ICSI compared with IVF cycles among patients with male factor infertility. This study used data from the society for assisted reproductive technologies clinical outcomes reporting system database from 2004 to 2013. Conventional IVF participants were one-to-one propensity score matched with ICSI participants. Among matched patients with male factor infertility $(n=2,184)$ no significant association was found with preterm delivery (OR $0.7795 \%$ CI $0.54,1.12$ ) or low birth weight (OR 1.06 95\% CI 0.70, 1.61).

Finally, one poor-quality RCT found that the mean birthweight was 2535 grams (SD 710) associated with IMSI versus 2789 grams (SD 575) associated with ICSI ( $p=0.492)$, not demonstrating a difference. ${ }^{253}$ Given the poor- and fair-quality of the included studies and the lack of quality RCT evidence, the SOE was rated as low for no difference in birthweight. 


\section{Congenital Anomalies}

Three studies explore congenital anomalies associated with infertility treatments for male infertility. One poor-quality RCT looked at congenital anomalies when comparing ICSI to IMSI for male factor infertility. The study reported zero cases of congenital anomalies for ICSI and $18.2 \%(2 / 11)$ for IMSI $(p=0.190) .{ }^{253}$ Additionally, a fair-quality observational study ${ }^{263}$ investigated rates of birth defects in patients who conceived through IVF and ICSI. This study found birth defects in $2.4 \%$ of infants conceived through IVF for male factor infertility, which was significantly lower than the $3.2 \%$ of infants with birth defects conceived through ICSI. However, these results should be interpreted with caution. The male infertility factor group in this study seems to include couples with and without female infertility indication.

Finally a third fair-quality observational study ${ }^{260}$ was conducted in Israel in a single outpatient infertility clinic of couples presenting with male factor infertility. The objective of the study was to determine the birth defect rates among ICSI and IMSI groups. Among 1,981 pregnancies, sixty-three pregnancies involving fetal malformation were reported with 3.9\% in the ICSI group and $2.3 \%$ in the IMSI group. However no significant association was found between the two groups OR 0.71 (95\% CI 0.39-1.22). Given the study limitations of these included studies with imprecise findings, the SOE was rated as insufficient.

\section{ART Unspecified for Male Factor Infertility}

\section{Live Birth Rates}

We identified one good-quality $\mathrm{RCT}^{119}$ and one good-quality observational study ${ }^{186}$ that investigated live birth rates in couples undergoing IVF with male factor infertility. The RCT investigated the effects of a female weight-loss intervention on live birth rates in patients with both female and male factor infertility. ${ }^{119}$ The weight-loss intervention resulted in significantly more natural conceptions (rate ratio of intervention $=1.61$; 95\% CI 1.16 to 2.24 ), but significantly less live births of a healthy singleton at term than the control group (rate ratio of intervention $=0.77 ; 95 \%$ CI 0.60 to 0.99 ). These results were not stratified by infertility diagnosis (i.e. included both male and female factor infertility), however results reporting live birth rates by method of conception suggest that the female weight loss intervention had no effect on live birth rates for patients with male factor infertility. This is because the proportion of women who underwent ovulation induction (suggesting female factor infertility) was $26 \%$ versus $40 \%$ in intervention versus control (95\% CI of RR does not cross 1.0 ), $24 \%$ versus $26 \%$ for IUI (primarily male factor; 95\% CI of RR crosses 1.0) and 20\% versus 28\% for IVF/ICSI (combined female and male factor infertility; 95\% CI just crosses 1.0).

The observational national cohort study from the Danish ART Registry looked at live birth rates in couples initiating fertility treatment with ART or IUI, and continuing to undergo either fertility treatment (regardless of initial treatment) for two years. ${ }^{186}$ Among male factor infertility patients (females $<35$ years old) whose first treatment was ART, two-year total live birthrates were $60.6 \%$ (across interventions: $56.3 \%$ for ART conception, $0.3 \%$ for IUI, and $5.0 \%$ for natural conception). Among male factor infertility patients (females $\geq 35$ years old) whose first treatment was ART, two-year birthrates were $37.3 \%$ for ART conception, $0.4 \%$ for IUI, and $5.2 \%$ for natural conception. For male factor infertility patients whose first treatment was IUI (females $<35$ years old), two-year birthrates were $20.2 \%$ for ART conception, $31.9 \%$ for IUI, and $8.5 \%$ for natural conception. For male factor infertility patients whose first treatment was IUI (females $\geq 35$ years old), two-year birthrates were $12.9 \%$ for ART conception, $25.1 \%$ for IUI, and $7.4 \%$ for natural conception. These results provide age-stratified probabilities of live birth for 
patients with male factor infertility initiating with either ART or IUI and continuing to undergo either fertility treatment for 2 years. ${ }^{186}$

\section{Birthweight}

One good-quality observational study ${ }^{236}$ looked at all singleton live births in Florida and Massachusetts between 2000 and 2010 and Michigan between 2000-2009 and used data from the States Monitoring Assisted Reproductive Technology (SMART) Collaborative. Both conventional and discordant-sibling pairs analyses were conducted. For the discordant sibling pairs the study population was restricted to singleton live births where one sibling was conceived through ART and the other was conceived naturally. In conventional analysis, when compared to naturally conceiving, those with male factor only indication for ART had significant associations with low birth weight (OR 1.15 95\% CI 1.04 to 1.27) and preterm birth (OR 1.19 95\% CI 1.10 to 1.29). ${ }^{236}$

\section{Long-Term Outcomes: Child}

Risk for cancer in children conceived after IVF or ICSI was evaluated in a fair-quality cohort study of 106,013 children born in Britain between 1992 and 2008. ${ }^{213}$ For 24,427 of these children, the cause of infertility was male factor only. The average duration of follow-up was 6.6 years, with a maximum of 15 years of follow-up. Cancer diagnoses were identified through the National Registry of Childhood Tumors. Cancer was diagnosed in 16 children born after assisted conception for male factor infertility. The standardized incidence ratio (SIR) for all cancers in children conceived with assisted conception where the cause of infertility was male factor only compared to the general population of the same age was 0.92 (95\% CI 0.53 to 1.49 )

Despite the large number of children in the cohort, the overall number of cancers was small. Conclusions about the risk are tenuous due to the inability to examine risk for individual cancer types by underlying cause of infertility (low SOE).

In addition to cancer, we identified one good-quality Danish cohort study that evaluated the risk of type 1 diabetes mellitus in children conceived from ART for diagnosed male infertility. There was no significant difference found between type 1 diabetes mellitus diagnoses in children born to patients with male factor infertility conceived with ART compared to children conceived with no fertility treatment (adjusted HR=0.83, 95\% CI 0.55 to 1.46 ) (moderate SOE). ${ }^{184}$

\section{Other Strategies}

\section{Exercise for Male Factor Infertility}

We identified one good-quality RCT that studied the effect of an exercise intervention for men with male factor infertility. Out of 197 patients in the exercise arm, there were 139 pregnancies and 127 live births, and in the non-exercise arm of 189 patients there were 5 pregnancies and 0 live births. Using the non-exercise arm as the reference value the authors calculated an odds ratio of live birth of 197.0 (95\% CI 5.9-2149.6). ${ }^{261}$ Given imprecise findings from one small study the SOE was rated as insufficient.

\section{Antioxidants for Male Factor Infertility}

We did not identify any eligible studies that evaluated the comparative effectiveness of antioxidants on live births for couples with male factor infertility. We did, however, identify a good-quality systematic review ${ }^{72}$ that conducted a meta-analysis with data from 4 small RCTs, 3 of which were published prior to 2007; this meta-analysis demonstrated a significant increase in 
live birth rate associated with vitamin $\mathrm{E}$ or zinc supplementation relative to placebo or no supplementation (OR 4.21; 95\% CI, 2.08 to 8.51). However, these results are based only 44 live births out of 277 couples, and the overall quality of evidence within the included studies was rated low.

\section{Strength of Evidence for Male Factor Infertility}

Table 27 summarizes the SOE for the findings described above. The SOE was judged to be insufficient or low for all outcomes except for the comparison of IVF versus ICSI for live birth.

Table 27. Strength of evidence for major outcomes-KQ 5 (male factor infertility)

\begin{tabular}{|c|c|c|c|c|}
\hline Comparison & Outcome & $\begin{array}{c}\text { Study Design } \\
\text { and Sample Size }\end{array}$ & Conclusion & $\begin{array}{l}\text { Strength of } \\
\text { Evidence } \\
\text { (Rationale) }^{\mathrm{a}}\end{array}$ \\
\hline $\begin{array}{l}\text { Single vS. } \\
\text { double IUI } \\
\text { rFSH vs. } \\
\text { clomiphene } \\
\text { citrate } \\
\text { IUI vs. IUI with } \\
\text { ovarian } \\
\text { hyperstimulati } \\
\text { on }\end{array}$ & Live birth & $\begin{array}{l}2 \mathrm{RCTs}^{208,221} \\
\text { (447 patients) } \\
2 \text { SRs }^{265,266} \text { (4 } \\
\text { studies, 1,278 } \\
\text { couples) }\end{array}$ & $\begin{array}{l}\text { Inconclusive. SOE was } \\
\text { insufficient given imprecise } \\
\text { evidence studies with varying } \\
\text { quality and differing strategies. }\end{array}$ & $\begin{array}{l}\text { Insufficient } \\
\text { (Imprecise; } \\
\text { findings from } \\
\text { studies with } \\
\text { varying quality } \\
\text { and differing } \\
\text { strategies) }\end{array}$ \\
\hline $\begin{array}{l}\text { ART IVF: ICSI } \\
\text { or assisted } \\
\text { hatching (1 } \\
\text { embryo } \\
\text { transferred) } \\
\text { vs. ICSI or } \\
\text { assisted } \\
\text { hatching } \\
\text { (multiple } \\
\text { embryos } \\
\text { transferred) } \\
\text { TESE vs. } \\
\text { ejaculated } \\
\text { OAT }\end{array}$ & Live birth & $\begin{array}{l}2 \text { Obs }^{123,257} \\
(272,717 \text { cycles })\end{array}$ & $\begin{array}{l}\text { Improvement. Greater live births } \\
\text { with multiple embryos transferred } \\
\text { compared to } 1 \text { embryo transferred }\end{array}$ & Low (Imprecise) \\
\hline \multirow[t]{2}{*}{$\begin{array}{l}\text { ART IVF: } \\
\text { TESE vs. } \\
\text { ejaculated } \\
\text { OAT }\end{array}$} & $\begin{array}{l}\text { Neonatal } \\
\text { outcomes: } \\
\text { Birthweight }\end{array}$ & $\begin{array}{l}1 \text { Obs }^{257} \\
\text { (191 cycles) }\end{array}$ & $\begin{array}{l}\text { Inconclusive. SOE was } \\
\text { insufficient given imprecise } \\
\text { evidence from one observational } \\
\text { study with potential limitations }\end{array}$ & $\begin{array}{l}\text { Insufficient } \\
\text { (Imprecise } \\
\text { findings with } \\
\text { high study } \\
\text { limitations) }\end{array}$ \\
\hline & $\begin{array}{l}\text { Neonatal } \\
\text { outcomes: } \\
\text { Congenital } \\
\text { anomalies }\end{array}$ & $\begin{array}{l}1 \text { Obs }^{257} \\
\text { (191 cycles) }\end{array}$ & $\begin{array}{l}\text { Inconclusive. SOE was } \\
\text { insufficient given imprecise } \\
\text { evidence from one observational } \\
\text { study with potential limitations }\end{array}$ & $\begin{array}{l}\text { Insufficient } \\
\text { (Imprecise } \\
\text { findings with } \\
\text { high study } \\
\text { limitations) }\end{array}$ \\
\hline \multirow[t]{2}{*}{$\begin{array}{l}\text { ART IVF: } \\
\text { Frozen vs. } \\
\text { fresh embryo } \\
\text { transfer }\end{array}$} & Live birth & $\begin{array}{l}\text { 1 } \mathrm{RCT}^{262} \\
(2,157 \text { patients })\end{array}$ & $\begin{array}{l}\text { No difference: no difference in } \\
\text { live birth rates between couples } \\
\text { using frozen embryo versus fresh } \\
\text { embryo transfer }\end{array}$ & $\begin{array}{l}\text { Low (one study, } \\
\text { heterogeneous } \\
\text { infertility } \\
\text { indication) }\end{array}$ \\
\hline & $\begin{array}{l}\text { Pregnancy } \\
\text { complications: } \\
\text { Ectopic } \\
\text { pregnancy }\end{array}$ & $\begin{array}{l}\text { 1 } \mathrm{RCT}^{262} \\
(2,157 \text { patients })\end{array}$ & $\begin{array}{l}\text { No difference: no difference in } \\
\text { ectopic pregnancy rates between } \\
\text { couples using frozen embryo } \\
\text { versus fresh embryo transfer }\end{array}$ & $\begin{array}{l}\text { Low (one study, } \\
\text { heterogeneous } \\
\text { infertility } \\
\text { indication) }\end{array}$ \\
\hline
\end{tabular}




\begin{tabular}{|c|c|c|c|c|}
\hline Comparison & Outcome & $\begin{array}{c}\text { Study Design } \\
\text { and Sample Size }\end{array}$ & Conclusion & $\begin{array}{l}\text { Strength of } \\
\text { Evidence } \\
\text { (Rationale) }^{\mathrm{a}}\end{array}$ \\
\hline & $\begin{array}{l}\text { Pregnancy } \\
\text { complications: } \\
\text { Multiple births }\end{array}$ & $\begin{array}{l}1 \mathrm{RCT}^{262} \\
\text { (2,157 patients) }\end{array}$ & $\begin{array}{l}\text { No difference: no difference in } \\
\text { multiple birth rates between } \\
\text { couples using frozen embryo } \\
\text { versus fresh embryo transfer }\end{array}$ & $\begin{array}{l}\text { Low (one study, } \\
\text { heterogeneous } \\
\text { infertility } \\
\text { indication) }\end{array}$ \\
\hline & $\begin{array}{l}\text { Pregnancy } \\
\text { complications: } \\
\text { Miscarriage }\end{array}$ & $\begin{array}{l}1 \mathrm{RCT}^{262} \\
\text { (2,157 patients) }\end{array}$ & $\begin{array}{l}\text { No difference: no difference in } \\
\text { miscarriage rates between couples } \\
\text { using frozen embryo versus fresh } \\
\text { embryo transfer }\end{array}$ & $\begin{array}{l}\text { Low (one study, } \\
\text { heterogeneous } \\
\text { infertility } \\
\text { indication) }\end{array}$ \\
\hline & $\begin{array}{l}\text { Neonatal } \\
\text { outcomes: } \\
\text { Birthweight }\end{array}$ & $\begin{array}{l}1 \mathrm{RCT}^{262} \\
\text { (2,157 patients) }\end{array}$ & $\begin{array}{l}\text { No difference: no difference in } \\
\text { low birthweight rates between } \\
\text { couples using frozen embryo } \\
\text { versus fresh embryo transfer }\end{array}$ & $\begin{array}{l}\text { Low (one study, } \\
\text { heterogeneous } \\
\text { infertility } \\
\text { indication) }\end{array}$ \\
\hline & $\begin{array}{l}\text { Neonatal } \\
\text { outcomes: } \\
\text { Congenital } \\
\text { anomalies }\end{array}$ & $\begin{array}{l}1 \mathrm{RCT}^{262} \\
\text { (2,157 patients) }\end{array}$ & $\begin{array}{l}\text { No difference: no difference in } \\
\text { congenital anomalies rates } \\
\text { between couples using frozen } \\
\text { embryo versus fresh embryo } \\
\text { transfer }\end{array}$ & $\begin{array}{l}\text { Low (one study, } \\
\text { heterogeneous } \\
\text { infertility } \\
\text { indication) }\end{array}$ \\
\hline \multirow[t]{4}{*}{$\begin{array}{l}\text { ART ICSI: } \\
\text { ICSI vS. } \\
\text { Laser-assisted } \\
\text { hatching }\end{array}$} & Live birth & $\begin{array}{l}\text { 1 RCT } 255 \\
\text { (182 patients) }\end{array}$ & $\begin{array}{l}\text { Inconclusive. SOE was } \\
\text { insufficient given imprecise } \\
\text { evidence from } 1 \text { low-quality trial }\end{array}$ & $\begin{array}{l}\text { Insufficient } \\
\text { (Imprecise } \\
\text { findings with } \\
\text { high study } \\
\text { limitations) }\end{array}$ \\
\hline & $\begin{array}{l}\text { Pregnancy } \\
\text { complications: } \\
\text { Miscarriage }\end{array}$ & $\begin{array}{l}1 \mathrm{RCT}^{264} \\
(62)\end{array}$ & $\begin{array}{l}\text { Inconclusive. SOE was } \\
\text { insufficient given imprecise } \\
\text { evidence from } 1 \text { small moderate- } \\
\text { quality trial }\end{array}$ & $\begin{array}{l}\text { Insufficient } \\
\text { (Imprecise } \\
\text { findings from } \\
\text { one small study } \\
\text { with moderate } \\
\text { limitations) } \\
\end{array}$ \\
\hline & $\begin{array}{l}\text { Pregnancy } \\
\text { complications: } \\
\text { Multiple births }\end{array}$ & $\begin{array}{l}1 \mathrm{RCT}^{255} \\
\text { (182 patients) }\end{array}$ & $\begin{array}{l}\text { Inconclusive. SOE was } \\
\text { insufficient given imprecise } \\
\text { evidence from } 1 \text { low-quality trial. }\end{array}$ & $\begin{array}{l}\text { Insufficient } \\
\text { (Imprecise } \\
\text { findings with } \\
\text { high study } \\
\text { limitations) }\end{array}$ \\
\hline & $\begin{array}{l}\text { Neonatal } \\
\text { outcomes: } \\
\text { Congenital } \\
\text { anomalies }\end{array}$ & $\begin{array}{l}1 \mathrm{RCT}^{255} \\
\text { (182 patients) }\end{array}$ & $\begin{array}{l}\text { Inconclusive. SOE was } \\
\text { insufficient given imprecise } \\
\text { evidence from } 1 \text { low-quality trial. }\end{array}$ & $\begin{array}{l}\text { Insufficient } \\
\text { (Imprecise } \\
\text { findings with } \\
\text { high study } \\
\text { limitations and a } \\
\text { suspected } \\
\text { reporting bias) } \\
\end{array}$ \\
\hline \multirow[t]{2}{*}{ IVF vs. ICSI } & Live birth & $\begin{array}{l}\text { 3 RCTs } 253,256,258 \\
\text { (497 patients) } \\
\\
2 \text { Obs }^{124,254} \\
\text { (771,661 cycles) }\end{array}$ & $\begin{array}{l}\text { No difference. Meta-analysis of } 3 \\
\text { RCTs produced a summary } \\
\text { estimate of the OR for live birth per } \\
\text { cycle associated with ICSI of } 1.218 \\
\text { (95\% CI, } 0.779 \text { to } 1.903 \text { ) relative to } \\
\text { IMSI and therefore does not } \\
\text { demonstrate a difference between } \\
\text { ICSI and IMSI. }\end{array}$ & $\begin{array}{l}\text { Moderate } \\
\text { (Moderate study } \\
\text { limitations) }\end{array}$ \\
\hline & $\begin{array}{l}\text { Pregnancy } \\
\text { complications: } \\
\text { Miscarriage }\end{array}$ & $\begin{array}{l}1 \mathrm{RCT}^{253} \\
\text { (121 patients) } \\
1 \mathrm{Obs}^{254}(499,135 \\
\text { cycles) } \\
1 \mathrm{SR}^{79}(6 \text { studies, } \\
552 \text { women) }\end{array}$ & $\begin{array}{l}\text { No difference. Both included } \\
\text { studies and an existing systematic } \\
\text { review supported no difference in } \\
\text { miscarriage. SOE was reduced } \\
\text { because of quality of included } \\
\text { studies and imprecision of findings. }\end{array}$ & $\begin{array}{l}\text { Low } \\
\text { (High study } \\
\text { limitations, } \\
\text { imprecise) }\end{array}$ \\
\hline
\end{tabular}




\begin{tabular}{|c|c|c|c|c|}
\hline Comparison & Outcome & $\begin{array}{c}\text { Study Design } \\
\text { and Sample Size }\end{array}$ & Conclusion & $\begin{array}{l}\text { Strength of } \\
\text { Evidence } \\
\text { (Rationale) }^{\mathrm{a}}\end{array}$ \\
\hline & $\begin{array}{l}\text { Pregnancy } \\
\text { complications: } \\
\text { Multiple births }\end{array}$ & $\begin{array}{l}1 \mathrm{RCT}^{253} \\
(121 \text { patients }) \\
1 \text { Obs }^{254}(499,135 \\
\text { cycles) }\end{array}$ & $\begin{array}{l}\text { Inconclusive. SOE was } \\
\text { insufficient given imprecise } \\
\text { evidence from } 1 \text { trial and } 1 \\
\text { observational study with moderate } \\
\text { study limitations }\end{array}$ & $\begin{array}{l}\text { Insufficient } \\
\text { (Imprecise with } \\
\text { moderate study } \\
\text { limitations) }\end{array}$ \\
\hline & $\begin{array}{l}\text { Neonatal } \\
\text { outcomes: } \\
\text { Birthweight }\end{array}$ & $\begin{array}{l}1 \mathrm{RCT}^{253} \\
\text { (121 patients) } \\
3 \mathrm{Obs}^{124,185,254} \\
\text { (862,062 cycles) }\end{array}$ & $\begin{array}{l}\text { No difference: No significant } \\
\text { differences in rates of low birth } \\
\text { weight between ICSI versus } \\
\text { conventional-IVF cycles }\end{array}$ & $\begin{array}{l}\text { Low (moderate } \\
\text { study } \\
\text { limitations) }\end{array}$ \\
\hline & $\begin{array}{l}\text { Neonatal } \\
\text { outcomes: } \\
\text { Congenital } \\
\text { anomalies }\end{array}$ & $\begin{array}{l}1 \mathrm{RCT}^{253} \\
(121 \text { patients) } \\
2 \mathrm{Obs}^{260,263} \\
(143,436)\end{array}$ & $\begin{array}{l}\text { Inconclusive. SOE was } \\
\text { insufficient given imprecise } \\
\text { evidence from studies with } \\
\text { significant study limitations. }\end{array}$ & $\begin{array}{l}\text { Insufficient } \\
\text { (Imprecise; } \\
\text { findings with } \\
\text { high study } \\
\text { limitations) }\end{array}$ \\
\hline \multirow[t]{2}{*}{$\begin{array}{l}\text { ART } \\
\text { unspecified }\end{array}$} & $\begin{array}{l}\text { Long-term } \\
\text { outcomes: } \\
\text { Child (cancer) }\end{array}$ & $\begin{array}{l}1 \text { Obs }^{213} \\
\text { (924,427 patients) }\end{array}$ & $\begin{array}{l}\text { No difference: The overall cancer } \\
\text { incidence was not elevated in } \\
\text { children born after assisted } \\
\text { conception for male factor } \\
\text { infertility. The SIR for all cancers in } \\
\text { children conceived with assisted } \\
\text { conception for male factor infertility } \\
\text { compared to the general } \\
\text { population of the same age was } \\
0.92(95 \% \mathrm{Cl}, 0.53 \text { to } 1.49) \text {. }\end{array}$ & $\begin{array}{l}\text { Low } \\
\text { (Moderate study } \\
\text { limitations) }\end{array}$ \\
\hline & $\begin{array}{l}\text { Long-term } \\
\text { outcomes: } \\
\text { Child (type } 1 \\
\text { diabetes } \\
\text { mellitus) }\end{array}$ & $\begin{array}{l}1 \text { Obs }^{184} \\
(565,116 \\
\text { pregnancies) }\end{array}$ & $\begin{array}{l}\text { No difference: No significant } \\
\text { difference found between type } 1 \\
\text { diabetes mellitus diagnoses in } \\
\text { children born to patients with male } \\
\text { factor infertility conceived with ART } \\
\text { compared to children conceived } \\
\text { with no fertility treatment }\end{array}$ & $\begin{array}{l}\text { Moderate } \\
\text { (Imprecise) }\end{array}$ \\
\hline $\begin{array}{l}\text { Other } \\
\text { strategies: } \\
\text { Exercise for } \\
\text { Male Infertility }\end{array}$ & Live birth & $\begin{array}{l}1 \mathrm{RCT}^{261} \\
\text { (386 patients) }\end{array}$ & $\begin{array}{l}\text { Inconclusive. SOE was } \\
\text { insufficient given imprecise } \\
\text { evidence from } 1 \text { small trial. }\end{array}$ & $\begin{array}{l}\text { Insufficient } \\
\text { (Imprecise; one } \\
\text { study) }\end{array}$ \\
\hline $\begin{array}{l}\text { Other } \\
\text { strategies: } \\
\text { Antioxidant } \\
\text { use for Male } \\
\text { Infertility }\end{array}$ & Live birth & $\begin{array}{l}1 \mathrm{SR}^{72} \text { ( } 4 \text { studies } \\
\text { of } 277 \text { couples) }\end{array}$ & $\begin{array}{l}\text { Improvement: Increase in live } \\
\text { birth rate associated with vitamin E } \\
\text { or zinc supplementation relative to } \\
\text { placebo or no supplementation }\end{array}$ & $\begin{array}{l}\text { Low (Imprecise, } \\
\text { small studies) }\end{array}$ \\
\hline
\end{tabular}

${ }^{a}$ Criteria for downgrading strength of evidence is described as Rationale; when these criteria are insufficient for understanding the final strength of evidence, additional explanation is provided.

Abbreviations: ART=assisted reproductive technology; $\mathrm{CI}=$ confidence interval; HR=hazard ratio; ICSI=intra-cytoplasmic sperm injection; IMSI=intracytoplasmic morphologically selected sperm injection; IUI=intrauterine insemination; IVF=in vitro fertilization; KQ=Key Question; N=number of patients/participants; NA=not applicable; NR=not reported; OAT=oligo-asthenoteratozoospermia; Obs=observational study; OR=odds ratio; RCT=randomized controlled trial; $\mathrm{rFSH=recombinant} \mathrm{follicle-}$ stimulating hormone; $\mathrm{RR}=$ =relative risk; $\mathrm{SD}=$ standard deviation; $\mathrm{SIR}=$ standardized incidence ratio; TESE=extracted testicular sperm 


\section{Key Question 6. Donors in Infertility}

\section{$\mathrm{KQ}$ 6. What are the short- and long-term health outcomes of donors in infertility? \\ KQ 6a. For female oocyte donors:}

1. Do specific aspects of the pre-donation evaluation identify potential donors at greater risk for short- or long-term adverse outcomes (e.g., OHSS, quality-of-life issues)?

2. Do short- and long-term outcomes differ among different stimulation/retrieval protocols?

$\mathrm{KQ}$ 6b. For male semen donors:

1. Are there long-term health, quality-of-life, or other adverse outcomes associated with donation?

\section{Description of Included Studies for KQ 6 (Donors)}

We identified one fair-quality $\mathrm{RCT}^{267}$ and four retrospective observational studies, 3 fair-268270 and 1 poor-quality, ${ }^{271}$ that addressed short- or long-term health outcomes of donors in infertility. Two of the studies were conducted in the United States. ${ }^{270,271}$ The remaining three studies were conducted in Europe. ${ }^{267-269}$ Four studies were conducted in a subspecialty practice, and the remaining study did not report the setting or the setting was unclear. ${ }^{271}$ None of the studies reported a funding source. We did not perform meta-analysis because of the lack of studies reporting results for similar outcomes and treatment comparisons. All of the included studies focused on oocyte donors. We did not find any studies evaluating male donors.

\section{Key Points for Donors}

Key findings for outcomes of sperm and oocyte donors included:

- For oocyte donors, observational studies suggest a lower incidence of OHSS with GnRH agonist trigger than with hCG trigger (low SOE). However, there was a lack of evidence on any long-term outcomes.

- There was a lack of evidence on any short or long-term outcomes for sperm donors.

\section{Detailed Synthesis for Oocyte Donors}

Included studies and their findings for the included studies are discussed below in terms of short- and long-term outcomes.

\section{Short-Term Outcomes for Oocyte Donors}

One included study was a cross-sectional survey published in 2009 (poor quality). ${ }^{271}$ The authors surveyed former oocyte donors who were registered on the Donor Sibling Registry, a U.S.-based worldwide registry designed to help donor-conceived individuals search for and contact their donor sibling. Of the 287 women with valid e-mail addresses who were invited via an email message to participate in the study, 155 (54\%) completed the 25-item questionnaire. The mean age of respondents at the time of the survey was 35.8 years, and the number of years since first donation was 9.4 years (SD 5.2). Details of the stimulation protocols and oocyte retrieval methods were not reported. Of the 155 respondents, 47 (30.3\%) reported some degree 
of OHSS, 18 (11.6\%) reported OHSS-related hospitalization and/or paracentesis. This study was rated as poor quality because of the high risk of bias due to the relatively low response rate and the self-selected nature of the study sample (insufficient SOE for all outcomes).

A randomized crossover trial conducted in Cyprus in 2008 compared outcomes associated with hCG versus GnRH agonist (leuprolide acetate) trigger among 50 oocyte donors. ${ }^{267}$ Both study protocols used recombinant FSH or hMG administered beginning on day 3 of each donor's menstrual cycle for donor stimulation. Among the 44 donors who received both triggering agents in 2 consecutive cycles, 3 cases (6.8\%) of mild-to-moderate OHSS were reported after administration of hCG. No cases of OHSS were reported after administration of the GnRH agonist leuprolide. The between-group difference in rates of OHSS was not statistically significant, but this trial may have been underpowered to detect a statistically significant effect for this clinical outcome. This study was rated as fair quality because of the small sample size and the exclusion from the analysis of patients who did not complete the trial (insufficient SOE).

Two publications reported retrospectively assessed outcomes observed among overlapping cohorts of oocyte donors treated at a private infertility clinic in Spain between 2001 and 2007. ${ }^{268,269}$ Among 1907 donors who collectively underwent 4052 stimulation cycles that reached oocyte retrieval, the stimulation protocol and triggering agents used were as follows: 1238 cycles with a GnRH agonist protocol and hCG trigger; 1295 cycles with a GnRH antagonist protocol and hCG trigger; and 1519 cycles with a GnRH antagonist protocol and a GnRH agonist trigger. The incidence of moderate or severe OHSS resulting from these three protocols were, respectively, 0.65 percent, 1.08 percent, and 0 percent (difference not statistically significant). ${ }^{268}$ The other publication from this cohort of patients reported an incidence of 13 cases of moderate or severe OHSS associated with 624 cycles of hCG triggering (2.1\%) and no cases of moderate or severe OHSS associated with 547 cycles of triggering with a GnRH agonist. ${ }^{269}$ These two studies were rated as fair quality because of their retrospective design. Together they suggest a lower incidence of OHSS with GnRH agonist trigger than with hCG trigger (low SOE).

The fifth study was a retrospective analysis of all attempts at oocyte donations by anonymous and known directed donors at a medical center in the United States from 1991 to $2007 .{ }^{270}$ The charts of 587 donors (481 anonymous and 106 directed) who participated in 973 stimulation cycles and 886 retrievals were reviewed. The age of the donors ranged from 20 to 42 years. Of the 886 stimulation cycles, $12(1.4 \%)$ were associated in mild or moderate OHSS that led to 2 outpatient office visits, and $4(0.5 \%)$ were associated with moderate OHSS that led to 3 or 4 office visits. There was a single case $(0.1 \%)$ of intraabdominal bleeding and 18 cases $(2.0 \%)$ of other complications such as cysts, hematomas, urinary tract infections, yeast infections, or "vague symptoms." This study was rated as fair quality because of its retrospective design and because details of the stimulation and retrieval protocols were not reported. In addition, the applicability of these findings is limited in that clinical practice have evolved since 1991 and how the protocol during the study period reflected these changes in practice is unclear (insufficient SOE).

\section{Long-Term Outcomes for Oocyte Donors}

The only included study with evidence on long-term outcomes was the 2009 cross-sectional survey referenced above by Kramer and colleagues (poor quality). ${ }^{271}$ The authors surveyed former oocyte donors who were registered on the Donor Sibling Registry, a U.S.-based worldwide registry designed to help donor-conceived individuals search for and contact their 
donor sibling. Of the 287 women with valid e-mail addresses who were invited via an email message to participate in the study, 155 (54\%) completed the 25-item questionnaire. The mean age of respondents at the time of the survey was 35.8 years, and the number of years since first donation was 9.4 years (SD 5.2). Details of the stimulation protocols and oocyte retrieval methods were not reported. Of the 155 respondents, and 41 (26.4\%) reported infertility and/or menstrual changes since donation. Fifteen of these women ( $9.6 \%$ of the total sample) reported new infertility problems; of these, only 4 reported having become pregnant. This study was rated as poor quality because of the high risk of bias due to the relatively low response rate and the self-selected nature of the study sample (insufficient SOE for all outcomes)

\section{Strength of Evidence for Donors}

Table 28 summarizes the SOE for the incidence of OHSS with GnRH agonist trigger versus hCG trigger. All other short- and long-term outcomes had insufficient SOE or were not evaluated in the limited set of included studies.

Table 28. Strength of evidence for major outcomes-KQ 6 (donor)

\begin{tabular}{|l|l|l|l|l|}
\hline \multicolumn{1}{|c|}{ Comparison } & \multicolumn{1}{|c|}{ Outcome } & \multicolumn{1}{|c|}{$\begin{array}{c}\text { Study Design } \\
\text { (Sample Size) }\end{array}$} & \multicolumn{1}{c|}{ Conclusion } & $\begin{array}{l}\text { Strength of } \\
\text { Evidence } \\
\text { (Rationale) }\end{array}$ \\
\hline $\begin{array}{l}\text { GnRH agonist } \\
\text { (leuprolide acetate) vs. } \\
\text { hCG trigger }\end{array}$ & $\begin{array}{l}\text { Short term adverse } \\
\text { effects of treatment: } \\
\text { OHSS }\end{array}$ & $\begin{array}{l}2 \text { Obs } 268,269 \\
(3824)\end{array}$ & $\begin{array}{l}\text { Improvement: Lower } \\
\text { incidence of OHSS with } \\
\text { GnRH agonist trigger than } \\
\text { with hCG trigger. }\end{array}$ & $\begin{array}{l}\text { Low } \\
\text { (Moderate } \\
\text { study } \\
\text { limitations, } \\
\text { imprecise) }\end{array}$ \\
\hline
\end{tabular}

${ }^{a}$ Criteria for downgrading strength of evidence is described as Rationale; when these criteria are insufficient for understanding the final strength of evidence, additional explanation is provided.

Abbreviations: GnRH=gonadotropin-releasing hormone; hCG= human chorionic gonadotropin; Obs=observational study;

OHSS=ovarian hyperstimulation syndrome

\section{Key Findings Across All Infertility Diagnoses}

Select key studies reported findings applicable across all KQs. Note these studies all adjusted for cause of infertility although did not report their findings for the specific causes of infertility and therefore are reported here rather than in the individual KQ sections. The findings from these studies are considered relevant to individuals with all included infertility diagnoses.

\section{Description of Included Studies Across All KQs}

We identified 26 articles ${ }^{26,125-127,136-138,272-290}$ described in 21 studies that addressed outcomes after treatment for infertility and adjusted for cause of infertility.

Among the 21 studies, 3 were an RCTs and all were rated as good quality. ${ }^{275,285,288}$ The remaining 18 studies were observational studies; 16 of these were good quality ${ }^{125,136,272,274,276-}$ $279,281-283,286,287,289,290$ and three were fair quality. ${ }^{273,280,284}$ Geographically, most of the 21 studies took place in the United States or the United Kingdom/Europe; 14 studies in the U.S. ${ }^{136,272,274,276-}$ 284,286,290 and seven studies in the U.K./Europe. ${ }^{125,273,275,285,287-289}$ Most of the studies (19 total) were conducted in or used data from fertility subspecialty clinics, while the remaining 2 did not specify the setting. ${ }^{272,282}$ Eight studies reported support by government funding, ${ }^{136,272,274,276,277,282,284,290}$ two studies reported industry funding, ${ }^{275,285}$ three studies reported non-government, non-industry funding, ${ }^{273,280,287}$ three studies reported a combination of 
funding sources, ${ }^{125,288,289}$ and the remaining four did not report a funding source or it was unclear. $185,248,249,251,286,291$

\section{Key Points for Any Infertility Diagnosis}

Key points for patients who undergo IVF/ICSI include:

- Clomiphene or gonadotropins ever use was not associated with increased risk of maternal cancer (low SOE).

- Women who undergo IVF demonstrated an increased risk of ovarian neoplasms and colorectal malignancies (low SOE) compared to women who do not undergo IVF. There is no evidence of a difference in invasive ovarian cancers (low SOE).

- For children born after ART, ICSI may be associated with an increased risk of autism compared to IVF (low SOE).

- In the United States, live birth rates after IVF/ICSI are lower for African-Americans than for other racial/ethnic groups after adjusting for other prognostic factors (low SOE).

- Elective single-embryo transfer is associated with lower live birth rates but a significant reduction in multiple birth rates compared to multiple-embryo transfer (low SOE for both outcomes).

- There was no difference in the odds of low birth weight between ICSI versus conventional IVF cycles (low SOE). However, among couples undergoing ART with a singleton pregnancy, frozen embryo transfers result in a higher average birthweight, with a subsequent reduction in the incidence of low birthweight and an increase in the incidence of macrosomia (low SOE).

\section{Long-Term Outcomes: Maternal}

\section{Cancer}

Two studies evaluated long-term risk for cancer in infertile women (by diagnosis) who were treated with clomiphene citrate, gonadotropins, or IVF and reported results in multiple publications, as follows:

- Brinton, 2015: Primary report ${ }^{136}$ and three companion papers $26,137,138$

- Spaan, 2015: Primary report ${ }^{125}$ and a companion paper ${ }^{126}$

One study compared the long-term risk for cancer in infertile women treated with clomiphene citrate versus gonadotropins, with risks for different cancers reported in four separate papers. ${ }^{26,136-138}$ This study was a prospective cohort of 12,193 women who sought treatment for infertility between 1965 and 1988 at five reproductive endocrinology practices in the United States. The analytic cohort comprised 9892 women successfully traced, with a median follow-up of approximately 30 years.

Tables 29 and 30 highlight the risk of cancer in relation to use of clomiphene citrate or gonadotropins in women by infertility diagnosis. 
Table 29. Cancer risk $(95 \% \mathrm{Cl})$ for ever use of clomiphene citrate by infertility diagnosis

\begin{tabular}{|l|l|l|l|}
\hline Cancer & Endometriosis $(\mathbf{N}=2196)$ & $\begin{array}{c}\text { Tubal or Peritoneal } \\
\text { Factor (N=3496) }\end{array}$ & Male Factor (N=2218) \\
\hline Ovarian & $1.01(0.43$ to 2.36$)$ & $0.98(0.46$ to 2.07$)$ & $1.18(0.52$ to 2.68$)$ \\
\hline Endometrial & $1.79(0.76$ to 4.23$)$ & $1.14(0.59$ to 2.21$)$ & $1.55(0.82$ to 2.96$)$ \\
\hline Breast & $1.23(0.92$ to 1.65$)$ & $1.12(0.87$ to 1.45$)$ & $1.25(0.92$ to 1.69$)$ \\
\hline Colon & $0.53(0.22$ to 1.27$)$ & $0.66(0.31$ to 1.40$)$ & $1.14(0.42$ to 3.06$)$ \\
\hline Lung & $1.68(0.71$ to 3.99$)$ & $1.78(0.90$ to 3.54$)$ & $1.35(0.51$ to 3.61$)$ \\
\hline Thyroid & $0.87(0.28$ to 2.67$)$ & $1.08(0.40$ to 2.88$)$ & $2.46(0.64$ to 9.49$)$ \\
\hline Melanoma & $1.10(0.42$ to 2.89$)$ & $0.98(0.36$ to 2.73$)$ & $1.10(0.27$ to 4.46$)$ \\
\hline
\end{tabular}

Abbreviations: $\mathrm{CI}=$ confidence interval; $\mathrm{N}=$ number of patients

Table 30. Cancer risk $(95 \% \mathrm{Cl})$ for ever use of gonadotropins by infertility diagnosis

\begin{tabular}{|l|l|l|l|}
\hline Cancer & Endometriosis $(\mathbf{N}=\mathbf{2 1 9 6 )}$ & $\begin{array}{c}\text { Tubal or Peritoneal } \\
\text { Factor (N=3496) }\end{array}$ & Male Factor (N=2218) \\
\hline Endometrial & $1.15(0.72$ to 1.83$)$ & $1.16(0.77$ to 1.74$)$ & $1.29(0.79$ to 2.11$)$ \\
\hline Breast & $2.74(0.96$ to 7.85$)$ & $0.94(0.32$ to 2.70$)$ & $0.74(0.22$ to 2.43$)$ \\
\hline
\end{tabular}

Abbreviations: $\mathrm{CI}=$ confidence interval; $\mathrm{N}=$ number of patients

While the findings from this cohort are suggestive that, in couples with infertility due to endometriosis, tubal factors, or male factors, that women treated with clomiphene citrate or gonadotropins are not at markedly increased risk for any common cancer, the size of the cohort was not adequate to detect modest increases in risk. In addition, the size of the cohort precluded conducting more detailed analyses in relation to the number of cycles received or age at treatment (low SOE).

Cancer risk in relation to ART treatment was examined in two good-quality observational cohort studies, described in four papers. ${ }^{125-127,289}$ Results from a study conducted in the Netherland were described in papers examining melanoma risk ${ }^{125}$, ovarian neoplasms, ${ }^{126}$ and colorectal cancers ${ }^{127}$. In this study, the underlying cause of infertility was adjusted for along with other potential confounders. The cohort was comprised of 19,158 women who received IVF treatment and 6006 infertile women who did not receive IVF treatment after a median follow-up of 15 years. IVF was not significantly associated with melanoma risk (standardized incidence ratio [(SIR] 1.27; 95\% CI, 0.75 to 2.15 ) when controlling for cause of infertility. ${ }^{125}$ IVF was associated with a statistically significant increased risk of all ovarian malignancies (HR 2.05; 95\% CI, 1.10 to 3.82). This risk was increased most notably for borderline ovarian tumors (HR 6.38; 95\% CI, 2.05 to 19.84) as compared to the increase seen in invasive ovarian cancer (HR 1.14; $95 \%$ CI, 0.54 to 2.41) (low SOE). ${ }^{126}$ For colorectal cancers, IVF was not associated with a significant risk of colorectal cancer compared to the general population (SIR 1.00; 95\% CI, 0.80 to 1.23), but was increased compared to infertile patients who did not receive IVF (HR 1.80, 95\% CI, 1.10-2.94). Interestingly, this may be due to a significantly lower risk for colorectal cancers in this group compared to the general population (SIR 0.58, 95\% CI, 0.36-0.88), which the authors speculated may have been due to a "healthy female effect", but could also have been a chance finding because of a small number of cases in the non-IVF group. ${ }^{127}$

The applicability of the findings may be limited since the study was conducted in the Netherlands and the IVF treatments were received in the period between 1983 and 1995 . With the evolution of IVF treatment over time, findings from this study may not reflect the risk for women currently undergoing IVF.

The second study, conducted in the UK, examined ovarian, breast and corpus uteri cancers in a cohort of 255,786 women who underwent ART and were followed for an average of 8.8 years. ${ }^{289}$ SIRs were calculated comparing the observed incidence rates to expected rates based on 
national cancer incidence rates in women and are summarized in Table 31. Breast cancer risk (invasive or in situ) was not significantly increased in any of the female infertility diagnoses categories, and was significantly lower among those with a male factor diagnosis. Both invasive and borderline ovarian cancers were significantly increased among women with a diagnosis of endometriosis or tubal disease. Corpus uteri cancer risk was significantly higher only among women with a diagnosis of ovulatory problems.

Table 31. Cancer risk (SIR 95\% Cl) among women who underwent assisted reproduction by infertility diagnosis

\begin{tabular}{|l|c|l|l|l|}
\hline Cancer & $\begin{array}{c}\text { Endometriosis } \\
\text { (181,279 Person- } \\
\text { Years) }\end{array}$ & $\begin{array}{c}\text { Tubal Disease } \\
\text { (710,522 Person- } \\
\text { Years) }\end{array}$ & $\begin{array}{c}\text { Ovulatory Problems } \\
\text { (311,523 Person- } \\
\text { Years) }\end{array}$ & $\begin{array}{c}\text { Male Factor } \\
\text { (757,063 Person- } \\
\text { Years) }\end{array}$ \\
\hline $\begin{array}{l}\text { Invasive breast } \\
\text { cancer }\end{array}$ & $0.95(0.82$ to 1.10$)$ & $0.94(0.87$ to 1.01$)$ & $0.91(0.81$ to 1.02$)$ & $0.89(0.83$ to 0.96$)$ \\
\hline $\begin{array}{l}\text { In situ breast } \\
\text { cancer }\end{array}$ & $1.25(0.81$ to 1.83$)$ & $1.11(0.89$ to 1.36$)$ & $1.05(0.75$ to 1.42$)$ & $1.18(0.95$ to 1.44$)$ \\
\hline $\begin{array}{l}\text { Invasive } \\
\text { ovarian tumors }\end{array}$ & $2.47(1.75$ to 3.39$)$ & $1.71(1.40$ to 2.08$)$ & $1.16(0.80$ to 1.63$)$ & $1.09(0.84$ to 1.39$)$ \\
\hline $\begin{array}{l}\text { Borderline } \\
\text { ovarian tumors }\end{array}$ & $2.03(1.18$ to 3.25$)$ & $1.62(1.21$ to 2.12$)$ & $1.52(0.96$ to 2.31$)$ & $0.96(0.66$ to 1.35$)$ \\
\hline $\begin{array}{l}\text { Corpus uteri } \\
\text { cancer }\end{array}$ & $0.75(0.35$ to 1.43$)$ & $1.23(0.93$ to 1.58$)$ & $1.59(1.13$ to 2.17$)$ & 0.91 (0.65 to 1.24$)$ \\
\hline
\end{tabular}

Abbreviations: $\mathrm{CI}=$ confidence interval; $\mathrm{N}=$ number of patients; $\mathrm{SIR}=$ standardized incidence ratio

\section{Short-Term Outcomes: Child}

\section{Birthweight}

A good-quality observational study reported examined birth outcomes among all live births in Massachusetts (2004 through 2008) that linked to ART cycles in the Society for Assisted Reproductive Technology Clinic Online Reporting System (SART CORS) and the Pregnancy to Early Life Longitudinal (PELL) data system. ${ }^{274}$. There was no difference in low birth weight by assisted hatching, donor/autologous source of oocytes/semen, number of embryos or ICSI (low SOE). ${ }^{274}$

In a good-quality NASS comparing fresh and frozen embryo transfer, singleton infants both after frozen/thawed transfers had an average birthweight 142.3 grams heavier than those born after fresh transfers. In terms of clinically relevant categories, this translated into a significantly lower risk for low birthweight after frozen/thawed transfer (aRR 0.52, 95\% CI 0.48 to 0.56), and a significantly increased for macrosomia (aRR 1.70, 95\% CI 1.64 to 1.76 ). ${ }^{284}$

Finally, analyses of the SART CORS database compared outcomes for ICSI versus conventional IVF. ${ }^{185}$ Keyhan, et al. reported on low birth weight $(<2500$ g) in relation to ICSI versus conventional IVF using propensity score matching. ${ }^{185}$ The propensity score matching, which included infertility diagnosis as one of the factors used to calculate propensity scores, resulted in 12,364 ICSI cycles and 12,364 IVF cycles. In the ICSI group, 9.4\% of the births were low birth weight compared to $9.7 \%$ in the IVF group (OR 0.92, 95\% CI 0.78-1.10, p=0.35) (low SOE).

\section{Congenital Anomalies}

A good-quality observational study ${ }^{277}$ using the States Monitoring ART (SMART) Collaborative and linked ART surveillance, birth certificates, and birth defects registry data for Florida, Massachusetts, and Michigan from 2000 to 2010 (n=64,861). The objective of this study 
was to examine the prevalence of birth defects among liveborn infants conceived with and without ART and to evaluate risks associated with certain ART procedures among ARTconceived infants. Overall, the prevalence ratio was significantly higher for ART versus nonART birth (adjusted risk ratio 1.28, 95\% CI 1.15 to 1.42, p<0.001) (low SOE).

\section{Long-Term Outcomes: Child}

\section{Neurodevelopmental Outcomes}

Risk of autism through age 5 was examined in one good-quality observational study involving 42,383 children conceived with ART in California between 1997 and 2006. ${ }^{272}$ Analyses did not examine associations with type of embryo fertilization (ICSI or conventional IVF) stratified by cause of infertility, but did control for infertility diagnosis in multivariable analyses. Among ART-conceived infants, use of ICSI was associated with a higher incidence of autism in both singleton births (HR 1.65; 95\% CI, 1.08 to 2.52) and multiple births (HR 1.71; 95\% CI, 1.10 to 2.66) in multivariable analyses that controlled for infertility diagnosis (low SOE). Results of ICSI versus IVF were not described for specific causes of infertility, although male factor infertility was the most common diagnosis in the cohort.

One fair-quality ${ }^{273}$ observational study compared outcomes between ART and no intervention/expectant management. Risk of neurological dysfunction at 2 years was assessed in a fair-quality observational study examining children conceived with IVF $(n=122)$, conceived naturally to subfertile parents $(n=87)$, or born to parents without fertility problems $(n=101){ }^{273}$ Outcomes reported were simple or complex minor neurological dysfunction. Results were stratified by underlying cause of infertility. None of the specific causes of infertility were related to the rates of neurological dysfunction (insufficient SOE).

\section{Subgroups of Interest (With Any Infertility Diagnosis)}

\section{Race}

One good-quality observational study assessed the association between race and IVF outcomes. $^{292}$ The live birth rate per patient was 34.7\% among whites, $19.8 \%$ among blacks, 33.3\% among South Asians, 31.3\% among those who reported mixed race, $28.4 \%$ among those who reported "other," and 36.1\% among those who reported "unknown" ( $<<0.001$ for black vs. white comparison; $\mathrm{p}>0.05$ for South Asian vs. white) (low SOE).

\section{Number of Embryos Transferred}

A fair-quality observational study using SART CORS data assessed 69,028 cycles to determine factors associated with elective single-embryo transfer (eSET) and controlled for infertility diagnosis. ${ }^{123}$ Findings from the study are summarized in Table 32, demonstrating increased live birth rate per cycle with 2 embryo transfer as compared to single-embryo transfer (low SOE) and that multiple live birth rates are significantly higher with a 2-embryo transfer than a single-embryo transfer, but do not increase further with 3- or 4-embryo transfers (low SOE). 
Table 32. Birth rates by number of embryos transferred

\begin{tabular}{|c|c|c|c|c|c|}
\hline $\begin{array}{l}\text { \# Embryos } \\
\text { Transferred }\end{array}$ & $\begin{array}{c}1 \text { Embryo } \\
\text { Transferred }\end{array}$ & $\begin{array}{l}2 \text { Embryos } \\
\text { Transferred }\end{array}$ & $\begin{array}{l}3 \text { Embryos } \\
\text { Transferred }\end{array}$ & $\begin{array}{l}\text { 4+ Embryos } \\
\text { Transferred }\end{array}$ & P Value \\
\hline Singleton birth & $\begin{array}{c}1,302 / 3,037 \\
(42.9 \%)\end{array}$ & $\begin{array}{c}13,779 / 42,396 \\
(32.5 \%)\end{array}$ & $\begin{array}{c}4,632 / 17,480 \\
(26.5 \%)\end{array}$ & $\begin{array}{c}1,424 / 6,115 \\
(23.3 \%)\end{array}$ & $p<0.0001$ \\
\hline Multiple birth & $\begin{array}{c}15 / 3,037 \\
(0.5 \%)\end{array}$ & $\begin{array}{c}8,055 / 42,396 \\
(19.0 \%)\end{array}$ & $\begin{array}{c}3,094 / 17,480 \\
(17.7 \%)\end{array}$ & $\begin{array}{c}923 / 6,115 \\
(15.1 \%)\end{array}$ & $p<0.0001$ \\
\hline $\begin{array}{l}\text { Total births } \\
\text { per cycle }\end{array}$ & $\begin{array}{c}1,318 / 3,037 \\
(43.4 \%)\end{array}$ & $\begin{array}{c}21,834 / 42,396 \\
(51.5 \%)\end{array}$ & $\begin{array}{c}7,726 / 17,480 \\
(44.2 \%)\end{array}$ & $\begin{array}{c}2,348 / 6,115 \\
(38.4 \%)\end{array}$ & $p<0.0001$ \\
\hline
\end{tabular}

A good-quality study from NASS assessed correlation between infertility clinic eSET rates and pregnancy outcomes - there was a significant linear decrease in multiple birth rates with increasing eSET rates with no significant differences in clinic-level live birth rates for women younger than 38 years. $^{283}$

\section{Number of Oocytes Retrieved}

A good-quality observational study used data from the Swedish National Quality Registry of Assisted Reproduction and Medical Birth/IVF Registry assessed the association between the number of oocytes retrieved and obstetric and neonatal outcomes among 27,359 women who delivered singleton babies after IVF. ${ }^{287}$ In multivariable models that adjusted for cause of infertility as well as multiple maternal characteristics, the number of oocytes retrieved (continuous) was not significantly associated with pre-term birth (OR 1.002, 95\% CI 0.9941.011), peri/neonatal death (OR 1.008, 95\% CI 0.975-1.043), or major birth defects (OR 1.009, 95\% CI 0.998-1.001).

\section{Fresh Versus Frozen IVF Cycles}

Five observational studies evaluated outcomes using fresh or frozen IVF cycles. A goodquality observational study using SART CORS data compared pregnancy outcomes in 509,938 IVF cycles, based on the transfer of a fresh or frozen blastocyst or non-blastocyst. ${ }^{286}$ Table 33 summarizes findings from multivariable models that controlled for infertility diagnosis and shows that the best outcomes in terms of live births and first trimester pregnancy loss for fresh blastocyst transfer, and the worst outcomes for frozen non-blastocyst transfers. The risk for ectopic pregnancies was lower for frozen transfers, whether blastocyst or non-blastocyst.

Table 33. Outcomes with fresh and frozen IVF cycles

\begin{tabular}{|l|c|c|c|}
\hline Cycle & $\begin{array}{c}\text { Live Birth } \\
\text { OR (95\% Cl) }\end{array}$ & $\begin{array}{c}\text { First-Trimester } \\
\text { Pregnancy Loss } \\
\text { OR (95\% Cl) }\end{array}$ & $\begin{array}{c}\text { Ectopic/Heterotopic } \\
\text { Pregnancy } \\
\text { OR (95\% Cl) }\end{array}$ \\
\hline Fresh blastocyst & Reference & Reference & Reference \\
\hline Fresh non-blastocyst & $0.82(0.80$ to 0.83$)$ & $1.15(1.12$ to 1.18$)$ & $1.04(0.98$ to 1.11$)$ \\
\hline Frozen blastocyst & $0.73(0.72$ to 0.75$)$ & $1.29(1.25$ to 1.32$)$ & $0.48(0.43$ to 0.53$)$ \\
\hline Frozen non-blastocyst & $0.64(0.62$ to 0.65$)$ & $1.34(1.30$ to 1.38$)$ & $0.67(0.60$ to 0.73$)$ \\
\hline
\end{tabular}

Abbreviations: $\mathrm{CI}=$ confidence interval; $\mathrm{OR}=$ odds ratio

Another good-quality SART CORS study found no difference in live birth or miscarriage rate for fresh or frozen oocytes in autologous cycles, but a significantly lower rate of live birth with frozen oocytes in donor cycles (adjusted Risk Ratio [aRR] 0.87, 95\% CI, 0.80 to 0.95), with no difference in miscarriage rates. ${ }^{281}$

A good-quality observational study examined birth outcomes among all live births in Massachusetts (2004 through 2008) that linked to ART cycles in the Society for Assisted Reproductive Technology Clinic Online Reporting System (SART CORS) and the Pregnancy to Early Life Longitudinal (PELL) data system ${ }^{274}$ and found lower birthweight with thawed 
embryos compared to fresh (adjusted OR and 95\% CI 0.79, 0.65 to 0.96). There was no difference in low birth weight by assisted hatching, donor/autologous source of oocytes/semen, number of embryos or ICSI. ${ }^{274}$

Finally, within frozen cycles, another good-quality SART CORS study compared the live birth rate in first-cycle frozen embryo transfers with and without assisted hatching using propensity score matching. ${ }^{282}$ The propensity score matching, which included infertility diagnosis as one of the factors used to calculated propensity scores, resulted in 70,738 assisted hatching cycles and 80,795 cycles without assisted hatching. The live birth rate was significantly lower in the assisted hatching cohort compared to the no assisted hatching cohort (34.2\% versus $35.4 \%, \mathrm{p}<0.001)$.

\section{Blastocyst or Cleavage Stage Embryo Transfer}

A good-quality observational study used CDC's National ART Surveillance System (NASS) to evaluate differences in birthweight in 124,154 infants born after embryo transfer at the blastocyst stage or the cleavage stage. ${ }^{290}$ The results of the multivariable linear model that controlled for infertility diagnosis, maternal demographics and pregnancy characteristics showed that infants born after blastocyst transfer had birth weights that were slightly but statistically significantly higher than those with cleavage stage transfer (5.73 grams, $\mathrm{p}=0.040$ ). The difference was larger for single-embryo transfer (19.26 grams, $\mathrm{p}=0.008$ ) than for double embryo transfer (4.03 grams, $\mathrm{p}=0.245$ ). Embryo transfer type was not associated with low birth weight (RR 1.0, 95\% CI 0.96-1.04).

\section{GnRH Suppression Protocols}

A good-quality observational study using SART data compared ectopic pregnancy rates outcome of ectopic pregnancy in patients who used different $\mathrm{GnRH}$ analog protocols for ovarian hyperstimulation prior to IVF in fresh autologous cycles. ${ }^{278}$ This study compared this outcome in women who received one of 3 treatments: (1) a luteal phase GnRH agonist protocol; 2) a GnRH agonist flare protocol, begun during the follicular phase; or 3) a $\mathrm{GnRH}$ antagonist suppression protocol, begun in the mid-follicular phase. In the overall population, as compared to luteal $\mathrm{GnRH}$ agonist cycles in which ectopic pregnancies occurred in 1.6\% of the cycles, a higher risk for ectopic pregnancy was reported for both $\mathrm{GnRH}$ antagonist cycles (2.4\%, OR 1.52 (95\% CI 1.39 to 1.65$)$ ) and GnRH agonist flare cycles (2.1\%, OR 1.25 (95\% CI 1.09 to 1.44$)$ ). In multivariate models, among women with unexplained infertility, compared to women receiving the luteal phase $\mathrm{GnRH}$ agonist protocol, those receiving the $\mathrm{GnRH}$ agonist flare protocol had a lower risk of ectopic pregnancy with an adjusted OR (95\% CI) of 0.60 (0.37 to 0.96), P=0.03. Similarly, in multivariate models, among women with unexplained infertility, compared to women receiving the $\mathrm{GnRH}$ antagonist protocol, those receiving the $\mathrm{GnRH}$ agonist flare protocol had a lower risk of ectopic pregnancy with an adjusted OR (95\% CI) of 0.50 (0.32 to 0.80), $\mathrm{P}=0.004$. $^{278}$ There were no significant differences across protocols for PCOS, endometriosis, or tubal factor diagnoses.

\section{Preimplantation Genetic Diagnosis (PGD) Cycles With ART Versus Non-PGD Cycles}

In a good-quality observational study using CDC's web-based NASS database (which includes data from clinics participating in SART CORS, as well as a smaller number of nonSART participating clinics who report directly to CDC), for women under age 35, the odds of 
live birth per transfer was lower for all types of PGD cycles when compared with non-PGD cycles. Among live-birth deliveries, in models adjusting for cause of infertility, the adjusted OR (95\% CI) for low birth weight among PGD-Genetic cycles was 0.73 (0.54 to 0.98) as compared to those resulting from non-PGD cycles. In contrast, the adjusted OR (95\% CI) for low birth weight among PGD-Aneuploidy cycles was 1.25 (1.01 to 1.54) compared with non-PGD cycles. Among live births, the adjusted OR (95\% CI) for multiple birth in PGD-Other cycles was 0.76 (0.60 to 0.97) compared with non-PGD cycles. Results for women 35-37 and $>37$ years of age for low birth weight among PGD-Genetic cycles as compared to those resulting from non-PGD cycles or for PGD-Aneuploidy cycles compared with non-PGD cycles were not statistically significantly different. Results for women 35-37 and >37 years of age for multiple births among PGD-Genetic cycles as compared to those resulting from non-PGD cycles or for PGDAneuploidy cycles compared with non-PGD cycles were also not statistically significantly different. $^{276}$

In another good-quality study from SART CORS, PGD was associated with a lower chance of live birth with donor oocyte cycles compared to non-PGD cycles (OR 0.65, 95\% CI, 0.53 to $0.80) .^{280}$

\section{Insurance Coverage}

A cohort study of the SART CORS database examined pregnancy in States with mandated insurance of IVF coverage as compared with States without mandated IVF coverage. In models adjusted for cause of infertility, there was no significant difference in the odds of live birth in states with mandated insurance IVF coverage as compared with states with non-mandated IVF coverage (aOR 1.02, 95\% CI 0.97 to 1.07). The odds of multiple birth or higher-order multiple birth were lower in States with mandated insurance IVF coverage as compared with States with non-mandated IVF coverage. The adjusted odds ratio (95\% CI) of multiple birth or higher order multiple-birth in States with mandated IVF coverage was 0.87 (0.80 to 0.94$)$ and 0.74 (0.53 to 1.03), respectively, as compared with States without mandated IVF coverage. There was no statistically significant difference in the odds of low birth weight in States with mandated IVF coverage as compared with States without mandated IVF coverage (aOR 0.95, 95\% CI 0.88 to 1.03). ${ }^{279}$

\section{Strength of Evidence (With Any Infertility Diagnosis)}

Table 34 summarizes the SOE findings which span all infertility diagnoses.

Table 34. Strength of evidence for major outcomes-across all infertility diagnoses

\begin{tabular}{|c|c|c|c|c|}
\hline Comparison & Outcome & $\begin{array}{l}\text { Study Design } \\
\text { (Sample Size) }\end{array}$ & Conclusion & $\begin{array}{l}\text { Strength of } \\
\text { Evidence } \\
\text { (Rationale) }^{\mathrm{a}}\end{array}$ \\
\hline $\begin{array}{l}\text { Clomiphene } \\
\text { citrate and } \\
\text { gonadotropin }\end{array}$ & $\begin{array}{l}\text { Long-term } \\
\text { outcomes: Maternal } \\
\text { cancer }\end{array}$ & $\begin{array}{l}1 \text { Obs }^{136} \\
\text { (9892 patients) }\end{array}$ & $\begin{array}{l}\text { No difference. Ever use of } \\
\text { clomiphene citrate was not } \\
\text { statistically significantly } \\
\text { associated with maternal } \\
\text { ovarian, breast, endometrial, } \\
\text { lung, thyroid, colon, or } \\
\text { melanoma cancer. } \\
\text { Gonadotropin use was not } \\
\text { associated with increased risk } \\
\text { for breast or endometrial } \\
\text { cancer }\end{array}$ & $\begin{array}{l}\text { Low } \\
\text { (Size of cohort not } \\
\text { sufficient to detect } \\
\text { modest increases } \\
\text { in risk) }\end{array}$ \\
\hline
\end{tabular}




\begin{tabular}{|c|c|c|c|c|}
\hline Comparison & Outcome & $\begin{array}{l}\text { Study Design } \\
\text { (Sample Size) }\end{array}$ & Conclusion & $\begin{array}{l}\text { Strength of } \\
\text { Evidence } \\
\text { (Rationale) }^{\mathrm{a}}\end{array}$ \\
\hline \multirow[t]{8}{*}{ ART: IVF } & Live birth (by race) & $\begin{array}{l}1 \text { Obs }^{292} \\
(13,473 \text { cycles })\end{array}$ & $\begin{array}{l}\text { Greater disparity. Lower live } \\
\text { birth rate for blacks as } \\
\text { compared to white }(p<0.001)\end{array}$ & $\begin{array}{l}\text { Low } \\
\text { (Imprecise, } 1 \\
\text { study) }\end{array}$ \\
\hline & $\begin{array}{l}\text { Live birth (by } \\
\text { number of embryos } \\
\text { transferred) }\end{array}$ & $\begin{array}{l}1 \text { Obs }^{123} \\
(69,028 \text { cycles })\end{array}$ & $\begin{array}{l}\text { Improvement. Increased live } \\
\text { birth rate per cycle with } 2 \\
\text { embryo transfer as compared } \\
\text { to single-embryo transfer }\end{array}$ & $\begin{array}{l}\text { Low } \\
\text { (Imprecise, findings } \\
\text { with moderate } \\
\text { study limitations) }\end{array}$ \\
\hline & $\begin{array}{l}\text { Pregnancy } \\
\text { complications: } \\
\text { Multiple births (by } \\
\text { number of embryos } \\
\text { transferred) }\end{array}$ & $\begin{array}{l}1 \text { Obs }^{123} \\
(69,028 \text { cycles })\end{array}$ & $\begin{array}{l}\text { Greater risk. Multiple live birth } \\
\text { rates are significantly higher } \\
\text { with a 2-embryo transfer than } \\
\text { a single-embryo transfer, but } \\
\text { do not increase further with 3- } \\
\text { or 4-embryo transfers }\end{array}$ & $\begin{array}{l}\text { Low } \\
\text { (Imprecise, findings } \\
\text { with moderate } \\
\text { study limitations) }\end{array}$ \\
\hline & $\begin{array}{l}\text { Neonatal } \\
\text { outcomes: } \\
\text { Birthweight }\end{array}$ & $\begin{array}{l}1 \mathrm{Obs}^{274} \\
(8,948)\end{array}$ & $\begin{array}{l}\text { No difference: No significant } \\
\text { difference in rates of low } \\
\text { birthweight using ART by } \\
\text { assisted hatching, source of } \\
\text { oocytes/semen, number of } \\
\text { embryos or ICSI }\end{array}$ & $\begin{array}{l}\text { Low } \\
\text { (Imprecise) }\end{array}$ \\
\hline & $\begin{array}{l}\text { Neonatal } \\
\text { outcomes: } \\
\text { Congenital } \\
\text { Anomalies }\end{array}$ & $\begin{array}{l}1 \mathrm{Obs}^{277} \\
(64,861)\end{array}$ & $\begin{array}{l}\text { Greater risk. Risk of birth } \\
\text { defects was greater in infants } \\
\text { conceived using ART } \\
\text { (adjusted risk ratio } 1.28,95 \% \\
\mathrm{Cl} 1.15 \text { to } 1.42 \text { ) }\end{array}$ & $\begin{array}{l}\text { Low } \\
\text { (1 study) }\end{array}$ \\
\hline & $\begin{array}{l}\text { Long-term } \\
\text { outcomes: Child } \\
\text { (Autism) }\end{array}$ & $\begin{array}{l}1 \mathrm{Obs}^{272} \\
(42,383)\end{array}$ & $\begin{array}{l}\text { Greater risk. Risk of autism } \\
\text { was greater in children } \\
\text { conceived with ART with ICSI } \\
\text { as compared to ART without } \\
\text { ICSI (HR } 1.65, p<0.05 \text { ) }\end{array}$ & $\begin{array}{l}\text { Low } \\
\text { (Imprecise) }\end{array}$ \\
\hline & $\begin{array}{l}\text { Long-term } \\
\text { outcomes: Child } \\
\text { (neurological) }\end{array}$ & $\begin{array}{l}1 \text { Obs }^{273} \\
\text { (310 patients) }\end{array}$ & $\begin{array}{l}\text { Inconclusive. SOE was } \\
\text { insufficient given imprecise } \\
\text { evidence from } 1 \text { small } \\
\text { observational study with } \\
\text { moderate risk of bias }\end{array}$ & $\begin{array}{l}\text { Insufficient } \\
\text { (imprecise findings } \\
\text { with moderate } \\
\text { study limitations, } \\
\text { small study) }\end{array}$ \\
\hline & $\begin{array}{l}\text { Long-term } \\
\text { outcomes: Maternal } \\
\text { (cancer) }\end{array}$ & $\begin{array}{l}2 \text { Obs }^{125,289} \\
(280,950)\end{array}$ & $\begin{array}{l}\text { Greater risk. IVF was } \\
\text { associated with a statistically } \\
\text { significant increased risk of all } \\
\text { ovarian neoplasms (HR } 2.05 \text {; } \\
95 \% \mathrm{Cl}, 1.20 \text { to } 3.82 \text { ) and } \\
\text { borderline ovarian tumors (HR } \\
6.28 ; 95 \% \mathrm{Cl}, 2.05 \text { to } 19.84 \text { ), } \\
\text { and colorectal cancer (HR } \\
1.80,95 \% \mathrm{Cl} 1.10 \text { to } 2.94) \\
\text { No difference: IVF however } \\
\text { was not associated with an } \\
\text { increased risk of invasive } \\
\text { ovarian cancer (HR } 1.14 ; 95 \% \\
\text { CI, } 0.54 \text { to } 2.41) \text {, or melanoma } \\
\text { (SIR } 1.27 ; 95 \% \mathrm{Cl}, 0.75 \text { to } \\
2.15 \text { ) }\end{array}$ & $\begin{array}{l}\text { Low } \\
\text { (Imprecise, older } \\
\text { study) }\end{array}$ \\
\hline $\begin{array}{l}\text { IVF+ICSI vs. } \\
\text { IVF }\end{array}$ & $\begin{array}{l}\text { Neonatal } \\
\text { outcomes: Birth } \\
\text { weight }\end{array}$ & $\begin{array}{l}1 \text { Obs }^{185} \\
(90,401 \text { cycles })\end{array}$ & $\begin{array}{l}\text { No difference: No significant } \\
\text { difference in the odds of low } \\
\text { birth weight between ICSI } \\
\text { versus conventional-IVF } \\
\text { cycles }\end{array}$ & $\begin{array}{l}\text { Low } \\
\text { (1 study with } \\
\text { moderate study } \\
\text { limitations) }\end{array}$ \\
\hline
\end{tabular}

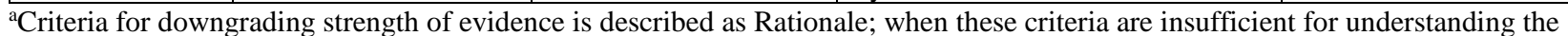
final strength of evidence, additional explanation is provided. 
Abbreviations: ART=assisted reproductive technology; $\mathrm{CI}=$ confidence interval; $\mathrm{HR}=$ hazards ratio; ICSI=intra-cytoplasmic sperm injection; IUI=intrauterine insemination; IVF=in vitro fertilization; KQ=Key Question; N=number of patients/participants; Obs=observational study; RCT=randomized controlled trial; SOE=strength of evidence

\section{Publication Bias}

As part of the Evidence-based Practice Center (EPC) Program, AHRQ sought to assess whether information from ClinicalTrials.gov would impact the conclusions of five ongoing systematic reviews. This infertility systematic review was part of this methods project (Augmenting Systematic Reviews with Information from ClinicalTrials.gov to Increase Transparency and Reduce Bias).

For this purpose, we searched the ClinicalTrials.gov registry of clinical studies to ascertain publication bias by identifying studies that have been completed but are as yet unpublished. Our search yielded 354 records of completed trials about treatments for infertility for screening (see Appendix A for our search strategy and Appendix $\mathrm{H}$ for details on our findings). Initial manual review identified 94 of these records as potentially relevant; subsequent review by a topic expert reduced this number to 66 . Of these 66 records, we were not able to identify publications for 12 studies that had expected completion dates 3 years or more prior to our search. During the search update period we again looked for publications covering these 12 studies. No new publications were found.

Of these 12 trials with unpublished results, two were considered potentially relevant to KQ 1 , 9 potentially relevant to KQ 3, and 1 potentially relevant to KQ 5. Implications for KQs 1, 2, and 4 are discussed in more detail in Appendix $\mathrm{H}$ as part of the augmenting transparency methods project.

For this current systematic review, the two trials identified as potentially relevant to KQ 1 had a combined sample size of 340 patients and were completed more than 3 years ago. These two "missing" trials are unlikely to have had a meaningful impact on our review's results especially given the presence of two systematic reviews for women with infertility from PCOS.

For KQ 3, there were nine potentially relevant unpublished studies where the underlying diagnosis of infertility was not listed. It is not clear whether these trials are specifically relevant to patients with infertility for unknown reasons as is required for inclusion in KQ 3 since the trials may focus on patients with identified infertility diagnoses. This diagnosis specification however is not available from the missing trials. The nine studies differed in terms of treatments evaluated and outcomes assessed. All studies were fairly small studies (planned enrollment varying between 60 and 242 individuals) and most of them focused on pregnancy rates rather than live births though could potentially be relevant based on information about miscarriages, ovarian hyperstimulation syndrome (OHSS), or other adverse events.

For KQ 5, there was one unpublished potentially relevant study which sought to determine whether couples with male factor infertility, specifically with elevated sperm DNA damage undergoing in vitro fertilization (IVF)/intra-cytoplasmic sperm injection (ICSI), should use testicular sperm extraction to improve their reproductive outcomes. Although this study did have live birth listed as the primary outcome, it had an enrollment of 25 males and so would not likely change any of our findings.

In summary, because of the relatively low number of unpublished studies identified through our ClinicalTrials.gov registry analysis as compared to our included set of studies, we do not believe these findings indicate significant publication bias in the evidence base that would impact our overall conclusions. 


\section{Discussion}

In this Comparative Effectiveness Review, we reviewed 151 studies described in 161 publications that directly compared infertility management strategies in couples with infertility due to polycystic ovary syndrome (PCOS; Key Question [KQ] 1) or endometriosis (KQ 2); unexplained infertility (KQ 3); tubal and peritoneal factor infertility (KQ 4); and male factor infertility (KQ 5). We also explored the comparative safety and effectiveness of management strategies for donors in infertility (KQ 6). Although the ultimate goal with any infertility management strategy is to improve live birth rates of healthy infants to a healthy couple, many studies initially identified in our review only reported on pregnancy rates or focused on other short-term outcomes and did not differentiate by the underlying causes of infertility. Our findings are based on those 151 studies which evaluated the comparative effectiveness of infertility management strategies in couples with a known cause of infertility (including unexplained infertility) and which evaluated the outcome of live birth or another long-term outcome.

\section{Key Findings and Strength of Evidence}

For women with infertility associated with PCOS, there was moderate strength of evidence (SOE) that letrozole compared to clomiphene results in higher live birth rates while reducing multiple births, with no difference in ectopic pregnancy or miscarriage (moderate SOE), or low birthweight and time to pregnancy (low SOE). There was moderate SOE clomiphene does not result in higher live birth rates compared to metformin, and low SOE for lack of differences in multiple birth, ectopic pregnancy, or time to pregnancy. Live birth rates are not different comparing laparoscopic ovarian drilling (LOD) with oral agents (moderate SOE).

For couples with endometriosis as the primary cause, there was insufficient evidence for specific comparisons/outcomes.

For couples with unexplained infertility, there is no difference between the oral agents of letrozole and anastrozole for the outcome of ectopic pregnancy (low SOE) but evidence is insufficient for other outcomes of interest. There is also no difference between differing adjunct treatments used in combination with oral agents and intrauterine insemination (IUI) for the outcomes of live birth, miscarriage and ovarian hyperstimulation syndrome (OHSS) (low SOE for all outcomes). Time to pregnancy was shorter with immediate in vitro fertilization (IVF) compared to strategies starting with clomiphene and IUI or gonadotropins and IUI followed by IVF if necessary (moderate SOE).

For couples with male factor infertility, live birth rate (moderate SOE) and miscarriage (low SOE) did not differ between intracytoplasmic sperm injection (ICSI) and intracytoplasmic morphological sperm injection (IMSI), a finding of limited applicability given the lack of clinical use of IMSI.

For oocyte donors, studies suggest a lower incidence of ovarian hyperstimulation syndrome (OHSS) with gonadotropin-releasing hormone $(\mathrm{GnRH})$ agonist trigger than with human chorionic gonadotropin (hCG) trigger (low SOE). However, there was a lack of evidence on any long-term outcomes

Findings applicable across all indications for infertility for couples undergoing assisted reproductive technology (ART) included: lower live birth rates for African-Americans compared to other racial/ethnic groups (low SOE); slightly lower live birth rates but significant reductions in multiple birth rates with elective single-embryo transfer compared to multiple-embryo transfer (low SOE); no increase in most maternal cancers after ART treatment after adjustment for 
infertility in general or specific causes (low SOE), and, for children born after ART, a possible increased risk of neurodevelopmental disorders after ICSI compared to IVF (low SOE) but no evidence of an increased risk of Type I diabetes.

\section{Findings in Relation to What Is Already Known}

The 2008 Agency for Healthcare Research and Quality (AHRQ) Evidence Report on "Effectiveness of ART"59 found that approximately 80 percent of the 478 included studies were performed outside the United States, and that the majority of RCTs did not report delivery rates and obstetric outcomes. In that review, most studies did not have sufficient power to detect clinically meaningful differences in live birth rates, and had still lower power to detect differences in less frequent outcomes such as multiple births and complications. In addition, the previous report focused on outcomes of specific treatments (ovulation induction, superovulation, and IVF/ICSI) rather than a wider range of potential treatments, and infertility diagnosis was considered as subgroup analyses, rather than the primary basis for comparing treatments.

Methods for evidence synthesis, in particular for rating strength of evidence, have also been revised since that report. Although an increasing number of studies are using live birth rate as the primary outcome, the majority of the literature, particularly randomized trials, is still based on pregnancy or ongoing pregnancy. Lack of precision for comparative estimates of rates for less common but important outcomes, such as complications, continues to be a major limitation.

To put the findings of our present systematic review in context, Tables 35 through 41 provide a comparison of the findings of this review, by KQ, with two major sets of guidelines/recommendations - those of the National Institute for Health and Care Excellence (NICE) in the United Kingdom (UK), and those of the American Society for Reproductive Medicine (ASRM). Recommendations for NICE and ASRM are presented verbatim. For the NICE guidelines, recommendations are taken from the 2013 document, ${ }^{17}$ except as noted in the tables. References to specific recommendations by ASRM are provided within the tables. For simplicity, we do not present the results of relevant Cochrane reviews here; the NICE guidance relied heavily on available Cochrane reviews, and the specific recommendations reflect judgments about overall strength of evidence. Sections or statements shaded in gray represent outcomes where our review's findings were different from those of the major guidelines/recommendations. Note that since our review focused on studies that reported live births - and not just pregnancies — several of the comparisons could not be made directly.

These tables demonstrate that in general, findings of our present review were concordant with the guidelines, with differences primarily attributable to differences in inclusion/exclusion criteria (particularly for publication dates and primary outcome of live birth vs. pregnancy).

For women with PCOS (Table 35), both NICE and ASRM support use of clomiphene citrate alone as first-line therapy, with the NICE guidance recommending ultrasound monitoring for dose adjustment to minimize risk of multiple pregnancy, followed by combination therapy with metformin or gonadotropins for women who do not conceive after a 3-6 month course of clomiphene alone. Both our review and NICE suggest letrozole may be superior to clomiphene as first line therapy, and that pretreatment with metformin may improve outcomes in women with PCOS being treated with gonadotropins.

For women with endometriosis (Table 36), ASRM concluded that evidence for surgical treatment of women with mild to moderate endometriosis was insufficient to recommend treatment, while the NICE guidance suggests some benefit, and our review was inconclusive. For 
those patients going directly to ART, surgical treatment of endometriosis, including endometrioma, prior to ART does not improve outcomes.

For women with unexplained infertility (Table 37), NICE recommends against use of oral agents entirely, while ASRM suggests clomiphene plus IUI may improve cycle fecundity compared to expectant management; our review found insufficient evidence. Based on our review, immediate IVF results in higher live birth rates and shorter time to pregnancy in women aged 38-42 compared with a trial of clomiphene and IUI or gonadotropins and IUI, with most live births ultimately resulting from IVF.

For women with suspected tubal factor infertility (Table 38), both NICE and ASRM recommend imaging for diagnosis (which is outside the scope of our review), although, when ART is readily available and affordable, proceeding directly to ART without a definitive diagnosis of tubal disease may be more efficient.

For male factor infertility (Table 39), our review found no relevant findings compared to the recommendations, primarily because of limited data on live birth outcomes.

For both male and female donors (Table 40), both NICE and ASRM recommend psychological evaluation and counseling, including, for females, the short term risks of ovarian stimulation and oocyte collection; our review found evidence on outcomes was limited only to the known short-term risks of these procedures, with no evidence on potential longer term risks.

For long-term outcomes in women and children after infertility treatment (Table 41), our review found limited or inconsistent evidence. Risks of adverse longer term maternal cancer outcomes were generally not increased after adjustment for the risk associated with infertility itself. ICSI however may be associated with an increased risk of neurodevelopmental disorders in children compared to those conceived through IVF. The NICE guidance was generally consistent with this assessment, and recommended that patients should be informed that any absolute risk was low, while there was still uncertainty about longer-term outcomes. 
Table 35. Report findings and major guidelines/recommendations-KQ 1. PCOS

\begin{tabular}{|c|c|c|c|}
\hline Intervention & Evidence Report Findings & NICE & ASRM \\
\hline $\begin{array}{l}\text { Selective estrogen } \\
\text { receptor } \\
\text { modulators }\end{array}$ & $\begin{array}{l}\text { Clomiphene citrate does not result in higher } \\
\text { live birth rates compared with metformin } \\
\text { (low SOE). Differences are also not found } \\
\text { in the rates of miscarriage, multiple birth, } \\
\text { ectopic pregnancy, or time to pregnancy } \\
\text { (low SOE for all outcomes) }\end{array}$ & $\begin{array}{l}\text { For women with WHO Group II ovulation } \\
\text { disorders (PCOS), offer as initial } \\
\text { treatment clomiphene, metformin, or a } \\
\text { combination, considering potential } \\
\text { adverse effects, ease and mode of use, } \\
\text { the woman's BMI, and monitoring } \\
\text { needed } \\
\text { For women who are taking clomiphene } \\
\text { citrate, offer ultrasound monitoring during } \\
\text { at least the first cycle of treatment to } \\
\text { ensure that they are taking a dose that } \\
\text { minimizes the risk of multiple pregnancy. } \\
\text { For women who are taking clomiphene } \\
\text { citrate, do not continue treatment for } \\
\text { longer than } 6 \text { months. } \\
\text { (Monitoring as part of treatment was not } \\
\text { included in our review.) }\end{array}$ & $\begin{array}{l}\text { Clomiphene citrate is an effective first-line } \\
\text { treatment for the majority of women with } \\
\text { anovulatory infertility. }{ }^{293} \\
\text { Failure to conceive after } 3 \text { to } 4 \text { successful CC- } \\
\text { induced ovulation cycles is indication for further } \\
\text { evaluation to exclude other contributing causes } \\
\text { of infertility, particularly in women }>35 \text { years of } \\
\text { age. } .^{293}\end{array}$ \\
\hline Insulin sensitizers & $\begin{array}{l}\text { Clomiphene citrate does not result in higher } \\
\text { live birth rates compared with metformin } \\
\text { (low SOE). Differences are also not found } \\
\text { in the rates of miscarriage, multiple birth, } \\
\text { ectopic pregnancy, or time to pregnancy } \\
\text { (low SOE for all outcomes) } \\
\text { The combination of metformin and } \\
\text { clomiphene does not significantly improve } \\
\text { live birth rates in women with higher BMI, } \\
\text { but precision is limited. }\end{array}$ & $\begin{array}{l}\text { For women with WHO Group II ovulation } \\
\text { disorders [PCOS] who are known to be } \\
\text { resistant to clomiphene citrate, consider } \\
\text { one of the following second-line } \\
\text { treatments, depending on clinical } \\
\text { circumstances and the woman's } \\
\text { preference: } \\
\text { - laparoscopic ovarian drilling or } \\
\text { combined treatment with } \\
\quad \text { clomiphene citrate and metformin } \\
\text { if not already offered as first-line } \\
\text { treatment or } \\
\text { - gonadotropins } \\
\text { Women prescribed metformin should be } \\
\text { informed of the side effects associated } \\
\text { with its use (such as nausea, vomiting } \\
\text { and other gastrointestinal disturbances). }\end{array}$ & $\begin{array}{l}\text { Combination therapies involving CC and other } \\
\text { agents (metformin, glucocorticoids, and } \\
\text { exogenous gonadotropins) may be effective } \\
\text { when treatment with CC alone fails to induce } \\
\text { ovulation. }{ }^{293} \\
\text { There is no evidence for improved live birth } \\
\text { rates or decreased pregnancy complications } \\
\text { with the use of metformin either before } \\
\text { conception or during pregnancy (Level A). }{ }^{294} \\
\text { Note there is a growing body of evidence on its } \\
\text { use both for PCOS patients who get treatment } \\
\text { and for patients with gestational diabetes. }\end{array}$ \\
\hline
\end{tabular}




\begin{tabular}{|c|c|c|c|}
\hline Intervention & Evidence Report Findings & NICE & ASRM \\
\hline $\begin{array}{l}\text { Aromatase } \\
\text { inhibitors }\end{array}$ & $\begin{array}{l}\text { Letrozole has a higher live birth rate than } \\
\text { clomiphene citrate alone and lower multiple } \\
\text { births (moderate SOE for both outcomes), } \\
\text { with no difference in ectopic pregnancy, } \\
\text { miscarriage, low birthweight, or time to } \\
\text { pregnancy (low SOE for these outcomes) }\end{array}$ & $\begin{array}{l}\text { In women with polycystic ovary } \\
\text { syndrome, letrozole appears to be } \\
\text { associated with a higher live birth rate, } \\
\text { lower rates of multiple pregnancy and } \\
\text { lower incidence of ovarian } \\
\text { hyperstimulation syndrome (OHSS) than } \\
\text { clomiphene citrate. }{ }^{295}\end{array}$ & No specific recommendations \\
\hline $\begin{array}{l}\text { Surgical } \\
\text { Management }\end{array}$ & $\begin{array}{l}\text { There was no difference between } \\
\text { laparoscopic ovarian drilling (LOD) and oral } \\
\text { agents for live birth (moderate SOE) or } \\
\text { miscarriage rates (low SOE). Multiple births } \\
\text { were reduced given LOD (moderate SOE) }\end{array}$ & $\begin{array}{l}\text { For women with WHO Group II ovulation } \\
\text { disorders [PCOS] who are known to be } \\
\text { resistant to clomiphene citrate, consider } \\
\text { one of the following second-line } \\
\text { treatments, depending on clinical } \\
\text { circumstances and the woman's } \\
\text { preference: } \\
\text { - LOD or } \\
\text { - combined treatment with } \\
\text { clomiphene citrate and } \\
\text { metformin if not already offered } \\
\text { as first-line treatment or } \\
\text { gonadotropins }\end{array}$ & No specific recommendations \\
\hline $\begin{array}{l}\text { Gonadotropins } \\
\text { alone }\end{array}$ & $\begin{array}{l}\text { Use of gonadotropins as primary therapy } \\
\text { does not improve outcomes compared to } \\
\text { oral agents }\end{array}$ & $\begin{array}{l}\text { Women with polycystic ovary syndrome } \\
\text { who are being treated with } \\
\text { gonadotropins should not be offered } \\
\text { treatment with gonadotropin-releasing } \\
\text { hormone agonist concomitantly because } \\
\text { it does not improve pregnancy rates, and } \\
\text { it is associated with an increased risk of } \\
\text { ovarian hyperstimulation. }\end{array}$ & No specific recommendations \\
\hline $\begin{array}{l}\text { Adjuncts to } \\
\text { gonadotropins }\end{array}$ & $\begin{array}{l}\text { Pretreatment with metformin prior to ART } \\
\text { may improve live birth rates and decrease } \\
\text { OHSS. } \\
\text { Use of GnRH antagonists as part of the } \\
\text { controlled ovarian hyperstimulation protocol } \\
\text { in IVF/ICSI reduces the incidence of OHSS } \\
\text { compared to GnRH agonists. }\end{array}$ & $\begin{array}{l}\text { The use of adjuvant growth hormone } \\
\text { treatment with gonadotropin-releasing } \\
\text { hormone agonist and/or human } \\
\text { menopausal gonadotropin during } \\
\text { ovulation induction in women with } \\
\text { polycystic ovary syndrome who do not } \\
\text { respond to clomiphene citrate is not } \\
\text { recommended because it does not } \\
\text { improve pregnancy rates. }\end{array}$ & No specific recommendations \\
\hline
\end{tabular}

aSections or statements shaded in gray represent outcomes where our review’s findings were different from those of the major guidelines/recommendations.

Abbreviations: ART=assisted reproductive technology; ASRM=American Society for Reproductive Medicine; BMI=body mass index; CC=clomiphene citrate; $\mathrm{GnRH=gonadotropin-releasing} \mathrm{hormone;} \mathrm{ICSI=intra-cytoplasmic} \mathrm{sperm} \mathrm{injection;} \mathrm{IVF=in} \mathrm{vitro} \mathrm{fertilization;} \mathrm{KQ=Key} \mathrm{Question;} \mathrm{NICE=National} \mathrm{Institute} \mathrm{for} \mathrm{Health} \mathrm{and} \mathrm{Care}$ Excellence (UK); OHSS=ovarian hyperstimulation syndrome; PCOS=polycystic ovary syndrome; SOE=strength of evidence; WHO=World Health Organization 
Table 36. Report findings and major guidelines/recommendations-KQ 2, endometriosis ${ }^{\mathrm{a}}$

\begin{tabular}{|c|c|c|c|}
\hline Intervention & Evidence Report Findings & NICE & ASRM $^{296}$ \\
\hline $\begin{array}{l}\text { Medical } \\
\text { management }\end{array}$ & $\begin{array}{l}\text { Strength of evidence was insufficient for all } \\
\text { comparisons. }\end{array}$ & $\begin{array}{l}\text { Medical treatment of minimal and mild } \\
\text { endometriosis diagnosed as the cause of } \\
\text { infertility in women does not enhance } \\
\text { fertility and should not be offered. }\end{array}$ & $\begin{array}{l}\text { There is no evidence that medical treatment of } \\
\text { endometriosis improves fertility } \\
\text { In younger women (under age } 35 \text { years) with } \\
\text { stage I/II endometriosis-associated infertility, } \\
\text { expectant management or superovulation with } \\
\text { IUI can be considered as first-line therapy. For } \\
\text { women } 35 \text { years of age or older, more } \\
\text { aggressive therapy (superovulation with IUI or } \\
\text { IVF) may be considered. } \\
\text { IVF success rates in women with endometriosis } \\
\text { appear to be diminished compared to women } \\
\text { with tubal factor infertility; however, IVF likely } \\
\text { maximizes cycle fecundity for those with } \\
\text { endometriosis. }\end{array}$ \\
\hline Surgery & $\begin{array}{l}\text { Strength of evidence was insufficient for all } \\
\text { comparisons. }\end{array}$ & $\begin{array}{l}\text { Women with minimal or mild } \\
\text { endometriosis who undergo laparoscopy } \\
\text { should be offered surgical ablation or } \\
\text { resection of endometriosis plus } \\
\text { laparoscopic adhesiolysis because this } \\
\text { improves the chance of pregnancy. } \\
\text { Women with ovarian endometriomas } \\
\text { should be offered laparoscopic } \\
\text { cystectomy because this improves the } \\
\text { chance of pregnancy. } \\
\text { Women with moderate or severe } \\
\text { endometriosis should be offered surgical } \\
\text { treatment because it improves the } \\
\text { chance of pregnancy. } \\
\text { Post-operative medical treatment does } \\
\text { not improve pregnancy rates in women } \\
\text { with moderate to severe endometriosis } \\
\text { and is not recommended. }\end{array}$ & $\begin{array}{l}\text { The benefit of laparoscopic treatment of } \\
\text { minimal or mild endometriosis is insufficient to } \\
\text { recommend laparoscopy solely to increase the } \\
\text { likelihood of pregnancy. } \\
\text { There is insufficient evidence to indicate that } \\
\text { resection of endometriomas prior to IVF } \\
\text { improves outcomes. } \\
\text { In women with stage III/IV endometriosis- } \\
\text { associated infertility, conservative surgical } \\
\text { therapy with laparoscopy or possible } \\
\text { laparotomy may be beneficial. Surgical } \\
\text { management of an endometrioma should } \\
\text { include resection or ablation, rather than } \\
\text { drainage, with resection preferred. For women } \\
\text { with stage III/IV endometriosis who fail to } \\
\text { conceive following conservative surgery or } \\
\text { because of advancing reproductive age, IVF-ET } \\
\text { is an effective alternative. }\end{array}$ \\
\hline
\end{tabular}

aSections or statements shaded in gray represent outcomes where our review’s findings were different from those of the major guidelines/recommendations.

Abbreviations: SRM=American Society for Reproductive Medicine; CC=clomiphene citrate; ET=embryo transfer; IUI=intrauterine insemination; IVF=in vitro fertilization; $\mathrm{KQ}=$ Key Question; NICE=National Institute for Health and Care Excellence (UK) 
Table 37. Report findings and major guidelines/recommendations-KQ 3, unexplained infertility ${ }^{\mathrm{a}}$

\begin{tabular}{|c|c|c|c|}
\hline Intervention & Evidence Report Findings & NICE & ASRM \\
\hline $\begin{array}{l}\text { Oral ovarian } \\
\text { stimulation }\end{array}$ & $\begin{array}{l}\text { There is no difference between the oral } \\
\text { agents of letrozole and anastrozole for the } \\
\text { outcome of ectopic pregnancy (low SOE) } \\
\text { but evidence is insufficient for other } \\
\text { outcomes of interest } \\
\text { There is no difference between differing } \\
\text { adjunct treatments used in combination } \\
\text { with oral agents and IUI for the outcomes } \\
\text { of live birth, miscarriage, and OHSS (low } \\
\text { SOE for all outcomes) }\end{array}$ & $\begin{array}{l}\text { Do not offer oral ovarian stimulation } \\
\text { agents (such as clomiphene citrate, } \\
\text { anastrozole or letrozole) to women with } \\
\text { unexplained infertility. }\end{array}$ & \multirow{2}{*}{$\begin{array}{l}\text { The treatment effects with non-ART treatment } \\
\text { for unexplained infertility generally are small. } \\
\text { Empiric treatment may do no more than } \\
\text { hasten conception in those couples who would } \\
\text { conceive eventually without treatment. }{ }^{297} \\
\text { Level I evidence from randomized clinical trials } \\
\text { supports short-term use of IUI, CC, } \\
\text { gonadotropins and IUI, and ART treatment for } \\
\text { unexplained infertility but is insufficient for } \\
\text { conclusions regarding CC/IUI treatment. } \\
\text { Clomiphene citrate treatment combined with } \\
\text { intercourse does not increase cycle fecundity } \\
\text { in couples with unexplained infertility } \\
\text { compared with expectant management; } \\
\text { however, clomiphene plus IUI does increase } \\
\text { fecundity compared to expectant } \\
\text { management. }\end{array}$} \\
\hline Clomiphene citrate & $\begin{array}{l}\text { There is no difference between letrozole } \\
\text { and clomiphene for outcomes of multiple } \\
\text { births or miscarriage (moderate SOE) }\end{array}$ & $\begin{array}{l}\text { Inform women with unexplained infertility } \\
\text { that clomiphene citrate as a stand-alone } \\
\text { treatment does not increase the chances } \\
\text { of a pregnancy or a live birth. }\end{array}$ & \\
\hline \multirow[t]{2}{*}{ IVF } & \multirow{2}{*}{$\begin{array}{l}\text { There are no differences between } \\
\text { immediate IVF versus other treatments } \\
\text { prior to IVF for the outcomes of live birth, } \\
\text { multiple births, ectopic pregnancy, } \\
\text { miscarriage, low birthweight, and OHSS } \\
\text { (low SOE for all outcomes). There is } \\
\text { however shorter time to pregnancy with } \\
\text { immediate IVF (moderate SOE) }\end{array}$} & $\begin{array}{l}\text { Advise women with unexplained infertility } \\
\text { who are having regular unprotected } \\
\text { sexual intercourse to try to conceive for a } \\
\text { total of } 2 \text { years (this can include up to } 1 \\
\text { year before their fertility investigations) } \\
\text { before IVF will be considered. }\end{array}$ & \multirow{2}{*}{$\begin{array}{l}\text { ART therapies are considerably more costly } \\
\text { than CC and IUI. } \\
\text { - Adverse effects of ART and ovarian } \\
\text { stimulation include multiple pregnancy } \\
\text { and ovarian hyperstimulation. } \\
\text { - When considering treatment options } \\
\text { for couples with unexplained infertility, } \\
\text { it is prudent to consider simple } \\
\text { treatment before complex treatment } \\
\text { and to balance what is known about } \\
\text { effectiveness against the cost and } \\
\text { adverse effects of different treatments. }\end{array}$} \\
\hline & & $\begin{array}{l}\text { Offer IVF treatment to women with } \\
\text { unexplained infertility who have not } \\
\text { conceived after } 2 \text { years (this can include } \\
\text { up to } 1 \text { year before their fertility } \\
\text { investigations) of regular unprotected } \\
\text { sexual intercourse. }\end{array}$ & \\
\hline
\end{tabular}

Abbreviations: ART=assisted reproductive technology; ASRM=American Society for Reproductive Medicine; CC=clomiphene citrate; FSH=follicle-stimulating hormone; ICSI=intra-cytoplasmic sperm injection; IUI=intrauterine insemination; IVF=in vitro fertilization; KQ=Key Question; NICE=National Institute for Health and Care Excellence (UK); OHSS=ovarian hyperstimulation syndrome; SOE=strength of evidence 
Table 38. Report findings and major guidelines/recommendations-KQ 4, tubal and peritoneal factor infertility

\begin{tabular}{|c|c|c|c|}
\hline Intervention & Evidence Report Findings & NICE & ASRM $^{298}$ \\
\hline Diagnosis & $\begin{array}{l}\text { Strength of evidence was insufficient for } \\
\text { all comparisons. }\end{array}$ & $\begin{array}{l}\text { Women who are not known to have } \\
\text { comorbidities (such as pelvic } \\
\text { inflammatory disease, previous ectopic } \\
\text { pregnancy or endometriosis) should be } \\
\text { offered HSG to screen for tubal occlusion } \\
\text { because this is a reliable test for ruling } \\
\text { out tubal occlusion, and it is less invasive } \\
\text { and makes more efficient use of } \\
\text { resources than laparoscopy. [2004] } \\
\text { Where appropriate expertise is available, } \\
\text { screening for tubal occlusion using } \\
\text { hysterosalpingo-contrast- } \\
\text { ultrasonography should be considered } \\
\text { because it is an effective alternative to } \\
\text { hysterosalpingography for women who } \\
\text { are not known to have comorbidities. } \\
\text { [2004] } \\
\text { Women who are thought to have } \\
\text { comorbidities should be offered } \\
\text { laparoscopy and dye so that tubal and } \\
\text { other pelvic pathology can be assessed } \\
\text { at the same time. [2004] }\end{array}$ & $\begin{array}{l}\text { There is good evidence to support HSG as the } \\
\text { standard firstline test to assess tubal patency, } \\
\text { but it is limited by false positive diagnoses of } \\
\text { proximal tubal blockage. } \\
\text { - The evidence is fair to recommend } \\
\text { tubal cannulation for proximal tubal } \\
\text { obstruction in young women with no } \\
\text { other significant infertility factors. } \\
\text { The evidence is fair to recommend } \\
\text { laparoscopic fimbrioplasty or } \\
\text { neosalpingostomy for the treatment of } \\
\text { mild hydrosalpinges in young women } \\
\text { with no other significant infertility } \\
\text { factors. }\end{array}$ \\
\hline
\end{tabular}




\begin{tabular}{|c|c|c|c|}
\hline Intervention & Evidence Report Findings & NICE & ASRM $^{298}$ \\
\hline Surgery & $\begin{array}{l}\text { Strength of evidence was insufficient for } \\
\text { all comparisons. }\end{array}$ & $\begin{array}{l}\text { For women with mild tubal disease, tubal } \\
\text { surgery may be more effective than no } \\
\text { treatment. In centres where appropriate } \\
\text { expertise is available it may be } \\
\text { considered as a treatment option. [2004] } \\
\text { For women with proximal tubal } \\
\text { obstruction, selective salpingography } \\
\text { plus tubal catheterisation, or } \\
\text { hysteroscopic tubal cannulation, may be } \\
\text { treatment options because these } \\
\text { treatments improve the chance of } \\
\text { pregnancy. [2004] }\end{array}$ & $\begin{array}{l}\text { There are no adequate trials comparing } \\
\text { pregnancy rates with tubal surgery vs. IVF. } \\
\text { However, IVF has a higher per-cycle } \\
\text { pregnancy rate. } \\
\text { The evidence is fair to recommend tubal } \\
\text { cannulation for proximal tubal obstruction in } \\
\text { young women with no other significant } \\
\text { infertility factors. } \\
\text { The evidence is fair to recommend } \\
\text { laparoscopic fimbrioplasty or } \\
\text { neosalpingostomy for the treatment of mild } \\
\text { hydrosalpinges in young women with no other } \\
\text { significant infertility factors. } \\
\text { Tubal anastomosis for reversal of tubal } \\
\text { sterilization has a significantly higher } \\
\text { cumulative pregnancy rate than IVF, and it is } \\
\text { more cost efficient, even in women } 40 \text { years of } \\
\text { age or older. } \\
\text { Laparoscopic salpingectomy or proximal tubal } \\
\text { ligation overcomes the detrimental effect of } \\
\text { hydrosalpinges on IVF pregnancy rates in } \\
\text { patients who are not candidates for corrective } \\
\text { tubal surgery. } \\
\text { There is good evidence for recommending } \\
\text { laparoscopic salpingectomy or proximal tubal } \\
\text { occlusion in cases of surgically irreparable } \\
\text { hydrosalpinges to improve IVF pregnancy } \\
\text { rates. } \\
\text { There is good evidence to support the } \\
\text { recommendation for microsurgical } \\
\text { anastomosis for tubal ligation reversal. }\end{array}$ \\
\hline
\end{tabular}

Abbreviations: ART=assisted reproductive technology; ASRM=American Society for Reproductive Medicine; HSG=hysterosalpingography; ICSI=intra-cytoplasmic sperm injection; IVF=in vitro fertilization; KQ=Key Question; NICE=National Institute for Health and Care Excellence (UK) 
Table 39. Report findings and major guidelines/recommendations-KQ 5, male factor infertilitya

\begin{tabular}{|c|c|c|c|}
\hline Intervention & Evidence Report Findings & NICE & ASRM \\
\hline Gonadotropins & No relevant findings & $\begin{array}{l}\text { Men with hypogonadotrophic } \\
\text { hypogonadism should be offered } \\
\text { gonadotrophin drugs because these are } \\
\text { effective in improving fertility. }\end{array}$ & No specific recommendations \\
\hline Surgical & No relevant findings & $\begin{array}{l}\text { Where appropriate expertise is available, } \\
\text { men with obstructive azoospermia } \\
\text { should be offered surgical correction of } \\
\text { epididymal blockage because it is likely } \\
\text { to restore patency of the duct and } \\
\text { improve fertility. Surgical correction } \\
\text { should be considered as an alternative to } \\
\text { surgical sperm recovery and IVF. } \\
\text { Men should not be offered surgery for } \\
\text { varicoceles as a form of fertility treatment } \\
\text { because it does not improve pregnancy } \\
\text { rates. }\end{array}$ & $\begin{array}{l}\text { Infertility due to obstructive azoospermia } \\
\text { may be treated effectively by surgical } \\
\text { reconstruction or by retrieval of sperm from } \\
\text { the epididymis or testis, followed by } \\
\text { IVF/ICSI. } \\
\text { When obstructive azoospermia results } \\
\text { from a vasectomy performed less than } 15 \\
\text { years before and there are no coexisting } \\
\text { female infertility factors, microsurgical } \\
\text { reconstruction of the reproductive tract } \\
\text { generally is preferred over sperm retrieval } \\
\text { and IVF/ICSI. }\end{array}$ \\
\hline ART & $\begin{array}{l}\text { Live birth rate (moderate SOE) and } \\
\text { miscarriage (low SOE) did not differ } \\
\text { between intracytoplasmic sperm injection } \\
\text { (ICSI) and intracytoplasmic morphological } \\
\text { sperm injection (IMSI). }\end{array}$ & $\begin{array}{l}\text { Couples should be informed that ICSI } \\
\text { improves fertilisation rates compared to } \\
\text { IVF alone, but once fertilisation is } \\
\text { achieved the pregnancy rate is no better } \\
\text { than with IVF. }\end{array}$ & No specific recommendations \\
\hline
\end{tabular}

a Sections or statements shaded in gray represent outcomes where our review’s findings were different from those of the major guidelines/recommendations.

Abbreviations: ASRM=American Society for Reproductive Medicine; ICSI=intra-cytoplasmic sperm injection; IVF=in vitro fertilization; KQ=Key Question; NICE=National Institute for Health and Care Excellence (UK) 
Table 40. Report findings and major guidelines/recommendations-KQ 6, donors

\begin{tabular}{|c|c|c|c|}
\hline Intervention & Evidence Report Findings & NICE & ASRM \\
\hline Male donors & $\begin{array}{l}\text { No evidence on short- or long-term } \\
\text { outcomes }\end{array}$ & $\begin{array}{l}\text { All potential semen donors should be } \\
\text { offered counselling from someone who is } \\
\text { independent of the treatment unit } \\
\text { regarding the implications for themselves } \\
\text { and their genetic children, including any } \\
\text { potential children resulting from donated } \\
\text { semen. }\end{array}$ & $\begin{array}{l}\text { Psychological evaluation and counseling by a } \\
\text { qualified mental health professional is strongly } \\
\text { recommended for all sperm donors. The } \\
\text { assessment should include a clinical interview } \\
\text { and, where appropriate, psychological testing. } \\
\text { Psychological consultation should be required } \\
\text { for individuals in whom there appear to be } \\
\text { factors that warrant further evaluation. In } \\
\text { cases of directed donation, psychological } \\
\text { evaluation and counseling are strongly } \\
\text { recommended for the donor and his partner (if } \\
\text { applicable) as well as for the recipient female } \\
\text { and her partner (if applicable). The potential } \\
\text { impact of the relationship between the donor } \\
\text { and recipient should be explored. The } \\
\text { psychological assessment also should address } \\
\text { the potential psychological risks and evaluate } \\
\text { for evidence of coercion (financial or } \\
\text { emotional). It is important to ascertain whether } \\
\text { the donor is well informed about the extent to } \\
\text { which information about him might be } \\
\text { disclosed and about any plans that may exist } \\
\text { relating to future contact. } 999\end{array}$ \\
\hline
\end{tabular}




\begin{tabular}{|c|c|c|c|}
\hline Female donors & $\begin{array}{l}\text { Limited evidence on short-term outcomes, } \\
\text { no evidence on long-term outcomes } \\
\text { Observational studies suggest a lower } \\
\text { incidence of OHSS with GnRH agonist } \\
\text { trigger than with hCG trigger (low SOE). }\end{array}$ & $\begin{array}{l}\text { Before donation is undertaken, oocyte } \\
\text { donors should be screened for both } \\
\text { infectious and genetic diseases in } \\
\text { accordance with the 'UK guidelines for } \\
\text { the medical and laboratory screening of } \\
\text { sperm, egg and embryo donors' (2008). } \\
\text { Oocyte donors should be offered } \\
\text { information regarding the potential risks } \\
\text { of ovarian stimulation and oocyte } \\
\text { collection. } \\
\text { Oocyte recipients and donors should be } \\
\text { offered counselling from someone who is } \\
\text { independent of the treatment unit } \\
\text { regarding the physical and psychological } \\
\text { implications of treatment for themselves } \\
\text { and their genetic children, including any } \\
\text { potential children resulting from donated } \\
\text { oocytes. }\end{array}$ & $\begin{array}{l}\text { Psychological evaluation and counseling by a } \\
\text { qualified mental health professional is strongly } \\
\text { recommended for the oocyte donor and her } \\
\text { partner (if applicable). The assessment should } \\
\text { include a clinical interview and, where } \\
\text { appropriate, psychological testing. } \\
\text { Psychological consultation should be required } \\
\text { for individuals in whom there appear to be } \\
\text { factors that warrant further evaluation. In } \\
\text { circumstances involving known donors, } \\
\text { psychological evaluation and counseling is } \\
\text { strongly recommended for the donor and her } \\
\text { partner, if applicable, as well as for the } \\
\text { recipient and her partner, if applicable. The } \\
\text { potential impact of the relationship between } \\
\text { the donor and recipient should be explored. } \\
\text { The psychological assessment also should } \\
\text { address the potential psychological risks and } \\
\text { evaluate for evidence of coercion (financial or } \\
\text { emotional). It is important to ascertain whether } \\
\text { the donor is well informed about the extent to } \\
\text { which information about her may be disclosed } \\
\text { and about any plans that may exist relating to } \\
\text { future contact } \\
\text { All oocyte donors should be advised } \\
\text { explicitly of the risks and adverse effects } \\
\text { of ovarian stimulation and retrieval, with } \\
\text { such counseling documented by informed } \\
\text { consent in the patient's permanent } \\
\text { medical record. }{ }^{299} \\
\text { Oocyte donors are exposed to the risks of } \\
\text { controlled ovarian stimulation, oocyte retrieval, } \\
\text { and anesthesia. } \\
\text { The risk of OHSS is estimated to occur in } 1 \%- \\
2 \% \text { of donation cycles and may be further } \\
\text { reduced by the use of GnRH agonists for } \\
\text { triggering final oocyte maturation. } \\
\text { The risk of serious acute complications } \\
\text { associated with these procedures is small } \\
\text { (<0.5\%). } \\
\text { As these are independent events, the } \\
\text { cumulative risk of multiple procedures should } \\
\text { be similarly low. } \\
\text { The preponderance of data does not } \\
\text { demonstrate a significant risk of future cancers }\end{array}$ \\
\hline
\end{tabular}




\begin{tabular}{|c|c|c|c|}
\hline Intervention & Evidence Report Findings & NICE & ASRM \\
\hline & & & $\begin{array}{l}\text { in women undergoing stimulation and egg } \\
\text { retrieval. } \\
\text { While the data are limited, available evidence } \\
\text { does not suggest that oocyte donation is } \\
\text { associated with changes in the donor's ovarian } \\
\text { reserve. } \\
\text { Currently, there are no clearly documented } \\
\text { long-term risks associated with oocyte } \\
\text { donation and as such no definitive data upon } \\
\text { which to base absolute recommendations. } \\
\text { However, because of the possible health risks } \\
\text { outlined in the preceding discussion, it is } \\
\text { prudent to limit the number of stimulated } \\
\text { cycles for a given oocyte donor to } 6 . .^{300}\end{array}$ \\
\hline
\end{tabular}

Abbreviations: ASRM=American Society for Reproductive Medicine; GnRH=gonadotropin-releasing hormone; hCG=human chorionic gonadotropin; ICSI=intra-cytoplasmic sperm injection; KQ=Key Question; NICE=National Institute for Health and Care Excellence (UK); OHSS=ovarian hyperstimulation syndrome; SOE=strength of evidence

\begin{tabular}{|c|c|c|c|}
\hline Intervention & Evidence Report Findings & NICE & ASRM \\
\hline $\begin{array}{l}\text { Ovulation } \\
\text { Induction Agents }\end{array}$ & $\begin{array}{l}\text { - Infertility itself is associated with an } \\
\text { increased risk of some cancers, and the } \\
\text { overall risk of cancer in women exposed to } \\
\text { oral ovulation agents, gonadotropins, and } \\
\text { IVF/ICSI is not increased after adjustment } \\
\text { for infertility in general or specific causes, } \\
\text { although there are some associations with } \\
\text { less common cancers (low SOE). } \\
\text { - For children born after ART, ICSI may be } \\
\text { associated with an increased risk of } \\
\text { neurodevelopmental disorders compared to } \\
\text { IVF, although evidence is inconsistent (low } \\
\text { SOE). }\end{array}$ & $\begin{array}{l}\text { Inform women who are offered ovulation } \\
\text { induction or ovarian stimulation that: } \\
\text { - No direct association has been found } \\
\text { between these treatments and invasive } \\
\text { cancer and } \\
\text { - No association has been found in the } \\
\text { short- to medium-term between these } \\
\text { treatments and adverse outcomes } \\
\text { (including cancer) in children born from } \\
\text { ovulation induction and } \\
\text { - Information about long-term health } \\
\text { outcomes in women and children is still } \\
\text { awaited. }\end{array}$ & No specific recommendations \\
\hline
\end{tabular}




\begin{tabular}{|c|c|c|c|}
\hline Intervention & Evidence Report Findings & NICE & ASRM \\
\hline IVF/ICSI & $\begin{array}{l}\text { - Infertility itself is associated with an } \\
\text { increased risk of some cancers, and the } \\
\text { overall risk of cancer in women exposed to } \\
\text { oral ovulation agents, gonadotropins, and } \\
\text { IVF/ICSI is not increased after adjustment } \\
\text { for infertility in general or specific causes, } \\
\text { although there are some associations with } \\
\text { less common cancers (low SOE).In one } \\
\text { large study from the United Kingdom, the } \\
\text { risk of colorectal cancer was not increased } \\
\text { among women undergoing ART compared } \\
\text { to the general population, but was } \\
\text { increased compared infertile women who } \\
\text { did not undergo ART (low SOE). } \\
\text { For children born after ART, ICSI may be } \\
\text { associated with an increased risk of } \\
\text { neurodevelopmental disorders compared to } \\
\text { IVF, although evidence is inconsistent (low } \\
\text { SOE). }\end{array}$ & $\begin{array}{l}\text { Inform women that while the absolute risks of } \\
\text { long-term adverse outcomes of IVF } \\
\text { treatment, with or without ICSI, are low, a } \\
\text { small increased risk of borderline ovarian } \\
\text { tumours cannot be excluded. } \\
\text { Inform people who are considering IVF } \\
\text { treatment that the absolute risks of long-term } \\
\text { adverse outcomes in children born as result } \\
\text { of IVF are low. }\end{array}$ & No specific recommendations \\
\hline
\end{tabular}

Abbreviations: ART=assisted reproductive technology; ASRM=American Society for Reproductive Medicine; ICSI=intra-cytoplasmic sperm injection; IVF=in vitro fertilization; KQs=Key Questions; NICE=National Institute for Health and Care Excellence (UK) 
In general, this review's findings are consistent with the guidelines cited above-there is a general consensus that the overall body of evidence for many aspects of infertility treatment across all patient groups is limited. One consistent limitation is the relative paucity of studies utilizing live birth per couple as the primary outcome. Where there are differences between our findings and these guidelines, or between guidelines, they can be attributed to the following:

- Differences in study inclusion/exclusion criteria, particularly in study dates and outcomes considered.

- Differences in grading individual study quality, which are then reflected in grading of the overall strength of evidence.

- Differences in the approach to guideline development - the NICE guidance follows the Grading of Recommendations Assessment, Development and Evaluation (GRADE) system similar to our approach, while the ASRM uses a less structured approach.

- Differences in settings, which affect the manner in which cost considerations are weighed in formulating recommendations. NICE guidance is designed for use within the UK National Health Service, and cost-effectiveness is considered from a health system perspective. Conversely, the ASRM guidelines are meant to be applied in the more diverse U.S. setting, where coverage of infertility services is much more varied, and the perspective of individual payers and patients, who are likely to bear a much higher proportion of the cost of care, must be considered.

\section{Applicability}

Table 42 summarizes the applicability scores across KQs.

Table 42. Potential issues with applicability of included studies ${ }^{\mathbf{a}}$

\begin{tabular}{|c|c|c|c|c|c|c|c|c|c|}
\hline & Issues & $\begin{array}{l}\mathrm{KQ} 1 \\
\mathrm{~N}=56\end{array}$ & $\begin{array}{c}\mathrm{KQ} 2 \\
\mathrm{~N}=7\end{array}$ & $\begin{array}{l}\mathrm{KQ} 3 \\
\mathrm{~N}=50\end{array}$ & $\begin{array}{c}\mathrm{KQ} 4 \\
\mathrm{~N}=8\end{array}$ & $\begin{array}{l}\mathrm{KQ} 5 \\
\mathrm{~N}=23\end{array}$ & $\begin{array}{c}\mathrm{KQ} 6 \\
\mathrm{~N}=5\end{array}$ & $\begin{array}{c}\text { Across } \\
\text { All KQs } \\
N=21\end{array}$ & $\begin{array}{c}\text { Total } \\
\mathrm{N}=151\end{array}$ \\
\hline \multirow[t]{2}{*}{ Population } & $\begin{array}{l}\text { Study population } \\
\text { demographics not } \\
\text { representative of } \\
\text { intended population }\end{array}$ & 1 & 1 & 0 & 1 & 1 & 0 & 0 & 4 \\
\hline & $\begin{array}{l}\text { Narrow or } \\
\text { unrepresentative } \\
\text { severity/stage/ } \\
\text { comorbidity }\end{array}$ & 1 & 0 & 0 & 0 & 0 & 0 & 1 & 2 \\
\hline \multirow[t]{2}{*}{ Intervention } & $\begin{array}{l}\text { Treatment protocol } \\
\text { not representative of } \\
\text { current practice }\end{array}$ & 2 & 1 & 4 & 1 & 0 & 0 & 1 & 9 \\
\hline & $\begin{array}{l}\text { Change in standard } \\
\text { of care }\end{array}$ & 0 & 0 & 1 & 0 & 0 & 0 & 1 & 2 \\
\hline Comparator & $\begin{array}{l}\text { Comparator not } \\
\text { representative of } \\
\text { current practice }\end{array}$ & 1 & 0 & 2 & 0 & 0 & 0 & 1 & 4 \\
\hline Outcomes & $\begin{array}{l}\text { Timing of outcome } \\
\text { assessment }\end{array}$ & 0 & 0 & 0 & 0 & 0 & 0 & 0 & 0 \\
\hline Setting & $\begin{array}{l}\text { Standards or access } \\
\text { to care vary from } \\
\text { U.S. setting }\end{array}$ & 32 & 5 & 27 & 3 & 11 & 3 & 2 & 77 \\
\hline
\end{tabular}




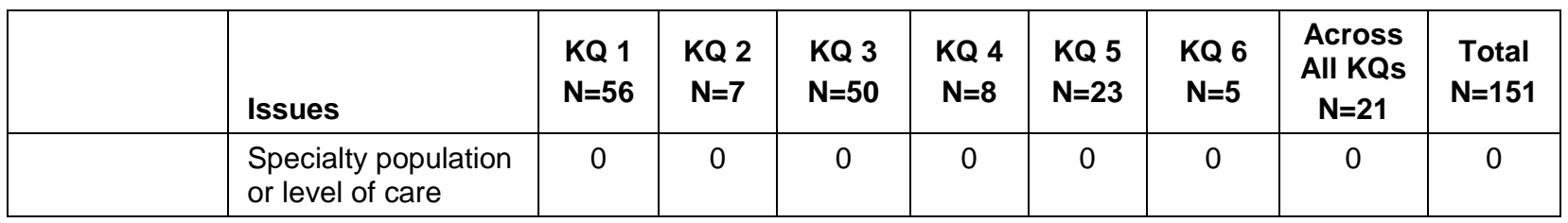

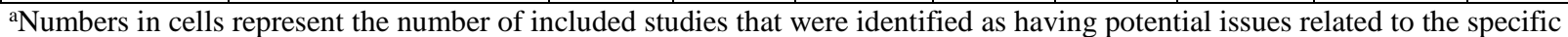
item. Columns represent numbers for each Key Question and then for all included studies.

Two broad issues relate to the overall applicability of the available evidence to clinical practice in the United States-one geographic and one temporal. Many of the randomized controlled trials (RCTs) meeting our criteria were performed outside of the United States. Leaving aside any issues related to differences in study oversight or reporting, the populations of these studies may differ from U.S. infertility patients in two potentially important ways.

The first issue is that there may be clinically relevant differences between populations in terms of non-clinical factors affecting outcomes. For example, live birth rates for AfricanAmerican women undergoing ART in the US are lower than for white women ${ }^{292}$, which may reflect issues related to socioeconomic status, insurance coverage, or other factors (such as wellestablished racial differences in the risk of many adverse pregnancy outcomes). Differences in access to infertility services between countries may lead to differences in the likelihood of treatment success. Although the estimate of any relative difference between two interventions derived from an unbiased RCT should in theory be independent of the probability of specific outcomes, the more clinically relevant absolute difference may be substantially different (e.g., the risk of preterm birth in African-American compared to white women is consistently elevated). To the extent that the probability of specific outcomes of interest may differ between populations because of differences in genetic risk, exposures to other factors affecting risk, or non-biological factors such as access to care, there may be substantial differences in estimates of absolute risk differences. For relatively uncommon but important outcomes, these differences might also affect precision of estimates - confidence intervals for any treatment effect will be wider in populations where the outcome is less common.

In addition to the potential impact of race/ethnicity, there may be important differences in the distribution of socioeconomic status between populations. Access to infertility diagnosis and treatment varies across countries, and certainly within the United States. ${ }^{301}$ Differences in socioeconomic status could affect applicability in several ways. Differences in access to care may lead to differences in the spectrum of severity of "disease" for U.S. patients who given the financial burden of treatment options they may wait longer to undergo evaluations. Although summary statistics of baseline characteristics may allow some judgment of comparability, there may be potentially important differences in the distribution that are obscured by the typical reporting of means and standard deviations (particularly if the underlying characteristic is not normally distributed), or by differences within a given stage. Socioeconomic status may also potentially affect some important outcomes independently of any specific treatment—for example, neurodevelopmental outcomes such as specific learning skills may be strongly correlated with parental socioeconomic status.

The second issue is that changes in practice over time have a major impact on applicability, particularly for long-term outcomes. The long lag time between exposure to infertility treatment and the potential development of longer term outcomes such as cancer means that data available today necessarily reflect women exposed to treatments at least 10 years in the past; even if the specific exposure is similar, there may be differences between past and current practice in potentially important attributes such as dosage, timing, patient selection criteria, use of 
adjunctive treatments, etc. For example, evidence that immediate use of IVF leads to shorter time to pregnancy than strategies where IVF is used only after a trial of agents such as clomiphene or gonadotropins has led to a change in guidelines. ${ }^{297}$ which now suggest that the cumulative exposure to gonadotropins during the course of treatment is likely to decrease compared to earlier cohorts of women, reducing any long-term risks.

In addition, there may be cohort effects in terms of other exposures that may affect the absolute risk of some outcomes (e.g., changes in the use of postmenopausal hormone replacement therapy or ages of mammography screening affecting breast cancer risk), which in turn would impact any additional absolute risk due to exposure to infertility treatments. Because of this phenomenon, there is likely to always be some unresolvable uncertainty about long-term outcomes for both parents undergoing current infertility treatments and their children.

\section{Implications for Clinical and Policy Decision Making}

Our review found considerable uncertainty about the comparative effectiveness of many available treatments for infertility, both those targeted at specific causes and those like ART that are used broadly in patients with a wide range of underlying diagnoses. A large component of this uncertainty is due to inconsistency in the choice of reported outcomes. This is especially true of the choice for primary measure of "success." Although there is a growing consensus that live birth rate per couple (either per cycle or per a number of treatment cycles) undergoing a particular intervention is the most relevant outcome for clinical decision making, ${ }^{22,302-304}$ many studies, particularly older ones, report intermediate outcomes such as clinical or ongoing pregnancy rates. There is some evidence that conclusions about relative effectiveness are similar whether ongoing pregnancy or live birth is used as the primary outcome. ${ }^{305}$ However, there may be relevant differences in absolute effectiveness, as discussed under Limitations. Although inclusion of studies using these intermediate outcomes would have certainly increased the potential pool of studies, our Technical Expert Panel felt that limiting included studies to those that reported live birth was important clinically. From a study design and feasibility perspective, intermediate outcomes reduce costs through smaller sample size requirements and shorter overall time to reaching endpoints (discussed in more detail under Research Recommendations), but feasible studies that do not sufficiently resolve uncertainty about specific clinical or policy decisions to allow confidence in those decisions are ultimately not an efficient use of resources.

Another source of uncertainty is a lack of consensus or clarity on the relative importance of different outcomes to patients, clinicians, and policymakers. Many clinical decisions regarding infertility treatment involve tradeoffs between, at least, the number of treatment cycles required, the cumulative probability of a successful outcome, and the relative probability of multiple gestations. Depending on the health system, these clinical tradeoffs can have financial implications for patients as well. There is a striking lack of evidence on the relative value patients place on different outcomes related to infertility treatment, with relatively few studies using standard methods of preference elicitation available. ${ }^{306-309}$ This makes decision analyses, both clinical and economic, difficult. This lack of data also inhibits design and testing of policies intended to optimize outcomes (e.g., insurance coverage for elective single-embryo transfer over multiple cycles for eligible patients in order to take away incentives for multiple-embryo transfer when patients pay out of pocket).

As discussed above in the applicability section, the combination of continuous changes in infertility treatment and the long time horizon needed to obtain evidence on long-term safety outcomes for both parents and children means that there will always be a degree of unresolvable 
uncertainty about long-term safety for patients making decisions now. To some extent, this is true of most clinical decision making (for example, estimates of the impact of specific interventions such as cancer screening on life expectancy are based on assumptions about treatment effectiveness, competing risks, etc., that do not reflect potential future changes). This is another area where more insight into relative preferences for both outcomes and timing of outcomes (e.g., is there a potential increased risk in the risk of cancer in 20 or 30 years that patients would be willing to trade off for an increased probability of a live birth within the next year) would be helpful.

A potential first step toward addressing these uncertainties within the U.S. context would be to create a formal structure for evaluating specific decisions relevant to infertility management, similar to the GRADE approach used by NICE. Achieving consensus on at least the relative importance of specific outcomes (potentially including costs), the ideal method for measuring those outcomes, and acceptable trade-offs between harms and benefits (such as the number of cases of OHSS per live birth, or number of preterm births attributable to multiple births per live birth), would be useful for structuring future reviews, guidelines development, and, through methods such as value-of-information analysis, ${ }^{310}$ prioritizing future research.

\section{Limitations of the Systematic Review Process}

Several aspects of the review process may have affected the results. First, there were constraints in our search strategy, developed in consultation with the Key Informants and TEP. We did not review evidence on the diagnostic evaluation of patients with infertility. Although this is obviously a critical question for patients, clinicians, and policymakers, it was outside of the scope of the review.

We limited the search to papers published after the cutoff date of the previous AHRQ Evidence Report on ART. ${ }^{59}$ This meant that studies completed prior to the cutoff date, which otherwise might have met inclusion criteria, were excluded. While we believe that the majority of these studies were included in the systematic reviews we used to supplement each $\mathrm{KQ}$, it is possible that potentially relevant articles were missed. Given broad changes in clinical practice (such as increasing use of ICSI even in the absence of specific male factor infertility and decreasing use of empiric gonadotropin therapy prior to ART in couples with unexplained infertility) over the past decade, the impact of missing earlier studies on conclusions about comparative effectiveness of currently used treatment alternatives is unclear.

We limited the outcomes to those considered most important by key stakeholders, using a formal prioritization process described in the Methods section, in an attempt to keep the scope of the review tractable. We specifically limited the review to articles that reported live birth as the primary pregnancy-related outcome, excluding studies that reported pregnancy rates alone (including studies reporting clinical or ongoing pregnancies). There is growing consensus that live birth is the most appropriate outcome for studies of infertility treatment effectiveness, ${ }^{22,302}$ particularly when expressed as the cumulative probability of live birth per couple over time rather than on a per-cycle basis, ${ }^{303,304}$ since this is the most clinically relevant information for a given couple. In 2010, the Cochrane group found that live birth is still infrequently reported in trials of infertility treatment, but there is some evidence suggesting that, in studies that report both clinical pregnancy and live birth outcomes, the magnitude and direction of effect are similar. ${ }^{305}$ Including otherwise eligible studies that reported clinical pregnancy rates alone may have provided some additional evidence relevant to the KQs. However, because the use of a surrogate or intermediate outcome such as clinical pregnancy rate affects the strength of 
evidence through its effect on directness, our overall assessment of strength of evidence would not likely have substantially changed.

Our review process was structured by KQ and, for each outcome, we required that outcomes be reported for the specific patient population covered by the KQ, or, in the case of observational studies, that the underlying diagnosis be included in multivariate analyses so that the reported overall measure of association accounted for variability based on diagnosis. This approach led to the exclusion of a number of studies of treatments used across multiple diagnoses, particularly those involving ART. The extent to which specific outcomes might differ based on underlying diagnosis is unclear. For long-term outcomes such as cancer in female patients, there is evidence that certain infertility diagnoses increase risk independently of any treatment effects (for example, PCOS and endometrial cancer, or endometriosis and ovarian cancer). Risks of some adverse outcomes (e.g., OHSS or ectopic pregnancy) differ in different populations (e.g., women with PCOS, tubal factor, or endometriosis infertility). However, there is less evidence that treatment effectiveness varies by diagnosis, although even when relative differences are similar, there still may be clinically important differences in the absolute probability of specific benefits and harms. One alternative approach to structuring the review would be to focus diagnosisspecific reviews only on treatments used prior to initiation of ART, and report comparative effectiveness of treatments used in ART under the assumption that outcomes are similar across patient populations. However, even if this approach expanded the evidence base, there still would be residual uncertainty surrounding quantitative estimates of outcome likelihood in specific patient populations.

Last, we did not include studies published in languages other than English, primarily due to resource limitations. However, given differences in the way infertility evaluation and treatment is financed in different countries, our judgment (discussed in more detail under Applicability) is that there may be important differences, both measurable and unmeasurable, between couples undergoing infertility in the United States compared with other countries. Inclusion of nonEnglish language studies would only make this problem worse.

\section{Research Recommendations}

In an era of constrained resources, future clinical research, especially comparative effectiveness research - which helps resolve current uncertainties regarding clinical or policy decisions — should receive priority. For most of the KQs, there are multiple areas of remaining uncertainty based on the existing evidence. In part because of the diversity of causes and treatment options, it is difficult to make specific recommendations for specific topics.

Before setting a specific agenda for future research in infertility, we believe a more general approach to identifying priorities would be helpful. Achieving consensus on the relative priority of specific outcomes, incorporating the perspective of multiple stakeholders (similar to the approach used for developing a research agenda for comparative effectiveness research for uterine fibroids. $^{311,312}$ Ideally, these outcome priorities would be used for subsequent evidence syntheses and guideline development.

As part of this consensus process, additional areas of discussion include:

- Formal consideration of the limits of acceptability for specific quantitative harms (e.g., preterm birth) and clinically meaningful differences in benefits (e.g., live birth).

- Formal discussion of the potential role of cost-effectiveness in decision making, including issues of willingness-to-pay and appropriate choice of outcome. This is particularly important because there are significant methodological challenges to the use 
of "standard” measures such as quality-adjusted life expectancy in the setting of infertility treatment.

- Issues related to study design, particularly from the patient stakeholder perspective. For example, in settings where patients and/or clinicians may have strong preferences for specific treatments, recruitment into RCTs may be difficult. ${ }^{313}$ In the uterine fibroid consensus process, patient stakeholders strongly preferred observational designs to randomized treatment assignment. ${ }^{311}$ Discussion of potential trade-offs between risk of bias, efficiency, ability to measure all relevant potential confounders and effect modifiers, appropriateness of alternative approaches such as Zelen randomization (where subjects are randomized prior to consent, then allowed to either receive the assigned treatment or choose the alternative ${ }^{314}$ ), and the likelihood that a specific study design would resolve a specific area of uncertainty should all be included.

- Issues related to data reporting. Particularly for ART and other treatments which are used for multiple indications, reporting of results separately by indication in both randomized trials and large observational studies would be extremely useful. Although these subgroup results may have insufficient power to detect clinically relevant differences within the context of individual studies (particularly RCTs), their routine publication would eventually allow synthesis of results using methods such as meta-analysis (including individual-level meta-analysis.)

Part of this process could include value-of-information analysis, a formal method for quantifying the impact of existing uncertainty on the likelihood of making the "wrong” decision. ${ }^{315-317}$ Although the approach has classically been used in the framework of costeffectiveness, the basic methods for illustrating the impact of uncertainty on the probability of making an optimal decision can also be applied using specific harms and benefits.

In addition to development of a specific consensus-driven approach to resolving uncertainty, other specific recommendations apply across all areas of infertility treatment. Empiric measurement of patient preferences using validated measures would have substantial impact. In the context of infertility treatment, where approaches such as the standard gamble or time tradeoff (which require trading off risk of immediate death or life expectancy versus specific health benefits) may be both conceptually difficult and counter-intuitive to patients, approaches such as discrete choice experiments (DCE) may be preferable (DCE, or conjoint analysis, also has the advantage of being able to explicitly incorporate costs to measure willingness-to-pay). ${ }^{318,319}$

The SART CORS and National ART Surveillance System (which includes data submitted through SART CORS (the majority of clinics providing ART) as well as a smaller number of non-SART participating clinics who report directly to CDC) databases are outstanding examples of what a large-scale, population-based registry can achieve in terms of providing data on treatment outcomes. However, the major limitation of the database in the past has been that data are only published on a per-cycle, rather than per-couple, basis. Recently the database methods have changed and now they are publicly reporting the cumulative success rate per patient. Results, however, are still reported at the clinic level, so patients who receive care at more than one clinic do not have the full range of outcomes captured, and there is no mechanism for prospectively collecting long-term outcomes of patients or children. Facilitating reporting of results so that outcomes are reported on a per-couple basis will substantially improve the ability to generate estimates of the likely outcome of specific ART-related decisions. 
Based on input from key informants and our Technical Expert Panel, we structured the review based on infertility diagnosis, and required studies to report outcomes specifically by diagnosis, or to adjust for diagnosis in multivariable analyses. As noted above, this led to exclusion of a number of papers, particularly those related to ART methods. There is clear evidence that the probability of some outcomes of interest, both short-term (e.g., OHSS) and long-term (certain cancers) differs based on underlying diagnosis. Although this may not be the case for all outcomes, we believe it would be helpful for future studies of interventions performed in patients with different underlying diagnoses to report results separately by diagnosis. Within an individual study powered on the basis of the total patients, estimates of diagnosis-specific outcomes may be too imprecise to confidently rule out clinically relevant differences - consistency of reporting would allow formal synthesis of estimates across studies.

We found very limited evidence on outcomes among sperm or oocyte donors. Oocyte donors, who undergo controlled ovarian hyperstimulation and oocyte retrieval in the same manner as patients undergoing IVF using their own eggs, have, in theory, at least the same risk of shortterm adverse events as patients. The frequency with which oocyte donors are used is increasing, and evidence from the SART CORS database suggests that the risk of certain pregnancy complications is lower when donor oocytes are used. ${ }^{37,38}$ If demand for donor oocytes continues to increase, much more evidence on the specific short- and long-term outcomes of donation (especially if a donor undergoes multiple cycles) is needed.

\section{Conclusion}

Recently there has been growing adaptation of more rigorous methods for evaluating treatments for infertility, particularly regarding treatments for PCOS and approaches to timing of interventions in patients undergoing ART. In addition, ongoing refinements to the SART CORS database continue to make it a valuable resource, particularly for data on short-term outcomes. However, given the diversity of infertility causes and treatments, there is considerable residual uncertainty about the optimal treatment options for specific patients. Consensus on which outcomes to report (such as encouraging reporting of live birth rates on a per couple basis as well as per cycle, and, for studies of treatment such as ART, reporting of both overall and diagnosisspecific outcomes) and which areas of uncertainty are most important to resolve (in order to prioritize research) is needed to improve the ability of patients and clinicians to make decisions about the most appropriate treatment. 


\section{References}

1. Gnoth C, Godehardt E, Frank-Herrmann P, et al. Definition and prevalence of subfertility and infertility. Hum Reprod. 2005 May;20(5):1144-7. doi: 10.1093/humrep/deh870. PMID: 15802321.

2. Chandra A, Copen CE, Stephen EH. Infertility and impaired fecundity in the United States, 1982-2010: data from the National Survey of Family Growth. Natl Health Stat Report. 2013 Aug 14(67):1-18. PMID: 24988820.

3. Simonsen SE, Baksh L, Stanford JB. Infertility treatment in a population-based sample: 2004-2005. Matern Child Health J. 2012 May;16(4):877-86. doi: 10.1007/s10995-011-0809-6. PMID: 21559776.

4. Greil AL, McQuillan J, Shreffler KM, et al. Race-ethnicity and medical services for infertility: stratified reproduction in a population-based sample of U.S. women. J Health Soc Behav. 2011 Dec;52(4):493-509. doi:

http://dx.doi.org/10.1177/002214651141823 6. PMID: 22031500.

5. Hammoud AO, Gibson M, Stanford J, et al. In vitro fertilization availability and utilization in the United States: a study of demographic, social, and economic factors. Fertil Steril. 2009 May;91(5):1630-5. doi: 10.1016/j.fertnstert.2007.10.038. PMID: 18539275.

6. Macaluso M, Wright-Schnapp TJ, Chandra A, et al. A public health focus on infertility prevention, detection, and management. Fertil Steril. 2010 Jan;93(1):16.e1-0. doi: 10.1016/j.fertnstert.2008.09.046. PMID: 18992879.

7. Sunderam S, Kissin DM, Flowers L, et al. Assisted reproductive technology surveillance-United States, 2009. MMWR Surveill Summ. 2012 Nov 2;61(7):1-23. PMID: 23114281.
8. Louis JF, Thoma ME, Sorensen DN, et al. The prevalence of couple infertility in the United States from a male perspective: evidence from a nationally representative sample. Andrology. 2013 Sep;1(5):741-8. doi: 10.1111/j.2047-2927.2013.00110.x. PMID: 23843214.

9. Eijkemans MJ, van Poppel F, Habbema DF, et al. Too old to have children? Lessons from natural fertility populations. Hum Reprod. 2014 Jun;29(6):1304-12. doi: 10.1093/humrep/deu056. PMID: 24676403.

10. Maheshwari A, Hamilton M, Bhattacharya $\mathrm{S}$. Effect of female age on the diagnostic categories of infertility. Hum Reprod. 2008 Mar;23(3):538-42. doi:

10.1093/humrep/dem431. PMID: 18308834.

11. Centers for Disease Control and Prevention, American Society for Reproductive Medicine, Society for Assisted Reproductive Technology. 2012 Assisted Reproductive Technology National Summary Report. Atlanta: U.S. Dept of Health and Human Services; 2014. www.cdc.gov/art/pdf/2012report/nationalsummary/art_2012_national_summary_repo rt.pdf. Accessed November 13, 2018.

12. Barton SE, Najita JS, Ginsburg ES, et al. Infertility, infertility treatment, and achievement of pregnancy in female survivors of childhood cancer: a report from the Childhood Cancer Survivor Study cohort. Lancet Oncol. 2013 Aug;14(9):87381. doi: 10.1016/s1470-2045(13)70251-1. PMID: 23856401.

13. Howard-Anderson J, Ganz PA, Bower JE, et al. Quality of life, fertility concerns, and behavioral health outcomes in younger breast cancer survivors: a systematic review. J Natl Cancer Inst. 2012 Mar 7;104(5):386405. doi: 10.1093/jnci/djr541. PMID: 22271773.

14. Letourneau JM, Ebbel EE, Katz PP, et al. Pretreatment fertility counseling and fertility preservation improve quality of life in reproductive age women with cancer. Cancer. 2012 Mar 15;118(6):1710-7. doi: 10.1002/cncr.26459. PMID: 21887678. 
15. Odisho AY, Nangia AK, Katz PP, et al. Temporal and geospatial trends in male factor infertility with assisted reproductive technology in the United States from 19992010. Fertil Steril. 2014 Aug;102(2):469-75. doi: 10.1016/j.fertnstert.2014.05.006. PMID: 24931206.

16. Practice Committee of the American Society for Reproductive Medicine. Diagnostic evaluation of the infertile female: a committee opinion. Fertil Steril. 2012 Aug;98(2):302-7. doi: 10.1016/j.fertnstert.2012.05.032. PMID: 22698637.

17. National Collaborating Centre for Women's and Children's Health (UK). Fertility: Assessment and Treatment for People with Fertility Problems. London (UK): RCOG Press; 2013 Feb. (NICE Clinical Guidelines, No. 156.) Available at: http://www.ncbi.nlm.nih.gov/pubmedhealth/ PMH0068976/. PMID: 25340218.

18. Hwang K, Walters RC, Lipshultz LI. Contemporary concepts in the evaluation and management of male infertility. Nat Rev Urol. 2011 Feb;8(2):86-94. doi: 10.1038/nrurol.2010.230. PMID: 21243017.

19. Beck S, Wojdyla D, Say L, et al. The worldwide incidence of preterm birth: a systematic review of maternal mortality and morbidity. Bull World Health Organ. 2010 Jan;88(1):31-8. doi: 10.2471/blt.08.062554. PMID: 20428351.

20. Legro RS, Myers E. Surrogate end-points or primary outcomes in clinical trials in women with polycystic ovary syndrome? Hum Reprod. 2004 Aug;19(8):1697-704. doi: 10.1093/humrep/deh322. PMID: 15192061.

21. Messerlian C, Maclagan L, Basso O. Infertility and the risk of adverse pregnancy outcomes: a systematic review and metaanalysis. Hum Reprod. 2013 Jan;28(1):12537. doi: 10.1093/humrep/des347. PMID: 23042798.

22. Barnhart KT. Live birth is the correct outcome for clinical trials evaluating therapy for the infertile couple. Fertil Steril. 2014 May;101(5):1205-8. doi: 10.1016/j.fertnstert.2014.03.026. PMID: 24786740 .
23. Legro RS, Wu X, Barnhart KT, et al. Improving the reporting of clinical trials of infertility treatments (IMPRINT): modifying the CONSORT statement. Hum Reprod. 2014 Oct 10;29(10):2075-82. doi: 10.1093/humrep/deu218. PMID: 25217611.

24. Kurta ML, Moysich KB, Weissfeld JL, et al. Use of fertility drugs and risk of ovarian cancer: results from a U.S.-based casecontrol study. Cancer Epidemiol Biomarkers Prev. 2012 Aug;21(8):1282-92. doi: 10.1158/1055-9965.epi-12-0426. PMID: 22707710.

25. Rizzuto I, Behrens RF, Smith LA. Risk of ovarian cancer in women treated with ovarian stimulating drugs for infertility. Cochrane Database of Systematic Reviews. 2013;8:CD008215. doi: http://dx.doi.org/10.1002/14651858.CD0082 15.pub2. PMID: 23943232.

26. Trabert B, Lamb EJ, Scoccia B, et al. Ovulation-inducing drugs and ovarian cancer risk: results from an extended followup of a large United States infertility cohort. Fertil Steril. 2013 Dec;100(6):1660-6. doi: 10.1016/j.fertnstert.2013.08.008. PMID: 24011610.

27. Kawwass JF, Crawford S, Kissin DM, et al. Tubal factor infertility and perinatal risk after assisted reproductive technology. Obstet Gynecol. 2013 Jun;121(6):1263-71. doi: 10.1097/AOG.0b013e31829006d9. PMID: 23812461.

28. Stern JE, Luke B, Tobias M, et al. Adverse pregnancy and birth outcomes associated with underlying diagnosis with and without assisted reproductive technology treatment. Fertil Steril. 2015 Jun;103(6):1438-45. doi: 10.1016/j.fertnstert.2015.02.027. PMID: 25813277.

29. Manipalviratn S, DeCherney A, Segars J. Imprinting disorders and assisted reproductive technology. Fertil Steril. 2009 Feb;91(2):305-15. doi: 10.1016/j.fertnstert.2009.01.002. PMID: 19201275. 
30. Batcheller A, Cardozo E, Maguire M, et al. Are there subtle genome-wide epigenetic alterations in normal offspring conceived by assisted reproductive technologies? Fertil Steril. 2011 Dec;96(6):1306-11. doi: 10.1016/j.fertnstert.2011.09.037. PMID: 22035969.

31. Greil AL, McQuillan J, Lowry M, et al. Infertility treatment and fertility-specific distress: A longitudinal analysis of a population-based sample of U.S. women. Soc Sci Med. 2011 Jul;73(1):87-94. doi: 10.1016/j.socscimed.2011.04.023. PMID: 21645954

32. Wilkins KM, Warnock JK, Serrano E. Depressive symptoms related to infertility and infertility treatments. Psychiatr Clin North Am. 2010 Jun;33(2):309-21. doi: 10.1016/j.psc.2010.01.009. PMID: 20385339.

33. Feinberg EC, Larsen FW, Wah RM, et al. Economics may not explain Hispanic underutilization of assisted reproductive technology services. Fertil Steril. 2007 Nov;88(5):1439-41. doi: 10.1016/j.fertnstert.2007.01.031. PMID: 17561005.

34. Fujimoto VY, Luke B, Brown MB, et al. Racial and ethnic disparities in assisted reproductive technology outcomes in the United States. Fertil Steril. 2010

Feb;93(2):382-90. doi: 10.1016/j.fertnstert.2008.10.061. PMID: 19081561.

35. McCarthy-Keith DM, Schisterman EF, Robinson RD, et al. Will decreasing assisted reproduction technology costs improve utilization and outcomes among minority women? Fertil Steril. 2010 Dec;94(7):25879. doi: 10.1016/j.fertnstert.2010.02.021. PMID: 20356585.

36. Wellons MF, Fujimoto VY, Baker VL, et al. Race matters: a systematic review of racial/ethnic disparity in Society for Assisted Reproductive Technology reported outcomes. Fertil Steril. 2012 Aug;98(2):4069. doi: 10.1016/j.fertnstert.2012.05.012. PMID: 22698638.
37. Kawwass JF, Monsour M, Crawford S, et al. Trends and outcomes for donor oocyte cycles in the United States, 2000-2010. JAMA. 2013 Dec 11;310(22):2426-34. doi: 10.1001/jama.2013.280924. PMID: 24135860.

38. Myers ER. Outcomes of donor oocyte cycles in assisted reproduction. JAMA. 2013 Dec 11;310(22):2403-4. doi: 10.1001/jama.2013.280925. PMID: 24135802.

39. Klitzman RL, Sauer MV. Kamakahi vs ASRM and the future of compensation for human eggs. Am J Obstet Gynecol. 2015 Mar 26doi: 10.1016/j.ajog.2015.03.046. PMID: 25816784.

40. De Melo-Martin I. The ethics of anonymous gamete donation: is there a right to know one's genetic origins? Hastings Cent Rep. 2014 Mar-Apr;44(2):28-35. doi: 10.1002/hast.285. PMID: 24532424.

41. Wu AK, Odisho AY, Washington SL, 3rd, et al. Out-of-pocket fertility patient expense: data from a multicenter prospective infertility cohort. J Urol. 2014 Feb;191(2):427-32. doi: 10.1016/j.juro.2013.08.083. PMID: 24018235.

42. Bitler M, Schmidt L. Health disparities and infertility: impacts of state-level insurance mandates. Fertil Steril. 2006 Apr;85(4):85865. doi: 10.1016/j.fertnstert.2005.11.038. PMID: 16580365.

43. Bitler MP, Schmidt L. Utilization of infertility treatments: the effects of insurance mandates. Demography. 2012 Feb;49(1):125-49. doi: 10.1007/s13524-0110078-4. PMID: 22167581.

44. Hamilton BH, McManus B. The effects of insurance mandates on choices and outcomes in infertility treatment markets. Health Econ. 2012 Aug;21(8):994-1016. doi: 10.1002/hec.1776. PMID: 21905150.

45. Kiatpongsan S, Huckman RS, Hornstein $\mathrm{MD}$. The Great Recession, insurance mandates, and the use of in vitro fertilization services in the United States. Fertil Steril. 2015 Feb;103(2):448-54. doi: http://dx.doi.org/10.1016/j.fertnstert.2014.10 .042. PMID: 25497450. 
46. Practice Committee of American Society for Reproductive Medicine. Elective singleembryo transfer. Fertil Steril. 2012 Apr;97(4):835-42. doi: 10.1016/j.fertnstert.2011.11.050. PMID: 22196716.

47. Garrido N, Bellver J, Remohi J, et al. Cumulative newborn rates increase with the total number of transferred embryos according to an analysis of 15,792 ovum donation cycles. Fertil Steril. 2012 Aug;98(2):341-6.e1-2. doi: 10.1016/j.fertnstert.2012.04.039. PMID: 22633263.

48. Dar S, Librach CL, Gunby J, et al. Increased risk of preterm birth in singleton pregnancies after blastocyst versus Day 3 embryo transfer: Canadian ART Register (CARTR) analysis. Hum Reprod. 2013 Apr;28(4):924-8. doi: 10.1093/humrep/des448. PMID: 23349411.

49. Barnhart KT. Introduction: are we ready to eliminate the transfer of fresh embryos in in vitro fertilization? Fertil Steril. 2014 Jul;102(1):1-2. doi: 10.1016/j.fertnstert.2014.05.024. PMID: 24890272.

50. Roque M, Lattes K, Serra S, et al. Fresh embryo transfer versus frozen embryo transfer in in vitro fertilization cycles: a systematic review and meta-analysis. Fertil Steril. 2013 Jan;99(1):156-62. doi: 10.1016/j.fertnstert.2012.09.003. PMID: 23040524.

51. Kulkarni AD, Jamieson DJ, Jones HW, Jr., et al. Fertility treatments and multiple births in the United States. N Engl J Med. 2013 Dec 5;369(23):2218-25. doi: 10.1056/NEJMoa1301467. PMID: 24304051.

52. Reindollar RH, Regan MM, Neumann PJ, et al. A randomized clinical trial to evaluate optimal treatment for unexplained infertility: the fast track and standard treatment (FASTT) trial. Fertil Steril. 2010 Aug;94(3):888-99. doi: 10.1016/j.fertnstert.2009.04.022. PMID: 19531445.
53. Huang LN, Tan J, Hitkari J, et al. Should IVF be used as first-line treatment or as a last resort? A debate presented at the 2013 Canadian Fertility and Andrology Society meeting. Reprod Biomed Online. 2015 Feb;30(2):128-36. doi: 10.1016/j.rbmo.2014.10.004. PMID: 25498596.

54. Kawwass JF, Kissin DM, Kulkarni AD, et al. Safety of assisted reproductive technology in the United States, 2000-2011. JAMA. 2015 Jan 6;313(1):88-90. doi: 10.1001/jama.2014.14488. PMID: 25562272.

55. Centers for Disease Control and Prevention. Reporting of Pregnancy Success Rates From Assisted Reproductive Technology (ART) Programs. Federal Register, 08/26/2015. Available at: https://www.federalregister.gov/documents/ 2015/08/26/2015-21108/reporting-ofpregnancy-success-rates-from-assistedreproductive-technology-art-programs. Accessed January 18, 2018.

56. Agency for Healthcare Research and Quality (AHRQ). Methods Guide for Effectiveness and Comparative Effectiveness Reviews. AHRQ Publication No. 10(14)-EHC063-EF. Rockville, MD: Agency for Healthcare Research and Quality. January 2014. Available at: https://www.effectivehealthcare.ahrq.gov/to pics/cer-methods-guide/overview. Accessed January 18, 2018.

57. Moher D, Liberati A, Tetzlaff J, et al. Preferred reporting items for systematic reviews and meta-analyses: the PRISMA statement. PLoS Med. 2009 Jul 21;6(7):e1000097. doi: http://dx.doi.org/10.1371/journal.pmed.1000 097. PMID: 19621072.

58. Agency for Healthcare Research and Quality (AHRQ). Evidence-based Practice Center Systematic Review Protocol. Project Title: Management of Infertility. Available at: https://effectivehealthcare.ahrq.gov/topics/in fertility/research-protocol. Accessed November 13, 2018. 
59. Myers ER, McCrory DC, Mills AA, et al. Effectiveness of Assisted Reproductive Technology. Evidence Report/Technology Assessment No. 167 (Prepared by the Duke University Evidence-based Practice Center under Contract No. 290-02-0025.) AHRQ Publication No. 08-E012. Rockville, MD: Agency for Healthcare Research and Quality. May 2008. Available at: http://www.ncbi.nlm.nih.gov/books/NBK38 549/. Accessed November 13, 2018.

60. Hamdan M, Dunselman G, Li TC, et al. The impact of endometrioma on IVF/ICSI outcomes: a systematic review and metaanalysis. Hum Reprod Update. $2015 \mathrm{Jul}$ 12;21(6):809-25. doi: 10.1093/humupd/dmv035. PMID: 26168799.

61. Osman A, Alsomait H, Seshadri S, et al. The effect of sperm DNA fragmentation on live birth rate after IVF or ICSI: a systematic review and meta-analysis. Reprod Biomed Online. 2015 Feb;30(2):120-7. doi: 10.1016/j.rbmo.2014.10.018. PMID: 25530036.

62. Siristatidis C, Sergentanis TN, Vogiatzi P, et al. In Vitro Maturation in Women with vs. without Polycystic Ovarian Syndrome: A Systematic Review and Meta-Analysis. PLoS One. 2015;10(8):e0134696. doi: 10.1371/journal.pone.0134696. PMID: 26241855.

63. Weiss Nienke S, Nahuis M, Bayram N, et al. Gonadotrophins for ovulation induction in women with polycystic ovarian syndrome. Cochrane Database of Systematic Reviews: John Wiley \& Sons, Ltd; 2015.

64. Barbosa MA, Teixeira DM, Navarro PA, et al. Impact of endometriosis and its staging on assisted reproduction outcome: systematic review and meta-analysis. Ultrasound Obstet Gynecol. 2014 Sep;44(3):261-78. doi: 10.1002/uog.13366. PMID: 24639087.

65. Duffy JM, Arambage K, Correa FJ, et al. Laparoscopic surgery for endometriosis. Cochrane Database Syst Rev. 2014;4:CD011031. doi: http://dx.doi.org/10.1002/14651858.CD0110 31.pub2. PMID: 24696265.
66. Franik S, Kremer JA, Nelen WL, et al. Aromatase inhibitors for subfertile women with polycystic ovary syndrome. Cochrane Database Syst Rev. 2014;2:Cd010287. doi: 10.1002/14651858.CD010287.pub2. PMID: 24563180 .

67. Goudarzi ZM, Fallahzadeh H, Aflatoonian A, et al. Laparoscopic ovarian electrocautery versus gonadotropin therapy in infertile women with clomiphene citrate-resistant polycystic ovary syndrome: A systematic review and meta-analysis. Iran J Reprod Med. 2014 Aug;12(8):531-8. PMID: 25408702.

68. Jin X, Ruiz Beguerie J. Laparoscopic surgery for subfertility related to endometriosis: a meta-analysis. Taiwan J Obstet Gynecol. 2014 Sep;53(3):303-8. doi: 10.1016/j.tjog.2013.02.004. PMID: 25286781.

69. Lin $\mathrm{H}, \mathrm{Li} \mathrm{Y}$, Li L, et al. Is a GnRH antagonist protocol better in PCOS patients? A meta-analysis of RCTs. PLoS One. 2014;9(3):e91796. doi: 10.1371/journal.pone.0091796. PMID: 24642641.

70. Liu A, Zheng C, Lang J, et al. Letrozole versus clomiphene citrate for unexplained infertility: a systematic review and metaanalysis. J Obstet Gynaecol Res. 2014 May;40(5):1205-16. doi: 10.1111/jog.12393. PMID: 24754848.

71. Palomba S, Falbo A, La Sala GB. Metformin and gonadotropins for ovulation induction in patients with polycystic ovary syndrome: a systematic review with metaanalysis of randomized controlled trials. Reprod Biol Endocrinol. 2014;12:3. doi: 10.1186/1477-7827-12-3. PMID: 24387273.

72. Showell MG, Mackenzie-Proctor R, Brown $\mathrm{J}$, et al. Antioxidants for male subfertility. Cochrane Database Syst Rev.

2014;12:Cd007411. doi: 10.1002/14651858.CD007411.pub3. PMID: 25504418.

73. Attia AM, Abou-Setta AM, Al-Inany HG. Gonadotrophins for idiopathic male factor subfertility. Cochrane Database Syst Rev. 2013;8:Cd005071. doi: 10.1002/14651858.CD005071.pub4. PMID: 23970458. 
74. Harb HM, Gallos ID, Chu J, et al. The effect of endometriosis on in vitro fertilisation outcome: a systematic review and metaanalysis. BJOG. 2013 Oct;120(11):1308-20. doi: 10.1111/1471-0528.12366. PMID: 23834505.

75. Li J, Chen Y, Liu C, et al. Intravenous immunoglobulin treatment for repeated IVF/ICSI failure and unexplained infertility: a systematic review and a meta-analysis. Am J Reprod Immunol. 2013 Dec;70(6):434-47. doi: 10.1111/aji.12170. PMID: 24238107.

76. Misso ML, Costello MF, Garrubba M, et al. Metformin versus clomiphene citrate for infertility in non-obese women with polycystic ovary syndrome: a systematic review and meta-analysis. Hum Reprod Update. 2013 Jan-Feb;19(1):2-11. doi: 10.1093/humupd/dms036. PMID: 22956412.

77. Palomba S, Falbo A, La Sala GB. Effects of metformin in women with polycystic ovary syndrome treated with gonadotrophins for in vitro fertilisation and intracytoplasmic sperm injection cycles: a systematic review and meta-analysis of randomised controlled trials. BJOG. 2013 Feb;120(3):267-76. doi: 10.1111/1471-0528.12070. PMID: 23194199.

78. Sun X, Zhang D, Zhang W. Effect of metformin on ovulation and reproductive outcomes in women with polycystic ovary syndrome: a meta-analysis of randomized controlled trials. Arch Gynecol Obstet. 2013 Aug;288(2):423-30. doi: 10.1007/s00404013-2756-5. PMID: 23430028.

79. Teixeira DM, Barbosa MA, Ferriani RA, et al. Regular (ICSI) versus ultra-high magnification (IMSI) sperm selection for assisted reproduction. Cochrane Database Syst Rev. 2013;7:Cd010167. doi: http://dx.doi.org/10.1002/14651858.CD0101 67.pub2. PMID: 23884963.

80. Thomopoulos C, Tsioufis C, Michalopoulou $\mathrm{H}$, et al. Assisted reproductive technology and pregnancy-related hypertensive complications: a systematic review. J Hum Hypertens. 2013 Mar;27(3):148-57. doi: 10.1038/jhh.2012.13. PMID: 22495105.
81. Farquhar C, Brown J, Marjoribanks J. Laparoscopic drilling by diathermy or laser for ovulation induction in anovulatory polycystic ovary syndrome. Cochrane Database Syst Rev. 2012;6:Cd001122. doi: http://dx.doi.org/10.1002/14651858.CD0011 22.pub4. PMID: 22696324.

82. Jefferys A, Siassakos D, Wardle P. The management of retrograde ejaculation: a systematic review and update. Fertil Steril. 2012 Feb;97(2):306-12. doi: 10.1016/j.fertnstert.2011.11.019. PMID: 22177462.

83. Misso ML, Wong JL, Teede HJ, et al. Aromatase inhibitors for PCOS: a systematic review and meta-analysis. Hum Reprod Update. 2012 May-Jun;18(3):30112. doi: 10.1093/humupd/dms003. PMID: 22431566.

84. Pandian Z, Gibreel A, Bhattacharya S. In vitro fertilisation for unexplained subfertility. Cochrane Database Syst Rev. 2012;4:Cd003357. doi: 10.1002/14651858.CD003357.pub3. PMID: 22513911.

85. Siebert TI, Viola MI, Steyn DW, et al. Is metformin indicated as primary ovulation induction agent in women with PCOS? A systematic review and meta-analysis. Gynecol Obstet Invest. 2012;73(4):304-13. doi: 10.1159/000335253. PMID: 22516925.

86. Tang T, Lord JM, Norman RJ, et al. Insulinsensitising drugs (metformin, rosiglitazone, pioglitazone, D-chiro-inositol) for women with polycystic ovary syndrome, oligo amenorrhoea and subfertility. Cochrane Database Syst Rev. 2012;5:Cd003053. doi: http://dx.doi.org/10.1002/14651858.CD0030 53.pub5. PMID: 22592687.

87. Veltman-Verhulst SM, Cohlen BJ, Hughes $\mathrm{E}$, et al. Intra-uterine insemination for unexplained subfertility. Cochrane Database Syst Rev. 2012;9:Cd001838. doi: 10.1002/14651858.CD001838.pub4. PMID: 22972053.

88. Al-Inany HG, Youssef MA, Aboulghar M, et al. Gonadotrophin-releasing hormone antagonists for assisted reproductive technology. Cochrane Database Syst Rev. 2011(5):Cd001750. doi: 10.1002/14651858.CD001750.pub3. PMID: 21563131. 
89. Johnson N. Metformin is a reasonable firstline treatment option for non-obese women with infertility related to anovulatory polycystic ovary syndrome--a meta-analysis of randomised trials. Aust N Z J Obstet Gynaecol. 2011 Apr;51(2):125-9. doi: 10.1111/j.1479-828X.2010.01274.X. PMID: 21466513.

90. Bhattacharya S, Johnson N, Tijani HA, et al. Female infertility. BMJ Clin Evid. 2010;2010. PMID: 21406133.

91. Bosteels J, Weyers S, Mathieu C, et al. The effectiveness of reproductive surgery in the treatment of female infertility: facts, views and vision. Facts Views Vis Obgyn. 2010;2(4):232-52. PMID: 25009712.

92. Hughes E, Brown J, Collins JJ, et al. Clomiphene citrate for unexplained subfertility in women. Cochrane Database Syst Rev. 2010(1):Cd000057. doi: 10.1002/14651858.CD000057.pub2. PMID: 20091498.

93. Johnson N, van Voorst S, Sowter MC, et al. Surgical treatment for tubal disease in women due to undergo in vitro fertilisation. Cochrane Database Syst Rev. 2010(1):Cd002125. doi: http://dx.doi.org/10.1002/14651858.CD0021 25.pub3. PMID: 20091531.

94. Noble G, Harvey C, Napier C, et al. Preimplantation genetic screening in advanced maternal age: A systematic review. Expert Review of Obstetrics and Gynecology. 2010;5(6):707-16.

95. Pandian Z, McTavish AR, Aucott L, et al. Interventions for 'poor responders' to controlled ovarian hyper stimulation $(\mathrm{COH})$ in in-vitro fertilisation (IVF). Cochrane Database Syst Rev. 2010(1):Cd004379. doi: 10.1002/14651858.CD004379.pub3. PMID: 20091563.

96. Parsanezhad ME, Zarei A, Sayadi M, et al. Surgical ovulation induction in women with polycystic ovary syndrome: A systematic review. Iranian Journal of Medical Sciences. 2010;35(2):225-41.
97. Woldringh GH, Besselink DE, Tillema AH, et al. Karyotyping, congenital anomalies and follow-up of children after intracytoplasmic sperm injection with non-ejaculated sperm: a systematic review. Hum Reprod Update. 2010 Jan-Feb;16(1):12-9. doi: 10.1093/humupd/dmp030. PMID: 19700489.

98. Duffy JM, Johnson N, Ahmad G, et al. Postoperative procedures for improving fertility following pelvic reproductive surgery. Cochrane Database Syst Rev. 2009(2):Cd001897. doi: http://dx.doi.org/10.1002/14651858.CD0018 97.pub2. PMID: 19370571.

99. Palomba S, Pasquali R, Orio F, Jr., et al. Clomiphene citrate, metformin or both as first-step approach in treating anovulatory infertility in patients with polycystic ovary syndrome (PCOS): a systematic review of head-to-head randomized controlled studies and meta-analysis. Clin Endocrinol (Oxf). 2009 Feb;70(2):311-21. doi: 10.1111/j.13652265.2008.03369.x. PMID: 18691273.

100. Anonymous. Guidelines for the number of embryos to transfer following in vitro fertilization No. 182, September 2006. Int J Gynaecol Obstet. 2008 Aug;102(2):203-16. PMID: 18773532.

101. Besselink DE, Farquhar C, Kremer JA, et al. Cervical insemination versus intra-uterine insemination of donor sperm for subfertility. Cochrane Database Syst Rev.

2008(2):Cd000317. doi: 10.1002/14651858.CD000317.pub3. PMID: 18425862.

102. Hart Roger J, Hickey M, Maouris P, et al. Excisional surgery versus ablative surgery for ovarian endometriomata. Cochrane Database of Systematic Reviews: John Wiley \& Sons, Ltd; 2008.

103. Pandian Z, Akande VA, Harrild K, et al. Surgery for tubal infertility. Cochrane Database Syst Rev. 2008(3):Cd006415. doi: http://dx.doi.org/10.1002/14651858.CD0064 15.pub2. PMID: 18646152. 
104. Polyzos NP, Tsappi M, Mauri D, et al. Aromatase inhibitors for infertility in polycystic ovary syndrome. The beginning or the end of a new era? Fertil Steril. 2008 Feb;89(2):278-80. doi: 10.1016/j.fertnstert.2007.10.016. PMID: 18275882.

105. Polyzos NP, Tzioras S, Mauri D, et al. Treatment of unexplained infertility with aromatase inhibitors or clomiphene citrate: a systematic review and meta-analysis. Obstet Gynecol Surv. 2008 Jul;63(7):472-9. doi: 10.1097/OGX.0b013e31817d4e8d. PMID: 18559123.

106. Bensdorp AJ, Cohlen BJ, Heineman MJ, et al. Intra-uterine insemination for male subfertility. Cochrane Database Syst Rev. 2007(4):Cd000360. doi: 10.1002/14651858.CD000360.pub4. PMID: 17943739.

107. Donoso P, Tournaye H, Devroey P. Which is the best sperm retrieval technique for nonobstructive azoospermia? A systematic review. Hum Reprod Update. 2007 NovDec;13(6):539-49. doi: 10.1093/humupd/dmm029. PMID: 17895238.

108. Hughes E, Brown J, Collins John J, et al. Ovulation suppression for endometriosis for women with subfertility. Cochrane Database of Systematic Reviews: John Wiley \& Sons, Ltd; 2007.

109. Hughes E, Brown J, Tiffin G, et al. Danazol for unexplained subfertility. Cochrane Database Syst Rev. 2007(1):Cd000069. doi: 10.1002/14651858.CD000069.pub2. PMID: 17253444.

110. Moll E, van der Veen F, van Wely M. The role of metformin in polycystic ovary syndrome: a systematic review. Hum Reprod Update. 2007 Nov-Dec;13(6):52737. doi: 10.1093/humupd/dmm026. PMID: 17767003.

111. Tso LO, Costello MF, Albuquerque LE, et al. Metformin treatment before and during IVF or ICSI in women with polycystic ovary syndrome. Cochrane Database Syst Rev. 2014;11:Cd006105. doi: http://dx.doi.org/10.1002/14651858.CD0061 05.pub3. PMID: 25406011.
112. Brown J, Farquhar C, Beck J, et al. Clomiphene and anti-oestrogens for ovulation induction in PCOS. Cochrane Database Syst Rev. 2009(4):Cd002249. doi: 10.1002/14651858.CD002249.pub4. PMID: 19821295.

113. Shea BJ, Grimshaw JM, Wells GA, et al. Development of AMSTAR: a measurement tool to assess the methodological quality of systematic reviews. BMC Med Res Methodol. 2007 Feb 15;7:10. doi: 10.1186/1471-2288-7-10. PMID: 17302989.

114. White CM, Ip S, McPheeters M, et al. AHRQ Methods for Effective Health Care. Using Existing Systematic Reviews To Replace De Novo Processes in Conducting Comparative Effectiveness Reviews. Methods Guide for Effectiveness and Comparative Effectiveness Reviews. Rockville (MD): Agency for Healthcare Research and Quality (US); 2008.

115. Owens DK, Lohr KN, Atkins D, et al. AHRQ series paper 5: grading the strength of a body of evidence when comparing medical interventions-Agency for Healthcare Research and Quality and the Effective Health Care Program. J Clin Epidemiol. 2010 May;63(5):513-23. PMID: 19595577.

116. Berkman ND, Lohr KN, Ansari M, et al. Grading the Strength of a Body of Evidence When Assessing Health Care Interventions for the Effective Health Care Program of the Agency for Healthcare Research and Quality: An Update. Methods Guide for Comparative Effectiveness Reviews (Prepared by the RTI-UNC Evidence-based Practice Center under Contract No. 2902007-10056-I). AHRQ Publication No. 13(14)-EHC130-EF. Rockville, MD: Agency for Healthcare Research and Quality. November 2013. Available at: https://effectivehealthcare.ahrq.gov/sites/def ault/files/pdf/methods-guidance-gradingevidence methods.pdf. Accessed November 13, 2018.

117. Myers ER, Moorman P, Gierisch JM, et al. Benefits and harms of breast cancer screening: a systematic review. JAMA. 2015;314(15):1615-34. PMID: 26501537. 
118. Atkins D, Chang SM, Gartlehner G, et al. Assessing applicability when comparing medical interventions: AHRQ and the Effective Health Care Program. J Clin Epidemiol. 2011 Nov;64(11):1198-207. PMID: 21463926.

119. Mutsaerts MA, van Oers AM, Groen H, et al. Randomized Trial of a Lifestyle Program in Obese Infertile Women. N Engl J Med. 2016 May 19;374(20):1942-53. doi: 10.1056/NEJMoa1505297. PMID: 27192672.

120. van Oers AM, Groen H, Mutsaerts MA, et al. Effectiveness of lifestyle intervention in subgroups of obese infertile women: a subgroup analysis of a RCT. Hum Reprod. 2016 Dec;31(12):2704-13. doi: 10.1093/humrep/dew252. PMID: 27798042.

121. van Oers AM, Mutsaerts MAQ, Burggraaff $\mathrm{JM}$, et al. Association between periconceptional weight loss and maternal and neonatal outcomes in obese infertile women. PLoS One. 2018;13(3):e0192670. doi: 10.1371/journal.pone.0192670. PMID: 29590118.

122. Butts SF, Owen C, Mainigi M, et al. Assisted hatching and intracytoplasmic sperm injection are not associated with improved outcomes in assisted reproduction cycles for diminished ovarian reserve: an analysis of cycles in the United States from 2004 to 2011. Fertil Steril. 2014

Oct;102(4):1041-7.e1. doi: 10.1016/j.fertnstert.2014.06.043. PMID: 25086790 .

123. Luke B, Brown MB, Grainger DA, et al. Practice patterns and outcomes with the use of single embryo transfer in the United States. Fertil Steril. 2010 Feb;93(2):490-8. doi: 10.1016/j.fertnstert.2009.02.077. PMID: 19376512.

124. Nangia AK, Luke B, Smith JF, et al. National study of factors influencing assisted reproductive technology outcomes with male factor infertility. Fertil Steril. 2011 Sep;96(3):609-14. doi: 10.1016/j.fertnstert.2011.06.026. PMID: 21733503.
125. Spaan M, van den Belt-Dusebout AW, Schaapveld M, et al. Melanoma risk after ovarian stimulation for in vitro fertilization. Hum Reprod. 2015 May;30(5):1216-28. doi: 10.1093/humrep/dev023. PMID: 25743782.

126. van Leeuwen FE, Klip H, Mooij TM, et al. Risk of borderline and invasive ovarian tumours after ovarian stimulation for in vitro fertilization in a large Dutch cohort. Hum Reprod. 2011 Dec;26(12):3456-65. doi: 10.1093/humrep/der322. PMID: 22031719.

127. Spaan M, van den Belt-Dusebout AW, Burger CW, et al. Risk of Colorectal Cancer After Ovarian Stimulation for In Vitro Fertilization. Clin Gastroenterol Hepatol. 2016 May;14(5):729-37.e5. doi: 10.1016/j.cgh.2015.12.018. PMID: 26687912.

128. Legro RS, Barnhart HX, Schlaff WD, et al. Clomiphene, metformin, or both for infertility in the polycystic ovary syndrome. N Engl J Med. 2007 Feb 8;356(6):551-66. doi: 10.1056/NEJMoa063971. PMID: 17287476.

129. Rausch ME, Legro RS, Barnhart HX, et al. Predictors of pregnancy in women with polycystic ovary syndrome. J Clin Endocrinol Metab. 2009 Sep;94(9):3458-66. doi: 10.1210/jc.2009-0545. PMID: 19509098.

130. Legro RS, Myers ER, Barnhart HX, et al. The Pregnancy in Polycystic Ovary Syndrome study: baseline characteristics of the randomized cohort including racial effects. Fertil Steril. 2006 Oct;86(4):914-33. doi: 10.1016/j.fertnstert.2006.03.037. PMID: 16963034.

131. Legro RS, Brzyski RG, Diamond MP, et al. Letrozole versus clomiphene for infertility in the polycystic ovary syndrome. N Engl J Med. 2014 Jul 10;371(2):119-29. doi: 10.1056/NEJMoa1313517. PMID: 25006718.

132. Polotsky AJ, Allshouse AA, Casson PR, et al. Impact of Male and Female Weight, Smoking, and Intercourse Frequency on Live Birth in Women With Polycystic Ovary Syndrome. J Clin Endocrinol Metab. 2015 Jun;100(6):2405-12. doi: 10.1210/jc.20151178. PMID: 25856211. 
133. Legro RS, Brzyski RG, Diamond MP, et al. The Pregnancy in Polycystic Ovary Syndrome II study: baseline characteristics and effects of obesity from a multicenter randomized clinical trial. Fertil Steril. 2014 Jan;101(1):258-69.e8. doi: 10.1016/j.fertnstert.2013.08.056. PMID: 24156957.

134. Legro RS, Kunselman AR, Brzyski RG, et al. The Pregnancy in Polycystic Ovary Syndrome II (PPCOS II) trial: rationale and design of a double-blind randomized trial of clomiphene citrate and letrozole for the treatment of infertility in women with polycystic ovary syndrome. Contemp Clin Trials. 2012 May;33(3):470-81. doi: 10.1016/j.cct.2011.12.005. PMID: 22265923.

135. Bhattacharya S, Harrild K, Mollison J, et al. Clomifene citrate or unstimulated intrauterine insemination compared with expectant management for unexplained infertility: pragmatic randomised controlled trial. BMJ. 2008;337:a716. doi: 10.1136/bmj.a716. PMID: 18687718.

136. Brinton LA, Moghissi KS, Scoccia B, et al. Effects of fertility drugs on cancers other than breast and gynecologic malignancies. Fertil Steril. 2015 Jul 29;104(4):980-8. doi: 10.1016/j.fertnstert.2015.06.045. PMID: 26232746.

137. Brinton LA, Scoccia B, Moghissi KS, et al. Long-term relationship of ovulationstimulating drugs to breast cancer risk. Cancer Epidemiol Biomarkers Prev. 2014 Apr;23(4):584-93. doi: 10.1158/10559965.epi-13-0996. PMID: 24700523.

138. Brinton LA, Westhoff CL, Scoccia B, et al. Fertility drugs and endometrial cancer risk: results from an extended follow-up of a large infertility cohort. Hum Reprod. 2013 Oct;28(10):2813-21. doi: 10.1093/humrep/det323. PMID: 23943795.

139. Custers IM, van Rumste MM, van der Steeg JW, et al. Long-term outcome in couples with unexplained subfertility and an intermediate prognosis initially randomized between expectant management and immediate treatment. Hum Reprod. 2012 Feb;27(2):444-50. doi: 10.1093/humrep/der389. PMID: 22114108.
140. Steures P, van der Steeg JW, Hompes PG, et al. Intrauterine insemination with controlled ovarian hyperstimulation versus expectant management for couples with unexplained subfertility and an intermediate prognosis: a randomised clinical trial. Lancet. $2006 \mathrm{Jul}$ 15;368(9531):216-21. doi: 10.1016/s01406736(06)69042-9. PMID: 16844491.

141. Nahuis MJ, Kose N, Bayram N, et al. Longterm outcomes in women with polycystic ovary syndrome initially randomized to receive laparoscopic electrocautery of the ovaries or ovulation induction with gonadotrophins. Hum Reprod. 2011 Jul;26(7):1899-904. doi: 10.1093/humrep/der141. PMID: 21576081.

142. Nahuis MJ, Oude Lohuis E, Kose N, et al. Long-term follow-up of laparoscopic electrocautery of the ovaries versus ovulation induction with recombinant FSH in clomiphene citrate-resistant women with polycystic ovary syndrome: an economic evaluation. Hum Reprod. 2012

Dec;27(12):3577-82. doi: 10.1093/humrep/des336. PMID: 23001778.

143. Rashidi M, Najmi Z, Mobasseri A. Advantages of Recombinant FollicleStimulating Hormone over Human Menopausal Gonadotropin in Intrauterine Insemination: A Randomized Clinical Trial in Polycystic Ovary Syndrome-Associated Infertility. Gynecol Obstet Invest. 2015 Jul 23;81(2):118-23. doi: 10.1159/000435773. PMID: 26228499.

144. Yazici G, Savas A, Tasdelen B, et al. Role of luteal phase support on gonadotropin ovulation induction cycles in patients with polycystic ovary syndrome. J Reprod Med. 2014 Jan-Feb;59(1-2):25-30. PMID: 24597283.

145. Ghanem ME, Elboghdady LA, Hassan M, et al. Clomiphene citrate co-treatment with low dose urinary FSH versus urinary FSH for clomiphene resistant PCOS: randomized controlled trial. J Assist Reprod Genet. 2013 Nov;30(11):1477-85. doi: 10.1007/s10815013-0090-2. PMID: 24014214.

146. An Y, Sun Z, Zhang Y, et al. The use of berberine for women with polycystic ovary syndrome undergoing IVF treatment. Clin Endocrinol (Oxf). 2014 Mar;80(3):425-31. doi: 10.1111/cen.12294. PMID: 23869585. 
147. Choi MH, Lee SH, Kim HO, et al. Comparison of assisted reproductive technology outcomes in infertile women with polycystic ovary syndrome: In vitro maturation, GnRH agonist, and GnRH antagonist cycles. Clin Exp Reprod Med. 2012 Dec;39(4):166-71. doi: 10.5653/cerm.2012.39.4.166. PMID: 23346527.

148. Mehrabian F, Eessaei F. The laparoscopic ovarian electrocautery versus gonadotropin therapy in infertile women with clomiphene citrate-resistant polycystic ovary syndrome; a randomized controlled trial. J Pak Med Assoc. 2012 Mar;62(3 Suppl 2):S42-4. PMID: 22768457.

149. Zheng X, Wang L, Zhen X, et al. Effect of hCG priming on embryonic development of immature oocytes collected from unstimulated women with polycystic ovarian syndrome. Reprod Biol Endocrinol. 2012;10:40. doi: 10.1186/1477-7827-10-40. PMID: 22621829.

150. Kim CH, Moon JW, Kang HJ, et al. Effectiveness of GnRH antagonist multiple dose protocol applied during early and late follicular phase compared with GnRH agonist long protocol in non-obese and obese patients with polycystic ovary syndrome undergoing IVF/ICSI. Clin Exp Reprod Med. 2012 Mar;39(1):22-7. doi: 10.5653/cerm.2012.39.1.22. PMID: 22563547.

151. Morin-Papunen L, Rantala AS, UnkilaKallio L, et al. Metformin improves pregnancy and live-birth rates in women with polycystic ovary syndrome (PCOS): a multicenter, double-blind, placebocontrolled randomized trial. J Clin Endocrinol Metab. 2012 May;97(5):1492500. doi: 10.1210/jc.2011-3061. PMID: 22419702.

152. Homburg R, Hendriks ML, Konig TE, et al. Clomifene citrate or low-dose FSH for the first-line treatment of infertile women with anovulation associated with polycystic ovary syndrome: a prospective randomized multinational study. Hum Reprod. 2012 Feb;27(2):468-73. doi: 10.1093/humrep/der401. PMID: 22128296.
153. Palomba S, Falbo A, Carrillo L, et al. Metformin reduces risk of ovarian hyperstimulation syndrome in patients with polycystic ovary syndrome during gonadotropin-stimulated in vitro fertilization cycles: a randomized, controlled trial. Fertil Steril. 2011 Dec;96(6):1384-90.e4. doi: 10.1016/j.fertnstert.2011.09.020. PMID: 21982727.

154. Abu Hashim H, Foda O, Ghayaty E, et al. Laparoscopic ovarian diathermy after clomiphene failure in polycystic ovary syndrome: is it worthwhile? A randomized controlled trial. Arch Gynecol Obstet. 2011 Nov;284(5):1303-9. doi: 10.1007/s00404011-1983-x. PMID: 21755338.

155. Kjotrod SB, Carlsen SM, Rasmussen PE, et al. Use of metformin before and during assisted reproductive technology in nonobese young infertile women with polycystic ovary syndrome: a prospective, randomized, double-blind, multi-centre study. Hum Reprod. 2011 Aug;26(8):204553. doi: 10.1093/humrep/der154. PMID: 21606131.

156. Abdellah MS. Reproductive outcome after letrozole versus laparoscopic ovarian drilling for clomiphene-resistant polycystic ovary syndrome. Int J Gynaecol Obstet. 2011 Jun;113(3):218-21. doi: 10.1016/j.ijgo.2010.11.026. PMID: 21457973.

157. Abu Hashim H, Ombar O, Abd Elaal I. Intrauterine insemination versus timed intercourse with clomiphene citrate in polycystic ovary syndrome: a randomized controlled trial. Acta Obstet Gynecol Scand. 2011 Apr;90(4):344-50. doi: 10.1111/j.1600-0412.2010.01063.x. PMID: 21306326.

158. Hosseini MA, Aleyasin A, Saeedi H, et al. Comparison of gonadotropin-releasing hormone agonists and antagonists in assisted reproduction cycles of polycystic ovarian syndrome patients. J Obstet Gynaecol Res. 2010 Jun;36(3):605-10. doi: 10.1111/j.14470756.2010.01247.x. PMID: 20598044. 
159. Stadtmauer LA, Sarhan A, Duran EH, et al. The impact of a gonadotropin-releasing hormone antagonist on gonadotropin ovulation induction cycles in women with polycystic ovary syndrome: a prospective randomized study. Fertil Steril. 2011 Jan;95(1):216-20. doi: 10.1016/j.fertnstert.2010.05.023. PMID: 20594551.

160. Johnson NP, Stewart AW, Falkiner J, et al. PCOSMIC: a multi-centre randomized trial in women with PolyCystic Ovary Syndrome evaluating Metformin for Infertility with Clomiphene. Hum Reprod. 2010 Jul;25(7):1675-83. doi: 10.1093/humrep/deq100. PMID: 20435692.

161. Tehraninejad ES, Nasiri R, Rashidi B, et al. Comparison of $\mathrm{GnRH}$ antagonist with long GnRH agonist protocol after OCP pretreatment in PCOs patients. Arch Gynecol Obstet. 2010 Sep;282(3):319-25. doi: 10.1007/s00404-010-1429-x. PMID: 20379731.

162. Aboulghar M, Saber W, Amin Y, et al. Prospective, randomized study comparing highly purified urinary follicle-stimulating hormone (FSH) and recombinant FSH for in vitro fertilization/intracytoplasmic sperm injection in patients with polycystic ovary syndrome. Fertil Steril. 2010

Nov;94(6):2332-4. doi: 10.1016/j.fertnstert.2010.01.051. PMID: 20188364.

163. Palomba S, Falbo A, Battista L, et al. Laparoscopic ovarian diathermy vs clomiphene citrate plus metformin as second-line strategy for infertile anovulatory patients with polycystic ovary syndrome: a randomized controlled trial. Am J Obstet Gynecol. 2010 Jun;202(6):577.e1-8. doi: 10.1016/j.ajog.2009.11.042. PMID: 20096821.

164. Kurzawa R, Ciepiela P, Baczkowski T, et al. Comparison of embryological and clinical outcome in GnRH antagonist vs. GnRH agonist protocols for in vitro fertilization in PCOS non-obese patients. A prospective randomized study. J Assist Reprod Genet. 2008 Aug;25(8):365-74. doi: 10.1007/s10815-008-9249-7. PMID: 18802744 .
165. Amer SA, Li TC, Metwally M, et al. Randomized controlled trial comparing laparoscopic ovarian diathermy with clomiphene citrate as a first-line method of ovulation induction in women with polycystic ovary syndrome. Hum Reprod. 2009 Jan;24(1):219-25. doi: 10.1093/humrep/den325. PMID: 18794162.

166. Zain MM, Jamaluddin R, Ibrahim A, et al. Comparison of clomiphene citrate, metformin, or the combination of both for first-line ovulation induction, achievement of pregnancy, and live birth in Asian women with polycystic ovary syndrome: a randomized controlled trial. Fertil Steril. 2009 Feb;91(2):514-21. doi: 10.1016/j.fertnstert.2007.12.002. PMID: 18321486.

167. Ge HS, Huang XF, Zhang W, et al. Exposure to human chorionic gonadotropin during in vitro maturation does not improve the maturation rate and developmental potential of immature oocytes from patients with polycystic ovary syndrome. Fertil Steril. 2008 Jan;89(1):98-103. doi: 10.1016/j.fertnstert.2007.02.021. PMID: 17524398.

168. Abu Hashim H, Mashaly AM, Badawy A. Letrozole versus laparoscopic ovarian diathermy for ovulation induction in clomiphene-resistant women with polycystic ovary syndrome: a randomized controlled trial. Arch Gynecol Obstet. 2010 Nov;282(5):567-71. doi: 10.1007/s00404010-1566-2. PMID: 20577748.

169. Zakherah MS, Nasr A, El Saman AM, et al. Clomiphene citrate plus tamoxifen versus laparoscopic ovarian drilling in women with clomiphene-resistant polycystic ovary syndrome. Int J Gynaecol Obstet. 2010 Mar;108(3):240-3. doi: 10.1016/j.ijgo.2009.10.004. PMID: 19944418.

170. Ghahiri A, Mogharehabed N, Mamourian $\mathrm{M}$. Letrozole as the first-line treatment of infertile women with poly cystic ovarian syndrome (PCOS) compared with clomiphene citrate: A clinical trial. Adv Biomed Res. 2016;5:6. doi: 10.4103/22779175.175237. PMID: 26962508. 
171. Wang Y, Chen Q, Wang N, et al. Controlled Ovarian Stimulation Using Medroxyprogesterone Acetate and hMG in Patients With Polycystic Ovary Syndrome Treated for IVF: A Double-Blind

Randomized Crossover Clinical Trial.

Medicine (Baltimore). 2016

Mar;95(9):e2939. doi:

10.1097/md.0000000000002939. PMID: 26945402.

172. Legro RS, Dodson WC, Kris-Etherton PM, et al. Randomized Controlled Trial of Preconception Interventions in Infertile Women With Polycystic Ovary Syndrome. J Clin Endocrinol Metab. 2015

Nov;100(11):4048-58. doi: 10.1210/jc.20152778. PMID: 26401593.

173. Kar S, Sanchita S. Clomiphene citrate, metformin or a combination of both as the first line ovulation induction drug for Asian Indian women with polycystic ovarian syndrome: A randomized controlled trial. J Hum Reprod Sci. 2015;8(4):197-201.

174. Chen ZJ, Shi Y, Sun Y, et al. Fresh versus Frozen Embryos for Infertility in the Polycystic Ovary Syndrome. N Engl J Med. 2016 Aug 11;375(6):523-33. doi: 10.1056/NEJMoa1513873. PMID: 27509101.

175. Wu XK, Wang YY, Liu JP, et al. Randomized controlled trial of letrozole, berberine, or a combination for infertility in the polycystic ovary syndrome. Fertil Steril. 2016 Sep 1;106(3):757-65.e1. doi: 10.1016/j.fertnstert.2016.05.022. PMID: 27336209.

176. Hossein-Rashidi B, Khandzad B, ShahrokhTehraninejad E, et al. Recombinant FSH Compared to Clomiphene Citrate as the First-Line in Ovulation Induction in Polycystic Ovary Syndrome Using Newly Designed Pens: A Randomized Controlled Trial. J Family Reprod Health. 2016 Mar;10(1):42-8. PMID: 27385973.

177. Jacob SL, Brewer C, Tang T, et al. A short course of metformin does not reduce OHSS in a GnRH antagonist cycle for women with PCOS undergoing IVF: a randomised placebo-controlled trial. Hum Reprod. 2016 Dec;31(12):2756-64. doi: 10.1093/humrep/dew268. PMID: 27816925.
178. Aghahosseini M, Aleyasin A, Chegini V, et al. Low-dose hCG as trigger day and $35 \mathrm{hr}$ later have different ovarian hyperstimulation syndrome occurrence in females undergoing In vitro fertilization: An RCT. Int J Reprod Biomed (Yazd). 2017 Nov;15(11):735-40. PMID: 29404536.

179. Amer SA, Smith J, Mahran A, et al. Doubleblind randomized controlled trial of letrozole versus clomiphene citrate in subfertile women with polycystic ovarian syndrome. Hum Reprod. 2017 Aug 1;32(8):1631-8. doi: 10.1093/humrep/dex227. PMID: 28854590.

180. de Wilde MA, Lamain-de Ruiter M, Veltman-Verhulst SM, et al. Increased rates of complications in singleton pregnancies of women previously diagnosed with polycystic ovary syndrome predominantly in the hyperandrogenic phenotype. Fertil Steril. 2017 Aug;108(2):333-40. doi: 10.1016/j.fertnstert.2017.06.015. PMID: 28778282.

181. Einarsson S, Bergh C, Friberg B, et al. Weight reduction intervention for obese infertile women prior to IVF: a randomized controlled trial. Hum Reprod. 2017 Aug 1;32(8):1621-30. doi: 10.1093/humrep/dex235. PMID: 28854592.

182. Emekci Ozay O, Ozay AC, Cagliyan E, et al. Myo-inositol administration positively effects ovulation induction and intrauterine insemination in patients with polycystic ovary syndrome: a prospective, controlled, randomized trial. Gynecol Endocrinol. 2017 Jul;33(7):524-8. doi: 10.1080/09513590.2017.1296127. PMID: 28277112.

183. Hassan A, Shehata N, Wahba A. Cost effectiveness of letrozole and purified urinary FSH in treating women with clomiphene citrate-resistant polycystic ovarian syndrome: a randomized controlled trial. Hum Fertil (Camb). 2017

Apr;20(1):37-42. doi: 10.1080/14647273.2016.1242783. PMID: 27825272. 
184. Kettner LO, Matthiesen NB, RamlauHansen $\mathrm{CH}$, et al. Fertility treatment and childhood type 1 diabetes mellitus: a nationwide cohort study of 565,116 live births. Fertil Steril. 2016 Dec;106(7):17516. doi: 10.1016/j.fertnstert.2016.09.009. PMID: 27773424.

185. Keyhan S, Truong T, Li YJ, et al. Preterm Delivery and Low Birth Weight Among Neonates Conceived With Intracytoplasmic Sperm Injection Compared With Conventional In Vitro Fertilization. Obstet Gynecol. 2018 Feb;131(2):262-8. doi: 10.1097/aog.0000000000002423. PMID: 29324596.

186. Malchau SS, Henningsen AA, Loft A, et al. The long-term prognosis for live birth in couples initiating fertility treatments. Hum Reprod. 2017 Jul 1;32(7):1439-49. doi: 10.1093/humrep/dex096. PMID: 28472455.

187. Weiss NS, Nahuis MJ, Bordewijk E, et al. Gonadotrophins versus clomifene citrate with or without intrauterine insemination in women with normogonadotropic anovulation and clomifene failure (MOVIN): a randomised, two-by-two factorial trial. Lancet. 2018 Feb 24;391(10122):75865. doi: 10.1016/s0140-6736(17)33308-1. PMID: 29273245.

188. Wu XK, Stener-Victorin E, Kuang HY, et al. Effect of Acupuncture and Clomiphene in Chinese Women With Polycystic Ovary Syndrome: A Randomized Clinical Trial. JAMA. 2017 Jun 27;317(24):2502-14. doi: 10.1001/jama.2017.7217. PMID: 28655015.

189. Ibrahim MH, Tawfic M, Hassan MM, et al. Letrozole versus laparoscopic ovarian drilling in infertile women with PCOS resistant to clomiphene citrate. Middle East Fertility Society Journal. 2017;22(4):251-4. doi: 10.1016/j.mefs.2017.02.003.

190. Mohammadi Yeganeh L, Moini A, Shiva M, et al. Methylprednisolone for prevention of ovarian hyperstimulation syndrome in patients with polycystic ovarian syndrome undergoing in-vitro fertilisation: a randomised controlled trial. J Obstet Gynaecol. 2018;38(2):241-6. doi: 10.1080/01443615.2017.1346593.
191. Topçu HO, Batioğlu AS, İslimye M. Tamoxifen versus clomiphene citrate for ovulation induction in women with polycystic ovary syndrome: A prospective randomized trial. J Reprod Med. 2017;62(5):507-12.

192. Zahran KM, Mostafa WA, Abbas AM, et al. Clomiphene citrate plus cabergoline versus clomiphene citrate for induction of ovulation in infertile euprolactinemic patients with polycystic ovary syndrome: A randomized clinical trial. Middle East Fertility Society Journal. 2018doi: 10.1016/j.mefs.2017.12.008.

193. Badawy A, Allam A, Abulatta M. Extending clomiphene treatment in clomipheneresistant women with PCOS: a randomized controlled trial. Reprod Biomed Online. 2008 Jun;16(6):825-9. PMID: 18549692.

194. Elsedeek M, Elgindy E. Comparison between two clomiphene citrate protocols for induction of ovulation in clomiphene resistant polycystic ovary syndrome. Middle East Fertility Society Journal. 2014;19(4):243-7.

195. Lim CE, Ng RW, Xu K, et al. Acupuncture for polycystic ovarian syndrome. Cochrane Database Syst Rev. 2016 May

3;5:Cd007689. doi: 10.1002/14651858.CD007689.pub3. PMID: 27136291.

196. Reavey J, Vincent K, Child T, et al. Human chorionic gonadotrophin priming for fertility treatment with in vitro maturation. Cochrane Database Syst Rev. 2016 Nov

16;11:Cd008720. doi: 10.1002/14651858.CD008720.pub2. PMID: 27852101.

197. Bordewijk EM, Nahuis M, Costello MF, et al. Metformin during ovulation induction with gonadotrophins followed by timed intercourse or intrauterine insemination for subfertility associated with polycystic ovary syndrome. Cochrane Database Syst Rev. 2017 Jan 24;1:Cd009090. doi: 10.1002/14651858.CD009090.pub2. PMID: 28118681. 
198. Brown J, Farquhar C. Clomiphene and other antioestrogens for ovulation induction in polycystic ovarian syndrome. Cochrane Database Syst Rev. 2016 Dec

15;12:Cd002249. doi: 10.1002/14651858.CD002249.pub5. PMID: 27976369.

199. Morley LC, Tang T, Yasmin E, et al. Insulin-sensitising drugs (metformin, rosiglitazone, pioglitazone, D-chiro-inositol) for women with polycystic ovary syndrome, oligo amenorrhoea and subfertility. Cochrane Database Syst Rev. 2017 Nov 29;11:Cd003053. doi: 10.1002/14651858.CD003053.pub6. PMID: 29183107.

200. Giudice LC, Kao LC. Endometriosis. Lancet. 2004 Nov 13-19;364(9447):178999. PMID: 15541453.

201. Anonymous. Revised American Fertility Society classification of endometriosis: 1985. Fertil Steril. 1985 Mar;43(3):351-2. PMID: 3979573.

202. Zhu S, Liu D, Huang W, et al. Postlaparoscopic oral contraceptive combined with Chinese herbal mixture in treatment of infertility and pain associated with minimal or mild endometriosis: a randomized controlled trial. BMC Complement Altern Med. 2014;14:222. doi: 10.1186/1472-688214-222. PMID: 24996447.

203. Stewart LM, Holman CD, Aboagye-Sarfo P, et al. In vitro fertilization, endometriosis, nulliparity and ovarian cancer risk. Gynecol Oncol. 2013 Feb;128(2):260-4. doi: 10.1016/j.ygyno.2012.10.023. PMID: 23116937.

204. Abu Hashim H, El Rakhawy M, Abd Elaal I. Randomized comparison of superovulation with letrozole vs. clomiphene citrate in an IUI program for women with recently surgically treated minimal to mild endometriosis. Acta Obstet Gynecol Scand. $2012 \mathrm{Mar}$;91(3):338-45. doi: 10.1111/j.1600-0412.2011.01346.x. PMID: 22181973.
205. Muller V, Kogan I, Yarmolinskaya M, et al. Dienogest treatment after ovarian endometrioma removal in infertile women prior to IVF. Gynecol Endocrinol. 2017;33(sup1):18-21. doi: 10.1080/09513590.2017.1415676. PMID: 29264985.

206. Yapca OE, Delibas IB, Karaca I, et al. Timelimited hydrotubation combined with clomiphene citrate treatment for unexplained infertility. Clin Exp Obstet Gynecol. 2015;42(3):311-4. PMID: 26152000.

207. Tartagni M, Cicinelli MV, Baldini D, et al. Dehydroepiandrosterone decreases the agerelated decline of the in vitro fertilization outcome in women younger than 40 years old. Reprod Biol Endocrinol. 2015;13:18. doi: 10.1186/s12958-015-0014-3. PMID: 25884390 .

208. Erdem M, Abay S, Erdem A, et al. Recombinant FSH increases live birth rates as compared to clomiphene citrate in intrauterine insemination cycles in couples with subfertility: a prospective randomized study. Eur J Obstet Gynecol Reprod Biol. 2015 Jun;189:33-7. doi: 10.1016/j.ejogrb.2015.03.023. PMID: 25855325.

209. Seckin B, Turkcapar F, Yildiz Y, et al. Effect of luteal phase support with vaginal progesterone in intrauterine insemination cycles with regard to follicular response: a prospective randomized study. J Reprod Med. 2014 May-Jun;59(5-6):260-6. PMID: 24937967.

210. Goldman MB, Thornton KL, Ryley D, et al. A randomized clinical trial to determine optimal infertility treatment in older couples: the Forty and Over Treatment Trial (FORT-T). Fertil Steril. 2014

Jun;101(6):1574-81.e1-2. doi: 10.1016/j.fertnstert.2014.03.012. PMID: 24796764.

211. Yildiz F, Bozkurt N, Erdem A, et al. Effect of Pertubation on Pregnancy Rates before Intrauterine Insemination Treatment in Patients with Unexplained Infertility. Int J Fertil Steril. 2014 Apr;8(1):77-84. PMID: 24695882. 
212. van Rumste MM, Custers IM, van Wely M, et al. IVF with planned single-embryo transfer versus IUI with ovarian stimulation in couples with unexplained subfertility: an economic analysis. Reprod Biomed Online. 2014 Mar;28(3):336-42. doi: 10.1016/j.rbmo.2013.10.021. PMID: 24456703.

213. Williams CL, Bunch KJ, Stiller CA, et al. Cancer risk among children born after assisted conception. N Engl J Med. 2013 Nov 7;369(19):1819-27. doi: 10.1056/NEJMoa1301675. PMID: 24195549 .

214. Majumdar G, Majumdar A. A prospective randomized study to evaluate the effect of hyaluronic acid sperm selection on the intracytoplasmic sperm injection outcome of patients with unexplained infertility having normal semen parameters. J Assist Reprod Genet. 2013 Nov;30(11):1471-5. doi: 10.1007/s10815-013-0108-9. PMID: 24085466.

215. Vitek WS, Galarraga O, Klatsky PC, et al. Management of the first in vitro fertilization cycle for unexplained infertility: a costeffectiveness analysis of split in vitro fertilization-intracytoplasmic sperm injection. Fertil Steril. 2013

Nov;100(5):1381-8. doi: 10.1016/j.fertnstert.2013.06.035. PMID: 23876534.

216. Rashidi M, Aaleyasin A, Aghahosseini M, et al. Advantages of recombinant folliclestimulating hormone over human menopausal gonadotropin for ovarian stimulation in intrauterine insemination: a randomized clinical trial in unexplained infertility. Eur J Obstet Gynecol Reprod Biol. 2013 Jul;169(2):244-7. doi: 10.1016/j.ejogrb.2013.03.002. PMID: 23541417.

217. Rubio C, Bellver J, Rodrigo L, et al. Preimplantation genetic screening using fluorescence in situ hybridization in patients with repetitive implantation failure and advanced maternal age: two randomized trials. Fertil Steril. 2013 Apr;99(5):1400-7. doi: 10.1016/j.fertnstert.2012.11.041. PMID: 23260857.
218. Ragni G, Levi-Setti PE, Fadini R, et al. Clomiphene citrate versus high doses of gonadotropins for in vitro fertilisation in women with compromised ovarian reserve: a randomised controlled non-inferiority trial. Reprod Biol Endocrinol. 2012;10:114. doi: 10.1186/1477-7827-10-114. PMID: 23249758.

219. Kim CH, Howles CM, Lee HA. The effect of transdermal testosterone gel pretreatment on controlled ovarian stimulation and IVF outcome in low responders. Fertil Steril. 2011 Feb;95(2):679-83. doi: 10.1016/j.fertnstert.2010.07.1077. PMID: 20801436.

220. Wiser A, Gonen O, Ghetler Y, et al. Addition of dehydroepiandrosterone (DHEA) for poor-responder patients before and during IVF treatment improves the pregnancy rate: a randomized prospective study. Hum Reprod. 2010 Oct;25(10):2496500. doi: 10.1093/humrep/deq220. PMID: 20729538.

221. Bagis T, Haydardedeoglu B, Kilicdag EB, et al. Single versus double intrauterine insemination in multi-follicular ovarian hyperstimulation cycles: a randomized trial. Hum Reprod. 2010 Jul;25(7):1684-90. doi: 10.1093/humrep/deq112. PMID: 20457669.

222. Badawy A, Shokeir T, Allam AF, et al. Pregnancy outcome after ovulation induction with aromatase inhibitors or clomiphene citrate in unexplained infertility. Acta Obstet Gynecol Scand. 2009;88(2):187-91. doi: 10.1080/00016340802638199. PMID: 19089782.

223. Kansal Kalra S, Ratcliffe S, Gracia CR, et al. Randomized controlled pilot trial of luteal phase recombinant FSH stimulation in poor responders. Reprod Biomed Online. 2008 Dec;17(6):745-50. PMID: 19079956.

224. Oyesanya OA, Olufowobi O, Ross W, et al. Prognosis of oocyte donation cycles: a prospective comparison of the in vitro fertilization-embryo transfer cycles of recipients who used shared oocytes versus those who used altruistic donors. Fertil Steril. 2009 Sep;92(3):930-6. doi: 10.1016/j.fertnstert.2008.07.1769. PMID: 18829002. 
225. Erdem A, Erdem M, Atmaca S, et al. Impact of luteal phase support on pregnancy rates in intrauterine insemination cycles: a prospective randomized study. Fertil Steril. 2009 Jun;91(6):2508-13. doi: 10.1016/j.fertnstert.2008.04.029. PMID: 18692788.

226. Gregoriou O, Vlahos NF, Konidaris S, et al. Randomized controlled trial comparing superovulation with letrozole versus recombinant follicle-stimulating hormone combined with intrauterine insemination for couples with unexplained infertility who had failed clomiphene citrate stimulation and intrauterine insemination. Fertil Steril. 2008 Sep;90(3):678-83. doi:

10.1016/j.fertnstert.2007.06.099. PMID: 17961561.

227. Morad AWA, Abdelhamid AA. Prospective randomized study for hydrotubation with or without lidocaine before intrauterine insemination in unexplained infertility. Middle East Fertility Society Journal. 2012;17(4):250-5.

228. Gibreel A, Badawy A, El-Refai W, et al. Endometrial scratching to improve pregnancy rate in couples with unexplained subfertility: A randomized controlled trial. J Obstet Gynaecol Res. 2013;39(3):680-4.

229. Ebrahimi M, Asbagh FA, Darvish S. The effect of luteal phase support on pregnancy rates of the stimulated intrauterine insemination cycles in couples with unexplained infertility. International Journal of Fertility and Sterility. 2010;4(2):51-6.

230. Demirol A, Gurgan T. Comparison of different gonadotrophin preparations in intrauterine insemination cycles for the treatment of unexplained infertility: a prospective, randomized study. Hum Reprod. 2007 Jan;22(1):97-100. doi: 10.1093/humrep/del335. PMID: 16954409.

231. Zarei A, Mahboubi M, Parsanezhad ME, et al. Effects of piroxicam administration on pregnancy outcome in intrauterine insemination (IUI) cycles: a randomized clinical trial. Clin Exp Obstet Gynecol. 2016;43(2):225-9. PMID: 27132415.
232. Khosravi D, Taheripanah R, Taheripanah A, et al. Comparison of oral dydrogesterone with vaginal progesteronefor luteal support in IUI cycles: a randomized clinical trial. Iran J Reprod Med. 2015 Jul;13(7):433-8. PMID: 26494991.

233. Diamond MP, Legro RS, Coutifaris C, et al. Letrozole, Gonadotropin, or Clomiphene for Unexplained Infertility. Acta Obstet Gynecol Scand. 2015 Sep 24;373(13):123040. doi: 10.1111/aogs.12781; 10.1056/NEJMoa1414827. PMID: 26398071.

234. Nada AM, ElSetohy KA, Banat MM, et al. Antagonist protocol versus clomiphene in unexplained infertility: A randomized controlled study. Taiwan J Obstet Gynecol. 2016 Jun;55(3):326-30. doi: 10.1016/j.tjog.2016.04.006. PMID: 27343309.

235. Youssef MA, van Wely M, Al-Inany H, et al. A mild ovarian stimulation strategy in women with poor ovarian reserve undergoing IVF: a multicenter randomized non-inferiority trial. Hum Reprod. 2016 Nov 11doi: 10.1093/humrep/dew282. PMID: 27836979.

236. Dhalwani NN, Boulet SL, Kissin DM, et al. Assisted reproductive technology and perinatal outcomes: conventional versus discordant-sibling design. Fertil Steril. 2016;106(3):710-6.e2.

237. Selman H, Rinaldi L. Effectiveness of corifollitropin alfa used for ovarian stimulation of poor responder patients. International Journal of Women's Health. 2016;8:609-15.

238. Farquhar CM, Liu E, Armstrong S, et al. Intrauterine insemination with ovarian stimulation versus expectant management for unexplained infertility (TUI): a pragmatic, open-label, randomised, controlled, two-centre trial. Lancet. 2018 Feb 3;391(10119):441-50. doi: 10.1016/s0140-6736(17)32406-6. PMID: 29174128. 
239. Levi Dunietz G, Holzman C, Zhang Y, et al. Assisted Reproductive Technology and Newborn Size in Singletons Resulting from Fresh and Cryopreserved Embryos Transfer. PLoS One. 2017;12(1):e0169869. doi: 10.1371/journal.pone.0169869. PMID: 28114395.

240. Nandi A, Bhide P, Hooper R, et al. Intrauterine insemination with gonadotropin stimulation or in vitro fertilization for the treatment of unexplained subfertility: a randomized controlled trial. Fertil Steril. 2017 Jun;107(6):1329-35.e2. doi: 10.1016/j.fertnstert.2017.03.028. PMID: 28501361.

241. Pourali L, Ayati S, Tavakolizadeh S, et al. Clomiphene citrate versus letrozole with gonadotropins in intrauterine insemination cycles: A randomized trial. Int J Reprod Biomed (Yazd). 2017 Jan;15(1):49-54. PMID: 28280800.

242. van Rijswijk J, Caanen MR, Mijatovic V, et al. Immobilization or mobilization after IUI: an RCT. Hum Reprod. 2017 Nov 1;32(11):2218-24. doi: 10.1093/humrep/dex302. PMID: 29040538.

243. Jahromi BN, Sadeghi S, Alipour S, et al. Effect of melatonin on the outcome of assisted reproductive technique cycles in women with diminished ovarian reserve: A double-blinded randomized clinical trial. Iranian Journal of Medical Sciences. 2017;42(1):73-8.

244. Danhof NA, van Wely M, Repping S, et al. Follicle stimulating hormone versus clomiphene citrate in intrauterine insemination for unexplained subfertility: a randomized controlled trial. Hum Reprod. 2018 Oct 1;33(10):1866-74. doi: 10.1093/humrep/dey268. PMID: 30137325.

245. Maher MA, Sayyed TM, Elkhouly N. Cervical mucus removal prior to intrauterine insemination: a randomized trial. BJOG. 2018 Jun;125(7):841-7. doi: 10.1111/14710528.15003. PMID: 29078018.

246. Harira M. Use of Letrozole versus clomiphene-estradiol for treating infertile women with unexplained infertility not responding well to clomiphene alone, comparative study. Middle East Fertility Society Journal. 2018doi: 10.1016/j.mefs.2018.05.008.
247. Yu R, Jin H, Huang X, et al. Comparison of modified agonist, mild-stimulation and antagonist protocols for in vitro fertilization in patients with diminished ovarian reserve. J Int Med Res. 2018;46(6):2327-37. doi: 10.1177/0300060518770346.

248. Kuzmin A, Linde V. Diagnostic and remedial capability of transcervical falloposcopy in conjunction with laparoscopy. Gynecol Endocrinol. 2014 Oct;30 Suppl 1:17-9. doi: 10.3109/09513590.2014.945771. PMID: 25200821.

249. Verhoeve HR, Moolenaar LM, Hompes P, et al. Cost-effectiveness of tubal patency tests. BJOG. 2013 Apr;120(5):583-93. doi: 10.1111/1471-0528.12121. PMID: 23331951.

250. Dreyer K, Lier MC, Emanuel MH, et al. Hysteroscopic proximal tubal occlusion versus laparoscopic salpingectomy as a treatment for hydrosalpinges prior to IVF or ICSI: an RCT. Hum Reprod. 2016 Sep;31(9):2005-16. doi: 10.1093/humrep/dew050. PMID: 27209341.

251. Grimstad FW, Nangia AK, Luke B, et al. Use of ICSI in IVF cycles in women with tubal ligation does not improve pregnancy or live birth rates. Hum Reprod. 2016 Dec;31(12):2750-5. doi: 10.1093/humrep/dew247. PMID: 27738114.

252. Qu F, Wang FF, Wu Y, et al.

Transcutaneous Electrical Acupoint Stimulation Improves the Outcomes of In Vitro Fertilization: A Prospective, Randomized and Controlled Study. Explore (NY). 2017 Sep - Oct;13(5):306-12. doi: 10.1016/j.explore.2017.06.004. PMID: 28915981.

253. La Sala GB, Nicoli A, Fornaciari E, et al. Intracytoplasmic morphologically selected sperm injection versus conventional intracytoplasmic sperm injection: a randomized controlled trial. Reprod Biol Endocrinol. 2015;13(1):97. doi: 10.1186/s12958-015-0096-y. PMID: 26307050 . 
254. Boulet SL, Mehta A, Kissin DM, et al. Trends in use of and reproductive outcomes associated with intracytoplasmic sperm injection. JAMA. 2015 Jan 20;313(3):25563. doi: 10.1001/jama.2014.17985. PMID: 25602996.

255. Razi MH, Halvaei I, Razi Y. Laser assisted zona hatching does not improve live birth rate in patients undergoing their first ICSI cycles. Iran J Reprod Med. 2013 Dec;11(12):1021-6. PMID: 24639729.

256. Leandri RD, Gachet A, Pfeffer J, et al. Is intracytoplasmic morphologically selected sperm injection (IMSI) beneficial in the first ART cycle? a multicentric randomized controlled trial. Andrology. 2013

Sep;1(5):692-7. doi: 10.1111/j.20472927.2013.00104.x. PMID: 23788532.

257. Tsai CC, Huang FJ, Wang LJ, et al. Clinical outcomes and development of children born after intracytoplasmic sperm injection (ICSI) using extracted testicular sperm or ejaculated extreme severe oligo-asthenoteratozoospermia sperm: a comparative study. Fertil Steril. 2011 Sep;96(3):567-71. doi: 10.1016/j.fertnstert.2011.06.080. PMID: 21880275.

258. Balaban B, Yakin K, Alatas C, et al. Clinical outcome of intracytoplasmic injection of spermatozoa morphologically selected under high magnification: a prospective randomized study. Reprod Biomed Online. 2011 May;22(5):472-6. doi: 10.1016/j.rbmo.2010.11.003. PMID: 21324747.

259. Belva F, Bonduelle M, Schiettecatte J, et al. Salivary testosterone concentrations in pubertal ICSI boys compared with spontaneously conceived boys. Hum Reprod. 2011 Feb;26(2):438-41. doi: 10.1093/humrep/deq345. PMID: 21138905.

260. Hershko-Klement A, Sukenik-Halevy R, Biron Shental T, et al. Intracytoplasmic morphologically selected sperm injection and congenital birth defects: a retrospective cohort study. Andrology. 2016 Sep;4(5):887-93. doi: 10.1111/andr.12221. PMID: 27317040.
261. Hajizadeh Maleki B, Tartibian B. Moderate aerobic exercise training for improving reproductive function in infertile patients: A randomized controlled trial. Cytokine. 2017 Apr;92:55-67. doi: 10.1016/j.cyto.2017.01.007. PMID: 28092795.

262. Shi Y, Sun Y, Hao C, et al. Transfer of Fresh versus Frozen Embryos in Ovulatory Women. N Engl J Med. 2018 Jan 11;378(2):126-36. doi: 10.1056/NEJMoa1705334. PMID: 29320646.

263. Xiong X, Dickey RP, Buekens P, et al. Use of Intracytoplasmic Sperm Injection and Birth Outcomes in Women Conceiving through In Vitro Fertilization. Paediatr Perinat Epidemiol. 2017 Mar;31(2):108-15. doi: 10.1111/ppe.12339. PMID: 28140471.

264. Rahman A, Francomano D, Sagnella F, et al. The effect on clinical results of adding recombinant LH in late phase of ovarian stimulation of patients with repeated implantation failure: A pilot study. Eur Rev Med Pharmacol Sci. 2017;21(23):5485-90.

265. Bensdorp AJ, Cohlen BJ, Heineman MJ, et al. Intra-uterine insemination for male subfertility. Cochrane Database Syst Rev. 2007(3):Cd000360. doi: 10.1002/14651858.CD000360.pub3. PMID: 17636632.

266. Cissen M, Bensdorp A, Cohlen BJ, et al. Assisted reproductive technologies for male subfertility. Cochrane Database Syst Rev. 2016;2:Cd000360. doi: 10.1016/j.fertnstert.2016.01.039

10.1002/14651858.CD000360.pub5. PMID: 26915339.

267. Sismanoglu A, Tekin HI, Erden HF, et al. Ovulation triggering with GnRH agonist vs. hCG in the same egg donor population undergoing donor oocyte cycles with $\mathrm{GnRH}$ antagonist: a prospective randomized crossover trial. J Assist Reprod Genet. 2009 May;26(5):251-6. doi: 10.1007/s10815-0099326-6. PMID: 19629674.

268. Bodri D, Guillen JJ, Polo A, et al. Complications related to ovarian stimulation and oocyte retrieval in 4052 oocyte donor cycles. Reprod Biomed Online. 2008 Aug;17(2):237-43. PMID: 18681998. 
269. Bodri D, Guillen JJ, Galindo A, et al. Triggering with human chorionic gonadotropin or a gonadotropin-releasing hormone agonist in gonadotropin-releasing hormone antagonist-treated oocyte donor cycles: findings of a large retrospective cohort study. Fertil Steril. 2009

Feb;91(2):365-71. doi: 10.1016/j.fertnstert.2007.11.049. PMID: 18367175 .

270. Maxwell KN, Cholst IN, Rosenwaks Z. The incidence of both serious and minor complications in young women undergoing oocyte donation. Fertil Steril. 2008 Dec;90(6):2165-71. doi: 10.1016/j.fertnstert.2007.10.065. PMID: 18249368.

271. Kramer W, Schneider J, Schultz N. US oocyte donors: a retrospective study of medical and psychosocial issues. Hum Reprod. 2009 Dec;24(12):3144-9. doi: 10.1093/humrep/dep309. PMID: 19729378.

272. Kissin DM, Zhang Y, Boulet SL, et al. Association of assisted reproductive technology (ART) treatment and parental infertility diagnosis with autism in ARTconceived children. Hum Reprod. 2015 Feb;30(2):454-65. doi: 10.1093/humrep/deu338. PMID: 25518976.

273. Schendelaar P, Middelburg KJ, Bos AF, et al. The Groningen ART cohort study: the effects of ovarian hyperstimulation and the IVF laboratory procedures on neurological condition at 2 years. Hum Reprod. 2011 Mar;26(3):703-12. doi: 10.1093/humrep/deq377. PMID: 21227942.

274. Luke B, Stern JE, Kotelchuck M, et al. Birth Outcomes by Infertility Treatment: Analyses of the Population-Based Cohort: Massachusetts Outcomes Study of Assisted Reproductive Technologies (MOSART). J Reprod Med. 2016 Mar-Apr;61(3-4):114-27. PMID: 27172633.

275. Toftager M, Bogstad J, Bryndorf T, et al. Risk of severe ovarian hyperstimulation syndrome in GnRH antagonist versus GnRH agonist protocol: RCT including 1050 first IVF/ICSI cycles. Hum Reprod. 2016 Jun;31(6):1253-64. doi: 10.1016/j.fertnstert.2016.03.037. PMID: 27060174 .
276. Chang J, Boulet SL, Jeng G, et al. Outcomes of in vitro fertilization with preimplantation genetic diagnosis: an analysis of the United States Assisted Reproductive Technology Surveillance Data, 2011-2012. Fertil Steril. 2016 Feb;105(2):394-400. doi: 10.1016/j.fertnstert.2015.10.018. PMID: 26551441.

277. Boulet SL, Kirby RS, Reefhuis J, et al. Assisted reproductive technology and birth defects among liveborn infants in Florida, Massachusetts, and Michigan, 2000-2010. JAMA Pediatrics. 2016;170(6).

278. Londra L, Moreau C, Strobino D, et al. Is the type of gonadotropin-releasing hormone suppression protocol for ovarian hyperstimulation associated with ectopic pregnancy in fresh autologous cycles for in vitro fertilization? Fertil Steril. 2016;106(3):666-72.

279. Provost MP, Thomas SM, Yeh JS, et al. State Insurance Mandates and Multiple Birth Rates After In Vitro Fertilization. Obstet Gynecol. 2016.

280. Barad DH, Darmon SK, Kushnir VA, et al. Impact of preimplantation genetic screening on donor oocyte-recipient cycles in the United States. Am J Obstet Gynecol. 2017 Nov;217(5):576.e1-.e8. doi: 10.1016/j.ajog.2017.07.023. PMID: 28735705.

281. Crawford S, Boulet SL, Kawwass JF, et al. Cryopreserved oocyte versus fresh oocyte assisted reproductive technology cycles, United States, 2013. Fertil Steril. 2017 Jan;107(1):110-8. doi: 10.1016/j.fertnstert.2016.10.002. PMID: 27842997.

282. Knudtson JF, Failor CM, Gelfond JA, et al. Assisted hatching and live births in firstcycle frozen embryo transfers. Fertil Steril. 2017 Oct;108(4):628-34. doi: 10.1016/j.fertnstert.2017.07.011. PMID: 28863938.

283. Mancuso AC, Boulet SL, Duran E, et al. Elective single embryo transfer in women less than age 38 years reduces multiple birth rates, but not live birth rates, in United States fertility clinics. Fertil Steril. 2016 Oct;106(5):1107-14. doi: 10.1016/j.fertnstert.2016.06.017. PMID: 27376458. 
284. Litzky JF, Boulet SL, Esfandiari N, et al. Effect of frozen/thawed embryo transfer on birthweight, macrosomia, and low birthweight rates in US singleton infants. Am J Obstet Gynecol. 2018;218(4):433.e1.e10. doi: 10.1016/j.ajog.2017.12.223.

285. Toftager M, Bogstad J, Lossl K, et al. Cumulative live birth rates after one ART cycle including all subsequent frozen-thaw cycles in 1050 women: secondary outcome of an RCT comparing GnRH-antagonist and GnRH-agonist protocols. Hum Reprod. 2017 Mar 1;32(3):556-67. doi: 10.1093/humrep/dew358. PMID: 28130435.

286. Wang ET, Kathiresan ASQ, Bresee C, et al. Abnormal implantation after fresh and frozen in vitro fertilization cycles. Fertil Steril. 2017 May;107(5):1153-8. doi: 10.1016/j.fertnstert.2017.03.012. PMID: 28433367.

287. Magnusson A, Wennerholm UB, Kallen K, et al. The association between the number of oocytes retrieved for IVF, perinatal outcome and obstetric complications. Hum Reprod. 2018 Oct 1;33(10):1939-47. doi: 10.1093/humrep/dey266. PMID: 30124838.

288. Peeraer K, Couck I, Debrock S, et al. Frozen-thawed embryo transfer in a natural or mildly hormonally stimulated cycle in women with regular ovulatory cycles: a RCT. Hum Reprod. 2015 Nov;30(11):255262. doi: 10.1093/humrep/dev224. PMID: 26364081.

289. Williams CL, Jones ME, Swerdlow AJ, et al. Risks of ovarian, breast, and corpus uteri cancer in women treated with assisted reproductive technology in Great Britain, 1991-2010: data linkage study including 2.2 million person years of observation. BMJ. 2018 Jul 11;362:k2644. doi: 10.1136/bmj.k2644. PMID: 29997145.

290. Litzky JF, Boulet SL, Esfandiari N, et al. Birthweight in infants conceived through in vitro fertilization following blastocyst or cleavage-stage embryo transfer: a national registry study. J Assist Reprod Genet. 2018;35(6):1027-37. doi: 10.1007/s10815018-1168-7.
291. Santos-Ribeiro S, Tournaye H, Polyzos NP. Trends in ectopic pregnancy rates following assisted reproductive technologies in the UK: a 12-year nationwide analysis including 160000 pregnancies. Hum Reprod. 2016 Feb;31(2):393-402. doi: 10.1093/humrep/dev315. PMID: 26724796.

292. Dhillon RK, Smith PP, Malhas R, et al. Investigating the effect of ethnicity on IVF outcome. Reprod Biomed Online. 2015 Jun 3;31(3):356-63. doi: 10.1016/j.rbmo.2015.05.015. PMID: 26208448.

293. Practice Committee of the American Society for Reproductive Medicine. Use of clomiphene citrate in infertile women: a committee opinion. Fertil Steril. 2013 Aug;100(2):341-8. doi: 10.1016/j.fertnstert.2013.05.033. PMID: 23809505.

294. Fauser BC, Tarlatzis BC, Rebar RW, et al. Consensus on women's health aspects of polycystic ovary syndrome (PCOS): the Amsterdam ESHRE/ASRM-Sponsored 3rd PCOS Consensus Workshop Group. Fertil Steril. 2012 Jan;97(1):28-38.e25. doi: 10.1016/j.fertnstert.2011.09.024. PMID: 22153789.

295. National Institute for Health and Care Excellence (NICE). Fertility: Evidence Update 74. A summary of selected new evidence relevant to NICE clinical guideline 156 "Assessment and treatment of people with fertility problems, 2013.” London (UK): NICE; March 2015. Available at: https://www.nice.org.uk/guidance/cg156/evi dence/fertility-evidence-update-2. Accessed November 13, 2018. PMID: 25340218.

296. Practice Committee of the American Society for Reproductive Medicine. Endometriosis and infertility: a committee opinion. Fertil Steril. 2012 Sep;98(3):591-8. doi: 10.1016/j.fertnstert.2012.05.031. PMID: 22704630 .

297. Practice Committee of the American Society for Reproductive Medicine. Effectiveness and treatment for unexplained infertility. Fertil Steril. 2006 Nov;86(5 Suppl 1):S1114. doi: 10.1016/j.fertnstert.2006.07.1475. PMID: 17055802. 
298. Practice Committee of the American Society for Reproductive Medicine. Role of tubal surgery in the era of assisted reproductive technology: a committee opinion. Fertil Steril. 2015 Jun;103(6):e37-43. doi: 10.1016/j.fertnstert.2015.03.032. PMID: 25958255.

299. . Recommendations for gamete and embryo donation: a committee opinion. Fertil Steril. 2013 Jan;99(1):47-62. doi: 10.1016/j.fertnstert.2012.09.037. PMID: 23095142.

300. Practice Committee of the American Society for Reproductive Medicine, Practice Committee of the Society for Assisted Reproductive Technology. Repetitive oocyte donation: a committee opinion. Fertil Steril. 2014 Oct;102(4):964-6. doi: 10.1016/j.fertnstert.2014.06.035. PMID: 25064399.

301. Adashi EY, Dean LA. Access to and use of infertility services in the United States: framing the challenges. Fertil Steril. 2016 May;105(5):1113-8. doi: 10.1016/j.fertnstert.2016.01.017. PMID: 26826275.

302. Harbin Consensus Conference Workshop G. Improving the Reporting of Clinical Trials of Infertility Treatments (IMPRINT): modifying the CONSORT statement. Fertil Steril. 2014 Oct;102(4):952-9 e15. doi: 10.1016/j.fertnstert.2014.08.002. PMID: 25225072.

303. Smith AD, Tilling K, Nelson SM, et al. Live-Birth Rate Associated With Repeat In Vitro Fertilization Treatment Cycles. JAMA. 2015 Dec 22-29;314(24):2654-62. doi: 10.1001/jama.2015.17296. PMID: 26717030 .

304. Myers ER. Repeated In Vitro Fertilization Cycles for Infertility. JAMA. 2015 Dec 2229;314(24):2627-9. doi: 10.1001/jama.2015.17297. PMID: 26717028.

305. Clarke JF, van Rumste MM, Farquhar CM, et al. Measuring outcomes in fertility trials: can we rely on clinical pregnancy rates? Fertil Steril. 2010 Oct;94(5):1647-51. doi: 10.1016/j.fertnstert.2009.11.018. PMID: 20056216.
306. Goldhaber-Fiebert JD, Brandeau ML. Evaluating Cost-effectiveness of Interventions That Affect Fertility and Childbearing: How Health Effects Are Measured Matters. Med Decis Making. 2015 Oct;35(7):818-46. doi: 10.1177/0272989x15583845. PMID: 25926281.

307. Myers ER. How Should We Estimate the Cost-effectiveness of Interventions That Affect Reproduction? Med Decis Making. 2015 Oct;35(7):812-4. doi: 10.1177/0272989x15602227. PMID: 26296618.

308. Walsh AP, Collins GS, Le Du M, et al. Pretreatment preferences and characteristics among patients seeking in vitro fertilisation. Reproductive Health. 2009;6(1).

309. Scotland GS, McNamee P, Peddie VL, et al. Safety versus success in elective single embryo transfer: women's preferences for outcomes of in vitro fertilisation. BJOG. 2007 Aug;114(8):977-83. doi: 10.1111/j.1471-0528.2007.01396.x. PMID: 17578474.

310. Myers E, Sanders GD, Ravi D, et al. Evaluating the Potential Use of Modeling and Value-of-Information Analysis for Future Research Prioritization Within the Evidence-based Practice Center Program. (Prepared by the Duke Evidence-based Practice Center under Contract No. 2902007-10066-I.) AHRQ Publication No. 11EHC030-EF. Rockville, MD: Agency for Healthcare Research and Quality. June 2011. Available at: https://effectivehealthcare.ahrq.gov/topics/v alue-of-information-modeling/research. Accessed November 13, 2018. 
311. Gliklich RE, Leavy MB, Velentgas P, et al. Identification of Future Research Needs in the Comparative Management of Uterine Fibroid Disease. A Report on the PrioritySetting Process, Preliminary Data Analysis, and Research Plan. Effective Healthcare Research Report No. 31. (Prepared by the Outcome DEcIDE Center, under Contract No. HHSA 290-2005-0035-I, TO5). AHRQ Publication No. 11-EHC023-EF. Rockville, MD: Agency for Healthcare Research and Quality. March 2011. Available at: https://effectivehealthcare.ahrq.gov/topics/ut erine-fibroids-2010/research. Accessed November 13, 2014.

312. Gliklich RE, Leavy MB, Velentgas P, et al. Incorporating stakeholder perspectives in developing a translation table framework for comparative effectiveness research. J Comp Eff Res. 2012 May;1(3):281-92. doi: 10.2217/cer.12.25. PMID: 24237409.

313. Vercellini P, Somigliana E, Cortinovis I, et al. "You can't always get what you want": from doctrine to practicability of study designs for clinical investigation in endometriosis. BMC Womens Health. 2015;15:89. doi: 10.1186/s12905-015-02484. PMID: 26490454.

314. Adamson J, Cockayne S, Puffer S, et al. Review of randomised trials using the postrandomised consent (Zelen's) design. Contemp Clin Trials. 2006 Aug;27(4):30519. doi: 10.1016/j.cct.2005.11.003. PMID: 16455306.
315. Tuffaha HW, Gordon LG, Scuffham PA. Value of information analysis in healthcare: a review of principles and applications. $\mathrm{J}$ Med Econ. 2014 Jun;17(6):377-83. doi: 10.3111/13696998.2014.907170. PMID: 24650041.

316. Carlson JJ, Thariani R, Roth J, et al. Valueof-information analysis within a stakeholder-driven research prioritization process in a US setting: an application in cancer genomics. Med Decis Making. 2013 May;33(4):463-71. doi: 10.1177/0272989x13484388. PMID: 23635833.

317. Minelli C, Baio G. Value of Information: A Tool to Improve Research Prioritization and Reduce Waste. PLoS Med. 2015 Sep;12(9):e1001882. doi: 10.1371/journal.pmed.1001882. PMID: 26418866.

318. van den Wijngaard L, Rodijk IC, van der Veen F, et al. Patient preference for a longacting recombinant FSH product in ovarian hyperstimulation in IVF: a discrete choice experiment. Hum Reprod. 2015 Feb;30(2):331-7. doi: http://dx.doi.org/10.1093/humrep/deu307. PMID: 25432926.

319. van Empel IW, Dancet EA, Koolman XH, et al. Physicians underestimate the importance of patient-centredness to patients: a discrete choice experiment in fertility care. Hum Reprod. 2011 Mar;26(3):584-93. doi: 10.1093/humrep/deq389. PMID: 21227936. 


\section{Acronyms and Abbreviations}

AHRQ

ART

ASRM

BMI

CDC

CI

DCE

DHEA

DOR

EHC

EPC

eSET

FASTT

FORT-T

$\mathrm{FSH}$

GnRH

GRADE

hCG

hMG

HR

ICTRP

ICSI

IMSI

IUI

IVF

IVM

KIs

KQ

NASS

NICE

$\mathrm{NIH}$

NR

NS

OAT

OHSS

OR
Agency for Healthcare Research and Quality

assisted reproductive technology

American Society for Reproductive Medicine

body mass index

Centers for Disease Control and Prevention

confidence interval

discrete choice experiments

dehydroepiandrosterone

diminished ovarian reserve

Effective Health Care

Evidence-based Practice Center

elective single-embryo transfer

Fast Track and Standard Treatment

Forty and Over Treatment Trial

follicle-stimulating hormone

gonadotropin-releasing hormone

Grading of Recommendations Assessment, Development, and Evaluation

human chorionic gonadotropin

human menopausal gonadotropin

hazard ratio

International Clinical Trials Registry Platform

intra-cytoplasmic sperm injection

intra-cytoplasmic morphologically selected sperm injection

intrauterine insemination

in vitro fertilization

in vitro maturation

Key Informants

Key Question

National ART Surveillance System

National Institute for Health and Care Excellence (UK)

National Institutes of Health

not reported

not statistically significant

oligo-astheno-teratozoospermia

ovarian hyperstimulation syndrome

odds ratio 
PCOS

PGS

PICOTS

PICSI

PRISMA

RCT

rFSH

RR

SART

SART CORS

SD

SIR

SOE

SRC

TEP

TESE

$\mathrm{uFSH}$

$\mathrm{UK}$

WHO polycystic ovary syndrome

preimplantation genetic screening

populations, interventions, comparators, outcomes, timing, settings

physiological intra-cytoplasmic sperm injection

Preferred Reporting Items for Systematic Reviews and Meta-Analyses

randomized controlled trial

recombinant follicle-stimulating hormone

relative risk

Society for Assisted Reproductive Technology

Society for Assisted Reproductive Technology Clinic Outcome Reporting System

standard deviation

standardized incidence ratio

strength of evidence

Scientific Resource Center

Technical Expert Panel

extracted testicular sperm

urinary follicle-stimulating hormone

United Kingdom

World Health Organization 


\section{Appendix A. Exact Search Strings}

\section{PubMed $^{\circledR}$ Search Strategy (October 3, 2018)}

\begin{tabular}{|c|c|}
\hline Set & Terms \\
\hline$\# 1$ & $\begin{array}{l}\text { "Infertility"[Mesh] OR "Anovulation"[Mesh] OR "infertility"[tiab] OR "infertile"[tiab] OR "subfertility"[tiab] OR } \\
\text { "subfertile"[tiab] OR "sub-fertility"[tiab] OR "sub-fertile"[tiab] OR “anovulation"[tiab] OR "aspermia"[tiab] OR } \\
\text { "asthenozoospermia"[tiab] OR "azoospermia"[tiab] OR "oligospermia"[tiab] OR "sertoli cell-only } \\
\text { syndrome"[tiab] }\end{array}$ \\
\hline$\# 2$ & 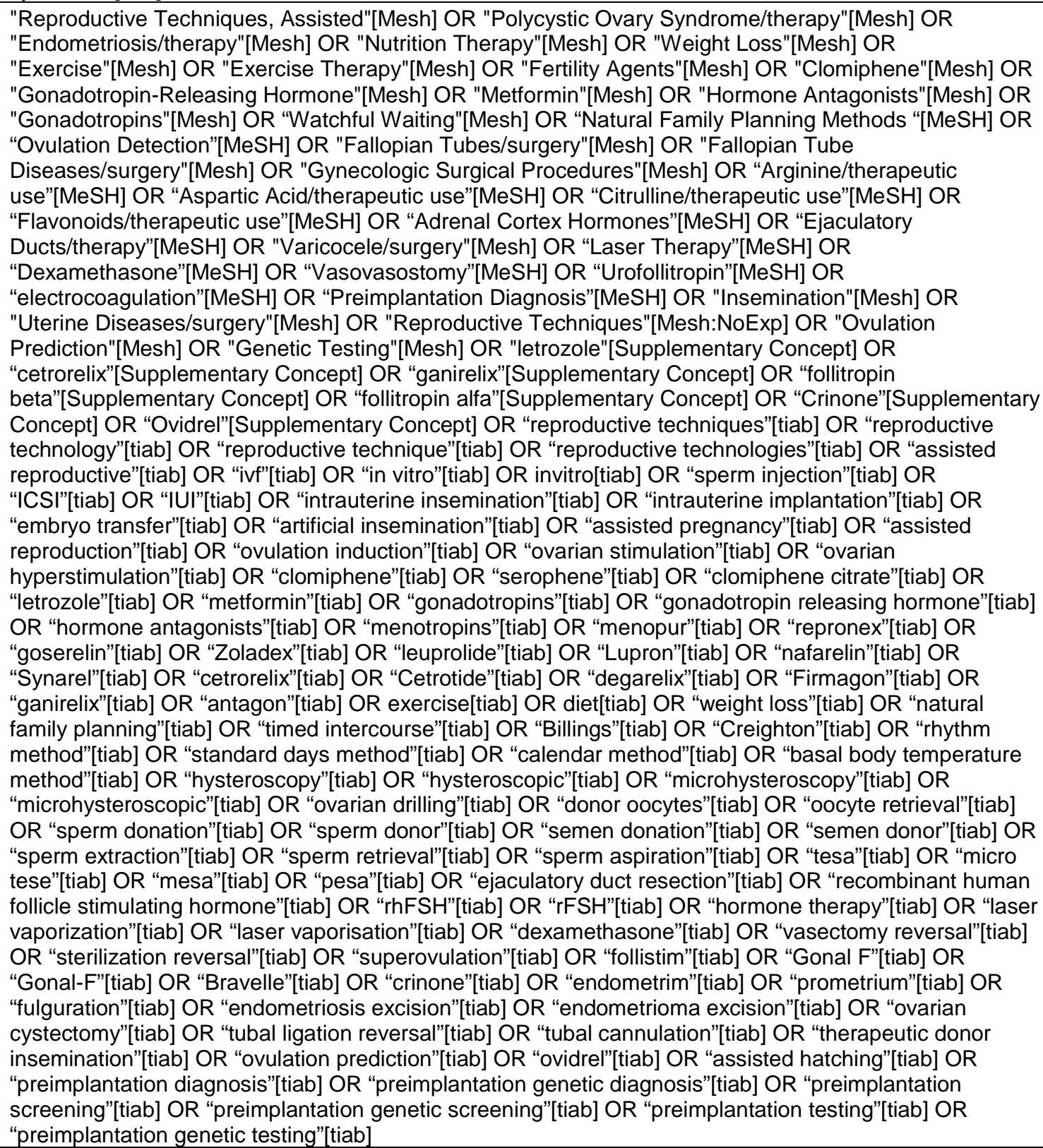 \\
\hline
\end{tabular}




\begin{tabular}{|c|l|}
\hline Set & \multicolumn{1}{c|}{ Terms } \\
\hline$\# 3$ & $\begin{array}{l}\text { (randomized controlled trial[pt] OR controlled clinical trial[pt] OR randomized[tiab] OR randomised[tiab] } \\
\text { OR randomization[tiab] OR randomisation[tiab] OR randomly[tiab] OR Clinical trial[pt] OR "clinical } \\
\text { trial"[tiab] OR "clinical trials"[tiab] OR "evaluation studies"[Publication Type] OR "evaluation studies as } \\
\text { topic"[MeSH Terms] OR "evaluation study"[tiab] OR evaluation studies[tiab] OR "intervention } \\
\text { studies"[MeSH Terms] OR "intervention study"[tiab] OR "intervention studies"[tiab] OR "case-control } \\
\text { studies"[MeSH Terms] OR "case-control"[tiab] OR "cohort studies"[MeSH Terms] OR cohort[tiab] OR }\end{array}$ \\
$\begin{array}{l}\text { "longitudinal studies"[MeSH Terms] OR "longitudinal"[tiab] OR longitudinally[tiab] OR "prospective"[tiab] } \\
\text { OR prospectively[tiab] OR "retrospective studies"[MeSH Terms] OR "retrospective"[tiab] OR "follow } \\
\text { up"[tiab] OR "comparative study"[Publication Type] OR "comparative study"[tiab] OR systematic[subset] } \\
\text { OR "meta-analysis"[Publication Type] OR "meta-analysis as topic"[MeSH Terms] OR "meta- } \\
\text { analysis"[tiab] OR "meta-analyses"[tiab]) NOT (Editorial[ptyp] OR Letter[ptyp] OR Case Reports[ptyp] OR } \\
\text { Comment[ptyp]) NOT (animals[mh] NOT humans[mh]) }\end{array}$ \\
\hline$\# 4$ & $\# 1$ AND \#2 AND \#3 \\
\hline$\# 5$ & Dates: 2007/01/01 - present \\
\hline$\# 6$ & Limit: English \\
\hline
\end{tabular}

\section{Embase $^{\circledR}$ Search Strategy (October 3, 2018)}

Platform: Embase.com

\begin{tabular}{|c|c|}
\hline Set & Terms \\
\hline$\# 1$ & $\begin{array}{l}\text { 'infertility'/exp OR 'anovulation'/exp OR “infertility":ab,ti OR "infertile":ab,ti OR "subfertility":ab,ti OR } \\
\text { "subfertile":ab,ti OR "sub-fertility":ab,ti OR "sub-fertile":ab,ti OR "anovulation":ab,ti OR "aspermia":ab,ti } \\
\text { OR "asthenozoospermia":ab,ti OR "azoospermia":ab,ti OR "oligospermia":ab,ti OR "sertoli cell-only } \\
\text { syndrome":ab,ti }\end{array}$ \\
\hline
\end{tabular}




\begin{tabular}{|c|c|}
\hline Set & Terms \\
\hline$\# 2$ & 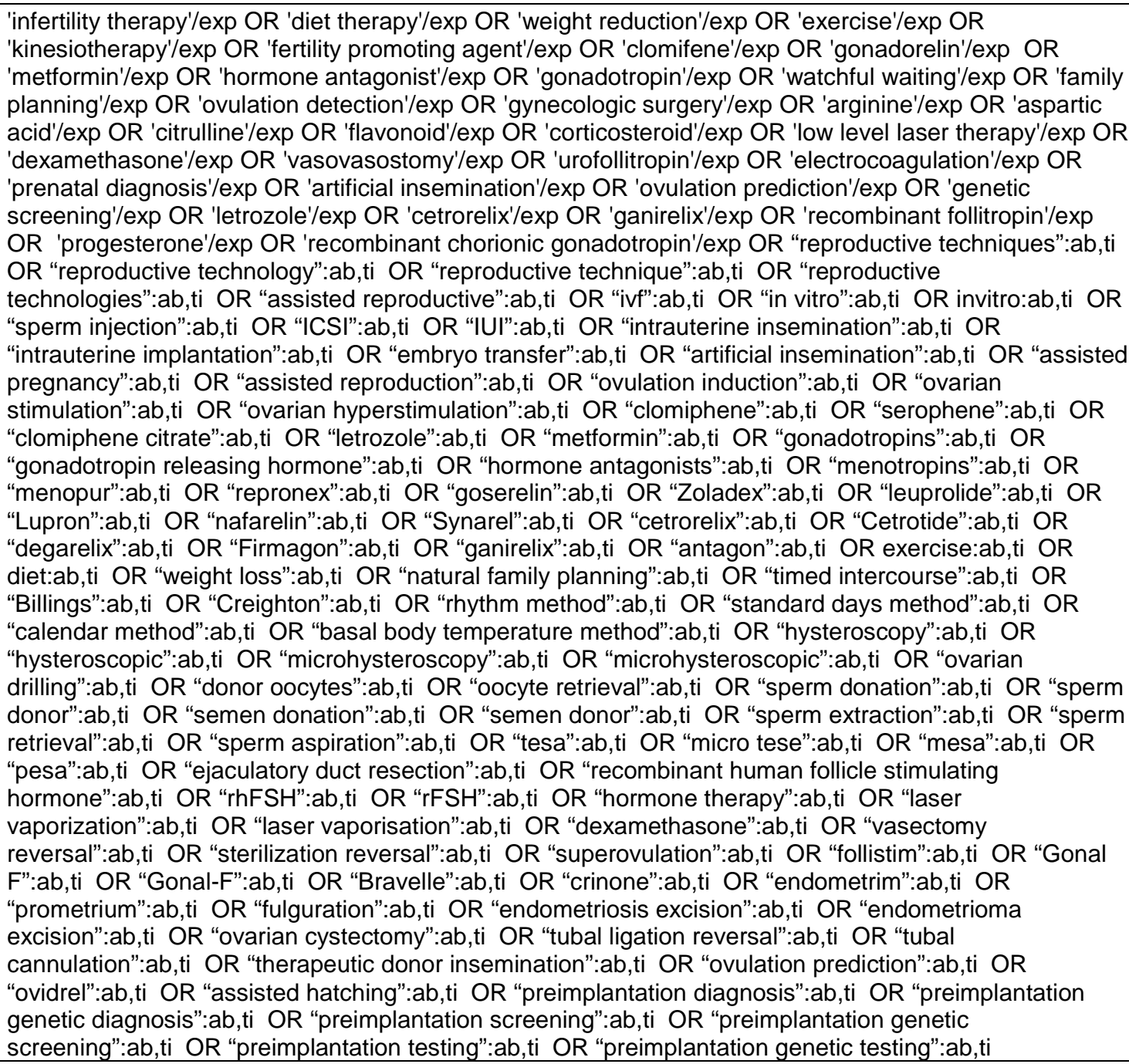 \\
\hline$\# 3$ & 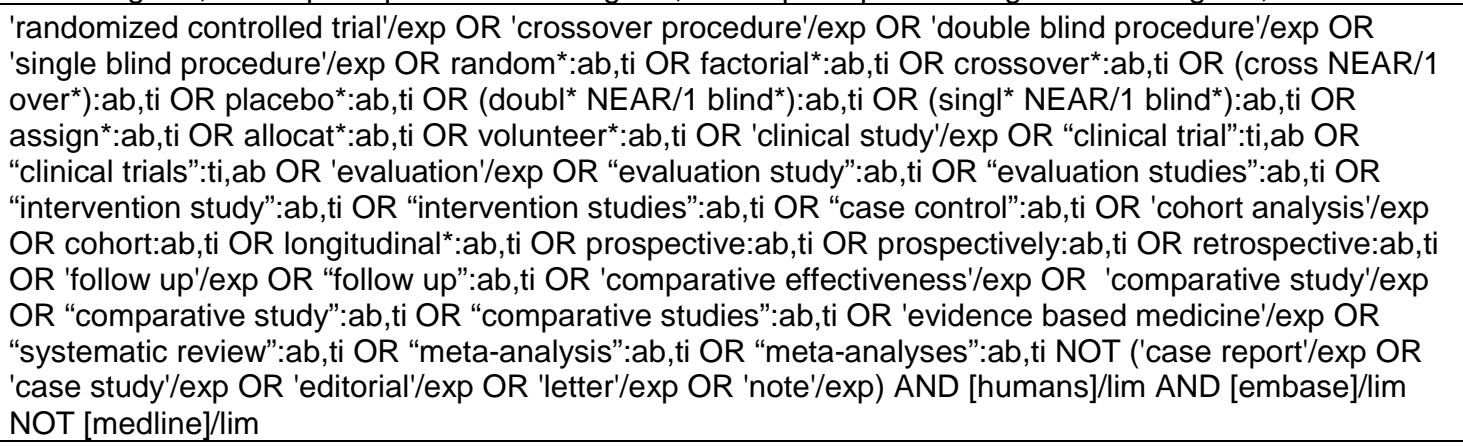 \\
\hline$\# 4$ & $\# 1$ AND \#2 AND \#3 \\
\hline$\# 5$ & \#4 AND [2007-2015]/py \\
\hline \#6 & \#5 AND [english]/lim \\
\hline
\end{tabular}




\section{Cochrane Search Strategy (October 3, 2018)}

Platform: Wiley

Database searched: Cochrane Database of Systematic Reviews

\begin{tabular}{|l|l|}
\hline Set & \multicolumn{1}{c|}{ Terms } \\
\hline$\# 1$ & $\begin{array}{l}\text { MeSH descriptor Infertility expolode all trees OR MeSH descriptor Anovulation explode all trees OR } \\
\text { "infertility":ab,ti,kw OR "infertile":ab,ti,kw OR "subfertility":ab,ti,kw OR "subfertile":ab,ti,kw OR "sub- } \\
\text { fertility":ab,ti,kw OR "sub-fertile":ab,ti,kw OR “anovulation":ab,ti,kw OR "aspermia":ab,ti,kw OR } \\
\text { "asthenozoospermia":ab,ti,kw OR "azoospermia":ab,ti,kw OR "oligospermia":ab,ti,kw OR "sertoli cell-only } \\
\text { syndrome":ab,ti,kw }\end{array}$ \\
\hline$\# 2$ & Dates: 2007/01/01 - present \\
\hline \#3 & Limit: Cochrane Reviews \\
\hline
\end{tabular}

\section{Grey Literature Searches}

ClinicalTrials.gov (December 16, 2015)

\begin{tabular}{|l|l|}
\hline \multicolumn{1}{|c|}{ Set } & \multicolumn{1}{c|}{ Terms } \\
\hline Condition & infertility OR infertile OR subfertility OR subfertile OR sub-fertility OR sub-fertile \\
\hline Limits & interventional studies \\
\hline
\end{tabular}

Total number of results exported: 858

Results were imported into Microsoft Excel and refined as follows:

1. Limited to studies with Completed status -482 records removed, 376 remaining

2. Limited to studies registered from 2005 forward - 22 records removed, 354 remaining

Total number of results for screening: 354 
ClinicalTrials.gov - Narrow search for the Appendix H. Supplemental Project to Assess the Transparency of Reporting for Trials Evaluating Treatment for Infertility (February 5, 2016)

\begin{tabular}{|l|l|}
\hline \multicolumn{1}{|c|}{ Set } & \multicolumn{1}{c|}{ Terms } \\
\hline Search terms & infertility OR infertile OR subfertility OR subfertile OR sub-fertility OR sub-fertile \\
\hline Condition terms & $\begin{array}{l}\text { polycystic ovary OR polycystic ovaries OR PCOS OR PCO } \\
\text { endometriosis OR endometrioma } \\
\text { unexplained OR ovarian reserve OR DOR OR ovarian response OR POR or responded OR } \\
\text { maternal age OR AMA OR reproductive age } \\
\text { tubal factor OR peritoneal factor OR pelvic adhesions OR pelvic adhesive OR hydrosalpinx } \\
\text { OR tubal obstruction OR tubal blockage } \\
\text { male factor OR male infertility OR Oligozoospermia OR Oligospermia OR Azoospermia OR } \\
\text { Asthenospermia OR Teratospermia } \\
\text { oocyte donor OR oocyte donation OR egg donation OR egg donor OR sperm donor OR } \\
\text { sperm donation OR donor eggs OR donor oocytes OR donor sperm OR oocyte recipient }\end{array}$ \\
\hline Limits & interventional studies \\
\hline
\end{tabular}

Total number of results: 494

WHO: International Clinical Trials Registry Platform Search Portal (January 27, 2016)

\begin{tabular}{|l|l|}
\hline \multicolumn{2}{|c|}{ KQs 1-6 } \\
\hline Condition & infertility OR infertile OR subfertility OR subfertile OR sub-fertility OR sub-fertile \\
\hline Recruiting status & All \\
\hline
\end{tabular}

Total number of results exported: 1708

Results were imported into EndNote ${ }^{\circledR}$ and refined as follows:

1. Removal of records originating from ClinicalTrials.gov (the ClinicalTrials.gov database was searched separately) -- 1013 records removed, 695 remaining

2. Keyword searches to identify records containing any of the following terms of interest: birth, delivery, ectopic, miscarriage, death, cancer, OHSS, ovarian hyperstimulation syndrome, time to pregnancy, costs- 442 records removed, 253 remaining

Total number of results for screening: 253 


\section{Appendix B. Data Abstraction Elements}

\section{Study Characteristics}

- Study Identifiers

o Study Name or Acronym

o NCT number or other trial registry identifier

o Last name of first author

- Additional Articles Used in This Abstraction

- Study Sites

o Single center, Multicenter, Unclear/Not reported

o Number of sites

- Geographic Location (Select all that apply)

o US, Canada, UK/Europe, Latin America, Middle East (including Israel), Asia, Africa, Australia/NZ, Unclear/Not reported

- Study Design

o RCT

o Observational

- Funding Source (Select all that apply)

o Government, Industry, Non-government/non-industry, Unclear/Not reported

- Setting (Select all that apply)

o Subspecialty practice (infertility specialist, urologist, etc.); General gynecology practice; Family practice/general internist/nurse practitioner/other nongynecologist primary care provider; Unclear/Not reported

- Study Definition of Infertility

o No pregnancy after 12 months of regular intercourse for women $<35$ years old or 6 months for women 35 and older; Other (specify); Not applicable; Not reported

- Study Enrollment/Study Completion

o $\mathrm{N}$ enrolled/included

o $\mathrm{N}$ completed

- Key Question Applicability (Select all that apply)

o KQ1, KQ2, KQ3, KQ4, KQ5, KQ6

- Baseline Characteristics - Record the following elements for Total Population, Women, Men, Arm 1, Arm 2, Arm 3, and Arm 4 (as applicable)

o Number of Patients ( $\mathrm{N}$ and \%)

0 Age in years

- Mean

- Median

- Standard Deviation

- Min

- Max

- $25 \% \mathrm{IQR}$

- $75 \%$ IQR

- Categorical

- Other, specify

o Race/Ethnicity ( $\mathrm{N}$ and \%) 
- Hispanic or Latino

- Black/African American

- American Indian or Alaska Native

- Asian

- Native Hawaiian or Pacific Islander

- White

- Multiracial

- Other (specify)

- Were there significant differences noted between groups in any baseline characteristic? (Yes/No)

0 If yes, please explain the differences

- Comments

\section{Intervention Characteristics}

- Is the comparison within a single intervention class or between classes?

- Intervention Descriptors

o Describe the intervention received by each patient group.

- Indicate components of the intervention (For each Arm)

o Oral Ovulation Induction with IUI

o Oral Ovulation Induction without IUI

o Surgical Management

o Gonadotropins with IUI

o Gonadotropins without IUI

o IVF

o ICSI

o No intervention / expectant management

- Indicate all intervention characteristics that are varied in this study

o IUI Details

- IUI methods

- Adjuvant treatments

o Oral Ovulation Induction Details

- Medication type

- Timing of medication

- Adjuvant treatments

- Dose

o Surgical Management Details

- Female - Surgical approach (e.g., laparoscopic vs. open)

- Female - Surgery vs. alternatives

- Male - Surgical repair

o Gonadotropin Details

- Ovarian stimulation (non-IVF) - medication type

- Ovarian stimulation (non-IVF) - timing

o IVF Details

- Pre-stimulation/adjuvant methods

- Down regulation methods

- Ovarian stimulation - medication type 
- Ovarian stimulation - monitoring

- Ovarian stimulation - poor responders

- Ovarian stimulation - natural cycle IVF

- Ovulation triggering methods

- Oocyte retrieval methods

- Sperm retrieval methods

- Laboratory phase methods

- Embryo transfer - stage of development

- Embryo transfer - \# of embryos

- Embryo transfer - transfer technique

- Luteal phase support

- Frozen embryos

- Prevention of ovarian hyperstimulation syndrome

o ICSI Details

- Sperm retrieval methods

- Sperm injection methods

- ICSI vs IVF

- Other (specify)

- Comments

\section{Outcomes}

- Select the outcome reported on this form:

o Live Birth

- Singleton (reported per cycle)

- Singleton (reported per patient)

- Multiple (reported per cycle)

- Multiple (reported per patient)

- Any (reported per cycle)

- Any (reported per patient)

o Pregnancy Complications

- Multiple births (and associated complications)

- Ectopic pregnancies

- Miscarriage

o Neonatal Outcomes

- Death

- Birthweight

- Congenital anomalies

o Time to Pregnancy

- Calendar time (months)

- Number of cycles

o Costs

- Patient

- Health system

- Societal

o Short-term Adverse Effects of Treatment

- OHSS 
- Surgical complications

o Long-term Outcomes - Child

- Neurodevelopment / other issues related to prematurity

- Specific issues related to infertility treatment (epigenetic changes, sex chromosomal abnormalities, etc.)

- Cancer (all types)

o Long-term Outcomes - Maternal

- Cancer

- Subsequent fertility

o Donor Women Outcomes

- Short-term - OHSS

- Short-term - Surgical Complications

- Short-term - Adverse effects of treatments

- Long-term - Downstream fertility

- Long-term - Cancer

- Long-term - Age at menopause

- Quality of Life

o Donor Men Outcomes

- Quality of life

- Short- and long-term health outcomes

- Any additional description / clarification of the outcome reported on this form

- Is this outcome form for a subgroup of interest? (Yes/No)

o What subpopulation is this outcome reported for on this form?

- Age

- Race/ethnicity

- Obesity/BMI

- Ovarian reserve

- History of prior treatment

- Primary vs. secondary infertility

- Maternal parity

- Insurance status

- Diagnostic criteria / evaluation

- Presence or absence of male factor infertility

- Other female causes of infertility

- Hypertension

- Diabetes

- Women without male partners (single women or lesbian couples)

- Anatomic cause of tubal occlusion (e.g. prior sterilization vs. adhesions)

- Cause of male infertility)

o Any additional description / clarification of subgroup reported on this form

- Total N Analyzed for this outcome

- Timepoint reported on this form

o Short-term

o Long-term

- Specify actual timing of the outcome (in months)

- For each arm: 
o N Analyzed (enter UNK if unknown)

o Unadjusted Result

- Number of patients with outcome

- \% of patients with outcome

- Events/denominator

- Odds ratio

- Hazard ratio

- Relative risk

- Mean

- Median

- Mean within group change

- Mean between group change

- Other (specify)

o Unadjusted Result Variability

- $95 \%$ CI

- IQR

- Standard Error (SE)

- Standard Deviation (SD)

- Other \% CI (specify)

- Other (specify)

o Unadjusted Result, p-value between groups

o Unadjusted Result, indicate reference group (for comparison between groups)

o Adjusted Result

- Number of patients with outcome

- \% of patients with outcome

- Events/denominator

- Odds ratio

- Hazard ratio

- Relative risk

- Mean

- Median

- Mean within group change

- Mean between group change

- Other (specify)

o Adjusted Result Variability

- $95 \% \mathrm{CI}$

- IQR

- Standard Error (SE)

- Standard Deviation (SD)

- Other \% CI (specify)

- Other (specify)

o Adjusted Result, p-value between groups

o Adjusted Result, indicate reference group (for comparison between groups)

o If adjusted data is recorded, indicate the adjustments applied

- Comments 


\section{Quality}

- $\quad$ Study Type (select one): RCT, Cohort, Case-control, Cross-sectional

- If RCT, select Yes/No/Unclear for each of the following questions:

o Selection Bias

- Was the allocation sequence generated adequately (e.g., random number table, computer-generated randomization)?

- Was the allocation of treatment adequately concealed (e.g., pharmacycontrolled randomization or use of sequentially numbered sealed envelopes)?

- Were participants analyzed within the groups they were originally assigned to?

- Does the design or analysis control account for important confounding and modifying variables through matching, stratification, multivariable analysis, or other approaches?

o Performance Bias

- Did researchers rule out any impact from a concurrent intervention or an unintended exposure that might bias results?

- Did the study maintain fidelity to the intervention protocol?

o Attrition Bias

- If attrition (overall or differential nonresponse, dropout, loss to follow-up, or exclusion of participants) was a concern, were missing data handled appropriately (e.g., intention-to-treat analysis and imputation)?

\section{o Detection Bias}

- In prospective studies, was the length of follow-up different between the groups, or in case-control studies, was the time period between the intervention/exposure and outcome the same for cases and controls?

- Were the outcome assessors blinded to the intervention or exposure status of participants?

- Were interventions/exposures assessed/defined using valid and reliable measures, implemented consistently across all study participants?

- Were outcomes assessed/defined using valid and reliable measures, implemented consistently across all study participants?

o Reporting Bias

- Were the potential outcomes prespecified by the researchers? Are all prespecified outcomes reported?

- If Cohort, select Yes/No/Unclear for each of the following questions:

o Selection Bias

- Were participants analyzed within the groups they were originally assigned to?

- Did the study apply inclusion/exclusion criteria uniformly to all comparison groups?

- Did the strategy for recruiting participants into the study differ across study groups?

- Does the design or analysis control account for important confounding and modifying variables through matching, stratification, multivariable analysis, or other approaches? 
o Performance Bias

- Did researchers rule out any impact from a concurrent intervention or an unintended exposure that might bias results?

- Did the study maintain fidelity to the intervention protocol?

o Attrition Bias

- If attrition (overall or differential nonresponse, dropout, loss to follow-up, or exclusion of participants) was a concern, were missing data handled appropriately (e.g., intention-to-treat analysis and imputation)?

o Detection Bias

- In prospective studies, was the length of follow-up different between the groups, or in case-control studies, was the time period between the intervention/exposure and outcome the same for cases and controls?

- Were the outcome assessors blinded to the intervention or exposure status of participants?

- Were interventions/exposures assessed/defined using valid and reliable measures, implemented consistently across all study participants?

- Were outcomes assessed/defined using valid and reliable measures, implemented consistently across all study participants?

- Were confounding variables assessed using valid and reliable measures, implemented consistently across all study participants?

o Reporting Bias

- Were the potential outcomes prespecified by the researchers? Are all prespecified outcomes reported?

- If Case-Control, select Yes/No/Unclear for each of the following questions:

o Selection Bias

- Were cases and controls selected appropriately (e.g., appropriate diagnostic criteria or definitions, equal application of exclusion criteria to case and controls, sampling not influenced by exposure status)

- Does the design or analysis control account for important confounding and modifying variables through matching, stratification, multivariable analysis, or other approaches?

o Performance Bias

- Did researchers rule out any impact from a concurrent intervention or an unintended exposure that might bias results?

- Did the study maintain fidelity to the intervention protocol?

o Attrition Bias

- If attrition (overall or differential nonresponse, dropout, loss to follow-up, or exclusion of participants) was a concern, were missing data handled appropriately (e.g., intention-to-treat analysis and imputation)?

o Detection Bias

- In prospective studies, was the length of follow-up different between the groups, or in case-control studies, was the time period between the intervention/exposure and outcome the same for cases and controls?

- Were the outcome assessors blinded to the intervention or exposure status of participants? 
- Were interventions/exposures assessed/defined using valid and reliable measures, implemented consistently across all study participants?

- Were outcomes assessed/defined using valid and reliable measures, implemented consistently across all study participants?

- Were confounding variables assessed using valid and reliable measures, implemented consistently across all study participants?

o Reporting Bias

- Were the potential outcomes prespecified by the researchers? Are all prespecified outcomes reported?

- If Cross-sectional, select Yes/No/Unclear for each of the following questions:

o Selection Bias

- Did the study apply inclusion/exclusion criteria uniformly to all comparison groups?

- Does the design or analysis control account for important confounding and modifying variables through matching, stratification, multivariable analysis, or other approaches?

o Performance Bias

- Did researchers rule out any impact from a concurrent intervention or an unintended exposure that might bias results?

o Attrition Bias

- If attrition (overall or differential nonresponse, dropout, loss to follow-up, or exclusion of participants) was a concern, were missing data handled appropriately (e.g., intention-to-treat analysis and imputation)?

o Detection Bias

- Were the outcome assessors blinded to the intervention or exposure status of participants?

- Were interventions/exposures assessed/defined using valid and reliable measures, implemented consistently across all study participants?

- Were outcomes assessed/defined using valid and reliable measures, implemented consistently across all study participants?

- Were confounding variables assessed using valid and reliable measures, implemented consistently across all study participants?

o Reporting Bias

- Were the potential outcomes prespecified by the researchers? Are all prespecified outcomes reported?

- Other Bias

o If applicable, describe any other concerns that may impact risk of bias

- Overall Study Rating (Good/Fair/Poor)

o Good (low risk of bias). These studies have the least bias, and the results are considered valid. These studies adhere to the commonly held concepts of high quality, including the following: a clear description of the population, setting, approaches, and comparison groups; appropriate measurement of outcomes; appropriate statistical and analytical methods and reporting; no reporting errors; a low dropout rate; and clear reporting of dropouts.

o Fair. These studies are susceptible to some bias, but not enough to invalidate the results. They do not meet all the criteria required for a rating of good quality 
because they have some deficiencies, but no flaw is likely to cause major bias. The study may be missing information, making it difficult to assess limitations and potential problems.

o Poor (high risk of bias). These studies have significant flaws that may have invalidated the results. They have serious errors in design, analysis, or reporting; large amounts of missing information; or discrepancies in reporting.

o If the study is rated as "Fair" or "Poor," provide rationale.

- Outcome-specific quality rating

o Do you think that any of the outcomes abstracted for this study should be assigned a quality rating DIFFERENT from the overall study rating? (No/Yes)

- If you think any of the abstracted outcomes should have a quality rating different from the overall study, please provide the outcome(s), rating(s) and rationale(s).

Applicability - Use the PICOS format to identify specific issues, if any, that may limit the applicability of the study.

- Population (P)

o Study population demographics not representative of intended population

o Narrow or unrepresentative severity/stage/comorbidity

- Intervention (I)

o Treatment protocol not representative of current practice

0 Change in standard of care

- Comparator (C)

o Comparator not representative of current practice

- Outcomes (O)

o Timing of outcome assessment

- Setting (S)

o Standards or access to care vary from US setting

o Specialty population or level of care

- Comments 


\section{Appendix C. List of Included Studies}

Abdellah MS. Reproductive outcome after letrozole versus laparoscopic ovarian drilling for clomipheneresistant polycystic ovary syndrome. Int J Gynaecol Obstet 2011;113(3):218-21. PMID: 21457973.

Aboulghar M, Saber W, Amin Y, et al. Prospective, randomized study comparing highly purified urinary follicle-stimulating hormone (FSH) and recombinant FSH for in vitro fertilization/intracytoplasmic sperm injection in patients with polycystic ovary syndrome. Fertil Steril 2010;94(6):2332-4. PMID: 20188364.

Abu Hashim H, El Rakhawy M and Abd Elaal I. Randomized comparison of superovulation with letrozole vs. clomiphene citrate in an IUI program for women with recently surgically treated minimal to mild endometriosis. Acta Obstet Gynecol Scand 2012;91(3):338-45. PMID: 22181973.

Abu Hashim H, Foda O, Ghayaty E, et al. Laparoscopic ovarian diathermy after clomiphene failure in polycystic ovary syndrome: is it worthwhile? A randomized controlled trial. Arch Gynecol Obstet 2011;284(5):1303-9. PMID: 21755338.

Abu Hashim H, Mashaly AM and Badawy A. Letrozole versus laparoscopic ovarian diathermy for ovulation induction in clomiphene-resistant women with polycystic ovary syndrome: a randomized controlled trial. Arch Gynecol Obstet 2010;282(5):567-71. PMID: 20577748.

Abu Hashim H, Ombar O and Abd Elaal I. Intrauterine insemination versus timed intercourse with clomiphene citrate in polycystic ovary syndrome: a randomized controlled trial. Acta Obstet Gynecol Scand 2011;90(4):344-50. PMID: 21306326.

Aghahosseini M, Aleyasin A, Chegini V, et al. Lowdose hCG as trigger day and $35 \mathrm{hr}$ later have different ovarian hyperstimulation syndrome occurrence in females undergoing In vitro fertilization: An RCT. Int J Reprod Biomed (Yazd) 2017;15(11):735-740. PMID: 29404536.

Amer SA, Li TC, Metwally M, et al. Randomized controlled trial comparing laparoscopic ovarian diathermy with clomiphene citrate as a first-line method of ovulation induction in women with polycystic ovary syndrome. Hum Reprod 2009;24(1):219-25. PMID: 18794162.
Amer SA, Smith J, Mahran A, et al. Double-blind randomized controlled trial of letrozole versus clomiphene citrate in subfertile women with polycystic ovarian syndrome. Hum Reprod 2017;32(8):1631-1638. PMID: 28854590.

An Y, Sun Z, Zhang Y, et al. The use of berberine for women with polycystic ovary syndrome undergoing IVF treatment. Clin Endocrinol (Oxf) 2014;80(3):425-31. PMID: 23869585.

Badawy A, Allam A and Abulatta M. Extending clomiphene treatment in clomiphene-resistant women with PCOS: a randomized controlled trial. Reprod Biomed Online 2008;16(6):825-9. PMID: 18549692.

Badawy A, Shokeir T, Allam AF, et al. Pregnancy outcome after ovulation induction with aromatase inhibitors or clomiphene citrate in unexplained infertility. Acta Obstet Gynecol Scand 2009;88(2):187-91. PMID: 19089782.

Bagis T, Haydardedeoglu B, Kilicdag EB, et al. Single versus double intrauterine insemination in multi-follicular ovarian hyperstimulation cycles: a randomized trial. Hum Reprod 2010;25(7):1684-90. PMID: 20457669.

Balaban B, Yakin K, Alatas C, et al. Clinical outcome of intracytoplasmic injection of spermatozoa morphologically selected under high magnification: a prospective randomized study. Reprod Biomed Online 2011;22(5):472-6. PMID: 21324747.

Barad DH, Darmon SK, Kushnir VA, et al. Impact of preimplantation genetic screening on donor oocyterecipient cycles in the United States. Am J Obstet Gynecol 2017;217(5):576.e1-576.e8. PMID: 28735705.

Belva F, Bonduelle M, Schiettecatte J, et al. Salivary testosterone concentrations in pubertal ICSI boys compared with spontaneously conceived boys. Hum Reprod 2011;26(2):438-41. PMID: 21138905.

Bhattacharya S, Harrild K, Mollison J, et al. Clomifene citrate or unstimulated intrauterine insemination compared with expectant management for unexplained infertility: pragmatic randomised controlled trial. Bmj 2008;337:a716. PMID: 18687718. 
Bodri D, Guillen JJ, Galindo A, et al. Triggering with human chorionic gonadotropin or a gonadotropinreleasing hormone agonist in gonadotropin-releasing hormone antagonist-treated oocyte donor cycles: findings of a large retrospective cohort study. Fertil Steril 2009;91(2):365-71. PMID: 18367175.

Bodri D, Guillen JJ, Polo A, et al. Complications related to ovarian stimulation and oocyte retrieval in 4052 oocyte donor cycles. Reprod Biomed Online 2008;17(2):237-43. PMID: 18681998.

Boulet SL, Kirby RS, Reefhuis J, et al. Assisted reproductive technology and birth defects among liveborn infants in Florida, Massachusetts, and Michigan, 2000-2010. JAMA Pediatrics 2016;170(6).

Boulet SL, Mehta A, Kissin DM, et al. Trends in use of and reproductive outcomes associated with intracytoplasmic sperm injection. Jama 2015;313(3):255-63. PMID: 25602996.

Brinton LA, Moghissi KS, Scoccia B, et al. Effects of fertility drugs on cancers other than breast and gynecologic malignancies. Fertil Steril 2015. PMID: 26232746.

Brinton LA, Scoccia B, Moghissi KS, et al. Longterm relationship of ovulation-stimulating drugs to breast cancer risk. Cancer Epidemiol Biomarkers Prev 2014;23(4):584-93. PMID: 24700523.

Brinton LA, Westhoff CL, Scoccia B, et al. Fertility drugs and endometrial cancer risk: results from an extended follow-up of a large infertility cohort. Hum Reprod 2013;28(10):2813-21. PMID: 23943795.

Butts SF, Owen C, Mainigi M, et al. Assisted hatching and intracytoplasmic sperm injection are not associated with improved outcomes in assisted reproduction cycles for diminished ovarian reserve: an analysis of cycles in the United States from 2004 to 2011. Fertil Steril 2014;102(4):1041-1047.e1. PMID: 25086790.

Chang J, Boulet SL, Jeng G, et al. Outcomes of in vitro fertilization with preimplantation genetic diagnosis: an analysis of the United States Assisted Reproductive Technology Surveillance Data, 20112012. Fertil Steril 2016;105(2):394-400. PMID: 26551441.

Chen ZJ, Shi Y, Sun Y, et al. Fresh versus Frozen Embryos for Infertility in the Polycystic Ovary Syndrome. N Engl J Med 2016;375(6):523-33. PMID: 27509101.
Choi MH, Lee SH, Kim HO, et al. Comparison of assisted reproductive technology outcomes in infertile women with polycystic ovary syndrome: In vitro maturation, GnRH agonist, and GnRH antagonist cycles. Clin Exp Reprod Med 2012;39(4):166-71. PMID: 23346527.

Crawford S, Boulet SL, Kawwass JF, et al. Cryopreserved oocyte versus fresh oocyte assisted reproductive technology cycles, United States, 2013. Fertil Steril 2017;107(1):110-118. PMID: 27842997.

Custers IM, van Rumste MM, van der Steeg JW, et al. Long-term outcome in couples with unexplained subfertility and an intermediate prognosis initially randomized between expectant management and immediate treatment. Hum Reprod 2012;27(2):44450. PMID: 22114108.

Danhof NA, van Wely M, Repping S, et al. Follicle stimulating hormone versus clomiphene citrate in intrauterine insemination for unexplained subfertility: a randomized controlled trial. Hum Reprod 2018;33(10):1866-1874. PMID: 30137325.

de Wilde MA, Lamain-de Ruiter M, VeltmanVerhulst SM, et al. Increased rates of complications in singleton pregnancies of women previously diagnosed with polycystic ovary syndrome predominantly in the hyperandrogenic phenotype. Fertil Steril 2017;108(2):333-340. PMID: 28778282.

Demirol A and Gurgan T. Comparison of different gonadotrophin preparations in intrauterine insemination cycles for the treatment of unexplained infertility: a prospective, randomized study. Hum Reprod 2007;22(1):97-100. PMID: 16954409.

Dhalwani NN, Boulet SL, Kissin DM, et al. Assisted reproductive technology and perinatal outcomes: conventional versus discordant-sibling design. Fertility and Sterility 2016;106(3):710-716.e2.

Diamond MP, Legro RS, Coutifaris C, et al. Letrozole, Gonadotropin, or Clomiphene for Unexplained Infertility. Acta Obstet Gynecol Scand 2015;373(13):1230-40. PMID: 26398071.

Dreyer K, Lier MC, Emanuel MH, et al. Hysteroscopic proximal tubal occlusion versus laparoscopic salpingectomy as a treatment for hydrosalpinges prior to IVF or ICSI: an RCT. Hum Reprod 2016;31(9):2005-16. PMID: 27209341.

Ebrahimi M, Asbagh FA and Darvish S. The effect of luteal phase support on pregnancy rates of the stimulated intrauterine insemination cycles in couples with unexplained infertility. International Journal of Fertility and Sterility 2010;4(2):51-56. 
Einarsson S, Bergh C, Friberg B, et al. Weight reduction intervention for obese infertile women prior to IVF: a randomized controlled trial. Hum Reprod 2017;32(8):1621-1630. PMID: 28854592.

Elsedeek M and Elgindy E. Comparison between two clomiphene citrate protocols for induction of ovulation in clomiphene resistant polycystic ovary syndrome. Middle East Fertility Society Journal 2014;19(4):243-247.

Emekci Ozay O, Ozay AC, Cagliyan E, et al. Myoinositol administration positively effects ovulation induction and intrauterine insemination in patients with polycystic ovary syndrome: a prospective, controlled, randomized trial. Gynecol Endocrinol 2017;33(7):524-528. PMID: 28277112.

Erdem A, Erdem M, Atmaca S, et al. Impact of luteal phase support on pregnancy rates in intrauterine insemination cycles: a prospective randomized study. Fertil Steril 2009;91(6):2508-13. PMID: 18692788.

Erdem M, Abay S, Erdem A, et al. Recombinant FSH increases live birth rates as compared to clomiphene citrate in intrauterine insemination cycles in couples with subfertility: a prospective randomized study. Eur J Obstet Gynecol Reprod Biol 2015;189:33-7. PMID: 25855325.

Farquhar CM, Liu E, Armstrong S, et al. Intrauterine insemination with ovarian stimulation versus expectant management for unexplained infertility (TUI): a pragmatic, open-label, randomised, controlled, two-centre trial. Lancet 2018;391(10119):441-450. PMID: 29174128.

Ge HS, Huang XF, Zhang W, et al. Exposure to human chorionic gonadotropin during in vitro maturation does not improve the maturation rate and developmental potential of immature oocytes from patients with polycystic ovary syndrome. Fertil Steril 2008;89(1):98-103. PMID: 17524398.

Ghahiri A, Mogharehabed N and Mamourian M. Letrozole as the first-line treatment of infertile women with poly cystic ovarian syndrome (PCOS) compared with clomiphene citrate: A clinical trial. Adv Biomed Res 2016;5:6. PMID: 26962508.

Ghanem ME, Elboghdady LA, Hassan M, et al. Clomiphene citrate co-treatment with low dose urinary FSH versus urinary FSH for clomiphene resistant PCOS: randomized controlled trial. J Assist Reprod Genet 2013;30(11):1477-85. PMID:

24014214.
Gibreel A, Badawy A, El-Refai W, et al. Endometrial scratching to improve pregnancy rate in couples with unexplained subfertility: A randomized controlled trial. Journal of Obstetrics and Gynaecology Research 2013;39(3):680-684.

Goldman MB, Thornton KL, Ryley D, et al. A randomized clinical trial to determine optimal infertility treatment in older couples: the Forty and Over Treatment Trial (FORT-T). Fertil Steril 2014;101(6):1574-81.e1-2. PMID: 24796764.

Gregoriou O, Vlahos NF, Konidaris S, et al. Randomized controlled trial comparing superovulation with letrozole versus recombinant follicle-stimulating hormone combined with intrauterine insemination for couples with unexplained infertility who had failed clomiphene citrate stimulation and intrauterine insemination. Fertil Steril 2008;90(3):678-83. PMID: 17961561.

Grimstad FW, Nangia AK, Luke B, et al. Use of ICSI in IVF cycles in women with tubal ligation does not improve pregnancy or live birth rates. Hum Reprod 2016;31(12):2750-2755. PMID: 27738114.

Hajizadeh Maleki B and Tartibian B. Moderate aerobic exercise training for improving reproductive function in infertile patients: A randomized controlled trial. Cytokine 2017;92:55-67. PMID: 28092795.

Harira M. Use of Letrozole versus clomipheneestradiol for treating infertile women with unexplained infertility not responding well to clomiphene alone, comparative study. Middle East Fertility Society Journal 2018.

Hassan A, Shehata N and Wahba A. Cost effectiveness of letrozole and purified urinary FSH in treating women with clomiphene citrate-resistant polycystic ovarian syndrome: a randomized controlled trial. Hum Fertil (Camb) 2017;20(1):3742. PMID: 27825272.

Hershko-Klement A, Sukenik-Halevy R, Biron Shental T, et al. Intracytoplasmic morphologically selected sperm injection and congenital birth defects: a retrospective cohort study. Andrology 2016;4(5):887-93. PMID: 27317040.

Homburg R, Hendriks ML, Konig TE, et al. Clomifene citrate or low-dose FSH for the first-line treatment of infertile women with anovulation associated with polycystic ovary syndrome: a prospective randomized multinational study. Hum Reprod 2012;27(2):468-73. PMID: 22128296. 
Hosseini MA, Aleyasin A, Saeedi H, et al. Comparison of gonadotropin-releasing hormone agonists and antagonists in assisted reproduction cycles of polycystic ovarian syndrome patients. J Obstet Gynaecol Res 2010;36(3):605-10. PMID: 20598044.

Hossein-Rashidi B, Khandzad B, ShahrokhTehraninejad E, et al. Recombinant FSH Compared to Clomiphene Citrate as the First-Line in Ovulation Induction in Polycystic Ovary Syndrome Using Newly Designed Pens: A Randomized Controlled Trial. J Family Reprod Health 2016;10(1):42-8. PMID: 27385973.

Ibrahim MH, Tawfic M, Hassan MM, et al. Letrozole versus laparoscopic ovarian drilling in infertile women with PCOS resistant to clomiphene citrate. Middle East Fertility Society Journal 2017;22(4):251254.

Jacob SL, Brewer C, Tang T, et al. A short course of metformin does not reduce OHSS in a GnRH antagonist cycle for women with PCOS undergoing IVF: a randomised placebo-controlled trial. Hum Reprod 2016;31(12):2756-2764. PMID: 27816925.

Jahromi BN, Sadeghi S, Alipour S, et al. Effect of melatonin on the outcome of assisted reproductive technique cycles in women with diminished ovarian reserve: A double-blinded randomized clinical trial. Iranian Journal of Medical Sciences 2017;42(1):7378.

Johnson NP, Stewart AW, Falkiner J, et al. PCOSMIC: a multi-centre randomized trial in women with PolyCystic Ovary Syndrome evaluating Metformin for Infertility with Clomiphene. Hum Reprod 2010;25(7):1675-83. PMID: 20435692.

Kansal Kalra S, Ratcliffe S, Gracia CR, et al. Randomized controlled pilot trial of luteal phase recombinant FSH stimulation in poor responders. Reprod Biomed Online 2008;17(6):745-50. PMID: 19079956.

Kar S and Sanchita S. Clomiphene citrate, metformin or a combination of both as the first line ovulation induction drug for Asian Indian women with polycystic ovarian syndrome: A randomized controlled trial. Journal of Human Reproductive Sciences 2015;8(4):197-201.

Kettner LO, Matthiesen NB, Ramlau-Hansen CH, et al. Fertility treatment and childhood type 1 diabetes mellitus: a nationwide cohort study of 565,116 live births. Fertil Steril 2016;106(7):1751-1756. PMID: 27773424.
Keyhan S, Truong T, Li YJ, et al. Preterm Delivery and Low Birth Weight Among Neonates Conceived With Intracytoplasmic Sperm Injection Compared With Conventional In Vitro Fertilization. Obstet Gynecol 2018;131(2):262-268. PMID: 29324596.

Khosravi D, Taheripanah R, Taheripanah A, et al. Comparison of oral dydrogesterone with vaginal progesteronefor luteal support in IUI cycles: a randomized clinical trial. Iran J Reprod Med 2015;13(7):433-8. PMID: 26494991.

Kim CH, Howles CM and Lee HA. The effect of transdermal testosterone gel pretreatment on controlled ovarian stimulation and IVF outcome in low responders. Fertil Steril 2011;95(2):679-83. PMID: 20801436.

Kim CH, Moon JW, Kang HJ, et al. Effectiveness of $\mathrm{GnRH}$ antagonist multiple dose protocol applied during early and late follicular phase compared with GnRH agonist long protocol in non-obese and obese patients with polycystic ovary syndrome undergoing IVF/ICSI. Clin Exp Reprod Med 2012;39(1):22-7. PMID: 22563547.

Kissin DM, Zhang Y, Boulet SL, et al. Association of assisted reproductive technology (ART) treatment and parental infertility diagnosis with autism in ARTconceived children. Hum Reprod 2015;30(2):454-65. PMID: 25518976.

Kjotrod SB, Carlsen SM, Rasmussen PE, et al. Use of metformin before and during assisted reproductive technology in non-obese young infertile women with polycystic ovary syndrome: a prospective, randomized, double-blind, multi-centre study. Hum Reprod 2011;26(8):2045-53. PMID: 21606131.

Knudtson JF, Failor CM, Gelfond JA, et al. Assisted hatching and live births in first-cycle frozen embryo transfers. Fertil Steril 2017;108(4):628-634. PMID: 28863938.

Kramer W, Schneider J and Schultz N. US oocyte donors: a retrospective study of medical and psychosocial issues. Hum Reprod 2009;24(12):31449. PMID: 19729378.

Kurzawa R, Ciepiela P, Baczkowski T, et al. Comparison of embryological and clinical outcome in GnRH antagonist vs. GnRH agonist protocols for in vitro fertilization in PCOS non-obese patients. A prospective randomized study. J Assist Reprod Genet 2008;25(8):365-74. PMID: 18802744.

Kuzmin A and Linde V. Diagnostic and remedial capability of transcervical falloposcopy in conjunction with laparoscopy. Gynecol Endocrinol 2014;30(Suppl 1):17-9. PMID: 25200821. 
La Sala GB, Nicoli A, Fornaciari E, et al. Intracytoplasmic morphologically selected sperm injection versus conventional intracytoplasmic sperm injection: a randomized controlled trial. Reprod Biol Endocrinol 2015;13(1):97. PMID: 26307050.

Leandri RD, Gachet A, Pfeffer J, et al. Is intracytoplasmic morphologically selected sperm injection (IMSI) beneficial in the first ART cycle? a multicentric randomized controlled trial. Andrology 2013;1(5):692-7. PMID: 23788532.

Legro RS, Barnhart HX, Schlaff WD, et al. Clomiphene, metformin, or both for infertility in the polycystic ovary syndrome. N Engl J Med 2007;356(6):551-66. PMID: 17287476.

Legro RS, Brzyski RG, Diamond MP, et al. Letrozole versus clomiphene for infertility in the polycystic ovary syndrome. N Engl J Med 2014;371(2):119-29. PMID: 25006718.

Legro RS, Dodson WC, Kris-Etherton PM, et al. Randomized Controlled Trial of Preconception Interventions in Infertile Women With Polycystic Ovary Syndrome. J Clin Endocrinol Metab 2015;100(11):4048-58. PMID: 26401593.

Levi Dunietz G, Holzman C, Zhang Y, et al. Assisted Reproductive Technology and Newborn Size in Singletons Resulting from Fresh and Cryopreserved Embryos Transfer. PLoS One 2017;12(1):e0169869. PMID: 28114395.

Litzky JF, Boulet SL, Esfandiari N, et al. Birthweight in infants conceived through in vitro fertilization following blastocyst or cleavage-stage embryo transfer: a national registry study. Journal of Assisted Reproduction and Genetics 2018;35(6):1027-1037.

Litzky JF, Boulet SL, Esfandiari N, et al. Effect of frozen/thawed embryo transfer on birthweight, macrosomia, and low birthweight rates in US singleton infants. American Journal of Obstetrics and Gynecology 2018;218(4):433.e1-433.e10.

Londra L, Moreau C, Strobino D, et al. Is the type of gonadotropin-releasing hormone suppression protocol for ovarian hyperstimulation associated with ectopic pregnancy in fresh autologous cycles for in vitro fertilization?. Fertility and Sterility 2016;106(3):666-672.

Luke B, Brown MB, Grainger DA, et al. Practice patterns and outcomes with the use of single embryo transfer in the United States. Fertil Steril 2010;93(2):490-8. PMID: 19376512.
Luke B, Brown MB, Spector LG, et al. Cancer in women after assisted reproductive technology Presented at the 70th Annual Meeting of the American Society for Reproductive Medicine, Honolulu, Hawaii, October 18-22, 2014. Fertility and Sterility 2015;104(5):1218-1226.

Luke B, Stern JE, Kotelchuck M, et al. Birth Outcomes by Infertility Treatment: Analyses of the Population-Based Cohort: Massachusetts Outcomes Study of Assisted Reproductive Technologies (MOSART). J Reprod Med 2016;61(3-4):114-27. PMID: 27172633.

Magnusson A, Wennerholm UB, Kallen K, et al. The association between the number of oocytes retrieved for IVF, perinatal outcome and obstetric complications. Hum Reprod 2018;33(10):1939-1947. PMID: 30124838.

Maher MA, Sayyed TM and Elkhouly N. Cervical mucus removal prior to intrauterine insemination: a randomized trial. Bjog 2018;125(7):841-847. PMID: 29078018.

Majumdar G and Majumdar A. A prospective randomized study to evaluate the effect of hyaluronic acid sperm selection on the intracytoplasmic sperm injection outcome of patients with unexplained infertility having normal semen parameters. J Assist Reprod Genet 2013;30(11):1471-5. PMID: 24085466.

Malchau SS, Henningsen AA, Loft A, et al. The long-term prognosis for live birth in couples initiating fertility treatments. Hum Reprod 2017;32(7):14391449. PMID: 28472455.

Mancuso AC, Boulet SL, Duran E, et al. Elective single embryo transfer in women less than age 38 years reduces multiple birth rates, but not live birth rates, in United States fertility clinics. Fertil Steril 2016;106(5):1107-1114. PMID: 27376458.

Maxwell KN, Cholst IN and Rosenwaks Z. The incidence of both serious and minor complications in young women undergoing oocyte donation. Fertil Steril 2008;90(6):2165-71. PMID: 18249368.

Mehrabian F and Eessaei F. The laparoscopic ovarian electrocautery versus gonadotropin therapy in infertile women with clomiphene citrate-resistant polycystic ovary syndrome; a randomized controlled trial. J Pak Med Assoc 2012;62(3 Suppl 2):S42-4. PMID: 22768457. 
Mohammadi Yeganeh L, Moini A, Shiva M, et al. Methylprednisolone for prevention of ovarian hyperstimulation syndrome in patients with polycystic ovarian syndrome undergoing in-vitro fertilisation: a randomised controlled trial. Journal of Obstetrics and Gynaecology 2018;38(2):241-246.

Morad AWA and Abdelhamid AA. Prospective randomized study for hydrotubation with or without lidocaine before intrauterine insemination in unexplained infertility. Middle East Fertility Society Journal 2012;17(4):250-255.

Morin-Papunen L, Rantala AS, Unkila-Kallio L, et al. Metformin improves pregnancy and live-birth rates in women with polycystic ovary syndrome (PCOS): a multicenter, double-blind, placebo-controlled randomized trial. J Clin Endocrinol Metab 2012;97(5):1492-500. PMID: 22419702.

Muller V, Kogan I, Yarmolinskaya M, et al. Dienogest treatment after ovarian endometrioma removal in infertile women prior to IVF. Gynecol Endocrinol 2017;33(sup1):18-21. PMID: 29264985.

Mutsaerts MA, van Oers AM, Groen H, et al. Randomized Trial of a Lifestyle Program in Obese Infertile Women. N Engl J Med 2016;374(20):194253. PMID: 27192672.

Nada AM, ElSetohy KA, Banat MM, et al. Antagonist protocol versus clomiphene in unexplained infertility: A randomized controlled study. Taiwan J Obstet Gynecol 2016;55(3):326-30. PMID: 27343309.

Nahuis MJ, Kose N, Bayram N, et al. Long-term outcomes in women with polycystic ovary syndrome initially randomized to receive laparoscopic electrocautery of the ovaries or ovulation induction with gonadotrophins. Hum Reprod 2011;26(7):1899904. PMID: 21576081.

Nahuis MJ, Oude Lohuis E, Kose N, et al. Long-term follow-up of laparoscopic electrocautery of the ovaries versus ovulation induction with recombinant FSH in clomiphene citrate-resistant women with polycystic ovary syndrome: an economic evaluation. Hum Reprod 2012;27(12):3577-82. PMID: 23001778.

Nandi A, Bhide P, Hooper R, et al. Intrauterine insemination with gonadotropin stimulation or in vitro fertilization for the treatment of unexplained subfertility: a randomized controlled trial. Fertil Steril 2017;107(6):1329-1335.e2. PMID: 28501361.
Nangia AK, Luke B, Smith JF, et al. National study of factors influencing assisted reproductive technology outcomes with male factor infertility. Fertil Steril 2011;96(3):609-14. PMID: 21733503.

Oyesanya OA, Olufowobi O, Ross W, et al. Prognosis of oocyte donation cycles: a prospective comparison of the in vitro fertilization-embryo transfer cycles of recipients who used shared oocytes versus those who used altruistic donors. Fertil Steril 2009;92(3):930-6. PMID: 18829002.

Palomba S, Falbo A, Battista L, et al. Laparoscopic ovarian diathermy vs clomiphene citrate plus metformin as second-line strategy for infertile anovulatory patients with polycystic ovary syndrome: a randomized controlled trial. Am J Obstet Gynecol 2010;202(6):577.e1-8. PMID: 20096821.

Palomba S, Falbo A, Carrillo L, et al. Metformin reduces risk of ovarian hyperstimulation syndrome in patients with polycystic ovary syndrome during gonadotropin-stimulated in vitro fertilization cycles: a randomized, controlled trial. Fertil Steril 2011;96(6):1384-1390.e4. PMID: 21982727.

Peeraer K, Couck I, Debrock S, et al. Frozen-thawed embryo transfer in a natural or mildly hormonally stimulated cycle in women with regular ovulatory cycles: a RCT. Hum Reprod 2015;30(11):2552-62. PMID: 26364081.

Polotsky AJ, Allshouse AA, Casson PR, et al. Impact of Male and Female Weight, Smoking, and Intercourse Frequency on Live Birth in Women With Polycystic Ovary Syndrome. J Clin Endocrinol Metab 2015;100(6):2405-12. PMID: 25856211.

Pourali L, Ayati S, Tavakolizadeh S, et al. Clomiphene citrate versus letrozole with gonadotropins in intrauterine insemination cycles: A randomized trial. Int J Reprod Biomed (Yazd) 2017;15(1):49-54. PMID: 28280800.

Provost MP, Thomas SM, Yeh JS, et al. State Insurance Mandates and Multiple Birth Rates After In Vitro Fertilization. Obstetrics and Gynecology 2016.

Qu F, Wang FF, Wu Y, et al. Transcutaneous Electrical Acupoint Stimulation Improves the Outcomes of In Vitro Fertilization: A Prospective, Randomized and Controlled Study. Explore (NY) 2017;13(5):306-312. PMID: 28915981. 
Ragni G, Levi-Setti PE, Fadini R, et al. Clomiphene citrate versus high doses of gonadotropins for in vitro fertilisation in women with compromised ovarian reserve: a randomised controlled non-inferiority trial. Reprod Biol Endocrinol 2012;10:114. PMID: 23249758.

Rahman A, Francomano D, Sagnella F, et al. The effect on clinical results of adding recombinant LH in late phase of ovarian stimulation of patients with repeated implantation failure: A pilot study.

European Review for Medical and Pharmacological Sciences 2017;21(23):5485-5490.

Rashidi M, Aaleyasin A, Aghahosseini M, et al. Advantages of recombinant follicle-stimulating hormone over human menopausal gonadotropin for ovarian stimulation in intrauterine insemination: a randomized clinical trial in unexplained infertility. Eur J Obstet Gynecol Reprod Biol 2013;169(2):2447. PMID: 23541417.

Rashidi M, Najmi Z and Mobasseri A. Advantages of Recombinant Follicle-Stimulating Hormone over Human Menopausal Gonadotropin in Intrauterine Insemination: A Randomized Clinical Trial in Polycystic Ovary Syndrome-Associated Infertility. Gynecol Obstet Invest 2015. PMID: 26228499.

Rausch ME, Legro RS, Barnhart HX, et al. Predictors of pregnancy in women with polycystic ovary syndrome. J Clin Endocrinol Metab 2009;94(9):3458-66. PMID: 19509098.

Razi MH, Halvaei I and Razi Y. Laser assisted zona hatching does not improve live birth rate in patients undergoing their first ICSI cycles. Iran J Reprod Med 2013;11(12):1021-6. PMID: 24639729.

Reindollar RH, Regan MM, Neumann PJ, et al. A randomized clinical trial to evaluate optimal treatment for unexplained infertility: the fast track and standard treatment (FASTT) trial. Fertil Steril 2010;94(3):888-99. PMID: 19531445.

Rubio C, Bellver J, Rodrigo L, et al. Preimplantation genetic screening using fluorescence in situ hybridization in patients with repetitive implantation failure and advanced maternal age: two randomized trials. Fertil Steril 2013;99(5):1400-7. PMID: 23260857.

Schendelaar P, Middelburg KJ, Bos AF, et al. The Groningen ART cohort study: the effects of ovarian hyperstimulation and the IVF laboratory procedures on neurological condition at 2 years. Hum Reprod 2011;26(3):703-12. PMID: 21227942.
Seckin B, Turkcapar F, Yildiz Y, et al. Effect of luteal phase support with vaginal progesterone in intrauterine insemination cycles with regard to follicular response: a prospective randomized study. J Reprod Med 2014;59(5-6):260-6. PMID: 24937967.

Selman H and Rinaldi L. Effectiveness of corifollitropin alfa used for ovarian stimulation of poor responder patients. International Journal of Women's Health 2016;8:609-615.

Shi Y, Sun Y, Hao C, et al. Transfer of Fresh versus Frozen Embryos in Ovulatory Women. N Engl J Med 2018;378(2):126-136. PMID: 29320646.

Sismanoglu A, Tekin HI, Erden HF, et al. Ovulation triggering with GnRH agonist vs. hCG in the same egg donor population undergoing donor oocyte cycles with GnRH antagonist: a prospective randomized cross-over trial. J Assist Reprod Genet 2009;26(5):251-6. PMID: 19629674.

Spaan M, van den Belt-Dusebout AW, Burger CW, et al. Risk of Colorectal Cancer After Ovarian Stimulation for In Vitro Fertilization. Clin Gastroenterol Hepatol 2016;14(5):729-737.e5. PMID: 26687912.

Spaan M, van den Belt-Dusebout AW, Schaapveld $\mathrm{M}$, et al. Melanoma risk after ovarian stimulation for in vitro fertilization. Hum Reprod 2015;30(5):121628. PMID: 25743782.

Stadtmauer LA, Sarhan A, Duran EH, et al. The impact of a gonadotropin-releasing hormone antagonist on gonadotropin ovulation induction cycles in women with polycystic ovary syndrome: a prospective randomized study. Fertil Steril 2011;95(1):216-20. PMID: 20594551.

Stewart LM, Holman CD, Aboagye-Sarfo P, et al. In vitro fertilization, endometriosis, nulliparity and ovarian cancer risk. Gynecol Oncol 2013;128(2):2604. PMID: 23116937.

Tartagni M, Cicinelli MV, Baldini D, et al. Dehydroepiandrosterone decreases the age-related decline of the in vitro fertilization outcome in women younger than 40 years old. Reprod Biol Endocrinol 2015;13:18. PMID: 25884390.

Tehraninejad ES, Nasiri R, Rashidi B, et al. Comparison of GnRH antagonist with long $\mathrm{GnRH}$ agonist protocol after OCP pretreatment in PCOs patients. Arch Gynecol Obstet 2010;282(3):319-25. PMID: 20379731. 
Toftager M, Bogstad J, Bryndorf T, et al. Risk of severe ovarian hyperstimulation syndrome in $\mathrm{GnRH}$ antagonist versus GnRH agonist protocol: RCT including 1050 first IVF/ICSI cycles. Hum Reprod 2016;31(6):1253-64. PMID: 27060174.

Toftager M, Bogstad J, Lossl K, et al. Cumulative live birth rates after one ART cycle including all subsequent frozen-thaw cycles in 1050 women: secondary outcome of an RCT comparing GnRHantagonist and GnRH-agonist protocols. Hum Reprod 2017;32(3):556-567. PMID: 28130435.

Topçu HO, Batioğlu AS and İslimye M. Tamoxifen versus clomiphene citrate for ovulation induction in women with polycystic ovary syndrome: A prospective randomized trial. Journal of Reproductive Medicine 2017;62(5):507-512.

Trabert B, Lamb EJ, Scoccia B, et al. Ovulationinducing drugs and ovarian cancer risk: results from an extended follow-up of a large United States infertility cohort. Fertil Steril 2013;100(6):1660-6. PMID: 24011610.

Tsai CC, Huang FJ, Wang LJ, et al. Clinical outcomes and development of children born after intracytoplasmic sperm injection (ICSI) using extracted testicular sperm or ejaculated extreme severe oligo-astheno-teratozoospermia sperm: a comparative study. Fertil Steril 2011;96(3):567-71. PMID: 21880275.

van Leeuwen FE, Klip H, Mooij TM, et al. Risk of borderline and invasive ovarian tumours after ovarian stimulation for in vitro fertilization in a large Dutch cohort. Hum Reprod 2011;26(12):3456-65. PMID: 22031719.

van Oers AM, Groen H, Mutsaerts MA, et al. Effectiveness of lifestyle intervention in subgroups of obese infertile women: a subgroup analysis of a RCT. Hum Reprod 2016;31(12):2704-2713. PMID: 27798042.

van Oers AM, Mutsaerts MAQ, Burggraaff JM, et al. Association between periconceptional weight loss and maternal and neonatal outcomes in obese infertile women. PLoS One 2018;13(3):e0192670. PMID: 29590118.

van Rijswijk J, Caanen MR, Mijatovic V, et al. Immobilization or mobilization after IUI: an RCT. Hum Reprod 2017;32(11):2218-2224. PMID: 29040538. van Rumste MM, Custers IM, van Wely M, et al. IVF with planned single-embryo transfer versus IUI with ovarian stimulation in couples with unexplained subfertility: an economic analysis. Reprod Biomed Online 2014;28(3):336-42. PMID: 24456703.

Verhoeve HR, Moolenaar LM, Hompes P, et al. Cost-effectiveness of tubal patency tests. Bjog 2013;120(5):583-93. PMID: 23331951.

Vitek WS, Galarraga O, Klatsky PC, et al. Management of the first in vitro fertilization cycle for unexplained infertility: a cost-effectiveness analysis of split in vitro fertilization-intracytoplasmic sperm injection. Fertil Steril 2013;100(5):1381-8. PMID: 23876534.

Wang Y, Chen Q, Wang N, et al. Controlled Ovarian Stimulation Using Medroxyprogesterone Acetate and hMG in Patients With Polycystic Ovary Syndrome Treated for IVF: A Double-Blind Randomized Crossover Clinical Trial. Medicine (Baltimore) 2016;95(9):e2939. PMID: 26945402.

Weiss NS, Nahuis MJ, Bordewijk E, et al. Gonadotrophins versus clomifene citrate with or without intrauterine insemination in women with normogonadotropic anovulation and clomifene failure (M-OVIN): a randomised, two-by-two factorial trial. Lancet 2018;391(10122):758-765. PMID: 29273245.

Williams CL, Bunch KJ, Stiller CA, et al. Cancer risk among children born after assisted conception. N Engl J Med 2013;369(19):1819-27. PMID: 24195549.

Williams CL, Jones ME, Swerdlow AJ, et al. Risks of ovarian, breast, and corpus uteri cancer in women treated with assisted reproductive technology in Great Britain, 1991-2010: data linkage study including 2.2 million person years of observation. Bmj 2018;362:k2644. PMID: 29997145.

Wiser A, Gonen O, Ghetler Y, et al. Addition of dehydroepiandrosterone (DHEA) for poor-responder patients before and during IVF treatment improves the pregnancy rate: a randomized prospective study. Hum Reprod 2010;25(10):2496-500. PMID: 20729538.

Wu XK, Stener-Victorin E, Kuang HY, et al. Effect of Acupuncture and Clomiphene in Chinese Women With Polycystic Ovary Syndrome: A Randomized Clinical Trial. Jama 2017;317(24):2502-2514. PMID: 28655015. 
Wu XK, Wang YY, Liu JP, et al. Randomized controlled trial of letrozole, berberine, or a combination for infertility in the polycystic ovary syndrome. Fertil Steril 2016;106(3):757-765.e1. PMID: 27336209.

Xiong X, Dickey RP, Buekens P, et al. Use of Intracytoplasmic Sperm Injection and Birth Outcomes in Women Conceiving through In Vitro Fertilization. Paediatr Perinat Epidemiol 2017;31(2):108-115. PMID: 28140471.

Yapca OE, Delibas IB, Karaca I, et al. Time-limited hydrotubation combined with clomiphene citrate treatment for unexplained infertility. Clin Exp Obstet Gynecol 2015;42(3):311-4. PMID: 26152000.

Yazici G, Savas A, Tasdelen B, et al. Role of luteal phase support on gonadotropin ovulation induction cycles in patients with polycystic ovary syndrome. J Reprod Med 2014;59(1-2):25-30. PMID: 24597283.

Yildiz F, Bozkurt N, Erdem A, et al. Effect of Pertubation on Pregnancy Rates before Intrauterine Insemination Treatment in Patients with Unexplained Infertility. Int J Fertil Steril 2014;8(1):77-84. PMID: 24695882.

Youssef MA, van Wely M, Al-Inany $\mathrm{H}$, et al. A mild ovarian stimulation strategy in women with poor ovarian reserve undergoing IVF: a multicenter randomized non-inferiority trial. Hum Reprod 2016. PMID: 27836979.

Yu R, Jin H, Huang X, et al. Comparison of modified agonist, mild-stimulation and antagonist protocols for in vitro fertilization in patients with diminished ovarian reserve. Journal of International Medical Research 2018;46(6):2327-2337.
Zahran KM, Mostafa WA, Abbas AM, et al. Clomiphene citrate plus cabergoline versus clomiphene citrate for induction of ovulation in infertile euprolactinemic patients with polycystic ovary syndrome: A randomized clinical trial. Middle East Fertility Society Journal 2018.

Zain MM, Jamaluddin R, Ibrahim A, et al. Comparison of clomiphene citrate, metformin, or the combination of both for first-line ovulation induction, achievement of pregnancy, and live birth in Asian women with polycystic ovary syndrome: a randomized controlled trial. Fertil Steril 2009;91(2):514-21. PMID: 18321486.

Zakherah MS, Nasr A, El Saman AM, et al. Clomiphene citrate plus tamoxifen versus laparoscopic ovarian drilling in women with clomiphene-resistant polycystic ovary syndrome. Int J Gynaecol Obstet 2010;108(3):240-3. PMID: 19944418.

Zarei A, Mahboubi M, Parsanezhad ME, et al. Effects of piroxicam administration on pregnancy outcome in intrauterine insemination (IUI) cycles: a randomized clinical trial. Clin Exp Obstet Gynecol 2016;43(2):225-9. PMID: 27132415.

Zheng X, Wang L, Zhen X, et al. Effect of hCG priming on embryonic development of immature oocytes collected from unstimulated women with polycystic ovarian syndrome. Reprod Biol Endocrinol 2012;10:40. PMID: 22621829.

Zhu S, Liu D, Huang W, et al. Post-laparoscopic oral contraceptive combined with Chinese herbal mixture in treatment of infertility and pain associated with minimal or mild endometriosis: a randomized controlled trial. BMC Complement Altern Med 2014;14:222. PMID: 24996447 


\section{Appendix D. List of Excluded Studies}

All studies listed below were reviewed in their full-text version and excluded for the reasons cited. Reasons for exclusion signify only the usefulness of the articles for this study and are not intended as criticisms of the articles.

\section{Not a full publication or full text not available:}

Abdalmageed OS, Farghaly TA, Ismail AM, et al. Impact of metformin on in vitro fertilization outcomes in overweight and obese polycystic ovary syndrome women: A prospective cohort Study. Fertility and Sterility 2016;106:e262.

Acharya KS, Keyhan S, Acharya CR, et al. Noncompliance with ASRM/SART guidelines continues to be high in 2013 compared to 2011-2012 in donor oocyte cycles with blastocyst transfer. Fertility and Sterility 2016;106:e323-e324.

AkçelIk Y, Çögendez E, D.B SI, et al. Comparison of clomiphene citrate and low dose recombinant fsh for induction of ovulation in infertilewomen with polycystic ovary syndrome. Turkiye Klinikleri Jinekoloji Obstetrik 2015;25(1):19-26.

Alasmari N. Randomized trial on the effect of prewashing the insemination catheter on the pregnancy outcome. Fertility and Sterility 2016;106:e346.

Algergawy A, Alhalwagy A, Shehata A, et al. Unexplained infertility: Laparoscopy first or art directly. Fertility and Sterility 2016;106:e42.

Barad DH, Darmon S, Kushnir VA, et al. Prevalence and effect of preimplantation genetic screening (PGS) on oocyte donation cycles in the United States: 2005 to 2013. Fertility and Sterility 2016;106:e330.

Beliveau LN, Vilos A, Tekpetey F, et al. Impact of the number of lead follicles at time of trigger on intrauterine insemination(IUI) pregnancy outcomes. Fertility and Sterility 2016;106:e182.

Çakar E, Taşan HA, Kumru P, et al. Is adding estradiol (E2) to progesterone for luteal phase support in antagonist protocol stimulated in vitro fertilisation (IVF) cycles beneficial?. Journal of the Turkish German Gynecology Association 2016;17:S212-S213.

Chan S, Greenstein Y, Dasig D, et al. Perinatal outcomes after fresh versus frozen embryo transfers. Fertility and Sterility 2016;106:e324-e325.

Chang TA, Jacoby ES, Su YT, et al. Utilization of intracytoplasmic sperm injection (ICSI) among fertility centers in the United States 2000-2013. Fertility and Sterility 2016;106:e312.

Chişu CG and Ancăr V. Ovarian hyperstimulation syndrome a complication of the treatment of ovarian stimulation. Obstetrica si Ginecologie 2012;60(1):35-40. 
Chiu Y, Gaskins AJ, Williams P, et al. Fruit and vegetable intake and their pesticide residues in relation to outcomes of assisted reproductive technology. Fertility and Sterility 2016;106:e27.

Christopoulos G, Vlismas A, Carby A, et al. GnRH agonist trigger with intensive luteal phase support versus human chorionic gonadotropin trigger in high responders: An observational study reporting pregnancy outcomes and incidence of ovarian hyperstimulation syndrome. BJOG: An International Journal of Obstetrics and Gynaecology 2016;123:69.

Collins G, Thakore S and Goldfarb JM. IVF outcomes in young patients with unexplained infertility: An analysis of 273,779 cycles from the 2011-2013 society for assisted reproductive technology clinic outcome reporting system registry. Fertility and Sterility 2016;106:e171.

Du T, Chen Q, Lyu Q, et al. Effects of different endometrial preparations on the outcomes of frozen embryo transfer cycles of in vitrofertilization/intracytoplasmic sperm injection: A study based on more than 30,000 cycles. Fertility and Sterility 2016;106:e345-e346.

Du T, Chen Q, Lyu Q, et al. Is blastocyst transfer associated with a significantly lower incidence of ectopic pregnancy? A strictly controlled retrospect cohort study based on more than 30,000 frozen embryo transfer cycles. Fertility and Sterility 2016;106:e105.

Elnashar I, Farghaly TA, Abdalbadie AS, et al. Low cost ovarian stimulation protocolis associated with lower pregnancy rate in normal responders in comparison to long protocol. Fertility and Sterility 2016;106:e194-e195.

Flatley C, Hillsley K and Kumar S. Intrapartum and neonatal consequences of assisted reproduction. Australian and New Zealand Journal of Obstetrics and Gynaecology 2016;56:5.

Hatirnaz S, Hatirnaz ES, Tan SL, et al. Outcomes of single vs double embryo transfer in in-vitro maturation cycles done in women with polycystic ovary syndrome. Fertility and Sterility 2016;106:e258-e259.

Hebisha SA, Aboelazm BA, Adel HM, et al. Impact of the oxytocin receptor antagonist (atosiban) administered shortly before embryo transfer on pregnancy outcome after intracytoplasmic sperm injection (ICSI). Fertility and Sterility 2016;106:e88-e89.

Herrero B, Lusignan M, Son W, et al. Effects of sperm quality on the success of intracytoplasmic sperm injection (ICSI) with testicular sperm in couples with recurrent ICSI failure with ejaculated sperm. Fertility and Sterility 2016;106:e226.

Hilton JL, Liu K, Laskin CA, et al. Effect of endometrial biopsy on in vitro fertilization clinical pregnancy rates-a randomized multicentre study. Fertility and Sterility 2016;106:e344.

Hipp H, Crawford S, Kawwass JF, et al. In-vitro fertilization cycles among women ages 40 and older. Fertility and Sterility 2016;106:e182-e183.

Humphries LA, Dodge LE, Kennedy EB, et al. Is younger better? donor age less than 25 does not predict more favorable outcomes after in vitro fertilization. Fertility and Sterility 2016;106:e176. 
Hurley EG and De Franco E. Influence of paternal age on perinatal outcomes in pregnancies achieved with assisted reproductive technologies. Fertility and Sterility 2016;106:e166.

Hurst BS, Merriam K, Marshburn P, et al. Optimal timing for intrauterine insemination (IUI) after administering HCG in ovulation induction IUI cycles. Fertility and Sterility 2016;106:e305e306.

Irani M, Gunnala V, Goldschlag DE, et al. Does trigger of final oocyte maturation with pure GNRH-agonist have adverse effects on pregnancy outcomes of donor/recipient cycles?. Fertility and Sterility 2016;106:e189.

Irani M, Setton R, Gunnala V, et al. Dose of human chorionic gonadotropin to trigger final oocyte maturation. Fertility and Sterility 2016;106:e262-e263.

Janati S, Dehghani Firouzabadi R, Mohseni F, et al. Evaluation effect of intrauterine human chorionic gonadotropin injection before embryo transfer in implantation and pregnancy rate in infertile patients and comparison with conventional embryo transfer in IVF/ICSI/ET cycles. Iranian Journal of Reproductive Medicine 2013;11:67-68.

Janitz A, Peck JD and Craig LB. Ethnic and racial differences in the utilization of infertility services: National survey of family growth(NSFG). Fertility and Sterility 2016;106:e112-e113.

Jayakumaran J, Silva C, Gangrade BK, et al. Ethnic differences in ovarian reserve and assisted reproductive technology outcomes. Fertility and Sterility 2016;106:e368-e369.

Kawwass JF, Crawford S, Hipp H, et al. Embryo donation: National trends and outcomes, 20002013. Fertility and Sterility 2016;106:e320.

Kawwass JF, Kulkarni A, Hipp H, et al. Assisted reproductive technology cycle and obstetric outcomes among underweight and overweight women. Fertility and Sterility 2016;106:e21.

Keyhan S, Acharya KS, Acharya CR, et al. Are we transferring too many embryos in the most favorable group of fresh autologous IVF cycles: A 2013 update. Fertility and Sterility 2016;106:e52-e53.

Keyhan S, Li Y, Truong T, et al. Perinatal outcomes following intracytoplasmic sperm injection (ICSI) versus conventional in vitro fertilization (IVF). Fertility and Sterility 2016;106:e165.

Khudhari A, Hemmings R, Phillips S, et al. How does art singletons differ from naturally conceived (NC) singletons; comparison of perinatal data of 872 art to 19317 (NC) singleton babies. Fertility and Sterility 2016;106:e176-e177.

Kjotrod S, Carlsen SM, Rasmussen PE, et al. Metformin treatment before and during IVF or ICSI in PCOS women with BMI < $28 \mathrm{~kg} / \mathrm{m}$ ?: A prospective, randomized, double-blind, multicenter study. Human Reproduction 2010;25:i286-i287.

Knudtson J, Failor C, Gelfond J, et al. The effect of assisted hatching on frozen embryo transfer live birth rate and clinic trends. Fertility and Sterility 2016;106:e141. 
Konar H, Sharma S, Chakraborty P, et al. Endometrial vascularity and increasing endometrial thickness can predict live birth: A retrospective analysis of 1575 Fet cycles. Fertility and Sterility 2016;106:e215-e216.

Kort J, Riestenberg C, Shah M, et al. Effect of BMI on live birth rates in euploid frozen single embryo transfers. Fertility and Sterility 2016;106:e265.

Kriplani A, Goel T, Mahey R, et al. Pregnancy rate after endometrial scratching in couples with unexplained infertility in ovulation induction \& IUI cycles-a randomised controlled trial. Fertility and Sterility 2016;106:e329.

Kushnir VA, Barad DH, Darmon S, et al. Contribution of third party reproduction to the birth cohort following art in the U.S.A. Fertility and Sterility 2016;106:e108.

Kushnir VA, Shapiro A, Barad DH, et al. Effect of race and ethnicity on fertility rates and utilization of art in the USA. Fertility and Sterility 2016;106:e367-e368.

Li X, Huang R, Fang C, et al. Live birth rate after fresh or frozen-thawed embryo transfers in relation to maternal age: A retrospective cochort study of 13426 cycles. Fertility and Sterility 2016;106:e142.

Londra LC and Mumford SL. Birth weight in singletons after autologous fresh transfer according to the ovarian hyperstimulation protocol used. Fertility and Sterility 2016;106:e170.

Luke B, Brown MB and Spector LG. Risk of maternal morbidity in IVF and non-IVF births: A US study in five states. Fertility and Sterility 2016;106:e104.

Luke B, Brown MB and Wantman E. Physician prediction model for live birth and multiple births after assisted reproductive technology. Fertility and Sterility 2016;106:e177-e178.

Mancuso A, Boulet S, Duran EH, et al. Live birth and multiple birth rates in women under age 38 by elective single embryo transfer (ESET) versus double embryo transfer (DET) in United States IVF clinics. Fertility and Sterility 2016;106:e104.

Marín D and Labarta E. Potential use of kisspeptin in ovarian stimulation treatments. Medicina Reproductiva y Embriologia Clinica 2015;2(3):115-125.

Maslow BL, Griffin D, Benadiva CA, et al. Prospective double-blind randomized placebo controlled clinical trial comparing pregnancy rates after co-administration of low dose hcg at the time of GNRH-agonist trigger or 35 hours later, for the prevention of ohss. Fertility and Sterility 2016;106:e58.

McClennen EL, Richter KS, Moon K, et al. The impact of race and ethnicity on assisted reproductive technology (ART) outcomes: A retrospective cohort study. Fertility and Sterility 2016;106:e99-e100. 
Mizuta S, Yamaguchi K, Nishiyama R, et al. Intracytoplasmic sperm injection (ICSI) outcome using immotile spermatozoa even after pentoxifyllin administration in non-obstructive azoospermic patients. Fertility and Sterility 2016;106:e225.

Mohanasundaram P, Balasubramanyam S and Varma T. GnRH agonist as luteal support in antagonist treated ART cycles: A prospective randomised controlled trial. BJOG: An International Journal of Obstetrics and Gynaecology 2016;123:68.

Mukherjee G, Konar H, Mandve P, et al. Progesterone supplementation in PCOS women undergoing clomiphene citrate stimulated IUI may improve pregnancy by increasing uterine Blood. Fertility and Sterility 2016;106:e260.

Orvieto R. Mono-ovulation in women with polycystic ovary syndrome: The role of step-up, ultra-low-dose gonadotrophin regimen. Reproductive BioMedicine Online 2016.

Pandian Z, Akande VA, Bhattacharya S, et al. Effectiveness of surgical treatment for tubal infertility. Cochrane Database of Systematic Reviews 2007.

Parneix I, Arvis P, Paillet S, et al. Long term outcome of couples undertaking their first IVF/ICSI attempt: A french study. Value in Health 2016;19(7):A406.

Plowden TC, Mumford SL, Kim K, et al. Racial disparities in elective single embryo transfer (ESET) utilization in the United States: A national study. Fertility and Sterility 2016;106:e366.

Puntoni M, Costa M, Paleari L, et al. Ovarian stimulation for infertility treatment and cancer risk: An Italian cohort study. Journal of Clinical Oncology 2016;34.

Putra DE, Birowo P, Widyahening IS, et al. The role of varicocele repair in nonobstructive azoospermic men: A systematic review. BJU International 2016;117:5-6.

Rodriguez-Purata J, Santistevan A, Sekhon L, et al. 1+ 1>2: A cost effectiveness analysis of single embryo transfer with PGS in two successive cycles vs a double embryo transfer with PGS in one. Fertility and Sterility 2016;106:e337.

Salem W, Ho J, Bendikson KA, et al. Modified natural cycle IVF increases value and access to care over traditional IVF for good prognosis patients: A decision analytic model and cost effectiveness analysis. Fertility and Sterility 2016;106:e74-e75.

Shapiro A, Barad DH, Darmon S, et al. Effect of race and ethnicity on live birth rates in thirdparty art cycles in the U.S. Fertility and Sterility 2016;106:e367.

Shapiro A, Barad DH, Darmon S, et al. Racial and ethnic disparities in the use of third party art in the U.S. Fertility and Sterility 2016;106:e108-e109.

Singh S, Singh S, Raman AK, et al. Efficacy of cabergoline in the prevention of ovarian hyperstimulation syndrome: A randomized, double-blind and placebo-controlled trial. International Journal of Infertility and Fetal Medicine 2017;8(2):54-60. 
Siristatidis CS, Bhattacharya S and Maheshwari A. In vitro maturation in sub fertile patients with polycystic ovarian syndrome undergoing assisted reproduction. Cochrane Database of Systematic Reviews 2007.

Smith MB, Hodes-Wertz B, Grifo J, et al. Are the biggest losing? analyzing the effect of body mass index (BMI) on pregnancy rates in euploid frozen embyro transfer (FET) cycles. Fertility and Sterility 2016;106:e102.

Souza PV, Simões AC, Zanini FE, et al. Infertility treatment of women over age 35 in Brazil: An economic evaluation of recombinant Vs. urinary gonadotropins. Value in Health 2016;19(7):A402.

Stevens JM, Schneiderman A, Maruniak K, et al. Euploid blastocyst transfer is aviable clinical option for male factor infertility with high sperm DNA fragmentation. Fertility and Sterility 2016;106:e233.

Styer AK, Mumford SL, Plowden T, et al. Racial disparities in live birth pregnancy outcomes following fresh elective single embryo transfer: A sart-cors analysis 2004-2013. Fertility and Sterility 2016;106:e98.

Vega MG, Zaghi S, Jindal SK, et al. Impact of ovarian aging on perinatal outcomes: Analysis of 135,252 art cycles reported to sart. Fertility and Sterility 2016;106:e167.

Venugopalan L. Immediate neonatal outcome and neurodevelopmental outcome in infants born after artificial reproduction techniques: A matched control study. BJOG: An International Journal of Obstetrics and Gynaecology 2016;123:230-231.

Volovsky M, Healey M, MacLachlan VB, et al. Intrauterine human chorionic gonadotropin (HCG) infusion prior to embryo transfer (ET) may be detrimental to pregnancy rate. Fertility and Sterility 2016;106:e52.

Wang AY. Increased rate of adverse neonatal outcomes among twins following assisted reproductive technology. Fertility and Sterility 2016;106:e174.

Wu X, Stener-Victorin E, Liu J, et al. Acupuncture and clomiphene for infertility in the polycystic ovary syndrome: A multicentre rondomized controlled trial. Fertility and Sterility 2016;106:e375.

Zhou X, McQueen D, Schufreider A, et al. Racial disparities: In vitro fertilization (IVF) outcomes in donor oocyte recipients. Fertility and Sterility 2016;106:e99.

\section{Not available in English:}

Chatillon-Boissier K, Genod A, Denis-Belicard E, et al. [Prospective randomised study of long versus short agonist protocol with poor responder patients during in vitro fertilization]. Gynecol Obstet Fertil 2012;40(11):652-7. PMID: 22342506. 
Emergui Zrihen Y, García Escribano PA, Escamilla Galindo EP, et al. Birth defects in women undergoing assisted reproduction techniques. Clinica e Investigacion en Ginecologia y Obstetricia 2017;44(4):152-156.

Gabriele V, Benabu JC, Ohl J, et al. Does fertility treatment increase the risk of breast cancer? Current knowledge and meta-analysis. Gynecologie Obstetrique Fertilite et Senologie 2017;45(5):299-308.

Ghafarnegad M, Arjmand N and Khazaeipour Z. Pregnancy rate of gonadotrophin therapy and laparoscopic ovarian electrocautery in polycystic ovary syndrome resistant to clomiphene citrate: A comparative study. Tehran University Medical Journal 2010;67(10):712-717.

Liu Z, Tang HL and Zhai SD. Aromatase inhibitors in ovulation induction for women with unexplained infertility: A systematic review. Chinese Journal of Evidence-Based Medicine 2011;11(11):1327-1334.

Moguel-Hernández A, Tietzsch-Escalante P, Iglesias-Leboreiro J, et al. Neonatal complications associated to assisted reproductive techniques at the Hospital Español of Mexico. Revista Mexicana de Pediatria 2017;84(5):182-188.

Zhao J, Gou J, Li DH, et al. Salpingectomy before IVF-ET for hydrosalpinx among Chinese Women: A systematic review. Chinese Journal of Evidence-Based Medicine 2011;11(9):10391046.

\section{Not original data from an RCT, SR/MA, or observational study with comparator:}

Aarts JW, Huppelschoten AG, van Empel IW, et al. How patient-centred care relates to patients' quality of life and distress: a study in 427 women experiencing infertility. Hum Reprod 2012;27(2):488-95. PMID: 22108249.

Abbott J. Surgical treatment is an excellent option for women with endometriosis and infertility. Australian and New Zealand Journal of Obstetrics and Gynaecology 2017;57(6):679-681.

Abd El Fattah EA. Uterine Cavity Abnormalities in Patients with Endometriosis in Alexandria: A Diagnostic Test Accuracy Study. Obstet Gynecol Int 2017;2017:5869028. PMID: 28638413.

Abu Hashim H, Al-Inany H, De Vos M, et al. Three decades after Gjonnaess's laparoscopic ovarian drilling for treatment of PCOS; what do we know? An evidence-based approach. Arch Gynecol Obstet 2013;288(2):409-22. PMID: 23543241.

Akanji Tijani $\mathrm{H}$ and Bhattacharya S. The role of intrauterine insemination in male infertility. Hum Fertil (Camb) 2010;13(4):226-32. PMID: 21117932.

Al-Malki AH, Alrabeeah K, Mondou E, et al. Testicular sperm aspiration (TESA) for infertile couples with severe or complete asthenozoospermia. Andrology 2017;5(2):226-231. PMID: 28187532. 
Almog B, Shalom-Paz E, Dufort D, et al. Promoting implantation by local injury to the endometrium. Fertil Steril 2010;94(6):2026-9. PMID: 20171615.

Alom M, Ziegelmann M, Savage J, et al. Office-based andrology and male infertility proceduresa cost-effective alternative. Transl Androl Urol 2017;6(4):761-772. PMID: 28904909.

Al-Ruthia YS, Al-Mandeel $\mathrm{H}$, AlSanawi $\mathrm{H}$, et al. The effect of metformin use on pregnancy rates among polycystic ovary syndrome patients undergoing in vitro fertilization: A retrospectivecohort study. Saudi Pharm J 2017;25(6):906-910. PMID: 28951677.

Al-Shaikh SFMH, Al-Mukhatar EJ, Al-Zubaidy AA, et al. Use of clomiphene or letrozole for treating women with polycystic ovary syndrome related subfertility in Hilla city. Middle East Fertility Society Journal 2017;22(2):105-110

Al-Taee H and Edan BJ. Estimation of Day-Specific Probabilities of Conception during Natural Cycle in Women from Babylon. Int J Fertil Steril 2018;11(4):314-317. PMID: 29043709.

Anderson BJ, Haimovici F, Ginsburg ES, et al. In vitro fertilization and acupuncture: clinical efficacy and mechanistic basis. Altern Ther Health Med 2007;13(3):38-48. PMID: 17515023.

Angioni S, Cela V, Sedda F, et al. Focusing on surgery results in infertile patients with deep endometriosis. Gynecol Endocrinol 2015:1-4. PMID: 26172932.

Anonymous. Clomifene gives the best live-birth rates in women with polycystic ovary syndrome. Nature Clinical Practice Endocrinology and Metabolism 2007;3(6):445.

Anonymous. Intrauterine insemination. Hum Reprod Update 2009;15(3):265-77. PMID: 19240042.

Arya S, Kupesic-Plavsic S, Mulla ZD, et al. Ovulation induction and controlled ovarian stimulation using letrozole gonadotropin combination: A single center retrospective cohort study. Eur J Obstet Gynecol Reprod Biol 2017;218:123-128. PMID: 28985546.

Awonuga AO, Wheeler K, Thakur M, et al. The value of delaying hCG administration to enable maturation of medium-sized follicles in patients undergoing superovulation for IVF/ICSI. Journal of Assisted Reproduction and Genetics 2018;35(2):289-295.

Banker M, Sorathiya D and Shah S. Vitamin D Deficiency Does Not Influence Reproductive Outcomes of IVF-ICSI: A Study of Oocyte Donors and Recipients. J Hum Reprod Sci 2017;10(2):79-85. PMID: 28904494.

Bar Hava I, Blueshtein M, Ganer Herman H, et al. Gonadotropin-releasing hormone analogue as sole luteal support in antagonist-based assisted reproductive technology cycles. Fertil Steril 2017;107(1):130-135.e1. PMID: 28228316.

Barcena P, Rodriguez M, Obradors A, et al. Should we worry about the clock? Relationship between time to ICSI and reproductive outcomes in cycles with fresh and vitrified oocytes. Hum Reprod 2016;31(6):1182-91. PMID: 27076502. 
Basatemur E and Sutcliffe A. Follow-up of children born after ART. Placenta 2008;29(Suppl B):135-40. PMID: 18790325.

Bedaiwy MA, Ryan E, Shaaban O, et al. Pregnancy outcome after metformin co-treatment in ovulation induction. Middle East Fertility Society Journal 2009;14(4):270-274.

Behbehani S, Hasson J, Polesello S, et al. Do trained reproductive endocrinologists perform better than their trainees? Comparing clinical pregnancy rates and live birth rates after transfer of single fresh blastocysts. J Assist Reprod Genet 2018. PMID: 29423789.

Benaglia L, Somigliana E, Iemmello R, et al. Endometrioma and oocyte retrieval-induced pelvic abscess: a clinical concern or an exceptional complication? Fertil Steril 2008;89(5):1263-6. PMID: 18339383.

Bendifallah S, Roman H, Mathieu d'Argent E, et al. Colorectal endometriosis-associated infertility: should surgery precede ART?. Fertil Steril 2017;108(3):525-531.e4. PMID: 28807397.

Bishop LA, Richter KS, Patounakis G, et al. Diminished ovarian reserve as measured by means of baseline follicle-stimulating hormone and antral follicle count is not associated with pregnancy loss in younger in vitro fertilization patients. Fertil Steril 2017;108(6):980-987. PMID: 29202975.

Cabry-Goubet R, Scheffler F, Belhadri-Mansouri N, et al. Effect of Gonadotropin Types and Indications on Homologous Intrauterine Insemination Success: A Study from 1251 Cycles and a Review of the Literature. Biomed Res Int 2017;2017:3512784. PMID: 29387719.

Cardellicchio L, Reschini M, Paffoni A, et al. Frozen-thawed blastocyst transfer in natural cycle: feasibility in everyday clinical practice. Archives of Gynecology and Obstetrics 2017;295(6):1509-1514.

Casamonti E, Vinci S, Serra E, et al. Short-term FSH treatment and sperm maturation: a prospective study in idiopathic infertile men. Andrology 2017;5(3):414-422. PMID: 28296254.

Ceelen M, van Weissenbruch MM, Vermeiden JP, et al. Growth and development of children born after in vitro fertilization. Fertil Steril 2008;90(5):1662-73. PMID: 18163998.

Çelik O, Acet M, Imren A, et al. DHEA supplementation improves endometrial HOXA-10 mRNA expression in poor responders. Journal of the Turkish-German Gynecological Association 2017;18(4):160-166.

Chambers GM, Paul RC, Harris K, et al. Assisted reproductive technology in Australia and New Zealand: cumulative live birth rates as measures of success. Med J Aust 2017;207(3):114-118. PMID: 28764619.

Chapuis A, Gala A, Ferrieres-Hoa A, et al. Sperm quality and paternal age: effect on blastocyst formation and pregnancy rates. Basic Clin Androl 2017;27:2. PMID: 28127436. 
Checa MA, Teixeira DM, González-Comadran M, et al. Luteal phase support for women trying to conceive by intrauterine insemination or sexual intercourse. Cochrane Database of Systematic Reviews 2016;2016(11).

Connell MT, Patounakis G, Healy MW, et al. Is the effect of premature elevated progesterone augmented by human chorionic gonadotropin versus gonadotropin-releasing hormone agonist trigger?. Fertil Steril 2016;106(3):584-589.e1. PMID: 27178228.

Craciunas L, Kollmann M, Tsampras N, et al. Oxytocin antagonists for assisted reproduction. Cochrane Database of Systematic Reviews 2016;2016(10).

Danhof NA, van Wely M, Koks CAM, et al. The SUPER study: protocol for a randomised controlled trial comparing follicle-stimulating hormone and clomiphene citrate for ovarian stimulation in intrauterine insemination. BMJ Open 2017;7(5):e015680. PMID: 28550023.

Davies MJ, Rumbold AR and Moore VM. Assisted reproductive technologies: a hierarchy of risks for conception, pregnancy outcomes and treatment decisions. J Dev Orig Health Dis 2017;8(4):443-447. PMID: 28721836.

Diergaarde B and Kurta ML. Use of fertility drugs and risk of ovarian cancer. Curr Opin Obstet Gynecol 2014;26(3):125-9. PMID: 24752005.

Eftekhar M, Deghani Firoozabadi R, Khani P, et al. Effect of Laparoscopic Ovarian Drilling on Outcomes of In Vitro Fertilization in Clomiphene-Resistant Women with Polycystic Ovary Syndrome. Int J Fertil Steril 2016;10(1):42-7. PMID: 27123199.

El Hachem H, Antaki R, Sylvestre C, et al. Clomiphene Citrate versus Letrozole for Ovarian Stimulation in Therapeutic Donor Sperm Insemination. Gynecol Obstet Invest 2016. PMID: 27852078.

Elizur SE and Tulandi T. Drugs in infertility and fetal safety. Fertil Steril 2008;89(6):1595-602. PMID: 18519067.

Elkhateeb RR, Mahran AE and Kamel HH. Long-term use of clomiphene citrate in induction of ovulation in PCO patients with clomiphene citrate resistance. Journal of Gynecology Obstetrics and Human Reproduction 2017;46(7):575-577.

Farhi J and Fisch B. Risk of major congenital malformations associated with infertility and its treatment by extent of iatrogenic intervention. Pediatr Endocrinol Rev 2007;4(4):352-7. PMID: 17643083.

Farquhar C, Rishworth JR, Brown J, et al. Assisted reproductive technology: an overview of Cochrane Reviews. Cochrane Database Syst Rev 2013;8:Cd010537. PMID: 23970457.

Fauque P. Ovulation induction and epigenetic anomalies. Fertil Steril 2013;99(3):616-23. PMID: 23714436. 
G.A R, Cheemakurthi R, Prathigudupu K, et al. Role of Lh polymorphisms and r-hLh supplementation in GnRh agonist treated ART cycles: A cross sectional study. European Journal of Obstetrics Gynecology and Reproductive Biology 2018;222:119-125.

Gizzo S, Garcia-Velasco JA, Heiman F, et al. A cost-effectiveness evaluation comparing originator follitropin alfa to the biosimilar for the treatment of infertility. International Journal of Women's Health 2016;8:683-689.

Gonzalez-Comadran M, Schwarze JE, Zegers-Hochschild F, et al. The impact of endometriosis on the outcome of Assisted Reproductive Technology. Reprod Biol Endocrinol 2017;15(1):8. PMID: 28118836.

Groen H, Tonch N, Simons AH, et al. Modified natural cycle versus controlled ovarian hyperstimulation IVF: a cost-effectiveness evaluation of three simulated treatment scenarios. Hum Reprod 2013;28(12):3236-46. PMID: 24166594.

Guo H, Wang Y, Chen Q, et al. Effect of Natural Cycle Endometrial Preparation for FrozenThawed Embryo Transfer in Patients with Advanced Endometriosis. Med Sci Monit 2016;22:4596-4603. PMID: 27889789.

Holschbach V, Weigert J, Dietrich JE, et al. Pregnancy rates of day 4 and day 5 embryos after culture in an integrated time-lapse incubator. Reprod Biol Endocrinol 2017;15(1):37. PMID: 28482910.

Humaidan P, Nelson SM, Devroey P, et al. Ovarian hyperstimulation syndrome: review and new classification criteria for reporting in clinical trials. Hum Reprod 2016;31(9):1997-2004. PMID: 27343272.

Jaganmohan C, Vannan M, Ali A, et al. Evaluation of clinical efficacy of metformin therapy in Polycystic Ovary Syndrome. Journal of Young Pharmacists 2017;9(2):277-279.

Jansen C, Elisen M, Leenstra CW, et al. Longer time interval between semen processing and intrauterine insemination does not affect pregnancy outcome. Fertil Steril 2017;108(5):764-769. PMID: 28923283.

Jiang S and Kuang Y. Clomiphene citrate is associated with favorable cycle characteristics but impaired outcomes of obese women with polycystic ovarian syndrome undergoing ovarian stimulation for in vitro fertilization. Medicine (Baltimore) 2017;96(32):e7540. PMID: 28796038.

Jiang S and Kuang Y. The effects of low-dose human chorionic gonadotropin combined with human menopausal gonadotropin protocol on women with hypogonadotropic hypogonadism undergoing ovarian stimulation for in vitro fertilization. Clin Endocrinol (Oxf) 2018;88(1):77-87. PMID: 28960429.

Jones T, Ho JR, Gualtieri M, et al. Clomiphene Stair-Step Protocol for Women With Polycystic Ovary Syndrome. Obstet Gynecol 2018;131(1):91-95. PMID: 29215516. 
Jungheim ES and Odibo AO. Fertility treatment in women with polycystic ovary syndrome: a decision analysis of different oral ovulation induction agents. Fertil Steril 2010;94(7):2659-64. PMID: 20451181.

Kang HJ, Melnick AP, Stewart JD, et al. Preimplantation genetic screening: who benefits?. Fertil Steril 2016;106(3):597-602. PMID: 27143516.

Karayiannis D, Kontogianni MD, Mendorou C, et al. Adherence to the Mediterranean diet and IVF success rate among non-obese women attempting fertility. Hum Reprod 2018;33(3):494502. PMID: 29390148.

Keane K, Cruzat VF, Wagle S, et al. Specific ranges of anti-Mullerian hormone and antral follicle count correlate to provide a prognostic indicator for IVF outcome. Reprod Biol 2017;17(1):51-59. PMID: 28132758.

Kuang H, Jin S, Hansen KR, et al. Identification and replication of prediction models for ovulation, pregnancy and live birth in infertile women with polycystic ovary syndrome. Hum Reprod 2015;30(9):2222-33. PMID: 26202922.

La Bastide-Van Gemert S, Seggers J, Haadsma ML, et al. Is ovarian hyperstimulation associated with higher blood pressure in 4-year-old IVF offspring? Part II: an explorative causal inference approach. Hum Reprod 2014;29(3):510-7. PMID: 24365798.

Leijdekkers JA, Eijkemans MJC, van Tilborg TC, et al. Predicting the cumulative chance of live birth over multiple complete cycles of in vitro fertilization: an external validation study. Hum Reprod 2018;33(9):1684-1695. PMID: 30085143.

Li H, Li L, Lu X, et al. Comparison of the effect of immediate versus delayed transfer following a stimulated IVF cycle on the ongoing pregnancy rate of frozen-thawed embryo transfer cycles: a study protocol for a randomised controlled trial. BMJ Open 2018;8(5):e020507. PMID: 29773699.

Li Y, Kuang H, Shen W, et al. Letrozole, berberine, or their combination for anovulatory infertility in women with polycystic ovary syndrome: study design of a double-blind randomised controlled trial. BMJ Open 2013;3(11):e003934. PMID: 24282248.

Li Z, Wang AY, Bowman M, et al. ICSI does not increase the cumulative live birth rate in nonmale factor infertility. Hum Reprod 2018;33(7):1322-1330. PMID: 29897449.

Loutradis D, Drakakis P, Vomvolaki E, et al. Different ovarian stimulation protocols for women with diminished ovarian reserve. J Assist Reprod Genet 2007;24(12):597-611. PMID: 18034299.

Maalouf W, Maalouf W, Campbell B, et al. Effect of ethnicity on live birth rates after in vitro fertilisation/intracytoplasmic sperm injection treatment: analysis of UK national database. Bjog 2016. PMID: 27539893. 
McLernon DJ, Steyerberg EW, Te Velde ER, et al. Predicting the chances of a live birth after one or more complete cycles of in vitro fertilisation: population based study of linked cycle data from 113873 women. Bmj 2016;355:i5735. PMID: 27852632.

Meijerink AM, Cissen M, Mochtar MH, et al. Prediction model for live birth in ICSI using testicular extracted sperm. Hum Reprod 2016;31(9):1942-51. PMID: 27406949.

Min JK, Hughes E, Young D, et al. Elective single embryo transfer following in vitro fertilization. J Obstet Gynaecol Can 2010;32(4):363-77. PMID: 20500945.

Moolenaar LM, Nahuis MJ, Hompes PG, et al. Cost-effectiveness of treatment strategies in women with PCOS who do not conceive after six cycles of clomiphene citrate. Reprod Biomed Online 2014;28(5):606-13. PMID: 24656560.

Mutsaerts MA, Groen H, ter Bogt NC, et al. The LIFESTYLE study: costs and effects of a structured lifestyle program in overweight and obese subfertile women to reduce the need for fertility treatment and improve reproductive outcome. A randomised controlled trial. BMC Womens Health 2010;10:22. PMID: 20579357.

Muzii L, Di Tucci C, Di Feliciantonio M, et al. Surgery versus expectant management in patients with endometrioma who seek pregnancy. Journal of Endometriosis and Pelvic Pain Disorders 2017;9(3):135-138.

Niederberger C. Re: Comparison of Microdissection Testicular Sperm Extraction, Conventional Testicular Sperm Extraction, and Testicular Sperm Aspiration for Nonobstructive Azoospermia: A Systematic Review and Meta-Analysis. J Urol 2016;195(5):1564-6. PMID: 27186769.

Niederberger C. Re: High-Intensity Exercise Training for Improving Reproductive Function in Infertile Patients: A Randomized Controlled Trial. J Urol 2018;199(2):331. PMID: 29357545.

Niederberger C. Re: Moderate Aerobic Exercise Training for Improving Reproductive Function in Infertile Patients: A Randomized Controlled Trial. J Urol 2017;198(2):241-242. PMID: 29370624.

Okuyama N, Obata R, Oka N, et al. Long-term clinical outcomes of testicular sperm extraction and intracytoplasmic sperm injection for infertile men. Reproductive Medicine and Biology 2018;17(1):82-88.

Omokanye LO, Olatinwo AO, Panti A, et al. Clomiphene resistant polycystic ovarian syndrome: Analysis of outcomes following laparoscopic ovarian drilling in infertile women in Ilorin, Northcentral, Nigeria. Annals of Tropical Medicine and Public Health 2017;10(5):1292-1298.

Ozer C, Caglar Aytac P, Goren MR, et al. Sperm retrieval by microdissection testicular sperm extraction and intracytoplasmic sperm injection outcomes in nonobstructive azoospermic patients with Klinefelter syndrome. Andrologia 2018.

Porreco RP and Heyborne KD. Immunogenesis of preeclampsia: lessons from donor gametes. Journal of Maternal-Fetal and Neonatal Medicine 2018;31(9):1220-1226. 
Qin N, Chen Q, Hong Q, et al. Flexibility in starting ovarian stimulation at different phases of the menstrual cycle for treatment of infertile women with the use of in vitro fertilization or intracytoplasmic sperm injection. Fertil Steril 2016. PMID: 27114329.

Roesner S, Von Wolff M, Eberhardt I, et al. In vitro maturation: a five-year experience. Acta Obstet Gynecol Scand 2012;91(1):22-7. PMID: 21995801.

Schieve LA, Devine O, Boyle CA, et al. Estimation of the contribution of non-assisted reproductive technology ovulation stimulation fertility treatments to US singleton and multiple births. Am J Epidemiol 2009;170(11):1396-407. PMID: 19854803.

Schneider J, Lahl J and Kramer W. Long-term breast cancer risk following ovarian stimulation in young egg donors: a call for follow-up, research and informed consent. Reprod Biomed Online 2017;34(5):480-485. PMID: 28473127.

Sedaghatpour D and Berookhim BM. The Role of Varicocele in Male Factor Subfertility. Curr Urol Rep 2017;18(9):73. PMID: 28718159.

Shi Y, Wei D, Liang X, et al. Live birth after fresh embryo transfer vs elective embryo cryopreservation/frozen embryo transfer in women with polycystic ovary syndrome undergoing IVF (FreFro-PCOS): study protocol for a multicenter, prospective, randomized controlled clinical trial. Trials 2014;15:154. PMID: 24885793.

Showell MG, Mackenzie-Proctor R, Jordan V, et al. Inositol for subfertile women with polycystic ovary syndrome. Cochrane Database of Systematic Reviews 2016;2016(9).

Stener-Victorin E. Acupuncture for Infertility in Women with Polycystic Ovary Syndrome: What Does It Add?. Seminars in Reproductive Medicine 2017;35(4):353-358.

Sterling L, Liu J, Okun N, et al. Pregnancy outcomes in women with polycystic ovary syndrome undergoing in vitro fertilization. Fertil Steril 2016;105(3):791-7.e2. PMID: 26654973.

Stern JE, Gopal D, Liberman RF, et al. Validation of birth outcomes from the Society for Assisted Reproductive Technology Clinic Outcome Reporting System (SART CORS): population-based analysis from the Massachusetts Outcome Study of Assisted Reproductive Technology (MOSART). Fertility and Sterility 2016;106(3):717-722.e2.

Tigges J, Godehardt E, Soepenberg T, et al. Determinants of cumulative ART live-birth rates in a single-center study: age, fertilization modality, and first-cycle outcome. Arch Gynecol Obstet 2016;294(5):1081-1089. PMID: 27488700.

Vause TD, Cheung AP, Sierra S, et al. Ovulation induction in polycystic ovary syndrome. J Obstet Gynaecol Can 2010;32(5):495-502. PMID: 20500959.

Wang A, Santistevan A, Hunter Cohn K, et al. Freeze-only versus fresh embryo transfer in a multicenter matched cohort study: contribution of progesterone and maternal age to success rates. Fertil Steril 2017;108(2):254-261.e4. PMID: 28579411. 
Wang C, Feng G, Zhang B, et al. Effect of different artificial shrinkage methods, when applied before blastocyst vitrification, on perinatal outcomes. Reprod Biol Endocrinol 2017;15(1):32. PMID: 28446183.

Wang ET, Kathiresan ASQ, Bresee C, et al. Abnormal implantation after fresh and frozen in vitro fertilization cycles. Fertil Steril 2017;107(5):1153-1158. PMID: 28433367.

Wintner EM, Hershko-Klement A, Tzadikevitch K, et al. Does the transfer of a poor quality embryo together with a good quality embryo affect the In Vitro Fertilization (IVF) outcome?. J Ovarian Res 2017;10(1):2. PMID: 28086935.

Wordsworth S, Buchanan J, Mollison J, et al. Clomifene citrate and intrauterine insemination as first-line treatments for unexplained infertility: are they cost-effective? Hum Reprod 2011;26(2):369-75. PMID: 21127355.

Xue LT, Wang RX, He B, et al. Effect of sperm DNA fragmentation on clinical outcomes for Chinese couples undergoing in vitro fertilization or intracytoplasmic sperm injection. J Int Med Res 2016;44(6):1283-1291. PMID: 28322098.

Zore T and Wang ET. Perinatal and Childhood Outcomes Associated with Infertility. Seminars in Reproductive Medicine 2017;35(3):304-310.

\section{Observational study sample size less than 100 subjects:}

Alhathal N, San Gabriel M and Zini A. Beneficial effects of microsurgical varicocoelectomy on sperm maturation, DNA fragmentation, and nuclear sulfhydryl groups: a prospective trial. Andrology 2016;4(6):1204-1208. PMID: 27565125.

Baker VL, Brown MB, Luke B, et al. Gonadotropin dose is negatively correlated with live birth rate: Analysis of more than 650,000 assisted reproductive technology cycles. Fertility and Sterility 2015.

Bo S, Hongmei S, Zhenhui H, et al. Effectiveness of two kind of medicine on non-obstructive azoospermia. Australasian Medical Journal 2017;10(9):785-788.

Bushaqer NJ, Dayoub NM, Alhattali KK, et al. Follicular aspiration vs. coasting for OHSS prevention. Saudi Medical Journal 2018;39(3):290-296.

Cao S, Zhao C, Zhang J, et al. Retrospective clinical analysis of two artificial shrinkage methods applied prior to blastocyst vitrification on the outcome of frozen embryo transfer. Journal of Assisted Reproduction and Genetics 2014;31(5):577-581.

Check JH, Pinto J, Liss JR, et al. Improved pregnancy outcome for women with decreased ovarian oocyte reserve and advanced reproductive age by performing in vitro fertilizationembryo transfer. Clin Exp Obstet Gynecol 2008;35(3):167-9. PMID: 18754283.

Colpin H and Bossaert G. Adolescents conceived by IVF: parenting and psychosocial adjustment. Hum Reprod 2008;23(12):2724-30. PMID: 18753143. 
Dharia Patel SP, Steinkampf MP, Whitten SJ, et al. Robotic tubal anastomosis: surgical technique and cost effectiveness. Fertil Steril 2008;90(4):1175-9. PMID: 18054354.

Feng C, Wang LQ, Dong MY, et al. Assisted reproductive technology may increase clinical mutation detection in male offspring. Fertil Steril 2008;90(1):92-6. PMID: 18258231.

Foix-L'Helias L, Grynberg M, Ducot B, et al. Growth development of French children born after in vitro maturation. PLoS One 2014;9(2):e89713. PMID: 24586979.

Genovese F, Teodoro MC, Rubbino G, et al. Laparoscopic ablation of endometriosis in infertile patients: A retrospective study. Journal of Endometriosis 2011;3(1):53-57.

Gilman AR, Younes G, Tannus S, et al. Does using testicular sperm retrieval rather than ejaculated spermatozoa improve reproductive outcomes in couples with previous ART failure and poor ovarian response? A case-controlled study. Andrology 2018;6(1):142-145. PMID: 29195015.

Grow D, Kawwass JF, Kulkarni AD, et al. GnRH agonist and GnRH antagonist protocols: Comparison of outcomes among goodprognosis patients using national surveillance data. Reproductive BioMedicine Online 2014;29(3):299-304.

Gunby J, Bissonnette F, Librach C, et al. Assisted reproductive technologies in Canada: 2005 results from the Canadian Assisted Reproductive Technologies Register. Fertil Steril 2009;91(5):1721-30. PMID: 18423460.

Haydardedeoglu B, Turunc T, Kilicdag EB, et al. The effect of prior varicocelectomy in patients with nonobstructive azoospermia on intracytoplasmic sperm injection outcomes: a retrospective pilot study. Urology 2010;75(1):83-6. PMID: 19913887.

Jeje EA, Alabi TO, Ojewola RW, et al. Male infertility: An audit of 70 cases in a single centre. African Journal of Urology 2016;22(3):223-226.

Kaarouch I, Bouamoud N, Madkour A, et al. Paternal age: Negative impact on sperm genome decays and IVF outcomes after 40 years. Molecular Reproduction and Development 2018;85(3):271-280.

Kahyaoglu S, Yumuşak OH, Ozyer S, et al. Clomiphene citrate treatment cycle outcomes of polycystic ovary syndrome patients based on basal high sensitive C-Reactive protein levels: A cross-sectional study. International Journal of Fertility and Sterility 2017;10(4):320-326.

Keltz MD, Rovner EM, Gonzalez E, et al. Fresh MESA improved embryo fertilization, cleavage, blastula formation and implantation rates after failed TESA in couples with obstructive azoospermia. J Assist Reprod Genet 2011;28(4):375-7. PMID: 21213037.

Kunicki M, Lukaszuk K, Liss J, et al. Granulocyte colony stimulating factor treatment of resistant thin endometrium in women with frozen-thawed blastocyst transfer. Syst Biol Reprod Med 2017;63(1):49-57. PMID: 27874292. 
Martin JR, Bromer JG, Sakkas D, et al. Live babies born per oocyte retrieved in a subpopulation of oocyte donors with repetitive reproductive success. Fertil Steril 2010;94(6):2064-8. PMID: 20303483.

Matalliotakis IM, Cakmak H, Mahutte N, et al. Women with advanced-stage endometriosis and previous surgery respond less well to gonadotropin stimulation, but have similar IVF implantation and delivery rates compared with women with tubal factor infertility. Fertil Steril 2007;88(6):1568-72. PMID: 17349642.

Maxwell SM, Colls P, Hodes-Wertz B, et al. Why do euploid embryos miscarry? A case-control study comparing the rate of aneuploidy within presumed euploid embryos that resulted in miscarriage or live birth using next-generation sequencing. Fertil Steril 2016;106(6):14141419.e5. PMID: 27692437.

Milone M, Sosa Fernandez LM, Sosa Fernandez LV, et al. Does Bariatric Surgery Improve Assisted Reproductive Technology Outcomes in Obese Infertile Women?. Obes Surg 2017;27(8):2106-2112. PMID: 28233264.

Munch EM, Sparks AE, Duran HE, et al. Lack of carbon air filtration impacts early embryo development. Journal of Assisted Reproduction and Genetics 2015;32(7):1009-1017.

Nasr-Esfahani MH, Razavi S, Javdan Z, et al. Artificial oocyte activation in severe teratozoospermia undergoing intracytoplasmic sperm injection. Fertil Steril 2008;90(6):2231-7. PMID: 18206146.

Noorashikin M, Ong FB, Omar MH, et al. Affordable ART for developing countries: a cost benefit comparison of low dose stimulation versus high dose GnRH antagonist protocol. J Assist Reprod Genet 2008;25(7):297-303. PMID: 18654847.

Oktem M, Guler I, Erdem M, et al. Comparison of The Effectiveness of Clomiphene Citrate versus Letrozole in Mild IVF in Poor Prognosis Subfertile Women with Failed IVF Cycles. Int J Fertil Steril 2015;9(3):285-91. PMID: 26644850.

Ou YC, Lan KC, Huang FJ, et al. Comparison of in vitro fertilization versus intracytoplasmic sperm injection in extremely low oocyte retrieval cycles. Fertil Steril 2010;93(1):96-100. PMID: 19108823.

Pabuccu E, Pabuccu R, Caglar G, et al. Different gonadotropin releasing hormone agonist doses for the final oocyte maturation in high-responder patients undergoing in vitro fertilization/intracytoplasmic sperm injection. Journal of Human Reproductive Sciences 2015;8(1):25-29.

Petrucco OM, Silber SJ, Chamberlain SL, et al. Live birth following day surgery reversal of female sterilisation in women older than 40 years: a realistic option in Australia? Med J Aust 2007;187(5):271-3. PMID: 17767430.

Reignier A, Lammers J, Splingart C, et al. Sperm cryopreservation and assisted reproductive technology outcome in patients with spinal cord injury. Andrologia 2018;50(1). 
Samavat J, Cantini G, Lotti F, et al. Massive Weight Loss Obtained by Bariatric Surgery Affects Semen Quality in Morbid Male Obesity: a Preliminary Prospective Double-Armed Study. Obes Surg 2018;28(1):69-76. PMID: 28702741.

Schwarze JE, Crosby JA and Zegers-Hochschild F. Effect of embryo freezing on perinatal outcome after assisted reproduction techniques: lessons from the Latin American Registry of Assisted Reproduction. Reproductive BioMedicine Online 2015;31(1):39-43.

Seifer DB, Frazier LM and Grainger DA. Disparity in assisted reproductive technologies outcomes in black women compared with white women. Fertil Steril 2008;90(5):1701-10. PMID: 17980873.

Shaban MM. Mini-dose long gonadotropin-releasing hormone (GnRH) agonist versus agonist flare stimulation protocol for in vitro fertilization poor responders. Middle East Fertility Society Journal 2014;19(4):287-292.

Shen C, Shu D, Zhao X, et al. Comparison of clinical outcomes between fresh embryo transfers and frozen-thawed embryo transfers. Iranian Journal of Reproductive Medicine 2014;12(6):409414.

Siristatidis C, Salamalekis G, Dafopoulos K, et al. Mild Versus Conventional Ovarian Stimulation for Poor Responders Undergoing IVF/ICSI. In Vivo 2017;31(2):231-237. PMID: 28358705.

Souza ALM, Sampaio M, Noronha GB, et al. Effect of follicular flushing on reproductive outcomes in patients with poor ovarian response undergoing assisted reproductive technology. Journal of Assisted Reproduction and Genetics 2017;34(10):1353-1357.

Spijkers S, Lens JW, Schats R, et al. Fresh and Frozen-Thawed Embryo Transfer Compared to Natural Conception: Differences in Perinatal Outcome. Gynecologic and Obstetric Investigation 2017;82(6):538-546.

Stoop D, Vercammen L, Polyzos NP, et al. Effect of ovarian stimulation and oocyte retrieval on reproductive outcome in oocyte donors. Fertil Steril 2012;97(6):1328-30. PMID: 22464081.

Tiegs AW, Sachdev NM, Grifo JA, et al. Paternal Age Is Not Associated with Pregnancy Outcomes after Single Thawed Euploid Blastocyst Transfer. Reproductive Sciences 2017;24(9):1319-1324.

Vartanyan EV, Tsaturova KA, Devyatova EA, et al. Improvement in quality of oocytes in polycystic ovarian syndrome in programs of in vitro fertilization. Gynecological Endocrinology 2017;33:8-11.

Vembu R and Reddy NS. Serum AMH level to predict the hyper response in women with PCOS and non-PCOS undergoing controlled ovarian stimulation in art. Journal of Human Reproductive Sciences 2017;10(2):91-94. 
Wu YG, Barad DH, Kushnir VA, et al. With low ovarian reserve, Highly Individualized Egg Retrieval (HIER) improves IVF results by avoiding premature luteinization. Journal of Ovarian Research 2018;11(1).

Yu X, Cai H, Zheng X, et al. Tubal restorative surgery for hydrosalpinges in women due to in vitro fertilization. Archives of Gynecology and Obstetrics 2018:1-5.

Zheng Y, Li Z, Xiong M, et al. Hormonal replacement treatment improves clinical pregnancy in frozen-thawed embryos transfer cycles: A retrospective cohort study. American Journal of Translational Research 2014;6(1):85-90.

\section{Not a study population of interest:}

Aboulghar MA, Amin YM, Al-Inany HG, et al. Prospective randomized study comparing luteal phase support for ICSI patients up to the first ultrasound compared with an additional three weeks. Hum Reprod 2008;23(4):857-62. PMID: 18270182.

Adams D, Fernandez R, Moore V, et al. Sperm donation perinatal outcomes in an Australian population cohort. Journal of Obstetrics and Gynaecology Research 2017;43(12):1830-1839.

Adams DH, Clark RA, Davies MJ, et al. A meta-analysis of neonatal health outcomes from oocyte donation. J Dev Orig Health Dis 2015:1-16. PMID: 26611736.

Al-Anbari LA. Thyroxine supplementation improve intrauterine insemination outcome in patients with subclinical hypothyroidism. Journal of Pharmaceutical Sciences and Research 2017;9(10):1768-1772.

Amer MI, Abd-El-Maeboud KH, Abdelfatah I, et al. Human amnion as a temporary biologic barrier after hysteroscopic lysis of severe intrauterine adhesions: pilot study. J Minim Invasive Gynecol 2010;17(5):605-11. PMID: 20576472.

Azargoon A, Ghorbani R and Aslebahar F. Vaginal progesterone on the prevention of preterm birth and neonatal complications in high risk women: A randomized placebo-controlled doubleblind study. International Journal of Reproductive BioMedicine 2016;14(5):309-316.

Balen A, Platteau P, Andersen AN, et al. Highly purified FSH is as efficacious as recombinant FSH for ovulation induction in women with WHO Group II anovulatory infertility: a randomized controlled non-inferiority trial. Hum Reprod 2007;22(7):1816-23. PMID: 17449511.

Bosteels J, Kasius J, Weyers S, et al. Hysteroscopy for treating subfertility associated with suspected major uterine cavity abnormalities. Cochrane Database Syst Rev 2015;2:Cd009461. PMID: 25701429.

Bosteels J, Weyers S, Mol BW, et al. Anti-adhesion barrier gels following operative hysteroscopy for treating female infertility: a systematic review and meta-analysis. Gynecol Surg 2014;11:113-127. PMID: 24795547. 
Du T, Chen H, Fu R, et al. Comparison of ectopic pregnancy risk among transfers of embryos vitrified on day 3, day 5, and day 6. Fertil Steril 2017;108(1):108-116.e1. PMID: 28602476.

Elenis E, Svanberg AS, Lampic C, et al. Adverse obstetric outcomes in pregnancies resulting from oocyte donation: a retrospective cohort case study in Sweden. BMC Pregnancy Childbirth 2015;15:247. PMID: 26450684.

Farrag A, Sagnella F, Pappalardo S, et al. The use of r-hFSH in treatment of idiopathic male factor infertility before ICSI. Eur Rev Med Pharmacol Sci 2015;19(12):2162-7. PMID: 26166637.

Gambadauro P, Iliadis S, Brann E, et al. Conception by means of in vitro fertilization is not associated with maternal depressive symptoms during pregnancy or postpartum. Fertil Steril 2017;108(2):325-332. PMID: 28651958.

Gerkowicz SA, Crawford SB, Hipp HS, et al. Assisted reproductive technology with donor sperm: national trends and perinatal outcomes. American Journal of Obstetrics and Gynecology 2018;218(4):421.e1-421.e10.

Goldberg AS, Sujana Kumar S, Greenblatt E, et al. Delaying Thyroxine Until Positive BetaHuman Chorionic Gonadotropin is Safe for Patients Receiving Fertility Therapy: Applying New ATA Guidelines to Subclinical Hypothyroidism. Journal of Obstetrics and Gynaecology Canada 2018;40(3):299-303.

Hamta A, Kazemnejad A, Fesharaki MG, et al. Effectiveness of cervical cerclage on preterm delivery and neonatal outcome in twin pregnancies: Propensity score matching analysis. Iranian Red Crescent Medical Journal 2017;19(5).

Herndon CN, Anaya Y, Noel M, et al. Outcomes from a university-based low-cost in vitro fertilization program providing access to care for a low-resource socioculturally diverse urban community. Fertil Steril 2017;108(4):642-649.e4. PMID: 28874259.

Ibanez L, Del Rio L, Diaz M, et al. Normalizing Ovulation Rate by Preferential Reduction of Hepato-Visceral Fat in Adolescent Girls With Polycystic Ovary Syndrome. J Adolesc Health 2017;61(4):446-453. PMID: 28712591.

Jayaprakasan K, Polanski L, Sahu B, et al. Surgical intervention versus expectant management for endometrial polyps in subfertile women. Cochrane Database Syst Rev 2014;8:Cd009592. PMID: 25172985.

Kaser DJ, Melamed A, Bormann CL, et al. Cryopreserved embryo transfer is an independent risk factor for placenta accreta. Fertil Steril 2015;103(5):1176-84.e2. PMID: 25747133.

Lee WL, Chang WH, Wang KC, et al. The risk of epithelial ovarian cancer of women with endometriosis may be varied greatly if diagnostic criteria are different: A nationwide populationbased cohort study. Medicine (United States) 2015;94(39):e1633. 
Li W, Xue X, Zhao W, et al. Blastocyst transfer is not associated with increased unfavorable obstetric and perinatal outcomes compared with cleavage-stage embryo transfer. Gynecological Endocrinology 2017;33(11):857-860.

Liffner S, Hammar M, Bladh M, et al. Men becoming fathers by intracytoplasmic sperm injection were more often born small for gestational age. Asian J Androl 2017;19(1):103-106. PMID: 27184547.

Magnusson A, Kallen K, Thurin-Kjellberg A, et al. The number of oocytes retrieved during IVF: a balance between efficacy and safety. Hum Reprod 2018;33(1):58-64. PMID: 29136154.

Marqueta B, Barri PN, Coroleu B, et al. Effect of non-cavity distorting intramural fibroids on assisted reproduction outcomes: A cohort study. Journal of Endometriosis 2016;8(3):111-115.

McDonnell R, Marjoribanks J and Hart RJ. Ovarian cyst aspiration prior to in vitro fertilization treatment for subfertility. Cochrane Database Syst Rev 2014;12:Cd005999. PMID: 25502626.

Melli MS, Tagavi S, Alizadeh M, et al. Comparison the effect of oxytocin and human chorionic gonadotropin on ovulation. Journal of Medical Sciences 2007;7(7):1126-1134.

Murugappan G, Farland LV, Missmer SA, et al. Gestational carrier in assisted reproductive technology. Fertil Steril 2018;109(3):420-428. PMID: 29428314.

Murugappan G, Shahine LK, Perfetto CO, et al. Intent to treat analysis of in vitro fertilization and preimplantation genetic screening versus expectant management in patients with recurrent pregnancy loss. Human Reproduction 2016;31(8):1668-1674.

Rezai M, Jamshidi M, Mohammadbeigi R, et al. Comparing the Effect of Metformin and Acarbose Accompanying Clomiphene on the Successful Ovulation Induction in Infertile Women with Polycystic Ovary Syndrome. Glob J Health Sci 2016;8(9):54516. PMID: 27157179.

Roy KK, Lingampally A, Kansal Y, et al. A Pilot Study Comparing Hysteroscopic Adhesiolysis by Conventional Resectoscope Versus Mini-resectoscope. Oman Med J 2017;32(6):492-498. PMID: 29218126.

Saad AS and Mohamed KAA. Diosmin versus cabergoline for prevention of ovarian hyperstimulation syndrome. Middle East Fertility Society Journal 2017;22(3):206-210.

Seyedoshohadaei F, Zandvakily F and Shahgeibi S. Comparison of the effectiveness of clomiphene citrate, tamoxifen and letrozole in ovulation induction in infertility due to isolated unovulation. Iran J Reprod Med 2012;10(6):531-6. PMID: 25246922.

Shechter-Maor G, Czuzoj-Shulman N, Spence AR, et al. The effect of assisted reproductive technology on the incidence of birth defects among livebirths. Archives of Gynecology and Obstetrics 2018:1-7. 
Styer AK, Jin S, Liu D, et al. Association of uterine fibroids and pregnancy outcomes after ovarian stimulation-intrauterine insemination for unexplained infertility. Fertil Steril 2017;107(3):756-762.e3. PMID: 28089575.

Supramaniam PR, Mittal M, McVeigh E, et al. The correlation between raised body mass index and assisted reproductive treatment outcomes: A systematic review and meta-analysis of the evidence. Reproductive Health 2018;15(1).

Swanton A, Lighten A, Granne I, et al. Do women with ovaries of polycystic morphology without any other features of PCOS benefit from short-term metformin co-treatment during IVF? A double-blind, placebo-controlled, randomized trial. Hum Reprod 2011;26(8):2178-84. PMID: 21593045.

Taerk E, Hughes E, Greenberg C, et al. Controlled ovarian hyperstimulation with intrauterine insemination is more successful after r-hCG administration than spontaneous LH Surge. Journal of Reproduction and Infertility 2017;18(3):316-322.

Taheripanah R, Vasef M, Zamaniyan M, et al. Comparison of cabergoline and quinagolide in prevention of severe ovarian hyperstimulation syndrome among patients undergoing intracytoplasmic sperm injection. International Journal of Fertility and Sterility 2018;12(1):1-5.

Takeda E, Suzumori N, Ebara T, et al. Psychological distress in post-partum women after noninvasive prenatal testing (NIPT) in Japan. Journal of Obstetrics and Gynaecology Research 2018;44(1):35-42.

Tarlatzi TB, Imbert R, Alvaro Mercadal B, et al. Does oocyte donation compared with autologous oocyte IVF pregnancies have a higher risk of preeclampsia?. Reprod Biomed Online 2017;34(1):11-18. PMID: 27793549.

Taylor F and Levine L. Clomiphene citrate and testosterone gel replacement therapy for male hypogonadism: efficacy and treatment cost. J Sex Med 2010;7(1 Pt 1):269-76. PMID: 19694928.

Tse WT, Law LW, Sahota DS, et al. Triplet pregnancy with fetal reduction: Experience in Hong Kong. Hong Kong Medical Journal 2017;23(4):326-332.

Van Oers AM, Mutsaerts MAQ, Burggraaff JM, et al. Cost-effectiveness analysis of lifestyle intervention in obese infertile women. Human Reproduction 2017;32(7):1418-1426.

Xu B, He YQ, Wang Y, et al. Frozen embryo transfer or fresh embryo transfer: Clinical outcomes depend on the number of oocytes retrieved. European Journal of Obstetrics Gynecology and Reproductive Biology 2017;215:50-54.

Xu W, Zhang Y, Yang Y, et al. Effect of early second-look hysteroscopy on reproductive outcomes after hysteroscopic adhesiolysis in patients with intrauterine adhesion, a retrospective study in China. Int J Surg 2018;50:49-54. PMID: 29203342. 
Yamatoya K, Saito K, Saito T, et al. Birthweights and Down syndrome in neonates that were delivered after frozen-thawed embryo transfer: The 2007-2012 Japan Society of Obstetrics and Gynecology National Registry data in Japan. 2017;16(2):228-234.

Yan L, Yu Q, Zhang YN, et al. Effect of type 3 intramural fibroids on in vitro fertilizationintracytoplasmic sperm injection outcomes: a retrospective cohort study. Fertil Steril 2018. PMID: 29605409.

Yin B, Zhu Y, Wu T, et al. Clinical outcomes for couples containing a reciprocal chromosome translocation carrier without preimplantation genetic diagnosis. Int J Gynaecol Obstet 2017;136(3):304-308. PMID: 28099679.

\section{No comparator of interest:}

Abbara A, Jayasena CN, Christopoulos G, et al. Efficacy of Kisspeptin-54 to Trigger Oocyte Maturation in Women at High Risk of Ovarian Hyperstimulation Syndrome (OHSS) During In Vitro Fertilization (IVF) Therapy. J Clin Endocrinol Metab 2015;100(9):3322-31. PMID: 26192876.

Abdullah UH, Lalani S, Syed F, et al. Association of Vitamin D with outcome after intra cytoplasmic sperm injection. Journal of Maternal-Fetal and Neonatal Medicine 2016:1-4.

Adams DH, Clark RA, Davies MJ, et al. A meta-analysis of sperm donation offspring health outcomes. J Dev Orig Health Dis 2016:1-12. PMID: 27573256.

Akamine K, Mekaru K, Gibo K, et al. Comparative study of obstetric and neonatal outcomes of live births between poor- and good-quality embryo transfers. Reproductive Medicine and Biology 2018.

Bak CW, Seok HH, Song SH, et al. Hormonal imbalances and psychological scars left behind in infertile men. J Androl 2012;33(2):181-9. PMID: 21546616.

Baker VL, Jones CE, Cometti B, et al. Factors affecting success rates in two concurrent clinical IVF trials: an examination of potential explanations for the difference in pregnancy rates between the United States and Europe. Fertil Steril 2010;94(4):1287-91. PMID: 19815197.

Daney de Marcillac F, Pinton A, Guillaume A, et al. What are the likely IVF/ICSI outcomes if there is a discrepancy between serum AMH and FSH levels? A multicenter retrospective study. Journal of Gynecology Obstetrics and Human Reproduction 2017;46(8):629-635.

De Brucker M, Camus M, Haentjens P, et al. Cumulative delivery rates after ICSI with donor spermatozoa in different age groups. Reprod Biomed Online 2014;28(5):599-605. PMID: 24631165.

De Pascalis L, Monti F, Agostini F, et al. Psychological vulnerability of singleton children after the 'vanishing' of a co-twin following assisted reproduction. Twin Res Hum Genet 2008;11(1):93-8. PMID: 18251681. 
Devranołlu B, Özdamar Ö, Köle E, et al. Do younger women with elevated basal follicular stimulating hormone levels undergoing gonadotropin-stimulated intrauterine insemination cycles represent compromised reproductive outcomes?. European Journal of Obstetrics Gynecology and Reproductive Biology 2016;199:141-145.

Dickey RP, Pridjian G, Xiong X, et al. Birth Weight by Gestational Age for 76,710 Twins Born in the United States as a Result of In Vitro Fertilization: 2006 to 2010. American Journal of Perinatology 2016.

El-Mazny A, Ramadan W, Kamel A, et al. Effect of hydrosalpinx on uterine and ovarian hemodynamics in women with tubal factor infertility. European Journal of Obstetrics Gynecology and Reproductive Biology 2016;199:55-59.

Fisher J, Wynter K, Hammarberg K, et al. Age, mode of conception, health service use and pregnancy health: a prospective cohort study of Australian women. BMC Pregnancy Childbirth 2013;13:88. PMID: 23565589.

Geisler ME, Ledwidge M, Bermingham M, et al. Intrauterine insemination-No more Mr. N.I.C.E. guy?. Eur J Obstet Gynecol Reprod Biol 2017;210:342-347. PMID: 28122315.

Ghahiri A, Mogharehabed N, Movahedi M, et al. Evaluation of intravenous hydroxylethyl starch, intravenous albumin $20 \%$, and oral cabergoline for prevention of ovarian hyperstimulation syndrome in patients undergoing ovulation induction. J Res Med Sci 2015;20(7):692-6. PMID: 26622260 .

Ghasemian F, Mirroshandel SA and Zahiri Z. Impact of hormonal changes on the semen quality and assisted reproductive outcomes in infertile men. Journal of Applied Biomedicine 2017;15(3):227-232.

Ghuman NK, Mair E, Pearce K, et al. Does age of the sperm donor influence live birth outcome in assisted reproduction?. Human Reproduction 2016;31(3):582-590.

Gianaroli L, Magli MC, Ferraretti AP, et al. Birefringence characteristics in sperm heads allow for the selection of reacted spermatozoa for intracytoplasmic sperm injection. Fertil Steril 2010;93(3):807-13. PMID: 19064263.

Green KA, Franasiak JM, Werner MD, et al. Cumulus cell transcriptome profiling is not predictive of live birth after in vitro fertilization: a paired analysis of euploid sibling blastocysts. Fertility and Sterility 2018;109(3):460-466.e2.

Griesinger G, Verweij PJM, Gates D, et al. Prediction of ovarian hyperstimulation syndrome in patients treated with corifollitropin alfa or $\mathrm{rFSH}$ in a GnRH antagonist protocol. PLoS ONE 2016;11(3).

Haaf T, Tresch A, Lambrecht A, et al. Outcome of intracytoplasmic sperm injection with and without polar body diagnosis of oocytes. Fertil Steril 2010;93(2):405-15. PMID: 19342037. 
Heo S, Kim KI, Lee J, et al. Effects of Korean herbal medicine on pregnancy outcomes of infertile women aged over 35: A retrospective study. European Journal of Integrative Medicine 2016;8(5):670-675.

Hessel M, Robben JC, D'Hauwers KW, et al. The influence of sperm motility and cryopreservation on the treatment outcome after intracytoplasmic sperm injection following testicular sperm extraction. Acta Obstet Gynecol Scand 2015. PMID: 26344577.

Hindryckx A, Peeraer K, Debrock S, et al. Has the prevalence of congenital abnormalities after intracytoplasmic sperm injection increased? The Leuven data 1994-2000 and a review of the literature. Gynecol Obstet Invest 2010;70(1):11-22. PMID: 20110727.

Hobo R, Nakagawa K, Usui C, et al. The Endometriosis Fertility Index Is Useful for Predicting the Ability to Conceive without Assisted Reproductive Technology Treatment after Laparoscopic Surgery, Regardless of Endometriosis. Gynecologic and Obstetric Investigation 2017.

Ishikawa T, Shiotani M, Izumi Y, et al. Fertilization and pregnancy using cryopreserved testicular sperm for intracytoplasmic sperm injection with azoospermia. Fertil Steril 2009;92(1):174-9. PMID: 18684442.

Jefferys AE, Griffith H, Wilson P, et al. Cohort study of perinatal outcomes of children born following surgical sperm recovery. Hum Fertil (Camb) 2016;19(3):207-11. PMID: 27662417.

Kamath MS, Antonisamy B, Mascarenhas M, et al. High-risk of preterm birth and low birth weight after oocyte donation IVF: analysis of 133,785 live births. Reproductive BioMedicine Online 2017;35(3):318-324.

Kanto S, Takahashi K, Maehara I, et al. Incidental testicular cancers that subsequently developed in oligozoospermic and azoospermic patients: report of three cases. Fertil Steril 2007;88(5):1374-6. PMID: 17408623.

Kawwass JF, Kulkarni AD, Hipp HS, et al. Extremities of body mass index and their association with pregnancy outcomes in women undergoing in vitro fertilization in the United States. Fertility and Sterility 2016

Kawwass JF, Monsour M, Crawford S, et al. Trends and outcomes for donor oocyte cycles in the United States, 2000-2010. Jama 2013;310(22):2426-34. PMID: 24135860.

Kedem A, Tsur A, Haas J, et al. Is the modified natural in vitro fertilization cycle justified in patients with "genuine" poor response to controlled ovarian hyperstimulation? Fertil Steril 2014;101(6):1624-8. PMID: 24680364.

Kersten FAM, Nelen W, van den Boogaard NM, et al. Implementing targeted expectant management in fertility care using prognostic modelling: a cluster randomized trial with a multifaceted strategy. Hum Reprod 2017;32(8):1648-1657. PMID: 28591847. 
Keyhan S, Acharya KS, Acharya CR, et al. How compliant are in vitro fertilization member clinics in following embryo transfer guidelines? An analysis of 59,689 fresh first in vitro fertilization autologous cycles from 2011 to 2012. Fertility and Sterility 2016;106(3):645-652.e1.

Kliebisch TK, Bielfeld AP, Krüssel JS, et al. The German Middleway as Precursor for Single Embryo TransferA Retrospective Data-analysis of the Düsseldorf University Hospitals Interdisciplinary Fertility Centre - UniKiD. Geburtshilfe und Frauenheilkunde 2016;76(6):690698.

Kling C, Hedderich J and Kabelitz D. Lymphocyte immunotherapy (LIT) after recurrent IVF implantation failure: Results of a prospective observational trial. Giornale Italiano di Ostetricia e Ginecologia 2014;36(6):513-518.

Kuang H, Jin S, Thomas T, et al. Predictors of participant retention in infertility treatment trials. Fertil Steril 2015;104(5):1236-43.e1-2. PMID: 26354094.

Kuiper DB, Seggers J, Schendelaar P, et al. Asthma and asthma medication use among 4-yearold offspring of subfertile couples - Association with IVF?. Reproductive BioMedicine Online 2015;31(5):711-714.

Kushnir VA, Barad DH, Albertini DF, et al. Effect of embryo banking on U.S. National Assisted Reproductive Technology live birth rates. PLoS ONE 2016;11(5).

La Marca A, Grisendi V, Giulini S, et al. Live birth rates in the different combinations of the Bologna criteria poor ovarian responders: A validation study. Journal of Assisted Reproduction and Genetics 2015;32(6):931-937.

La Sala GB, Nicoli A, Capodanno F, et al. The effect of the 2004 Italian legislation on perinatal outcomes following assisted reproduction technology. J Perinat Med 2009;37(1):43-7. PMID: 18759685.

Lee HL, McCulloh DH, Hodes-Wertz B, et al. In vitro fertilization with preimplantation genetic screening improves implantation and live birth in women age 40 through 43. J Assist Reprod Genet 2015;32(3):435-44. PMID: 25578536.

Leiva P, Schwarze JE, Vasquez P, et al. There is no association between the presence of antithyroid antibodies and increased reproductive loss in pregnant women after ART: A systematic review and meta-analysis. Jornal Brasileiro de Reproducao Assistida 2017;21(4):361-365.

Liu SM, Zhou YZ, Wang HB, et al. Factors Associated with Effectiveness of Treatment and Reproductive Outcomes in Patients with Thin Endometrium Undergoing Estrogen Treatment. Chin Med J (Engl) 2015;128(23):3173-7. PMID: 26612292.

Luke B, Stern JE, Kotelchuck M, et al. Birth outcomes by infertility diagnosis: Analyses of the Massachusetts outcomes study of assisted reproductive technologies (MOSART). Journal of Reproductive Medicine 2015;60(6):480-490. 
Maher MA, Abdelaziz A and Shehata YA. Effect of follicular diameter at the time of ovulation triggering on pregnancy outcomes during intrauterine insemination. Int J Gynaecol Obstet 2017;139(2):174-179. PMID: 28771718.

Mahesan AM, Sadek S, Moussavi V, et al. Clinical outcomes following ICSI cycles using surgically recovered sperm and the impact of maternal age: 2004-2015 SART CORS registry. J Assist Reprod Genet 2018. PMID: 29926376.

Maheux-Lacroix S, Nesbitt-Hawes E, Deans R, et al. Endometriosis fertility index predicts live births following surgical resection of moderate and severe endometriosis. Hum Reprod 2017;32(11):2243-2249. PMID: 29040471.

Maignien C, Santulli P, Gayet V, et al. Prognostic factors for assisted reproductive technology in women with endometriosis-related infertility. Am J Obstet Gynecol 2017;216(3):280.e1-280.e9. PMID: 27899313.

Malak M, Tawfeeq T, Holzer H, et al. Risk factors for ectopic pregnancy after in vitro fertilization treatment. J Obstet Gynaecol Can 2011;33(6):617-9. PMID: 21846451.

Mazzilli R, Cimadomo D, Vaiarelli A, et al. Effect of the male factor on the clinical outcome of intracytoplasmic sperm injection combined with preimplantation aneuploidy testing: observational longitudinal cohort study of 1,219 consecutive cycles. Fertil Steril 2017;108(6):961-972.e3. PMID: 28985908.

McPherson NO, Zander-Fox D, Vincent AD, et al. Combined advanced parental age has an additive negative effect on live birth rates-data from 4057 first IVF/ICSI cycles. J Assist Reprod Genet 2018;35(2):279-287. PMID: 28980182.

Meijerink AM, Ramos L, Janssen AJ, et al. Behavioral, cognitive, and motor performance and physical development of five-year-old children who were born after intracytoplasmic sperm injection with the use of testicular sperm. Fertil Steril 2016;106(7):1673-1682.e5. PMID: 27793367.

Miller N, Biron-Shental T, Pasternak Y, et al. Fertility outcomes after extended searches for ejaculated spermatozoa in men with virtual azoospermia. Fertil Steril 2017;107(6):1305-1311. PMID: 28483501.

Mukhtar HB, Shaman A, Mirghani HO, et al. The Outcome of Assisted Reproductive Techniques among Couples with Male Factors at Prince Khalid Bin Sultan Fertility Centre, Kingdom of Saudi Arabia. Open Access Maced J Med Sci 2017;5(5):603-607. PMID: 28932299.

Munne S, Blazek J, Large M, et al. Detailed investigation into the cytogenetic constitution and pregnancy outcome of replacing mosaic blastocysts detected with the use of high-resolution next-generation sequencing. Fertil Steril 2017;108(1):62-71.e8. PMID: 28579407.

Niinimaki M, Veleva Z and Martikainen H. Embryo quality is the main factor affecting cumulative live birth rate after elective single embryo transfer in fresh stimulation cycles. Eur $\mathrm{J}$ Obstet Gynecol Reprod Biol 2015;194:131-5. PMID: 26366790. 
Panchaud A, Rousson V, Vial T, et al. Pregnancy outcomes in women on metformin for diabetes or other indications among those seeking teratology information services. Br J Clin Pharmacol 2018;84(3):568-578. PMID: 29215149.

Patel A, Sharma PSVN, Narayan P, et al. Prevalence and predictors of infertility-specific stress in women diagnosed with primary infertility: A clinic-based study. Journal of Human Reproductive Sciences 2016;9(1):28-34.

Pinborg A, Hougaard CO, Nyboe Andersen A, et al. Prospective longitudinal cohort study on cumulative 5-year delivery and adoption rates among 1338 couples initiating infertility treatment. Hum Reprod 2009;24(4):991-9. PMID: 19136480.

Provost MP, Acharya KS, Acharya CR, et al. Pregnancy outcomes decline with increasing body mass index: analysis of 239,127 fresh autologous in vitro fertilization cycles from the 2008-2010 Society for Assisted Reproductive Technology registry. Fertil Steril 2016;105(3):663-9. PMID: 26627120.

Purcell K, Schembri M, Frazier LM, et al. Asian ethnicity is associated with reduced pregnancy outcomes after assisted reproductive technology. Fertil Steril 2007;87(2):297-302. PMID: 17081529 .

Rago R, Gallo M, Dal Lago A, et al. Controlled, prospective, observational study on the efficiency and tolerability of a combination of potential Nrf2-inducing antioxidants and micronutrients as pre-treatment for ICSI in dyspermic patients with previous failure. Eur Rev Med Pharmacol Sci 2017;21(7):1645-1652. PMID: 28429340.

Regidor PA and Schindler AE. Myoinositol as a Safe and Alternative Approach in the Treatment of Infertile PCOS Women: A German Observational Study. International Journal of Endocrinology 2016;2016.

Seifer DB, Tal O, Wantman E, et al. Prognostic indicators of assisted reproduction technology outcomes of cycles with ultralow serum antimullerian hormone: a multivariate analysis of over 5,000 autologous cycles from the Society for Assisted Reproductive Technology Clinic Outcome Reporting System database for 2012-2013. Fertil Steril 2016;105(2):385-93.e3. PMID: 26515380.

Selçuk S, Özkaya E, Eser A, et al. Characteristics and outcomes of in vitro fertilization in different phenotypes of polycystic ovary syndrome. Turk Jinekoloji ve Obstetrik Dernegi Dergisi 2016;13(1):1-6.

Senapati S, Sammel MD, Morse C, et al. Impact of endometriosis on in vitro fertilization outcomes: an evaluation of the Society for Assisted Reproductive Technologies Database. Fertil Steril 2016. PMID: 27060727.

Stephen EH, Chandra A and King RB. Supply of and demand for assisted reproductive technologies in the United States: clinic- and population-based data, 1995-2010. Fertility and Sterility 2016;105(2):451-458 
Stern JE, McLain AC, Buck Louis GM, et al. Accuracy of self-reported survey data on assisted reproductive technology treatment parameters and reproductive history. American Journal of Obstetrics and Gynecology 2016;215(2):219.e1-219.e6.

Styer AK, Luke B, Vitek W, et al. Factors associated with the use of elective single-embryo transfer and pregnancy outcomes in the United States, 2004-2012. Fertility and Sterility 2016;106(1):80-89.

Tsai YR, Huang FJ, Lin PY, et al. Clinical outcomes and development of children born to couples with obstructive and nonobstructive azoospermia undergoing testicular sperm extractionintracytoplasmic sperm injection: A comparative study. Taiwan J Obstet Gynecol 2015;54(2):155-9. PMID: 25951720.

Tworoger SS, Fairfield KM, Colditz GA, et al. Association of oral contraceptive use, other contraceptive methods, and infertility with ovarian cancer risk. Am J Epidemiol 2007;166(8):894-901. PMID: 17656616.

Valsangkar S, Bodhare T, Bele S, et al. An evaluation of the effect of infertility on marital, sexual satisfaction indices and health-related quality of life in women. Journal of Human Reproductive Sciences 2011;4(2):80-85.

Vega M, Zaghi S, Buyuk E, et al. Not all twins are monozygotic after elective single embryo transfer: analysis of 32,600 elective single embryo transfer cycles as reported to the Society for Assisted Reproductive Technology. Fertil Steril 2018;109(1):118-122. PMID: 29307392.

Verkuijlen J, Verhaak C, Nelen WL, et al. Psychological and educational interventions for subfertile men and women. Cochrane Database Syst Rev 2016;3:Cd011034. PMID: 27031818.

Volgsten H, Schmidt L, Skoog Svanberg A, et al. Psychiatric disorders in women and men up to five years after undergoing assisted reproductive technology treatment - a prospective cohort study. Hum Fertil (Camb) 2018:1-6. PMID: 29768933.

Wang H, Gao H, Chi H, et al. Effect of Levothyroxine on Miscarriage Among Women With Normal Thyroid Function and Thyroid Autoimmunity Undergoing In Vitro Fertilization and Embryo Transfer: A Randomized Clinical Trial. Jama 2017;318(22):2190-2198. PMID: 29234808.

Wei D, Zhang B, Shi Y, et al. Effect of Preconception Impaired Glucose Tolerance on Pregnancy Outcomes in Women With Polycystic Ovary Syndrome. J Clin Endocrinol Metab 2017;102(10):3822-3829. PMID: 28938429.

Weiss NS, van Vliet MN, Limpens J, et al. Endometrial thickness in women undergoing IUI with ovarian stimulation. How thick is too thin? A systematic review and meta-analysis. Hum Reprod 2017;32(5):1009-1018. PMID: 28333207.

Xu HJ, Ji XW, Hong Y, et al. Mini-dose GnRH-a long versus short protocol in patients >/= 35 years old undergoing in vitro fertilization. Gynecol Endocrinol 2014;30(7):498-501. PMID: 24693851. 
Yu HT, Huang HY, Tseng HJ, et al. Bilaterality of ovarian endometriomas does not affect the outcome of in vitro fertilization/intracytoplasmic sperm injection in infertile women after laparoscopic cystectomy. Biomedical Journal 2017;40(5):295-299.

Zhang QF, Chen GY, Liu Y, et al. Relationship between resistin and IL-23 levels in follicular fluid in infertile patients with endometriosis undergoing IVF-ET. Advances in Clinical and Experimental Medicine 2017;26(9):1431-1435.

Ziebe S, Loft A, Povlsen BB, et al. A randomized clinical trial to evaluate the effect of granulocyte-macrophage colony-stimulating factor (GM-CSF) in embryo culture medium for in vitro fertilization. Fertil Steril 2013;99(6):1600-9. PMID: 23380186.

\section{No outcomes of interest:}

Abhyankar N, Kathrins M and Niederberger C. Use of testicular versus ejaculated sperm for intracytoplasmic sperm injection among men with cryptozoospermia: a meta-analysis. Fertility and Sterility 2016;105(6):1469-1475.e1.

Abu Hashim H, Bazeed M and Abd Elaal I. Minimal stimulation or clomiphene citrate as firstline therapy in women with polycystic ovary syndrome: a randomized controlled trial. Gynecol Endocrinol 2012;28(2):87-90. PMID: 21770837.

Abu Hashim H. Predictors of success of laparoscopic ovarian drilling in women with polycystic ovary syndrome: an evidence-based approach. Arch Gynecol Obstet 2015;291(1):11-8. PMID: 25186279.

Adesiyun AG, Cole B and Ogwuche P. Hydrotubation in the management of female infertility: outcome in low resource settings. East Afr Med J 2009;86(1):31-6. PMID: 19530546.

Aflatoonian A, Baradaran Bagheri R and Hosseinisadat R. The effect of endometrial injury on pregnancy rate in frozen-thawed embryo transfer: A randomized control trial. Int J Reprod Biomed (Yazd) 2016;14(7):453-158. PMID: 27525329.

Aghahosseini M, Aleyassin A, Khodaverdi S, et al. Estradiol supplementation during the luteal phase in poor responder patients undergoing in vitro fertilization: a randomized clinical trial. $\mathrm{J}$ Assist Reprod Genet 2011;28(9):785-90. PMID: 21720788.

Agrawal K, Gainder S, Dhaliwal LK, et al. Ovulation Induction Using Clomiphene Citrate Using Stair - Step Regimen versus Traditional Regimen in Polycystic Ovary Syndrome Women - A Randomized Control Trial. J Hum Reprod Sci 2017;10(4):261-264. PMID: 29430152.

Ahinko-Hakamaa K, Huhtala $\mathrm{H}$ and Tinkanen H. Success in intrauterine insemination: the role of etiology. Acta Obstet Gynecol Scand 2007;86(7):855-60. PMID: 17611832.

Ahmad G, Watson AJ and Metwally M. Laparoscopy or laparotomy for distal tubal surgery? A meta-analysis. Hum Fertil (Camb) 2007;10(1):43-7. PMID: 17454208. 
Akarsu S, Demir S, Gode F, et al. Comparison of corifollitropin alfa and daily recombinant follicle-stimulating hormone in poor responder patients undergoing in vitro fertilization cycles. Turk J Obstet Gynecol 2017;14(4):199-202. PMID: 29379660.

Aleyasin A, Abediasl Z, Nazari A, et al. Granulocyte colony-stimulating factor in repeated IVF failure, a randomized trial. Reproduction 2016;151(6):637-42. PMID: 26980809.

AlKudmani B, Gat I, Buell D, et al. In Vitro Fertilization Success Rates after Surgically Treated Endometriosis and Effect of Time Interval between Surgery and In Vitro Fertilization. J Minim Invasive Gynecol 2018;25(1):99-104. PMID: 28807807.

Alnemr AAA, Ammar IMM, Aboelfath AMK, et al. Effect of estradiol valerate on the pregnancy rate in patients receiving letrozole for induction of ovulation. Middle East Fertility Society Journal 2017.

Ammar IMM and Salem MAA. Effect of oral Tadalafil on endometrial thickness in patients receiving Clomiphene citrate for ovulation induction. Middle East Fertility Society Journal 2017

Amrane S, Brown MB, Lobo RA, et al. Factors associated with short interpregnancy interval among women treated with in vitro fertilization. Journal of Assisted Reproduction and Genetics 2018;35(9):1595-1602.

Anonymous. Corifollitropin alfa for ovarian stimulation in IVF: a randomized trial in lowerbody-weight women. Reprod Biomed Online 2010;21(1):66-76. PMID: 20483664.

Arentz S, Abbott JA, Smith CA, et al. Herbal medicine for the management of polycystic ovary syndrome (PCOS) and associated oligo/amenorrhoea and hyperandrogenism; a review of the laboratory evidence for effects with corroborative clinical findings. BMC Complement Altern Med 2014;14(1):511. PMID: 25524718.

Attia AM, Al-Inany HG and Proctor ML. Gonadotrophins for idiopathic male factor subfertility: A Cochrane systematic review. Middle East Fertility Society Journal 2007;12(2):77-85.

Attia AM, Al-Inany HG, Farquhar C, et al. Gonadotrophins for idiopathic male factor subfertility. Cochrane Database Syst Rev 2007;(4):Cd005071. PMID: 17943837.

Aubuchon M, Kunselman AR, Schlaff WD, et al. Metformin and/or clomiphene do not adversely affect liver or renal function in women with polycystic ovary syndrome. J Clin Endocrinol Metab 2011;96(10):E1645-9. PMID: 21832111.

Azadi L, Abbasi H, Deemeh MR, et al. Zaditen (Ketotifen), as mast cell blocker, improves sperm quality, chromatin integrity and pregnancy rate after varicocelectomy. Int J Androl 2011;34(5 Pt 1):446-52. PMID: 20874726.

Badawy A, Khiary M, Ragab A, et al. Laparoscopy--or not--for management of unexplained infertility. J Obstet Gynaecol 2010;30(7):712-5. PMID: 20925617. 
Bahaa Eldin AM, Abdelmaabud KH, Laban M, et al. Endometrial Injury May Increase the Pregnancy Rate in Patients Undergoing Intrauterine Insemination: An Interventional Randomized Clinical Trial. Reprod Sci 2016. PMID: 27146582.

Baker VL, Luke B, Brown MB, et al. Multivariate analysis of factors affecting probability of pregnancy and live birth with in vitro fertilization: an analysis of the Society for Assisted Reproductive Technology Clinic Outcomes Reporting System. Fertil Steril 2010;94(4):1410-6. PMID: 19740463.

Barbosa MW, Silva LR, Navarro PA, et al. Dydrogesterone vs progesterone for luteal-phase support: systematic review and meta-analysis of randomized controlled trials. Ultrasound Obstet Gynecol 2016;48(2):161-70. PMID: 26577241.

Basirat Z, Adib Rad H, Esmailzadeh S, et al. Comparison of pregnancy rate between fresh embryo transfers and frozen-thawed embryo transfers following ICSI treatment. Int J Reprod Biomed (Yazd) 2016;14(1):39-46. PMID: 27141547.

Basirat Z, Zeinalzadeh M, Mahouti T, et al. Impact of dexamethasone on pregnancy outcome in PCOs women candidate for IVF/ICSI, a single-blind randomized clinical trial study. Middle East Fertility Society Journal 2016.

Belanoff C, Declercq ER, Diop H, et al. Severe Maternal Morbidity and the Use of Assisted Reproductive Technology in Massachusetts. Obstet Gynecol 2016;127(3):527-34. PMID: 26855105.

Belloc S, Cohen-Bacrie P, Benkhalifa M, et al. Effect of maternal and paternal age on pregnancy and miscarriage rates after intrauterine insemination. Reprod Biomed Online 2008;17(3):392-7. PMID: 18765010.

Benschop L, Farquhar C, van der Poel N, et al. Interventions for women with endometrioma prior to assisted reproductive technology. Cochrane Database Syst Rev 2010;(11):Cd008571. PMID: 21069706.

Beydoun HA, Ugwu B, Indika S, et al. Timing of oocyte retrieval and embryo transfer with pregnancy duration. J Womens Health (Larchmt) 2011;20(11):1669-75. PMID: 21970598.

Bhadarka HK, Patel NH, Patel NH, et al. Impact of embryo co-culture with cumulus cells on pregnancy \& implantation rate in patients undergoing in vitro fertilization using donor oocyte. Indian Journal of Medical Research 2017;146(September):341-345.

Bleau N, Agdi M, Son WY, et al. A comparison of outcomes from in vitro fertilization cycles stimulated with follicle stimulating hormone plus either recombinant luteinizing hormone or human menopausal gonadotropins in subjects treated with long gonadotropin releasing hormone agonist protocols. International Journal of Fertility and Sterility 2017;11(2):79-84.

Boomsma CM, Heineman MJ, Cohlen BJ, et al. Semen preparation techniques for intrauterine insemination. Cochrane Database Syst Rev 2007;(4):Cd004507. PMID: 17943816. 
Bourdon M, Santulli P, De Ziegler D, et al. Does GnRH Agonist Triggering Control Painful Symptom Scores during Assisted Reproductive Technology? A Retrospective Study.

Reproductive Sciences 2017;24(9):1325-1333.

Bouwmans CA, Lintsen BA, Al M, et al. Absence from work and emotional stress in women undergoing IVF or ICSI: an analysis of IVF-related absence from work in women and the contribution of general and emotional factors. Acta Obstet Gynecol Scand 2008;87(11):1169-75. PMID: 18951267.

Boza A, Cakar E, Boza B, et al. Microdose flare-up gonadotropin-releasing hormone (GnRH) agonist versus GnRH antagonist protocols in poor ovarian responders undergoing intracytoplasmic sperm injection. Journal of Reproduction and Infertility 2016;17(3):163-168.

Bracewell-Milnes T, Saso S, Bora S, et al. Investigating psychosocial attitudes, motivations and experiences of oocyte donors, recipients and egg sharers: a systematic review. Hum Reprod Update 2016. PMID: 27016289.

Brandes M, Hamilton CJ, van der Steen JO, et al. Unexplained infertility: overall ongoing pregnancy rate and mode of conception. Hum Reprod 2011;26(2):360-8. PMID: 21163857.

Brandes M, van der Steen JO, Bokdam SB, et al. When and why do subfertile couples discontinue their fertility care? A longitudinal cohort study in a secondary care subfertility population. Hum Reprod 2009;24(12):3127-35. PMID: 19783833.

Brodin T, Hadziosmanovic N, Berglund L, et al. Comparing four ovarian reserve markers associations with ovarian response and live births after assisted reproduction. Acta Obstet Gynecol Scand 2015. PMID: 26184379.

Broekmans FJ, Verweij PJ, Eijkemans MJ, et al. Prognostic models for high and low ovarian responses in controlled ovarian stimulation using a GnRH antagonist protocol. Hum Reprod 2014;29(8):1688-97. PMID: 24903202.

Bryniarski P, Taborowski P, Rajwa P, et al. The comparison of laparoscopic and microsurgical varicocoelectomy in infertile men with varicocoele on paternity rate 12 months after surgery: a prospective randomized controlled trial. Andrology 2017;5(3):445-450. PMID: 28346969.

Carbillon L, Gronier H, Cedrin-Durnerin I, et al. The impact of ovulation induction and ovarian stimulation on the risk of pregnancy-induced hypertension and on neonatal outcomes: A case/control study. Eur J Obstet Gynecol Reprod Biol 2017;217:137-143. PMID: 28898686.

Cavalcante MB, Silva Costa FDA, Barini R, et al. Granulocyte colony-stimulating factor and reproductive medicine: A review. Iranian Journal of Reproductive Medicine 2015;13(4):195202.

Cayan S, Shavakhabov S and Kadioglu A. Treatment of palpable varicocele in infertile men: a meta-analysis to define the best technique. J Androl 2009;30(1):33-40. PMID: 18772487. 
Ceyhan ST, Tekin YB, Sakinci M, et al. What should be the protocol selection after failure of invitro fertilization at normoresponder patients: Agonist or antagonist? Turk Jinekoloji ve Obstetrik Dernegi Dergisi 2014;11(4):198-202.

Chang $\mathrm{X}$ and Wu J. Effects of luteal estradiol pre-treatment on the outcome of IVF in poor ovarian responders. Gynecol Endocrinol 2013;29(3):196-200. PMID: 23194136.

Chiaffarino F, Baldini MP, Scarduelli C, et al. Prevalence and incidence of depressive and anxious symptoms in couples undergoing assisted reproductive treatment in an Italian infertility department. Eur J Obstet Gynecol Reprod Biol 2011;158(2):235-41. PMID: 21641108.

Chua ME, Escusa KG, Luna S, et al. Revisiting oestrogen antagonists (clomiphene or tamoxifen) as medical empiric therapy for idiopathic male infertility: a meta-analysis. Andrology 2013;1(5):749-57. PMID: 23970453.

Darwish AM, Metwally A, Shaaban MM, et al. Monopolar versus bipolar laparoscopic ovarian drilling in clomiphene-resistant polycystic ovaries (PCO): a preliminary study. Gynecological Surgery 2016:1-7.

Davar R, Eftekhar M and Tayebi N. Transfer of cryopreserved-thawed embryos in a cycle using exogenous steroids with or without prior gonadotropihin-releasing hormone agonist. Journal of Medical Sciences 2007;7(5):880-883.

Davar R, Janati S, Mohseni F, et al. A comparison of the effects of transdermal estradiol and estradiol valerate on endometrial receptivity in frozen-thawed embryo transfer cycles: A randomized clinical trial. Journal of Reproduction and Infertility 2016;17(2):97-103.

Davar R, Mojtahedi MF and Miraj S. Effects of single dose GnRH agonist as luteal support on pregnancy outcome in frozen-thawed embryo transfer cycles: An RCT. Iranian Journal of Reproductive Medicine 2015;13(8):483-488.

Devroey P, Boostanfar R, Koper NP, et al. A double-blind, non-inferiority RCT comparing corifollitropin alfa and recombinant FSH during the first seven days of ovarian stimulation using a GnRH antagonist protocol. Hum Reprod 2009;24(12):3063-72. PMID: 19684043.

Ding H, Tian J, Du W, et al. Open non-microsurgical, laparoscopic or open microsurgical varicocelectomy for male infertility: a meta-analysis of randomized controlled trials. BJU Int 2012;110(10):1536-42. PMID: 22642226.

Ebrahimi M, Akbari-Asbagh F and Ghalandar-Attar M. Letrozole+ GnRH antagonist stimulation protocol in poor ovarian responders undergoing intracytoplasmic sperm injection cycles: An RCT. Int J Reprod Biomed (Yazd) 2017;15(2):101-108. PMID: 28462402.

Elsetohy KA, Askalany AH, Hassan M, et al. Routine office hysteroscopy prior to ICSI vs. ICSI alone in patients with normal transvaginal ultrasound: a randomized controlled trial. Arch Gynecol Obstet 2015;291(1):193-9. PMID: 25082070. 
Engmann L, DiLuigi A, Schmidt D, et al. The use of gonadotropin-releasing hormone (GnRH) agonist to induce oocyte maturation after cotreatment with $\mathrm{GnRH}$ antagonist in high-risk patients undergoing in vitro fertilization prevents the risk of ovarian hyperstimulation syndrome: a prospective randomized controlled study. Fertil Steril 2008;89(1):84-91. PMID: 17462639.

Fan W, Li SW, Li L, et al. Outcome of conventional IVF and ICSI on sibling oocytes in the case of isolated teratozoospermia. J Assist Reprod Genet 2012;29(9):905-10. PMID: 22733316.

Farland LV, Grodstein F, Srouji SS, et al. Infertility, fertility treatment, and risk of hypertension. Fertil Steril 2015;104(2):391-7. PMID: 26049054.

Farquhar C, Rishworth JR, Brown J, et al. Assisted reproductive technology: an overview of Cochrane reviews. Cochrane Database Syst Rev 2014;12:Cd010537. PMID: 25532533.

Fatemi F, Mohammadzadeh A, Sadeghi MR, et al. Role of vitamin E and D3 supplementation in Intra-Cytoplasmic Sperm Injection outcomes of women with polycystic ovarian syndrome: A double blinded randomized placebo-controlled trial. Clin Nutr ESPEN 2017;18:23-30. PMID: 29132734.

Fawzy M, AbdelRahman MY, Zidan MH, et al. Humid versus dry incubator: a prospective, randomized, controlled trial. Fertil Steril 2017;108(2):277-283. PMID: 28651960.

Flower A, Liu JP, Lewith G, et al. Chinese herbal medicine for endometriosis. Cochrane Database Syst Rev 2012;5:Cd006568. PMID: 22592712.

Forman EJ, Hong KH, Ferry KM, et al. In vitro fertilization with single euploid blastocyst transfer: a randomized controlled trial. Fertil Steril 2013;100(1):100-7.e1. PMID: 23548942.

Gameiro S, van den Belt-Dusebout AW, Bleiker E, et al. Do children make you happier? Sustained child-wish and mental health in women 11-17 years after fertility treatment. Hum Reprod 2014;29(10):2238-46. PMID: 25205751.

Garalejic E, Arsic B, Radakovic J, et al. A preliminary evaluation of influence of body mass index on in vitro fertilization outcome in non-obese endometriosis patients. BMC Womens Health 2017;17(1):112. PMID: 29145852.

Ghanem ME, Sadek EE, Elboghdady LA, et al. The effect of luteal phase support protocol on cycle outcome and luteal phase hormone profile in long agonist protocol intracytoplasmic sperm injection cycles: a randomized clinical trial. Fertil Steril 2009;92(2):486-93. PMID: 19464001.

Glujovsky D, Riestra B, Sueldo C, et al. Vitrification versus slow freezing for women undergoing oocyte cryopreservation. Cochrane Database Syst Rev 2014;9:Cd010047. PMID: 25192224.

Goldman RH, Batsis M, Petrozza JC, et al. Patient-specific predictions of outcome after gonadotropin ovulation induction/intrauterine insemination. Fertil Steril 2014;101(6):164955.e1-2. PMID: 24690238. 
Gopinath PM, Kalra B, Saxena A, et al. Fixed dose combination therapy of antioxidants in treatment of idiopathic oligoasthenozoospermia: Results of a randomized, double-blind, placebocontrolled clinical trial. International Journal of Infertility and Fetal Medicine 2013;4(1):6-13.

Gupta S, Satwik R, Majumdar A, et al. Clomiphene based ovarian stimulation in a commercial donor program. Journal of Human Reproductive Sciences 2015;8(3):142-145.

Hardarson T, Bungum M, Conaghan J, et al. Noninferiority, randomized, controlled trial comparing embryo development using media developed for sequential or undisturbed culture in a time-lapse setup. Fertil Steril 2015;104(6):1452-9.e1-4. PMID: 26409153.

$\mathrm{He} \mathrm{Y}, \mathrm{Wu} \mathrm{H}$, et al. Administration of atosiban in patients with endometriosis undergoing frozenthawed embryo transfer: a prospective, randomized study. Fertility and sterility 2016 PMID: 27143518.

Hendawy SF, Samaha HE and Elkholy MF. Letrozole versus Clomiphene Citrate for Induction of Ovulation in Patients with Polycystic Ovarian Syndrome Undergoing Intrauterine Insemination. Clin Med Insights Reprod Health 2011;5:11-6. PMID: 24453507.

Heredia M, Tenias JM, Rocio R, et al. Quality of life and predictive factors in patients undergoing assisted reproduction techniques. Eur J Obstet Gynecol Reprod Biol 2013;167(2):176-80. PMID: 23347604.

Hipp H, Crawford S, Kawwass JF, et al. First trimester pregnancy loss after fresh and frozen in vitro fertilization cycles. Fertil Steril 2016;105(3):722-8. PMID: 26627121.

Ho YK, Lee TH, Lee CI, et al. Effects of growth hormone plus gonadotropins on controlled ovarian stimulation in infertile women of advanced age, poor responders, and previous in vitro fertilization failure patients. Taiwan J Obstet Gynecol 2017;56(6):806-810. PMID: 29241924.

Hobeika E, Singh S, Malik S, et al. Initial maternal serum human chorionic gonadotropin levels in pregnancies achieved after assisted reproductive technology are higher after preimplantation genetic screening and after frozen embryo transfer: a retrospective cohort. Journal of Assisted Reproduction and Genetics 2017;34(10):1333-1340.

Hossein-Rashidi B and Nemati M. Effects of Vitex agnus-castus extract on the secretory function of pituitary-gonadal axis and pregnancy rate in patients with premature ovarian aging (POA). Journal of Herbal Medicine 2017;10:24-30.

Hotaling JM, Smith JF, Rosen M, et al. The relationship between isolated teratozoospermia and clinical pregnancy after in vitro fertilization with or without intracytoplasmic sperm injection: a systematic review and meta-analysis. Fertil Steril 2011;95(3):1141-5. PMID: 21030014.

Huang P, Wei L, Li X, et al. Effects of intrauterine perfusion of human chorionic gonadotropin in women with different implantation failure numbers. American Journal of Reproductive Immunology 2018;79(2). 
Ikenaga H, Tanaka Y, Shiotani M, et al. Phase III trial comparing the efficacy and safety of recombinant- or urine-derived human chorionic gonadotropin for ovulation triggering in Japanese women diagnosed with anovulation or oligo-ovulation and undergoing ovulation induction with follitropin-alfa. Reprod Med Biol 2017;16(1):45-51. PMID: 29259450.

Iltemir Duvan ZC, Namli Kalem M, Onaran Y, et al. The effect of coasting on intracytoplasmic sperm injection outcome in antagonist and agonist cycle. International Journal of Fertility and Sterility 2017;11(1):1-6.

Ismail Madkour WA, Noah B, Zaheer H, et al. Does sequential embryo transfer improve pregnancy rate in patients with repeated implantation failure? A randomized control study. Middle East Fertility Society Journal 2015;20(4):255-261.

Jamilian M, Zadeh Modarres S, Amiri Siavashani M, et al. The Influences of Chromium Supplementation on Glycemic Control, Markers of Cardio-Metabolic Risk, and Oxidative Stress in Infertile Polycystic ovary Syndrome Women Candidate for In vitro Fertilization: a Randomized, Double-Blind, Placebo-Controlled Trial. Biol Trace Elem Res 2018. PMID: 29307112.

Jin R, Bao J, Tang D, et al. Outcomes of intracytoplasmic sperm injection using the zona pellucida-bound sperm or manually selected sperm. J Assist Reprod Genet 2016;33(5):597-601. PMID: 26941098.

Kamal A, Fahmy I, Mansour R, et al. Does the outcome of ICSI in cases of obstructive azoospermia depend on the origin of the retrieved spermatozoa or the cause of obstruction? A comparative analysis. Fertil Steril 2010;94(6):2135-40. PMID: 20188354.

Kandil M, Rezk M, Al-Halaby A, et al. Impact of Ultrasound-Guided Transvaginal Ovarian Needle Drilling Versus Laparoscopic Ovarian Drilling on Ovarian Reserve and Pregnancy Rate in Polycystic Ovary Syndrome: A Randomized Clinical Trial. J Minim Invasive Gynecol 2018. PMID: 29476810.

Kaponis A, Taniguchi F, Azuma Y, et al. Current treatment of endometrioma. Obstet Gynecol Surv 2015;70(3):183-95. PMID: 25769433.

Kasia JM, Ngowa JDK, Mimboe YS, et al. Laparoscopic fimbrioplasty and neosalpingostomy in female infertility: A review of 402 cases at the gynecological endoscopic surgery and human reproductive teaching hospital in Yaoundé-Cameroon. Journal of Reproduction and Infertility 2016;17(2):104-109.

Kaur P, Swarankar ML, Maheshwari M, et al. A comparative study between cleavage stage embryo transfer at day 3 and blastocyst stage transfer at day 5 in in-vitro fertilization/intracytoplasmic sperm injection on clinical pregnancy rates. J Hum Reprod Sci 2014;7(3):194-7. PMID: 25395745.

Kayatas S, Boza A, Api M, et al. Body composition: A predictive factor of cycle fecundity. Clin Exp Reprod Med 2014;41(2):75-9. PMID: 25045631. 
Kazemi A, Delavar MZ and Kheirabadi G. Psychiatric Symptoms Associated with OocyteDonation. Psychiatric Quarterly 2016:1-6.

Ketabchi AA. Intracytoplasmic sperm injection outcomes with freshly ejaculated sperms and testicular or epididymal sperm extraction in patients with idiopathic cryptozoospermia. NephroUrology Monthly 2016;8(6).

Khairy M, Shah H and Rajkhowa M. Transmyometrial versus very difficult transcervical embryo transfer: Efficacy and safety. Reproductive BioMedicine Online 2016;32(5):513-517.

Kim HJ, Seo JT, Kim KJ, et al. Clinical significance of subclinical varicocelectomy in male infertility: systematic review and meta-analysis. Andrologia 2015. PMID: 26589369.

Kocak M, Dilbaz B, Demir B, et al. Lyophilised hMG versus rFSH in women with unexplained infertility undergoing a controlled ovarian stimulation with intrauterine insemination: a prospective, randomised study. Gynecol Endocrinol 2010;26(6):429-34. PMID: 20175705.

Kosus A, Kosus N, Duran M, et al. Does assisted reproductive technology itself or polycystic ovary syndrome as a cause of infertility have any effect on first trimester serum screening results? J Reprod Med 2012;57(3-4):129-35. PMID: 22523872.

Kotb MM, Hassan AM and AwadAllah AM. Does dehydroepiandrosterone improve pregnancy rate in women undergoing IVF/ICSI with expected poor ovarian response according to the Bologna criteria? A randomized controlled trial. Eur J Obstet Gynecol Reprod Biol 2016;200:115. PMID: 26963897.

Kroese AC, de Lange NM, Collins J, et al. Surgery or embolization for varicoceles in subfertile men. Cochrane Database Syst Rev 2012;10:Cd000479. PMID: 23076888.

Kroon B, Hart RJ, Wong BM, et al. Antibiotics prior to embryo transfer in ART. Cochrane Database Syst Rev 2012;3:Cd008995. PMID: 22419341.

Kwon SK, Kim CH, Lee KH, et al. Luteal estradiol supplementation in gonadotropin-releasing hormone antagonist cycles for infertile patients in vitro fertilization. Clin Exp Reprod Med 2013;40(3):131-4. PMID: 24179871.

la Cour Freiesleben N, Lossl K, Bogstad J, et al. Individual versus standard dose of rFSH in a mild stimulation protocol for intrauterine insemination: a randomized study. Hum Reprod 2009;24(10):2523-30. PMID: 19602518.

Labarta E and Bosch E. Comparative study of highly purified HMG versus recombinant FSH + recombinant LH in ovulation induction for intrauterine insemination: A randomized controlled trial. Medicina Reproductiva y Embriologia Clinica 2016;3(1):4-11.

Lamb JD, Huddleston HG, Purcell KJ, et al. Asian ethnicity is associated with decreased pregnancy rates following intrauterine insemination. Reprod Biomed Online 2009;19(2):252-6. PMID: 19712563. 
Lefebvre J, Antaki R, Kadoch IJ, et al. 450 IU versus 600 IU gonadotropin for controlled ovarian stimulation in poor responders: a randomized controlled trial. Fertil Steril 2015;104(6):1419-25. PMID: 26361207.

Legro RS, Kunselman AR, Brzyski RG, et al. The Pregnancy in Polycystic Ovary Syndrome II (PPCOS II) trial: rationale and design of a double-blind randomized trial of clomiphene citrate and letrozole for the treatment of infertility in women with polycystic ovary syndrome. Contemp Clin Trials 2012;33(3):470-81. PMID: 22265923.

Lehert P, Schertz JC and Ezcurra D. Recombinant human follicle-stimulating hormone produces more oocytes with a lower total dose per cycle in assisted reproductive technologies compared with highly purified human menopausal gonadotrophin: a meta-analysis. Reprod Biol Endocrinol 2010;8:112. PMID: 20846363.

Li L, Zhang Y, Zeng D, et al. Depression in Chinese men undergoing different assisted reproductive technique treatments: prevalence and risk factors. J Assist Reprod Genet 2013;30(9):1161-7. PMID: 23907732.

Li Y, Deng B, Ouyang N, et al. Telomere length is short in PCOS and oral contraceptive does not affect the telomerase activity in granulosa cells of patients with PCOS. J Assist Reprod Genet 2017;34(7):849-859. PMID: 28477298.

Lim CE, Ng RW, Xu K, et al. Acupuncture for polycystic ovarian syndrome. Cochrane Database Syst Rev 2016;5:Cd007689. PMID: 27136291.

Lin LT, Cheng JT, Wang PH, et al. Dehydroepiandrosterone as a potential agent to slow down ovarian aging. Journal of Obstetrics and Gynaecology Research 2017;43(12):1855-1862.

Lin PY, Huang FJ, Kung FT, et al. Comparison of the offspring sex ratio between fresh and vitrification-thawed blastocyst transfer. Fertil Steril 2009;92(5):1764-6. PMID: 19523616.

Lintsen AM, Eijkemans MJ, Hunault CC, et al. Predicting ongoing pregnancy chances after IVF and ICSI: a national prospective study. Hum Reprod 2007;22(9):2455-62. PMID: 17636281.

Liu XH, Yan LY, Lu CL, et al. ART do not increase the risk of Y-chromosome microdeletion in 19 candidate genes at AZF regions. Reprod Fertil Dev 2014;26(6):778-86. PMID: 23751883.

Lu D, Song H, Li Y, et al. Pentoxifylline for endometriosis. Cochrane Database Syst Rev 2012;1:Cd007677. PMID: 22258970.

Lu E, Barfield WD, Wilber N, et al. Surveillance of births conceived with various infertility therapies in Massachusetts, January-March 2005. Public Health Rep 2008;123(2):173-7. PMID: 18457069.

Luke B, Brown MB, Grainger DA, et al. The sex ratio of singleton offspring in assistedconception pregnancies. Fertil Steril 2009;92(5):1579-85. PMID: 18950756. 
Luo S, Li S, Jin S, et al. Effectiveness of GnRH antagonist in the management of subfertile couples undergoing controlled ovarian stimulation and intrauterine insemination: a metaanalysis. PLoS One 2014;9(10):e109133. PMID: 25299186.

Lv D, Song H, Li Y, et al. Pentoxifylline versus medical therapies for subfertile women with endometriosis. Cochrane Database Syst Rev 2009;(3):Cd007677. PMID: 19588441.

Lyerly AD, Steinhauser K, Voils C, et al. Fertility patients' views about frozen embryo disposition: results of a multi-institutional U.S. survey. Fertil Steril 2010;93(2):499-509. PMID: 19061998.

Maged AM, Elsawah H, Abdelhafez A, et al. The adjuvant effect of metformin and N acetylcysteine to clomiphene citrate in induction of ovulation in patients with Polycystic Ovary Syndrome. Gynecological Endocrinology 2015;31(8):635-638.

Malik N, Kriplani A, Agarwal N, et al. Dehydroepiandrosterone as an adjunct to gonadotropins in infertile Indian women with premature ovarian aging: A pilot study. J Hum Reprod Sci 2015;8(3):135-41. PMID: 26538855.

Marmar JL, Agarwal A, Prabakaran S, et al. Reassessing the value of varicocelectomy as a treatment for male subfertility with a new meta-analysis. Fertil Steril 2007;88(3):639-48. PMID: 17434508.

McGovern PG, Carson SA, Barnhart HX, et al. Medication adherence and treatment success in the National Institute of Child Health and Human Development-Reproductive Medicine Network's Pregnancy in Polycystic Ovary Syndrome Trial. Fertil Steril 2008;90(4):1283-6. PMID: 18082737.

Michels KA, Mumford SL, Sundaram R, et al. Differences in infant feeding practices by mode of conception in a United States cohort. Fertility and Sterility 2016;105(4):1014-1022.e1.

Moll E, van Wely M, Lambalk CB, et al. Health-related quality of life in women with newly diagnosed polycystic ovary syndrome randomized between clomifene citrate plus metformin or clomifene citrate plus placebo. Hum Reprod 2012;27(11):3273-8. PMID: 22926838.

Monteleone PAA, Peregrino PFM, Baracat EC, et al. Transfer of 2 Embryos Using a DoubleEmbryo Transfer Protocol Versus 2 Sequential Single-Embryo Transfers: The Impact on Multiple Pregnancy. Reproductive Sciences 2018.

Mourad SM, Nelen WL, Akkermans RP, et al. Determinants of patients' experiences and satisfaction with fertility care. Fertil Steril 2010;94(4):1254-60. PMID: 19732885.

Mui Lam P, Chun Cheung M, Ping Cheung L, et al. Effects of early luteal-phase vaginal progesterone supplementation on the outcome of in vitro fertilization and embryo transfer. Gynecol Endocrinol 2008;24(12):674-80. PMID: 19172535. 
Mutsaerts MA, Kuchenbecker WK, Mol BW, et al. Dropout is a problem in lifestyle intervention programs for overweight and obese infertile women: a systematic review. Hum Reprod 2013;28(4):979-86. PMID: 23427235.

Naeem A, Amjad F and Memon AS. Comparison of letrozole versus clomiphene citrate on ovulation and achieving a successful pregnancy. Pakistan Journal of Medical and Health Sciences 2017;11(3):1143-1145

Nahuis MJ, Oude Lohuis EJ, Bayram N, et al. Pregnancy complications and metabolic disease in women with clomiphene citrate-resistant anovulation randomized to receive laparoscopic electrocautery of the ovaries or ovulation induction with gonadotropins: a 10-year follow-up. Fertil Steril 2014;101(1):270-4. PMID: 24268702.

Nordhoff V, Schuring AN, Krallmann C, et al. Optimizing TESE-ICSI by laser-assisted selection of immotile spermatozoa and polarization microscopy for selection of oocytes. Andrology 2013;1(1):67-74. PMID: 23258632.

Ob’edkova K, Kogan I, Krikheli I, et al. Growth hormone co-treatment in IVF/ICSI cycles in poor responders. Gynecological Endocrinology 2017;33:15-17.

Okhowat J, Murtinger M, Schuff M, et al. Massage therapy improves in vitro fertilization outcome in patients undergoing blastocyst transfer in a cryo-cycle. Altern Ther Health Med 2015;21(2):16-22. PMID: 25830275.

Olgan S and Dirican EK. The Effect of Instant Urinary Catheterization on Pregnancy Outcomes after Intracytoplasmic Sperm Injection and Embryo Transfer. Gynecologic and Obstetric Investigation 2017;82(6):563-568.

Oliveira JB, Baruffi R, Petersen CG, et al. Administration of single-dose GnRH agonist in the luteal phase in ICSI cycles: a meta-analysis. Reprod Biol Endocrinol 2010;8:107. PMID: 20825643.

Ostrowski KA, Polackwich AS, Kent J, et al. Higher outcomes of vasectomy reversal in men with the same female partner as before vasectomy. J Urol 2015;193(1):245-7. PMID: 25088953.

Ozekinci M, Seven A, Olgan S, et al. Does obesity have detrimental effects on IVF treatment outcomes? BMC Womens Health 2015;15:61. PMID: 26285703.

Ozgur K, Berkkanoglu M, Bulut H, et al. Agonist depot versus OCP programming of frozen embryo transfer: a retrospective analysis of freeze-all cycles. Journal of Assisted Reproduction and Genetics 2016;33(2):207-214.

Ozmen B, Sonmezer M, Atabekoglu CS, et al. Use of aromatase inhibitors in poor-responder patients receiving GnRH antagonist protocols. Reprod Biomed Online 2009;19(4):478-85. PMID: 19909587. 
Pacchiarotti A, Carlomagno G, Antonini G, et al. Effect of myo-inositol and melatonin versus myo-inositol, in a randomized controlled trial, for improving in vitro fertilization of patients with polycystic ovarian syndrome. Gynecol Endocrinol 2016;32(1):69-73. PMID: 26507336.

Pacchiarotti A, Sbracia M, Frega A, et al. Urinary hMG (Meropur) versus recombinant FSH plus recombinant LH (Pergoveris) in IVF: a multicenter, prospective, randomized controlled trial. Fertil Steril 2010;94(6):2467-9. PMID: 20537626.

Papaleo E, Pagliardini L, Vanni VS, et al. A direct healthcare cost analysis of the cryopreserved versus fresh transfer policy at the blastocyst stage. Reprod Biomed Online 2017;34(1):19-26. PMID: 27746110.

Paredes Palma JC, López Byhen E, Ibáñez L, et al. Comparative treatment between sitagliptin vs. metformin, alone or in combination, in patients with polycystic ovary syndrome. A clinical entity at high risk for developing diabetes mellitus and gestational diabetes: A pilot study. Revista Medica del Hospital General de Mexico 2018;81(1):15-26.

Park JH, Jee BC and Kim SH. Comparison of normal and abnormal fertilization of in vitromatured human oocyte according to insemination method. Journal of Obstetrics and Gynaecology Research 2016;42(4):417-421.

Park YS, Lee SH, Lim CK, et al. Paternal age as an independent factor does not affect embryo quality and pregnancy outcomes of testicular sperm extraction-intracytoplasmic sperm injection in azoospermia. Andrologia 2018;50(2).

Peeraer K, Luyten J, Tomassetti C, et al. Cost-effectiveness of ovarian stimulation with gonadotrophin and clomiphene citrate in an intrauterine insemination programme for subfertile couples. Reprod Biomed Online 2018;36(3):302-310. PMID: 29395750.

Peyvandi S, Rokhgireh S and Esmaeeli N. A survey of acupuncture effect on the result of invitro fertilization. World Journal of Acupuncture - Moxibustion 2016;26(3):55-58.

Porat-Katz A, Eldar-Geva T, Kahane A, et al. Using propensity score matching to evaluate the effect of complementary medicine on clinical and embryologic outcomes of in vitro fertilization. Int J Gynaecol Obstet 2017;138(2):212-218. PMID: 28598500.

Poulsen V, Ingerslev HJ and Kirkegaard K. Elective embryo transfers on Day 6 reduce implantation compared with transfers on Day 5. Hum Reprod 2017;32(6):1238-1243. PMID: 28398477.

Proctor M, Johnson N, van Peperstraten Arno M, et al. Techniques for surgical retrieval of sperm prior to intra-cytoplasmic sperm injection (ICSI) for azoospermia. CD002807.

Purandare N, Emerson G, Kirkham C, et al. The duration of gonadotropin stimulation does not alter the clinical pregnancy rate in IVF or ICSI cycles. Irish Journal of Medical Science 2017;186(3):653-657. 
Qu F, Zhang D, Chen LT, et al. Auricular acupressure reduces anxiety levels and improves outcomes of in vitro fertilization: a prospective, randomized and controlled study. Sci Rep 2014;4:5028. PMID: 24848522.

Qublan HS, Yannakoula EK, Al-Qudah MA, et al. Dietary intervention versus metformin to improve the reproductive outcome in women with polycystic ovary syndrome. A prospective comparative study. Saudi Med J 2007;28(11):1694-9. PMID: 17965792.

Raguz N, McDonald SW, Metcalfe A, et al. Mental health outcomes of mothers who conceived using fertility treatment. Reproductive Health 2015;11(1).

Ramezanali F, Ashrafi M, Hemat M, et al. Assisted reproductive outcomes in women with different polycystic ovary syndrome phenotypes: The predictive value of anti-Müllerian hormone. Reproductive BioMedicine Online 2016;32(5):503-512.

Rancourt RC, Harris HR and Michels KB. Methylation levels at imprinting control regions are not altered with ovulation induction or in vitro fertilization in a birth cohort. Hum Reprod 2012;27(7):2208-16. PMID: 22587996.

Rashidi B, Montazeri A, Ramezanzadeh F, et al. Health-related quality of life in infertile couples receiving IVF or ICSI treatment. BMC Health Serv Res 2008;8:186. PMID: 18803838.

Ribeiro MA, Gameiro LF, Scarano WR, et al. Aromatase inhibitors in the treatment of oligozoospermic or azoospermic men: a systematic review of randomized controlled trials. JBRA Assist Reprod 2016;20(2):82-8. PMID: 27244767.

Ried K and Stuart K. Efficacy of Traditional Chinese Herbal Medicine in the management of female infertility: a systematic review. Complement Ther Med 2011;19(6):319-31. PMID: 22036524.

Rubio I, Galan A, Larreategui Z, et al. Clinical validation of embryo culture and selection by morphokinetic analysis: a randomized, controlled trial of the EmbryoScope. Fertil Steril 2014;102(5):1287-1294.e5. PMID: 25217875.

Safdarian L, Mohammadi FS, Alleyassin A, et al. Clinical outcome with half-dose depot triptorelin is the same as reduced-dose daily buserelin in a long protocol of controlled ovarian stimulation for ICSI/embryo transfer: a randomized double-blind clinical trial (NCT00461916). Hum Reprod 2007;22(9):2449-54. PMID: 17635844.

Salamun V, Jensterle M, Janez A, et al. Liraglutide increases IVF pregnancy rates in obese PCOS women with poor response to first-line reproductive treatments: a pilot randomized study. Eur J Endocrinol 2018;179(1):1-11. PMID: 29703793.

Schorsch M, Gomez R, Hahn T, et al. Success Rate of Inseminations Dependent on Maternal Age? An Analysis of 4246 Insemination Cycles. Geburtshilfe Frauenheilkd 2013;73(8):808-811. PMID: 24771935. 
Scott RT, Jr., Upham KM, Forman EJ, et al. Cleavage-stage biopsy significantly impairs human embryonic implantation potential while blastocyst biopsy does not: a randomized and paired clinical trial. Fertil Steril 2013;100(3):624-30. PMID: 23773313.

Senocak GC, Yapca OE and Borekci B. Comparison of pregnancy rates between patients with and without local endometrial scratching before intrauterine insemination. J Gynecol Obstet Hum Reprod 2017;46(9):687-690. PMID: 28964963.

Setti AS, Braga DP, Figueira RC, et al. Intracytoplasmic morphologically selected sperm injection results in improved clinical outcomes in couples with previous ICSI failures or male factor infertility: a meta-analysis. Eur J Obstet Gynecol Reprod Biol 2014;183:96-103. PMID: 25461360.

Shokeir T, Ebrahim M and El-Mogy H. Hysteroscopic-guided local endometrial injury does not improve natural cycle pregnancy rate in women with unexplained infertility: Randomized controlled trial. J Obstet Gynaecol Res 2016;42(11):1553-1557. PMID: 27363928.

Shoshany O, Abhyankar N, Mufarreh N, et al. Outcomes of anastrozole in oligozoospermic hypoandrogenic subfertile men. Fertil Steril 2017;107(3):589-594. PMID: 28069178.

Sicchieri F, Silva AB, Silva A, et al. Prognostic factors in intrauterine insemination cycles. JBRA Assist Reprod 2018;22(1):2-7. PMID: 29327861.

Siristatidis CS, Maheshwari A and Bhattacharya S. In vitro maturation in sub fertile women with polycystic ovarian syndrome undergoing assisted reproduction. Cochrane Database Syst Rev 2009 (1):Cd006606. PMID: 19160291.

Siristatidis CS, Vrachnis N, Creatsa M, et al. In vitro maturation in subfertile women with polycystic ovarian syndrome undergoing assisted reproduction. Cochrane Database Syst Rev 2013;10:Cd006606. PMID: 24101529.

Smith JF, Eisenberg ML, Glidden D, et al. Socioeconomic disparities in the use and success of fertility treatments: analysis of data from a prospective cohort in the United States. Fertil Steril 2011;96(1):95-101. PMID: 21616487.

Soria M, Pradillo G, Garcia J, et al. Pregnancy predictors after intrauterine insemination: analysis of 3012 cycles in 1201 couples. J Reprod Infertil 2012;13(3):158-66. PMID: 23926541.

Soritsa D, Saare M, Laisk-Podar T, et al. Pregnancy rate in endometriosis patients according to the severity of the disease after using a combined approach of laparoscopy, GnRH agonist treatment and in vitro fertilization. Gynecol Obstet Invest 2015;79(1):34-9. PMID: 25277802.

Stevenson EL and Sloane R. Certain Less Invasive Infertility Treatments Associated with Different Levels of Pregnancy-Related Anxiety in Pregnancies Conceived via In Vitro Fertilization. J Reprod Infertil 2017;18(1):190-196. PMID: 28377899. 
Steward RG, Gill I, Williams DB, et al. Cetrorelix lowers premature luteinization rate in gonadotropin ovulation induction-intrauterine insemination cycles: a randomized-controlled clinical trial. Fertil Steril 2011;95(1):434-6. PMID: 20810107.

Stochino-Loi E, Darwish B, Mircea O, et al. Does preoperative antimullerian hormone level influence postoperative pregnancy rate in women undergoing surgery for severe endometriosis?. Fertil Steril 2017;107(3):707-713.e3. PMID: 28089574.

Sun N, Wei L, Chen D, et al. Clinical observation of fallopian tube obstruction recanalization by ozone. Pakistan Journal of Medical Sciences 2017;33(2):290-294.

Sun XL, Wang JL, Peng YP, et al. Bilateral is superior to unilateral varicocelectomy in infertile males with left clinical and right subclinical varicocele: a prospective randomized controlled study. International Urology and Nephrology 2018;50(2):205-210.

Sutcliffe AG, Manning JT, Katalanic A, et al. Perturbations in finger length and digit ratio (2D:4D) in ICSI children. Reprod Biomed Online 2010;20(1):138-43. PMID: 20158999.

Taiyeb AM, Ridha-Albarzanchi MT, Taiyeb SM, et al. Improvement in pregnancy outcomes in couples with immunologically male infertility undergoing prednisolone treatment and conventional in vitro fertilization preceded by sperm penetration assay: a randomized controlled trial. Endocrine 2017;58(3):448-457. PMID: 29030775.

Takemura Y, Osuga Y, Fujimoto A, et al. Increased risk of placenta previa is associated with endometriosis and tubal factor infertility in assisted reproductive technology pregnancy. Gynecol Endocrinol 2013;29(2):113-5. PMID: 22835092.

Tendais I and Figueiredo B. Parents' anxiety and depression symptoms after successful infertility treatment and spontaneous conception: Does singleton/twin pregnancy matter?. Human Reproduction 2016;31(10):2303-2312.

Tjon-Kon-Fat RI, Tajik P, Zafarmand MH, et al. IVF or IUI as first-line treatment in unexplained subfertility: the conundrum of treatment selection markers. Hum Reprod 2017;32(5):1028-1032. PMID: 28333222.

Tredway D, Schertz JC, Bock D, et al. Anastrozole vs. clomiphene citrate in infertile women with ovulatory dysfunction: a phase II, randomized, dose-finding study. Fertil Steril 2011;95(5):1720-4.e1-8. PMID: 21300344.

Udawath M. A comparative study between clomiphene citrate with timed intercourse Vs clomiphene citrate with IUI. International Journal of Pharma and Bio Sciences 2014;5(3):P451P453.

Unfer V, Nestler JE, Kamenov ZA, et al. Effects of Inositol(s) in Women with PCOS: A Systematic Review of Randomized Controlled Trials. Int J Endocrinol 2016;2016:1849162. PMID: 27843451. 
van der Hoorn ML, Lashley EE, Bianchi DW, et al. Clinical and immunologic aspects of egg donation pregnancies: a systematic review. Hum Reprod Update 2010;16(6):704-12. PMID: 20543201.

van Eekelen R, Tjon-Kon-Fat RI, Bossuyt PMM, et al. Natural conception rates in couples with unexplained or mild male subfertility scheduled for fertility treatment: a secondary analysis of a randomized controlled trial. Hum Reprod 2018. PMID: 29538638.

Verberg MF, Eijkemans MJ, Heijnen EM, et al. Why do couples drop-out from IVF treatment? A prospective cohort study. Hum Reprod 2008;23(9):2050-5. PMID: 18544578.

Wadhwa L, Khanna R, Gupta T, et al. Evaluation of Role of GnRH Antagonist in Intrauterine Insemination (IUI) Cycles with Mild Ovarian Hyperstimulation (MOH): A Prospective Randomised Study. Journal of Obstetrics and Gynecology of India 2016:1-7.

Walschaerts M, Bujan L, Parinaud J, et al. Treatment discontinuation in couples consulting for male infertility after failing to conceive. Fertil Steril 2013;99(5):1319-23. PMID: 23260858.

Wang YA, Chughtai AA, Farquhar CM, et al. Increased incidence of gestational hypertension and preeclampsia after assisted reproductive technology treatment. Fertility and Sterility 2016;105(4):920-926.e2.

Weiss A, Neril R, Geslevich J, et al. Lag time from ovulation trigger to oocyte aspiration and oocyte maturity in assisted reproductive technology cycles: a retrospective study. Fertil Steril 2014;102(2):419-23. PMID: 24880653.

Wongtra-Ngan S, Vutyavanich T and Brown J. Follicular flushing during oocyte retrieval in assisted reproductive techniques. Cochrane Database Syst Rev 2010;(9):Cd004634. PMID: 20824839.

Wu K, Zhao H, Liu H, et al. Day 3 ET, single blastocyst transfer (SBT) or frozen-thawed embryo transfer (FET): which is preferable for high responder patients in IVF/ICSI cycles? J Assist Reprod Genet 2014;31(3):275-8. PMID: 24390677.

Xi W, Liu S, Mao H, et al. Use of letrozole and clomiphene citrate combined with gonadotropins in clomiphene-resistant infertile women with polycystic ovary syndrome: a prospective study. Drug Des Devel Ther 2015;9:6001-8. PMID: 26648691.

Xiao J, Chen S, Zhang C, et al. Effectiveness of GnRH antagonist in the treatment of patients with polycystic ovary syndrome undergoing IVF: a systematic review and meta analysis. Gynecol Endocrinol 2013;29(3):187-91. PMID: 23194095.

Xydias G, Liarmakopoulou S, Argyriou A, et al. A controlled trial of natural cycles with and without GnRH antagonist administration in poor responder women: Preliminary results. Review of Clinical Pharmacology and Pharmacokinetics, International Edition 2007;21(1):91-92. 
Yavangi M, Rabiee S, Nazari S, et al. Comparison of the effect of oestrogen plus Foeniculum vulgare seed and oestrogen alone on increase in endometrial thickness in infertile women. Journal of Clinical and Diagnostic Research 2018;12(1):QC01-QC04.

Ying L, Wu LH and Loke AY. Gender differences in emotional reactions to in vitro fertilization treatment: a systematic review. J Assist Reprod Genet 2016;33(2):167-79. PMID: 26712577.

Ying L, Wu LH and Loke AY. The effects of psychosocial interventions on the mental health, pregnancy rates, and marital function of infertile couples undergoing in vitro fertilization: a systematic review. J Assist Reprod Genet 2016. PMID: 26979745.

Youssef T and Abdalla E. Single incision transumbilical laparoscopic varicocelectomy versus the conventional laparoscopic technique: A randomized clinical study. Int J Surg 2015;18:178-83. PMID: 25937155.

Zhang H, Chu Y, Zhou P, et al. Dehydroepiandrosterone plus climen supplementation shows better effects than dehydroepiandrosterone alone on infertility patients with diminished ovarian reserve of low-FSH level undergoing in-vitro fertilization cycles: a randomized controlled trial. Reprod Biol Endocrinol 2016;14:9. PMID: 26879683.

Zhang H, Li H, Hou Y, et al. Microscopic Retroperitoneal Varicocelectomy With Artery and Lymphatic Sparing: An Alternative Treatment for Varicocele in Infertile Men. Urology 2015;86(3):511-5. PMID: 26151891.

Zhang $\mathrm{H}$, Zheng $\mathrm{Y}$, Wu Y, et al. A prospective randomized comparison of early embryo cleavage kinetics between two media culture systems. Pakistan Journal of Medical Sciences 2016;32(6):1375-1379.

Zhu L, Quan S, Xing Fq, et al. Application of Ultra-low-dose Incremental Gn Protocol in Controlled Ovarian Hyperstimulation of the Patients with Ovary Hyperreaction. Journal of Reproduction and Contraception 2009;20(3):145-152.

\section{Outcomes not reported by underlying diagnosis or by using a multivariate model that includes diagnosis as one of the covariates:}

Aaleyasin A, Aghahosseini M, Rashidi M, et al. In vitro fertilization outcome following embryo transfer with or without preinstillation of human chorionic gonadotropin into the uterine cavity: a randomized controlled trial. Gynecol Obstet Invest 2015;79(3):201-5. PMID: 25531413.

Aanesen A and Westerbotn M. Prospective study of a Swedish infertile cohort 2005-08: population characteristics, treatments and pregnancy rates. Fam Pract 2014;31(3):290-7. PMID: 24591683.

Abdalla $\mathrm{H}$ and Nicopoullos JD. The effect of duration of coasting and estradiol drop on the outcome of assisted reproduction: 13 years of experience in 1,068 coasted cycles to prevent ovarian hyperstimulation. Fertil Steril 2010;94(5):1757-63. PMID: 19939366. 
Abou-Setta AM, D'Angelo A, Hart R, et al. Post embryo transfer interventions for in vitro fertilization / intracytoplasmic sperm injection patients. Cochrane Database of Systematic Reviews 2007(2).

Abou-Setta AM, Mansour RT, Al-Inany HG, et al. Among women undergoing embryo transfer, is the probability of pregnancy and live birth improved with ultrasound guidance over clinical touch alone? A systemic review and meta-analysis of prospective randomized trials. Fertil Steril 2007;88(2):333-41. PMID: 17559845.

Abou-Setta AM, Peters LR, D'Angelo A, et al. Post-embryo transfer interventions for assisted reproduction technology cycles. Cochrane Database Syst Rev 2014;8:Cd006567. PMID: 25157849.

Absalan F, Ghannadi A and Kazerooni M. Reproductive outcome following thawed embryo transfer in management of ovarian hyperstimulation syndrome. Journal of Reproduction and Infertility 2013;14(3):133-137.

Acharya KS, Acharya CR, Provost MP, et al. Ectopic pregnancy rate increases with the number of retrieved oocytes in autologous in vitro fertilization with non-tubal infertility but not donor/recipient cycles: an analysis of 109,140 clinical pregnancies from the Society for Assisted Reproductive Technology registry. Fertil Steril 2015. PMID: 26171996.

Acharya KS, Keyhan S, Acharya CR, et al. Do donor oocyte cycles comply with ASRM/SART embryo transfer guidelines? An analysis of 13,393 donor cycles from the SART registry. Fertility and Sterility 2016;106(3):603-607.

Acharya KS, Provost MP, Yeh JS, et al. Ectopic pregnancy rates in frozen versus fresh embryo transfer in in vitro fertilization: A systematic review and meta-analysis. Middle East Fertility Society Journal 2014;19(4):233-238.

Adler-Levy Y, Lunenfeld E and Levy A. Obstetric outcome of twin pregnancies conceived by in vitro fertilization and ovulation induction compared with those conceived spontaneously. Eur $\mathrm{J}$ Obstet Gynecol Reprod Biol 2007;133(2):173-8. PMID: 17056174.

Aflatoonian A, Amouzegar H and Dehghani Firouzabadi R. Efficacy of 17alpha- hydroxy progestrone on decreasing preterm labor in ART pregnancies: A randomized clinical trial. Iran J Reprod Med 2013;11(10):785-90. PMID: 24639698.

Aflatoonian A, Hosseinisadat A, Baradaran R, et al. Pregnancy outcome of "delayed start" GnRH antagonist protocol versus GnRH antagonist protocol in poor responders: A clinical trial study. Int J Reprod Biomed (Yazd) 2017;15(4):231-238. PMID: 28835940.

Aflatoonian A, Mansoori Moghaddam F, Mashayekhy M, et al. Comparison of early pregnancy and neonatal outcomes after frozen and fresh embryo transfer in ART cycles. J Assist Reprod Genet 2010;27(12):695-700. PMID: 20886367.

Aflatoonian A, Mansoori-Torshizi M, Mojtahedi MF, et al. Fresh versus frozen embryo transfer after gonadotropin-releasing hormone agonist trigger in gonadotropin-releasing hormone 
antagonist cycles among high responder women: A randomized, multi-center study. International Journal of Reproductive BioMedicine 2018;16(1):9-18.

Aghssa MM, Azargoon A, Ramezanzadeh F, et al. A comparison of the efficacy, tolerability, and convenience of two formulations of follitropin-alpha in Iranian woman undergoing intracytoplasmic sperm injection cycles. Fertil Steril 2008;90(4):1043-8. PMID: 18053995.

Ahmad G, Brown J, Duffy JM, et al. Growth hormone for in vitro fertilization. Cochrane Database Syst Rev 2009;(4):Cd000099. PMID: 19821264.

Ahmadi SE, Montazeri A, Mozafari R, et al. Health-Related Quality of Life and Primi-Gravid: A Comparative Study of Natural Conception and Conception by Assisted Reproduction Technologies (ARTs). Int J Fertil Steril 2014;8(2):167-74. PMID: 25083182.

Ahmadi SE, Nateghi MR, Gourabi H, et al. Frequency of hearing defect and ear abnormalities in newborns conceived by assisted reproductive techniques in Royan institute. International Journal of Fertility and Sterility 2010;4(2):79-84.

Akhtar MA, Eljabu H, Hopkisson J, et al. Aspirin and heparin as adjuvants during IVF do not improve live birth rates in unexplained implantation failure. Reprod Biomed Online 2013;26(6):586-94. PMID: 23518029.

Akhtar MA, Sur S, Raine-Fenning N, et al. Heparin for assisted reproduction. Cochrane Database Syst Rev 2013;8:Cd009452. PMID: 23955506.

Akhtar MA, Sur S, Raine-Fenning N, et al. Heparin for assisted reproduction: summary of a Cochrane review. Fertil Steril 2015;103(1):33-4. PMID: 25282470.

Albuquerque LE, Tso LO, Saconato H, et al. Depot versus daily administration of gonadotrophin-releasing hormone agonist protocols for pituitary down regulation in assisted reproduction cycles. Cochrane Database Syst Rev 2013;1:Cd002808. PMID: 23440788.

Aleyasin A, Aghahosseini M, Safdarian L, et al. Can letrozole plus HMG protocol improve pregnancy outcomes in frozen-thawed embryo transfer? An RCT. International Journal of Reproductive BioMedicine 2017;15(2):83-86.

Al-Inany $\mathrm{H}$ and van Gelder P. Success of frozen embryo transfer: Does the type of gonadotropin influence the outcome? Int J Womens Health 2010;2:89-98. PMID: 21072302.

Al-Inany HG, Abou-Setta AM and Aboulghar M. Gonadotrophin-releasing hormone antagonists for assisted conception: a Cochrane review. Reprod Biomed Online 2007;14(5):640-9. PMID: 17509210.

Al-Inany HG, Abou-Setta AM, Aboulghar MA, et al. Highly purified hMG achieves better pregnancy rates in IVF cycles but not ICSI cycles compared with recombinant FSH: a metaanalysis. Gynecol Endocrinol 2009;25(6):372-8. PMID: 19340668. 
Allersma T, Farquhar C and Cantineau AE. Natural cycle in vitro fertilisation (IVF) for subfertile couples. Cochrane Database Syst Rev 2013;8:Cd010550. PMID: 23990351.

Alleyassin A, Ghasemi M, Aghahosseini M, et al. Final oocyte maturation with a dual trigger compared to human chorionic gonadotropin trigger in antagonist co-treated cycles: A randomized clinical trial. Middle East Fertility Society Journal 2018.

Alsbjerg B, Polyzos NP, Elbaek HO, et al. Increasing vaginal progesterone gel supplementation after frozen-thawed embryo transfer significantly increases the delivery rate. Reprod Biomed Online 2013;26(2):133-7. PMID: 23265957.

Altmae S, Mendoza-Tesarik R, Mendoza C, et al. Effect of Growth Hormone on Uterine Receptivity in Women With Repeated Implantation Failure in an Oocyte Donation Program: A Randomized Controlled Trial. J Endocr Soc 2018;2(1):96-105. PMID: 29379897.

Al-Turki HA. Obstetric management after infertility treatment. Ann Afr Med 2010;9(2):77-80. PMID: 20587928.

Alvarez C, Marti-Bonmati L, Novella-Maestre E, et al. Dopamine agonist cabergoline reduces hemoconcentration and ascites in hyperstimulated women undergoing assisted reproduction. $\mathrm{J}$ Clin Endocrinol Metab 2007;92(8):2931-7. PMID: 17456571.

Amir H, Yaniv D, Hasson J, et al. Cabergoline for reducing ovarian hyperstimulation syndrome in assisted reproductive technology treatment cycles. A prospective randomized controlled trial. J Reprod Med 2015;60(1-2):48-54. PMID: 25745751.

Ammar AR, Mousa KS, Rabei NH, et al. Effect of ultrasound guided embryo transfer on pregnancy rates. New York Science Journal 2013;6(12):79-83.

Angel M, Ghose S and Gowda M. A randomized trial comparing the ovulation induction efficacy of clomiphene citrate and letrozole. J Nat Sci Biol Med 2014;5(2):450-2. PMID: 25097433.

Ardalan M, Rafati A, Nejat F, et al. Risk factors associated with craniosynostosis: a case control study. Pediatr Neurosurg 2012;48(3):152-6. PMID: 23428561.

Armstrong S, Arroll N, Cree LM, et al. Time-lapse systems for embryo incubation and assessment in assisted reproduction. Cochrane Database Syst Rev 2015;2:Cd011320. PMID: 25721906.

Asante A, Leonard PH, Weaver AL, et al. Fertility drug use and the risk of ovarian tumors in infertile women: a case-control study. Fertil Steril 2013;99(7):2031-6. PMID: 23552324.

Asato K, Mekaru K, Heshiki C, et al. Subchorionic hematoma occurs more frequently in in vitro fertilization pregnancy. Eur J Obstet Gynecol Reprod Biol 2014;181:41-4. PMID: 25126980.

Ashrafi M, Tehraninejad ES, Haghiri M, et al. The effect of endometrial scratch injury on pregnancy outcome in women with previous intrauterine insemination failure: A randomized clinical trial. Journal of Obstetrics and Gynaecology Research 2017;43(9):1421-1427. 
Athar R, Mehrnoosh M, Farzaneh A, et al. Assessing an optimal regimen in treatment of infertility (clomiphene citrate, tamoxifen and vit. E versus estrogen, letrozole and tamoxifen): A double blind control trial. International Journal of Pharmacology 2015;11(4):377-381.

Baker VL, Fujimoto VY, Kettel LM, et al. Clinical efficacy of highly purified urinary FSH versus recombinant FSH in volunteers undergoing controlled ovarian stimulation for in vitro fertilization: a randomized, multicenter, investigator-blind trial. Fertil Steril 2009;91(4):1005-11. PMID: 18367182.

Bakos HW, Henshaw RC, Mitchell M, et al. Paternal body mass index is associated with decreased blastocyst development and reduced live birth rates following assisted reproductive technology. Fertil Steril 2011;95(5):1700-4. PMID: 21145051.

Balakier H, Mandel R, Sojecki A, et al. Laser zona thinning in women aged <or=37 years: a randomized study. Fertil Steril 2009;91(4 Suppl):1479-82. PMID: 18793768.

Bamberg C, Fotopoulou C, Neissner P, et al. Maternal characteristics and twin gestation outcomes over 10 years: impact of conception methods. Fertil Steril 2012;98(1):95-101. PMID: 22608318.

Barbosa MW, Sotiriadis A, Papatheodorou SI, et al. High miscarriage rate in women submitted to Essure for hydrosalpinx before embryo transfer: a systematic review and meta-analysis. Ultrasound Obstet Gynecol 2016. PMID: 27152454.

Bassiouny YA, Dakhly DMR, Bayoumi YA, et al. Does the addition of growth hormone to the in vitro fertilization/intracytoplasmic sperm injection antagonist protocol improve outcomes in poor responders? A randomized, controlled trial. Fertility and Sterility 2016;105(3):697-702.

Baum M, Yerushalmi GM, Maman E, et al. Does local injury to the endometrium before IVF cycle really affect treatment outcome? Results of a randomized placebo controlled trial. Gynecol Endocrinol 2012;28(12):933-6. PMID: 22943664.

Bay B, Ingerslev HJ, Lemmen JG, et al. Preimplantation genetic diagnosis: a national multicenter obstetric and neonatal follow-up study. Fertil Steril 2016;106(6):1363-1369.e1. PMID: 27542705.

Bay B, Lykke E, Hvidtjørn D, et al. Fertility treatment and risk of childhood and adolescent mental disorders: Register based cohort study. BMJ (Online) 2013;347(7918).

Bay B, Mortensen EL and Kesmodel US. Assisted reproduction and child neurodevelopmental outcomes: a systematic review. Fertil Steril 2013;100(3):844-53. PMID: 23810272.

Bay B, Mortensen EL and Kesmodel US. Fertility treatment and child intelligence, attention, and executive functions in 5-year-old singletons: a cohort study. Bjog 2014;121(13):1642-51. PMID: 24910085. 
Bay B, Mortensen EL and Kesmodel US. Is subfertility or fertility treatment associated with long-term growth in the offspring? A cohort study. Fertil Steril 2014;102(4):1117-23. PMID: 25123636.

Bay B, Mortensen EL, Golombok S, et al. Long-awaited pregnancy: intelligence and academic performance in offspring of infertile parents-a cohort study. Fertil Steril 2016;106(5):10331040.e1. PMID: 27379706.

Bay B, Mortensen EL, Hvidtjorn D, et al. Fertility treatment and risk of childhood and adolescent mental disorders: register based cohort study. Bmj 2013;347:f3978. PMID: 23833075.

Bay B. Fertility treatment: long-term growth and mental development of the children. Dan Med J 2014;61(10):B4947. PMID: 25283630.

Bechoua S, Astruc K, Thouvenot S, et al. How to demonstrate that eSET does not compromise the likelihood of having a baby? Hum Reprod 2009;24(12):3073-81. PMID: 19752013.

Becker GF, Passos EP and Moulin CC. Short-term effects of a hypocaloric diet with low glycemic index and low glycemic load on body adiposity, metabolic variables, ghrelin, leptin, and pregnancy rate in overweight and obese infertile women: a randomized controlled trial. Am J Clin Nutr 2015;102(6):1365-72. PMID: 26561614.

Beck-Fruchter R, Lavee M, Weiss A, et al. Rescue intracytoplasmic sperm injection: a systematic review. Fertil Steril 2014;101(3):690-8. PMID: 24444597.

Bellavia M, de Geyter C, Streuli I, et al. Randomized controlled trial comparing highly purified (HP-hCG) and recombinant hCG (r-hCG) for triggering ovulation in ART. Gynecol Endocrinol 2013;29(2):93-7. PMID: 23116325.

Bellver J, Ayllon Y, Ferrando M, et al. Female obesity impairs in vitro fertilization outcome without affecting embryo quality. Fertil Steril 2010;93(2):447-54. PMID: 19171335.

Beltran Anzola A, Pauly V, Montjean D, et al. No difference in congenital anomalies prevalence irrespective of insemination methods and freezing procedure: cohort study over fourteen years of an ART population in the south of France. J Assist Reprod Genet 2017;34(7):867-876. PMID: 28444613.

Belva F, Roelants M, De Schepper J, et al. Blood pressure in ICSI-conceived adolescents. Hum Reprod 2012;27(10):3100-8. PMID: 22814483.

Bensdorp AJ, Tjon-Kon-Fat RI, Bossuyt PM, et al. Prevention of multiple pregnancies in couples with unexplained or mild male subfertility: randomised controlled trial of in vitro fertilisation with single embryo transfer or in vitro fertilisation in modified natural cycle compared with intrauterine insemination with controlled ovarian hyperstimulation. Bmj 2015;350:g7771. PMID: 25576320. 
Besselink DE, Marjoribanks J, Farquhar C, et al. Cervical insemination versus intra-uterine insemination of donor sperm for subfertility. Cochrane Database of Systematic Reviews 2007;(3).

Best D, Avenell A and Bhattacharya S. How effective are weight-loss interventions for improving fertility in women and men who are overweight or obese? A systematic review and meta-analysis of the evidence. Hum Reprod Update 2017;23(6):681-705. PMID: 28961722.

Beukers F, Houtzager BA, Paap MC, et al. Parental psychological distress and anxiety after a successful IVF/ICSI procedure with and without preimplantation genetic screening: follow-up of a randomised controlled trial. Early Hum Dev 2012;88(9):725-30. PMID: 22460061.

Beukers F, van der Heide M, Middelburg KJ, et al. Morphologic abnormalities in 2-year-old children born after in vitro fertilization/intracytoplasmic sperm injection with preimplantation genetic screening: follow-up of a randomized controlled trial. Fertil Steril 2013;99(2):408-13. PMID: 23127590.

Bhandari S, Ganguly I, Agarwal P, et al. Relationship of number of embryos transferred with perinatal outcome of singleton pregnancy. Journal of Reproduction and Infertility 2017;18(1):179-184.

Bhatt $\mathrm{T}$ and Baibergenova A. A comparison of the cost-effectiveness of in vitro fertilization strategies and stimulated intrauterine insemination in a Canadian health economic model. J Obstet Gynaecol Can 2008;30(5):411-20. PMID: 18505665.

Bjercke S, Tanbo T, Abyholm T, et al. Clinical outcome following stimulation with highly purified hMG or recombinant FSH in patients undergoing their first treatment cycle of IVF or ICSI. Acta Obstet Gynecol Scand 2010;89(8):1053-60. PMID: 20602599.

Bjornholt SM, Kjaer SK, Nielsen TS, et al. Risk for borderline ovarian tumours after exposure to fertility drugs: results of a population-based cohort study. Hum Reprod 2015;30(1):222-31.

PMID: 25376453.

Blake DA, Farquhar CM, Johnson N, et al. Cleavage stage versus blastocyst stage embryo transfer in assisted conception. Cochrane Database Syst Rev 2007;(4):Cd002118. PMID: 17943767.

Bodri D, Kawachiya S, Kondo M, et al. Oocyte retrieval timing based on spontaneous luteinizing hormone surge during natural cycle in vitro fertilization treatment. Fertil Steril 2014;101(4):1001-7.e2. PMID: 24534290.

Boivin J and Schmidt L. Use of complementary and alternative medicines associated with a 30\% lower ongoing pregnancy/live birth rate during 12 months of fertility treatment. Hum Reprod 2009;24(7):1626-31. PMID: 19359338.

Bontekoe S, Heineman MJ, Johnson N, et al. Adherence compounds in embryo transfer media for assisted reproductive technologies. Cochrane Database Syst Rev 2014;2:Cd007421. PMID: 24567053. 
Bontekoe S, Mantikou E, van Wely M, et al. Low oxygen concentrations for embryo culture in assisted reproductive technologies. Cochrane Database Syst Rev 2012;7:Cd008950. PMID: 22786519.

Boomsma CM, Keay SD and Macklon NS. Peri-implantation glucocorticoid administration for assisted reproductive technology cycles. Cochrane Database Syst Rev 2007;(1):Cd005996. PMID: 17253574.

Boomsma CM, Keay SD and Macklon NS. Peri-implantation glucocorticoid administration for assisted reproductive technology cycles. Cochrane Database Syst Rev 2012;6:Cd005996. PMID: 22696356.

Boostanfar R, Gates D, Guan Y, et al. Efficacy and safety of frozen-thawed embryo transfer in women aged 35 to 42 years from the PURSUE randomized clinical trial. Fertil Steril 2016. PMID: 27090863.

Boostanfar R, Shapiro B, Levy M, et al. Large, comparative, randomized double-blind trial confirming noninferiority of pregnancy rates for corifollitropin alfa compared with recombinant follicle-stimulating hormone in a gonadotropin-releasing hormone antagonist controlled ovarian stimulation protocol in older patients undergoing in vitro fertilization. Fertility and Sterility 2015;104(1):94-103.e1.

Brandes M, Hamilton CJ, Bergevoet KA, et al. Origin of multiple pregnancies in a subfertile population. Acta Obstet Gynecol Scand 2010;89(9):1149-54. PMID: 20804340.

Brinton LA, Trabert B, Shalev V, et al. In vitro fertilization and risk of breast and gynecologic cancers: a retrospective cohort study within the Israeli Maccabi Healthcare Services. Fertil Steril 2013;99(5):1189-96. PMID: 23375197.

Bromer JG, Cetinkaya MB and Arici A. Pretreatments before the induction of ovulation in assisted reproduction technologies: evidence-based medicine in 2007. Ann N Y Acad Sci 2008;1127:31-40. PMID: 18443327.

Bromer JG, Sakkas D, Siano LJ, et al. Reproductive efficiency of women over the age of 40 and the low risk of multiple pregnancies. Reprod Biomed Online 2009;19(Suppl 4):4316. PMID: 20034414.

Brown J and Farquhar C. Endometriosis: an overview of Cochrane Reviews. Cochrane Database Syst Rev 2014;3:Cd009590. PMID: 24610050.

Brown J, Buckingham K, Abou-Setta AM, et al. Ultrasound versus 'clinical touch' for catheter guidance during embryo transfer in women. Cochrane Database Syst Rev 2010;(1):Cd006107. PMID: 20091584.

Brown J, Daya S and Matson P. Day three versus day two embryo transfer following in vitro fertilization or intracytoplasmic sperm injection. Cochrane Database Syst Rev 2016;12:Cd004378. PMID: 27976360. 
Brown JA, Buckingham K, Abou-Setta A, et al. Ultrasound versus 'clinical touch' for catheter guidance during embryo transfer in women. Cochrane Database Syst Rev 2007;(1):Cd006107. PMID: 17253582.

Busso C, Fernandez-Sanchez M, Garcia-Velasco JA, et al. The non-ergot derived dopamine agonist quinagolide in prevention of early ovarian hyperstimulation syndrome in IVF patients: a randomized, double-blind, placebo-controlled trial. Hum Reprod 2010;25(4):995-1004. PMID: 20139430.

Calderon-Margalit R, Friedlander Y, Yanetz R, et al. Cancer risk after exposure to treatments for ovulation induction. Am J Epidemiol 2009;169(3):365-75. PMID: 19037008.

Camprubi C, Iglesias-Platas I, Martin-Trujillo A, et al. Stability of genomic imprinting and gestational-age dynamic methylation in complicated pregnancies conceived following assisted reproductive technologies. Biol Reprod 2013;89(3):50. PMID: 23884645.

Cantineau AE, Cohlen BJ and Heineman MJ. Intra-uterine insemination versus fallopian tube sperm perfusion for non-tubal infertility. Cochrane Database Syst Rev 2009;(2):Cd001502. PMID: 19370567.

Cantineau AE, Cohlen BJ and Heineman MJ. Ovarian stimulation protocols (anti-oestrogens, gonadotrophins with and without GnRH agonists/antagonists) for intrauterine insemination (IUI) in women with subfertility. Cochrane Database Syst Rev 2007;(2):Cd005356. PMID: 17443584.

Cantineau AE, Cohlen BJ, Heineman MJ, et al. Intrauterine insemination versus fallopian tube sperm perfusion for non-tubal infertility. Cochrane Database Syst Rev 2013;10:Cd001502. PMID: 24174382.

Cantineau AE, Cohlen BJ, Klip H, et al. The addition of GnRH antagonists in intrauterine insemination cycles with mild ovarian hyperstimulation does not increase live birth rates--a randomized, double-blinded, placebo-controlled trial. Hum Reprod 2011;26(5):1104-11. PMID: 21339196.

Cantineau AE, Janssen MJ and Cohlen BJ. Synchronised approach for intrauterine insemination in subfertile couples. Cochrane Database Syst Rev 2010;(4):Cd006942. PMID: 20393953.

Cantineau AE, Janssen MJ, Cohlen BJ, et al. Synchronised approach for intrauterine insemination in subfertile couples. Cochrane Database Syst Rev 2014;12:Cd006942. PMID: 25528596.

Carizza C, Abdelmassih V, Abdelmassih S, et al. Cabergoline reduces the early onset of ovarian hyperstimulation syndrome: a prospective randomized study. Reprod Biomed Online 2008;17(6):751-5. PMID: 19079957.

Carney SK, Das S, Blake D, et al. Assisted hatching on assisted conception (in vitro fertilisation (IVF) and intracytoplasmic sperm injection (ICSI). Cochrane Database Syst Rev 2012;12:Cd001894. PMID: 23235584. 
Carrasco B, Boada M, Rodriguez I, et al. Does culture medium influence offspring birth weight?. Fertil Steril 2013;100(5):1283-8. PMID: 23916797.

Carson C, Kurinczuk JJ, Sacker A, et al. Cognitive development following ART: effect of choice of comparison group, confounding and mediating factors. Hum Reprod 2010;25(1):244-52. PMID: 19828556.

Carson C, Redshaw M, Sacker A, et al. Effects of pregnancy planning, fertility, and assisted reproductive treatment on child behavioral problems at 5 and 7 years: evidence from the Millennium Cohort Study. Fertil Steril 2013;99(2):456-63. PMID: 23158833.

Carson C, Sacker A, Kelly Y, et al. Asthma in children born after infertility treatment: findings from the UK Millennium Cohort Study. Hum Reprod 2013;28(2):471-9. PMID: 23223378.

Caserta D, Bordi G, Stegagno M, et al. Maternal and perinatal outcomes in spontaneous versus assisted conception twin pregnancies. Eur J Obstet Gynecol Reprod Biol 2014;174:64-9. PMID: 24405729.

Caserta D, Marci R, Tatone C, et al. IVF pregnancies: neonatal outcomes after the new Italian law on assisted reproduction technology (law 40/2004). Acta Obstet Gynecol Scand 2008;87(9):935-9. PMID: 18720037.

Catford SR, McLachlan RI, O'Bryan MK, et al. Long-term follow-up of ICSI-conceived offspring compared with spontaneously conceived offspring: a systematic review of health outcomes beyond the neonatal period. Andrology 2018.

Cedrin-Durnerin I, Guivarc'h-Leveque A and Hugues JN. Pretreatment with estrogen does not affect IVF-ICSI cycle outcome compared with no pretreatment in GnRH antagonist protocol: a prospective randomized trial. Fertil Steril 2012;97(6):1359-64.e1. PMID: 22464760.

Ceelen M, van Weissenbruch MM, Prein J, et al. Growth during infancy and early childhood in relation to blood pressure and body fat measures at age 8-18 years of IVF children and spontaneously conceived controls born to subfertile parents. Hum Reprod 2009;24(11):2788-95. PMID: 19648588.

Ceelen M, van Weissenbruch MM, Roos JC, et al. Body composition in children and adolescents born after in vitro fertilization or spontaneous conception. J Clin Endocrinol Metab 2007;92(9):3417-23. PMID: 17595253.

Ceelen M, van Weissenbruch MM, Vermeiden JP, et al. Cardiometabolic differences in children born after in vitro fertilization: follow-up study. J Clin Endocrinol Metab 2008;93(5):1682-8. PMID: 18285409.

Ceelen M, van Weissenbruch MM, Vermeiden JP, et al. Pubertal development in children and adolescents born after IVF and spontaneous conception. Hum Reprod 2008;23(12):2791-8. PMID: 18689849. 
Chaabane S, Sheehy O, Monnier P, et al. Association between ovarian stimulators with or without intrauterine insemination, and assisted reproductive technologies on multiple births. Am J Obstet Gynecol 2015. PMID: 26079626.

Chaabane S, Sheehy O, Monnier P, et al. Ovarian Stimulators, Intrauterine Insemination, and Assisted Reproductive Technologies Use and the Risk of Major Congenital Malformations-The AtRISK Study. Birth Defects Res B Dev Reprod Toxicol 2016;107(3):136-47. PMID: 27295407.

Chachamovich J, Chachamovich E, Fleck MP, et al. Congruence of quality of life among infertile men and women: findings from a couple-based study. Hum Reprod 2009;24(9):2151-7. PMID: 19451130.

Chachamovich JR, Chachamovich E, Ezer H, et al. Investigating quality of life and healthrelated quality of life in infertility: a systematic review. J Psychosom Obstet Gynaecol 2010;31(2):101-10. PMID: 20443659.

Chambers GM, Lee E, Hoang VP, et al. Hospital utilization, costs and mortality rates during the first 5 years of life: a population study of ART and non-ART singletons. Hum Reprod 2014;29(3):601-10. PMID: 24310618.

Chambers GM, Sullivan EA, Shanahan M, et al. Is in vitro fertilisation more effective than stimulated intrauterine insemination as a first-line therapy for subfertility? A cohort analysis. Aust N Z J Obstet Gynaecol 2010;50(3):280-8. PMID: 20618248.

Chan $\mathrm{CH}$, Chan $\mathrm{CL}$, Ng EH, et al. Incorporating spirituality in psychosocial group intervention for women undergoing in vitro fertilization: a prospective randomized controlled study. Psychol Psychother 2012;85(4):356-73. PMID: 23080528.

Chan RVP, Yonekawa Y, Morrison MA, et al. Association between assisted reproductive technology and advanced retinopathy of prematurity. 2010;4:1385-1390.

Chang HJ, Lee JR, Jee BC, et al. Impact of blastocyst transfer on offspring sex ratio and the monozygotic twinning rate: a systematic review and meta-analysis. Fertil Steril 2009;91(6):2381-90. PMID: 18718582.

Chavarro JE, Ehrlich S, Colaci DS, et al. Body mass index and short-term weight change in relation to treatment outcomes in women undergoing assisted reproduction. Fertil Steril 2012;98(1):109-16. PMID: 22607889.

Check JH, Jamison T, Check D, et al. Live delivery and implantation rates of donor oocyte recipients in their late forties are similar to younger recipients. J Reprod Med 2011;56(3-4):14952. PMID: 21542533.

Check JH, Liss J and Cohen R. A comparison of three types of therapies for three different ovulation disorders in establishing pregnancies and evaluation of laboratory parameters that could influence the outcome. Clin Exp Obstet Gynecol 2013;40(3):317-8. PMID: 24283155. 
Check JH, Wilson C, Levine $\mathrm{K}$, et al. Improved implantation and live delivered pregnancy rates following transfer of embryos derived from donor oocytes by single injection of leuprolide in mid-luteal phase. Clinical and Experimental Obstetrics and Gynecology 2015;42(4):429-430.

Chen H, Wang Y, Lyu Q, et al. Comparison of live-birth defects after luteal-phase ovarian stimulation vs. conventional ovarian stimulation for in vitro fertilization and vitrified embryo transfer cycles. Fertil Steril 2015;103(5):1194-1201.e2. PMID: 25813280.

Chen Y, Zhang Y, Hu M, et al. Timing of human chorionic gonadotropin (hCG) hormone administration in IVF/ICSI protocols using GnRH agonist or antagonists: a systematic review and meta-analysis. Gynecol Endocrinol 2014;30(6):431-7. PMID: 24731070.

Cheong Y, Nardo LG, Rutherford T, et al. Acupuncture and herbal medicine in in vitro fertilisation: a review of the evidence for clinical practice. Hum Fertil (Camb) 2010;13(1):3-12. PMID: 20053149.

Cheong YC, Dix S, Hung Yu Ng E, et al. Acupuncture and assisted reproductive technology. Cochrane Database Syst Rev 2013;7:Cd006920. PMID: 23888428.

Cheong YC, Hung Yu Ng E and Ledger WL. Acupuncture and assisted conception. Cochrane Database Syst Rev 2008;(4):Cd006920. PMID: 18843737.

Cheung CS, Chan $\mathrm{CH}$ and Ng EH. Stress and anxiety-depression levels following first-trimester miscarriage: a comparison between women who conceived naturally and women who conceived with assisted reproduction. Bjog 2013;120(9):1090-7. PMID: 23631687.

Chiamchanya C and Su-angkawatin W. Study of the causes and the results of treatment in infertile couples at Thammasat Hospital between 1999-2004. J Med Assoc Thai 2008;91(6):80512. PMID: 18697377.

Chiarelli L, Mirea L, Yang J, et al. Neonatal Outcomes in Very Preterm Singleton Infants Conceived Using Assisted Reproductive Technologies. American Journal of Perinatology 2015;32(6):515-522.

Chibber R and Al-Sibai MH. Pregnancy following infertility: a concern? J Obstet Gynaecol 2007;27(8):806-9. PMID: 18097899.

Child T, Leonard SA, Evans JS, et al. Systematic review of the clinical efficacy of vaginal progesterone for luteal phase support in assisted reproductive technology cycles. Reproductive BioMedicine Online 2018

Chou PY, Wu MH, Pan HA, et al. Use of an oxytocin antagonist in in vitro fertilization-embryo transfer for women with repeated implantation failure: a retrospective study. Taiwan J Obstet Gynecol 2011;50(2):136-40. PMID: 21791296.

Christiansen T, Erb K, Rizvanovic A, et al. Costs of medically assisted reproduction treatment at specialized fertility clinics in the Danish public health care system: results from a 5-year followup cohort study. Acta Obstet Gynecol Scand 2014;93(1):64-72. PMID: 24266525. 
Chughtai AA, Wang AY, Hilder L, et al. Gestational age-specific perinatal mortality rates for assisted reproductive technology (ART) and other births. Human Reproduction 2018;33(2):320327.

Cil AP, Bang H and Oktay K. Age-specific probability of live birth with oocyte cryopreservation: an individual patient data meta-analysis. Fertil Steril 2013;100(2):492-9.e3. PMID: 23706339.

Clark NA, Will M, Moravek MB, et al. A systematic review of the evidence for complementary and alternative medicine in infertility. Int J Gynaecol Obstet 2013;122(3):202-6. PMID: 23796256.

Clarke JF, van Rumste MM, Farquhar CM, et al. Measuring outcomes in fertility trials: can we rely on clinical pregnancy rates? Fertil Steril 2010;94(5):1647-51. PMID: 20056216.

Clayton HB, Schieve LA, Peterson HB, et al. A comparison of heterotopic and intrauterine-only pregnancy outcomes after assisted reproductive technologies in the United States from 1999 to 2002. Fertil Steril 2007;87(2):303-9. PMID: 17113092.

Coates A, Kung A, Mounts E, et al. Optimal euploid embryo transfer strategy, fresh versus frozen, after preimplantation genetic screening with next generation sequencing: a randomized controlled trial. Fertil Steril 2017;107(3):723-730.e3. PMID: 28139240.

Colaci DS, Afeiche M, Gaskins AJ, et al. Men's body mass index in relation to embryo quality and clinical outcomes in couples undergoing in vitro fertilization. Fertil Steril 2012;98(5):11939.e1. PMID: 22884013.

Connell MT, Szatkowski JM, Terry N, et al. Timing luteal support in assisted reproductive technology: a systematic review. Fertil Steril 2015;103(4):939-946.e3. PMID: 25638420.

Corchia C, Da Frè M, Di Lallo D, et al. Mortality and major morbidities in very preterm infants born from assisted conception or naturally conceived: Results of the area-based ACTION study. BMC Pregnancy and Childbirth 2014;14(1).

Court KA, Dare AJ, Weston-Webb M, et al. Establishment of lipiodol as a fertility treatment prospective study of the complete innovative treatment data set. Aust N Z J Obstet Gynaecol 2014;54(1):13-9. PMID: 24033087.

Craciunas L, Tsampras N and Fitzgerald C. Cervical mucus removal before embryo transfer in women undergoing in vitro fertilization/intracytoplasmic sperm injection: a systematic review and meta-analysis of randomized controlled trials. Fertil Steril 2014;101(5):1302-7. PMID: 24602754.

Crawford S, Boulet SL, Jamieson DJ, et al. Assisted reproductive technology use, embryo transfer practices, and birth outcomes after infertility insurance mandates: New Jersey and Connecticut. Fertil Steril 2016;105(2):347-55. PMID: 26515377. 
Crawford S, Boulet SL, Mneimneh AS, et al. Costs of achieving live birth from assisted reproductive technology: a comparison of sequential single and double embryo transfer approaches. Fertil Steril 2016;105(2):444-50. PMID: 26604068.

Cusido M, Fabregas R, Pere BS, et al. Ovulation induction treatment and risk of borderline ovarian tumors. Gynecol Endocrinol 2007;23(7):373-6. PMID: 17701767.

Custers IM, Flierman PA, Maas P, et al. Immobilisation versus immediate mobilisation after intrauterine insemination: randomised controlled trial. Bmj 2009;339:b4080. PMID: 19875843.

Custers IM, Konig TE, Broekmans FJ, et al. Couples with unexplained subfertility and unfavorable prognosis: a randomized pilot trial comparing the effectiveness of in vitro fertilization with elective single embryo transfer versus intrauterine insemination with controlled ovarian stimulation. Fertil Steril 2011;96(5):1107-11.e1. PMID: 21890134.

Dain L, Ojha K, Bider D, et al. Effect of local endometrial injury on pregnancy outcomes in ovum donation cycles. Fertil Steril 2014;102(4):1048-54. PMID: 25064410.

Damgaard IN, Jensen TK, Petersen JH, et al. Risk factors for congenital cryptorchidism in a prospective birth cohort study. PLoS One 2008;3(8):e3051. PMID: 18725961.

D'Angelo A and Amso N. Embryo freezing for preventing ovarian hyperstimulation syndrome. Cochrane Database Syst Rev 2007;(3):Cd002806. PMID: 17636707.

D'Angelo A, Brown J and Amso NN. Coasting (withholding gonadotrophins) for preventing ovarian hyperstimulation syndrome. Cochrane Database Syst Rev 2011;(2):Cd002811. PMID: 21328256.

D'Angelo A, Brown J and Amso NN. Coasting (withholding gonadotrophins) for preventing ovarian hyperstimulation syndrome. Cochrane Database Syst Rev 2011;(6):Cd002811. PMID: 21678336.

D'Angelo DV, Whitehead N, Helms K, et al. Birth outcomes of intended pregnancies among women who used assisted reproductive technology, ovulation stimulation, or no treatment. Fertil Steril 2011;96(2):314-320.e2. PMID: 21718990.

Dankert T, Kremer JA, Cohlen BJ, et al. A randomized clinical trial of clomiphene citrate versus low dose recombinant FSH for ovarian hyperstimulation in intrauterine insemination cycles for unexplained and male subfertility. Hum Reprod 2007;22(3):792-7. PMID: 17110396.

Das S, Blake D, Farquhar C, et al. Assisted hatching on assisted conception (IVF and ICSI). Cochrane Database Syst Rev 2009;(2):Cd001894. PMID: 19370570.

Datta AK, Vitthala S, Tozer A, et al. Controlled ovarian hyperstimulation for low responders in in vitro fertilization/intracytoplasmic sperm injection: a low-dose flare protocol. Fertil Steril 2011;95(5):1809-12. PMID: 21168131. 
Davar R, Ghandi S and Tayebi N. Does transabdominal ultrasound-guided embryo transfer improve pregnancy rates in ART cycles?. Iranian Journal of Reproductive Medicine 2007;5(3):95-98.

Davies MJ, Moore VM, Willson KJ, et al. Reproductive technologies and the risk of birth defects. N Engl J Med 2012;366(19):1803-13. PMID: 22559061.

Dayal MB, Gindoff P, Dubey A, et al. Does ethnicity influence in vitro fertilization (IVF) birth outcomes? Fertil Steril 2009;91(6):2414-8. PMID: 18691706.

Dayan N, Filion KB, Okano M, et al. Cardiovascular Risk Following Fertility Therapy: Systematic Review and Meta-Analysis. J Am Coll Cardiol 2017;70(10):1203-1213. PMID: 28859782.

De Brucker M, Tournaye H, Haentjens P, et al. Assisted reproduction counseling in women aged 40 and above: a cohort study. J Assist Reprod Genet 2013;30(11):1431-8. PMID: 23982590.

de Klerk C, Macklon NS, Heijnen EM, et al. The psychological impact of IVF failure after two or more cycles of IVF with a mild versus standard treatment strategy. Hum Reprod 2007;22(9):2554-8. PMID: 17586832.

De Neubourg D, Bogaerts K, Blockeel C, et al. How do cumulative live birth rates and cumulative multiple live birth rates over complete courses of assisted reproductive technology treatment per woman compare among registries?. Human Reproduction 2016;31(1):93-99.

De Vos A, Janssens R, Van de Velde H, et al. The type of culture medium and the duration of in vitro culture do not influence birthweight of ART singletons. Hum Reprod 2015;30(1):20-7. PMID: 25406185.

Debrock S, Spiessens C, Peeraer K, et al. Higher implantation rate using modified quarter laserassisted zona thinning in repeated implantation failure. Gynecol Obstet Invest 2009;67(2):12733. PMID: 19005260.

Declercq E, Luke B, Belanoff C, et al. Perinatal outcomes associated with assisted reproductive technology: the Massachusetts Outcomes Study of Assisted Reproductive Technologies (MOSART). Fertil Steril 2015;103(4):888-95. PMID: 25660721.

Deemeh MR, Tavalaee M and Nasr-Esfahani MH. Health of children born through artificial oocyte activation: a pilot study. Reprod Sci 2015;22(3):322-8. PMID: 25028175.

Dehghani Firouzabadi R, Janati S and Razi MH. The effect of intrauterine human chorionic gonadotropin injection before embryo transfer on the implantation and pregnancy rate in infertile patients: A randomized clinical trial. Int J Reprod Biomed (Yazd) 2016;14(10):657-664. PMID: 27921090.

Delaroche L, Yazbeck C, Gout C, et al. Intracytoplasmic morphologically selected sperm injection (IMSI) after repeated IVF or ICSI failures: a prospective comparative study. Eur J Obstet Gynecol Reprod Biol 2013;167(1):76-80. PMID: 23276454. 
Delbaere I, Goetgeluk S, Derom C, et al. Umbilical cord anomalies are more frequent in twins after assisted reproduction. Hum Reprod 2007;22(10):2763-7. PMID: 17720701.

Deltombe-Bodart S, Deruelle P, Drumez E, et al. Obstetrical and perinatal complications of twin pregnancies: is there a link with the type of infertility treatment?. Acta Obstet Gynecol Scand 2017;96(7):844-851. PMID: 28369714.

Depalo R, Lorusso F, Palmisano M, et al. Follicular growth and oocyte maturation in GnRH agonist and antagonist protocols for in vitro fertilisation and embryo transfer. Gynecol Endocrinol 2009;25(5):328-34. PMID: 19340626.

Derks RS, Farquhar C, Mol BW, et al. Techniques for preparation prior to embryo transfer. Cochrane Database Syst Rev 2009;(4):Cd007682. PMID: 19821435.

Dessolle L, Darai E, Cornet D, et al. Determinants of pregnancy rate in the donor oocyte model: a multivariate analysis of 450 frozen-thawed embryo transfers. Hum Reprod 2009;24(12):30829. PMID: 19726449.

Devroey P, Pellicer A, Nyboe Andersen A, et al. A randomized assessor-blind trial comparing highly purified hMG and recombinant FSH in a GnRH antagonist cycle with compulsory singleblastocyst transfer. Fertil Steril 2012;97(3):561-71. PMID: 22244781.

Donckers J, Evers JL and Land JA. The long-term outcome of 946 consecutive couples visiting a fertility clinic in 2001-2003. Fertil Steril 2011;96(1):160-4. PMID: 21550038.

Dong J, Wang Y, Chai WR, et al. The pregnancy outcome of progestin-primed ovarian stimulation using 4 versus $10 \mathrm{mg}$ of medroxyprogesterone acetate per day in infertile women undergoing in vitro fertilisation: a randomised controlled trial. Bjog 2017;124(7):1048-1055. PMID: 28276192.

Drakeley AJ, Jorgensen A, Sklavounos J, et al. A randomized controlled clinical trial of 2295 ultrasound-guided embryo transfers. Hum Reprod 2008;23(5):1101-6. PMID: 18325883.

Drakopoulos P, Vuong TNL, Ho NAV, et al. Corifollitropin alfa followed by highly purified HMG versus recombinant FSH in young poor ovarian responders: a multicentre randomized controlled clinical trial. Hum Reprod 2017;32(11):2225-2233. PMID: 29040589.

Dude AM, Yeh JS and Muasher SJ. Donor oocytes are associated with preterm birth when compared to fresh autologous in vitro fertilization cycles in singleton pregnancies. Fertility and Sterility 2016;106(3):660-665.

Duffy JM, Ahmad G, Mohiyiddeen L, et al. Growth hormone for in vitro fertilization. Cochrane Database Syst Rev 2010;(1):Cd000099. PMID: 20091500.

Dukhovny D, Hwang SS, Gopal D, et al. Length of stay and cost of birth hospitalization: effects of subfertility and ART. Journal of Perinatology 2018 
Dunietz GL, Holzman C, Zhang Y, et al. Assisted reproduction and risk of preterm birth in singletons by infertility diagnoses and treatment modalities: a population-based study. Journal of Assisted Reproduction and Genetics 2017;34(11):1529-1535.

Ebner T, Montag M, Montag M, et al. Live birth after artificial oocyte activation using a readyto-use ionophore: A prospective multicentre study. Reproductive BioMedicine Online 2015;30(4):359-365.

Eftekhar M, Hosseinisadat R, Baradaran R, et al. Effect of granulocyte colony stimulating factor (G-CSF) on IVF outcomes in infertile women: An RCT. Int J Reprod Biomed (Yazd) 2016;14(5):341-6. PMID: 27326420.

Eftekhar M, Rahsepar M and Rahmani E. Effect of progesterone supplementation on natural frozen-thawed embryo transfer cycles: A randomized controlled trial. International Journal of Fertility and Sterility 2013;7(1):13-20.

Eindhoven SC, van Uitert EM, Laven JS, et al. The influence of IVF/ICSI treatment on human embryonic growth trajectories. Hum Reprod 2014;29(12):2628-36. PMID: 25316447.

El Kissi Y, Amamou B, Hidar S, et al. Quality of life of infertile Tunisian couples and differences according to gender. Int J Gynaecol Obstet 2014;125(2):134-7. PMID: 24568955.

El-Chaar D, Yang Q, Gao J, et al. Risk of birth defects increased in pregnancies conceived by assisted human reproduction. Fertil Steril 2009;92(5):1557-61. PMID: 18973885.

El-Khayat W and Elsadek M. Calcium infusion for the prevention of ovarian hyperstimulation syndrome: a double-blind randomized controlled trial. Fertil Steril 2015;103(1):101-5. PMID: 25552412.

El-Khayat W, Elsadek M and Saber W. Comparing the effect of office hysteroscopy with endometrial scratch versus office hysteroscopy on intrauterine insemination outcome: a randomized controlled trial. Eur J Obstet Gynecol Reprod Biol 2015;194:96-100. PMID: 26344351.

El-Toukhy T, Campo R, Khalaf Y, et al. Hysteroscopy in recurrent in-vitro fertilisation failure (TROPHY): a multicentre, randomised controlled trial. Lancet 2016;387(10038):2614-21. PMID: 27132053.

El-Toukhy T, Sunkara S and Khalaf Y. Local endometrial injury and IVF outcome: a systematic review and meta-analysis. Reprod Biomed Online 2012;25(4):345-54. PMID: 22885017.

El-Toukhy T, Sunkara SK, Khairy M, et al. A systematic review and meta-analysis of acupuncture in in vitro fertilisation. Bjog 2008;115(10):1203-13. PMID: 18652588.

El-Toukhy T, Wharf E, Walavalkar R, et al. Delayed blastocyst development does not influence the outcome of frozen-thawed transfer cycles. Bjog 2011;118(13):1551-6. PMID: 21895955. 
Eldar-Geva T, Srebnik N, Altarescu G, et al. Neonatal outcome after preimplantation genetic diagnosis. Fertil Steril 2014;102(4):1016-21. PMID: 25064409.

Elzeiny H, Garrett C, Toledo M, et al. A randomised controlled trial of intra-uterine insemination versus in vitro fertilisation in patients with idiopathic or mild male infertility. Aust $\mathrm{N} \mathrm{Z} \mathrm{J} \mathrm{Obstet}$ Gynaecol 2014;54(2):156-61. PMID: 24579923.

Ercan CM, Kerimoglu OS, Sakinci M, et al. Pregnancy outcomes in a university hospital after legal requirement for single-embryo transfer. Eur J Obstet Gynecol Reprod Biol 2014;175:163-6. PMID: 24485667.

Erez O, Mayer A, Shoham-Vardi I, et al. Primiparity, assisted reproduction, and preterm birth in twin pregnancies: a population based study. Arch Gynecol Obstet 2008;277(4):311-7. PMID: 17972089.

Esinler I, Bozdag G and Yarali H. Impact of isolated obesity on ICSI outcome. Reprod Biomed Online 2008;17(4):583-7. PMID: 18854116.

Eskandar M, Abou-Setta AM, Almushait MA, et al. Ultrasound guidance during embryo transfer: a prospective, single-operator, randomized, controlled trial. Fertil Steril 2008;90(4):1187-90. PMID: 18439602.

Espinós JJ, Polo A, Sánchez-Hernández J, et al. Weight decrease improves live birth rates in obese women undergoing IVF: a pilot study. Reproductive BioMedicine Online 2017;35(4):417424.

Esteves SC, Sanchez-Martin F, Sanchez-Martin P, et al. Comparison of reproductive outcome in oligozoospermic men with high sperm DNA fragmentation undergoing intracytoplasmic sperm injection with ejaculated and testicular sperm. Fertil Steril 2015;104(6):1398-405. PMID: 26428305.

Esteves SC, Schertz JC, Verza S, Jr., et al. A comparison of menotropin, highly-purified menotropin and follitropin alfa in cycles of intracytoplasmic sperm injection. Reprod Biol Endocrinol 2009;7:111. PMID: 19828024.

Evenson KR, Calhoun KC, Herring AH, et al. Association of physical activity in the past year and immediately after in vitro fertilization on pregnancy. Fertil Steril 2014;101(4):1047-1054.e5. PMID: 24524834.

Fadini R, Mignini Renzini M, Guarnieri T, et al. Comparison of the obstetric and perinatal outcomes of children conceived from in vitro or in vivo matured oocytes in in vitro maturation treatments with births from conventional ICSI cycles. Hum Reprod 2012;27(12):3601-8. PMID: 23042796.

Fallah R, Akhavan Karbasi S, Galalian MT, et al. Comparison of developmental status of 5-yearold singleton children born through assisted and natural conceptions. Iran J Reprod Med 2013;11(5):365-70. PMID: 24639767. 
Fallah R, Jalalian MT, Golestan M, et al. Comparison of growth parameters of 5-year-old singleton children born in assisted versus natural conception. Ann Acad Med Singapore 2013;42(2):80-4. PMID: 23515690.

Fan Y, Zhang X, Hao Z, et al. Effectiveness of mild ovarian stimulation versus GnRH agonist protocol in women undergoing assisted reproductive technology: a meta-analysis. Gynecological Endocrinology 2017;33(10):746-756.

Farhangniya M, Dortaj Rabori E, Mozafari Kermani R, et al. Comparison of congenital abnormalities of infants conceived by assisted reproductive techniques versus infants with natural conception in Tehran. International Journal of Fertility and Sterility 2013;7(3):217-224.

Farhi A, Reichman B, Boyko V, et al. Maternal and neonatal health outcomes following assisted reproduction. Reprod Biomed Online 2013;26(5):454-61. PMID: 23518031.

Farhi J, Ben-Haroush A, Lande Y, et al. In vitro fertilization cycle outcome after coasting in gonadotropin-releasing hormone $(\mathrm{GnRH})$ agonist versus $\mathrm{GnRH}$ antagonist protocols. Fertil Steril 2009;91(2):377-82. PMID: 18321490.

Farquhar CM, Brown J, Arroll N, et al. A randomized controlled trial of fallopian tube sperm perfusion compared with standard intrauterine insemination for women with non-tubal infertility. Hum Reprod 2013;28(8):2134-9. PMID: 23578949.

Feinberg EC, Beltsos AN, Nicolaou E, et al. Endometrin as luteal phase support in assisted reproduction. Fertil Steril 2013;99(1):174-8. PMID: 23137759.

Fisher JR, Rowe H and Hammarberg K. Admissions for early parenting difficulties among women with infants conceived by assisted reproductive technologies: a prospective cohort study. Fertil Steril 2012;97(6):1410-6. PMID: 22464762.

Forman EJ, Hong KH, Franasiak JM, et al. Obstetrical and neonatal outcomes from the BEST Trial: single embryo transfer with aneuploidy screening improves outcomes after in vitro fertilization without compromising delivery rates. Am J Obstet Gynecol 2014;210(2):157.e1-6. PMID: 24145186.

Forman EJ, Tao X, Ferry KM, et al. Single embryo transfer with comprehensive chromosome screening results in improved ongoing pregnancy rates and decreased miscarriage rates. Hum Reprod 2012;27(4):1217-22. PMID: 22343551.

Franasiak JM, Dondik Y, Molinaro TA, et al. Blastocyst transfer is not associated with increased rates of monozygotic twins when controlling for embryo cohort quality. Fertil Steril 2015;103(1):95-100. PMID: 25455537.

Frattarelli JL, Miller KA, Miller BT, et al. Male age negatively impacts embryo development and reproductive outcome in donor oocyte assisted reproductive technology cycles. Fertil Steril 2008;90(1):97-103. PMID: 17765235. 
Frederiksen Y, Farver-Vestergaard I, Skovgard NG, et al. Efficacy of psychosocial interventions for psychological and pregnancy outcomes in infertile women and men: a systematic review and meta-analysis. BMJ Open 2015;5(1):e006592. PMID: 25631310.

Friedler S, Ben-Ami I, Gidoni Y, et al. Effect of seminal plasma application to the vaginal vault in in vitro fertilization or intracytoplasmic sperm injection treatment cycles-a double-blind, placebo-controlled, randomized study. J Assist Reprod Genet 2013;30(7):907-11. PMID: 23812801.

Fuentes A, Munoz A, Barnhart K, et al. Recent cigarette smoking and assisted reproductive technologies outcome. Fertil Steril 2010;93(1):89-95. PMID: 18973890.

Fujii M, Matsuoka R, Bergel E, et al. Perinatal risk in singleton pregnancies after in vitro fertilization. Fertil Steril 2010;94(6):2113-7. PMID: 20117775.

Fujimoto A, Fujiwara T, Oishi H, et al. Predictive factors of successful pregnancy after assisted reproductive technology in women aged 40 years and older. Reproductive Medicine and Biology 2009;8(4):145-149.

Funabiki M, Taguchi S, Hayashi T, et al. Vaginal preparation with povidone iodine disinfection and saline douching as a safe and effective method in prevention of oocyte pickup-associated pelvic inflammation without spoiling the reproductive outcome: evidence from a large cohort study. Clin Exp Obstet Gynecol 2014;41(6):689-90. PMID: 25551964.

Fujii M, Matsuoka R, Bergel E, et al. Perinatal risk in singleton pregnancies after in vitro fertilization. Fertil Steril 2010;94(6):2113-7. PMID: 20117775.Gaikwad S, Garrido N, Cobo A, et al. Bed rest after embryo transfer negatively affects in vitro fertilization: a randomized controlled clinical trial. Fertil Steril 2013;100(3):729-35. PMID: 23755954.

Gameiro S, Nazare B, Fonseca A, et al. Changes in marital congruence and quality of life across the transition to parenthood in couples who conceived spontaneously or with assisted reproductive technologies. Fertil Steril 2011;96(6):1457-62. PMID: 21982288.

Garcia-Velasco JA, Bermejo A, Ruiz F, et al. Cycle scheduling with oral contraceptive pills in the GnRH antagonist protocol vs the long protocol: a randomized, controlled trial. Fertil Steril 2011;96(3):590-3. PMID: 21718992.

Gaskins AJ, Afeiche MC, Hauser R, et al. Paternal physical and sedentary activities in relation to semen quality and reproductive outcomes among couples from a fertility center. Hum Reprod 2014;29(11):2575-82. PMID: 25164027.

Gelbaya TA, Tsoumpou I and Nardo LG. The likelihood of live birth and multiple birth after single versus double embryo transfer at the cleavage stage: a systematic review and metaanalysis. Fertil Steril 2010;94(3):936-45. PMID: 19446809.

Geng L, Li W, Li X, et al. Controlled ovarian hyperstimulation interventions in infertile women aged 40 years or older undergoing in vitro fertilization. Int J Gynaecol Obstet 2009;106(3):1937. PMID: 19464684. 
Gennari A, Costa M, Puntoni M, et al. Breast cancer incidence after hormonal treatments for infertility: systematic review and meta-analysis of population-based studies. Breast Cancer Res Treat 2015;150(2):405-13. PMID: 25744295.

George K, Kamath MS and Tharyan P. Minimally invasive versus open surgery for reversal of tubal sterilization. Cochrane Database Syst Rev 2013;2:Cd009174. PMID: 23450598.

George K, Kamath MS, Nair R, et al. Ovulation triggers in anovulatory women undergoing ovulation induction. Cochrane Database Syst Rev 2014;1:Cd006900. PMID: 24482059.

George K, Nair R and Tharyan P. Ovulation triggers in anovulatory women undergoing ovulation induction. Cochrane Database Syst Rev 2008;(3):Cd006900. PMID: 18646175.

Gerli S, Bini V and Di Renzo GC. Cost-effectiveness of recombinant follicle-stimulating hormone (FSH) versus human FSH in intrauterine insemination cycles: a statistical modelderived analysis. Gynecol Endocrinol 2008;24(1):18-23. PMID: 18224540.

Ghahiri A, Firozmand A, Ghasemi M, et al. A comparative cohort study for detecting the incidence of trisomy 21 in ART and non-ART neonates. Iran J Reprod Med 2014;12(6):435-8. PMID: 25071853.

Gibbons WE, Cedars M and Ness RB. Toward understanding obstetrical outcome in advanced assisted reproduction: varying sperm, oocyte, and uterine source and diagnosis. Fertil Steril 2011;95(5):1645-9.e1. PMID: 21130432.

Gibreel A, El-Adawi N, Elgindy E, et al. Endometrial scratching for women with previous IVF failure undergoing IVF treatment. Gynecol Endocrinol 2015;31(4):313-6. PMID: 25561347.

Gibreel A, Maheshwari A and Bhattacharya S. Clomiphene citrate in combination with gonadotropins for controlled ovarian stimulation in women undergoing in vitro fertilization. Cochrane Database Syst Rev 2012;11:Cd008528. PMID: 23152261.

Glujovsky D, Blake D, Farquhar C, et al. Cleavage stage versus blastocyst stage embryo transfer in assisted reproductive technology. Cochrane Database Syst Rev 2012;7:Cd002118. PMID: 22786480 .

Gnoth C, Maxrath B, Skonieczny T, et al. Final ART success rates: a 10 years survey. Hum Reprod 2011;26(8):2239-46. PMID: 21659314.

Goel T, Mahey R, Bhatla N, et al. Pregnancy after endometrial scratching in infertile couples undergoing ovulation induction and intrauterine insemination cycles-a randomized controlled trial. J Assist Reprod Genet 2017;34(8):1051-1058. PMID: 28551840.

Gokce MI, Gulpinar O, Suer E, et al. Effect of performing varicocelectomy before intracytoplasmic sperm injection on clinical outcomes in non-azoospermic males. Int Urol Nephrol 2013;45(2):367-72. PMID: 23392963. 
Goldman RH, Batsis M, Hacker MR, et al. Outcomes after intrauterine insemination are independent of provider type. Am J Obstet Gynecol 2014;211(5):492.e1-9. PMID: 24881820.

Golombok S, Owen L, Blake L, et al. Parent-child relationships and the psychological well-being of 18-year-old adolescents conceived by in vitro fertilisation. Hum Fertil (Camb) 2009;12(2):6372. PMID: 19802956.

Gomez-Palomares JL, Acevedo-Martin B, Chavez M, et al. Multifollicular recruitment in combination with gonadotropin-releasing hormone antagonist increased pregnancy rates in intrauterine insemination cycles. Fertil Steril 2008;89(3):620-4. PMID: 17678911.

Gong M, Shi H, Zhang YG, et al. Prenatal screening at 11-13+6 weeks in assisted reproductive technology singleton pregnancies and those conceived naturally. J Obstet Gynaecol Res 2015. PMID: 26177681.

Gonzalez-Comadran M, Urresta Avila J, Saavedra Tascon A, et al. The impact of donor insemination on the risk of preeclampsia: a systematic review and meta-analysis. Eur J Obstet Gynecol Reprod Biol 2014;182:160-6. PMID: 25282539.

Gordts S, Van Turnhout C, Campo R, et al. A prospective randomised study comparing a GnRHantagonist versus a GnRH-agonist short protocol for ovarian stimulation in patients referred for IVF. Facts Views Vis Obgyn 2012;4(2):82-7. PMID: 24753894.

Goudge CS, Nagel TC and Damario MA. Duration of progesterone-in-oil support after in vitro fertilization and embryo transfer: a randomized, controlled trial. Fertil Steril 2010;94(3):946-51. PMID: 19523613.

Gourounti K. Psychological Stress and Adjustment in Pregnancy Following Assisted Reproductive Technology and Spontaneous Conception: A Systematic Review. Women Health 2015. PMID: 26212077.

Grady R, Alavi N, Vale R, et al. Elective single embryo transfer and perinatal outcomes: a systematic review and meta-analysis. Fertil Steril 2012;97(2):324-31. PMID: 22177461.

Graziano A, Caserta D, Piva I, et al. The addition of GnRH antagonists in intrauterine insemination cycles: a pilot study. Eur Rev Med Pharmacol Sci 2013;17(12):1604-10. PMID: 23832726.

Greco E, Litwicka K, Arrivi C, et al. The endometrial preparation for frozen-thawed euploid blastocyst transfer: a prospective randomized trial comparing clinical results from natural modified cycle and exogenous hormone stimulation with GnRH agonist. Journal of Assisted Reproduction and Genetics 2016;33(7):873-884.

Griesinger G, Kolibianakis EM, Diedrich K, et al. Ovarian stimulation for IVF has no quantitative association with birthweight: a registry study. Hum Reprod 2008;23(11):2549-54. PMID: 18684734. 
Griffin D, Benadiva C, Kummer N, et al. Dual trigger of oocyte maturation with gonadotropinreleasing hormone agonist and low-dose human chorionic gonadotropin to optimize live birth rates in high responders. Fertil Steril 2012;97(6):1316-20. PMID: 22480822.

Groenewoud ER, Cohlen BJ, Al-Oraiby A, et al. A randomized controlled, non-inferiority trial of modified natural versus artificial cycle for cryo-thawed embryo transfer. Hum Reprod 2016;31(7):1483-92. PMID: 27179265.

Groenewoud ER, Macklon NS and Cohlen BJ. Cryo-thawed embryo transfer: natural versus artificial cycle. A non-inferiority trial. (ANTARCTICA trial). BMC Womens Health 2012;12:27. PMID: 22950651.

Gronwald J, Glass K, Rosen B, et al. Treatment of infertility does not increase the risk of ovarian cancer among women with a BRCA1 or BRCA2 mutation. Fertil Steril 2016;105(3):781-5. PMID: 26698676.

Gunby J, Bissonnette F, Librach C, et al. Assisted reproductive technologies (ART) in Canada: 2006 results from the Canadian ART Register. Fertil Steril 2010;93(7):2189-201. PMID: 19439295.

Gunby J, Bissonnette F, Librach C, et al. Assisted reproductive technologies (ART) in Canada: 2007 results from the Canadian ART Register. Fertil Steril 2011;95(2):542-7.e1-10. PMID: 20656287.

Guo XY, Liu XM, Jin L, et al. Cardiovascular and metabolic profiles of offspring conceived by assisted reproductive technologies: a systematic review and meta-analysis. Fertil Steril 2017;107(3):622-631.e5. PMID: 28104241.

Gupta S, Fox NS, Rebarber A, et al. Biochemical screening for aneuploidy in patients with donor oocyte pregnancies compared with autologous pregnancies. J Matern Fetal Neonatal Med 2014;27(14):1418-21. PMID: 24228730.

Gutarra-Vilchez RB, Urrutia G, Glujovsky D, et al. Vasodilators for women undergoing fertility treatment. Cochrane Database Syst Rev 2014;10:Cd010001. PMID: 25310622.

Haagen EC, Nelen WL, Adang EM, et al. Guideline adherence is worth the effort: a costeffectiveness analysis in intrauterine insemination care. Hum Reprod 2013;28(2):357-66. PMID: 23202990.

Haahr T, Roque M, Esteves SC, et al. GnRH Agonist Trigger and LH Activity Luteal Phase Support versus hCG Trigger and Conventional Luteal Phase Support in Fresh Embryo Transfer IVF/ICSI Cycles-A Systematic PRISMA Review and Meta-analysis. Front Endocrinol (Lausanne) 2017;8:116. PMID: 28638367.

Haas J, Baum M, Meridor K, et al. Is severe OHSS associated with adverse pregnancy outcomes? Evidence from a case-control study. Reprod Biomed Online 2014;29(2):216-21. PMID: 24934625. 
Habroe M, Schmidt L and Evald BE. Does childbirth after fertility treatment influence sense of coherence? A longitudinal study of 1,934 men and women. Acta Obstet Gynecol Scand 2007;86(10):1215-21. PMID: 17851813.

Haddad G, Saguan DA, Maxwell R, et al. Intramuscular route of progesterone administration increases pregnancy rates during non-downregulated frozen embryo transfer cycles. J Assist Reprod Genet 2007;24(10):467-70. PMID: 17721816.

Hagemann AR, Lanzendorf SE, Jungheim ES, et al. A prospective, randomized, double-blinded study of assisted hatching in women younger than 38 years undergoing in vitro fertilization. Fertil Steril 2010;93(2):586-91. PMID: 19268926.

Halliday JL, Ukoumunne OC, Baker HW, et al. Increased risk of blastogenesis birth defects, arising in the first 4 weeks of pregnancy, after assisted reproductive technologies. Hum Reprod 2010;25(1):59-65. PMID: 19850591.

Hammarberg K, Fisher JR and Wynter KH. Psychological and social aspects of pregnancy, childbirth and early parenting after assisted conception: a systematic review. Hum Reprod Update 2008;14(5):395-414. PMID: 18653674.

Hammarberg K, Rowe HJ and Fisher JR. Early post-partum adjustment and admission to parenting services in Victoria, Australia after assisted conception. Hum Reprod 2009;24(11):2801-9. PMID: 19661124.

Han Y, Luo H and Zhang Y. Congenital anomalies in infants conceived by infertile women through assisted reproductive technology: A cohort study 2004-2014. Exp Ther Med 2018;16(4):3179-3185. PMID: 30214541.

Hannibal CG, Jensen A, Sharif H, et al. Malignant melanoma risk after exposure to fertility drugs: results from a large Danish cohort study. Cancer Causes Control 2008;19(7):759-65. PMID: 18340541.

Hannibal CG, Jensen A, Sharif H, et al. Risk of thyroid cancer after exposure to fertility drugs: results from a large Danish cohort study. Hum Reprod 2008;23(2):451-6. PMID: 18065402.

Hansen M, Kurinczuk JJ, de Klerk N, et al. Assisted reproductive technology and major birth defects in Western Australia. Obstet Gynecol 2012;120(4):852-63. PMID: 22996103.

Hargreave M, Jensen A, Nielsen TS, et al. Maternal use of fertility drugs and risk of cancer in children--a nationwide population-based cohort study in Denmark. Int J Cancer 2015;136(8):1931-9. PMID: 25257918.

Hargreave M, Jensen A, Toender A, et al. Fertility treatment and childhood cancer risk: a systematic meta-analysis. Fertil Steril 2013;100(1):150-61. PMID: 23562045.

Hargreave M, Kjaer SK, Jorgensen ME, et al. Type 1 diabetes risk in children born to women with fertility problems: a cohort study in 1.5 million Danish children. Acta Obstet Gynecol Scand 2016;95(12):1441-1446. PMID: 27681848. 
Harju M, Keski-Nisula L, Raatikainen K, et al. Maternal fecundity and asthma among offspringis the risk programmed preconceptionally? Retrospective observational study. Fertil Steril 2013;99(3):761-767.e1. PMID: 23148921.

Harlev A, Walfisch A, Oran E, et al. The effect of fertility treatment on adverse perinatal outcomes in women aged at least 40 years. International Journal of Gynecology and Obstetrics 2018;140(1):98-104.

Hashimoto K, Ogawa K, Horikawa R, et al. Gross motor function and general development of babies born after assisted reproductive technology. Journal of Obstetrics and Gynaecology Research 2016;42(3):266-272.

Hatirnaz S and Kanat Pektas M. Day 3 embryo transfer versus day 5 blastocyst transfers: A prospective randomized controlled trial. Turk J Obstet Gynecol 2017;14(2):82-88. PMID: 28913142.

Hauzman EE, Zapata A, Bermejo A, et al. Cycle scheduling for in vitro fertilization with oral contraceptive pills versus oral estradiol valerate: a randomized, controlled trial. Reprod Biol Endocrinol 2013;11:96. PMID: 24074027.

Hayashi M, Nakai A, Satoh S, et al. Adverse obstetric and perinatal outcomes of singleton pregnancies may be related to maternal factors associated with infertility rather than the type of assisted reproductive technology procedure used. Fertil Steril 2012;98(4):922-8. PMID: 22763098.

Haydardedeoglu B, Gjemalaj F, Aytac PC, et al. Direct aspiration versus follicular flushing in poor responders undergoing intracytoplasmic sperm injection: a randomised controlled trial. BJOG: An International Journal of Obstetrics and Gynaecology 2017;124(8):1190-1196.

Heijnen EM, Eijkemans MJ, De Klerk C, et al. A mild treatment strategy for in-vitro fertilisation: a randomised non-inferiority trial. Lancet 2007;369(9563):743-9. PMID: 17336650.

Heisey AS, Bell EM, Herdt-Losavio ML, et al. Surveillance of congenital malformations in infants conceived through assisted reproductive technology or other fertility treatments. Birth Defects Research Part A - Clinical and Molecular Teratology 2015;103(2):119-126.

Helmy MEE, Maher MA, Elkhouly NI, et al. A randomized trial of local endometrial injury during ovulation induction cycles. Int J Gynaecol Obstet 2017;138(1):47-52. PMID: 28397981.

Henne MB, Stegmann BJ, Neithardt AB, et al. The combined effect of age and basal folliclestimulating hormone on the cost of a live birth at assisted reproductive technology. Fertil Steril 2008;89(1):104-10. PMID: 17669406.

Henningsen AA, Bergh C, Skjaerven R, et al. Trends over time in congenital malformations in live-born children conceived after assisted reproductive technology. Acta Obstet Gynecol Scand 2018. PMID: 29572867. 
Henningsen AA, Gissler M, Skjaerven R, et al. Trends in perinatal health after assisted reproduction: a Nordic study from the CoNARTaS group. Hum Reprod 2015;30(3):710-6. PMID: 25605701.

Henningsen AK, Pinborg A, Lidegaard O, et al. Perinatal outcome of singleton siblings born after assisted reproductive technology and spontaneous conception: Danish national siblingcohort study. Fertil Steril 2011;95(3):959-63. PMID: 20813359.

Herbert DL, Lucke JC and Dobson AJ. Birth outcomes after spontaneous or assisted conception among infertile Australian women aged 28 to 36 years: a prospective, population-based study. Fertil Steril 2012;97(3):630-8. PMID: 22265037.

Hernandez Torres E, Navarro-Espigares JL, Clavero A, et al. Economic evaluation of elective single-embryo transfer with subsequent single frozen embryo transfer in an in vitro fertilization/intracytoplasmic sperm injection program. Fertility and Sterility 2015;103(3):699706.

Hernandez-Diaz S, Werler MM and Mitchell AA. Gestational hypertension in pregnancies supported by infertility treatments: role of infertility, treatments, and multiple gestations. Fertil Steril 2007;88(2):438-45. PMID: 17449034.

Hill MJ, Hong S and Frattarelli JL. Body mass index impacts in vitro fertilization stimulation. ISRN Obstet Gynecol 2011;2011:929251. PMID: 21637365.

Hill MJ, Whitcomb BW, Lewis TD, et al. Progesterone luteal support after ovulation induction and intrauterine insemination: a systematic review and meta-analysis. Fertil Steril 2013;100(5):1373-80. PMID: 23876537.

Ho JR, Woo I, Louie K, et al. A comparison of live birth rates and perinatal outcomes between cryopreserved oocytes and cryopreserved embryos. Journal of Assisted Reproduction and Genetics 2017;34(10):1359-1366.

Hollingsworth B, Harris A and Mortimer D. The cost effectiveness of intracyctoplasmic sperm injection (ICSI). J Assist Reprod Genet 2007;24(12):571-7. PMID: 18008157.

Hong KH, Forman EJ, Werner MD, et al. Endometrial infusion of human chorionic gonadotropin at the time of blastocyst embryo transfer does not impact clinical outcomes: a randomized, double-blind, placebo-controlled trial. Fertil Steril 2014;102(6):1591-5.e2. PMID: 25234040.

Hong SB, Lee NR, Kim SK, et al. In vitro fertilization outcomes in women with surgery induced diminished ovarian reserve after endometrioma operation: Comparison with diminished ovarian reserve without ovarian surgery. Obstet Gynecol Sci 2017;60(1):63-68. PMID: 28217673.

Hsieh YY, Chang CC and Tsai HD. Comparisons of different dosages of gonadotropin-releasing hormone $(\mathrm{GnRH})$ antagonist, short-acting form and single, half-dose, long-acting form of $\mathrm{GnRH}$ agonist during controlled ovarian hyperstimulation and in vitro fertilization. Taiwan J Obstet Gynecol 2008;47(1):66-74. PMID: 18400585. 
Huang B, Hu D, Qian K, et al. Is frozen embryo transfer cycle associated with a significantly lower incidence of ectopic pregnancy? An analysis of more than 30,000 cycles. Fertil Steril 2014;102(5):1345-9. PMID: 25241365.

Huang B, Qian K, Li Z, et al. Neonatal outcomes after early rescue intracytoplasmic sperm injection: an analysis of a 5-year period. Fertil Steril 2015;103(6):1432-7.e1. PMID: 25813286.

Huang YL, Wu AH and Liu JQ. Selective short-term fertilization combined with early rescue ICSI: An optimal strategy for patients at high risk for fertilization failure. Journal of Reproduction and Contraception 2014;25(4):207-217.

Huang Z, Li J, Wang L, et al. Brief co-incubation of sperm and oocytes for in vitro fertilization techniques. Cochrane Database Syst Rev 2013;4:Cd009391. PMID: 23633370.

Humaidan P, Polyzos NP, Alsbjerg B, et al. GnRHa trigger and individualized luteal phase hCG support according to ovarian response to stimulation: two prospective randomized controlled multi-centre studies in IVF patients. Hum Reprod 2013;28(9):2511-21. PMID: 23753114.

Hvidtjorn D, Schieve L, Schendel D, et al. Cerebral palsy, autism spectrum disorders, and developmental delay in children born after assisted conception: a systematic review and metaanalysis. Arch Pediatr Adolesc Med 2009;163(1):72-83. PMID: 19124707.

Hwang SS, Dukhovny D, Gopal D, et al. Health of infants after ART-Treated, subfertile, and fertile deliveries. Pediatrics 2018;142(2).

Inal ZHO, Görkemli H and Inal HA. The effect of local injury to the endometrium for implantation and pregnancy rates in ICSI -ET cycles with implantation failure: A randomised controlled study. European Journal of General Medicine 2012;9(4):223-229.

Insua MF, Cobo AC, Larreategui Z, et al. Obstetric and perinatal outcomes of pregnancies conceived with embryos cultured in a time-lapse monitoring system. Fertil Steril 2017;108(3):498-504. PMID: 28865550.

Isikoglu M, Ozgur K and Oehninger S. Extension of GnRH agonist through the luteal phase to improve the outcome of intracytoplasmic sperm injection. J Reprod Med 2007;52(7):639-44. PMID: 17847764.

Jarahzadeh MH, Jouya R, Mousavi FS, et al. Propofol or thiopental sodium in patients undergoing reproductive assisted technologies: Differences in hemodynamic recovery and outcome of oocyte retrieval: A randomized clinical trial. Iranian Journal of Reproductive Medicine 2014;12(1):77-82.

Jayaprakasan K, Hopkisson J, Campbell B, et al. A randomised controlled trial of 300 versus 225 IU recombinant FSH for ovarian stimulation in predicted normal responders by antral follicle count. Bjog 2010;117(7):853-62. PMID: 20353458. 
Jayaprakasan K, Pandian D, Hopkisson J, et al. Effect of ethnicity on live birth rates after in vitro fertilisation or intracytoplasmic sperm injection treatment. Bjog 2014;121(3):300-6. PMID: 24206211.

Jellad S, Haj Hassine A, Basly M, et al. Vascular endothelial growth factor antagonist reduces the early onset and the severity of ovarian hyperstimulation syndrome. Journal of Gynecology Obstetrics and Human Reproduction 2017;46(1):87-91.

Jensen A, Sharif $\mathrm{H}$ and Kjaer SK. Use of fertility drugs and risk of uterine cancer: results from a large Danish population-based cohort study. Am J Epidemiol 2009;170(11):1408-14. PMID: 19884127.

Jensen A, Sharif H, Frederiksen K, et al. Use of fertility drugs and risk of ovarian cancer: Danish Population Based Cohort Study. Bmj 2009;338:b249. PMID: 19196744.

Jensen A, Sharif H, Svare EI, et al. Risk of breast cancer after exposure to fertility drugs: results from a large Danish cohort study. Cancer Epidemiol Biomarkers Prev 2007;16(7):1400-7.

PMID: 17585058.

Jia Y, Geng LH and Zhong Y. Birth defects in assisted reproductive technology and spontaneously conceived children: A meta-analysis. Journal of Reproduction and Contraception 2013;24(4):237-252.

Jie Z, Yiling D and Ling Y. Association of assisted reproductive technology with adverse pregnancy outcomes. Iran J Reprod Med 2015;13(3):169-80. PMID: 26000008.

Johansson M, Adolfsson A, Berg M, et al. Gender perspective on quality of life, comparisons between groups 4-5.5 years after unsuccessful or successful IVF treatment. Acta Obstet Gynecol Scand 2010;89(5):683-91. PMID: 20302532.

Johansson M, Adolfsson A, Berg M, et al. Quality of life for couples 4-5.5 years after unsuccessful IVF treatment. Acta Obstet Gynecol Scand 2009;88(3):291-300. PMID: 19172440.

Jongbloed-Pereboom M, Middelburg KJ, Heineman MJ, et al. The Groningen assisted reproductive technologies cohort study: developmental status and behavior at 2 years. Fertil Steril 2011;95(7):2283-9. PMID: 21458797.

Jongbloed-Pereboom M, Middelburg KJ, Heineman MJ, et al. The impact of IVF/ICSI on parental well-being and anxiety 1 year after childbirth. Hum Reprod 2012;27(8):2389-95. PMID: 22611166.

Joshi N, Kissin D, Anderson JE, et al. Trends and correlates of good perinatal outcomes in assisted reproductive technology. Obstet Gynecol 2012;120(4):843-51. PMID: 22996102.

Jungheim ES, Schon SB, Schulte MB, et al. IVF outcomes in obese donor oocyte recipients: a systematic review and meta-analysis. Hum Reprod 2013;28(10):2720-7. PMID: 23847110. 
Kaartinen N, Kananen K, Huhtala H, et al. The freezing method of cleavage stage embryos has no impact on the weight of the newborns. Journal of Assisted Reproduction and Genetics 2016;33(3):393-399.

Kaartinen NM, Kananen KM, Rodriguez-Wallberg KA, et al. Male gender explains increased birthweight in children born after transfer of blastocysts. Hum Reprod 2015. PMID: 26253840.

Kamath MS, Kirubakaran R, Mascarenhas M, et al. Perinatal outcomes after stimulated versus natural cycle IVF: a systematic review and meta-analysis. Reprod Biomed Online 2018;36(1):94101. PMID: 29111312.

Kamath MS, Mascarenhas M, B K, et al. Uterine flushing with supernatant embryo culture medium in vitrified warmed blastocyst transfer cycles: a randomized controlled trial. J Assist Reprod Genet 2015;32(2):225-31. PMID: 25428435.

Kan A, Leung P, Luo K, et al. Do Asian women do as well as their Caucasian counterparts in IVF treatment: Cohort study. J Obstet Gynaecol Res 2015;41(6):946-51. PMID: 25510340.

Kansal Kalra S, Ratcliffe SJ, Milman L, et al. Perinatal morbidity after in vitro fertilization is lower with frozen embryo transfer. Fertil Steril 2011;95(2):548-53. PMID: 20663500.

Karavani G, Ben-Meir A, Shufaro Y, et al. Transvaginal ultrasound to guide embryo transfer: a randomized controlled trial. Fertil Steril 2017;107(5):1159-1165. PMID: 28347493.

Karipcin FS, Moragianni VA, Milette B, et al. Effect of steroid and antibiotic treatment during assisted hatching on IVF outcomes. Hum Fertil (Camb) 2012;15(4):205-9. PMID: 23190298.

Kasdallah N, Salem HB, Kbaier H, et al. Premature birth, low birth weight and birth defects after assisted reproductive therapies. A 18-year comparative study. Tunisie Medicale 2017;95(2):103108.

Kaser DJ, Goldman MB, Fung JL, et al. When is clomiphene or gonadotropin intrauterine insemination futile? Results of the Fast Track and Standard Treatment Trial and the Forty and Over Treatment Trial, two prospective randomized controlled trials. Fertil Steril 2014;102(5):1331-1337.e1. PMID: 25226857.

Katz P, Showstack J, Smith JF, et al. Costs of infertility treatment: results from an 18-month prospective cohort study. Fertil Steril 2011;95(3):915-21. PMID: 21130988.

Kaur H, Krishna D, Shetty N, et al. Effect of Pre-ovulatory Single Dose GnRH agonist Therapy on IVF Outcome in GnRH Antagonist Cycles; A Prospective Study. J Reprod Infertil 2012;13(4):225-31. PMID: 23926550.

Kaveh M, Ghajarzadeh M, Tanha FD, et al. Pregnancy complications and neonatal outcomes in multiple pregnancies: A comparison between assisted reproductive techniques and spontaneous conception. International Journal of Fertility and Sterility 2015;8(4):367-372. 
Kawahara S, Ueda A, Nakahori T, et al. Treatment period and medical care costs to achieve the first live birth by assisted reproductive technology are lower in the single embryo transfer period than in the double embryo transfer period: a retrospective analysis of women younger than 40 years of age. 2017;16(2):139-142.

Keenan JA, Gissler M and Finger R. Assisted reproduction using donated embryos: outcomes from surveillance systems in six countries. Hum Reprod 2012;27(3):747-52. PMID: 22258660.

Kermani RM, Farhangniya M, Shahzadeh Fazeli SA, et al. Congenital malformations in singleton infants conceived by assisted reproductive technologies and singleton infants by natural conception in Tehran, Iran. International Journal of Fertility and Sterility 2018;11(4):304308.

Kermani RM, Zoljalali S, Kouhpayezadeh J, et al. Evaluation of the growth process of infants conceived by assisted reproductive techniques at royan institute from birth to 9 months. Iranian Journal of Pediatrics 2011;21(4):449-454.

Kessous R, Davidson E, Meirovitz M, et al. The risk of female malignancies after fertility treatments: a cohort study with 25-year follow-up. Journal of Cancer Research and Clinical Oncology 2016;142(1):287-293.

Kettner LO, Kesmodel US, Ramlau-Hansen CH, et al. Fertility Treatment and Childhood Epilepsy: A Nationwide Cohort Study. Epidemiology 2017;28(3):412-418. PMID: 28072588.

Kettner LO, Ramlau-Hansen CH, Kesmodel US, et al. Parental Infertility, Fertility Treatment, and Childhood Epilepsy: A Population-Based Cohort Study. Paediatr Perinat Epidemiol 2016;30(5):488-95. PMID: 27237870.

Khan N, Richter KS, Newsome TL, et al. Matched-samples comparison of intramuscular versus vaginal progesterone for luteal phase support after in vitro fertilization and embryo transfer. Fertil Steril 2009;91(6):2445-50. PMID: 18555224.

Kim CH, Ahn JW, You RM, et al. Combined administration of gonadotropin-releasing hormone agonist with human chorionic gonadotropin for final oocyte maturation in GnRH antagonist cycles for in vitro fertilization. J Reprod Med 2014;59(1-2):63-8. PMID: 24597289.

Kim CH, Jeon GH, Cheon YP, et al. Comparison of GnRH antagonist protocol with or without oral contraceptive pill pretreatment and GnRH agonist low-dose long protocol in low responders undergoing IVF/intracytoplasmic sperm injection. Fertil Steril 2009;92(5):1758-60. PMID: 19523618.

Kim CH, You RM, Kang HJ, et al. GnRH antagonist multiple dose protocol with oral contraceptive pill pretreatment in poor responders undergoing IVF/ICSI. Clin Exp Reprod Med 2011;38(4):228-33. PMID: 22384447.

Klemetti R, Gissler M, Sevon T, et al. Resource allocation of in vitro fertilization: a nationwide register-based cohort study. BMC Health Serv Res 2007;7:210. PMID: 18154645. 
Knoester M, Helmerhorst FM, Vandenbroucke JP, et al. Cognitive development of singletons born after intracytoplasmic sperm injection compared with in vitro fertilization and natural conception. Fertil Steril 2008;90(2):289-96. PMID: 17980875.

Kolibianakis EM, Venetis CA, Bosdou JK, et al. Corifollitropin alfa compared with follitropin beta in poor responders undergoing ICSI: a randomized controlled trial. Hum Reprod 2015;30(2):432-40. PMID: 25492411.

Koning AM, Kuchenbecker WK, Groen H, et al. Economic consequences of overweight and obesity in infertility: a framework for evaluating the costs and outcomes of fertility care. Hum Reprod Update 2010;16(3):246-54. PMID: 20056674.

Koning AM, Mutsaerts MA, Kuchenbecker WK, et al. Complications and outcome of assisted reproduction technologies in overweight and obese women. Hum Reprod 2012;27(2):457-67. PMID: 22144420.

Krieger Y, Wainstock T, Sheiner E, et al. Long-term pediatric skin eruption-related hospitalizations in offspring conceived via fertility treatment. International Journal of Dermatology 2018;57(3):317-323.

Kuang Y, Chen Q, Fu Y, et al. Medroxyprogesterone acetate is an effective oral alternative for preventing premature luteinizing hormone surges in women undergoing controlled ovarian hyperstimulation for in vitro fertilization. Fertil Steril 2015;104(1):62-70.e3. PMID: 25956370.

Kuiper D, Hoek A, la Bastide-van Gemert S, et al. Cardiovascular health of 9-year-old IVF offspring: no association with ovarian hyperstimulation and the in vitro procedure. Hum Reprod 2017;32(12):2540-2548. PMID: 29087467.

Kumbasar S, Gul O and Sik A. Evaluation of the effect of indomethacin and piroxicam administration before embryo transfer on pregnancy rate. J Obstet Gynaecol Res 2017;43(3):536542. PMID: 28160506.

Kumaran A, Narayan P, Pai P, et al. Oocyte retrieval at 140-mmHg negative aspiration pressure: A promising alternative to flushing and aspiration in assisted reproduction in women with low ovarian reserve. Journal of Human Reproductive Sciences 2015;8(2):98-102.

Kung HF, Chen MJ, Guua HF, et al. Luteal phase support with decapeptyl improves pregnancy outcomes in intracytoplasmic sperm injection with basal follicle-stimulating hormone $>8$ $\mathrm{mIU} / \mathrm{mL}$ or mature oocytes $\leq 3$. Journal of the Chinese Medical Association 2014.

Kung HF, Chen MJ, Guua HF, et al. Luteal phase support with decapeptyl improves pregnancy outcomes in intracytoplasmic sperm injection with higher basal follicle-stimulating hormone or lower mature oocytes. Journal of the Chinese Medical Association 2014;77(10):524-530.

Kupka MS, Ferraretti AP, de Mouzon J, et al. Assisted reproductive technology in Europe, 2010: results generated from European registers by ESHREdagger. Hum Reprod 2014;29(10):2099-

113. PMID: 25069504. 
Kurta ML, Moysich KB, Weissfeld JL, et al. Use of fertility drugs and risk of ovarian cancer: results from a U.S.-based case-control study. Cancer Epidemiol Biomarkers Prev 2012;21(8):1282-92. PMID: 22707710.

Kushnir VA, Darmon SK, Albertini DF, et al. Effectiveness of in vitro fertilization with preimplantation genetic screening: a reanalysis of United States assisted reproductive technology data 2011-2012. Fertility and Sterility 2016;106(1):75-79.

Kwan I, Bhattacharya S, Kang A, et al. Monitoring of stimulated cycles in assisted reproduction (IVF and ICSI). Cochrane Database Syst Rev 2014;8:Cd005289. PMID: 25150465.

Kwan I, Bhattacharya S, Knox F, et al. Pain relief for women undergoing oocyte retrieval for assisted reproduction. Cochrane Database Syst Rev 2013;1:Cd004829. PMID: 23440796.

Kwan I, Bhattacharya S, McNeil A, et al. Monitoring of stimulated cycles in assisted reproduction (IVF and ICSI). Cochrane Database Syst Rev 2008;(2):Cd005289. PMID: 18425917.

Kwon H, Choi DH and Kim EK. Absolute position versus relative position in embryo transfer: A randomized controlled trial. Reproductive Biology and Endocrinology 2015;13(1).

Kyrou D, Kolibianakis EM, Devroey P, et al. Is the use of donor sperm associated with a higher incidence of preeclampsia in women who achieve pregnancy after intrauterine insemination? Fertil Steril 2010;93(4):1124-7. PMID: 19232411.

La Sala GB, Villani MT, Nicoli A, et al. The effect of legislation on outcomes of assisted reproduction technology: lessons from the 2004 Italian law. Fertil Steril 2008;89(4):854-9. PMID: 17681340.

Lamb JD, Shen S, McCulloch C, et al. Follicle-stimulating hormone administered at the time of human chorionic gonadotropin trigger improves oocyte developmental competence in in vitro fertilization cycles: a randomized, double-blind, placebo-controlled trial. Fertil Steril 2011;95(5):1655-60. PMID: 21315341.

Langen ES, Shahine LK, Lamb JD, et al. Asian ethnicity and poor outcomes after in vitro fertilization blastocyst transfer. Obstet Gynecol 2010;115(3):591-6. PMID: 20177291.

Lathi RB, Chi YY, Liu J, et al. Frozen blastocyst embryo transfer using a supplemented natural cycle protocol has a similar live birth rate compared to a programmed cycle protocol. Journal of Assisted Reproduction and Genetics 2015;32(7):1057-1062.

Lazaraviciute G, Kauser M, Bhattacharya S, et al. A systematic review and meta-analysis of DNA methylation levels and imprinting disorders in children conceived by IVF/ICSI compared with children conceived spontaneously. Hum Reprod Update 2014;20(6):840-52. PMID: 24961233.

Le W, Su SH, Shi LH, et al. Effect of male body mass index on clinical outcomes following assisted reproductive technology: a meta-analysis. Andrologia 2015. PMID: 26276351. 
Ledger W, Wiebinga C, Anderson P, et al. Costs and outcomes associated with IVF using recombinant FSH. Reprod Biomed Online 2009;19(3):337-42. PMID: 19778478.

Lee E, Illingworth $\mathrm{P}$, Wilton L, et al. The clinical effectiveness of preimplantation genetic diagnosis for aneuploidy in all 24 chromosomes (PGD-A): Systematic review. Human Reproduction 2015;30(2):473-483.

Lee MW, Kumolosasi E, Mohd Razi ZR, et al. Efficacy of letrozole and clomiphene in patients with multiple-cause infertility undergoing intrauterine insemination. International Journal of Pharmacy and Pharmaceutical Sciences 2012;4(SUPPL.3):264-269.

Legge A, Bouzayen R, Hamilton L, et al. The impact of maternal body mass index on in vitro fertilization outcomes. J Obstet Gynaecol Can 2014;36(7):613-9. PMID: 25184980.

Lepecka-Klusek C and Jakiel G. The socio-economic conditioning of difficulties in adaptation to pregnancy following assisted reproductive techniques. Eur J Obstet Gynecol Reprod Biol 2009;143(1):50-4. PMID: 19128868.

Lerner-Geva L, Rabinovici J, Olmer L, et al. Are infertility treatments a potential risk factor for cancer development? Perspective of 30 years of follow-up. Gynecol Endocrinol 2012;28(10):809-14. PMID: 22475084.

Levi Setti PE, Albani E, Novara P, et al. Results of in vitro fertilization in Italy after the introduction of a new law. Fertil Steril 2008;90(4):1081-6. PMID: 18001725.

Levi-Setti PE, Cirillo F, Smeraldi A, et al. No advantage of fresh blastocyst versus cleavage stage embryo transfer in women under the age of 39: a randomized controlled study. J Assist Reprod Genet 2017. PMID: 29168022.

Levi Setti PE, Moioli M, Smeraldi A, et al. Obstetric outcome and incidence of congenital anomalies in 2351 IVF/ICSI babies. J Assist Reprod Genet 2016. PMID: 27116010.

Li L, Long XL, Du HZ, et al. Incidence and potential causes affecting monozygotic twin formation following in vitro fertilization and embryo transfer. Journal of Reproduction and Contraception 2012;23(2):93-101.

Li Y, Yang D and Zhang Q. Impact of overweight and underweight on IVF treatment in Chinese women. Gynecol Endocrinol 2010;26(6):416-22. PMID: 20170351.

Li Z, Wang YA, Ledger W, et al. Clinical outcomes following cryopreservation of blastocysts by vitrification or slow freezing: a population-based cohort study. Hum Reprod 2014;29(12):2794801. PMID: 25316444.

Liberman RF, Getz KD, Heinke D, et al. Assisted Reproductive Technology and Birth Defects: Effects of Subfertility and Multiple Births. Birth Defects Res 2017;109(14):1144-1153. PMID: 28635008. 
Lin H, Li Y, Li L, et al. Effect of delayed initiation of gonadotropin in luteal long protocol on in vitro fertilization. Gynecol Endocrinol 2013;29(9):846-50. PMID: 23865696.

Lin MH, Wu FS, Lee RK, et al. Dual trigger with combination of gonadotropin-releasing hormone agonist and human chorionic gonadotropin significantly improves the live-birth rate for normal responders in GnRH-antagonist cycles. Fertil Steril 2013;100(5):1296-302. PMID: 23993928.

Lind JN, Tinker SC, Broussard CS, et al. Maternal medication and herbal use and risk for hypospadias: data from the National Birth Defects Prevention Study, 1997-2007. Pharmacoepidemiol Drug Saf 2013;22(7):783-93. PMID: 23620412.

Liu W, Tal R, Chao H, et al. Effect of local endometrial injury in proliferative vs. luteal phase on IVF outcomes in unselected subfertile women undergoing in vitro fertilization. Reprod Biol Endocrinol 2017;15(1):75. PMID: 28938896.

Liu Y, Chen Q, Yu S, et al. Progestin-primed ovarian stimulation with or without clomiphene citrate supplementation in normal ovulatory women undergoing in vitro fertilization/intracytoplasmic sperm injection: A prospective randomized controlled trial. Clin Endocrinol (Oxf) 2018;88(3):442-452. PMID: 29247457.

Lockwood G, Cometti B, Bogstad J, et al. A randomized controlled trial comparing the efficacy and safety of two HMG preparations gaining their LH bioactivity from different HCG sources. Reproductive BioMedicine Online 2017;35(1):17-27.

Lockwood G, Griesinger G and Cometti B. Subcutaneous progesterone versus vaginal progesterone gel for luteal phase support in in vitro fertilization: a noninferiority randomized controlled study. Fertil Steril 2014;101(1):112-119.e3. PMID: 24140033.

Lodigiani C, Dentali F, Banfi E, et al. The effect of parnaparin sodium on in vitro fertilization outcome: A prospective randomized controlled trial. Thromb Res 2017;159:116-121. PMID: 28826740.

Lopez-Regalado ML, Clavero A, Gonzalvo MC, et al. Randomised clinical trial comparing elective single-embryo transfer followed by single-embryo cryotransfer versus double embryo transfer. Eur J Obstet Gynecol Reprod Biol 2014;178:192-8. PMID: 24798072.

Ludwig AK, Katalinic A, Jendrysik J, et al. Spontaneous pregnancy after successful ICSI treatment: evaluation of risk factors in 899 families in Germany. Reprod Biomed Online 2008;17(3):403-9. PMID: 18765012.

Lukaszuk K, Liss J, Kunicki M, et al. Estradiol valerate pretreatment in short protocol GnRHagonist cycles versus combined pretreatment with oral contraceptive pills in long protocol GnRH-agonist cycles: A randomised controlled trial. BioMed Research International 2015;2015.

Luke B, Brown MB, Spector LG, et al. Cancer in women after assisted reproductive technology Presented at the 70th Annual Meeting of the American Society for Reproductive Medicine, Honolulu, Hawaii, October 18-22, 2014. Fertility and Sterility 2015;104(5):1218-1226. 
Luke B, Brown MB, Stern JE, et al. Using the Society for Assisted Reproductive Technology Clinic Outcome System morphological measures to predict live birth after assisted reproductive technology. Fertil Steril 2014;102(5):1338-44. PMID: 25217871.

Luke B, Gopal D, Cabral H, et al. Perinatal outcomes of singleton siblings: the effects of changing maternal fertility status. J Assist Reprod Genet 2016;33(9):1203-13. PMID: 27318927.

Luke B, Gopal D, Cabral H, et al. Pregnancy, birth, and infant outcomes by maternal fertility status: the Massachusetts Outcomes Study of Assisted Reproductive Technology. Am J Obstet Gynecol 2017;217(3):327.e1-327.e14. PMID: 28400311.

Luke B, Stern JE, Kotelchuck M, et al. Adverse pregnancy outcomes after in vitro fertilization: Effect of number of embryos transferred and plurality at conception. Fertility and Sterility 2015;104(1):79-86.

Lundberg FE, Iliadou AN, Rodriguez-Wallberg K, et al. Ovarian stimulation and risk of breast cancer in Swedish women. Fertil Steril 2017;108(1):137-144. PMID: 28600105.

Lung FW, Chiang TL, Lin SJ, et al. Assisted reproductive technology has no association with autism spectrum disorders: The Taiwan Birth Cohort Study. Autism 2017.

Lyall K, Baker A, Hertz-Picciotto I, et al. Infertility and its treatments in association with autism spectrum disorders: a review and results from the CHARGE study. Int J Environ Res Public Health 2013;10(8):3715-34. PMID: 23965925.

Lyall K, Pauls DL, Spiegelman D, et al. Fertility therapies, infertility and autism spectrum disorders in the Nurses' Health Study II. Paediatr Perinat Epidemiol 2012;26(4):361-72. PMID: 22686388.

Ma NZ, Chen L, Dai W, et al. Influence of endometrial thickness on treatment outcomes following in vitro fertilization/intracytoplasmic sperm injection. Reprod Biol Endocrinol 2017;15(1):5. PMID: 28056983.

Madani T, Mohammadi Yeganeh L, Ezabadi Z, et al. Comparing the efficacy of urinary and recombinant hCG on oocyte/follicle ratio to trigger ovulation in women undergoing intracytoplasmic sperm injection cycles: a randomized controlled trial. J Assist Reprod Genet 2013;30(2):239-45. PMID: 23274511.

Madaschi C, Braga DP, Figueira Rde C, et al. Effect of acupuncture on assisted reproduction treatment outcomes. Acupunct Med 2010;28(4):180-4. PMID: 20959311.

Maher MA. Luteal phase support may improve pregnancy outcomes during intrauterine insemination cycles. Eur J Obstet Gynecol Reprod Biol 2011;157(1):57-62. PMID: 21514032.

Maheshwari A, Gibreel A, Siristatidis CS, et al. Gonadotrophin-releasing hormone agonist protocols for pituitary suppression in assisted reproduction. Cochrane Database Syst Rev 2011;(8):Cd006919. PMID: 21833958. 
Maheshwari A, Kalampokas T, Davidson J, et al. Obstetric and perinatal outcomes in singleton pregnancies resulting from the transfer of blastocyst-stage versus cleavage-stage embryos generated through in vitro fertilization treatment: a systematic review and meta-analysis. Fertil Steril 2013;100(6):1615-21.e1-10. PMID: 24083875.

Maheshwari A, Pandey S, Shetty A, et al. Obstetric and perinatal outcomes in singleton pregnancies resulting from the transfer of frozen thawed versus fresh embryos generated through in vitro fertilization treatment: a systematic review and meta-analysis. Fertil Steril 2012;98(2):368-77.e1-9. PMID: 22698643.

Maheshwari A, Raja EA and Bhattacharya S. Obstetric and perinatal outcomes after either fresh or thawed frozen embryo transfer: an analysis of 112,432 singleton pregnancies recorded in the Human Fertilisation and Embryology Authority anonymized dataset. Fertil Steril 2016;106(7):1703-1708. PMID: 27678031.

Mahmoud Youssef MA, van Wely M, Aboulfoutouh I, et al. Is there a place for corifollitropin alfa in IVF/ICSI cycles? A systematic review and meta-analysis. Fertil Steril 2012;97(4):876-85. PMID: 22277766.

Mahran A, Ibrahim M and Bahaa $\mathrm{H}$. The effect of endometrial injury on first cycle IVF/ICSI outcome: A randomized controlled trial. International Journal of Reproductive BioMedicine 2016;14(3):193-198.

Maimburg RD and Vaeth M. Do children born after assisted conception have less risk of developing infantile autism? Hum Reprod 2007;22(7):1841-3. PMID: 17456530.

Mak JSM, Chung CHS, Chung JPW, et al. The effect of endometrial scratch on natural-cycle cryopreserved embryo transfer outcomes: a randomized controlled study. Reproductive BioMedicine Online 2017;35(1):28-36.

Malizia BA, Dodge LE, Penzias AS, et al. The cumulative probability of liveborn multiples after in vitro fertilization: a cohort study of more than 10,000 women. Fertil Steril 2013;99(2):393-9. PMID: 23141053.

Mamata D, Ray SK, Pratap K, et al. Impact of different controlled ovarian stimulation protocols on the physical and psychological burdens in women undergoing in vitro fertilization/intra cytoplasmic sperm injection. J Hum Reprod Sci 2015;8(2):86-92. PMID: 26157299.

Mamidala MP, Polinedi A, Kumar PT, et al. Maternal hormonal interventions as a risk factor for Autism Spectrum Disorder: an epidemiological assessment from India. J Biosci 2013;38(5):88792. PMID: 24296891.

Manders M, McLindon L, Schulze B, et al. Timed intercourse for couples trying to conceive. Cochrane Database Syst Rev 2015;3:Cd011345. PMID: 25775926.

Manheimer E, Zhang G, Udoff L, et al. Effects of acupuncture on rates of pregnancy and live birth among women undergoing in vitro fertilisation: systematic review and meta-analysis. Bmj 2008;336(7643):545-9. PMID: 18258932. 
Marino JL, Moore VM, Willson KJ, et al. Perinatal outcomes by mode of assisted conception and sub-fertility in an Australian data linkage cohort. PLoS One 2014;9(1):e80398. PMID: 24416127.

Marschalek J, Franz M, Gonen Y, et al. The effect of slow release insemination on pregnancy rates: report of two randomized controlled pilot studies and meta-analysis. Arch Gynecol Obstet 2017;295(4):1025-1032. PMID: 28197716.

Martin AS, Chang J, Zhang Y, et al. Perinatal outcomes among singletons after assisted reproductive technology with single-embryo or double-embryo transfer versus no assisted reproductive technology. Fertil Steril 2017;107(4):954-960. PMID: 28292615.

Martin AS, Monsour M, Kissin DM, et al. Trends in severe maternal morbidity after assisted reproductive technology in the United States, 2008-2012. Obstetrics and Gynecology 2016;127(1):59-66.

Martin-Johnston M, Beltsos AN, Grotjan HE, et al. Adding human menopausal gonadotrophin to antagonist protocols - is there a benefit? Reprod Biomed Online 2007;15(2):161-8. PMID: 17697491.

Martins WP, Ferriani RA, Navarro PA, et al. GnRH agonist during luteal phase in women undergoing assisted reproductive techniques: systematic review and meta-analysis of randomized controlled trials. Ultrasound Obstet Gynecol 2016;47(2):144-51. PMID: 25854891.

Martins WP, Vieira AD, Figueiredo JB, et al. FSH replaced by low-dose hCG in the late follicular phase versus continued FSH for assisted reproductive techniques. Cochrane Database Syst Rev 2013;3:Cd010042. PMID: 23543584.

Mastenbroek S, Twisk M, van Echten-Arends J, et al. In vitro fertilization with preimplantation genetic screening. N Engl J Med 2007;357(1):9-17. PMID: 17611204.

Matorras R, Andres M, Mendoza R, et al. Prevention of ovarian hyperstimulation syndrome in GnRH agonist IVF cycles in moderate risk patients: randomized study comparing hydroxyethyl starch versus cabergoline and hydroxyethyl starch. Eur J Obstet Gynecol Reprod Biol 2013;170(2):439-43. PMID: 23932181.

Matsubara K, Murakami N, Fukami M, et al. Risk assessment of medically assisted reproduction and advanced maternal ages in the development of Prader-Willi syndrome due to UPD(15)mat. Clinical Genetics 2016;89(5):614-619.

McAvey B, Zapantis A, Jindal SK, et al. How many eggs are needed to produce an assisted reproductive technology baby: is more always better? Fertil Steril 2011;96(2):332-5. PMID: 21718991.

McDowell S, Kroon B, Ford E, et al. Advanced sperm selection techniques for assisted reproduction. Cochrane Database Syst Rev 2014;10:Cd010461. PMID: 25348679. 
McQueen DB, Schufreider A, Lee SM, et al. Racial disparities in in vitro fertilization outcomes. Fertil Steril 2015;104(2):398-402.e1. PMID: 26049056.

Meijerink AM, Oomen RE, Fleischer K, et al. Effect of maternal and treatment related factors on the prevalence of birth defects after PESA-ICSI and TESE-ICSI: a retrospective cohort study. Acta Obstet Gynecol Scand 2015. PMID: 26265143.

Melnick AP, Murphy EM, Masbou AK, et al. Autologous endometrial coculture biopsy: Is timing everything? Fertility and Sterility 2015;104(1):104-109.e1.

Merritt MA, De Pari M, Vitonis AF, et al. Reproductive characteristics in relation to ovarian cancer risk by histologic pathways. Hum Reprod 2013;28(5):1406-17. PMID: 23315066.

Merritt TA, Goldstein M, Philips R, et al. Impact of ART on pregnancies in California: an analysis of maternity outcomes and insights into the added burden of neonatal intensive care. $\mathrm{J}$ Perinatol 2014;34(5):345-50. PMID: 24556981.

Mersereau J, Stanhiser J, Coddington C, et al. Patient and cycle characteristics predicting high pregnancy rates with single-embryo transfer: an analysis of the Society for Assisted Reproductive Technology outcomes between 2004 and 2013. Fertil Steril 2017;108(5):750-756. PMID: 28923285.

Mersereau JE, Pergament E, Zhang X, et al. Preimplantation genetic screening to improve in vitro fertilization pregnancy rates: a prospective randomized controlled trial. Fertil Steril 2008;90(4):1287-9. PMID: 18061593.

Messerlian C, Platt RW, Tan SL, et al. Low-technology assisted reproduction and the risk of preterm birth in a hospital-based cohort. Fertil Steril 2015;103(1):81-8.e2. PMID: 25456793.

Mesut N, Ciray HN, Mesut A, et al. Cryopreservation of blastocysts is the most feasible strategy in good responder patients. Fertil Steril 2011;96(5):1121-5.e1. PMID: 21890133.

Meyer LR, Klipstein S, Hazlett WD, et al. A prospective randomized controlled trial of preimplantation genetic screening in the "good prognosis" patient. Fertil Steril 2009;91(5):17318. PMID: 18804207.

Michnova L, Dostal J, Kudela M, et al. Vaginal use of micronized progesterone for luteal support.A randomized study comparing Utrogestan(R) and Crinone(R) 8. Biomed Pap Med Fac Univ Palacky Olomouc Czech Repub 2017;161(1):86-91. PMID: 28323291.

Middelburg KJ, Heineman MJ, Bos AF, et al. The Groningen ART cohort study: ovarian hyperstimulation and the in vitro procedure do not affect neurological outcome in infancy. Hum Reprod 2009;24(12):3119-26. PMID: 19749193.

Miller N, Biron-Shental T, Sukenik-Halevy R, et al. Oocyte activation by calcium ionophore and congenital birth defects: a retrospective cohort study. Fertil Steril 2016;106(3):590-596.e2.

PMID: 27143515. 
Miralpeix E, Gonzalez-Comadran M, Sola I, et al. Efficacy of luteal phase support with vaginal progesterone in intrauterine insemination: a systematic review and meta-analysis. J Assist Reprod Genet 2014;31(1):89-100. PMID: 24189966.

Moaddab A, Chervenak FA, McCullough LB, et al. Effect of advanced maternal age on maternal and neonatal outcomes in assisted reproductive technology pregnancies. European Journal of Obstetrics Gynecology and Reproductive Biology 2017;216:178-183.

Mogili KD, Selliah HY, Chandy A, et al. Do poor responders have poor perinatal outcomes? A retrospective analysis of 1386 assisted reproductive technology cycles. Middle East Fertility Society Journal 2017.

Morency AM, Shah PS, Seaward PGR, et al. Obstetrical and neonatal outcomes of triplet birthsspontaneous versus assisted reproductive technology conception. Journal of Maternal-Fetal and Neonatal Medicine 2016;29(6):938-943.

Moro F, Scarinci E, Palla C, et al. Highly purified hMG versus recombinant FSH plus recombinant $\mathrm{LH}$ in intrauterine insemination cycles in women $>/=35$ years: a RCT. Hum Reprod 2015;30(1):179-85. PMID: 25398971.

Moses XJ, Torres T, Rasmussen A, et al. Congenital anomalies identified at birth among infants born following assisted reproductive technology in Colorado. Birth Defects Res A Clin Mol Teratol 2014;100(2):92-9. PMID: 24532453.

Mounce G, McVeigh E, Turner K, et al. Randomized, controlled pilot trial of natural versus hormone replacement therapy cycles in frozen embryo replacement in vitro fertilization. Fertil Steril 2015;104(4):915-920.e1. PMID: 26255087.

Moustafa MK, Sheded SA and El Aziz Mousta MA. Elective single embryo transfer versus double embryo transfer in assisted reproduction. Reprod Biomed Online 2008;17(1):82-7. PMID: 18616895.

Mullin CM, Fino ME, Talebian S, et al. Comparison of pregnancy outcomes in elective single blastocyst transfer versus double blastocyst transfer stratified by age. Fertil Steril 2010;93(6):1837-43. PMID: 19249756.

Murray A. Biological risk versus socio-economic advantage: low birth-weight, multiple births and income variations among Irish infants born following fertility treatments. Ir J Med Sci 2014;183(4):667-70. PMID: 24913735.

Musella M, Milone M, Bellini M, et al. Effect of bariatric surgery on obesity-related infertility. Surg Obes Relat Dis 2012;8(4):445-9. PMID: 22057155.

Mussa A, Molinatto C, Cerrato F, et al. Assisted Reproductive Techniques and Risk of Beckwith-Wiedemann Syndrome. Pediatrics 2017;140(1). PMID: 28634246.

Naether OGJ, Tandler-Schneider A and Bilger W. Individualized recombinant human folliclestimulating hormone dosing using the CONSORT calculator in assisted reproductive technology: 
A large, multicenter, observational study of routine clinical practice. Drug, Healthcare and Patient Safety 2015;7:69-76.

Nakashima A, Araki R, Tani H, et al. Implications of assisted reproductive technologies on term singleton birth weight: an analysis of 25,777 children in the national assisted reproduction registry of Japan. Fertil Steril 2013;99(2):450-5. PMID: 23058683.

Nardelli AA, Stafinski T, Motan T, et al. Assisted reproductive technologies (ARTs): evaluation of evidence to support public policy development. Reprod Health 2014;11(1):76. PMID: 25376649 .

Narkwichean A, Maalouf W, Baumgarten M, et al. Efficacy of Dehydroepiandrosterone (DHEA) to overcome the effect of ovarian ageing (DITTO): A proof of principle double blinded randomized placebo controlled trial. Eur J Obstet Gynecol Reprod Biol 2017;218:39-48. PMID: 28934714.

Nastri CO, Ferriani RA, Raine-Fenning N, et al. Endometrial scratching performed in the nontransfer cycle and outcome of assisted reproduction: a randomized controlled trial. Ultrasound Obstet Gynecol 2013;42(4):375-82. PMID: 23754314.

Nastri CO, Gibreel A, Raine-Fenning N, et al. Endometrial injury in women undergoing assisted reproductive techniques. Cochrane Database Syst Rev 2012;7:Cd009517. PMID: 22786529.

Nastri CO, Lensen SF, Gibreel A, et al. Endometrial injury in women undergoing assisted reproductive techniques. Cochrane Database Syst Rev 2015;3:Cd009517. PMID: 25803542.

Navali N, Gassemzadeh A, Farzadi L, et al. Intrauterine administration of hCG immediately after oocyte retrieval and the outcome of ICSI: a randomized controlled trial. Hum Reprod 2016. PMID: 27680029.

$\mathrm{Ng} \mathrm{EH,} \mathrm{Li} \mathrm{RH,} \mathrm{Chen} \mathrm{L,} \mathrm{et} \mathrm{al.} \mathrm{A} \mathrm{randomized} \mathrm{double} \mathrm{blind} \mathrm{comparison} \mathrm{of} \mathrm{atosiban} \mathrm{in} \mathrm{patients}$ undergoing IVF treatment. Hum Reprod 2014;29(12):2687-94. PMID: 25336707.

Nicopoullos JD and Abdalla H. Poor response cycles: when should we cancel? Comparison of outcome between egg collection, intrauterine insemination conversion, and follow-up cycles after abandonment. Fertil Steril 2011;95(1):68-71. PMID: 20646690.

Noori S, Nedaeifard L, Agarasouli Z, et al. Prelinguistic behavior of infants of assisted reproductive techniques. Iranian Journal of Pediatrics 2012;22(4):535-538.

Norian JM, Levens ED, Richter KS, et al. Conversion from assisted reproductive technology to intrauterine insemination in low responders: is it advantageous? Fertil Steril 2010;94(6):2073-7. PMID: 20171626.

Noriega-Portella L, Noriega-Hoces L, Delgado A, et al. Effect of letrozole at $2.5 \mathrm{mg}$ or 5.0 $\mathrm{mg}$ /day on ovarian stimulation with gonadotropins in women undergoing intrauterine insemination. Fertil Steril 2008;90(5):1818-25. PMID: 18083169. 
Nouri K, Tempfer CB, Walch K, et al. Predictive value of the time interval between embryo loading and transfer for IVF/ICSI success: A prospective cohort study. Reproductive Biology and Endocrinology 2015;13(1).

Nyboe Andersen A, Nelson SM, Fauser BC, et al. Individualized versus conventional ovarian stimulation for in vitro fertilization: a multicenter, randomized, controlled, assessor-blinded, phase 3 noninferiority trial. Fertil Steril 2017;107(2):387-396.e4. PMID: 27912901.

Nyfløt LT, Sandven I, Oldereid NB, et al. Assisted reproductive technology and severe postpartum haemorrhage: a case-control study. BJOG: An International Journal of Obstetrics and Gynaecology 2017;124(8):1198-1205.

Oktem M, Altinkaya SO, Yilmaz SA, et al. Effect of luteal phase support after ovulation induction and intrauterine insemination. Gynecological Endocrinology 2014;30(12):909-912.

O'Neill KE, Tuuli M, Odibo AO, et al. Sex-related growth differences are present but not enhanced in in vitro fertilization pregnancies. Fertil Steril 2014;101(2):407-12. PMID: 24220702.

Ooki S. Birth defects after assisted reproductive technology according to the method of treatment in Japan: nationwide data between 2004 and 2012. Environmental Health and Preventive Medicine 2015;20(6):460-465.

Opdahl S, Henningsen AA, Tiitinen A, et al. Risk of hypertensive disorders in pregnancies following assisted reproductive technology: a cohort study from the CoNARTaS group. Hum Reprod 2015;30(7):1724-31. PMID: 25924655.

Orgeas CC, Sanner K, Hall P, et al. Breast cancer incidence after hormonal infertility treatment in Sweden: a cohort study. Am J Obstet Gynecol 2009;200(1):72.e1-7. PMID: 19121659.

Pal L, Jindal S, Witt BR, et al. Less is more: increased gonadotropin use for ovarian stimulation adversely influences clinical pregnancy and live birth after in vitro fertilization. Fertil Steril 2008;89(6):1694-701. PMID: 18440515.

Palomba S, Falbo A, Valli B, et al. Physical activity before IVF and ICSI cycles in infertile obese women: an observational cohort study. Reprod Biomed Online 2014;29(1):72-9. PMID: 24813759 .

Pandey S, McLernon DJ, Scotland G, et al. Cost of fertility treatment and live birth outcome in women of different ages and BMI. Hum Reprod 2014;29(10):2199-211. PMID: 25061026.

Pandian Z, Bhattacharya S, Ozturk O, et al. Number of embryos for transfer following in-vitro fertilisation or intra-cytoplasmic sperm injection. Cochrane Database Syst Rev 2009;(2):Cd003416. PMID: 19370588.

Pandian Z, Marjoribanks J, Ozturk O, et al. Number of embryos for transfer following in vitro fertilisation or intra-cytoplasmic sperm injection. Cochrane Database Syst Rev 2013;7:Cd003416. PMID: 23897513. 
Pantos K, Makrakis E, Chronopoulou M, et al. Day 4 versus day 3 embryo transfer: a prospective study of clinical outcomes. Fertil Steril 2008;89(3):573-7. PMID: 17517409.

Papanikolaou EG, Fatemi H, Camus M, et al. Higher birth rate after recombinant hCG triggering compared with urinary-derived hCG in single-blastocyst IVF antagonist cycles: a randomized controlled trial. Fertil Steril 2010;94(7):2902-4. PMID: 20580358.

Papanikolaou EG, Pados G, Grimbizis G, et al. GnRH-agonist versus GnRH-antagonist IVF cycles: is the reproductive outcome affected by the incidence of progesterone elevation on the day of HCG triggering? A randomized prospective study. Hum Reprod 2012;27(6):1822-8. PMID: 22422777.

Pappo I, Lerner-Geva L, Halevy A, et al. The possible association between IVF and breast cancer incidence. Ann Surg Oncol 2008;15(4):1048-55. PMID: 18214616.

Parsanezhad ME, Jahromi BN, Rezaee S, et al. The effect of four different gonadotropin protocols on oocyte and embryo quality and pregnancy outcomes in IVF/ICSI cycles; a randomized controlled trial. Iranian Journal of Medical Sciences 2017;42(1):57-65.

Pavone M, Innes J, Hirshfeld-Cytron J, et al. Comparing thaw survival, implantation and live birth rates from cryopreserved zygotes, embryos and blastocysts. Journal of Human Reproductive Sciences 2011;4(1):23-28.

Peeraer K, Debrock S, De Loecker P, et al. Low-dose human menopausal gonadotrophin versus clomiphene citrate in subfertile couples treated with intrauterine insemination: a randomized controlled trial. Hum Reprod 2015;30(5):1079-88. PMID: 25788569.

Peeraer K, D'Hooghe T, Laurent P, et al. Impact of luteal phase support with vaginal progesterone on the clinical pregnancy rate in intrauterine insemination cycles stimulated with gonadotropins: a randomized multicenter study. Fertil Steril 2016;106(6):1490-1495. PMID: 27565253.

Pelkonen S, Gissler M, Koivurova S, et al. Physical health of singleton children born after frozen embryo transfer using slow freezing: a 3-year follow-up study. Hum Reprod 2015. PMID: 26293785.

Pelkonen S, Hartikainen AL, Ritvanen A, et al. Major congenital anomalies in children born after frozen embryo transfer: a cohort study 1995-2006. Hum Reprod 2014;29(7):1552-7. PMID: 24812318.

Pelkonen S, Koivunen R, Gissler M, et al. Perinatal outcome of children born after frozen and fresh embryo transfer: the Finnish cohort study 1995-2006. Hum Reprod 2010;25(4):914-23. PMID: 20124395.

Peterson BD, Newton CR and Feingold T. Anxiety and sexual stress in men and women undergoing infertility treatment. Fertil Steril 2007;88(4):911-4. PMID: 17433317. 
Peterson BD, Sejbaek CS, Pirritano M, et al. Are severe depressive symptoms associated with infertility-related distress in individuals and their partners? Hum Reprod 2014;29(1):76-82.

PMID: 24256990.

Petersen GL, Schmidt L, Pinborg A, et al. The influence of female and male body mass index on live births after assisted reproductive technology treatment: a nationwide register-based cohort study. Fertil Steril 2013;99(6):1654-62. PMID: 23394781.

Pinborg A, Loft A, Aaris Henningsen AK, et al. Infant outcome of 957 singletons born after frozen embryo replacement: the Danish National Cohort Study 1995-2006. Fertil Steril 2010;94(4):1320-7. PMID: 19647236.

Pinborg A, Wennerholm UB, Romundstad LB, et al. Why do singletons conceived after assisted reproduction technology have adverse perinatal outcome? Systematic review and meta-analysis. Hum Reprod Update 2013;19(2):87-104. PMID: 23154145.

Pochiraju M and Nirmalan PK. Type of conception and outcomes in women with singleton pregnancy. J Clin Diagn Res 2014;8(2):103-5. PMID: 24701495.

Poikkeus P, Gissler M, Unkila-Kallio L, et al. Obstetric and neonatal outcome after single embryo transfer. Hum Reprod 2007;22(4):1073-9. PMID: 17251357.

Poikkeus P, Saisto T, Punamaki RL, et al. Birth experience of women conceiving with assisted reproduction: a prospective multicenter study. Acta Obstet Gynecol Scand 2014;93(9):880-7. PMID: 24909073.

Polinder S, Heijnen EM, Macklon NS, et al. Cost-effectiveness of a mild compared with a standard strategy for IVF: a randomized comparison using cumulative term live birth as the primary endpoint. Hum Reprod 2008;23(2):316-23. PMID: 18033807.

Poon WB and Lian WB. Perinatal outcomes of intrauterine insemination/clomiphene pregnancies represent an intermediate risk group compared with in vitro fertilisation/intracytoplasmic sperm injection and naturally conceived pregnancies. J Paediatr Child Health 2013;49(9):733-40. PMID: 23758121.

Poustie VJ, Dodd S and Drakeley AJ. Low-dose aspirin for in vitro fertilisation. Cochrane Database Syst Rev 2007;(4):Cd004832. PMID: 17943828.

Pouwer AW, Farquhar C and Kremer JA. Long-acting FSH versus daily FSH for women undergoing assisted reproduction. Cochrane Database Syst Rev 2012;6:Cd009577. PMID: 22696386.

Pouwer AW, Farquhar C and Kremer JA. Long-acting FSH versus daily FSH for women undergoing assisted reproduction. Cochrane Database Syst Rev 2015;7:Cd009577. PMID: 26171903.

Provost MP, Acharya KS, Acharya CR, et al. Pregnancy outcomes decline with increasing recipient body mass index: An analysis of 22,317 fresh donor/recipient cycles from the 2008- 
2010 Society for Assisted Reproductive Technology Clinic Outcome Reporting System registry. Fertility and Sterility 2015.

Puumala SE, Ross JA, Feusner JH, et al. Parental infertility, infertility treatment and hepatoblastoma: a report from the Children's Oncology Group. Hum Reprod 2012;27(6):164956. PMID: 22473396.

Puumala SE, Ross JA, Olshan AF, et al. Reproductive history, infertility treatment, and the risk of acute leukemia in children with down syndrome: a report from the Children's Oncology Group. Cancer 2007;110(9):2067-74. PMID: 17849462.

Qin J, Sheng X, Wu D, et al. Adverse Obstetric Outcomes Associated With In Vitro Fertilization in Singleton Pregnancies. Reprod Sci 2017;24(4):595-608. PMID: 27620916.

Qin J, Wang H, Sheng X, et al. Pregnancy-related complications and adverse pregnancy outcomes in multiple pregnancies resulting from assisted reproductive technology: a metaanalysis of cohort studies. Fertil Steril 2015;103(6):1492-508.e1-7. PMID: 25910567.

Qu F, Zhou J and Ren RX. Effects of acupuncture on the outcomes of in vitro fertilization: a systematic review and meta-analysis. J Altern Complement Med 2012;18(5):429-39. PMID: 22540969.

Qu F, Zhou J, Bovey M, et al. Does acupuncture improve the outcome of in vitro fertilization? Guidance for future trials. European Journal of Integrative Medicine 2012;4(3):e234-e244.

Qublan HS, Al-Khaderei S, Abu-Salem AN, et al. Metformin in the treatment of clomiphene citrate-resistant women with polycystic ovary syndrome undergoing in vitro fertilisation treatment: a randomised controlled trial. J Obstet Gynaecol 2009;29(7):651-5. PMID: 19757275.

Quinn MM, Rosen MP, Allen IE, et al. Decreased clinical pregnancy and live birth rates after short interval from delivery to subsequent assisted reproductive treatment cycle. Hum Reprod 2018;33(7):1316-1321. PMID: 29912323.

Raatikainen K, Kuivasaari-Pirinen P, Hippelainen M, et al. Comparison of the pregnancy outcomes of subfertile women after infertility treatment and in naturally conceived pregnancies. Hum Reprod 2012;27(4):1162-9. PMID: 22333986.

Rabati BK and Zeidi SN. Investigation of pregnancy outcome and ovarian hyper stimulation syndrome prevention in agonist and antagonist gonadotropin-releasing hormone protocol. Journal of Research in Medical Sciences 2012;17(11):1063-1066.

Raisanen S, Randell K, Nielsen HS, et al. Socioeconomic status affects the prevalence, but not the perinatal outcomes, of in vitro fertilization pregnancies. Hum Reprod 2013;28(11):3118-25. PMID: 23892321.

Raisanen S, Sokka A, Georgiadis L, et al. Infertility treatment and umbilical cord length-novel markers of childhood epilepsy? PLoS One 2013;8(2):e55394. PMID: 23418441. 
Rama Raju GA, Suryanarayana K, Jaya Prakash G, et al. Comparison of follitropin-beta administered by a pen device with conventional syringe in an ART programme - a retrospective study. J Clin Pharm Ther 2008;33(4):401-7. PMID: 18613858.

Rashidi BH, Tanha FD, Rahmanpour H, et al. Luteal Phase Support in the Intrauterine Insemination (IUI) Cycles: A Randomized Double Blind, Placebo Controlled Study. J Family Reprod Health 2014;8(4):149-53. PMID: 25530766.

Ratson R, Sheiner E, Davidson E, et al. Fertility treatments and the risk for ophthalmic complications: a cohort study with 25-year follow-up. Journal of Maternal-Fetal and Neonatal Medicine 2016;29(19):3094-3097.

Reid SM, Jaques AM, Susanto C, et al. Cerebral palsy and assisted reproductive technologies: a case-control study. Dev Med Child Neurol 2010;52(7):e161-6. PMID: 20015250.

Reigstad MM, Larsen IK, Myklebust TA, et al. Cancer risk among parous women following assisted reproductive technology. Hum Reprod 2015;30(8):1952-63. PMID: 26113657.

Reigstad MM, Larsen IK, Myklebust TA, et al. Risk of breast cancer following fertility treatment--a registry based cohort study of parous women in Norway. Int J Cancer 2015;136(5):1140-8. PMID: 25042052.

Reigstad MM, Larsen IK, Myklebust TA, et al. Risk of cancer in children conceived by assisted reproductive technology. Pediatrics 2016;137(3).

Reis S, Xavier MR, Coelho R, et al. Psychological impact of single and multiple courses of assisted reproductive treatments in couples: a comparative study. Eur J Obstet Gynecol Reprod Biol 2013;171(1):61-6. PMID: 23928476.

Repokari L, Punamaki RL, Unkila-Kallio L, et al. Infertility treatment and marital relationships: a 1-year prospective study among successfully treated ART couples and their controls. Hum Reprod 2007;22(5):1481-91. PMID: 17307807.

Rettenbacher M, Andersen AN, Garcia-Velasco JA, et al. A multi-centre phase 3 study comparing efficacy and safety of Bemfola ${ }^{\circledR}$ versus Gonal-f ${ }^{\circledR}$ in women undergoing ovarian stimulation for IVF. Reproductive BioMedicine Online 2015;30(5):504-513.

Revelli A, Pittatore G, Casano S, et al. Efficacy and safety of late-start Corifollitropin-alfa administration for controlled ovarian hyperstimulation in IVF: a cohort, case-control study. Journal of Assisted Reproduction and Genetics 2015;32(3):429-434.

Rimm AA, Katayama AC and Katayama KP. A meta-analysis of the impact of IVF and ICSI on major malformations after adjusting for the effect of subfertility. J Assist Reprod Genet 2011;28(8):699-705. PMID: 21625967.

Rinaldi L, Lisi F and Selman H. Mild/minimal stimulation protocol for ovarian stimulation of patients at high risk of developing ovarian hyperstimulation syndrome. J Endocrinol Invest 2014;37(1):65-70. PMID: 24464452. 
Rittenberg V, Seshadri S, Sunkara SK, et al. Effect of body mass index on IVF treatment outcome: an updated systematic review and meta-analysis. Reprod Biomed Online 2011;23(4):421-39. PMID: 21885344.

Rizzuto I, Behrens RF and Smith LA. Risk of ovarian cancer in women treated with ovarian stimulating drugs for infertility. Cochrane Database Syst Rev 2013;8:Cd008215. PMID: 23943232.

Roca de Bes M, Gutierrez Maldonado J and Gris Martinez JM. Psychosocial risks associated with multiple births resulting from assisted reproduction: a Spanish sample. Fertil Steril 2009;92(3):1059-66. PMID: 18973888.

Rockliff HE, Lightman SL, Rhidian E, et al. A systematic review of psychosocial factors associated with emotional adjustment in in vitro fertilization patients. Hum Reprod Update 2014;20(4):594-613. PMID: 24676468.

Rodriguez Barredo DB, Tur Padro R, Mancini F, et al. Elective single embryo transfer and cumulative pregnancy rate: five-year experience in a Southern European Country. Gynecol Endocrinol 2012;28(6):425-8. PMID: 22114913.

Romany L, Garrido N, Cobo A, et al. Obstetric and perinatal outcome of babies born from sperm selected by MACS from a randomized controlled trial. J Assist Reprod Genet 2017;34(2):201207. PMID: 27882439.

Romany L, Garrido N, Motato Y, et al. Removal of annexin V-positive sperm cells for intracytoplasmic sperm injection in ovum donation cycles does not improve reproductive outcome: a controlled and randomized trial in unselected males. Fertil Steril 2014;102(6):156775.e1. PMID: 25305728.

Romero Nieto MI, Lorente Gonzalez J, Arjona-Berral JE, et al. Luteal phase support with progesterone in intrauterine insemination: a prospective randomized study. Gynecol Endocrinol 2014;30(3):197-201. PMID: 24397361.

Romundstad LB, Romundstad PR, Sunde A, et al. Effects of technology or maternal factors on perinatal outcome after assisted fertilisation: a population-based cohort study. Lancet 2008;372(9640):737-43. PMID: 18674812.

Roy TK, Bradley CK, Bowman MC, et al. Single-embryo transfer of vitrified-warmed blastocysts yields equivalent live-birth rates and improved neonatal outcomes compared with fresh transfers. Fertil Steril 2014;101(5):1294-301. PMID: 24582521.

Rudant J, Amigou A, Orsi L, et al. Fertility treatments, congenital malformations, fetal loss, and childhood acute leukemia: the ESCALE study (SFCE). Pediatr Blood Cancer 2013;60(2):301-8. PMID: 22610722.

Safari S, Razi MH, Safari S, et al. Routine use of EmbryoGlue((R)) as embryo transfer medium does not improve the ART outcomes. Arch Gynecol Obstet 2015;291(2):433-7. PMID: 25151028. 
Sagnella F, Moro F, Lanzone A, et al. A prospective randomized noninferiority study comparing recombinant FSH and highly purified menotropin in intrauterine insemination cycles in couples with unexplained infertility and/or mild-moderate male factor. Fertil Steril 2011;95(2):689-94. PMID: 20869704.

Sagoskin AW, Levy MJ, Tucker MJ, et al. Laser assisted hatching in good prognosis patients undergoing in vitro fertilization-embryo transfer: a randomized controlled trial. Fertil Steril 2007;87(2):283-7. PMID: 17094975.

Sagot P, Bechoua S, Ferdynus C, et al. Similarly increased congenital anomaly rates after intrauterine insemination and IVF technologies: a retrospective cohort study. Hum Reprod 2012;27(3):902-9. PMID: 22215631.

Saharkhiz N, Zamaniyan M, Salehpour S, et al. A comparative study of dydrogesterone and micronized progesterone for luteal phase support during in vitro fertilization (IVF) cycles. Gynecol Endocrinol 2016;32(3):213-7. PMID: 26486011.

Sahin S, Ozay A, Ergin E, et al. The risk of ectopic pregnancy following GnRH agonist triggering compared with hCG triggering in GnRH antagonist IVF cycles. Arch Gynecol Obstet 2015;291(1):185-91. PMID: 25078054.

Saldeen P, Abou-Setta AM, Bergh T, et al. A prospective randomized controlled trial comparing two embryo transfer catheters in an ART program. Fertil Steril 2008;90(3):599-603. PMID: 18054000 .

Sandin S, Nygren KG, Iliadou A, et al. Autism and mental retardation among offspring born after in vitro fertilization. Jama 2013;310(1):75-84. PMID: 23821091.

Sanner K, Conner P, Bergfeldt K, et al. Ovarian epithelial neoplasia after hormonal infertility treatment: long-term follow-up of a historical cohort in Sweden. Fertil Steril 2009;91(4):1152-8. PMID: 18371964.

Schendelaar P, Heineman KR, Heineman MJ, et al. Movement variation in infants born following IVF/ICSI with and without ovarian hyperstimulation. Early Hum Dev 2013;89(7):50713. PMID: 23582573.

Schendelaar P, La Bastide-Van Gemert S, Heineman MJ, et al. Subfertility factors rather than assisted conception factors affect cognitive and behavioural development of 4-year-old singletons. Reprod Biomed Online 2016. PMID: 27665057.

Schendelaar P, Van den Heuvel ER, Heineman MJ, et al. Increased time to pregnancy is associated with less optimal neurological condition in 4-year-old singletons, in vitro fertilization itself is not. Hum Reprod 2014;29(12):2773-86. PMID: 25316454.

Schieve LA, Cohen B, Nannini A, et al. A population-based study of maternal and perinatal outcomes associated with assisted reproductive technology in Massachusetts. Matern Child Health J 2007;11(6):517-25. PMID: 17345154. 
Schieve LA, Drews-Botsch C, Harris S, et al. Maternal and Paternal Infertility Disorders and Treatments and Autism Spectrum Disorder: Findings from the Study to Explore Early Development. J Autism Dev Disord 2017;47(12):3994-4005. PMID: 28900768.

Schliep KC, Mumford SL, Ahrens KA, et al. Effect of male and female body mass index on pregnancy and live birth success after in vitro fertilization. Fertil Steril 2015;103(2):388-95. PMID: 25497445.

Schwarze JE, Crosby JA and Zegers-Hochschild F. Addition of neither recombinant nor urinary luteinizing hormone was associated with an improvement in the outcome of autologous in vitro fertilization/intracytoplasmatic sperm injection cycles under regular clinical settings: a multicenter observational analysis. Fertil Steril 2016;106(7):1714-1717.e1. PMID: 27678033.

Scott RT, Jr., Upham KM, Forman EJ, et al. Blastocyst biopsy with comprehensive chromosome screening and fresh embryo transfer significantly increases in vitro fertilization implantation and delivery rates: a randomized controlled trial. Fertil Steril 2013;100(3):697-703. PMID: 23731996.

Seggers J, Haadsma ML, La Bastide-Van Gemert S, et al. Is ovarian hyperstimulation associated with higher blood pressure in 4-year-old IVF offspring? Part I: multivariable regression analysis. Hum Reprod 2014;29(3):502-9. PMID: 24365797.

Seggers J, Pontesilli M, Ravelli AC, et al. Effects of in vitro fertilization and maternal characteristics on perinatal outcomes: a population-based study using siblings. Fertil Steril 2016;105(3):590-598.e2. PMID: 26658132.

Selman H, Pacchiarotti A, Rinaldi L, et al. Simultaneous administration of human acidic and recombinant less acidic follicle-stimulating hormone for ovarian stimulation improves oocyte and embryo quality, and clinical outcome in patients with repeated IVF failures. Eur Rev Med Pharmacol Sci 2013;17(13):1814-9. PMID: 23852909.

Seow KM, Lin YH, Bai CH, et al. Clinical outcome according to timing of cabergoline initiation for prevention of OHSS: a randomized controlled trial. Reprod Biomed Online 2013;26(6):5628. PMID: 23608246.

Serafini P, Yadid I, Motta EL, et al. Ovarian stimulation with daily late follicular phase administration of low-dose human chorionic gonadotropin for in vitro fertilization: a prospective, randomized trial. Fertil Steril 2006;86(4):830-8. PMID: 16963040.

Sergentanis TN, Diamantaras AA, Perlepe C, et al. IVF and breast cancer: a systematic review and meta-analysis. Hum Reprod Update 2014;20(1):106-23. PMID: 23884897.

Serna J, Cholquevilque JL, Cela V, et al. Estradiol supplementation during the luteal phase of IVF-ICSI patients: a randomized, controlled trial. Fertil Steril 2008;90(6):2190-5. PMID: 18191847. 
Seshadri S, Sunkara SK, Khalaf Y, et al. Effect of heparin on the outcome of IVF treatment: a systematic review and meta-analysis. Reprod Biomed Online 2012;25(6):572-84. PMID: 23069743.

Seto MTY, Cheung KW, Lo TK, et al. Pregnancy outcomes of women randomized to receive real versus placebo acupuncture on the day of fresh or frozen-thawed embryo transfer. Eur J Obstet Gynecol Reprod Biol 2017;218:119-122. PMID: 28982045.

Shah DK, Missmer SA, Berry KF, et al. Effect of obesity on oocyte and embryo quality in women undergoing in vitro fertilization. Obstet Gynecol 2011;118(1):63-70. PMID: 21691164.

Shahine LK, Lamb JD, Lathi RB, et al. Poor prognosis with in vitro fertilization in Indian women compared to Caucasian women despite similar embryo quality. PLoS One 2009;4(10):e7599. PMID: 19855835.

Shahine LK, Milki AA, Westphal LM, et al. Day 2 versus day 3 embryo transfer in poor responders: a prospective randomized trial. Fertil Steril 2011;95(1):330-2. PMID: 20813357.

Shahrokh Tehraninejad E, Azimi Nekoo E, Ghaffari F, et al. Zygote intrafallopian tube transfer versus intrauterine cleavage or blastocyst stage transfer after intracytoplasmic sperm injection cycles in patients with repeated implantation failure: A prospective follow-up study. Journal of Obstetrics and Gynaecology Research 2015.

Shahrokh Tehraninejad E, Farshbaf Taghinejad M, Hossein Rashidi B, et al. Controlled ovarian stimulation with r-FSH plus r-LH vs. HMG plus r-FSH in patients candidate for IVF/ICSI cycles: An RCT. Int J Reprod Biomed (Yazd) 2017;15(7):435-440. PMID: 29177245.

Shahrokh-Tehraninejad E, Vosoog Z and Farajzadeh-Vajari F. Comparison of Outcomes of IVF Cycles Between Transferred Frozen Thawed Embryos and Fresh Embryos by a 2 Year Survey. J Reprod Infertil 2017;18(4):386-389. PMID: 29201669.

Shaltout A, Shohyab A and Youssef MA. Can dopamine agonist at a low dose reduce ovarian hyperstimulation syndrome in women at risk undergoing ICSI treatment cycles? A randomized controlled study. Eur J Obstet Gynecol Reprod Biol 2012;165(2):254-8. PMID: 22948132.

Shapiro AJ, Darmon SK, Barad DH, et al. Effect of race and ethnicity on utilization and outcomes of assisted reproductive technology in the USA. Reproductive Biology and Endocrinology 2017;15(1).

Sharara FI, Mouhayar Y, Goodwin M, et al. A prospective, randomized study comparing morning to evening administration of gonadotropins in ART. J Assist Reprod Genet 2018;35(4):705-709. PMID: 29264689.

Sharma S, Ghosh S, Singh S, et al. Congenital malformations among babies born following letrozole or clomiphene for infertility treatment. PLoS One 2014;9(10):e108219. PMID: 25272289. 
Sher G, Keskintepe L, Keskintepe M, et al. Genetic analysis of human embryos by metaphase comparative genomic hybridization (mCGH) improves efficiency of IVF by increasing embryo implantation rate and reducing multiple pregnancies and spontaneous miscarriages. Fertil Steril 2009;92(6):1886-94. PMID: 19135663.

Shohayeb A and El-Khayat W. Does a single endometrial biopsy regimen (S-EBR) improve ICSI outcome in patients with repeated implantation failure? A randomised controlled trial. Eur J Obstet Gynecol Reprod Biol 2012;164(2):176-9. PMID: 22835632.

Showell MG, Brown J, Clarke J, et al. Antioxidants for female subfertility. Cochrane Database Syst Rev 2013;8:Cd007807. PMID: 23913583.

Sifer C, El Khattabi L, Dupont C, et al. Could sperm grade under high magnification condition predict IMSI clinical outcome? Eur J Obstet Gynecol Reprod Biol 2014;181:189-94. PMID: 25150959.

Silber SJ, Kato K, Aoyama N, et al. Intrinsic fertility of human oocytes. Fertil Steril 2017;107(5):1232-1237. PMID: 28433372.

Silva Idos S, Wark PA, McCormack VA, et al. Ovulation-stimulation drugs and cancer risks: a long-term follow-up of a British cohort. Br J Cancer 2009;100(11):1824-31. PMID: 19436296.

Silverberg KM, Vaughn TC, Hansard LJ, et al. Vaginal (Crinone 8\%) gel vs. intramuscular progesterone in oil for luteal phase support in in vitro fertilization: a large prospective trial. Fertil Steril 2012;97(2):344-8. PMID: 22188983.

Sim KA, Dezarnaulds GM, Denyer GS, et al. Weight loss improves reproductive outcomes in obese women undergoing fertility treatment: A randomized controlled trial. Clinical Obesity 2014;4(2):61-68.

Singh N, Toshyan V, Kumar S, et al. Does endometrial injury enhances implantation in recurrent in-vitro fertilization failures? A prospective randomized control study from tertiary care center. J Hum Reprod Sci 2015;8(4):218-23. PMID: 26752857.

Siristatidis C, Komitopoulou MA, Makris A, et al. Morphokinetic parameters of early embryo development via time lapse monitoring and their effect on embryo selection and ICSI outcomes: a prospective cohort study. J Assist Reprod Genet 2015;32(4):563-70. PMID: 25617087.

Siristatidis C, Sergentanis TN, Kanavidis P, et al. Controlled ovarian hyperstimulation for IVF: impact on ovarian, endometrial and cervical cancer--a systematic review and meta-analysis. Hum Reprod Update 2013;19(2):105-23. PMID: 23255514.

Siristatidis C, Vogiatzi P, Salamalekis G, et al. Granulocyte macrophage colony stimulating factor supplementation in culture media for subfertile women undergoing assisted reproduction technologies: a systematic review. Int J Endocrinol 2013;2013:704967. PMID: 23509457.

Siristatidis CS, Basios G, Pergialiotis V, et al. Aspirin for in vitro fertilisation. Cochrane Database of Systematic Reviews 2016;2016(11). 
Siristatidis CS, Dodd SR and Drakeley AJ. Aspirin for in vitro fertilisation. Cochrane Database Syst Rev 2011;(8):Cd004832. PMID: 21833951.

Sites CK, Wilson D, Barsky M, et al. Embryo cryopreservation and preeclampsia risk. Fertil Steril 2017;108(5):784-790. PMID: 28974308.

Smit JG, Kasius JC, Eijkemans MJ, et al. Hysteroscopy before in-vitro fertilisation (inSIGHT): a multicentre, randomised controlled trial. Lancet 2016. PMID: 27132052.

Smulders B, van Oirschot SM, Farquhar C, et al. Oral contraceptive pill, progestogen or estrogen pre-treatment for ovarian stimulation protocols for women undergoing assisted reproductive techniques. Cochrane Database Syst Rev 2010;(1):Cd006109. PMID: 20091585.

Sohrabvand F, Golestan B, Kashani H, et al. Comparison of ART outcomes between two COH potocols: Gonal-F versus gonal-F plus HMG. International Journal of Fertility and Sterility 2010;3(4):161-164.

Soliman BS and Harira M. Local endometrial scratching under ultrasound-guidance after failed intrauterine insemination and cycle outcome: A randomized controlled trial. Middle East Fertility Society Journal 2017;22(1):60-66.

Souter I, Dimitriadis I, Baltagi LM, et al. Elevated day 3 follicle-stimulating hormone in younger women: is gonadotropin stimulation/intrauterine insemination a good option? Am J Obstet Gynecol 2014;211(1):62.e1-8. PMID: 24487006.

Spangmose AL, Malchau SS, Schmidt L, et al. Academic performance in adolescents born after ART-a nationwide registry-based cohort study. Hum Reprod 2017;32(2):447-456. PMID: 28057876.

Stadtmauer L, Harrison DD, Boyd J, et al. Pilot study evaluating a progesterone vaginal ring for luteal-phase replacement in donor oocyte recipients. Fertil Steril 2009;92(5):1600-5. PMID: 18990373.

Stadtmauer L, Silverberg KM, Ginsburg ES, et al. Progesterone vaginal ring versus vaginal gel for luteal support with in vitro fertilization: a randomized comparative study. Fertil Steril 2013;99(6):1543-9. PMID: 23465817.

Staessen C, Verpoest W, Donoso P, et al. Preimplantation genetic screening does not improve delivery rate in women under the age of 36 following single-embryo transfer. Hum Reprod 2008;23(12):2818-25. PMID: 18930977.

Stanford JB, Simonsen SE and Baksh L. Fertility treatments and adverse perinatal outcomes in a population-based sampling of births in Florida, Maryland, and Utah: A cross-sectional study. BJOG: An International Journal of Obstetrics and Gynaecology 2016;123(5):718-729.

Stenbaek DS, Toftager M, Hjordt LV, et al. Mental distress and personality in women undergoing $\mathrm{GnRH}$ agonist versus $\mathrm{GnRH}$ antagonist protocols for assisted reproductive technology. Hum Reprod 2015;30(1):103-10. PMID: 25398970. 
Stern JE, Brown MB, Luke B, et al. Cycle 1 as predictor of assisted reproductive technology treatment outcome over multiple cycles: an analysis of linked cycles from the Society for Assisted Reproductive Technology Clinic Outcomes Reporting System online database. Fertil Steril 2011;95(2):600-5. PMID: 20643404.

Stern JE, Goldman MB, Hatasaka H, et al. Optimizing the number of blastocyst stage embryos to transfer on day 5 or 6 in women 38 years of age and older: a Society for Assisted Reproductive Technology database study. Fertil Steril 2009;91(1):157-66. PMID: 18222441.

Stern JE, Lieberman ES, Macaluso M, et al. Is cryopreservation of embryos a legitimate surrogate marker of embryo quality in studies of assisted reproductive technology conducted using national databases? Fertil Steril 2012;97(4):890-3. PMID: 22265032.

Stern JE, Liu CL, Cabral HJ, et al. Birth outcomes of singleton vaginal deliveries to ARTtreated, subfertile, and fertile primiparous women. Journal of Assisted Reproduction and Genetics 2018;35(9):1585-1593.

Stewart LM, Holman CD, Finn JC, et al. Association between in-vitro fertilization, birth and melanoma. Melanoma Res 2013;23(6):489-95. PMID: 24048222.

Stewart LM, Holman CD, Finn JC, et al. In vitro fertilization is associated with an increased risk of borderline ovarian tumours. Gynecol Oncol 2013;129(2):372-6. PMID: 23385152.

Stewart LM, Holman CD, Hart R, et al. How effective is in vitro fertilization, and how can it be improved? Fertil Steril 2011;95(5):1677-83. PMID: 21316660.

Stewart LM, Holman CD, Hart R, et al. In vitro fertilization and breast cancer: is there cause for concern? Fertil Steril 2012;98(2):334-40. PMID: 22633651.

Stewart LM, Holman CD, Semmens JB, et al. Hospital mental health admissions in women after unsuccessful infertility treatment and in vitro fertilization: an Australian population-based cohort study. PLoS One 2015;10(3):e0120076. PMID: 25807258.

Stillman RJ, Richter KS, Banks NK, et al. Elective single embryo transfer: a 6-year progressive implementation of 784 single blastocyst transfers and the influence of payment method on patient choice. Fertil Steril 2009;92(6):1895-906. PMID: 18976755.

Stimpfel M, Vrtacnik-Bokal E, Pozlep B, et al. Comparison of GnRH agonist, GnRH antagonist, and GnRH antagonist mild protocol of controlled ovarian hyperstimulation in good prognosis patients. International Journal of Endocrinology 2015;2015.

Storeng R, Vangen S, Omland AK, et al. Infertility treatment and the risk of cancer. Tidsskr Nor Laegeforen 2012;132(22):2494-9. PMID: 23338030.

Styer AK, Wright DL, Wolkovich AM, et al. Single-blastocyst transfer decreases twin gestation without affecting pregnancy outcome. Fertil Steril 2008;89(6):1702-8. PMID: 17644095. 
Sun Y, Vestergaard M, Christensen J, et al. Epilepsy and febrile seizures in children of treated and untreated subfertile couples. Hum Reprod 2007;22(1):215-20. PMID: 16936302.

Sunkara SK, Antonisamy B, Selliah HY, et al. Pre-term birth and low birth weight following preimplantation genetic diagnosis: analysis of 88010 singleton live births following PGD and IVF cycles. Hum Reprod 2017;32(2):432-438. PMID: 27979918.

Sutcliffe AG, Melhuish E, Barnes J, et al. Health and development of children born after assisted reproductive technology and sub-fertility compared to naturally conceived children: data from a national study. Pediatr Rep 2014;6(1):5118. PMID: 24711911.

Sydsjo G, Lampic C, Bladh M, et al. Relationships in oocyte recipient couples - a Swedish national prospective follow-up study. Reprod Health 2014;11:38. PMID: 24885541.

Sydsjo G, Svanberg AS, Bladh M, et al. Relationships in couples treated with sperm donation - a national prospective follow-up study. Reprod Health 2014;11:62. PMID: 25100133.

Sydsjo G, Wadsby M, Sydsjo A, et al. Relationship and parenthood in IVF couples with twin and singleton pregnancies compared with spontaneous singleton primiparous couples--a prospective 5-year follow-up study. Fertil Steril 2008;89(3):578-85. PMID: 17517400.

Taheripanah R, Zamaniyan M, Moridi A, et al. Comparing the effect of gonadotropin-releasing hormone agonist and human chorionic gonadotropin on final oocytes for ovulation triggering among infertile women undergoing intrauterine insemination: An RCT. Int J Reprod Biomed (Yazd) 2017;15(6):351-356. PMID: 29177238.

Taketani Y, Kelly E, Yoshimura Y, et al. Recombinant follicle-stimulating hormone (follitropin alfa) for ovulation induction in Japanese patients with anti-estrogen-ineffective oligo-or anovulatory infertility: Results of a phase II dose-response study. Reproductive Medicine and Biology 2010;9(2):91-97.

Tang H, Hunter T, Hu Y, et al. Cabergoline for preventing ovarian hyperstimulation syndrome. Cochrane Database Syst Rev 2012;2:Cd008605. PMID: 22336848.

Tang H, Mourad S, Zhai SD, et al. Dopamine agonists for preventing ovarian hyperstimulation syndrome. Cochrane Database of Systematic Reviews 2016;2016(11).

Tararbit K, Houyel L, Bonnet D, et al. Risk of congenital heart defects associated with assisted reproductive technologies: a population-based evaluation. Eur Heart J 2011;32(4):500-8. PMID: 21138932.

Tararbit K, Lelong N, Thieulin AC, et al. The risk for four specific congenital heart defects associated with assisted reproductive techniques: a population-based evaluation. Hum Reprod 2013;28(2):367-74. PMID: 23178272.

Tarlatzis BC, Griesinger G, Leader A, et al. Comparative incidence of ovarian hyperstimulation syndrome following ovarian stimulation with corifollitropin alfa or recombinant FSH. Reprod Biomed Online 2012;24(4):410-9. PMID: 22386594. 
Tartagni M, De Pergola G, Damiani GR, et al. Potential benefit of dehydroepiandrosterone supplementation for infertile but not poor responder patients in a IVF program. Minerva Ginecol 2015;67(1):7-12. PMID: 24867068.

Tehraninejad ES, Hafezi M, Arabipoor A, et al. Comparison of cabergoline and intravenous albumin in the prevention of ovarian hyperstimulation syndrome: a randomized clinical trial. $\mathrm{J}$ Assist Reprod Genet 2012;29(3):259-64. PMID: 22231013.

Tehraninejad ES, Tanha FD, Ghajarzadeh M, et al. Stimulation of the endometrium with highgrade blastocyst culture supernatant (SEHB) can improve pregnancy outcome for couples undergoing intracytoplasmic sperm injection (ICSI): a randomized clinical trial. Arch Gynecol Obstet 2012;285(4):1167-71. PMID: 22101499.

Thijssen A, Meier A, Panis K, et al. 'Fertility Awareness-Based Methods' and subfertility: a systematic review. Facts Views Vis Obgyn 2014;6(3):113-23. PMID: 25374654.

Thomsen L, Humaidan P, Bungum L, et al. The impact of male overweight on semen quality and outcome of assisted reproduction. Asian J Androl 2014;16(5):749-54. PMID: 24759576.

Thum MY, El-Sheikhah A, Faris R, et al. The influence of body mass index to in-vitro fertilisation treatment outcome, risk of miscarriage and pregnancy outcome. J Obstet Gynaecol 2007;27(7):699-702. PMID: 17999296.

Tjon-Kon-Fat RI, Bensdorp AJ, Bossuyt PM, et al. Is IVF-served two different ways-more costeffective than IUI with controlled ovarian hyperstimulation? Hum Reprod 2015. PMID: 26269539.

Tournaye H, Sukhikh GT, Kahler E, et al. A Phase III randomized controlled trial comparing the efficacy, safety and tolerability of oral dydrogesterone versus micronized vaginal progesterone for luteal support in in vitro fertilization. Hum Reprod 2017;32(5):1019-1027. PMID: 28333318.

Udell JA, Lu H and Redelmeier DA. Long-term cardiovascular risk in women prescribed fertility therapy. J Am Coll Cardiol 2013;62(18):1704-12. PMID: 23916924.

Ueno S, Bodri D, Uchiyama K, et al. Developmental potential of zona pellucida-free oocytes obtained following mild in vitro fertilization. Fertil Steril 2014;102(6):1602-7. PMID: 25256934.

Uk A, Collardeau-Frachon S, Scanvion Q, et al. Assisted Reproductive Technologies and imprinting disorders: Results of a study from a French congenital malformations registry. Eur J Med Genet 2018;61(9):518-523. PMID: 29775803.

Urman B, Ata B, Yakin K, et al. Luteal phase empirical low molecular weight heparin administration in patients with failed ICSI embryo transfer cycles: a randomized open-labeled pilot trial. Hum Reprod 2009;24(7):1640-7. PMID: 19357135.

Valent AM, Hall ES and DeFranco EA. The influence of obesity on perinatal outcomes in pregnancies achieved with assisted reproductive technology: A population-based retrospective cohort study. Obstetric Medicine 2016;9(1):34-39. 
Valenzuela-Alcaraz B, Crispi F, Bijnens B, et al. Assisted reproductive technologies are associated with cardiovascular remodeling in utero that persists postnatally. Circulation 2013;128(13):1442-50. PMID: 23985787.

Valenzuela-Alcaraz B, Crispi F, Manau D, et al. Differential effect of mode of conception and infertility treatment on fetal growth and prematurity. J Matern Fetal Neonatal Med 2016:1-6. PMID: 26856245.

van den Boogaard NM, Bensdorp AJ, Oude Rengerink K, et al. Prognostic profiles and the effectiveness of assisted conception: secondary analyses of individual patient data. Hum Reprod Update 2014;20(1):141-51. PMID: 24173882.

van der Linden M, Buckingham K, Farquhar C, et al. Luteal phase support for assisted reproduction cycles. Cochrane Database Syst Rev 2011;(10):Cd009154. PMID: 21975790.

van der Linden M, Buckingham K, Farquhar C, et al. Luteal phase support for assisted reproduction cycles. Cochrane Database Syst Rev 2015;7:Cd009154. PMID: 26148507.

van der Poel N, Farquhar C, Abou-Setta AM, et al. Soft versus firm catheters for intrauterine insemination. Cochrane Database Syst Rev 2010;(11):Cd006225. PMID: 21069687.

Van Dorp W, Rietveld AM, Laven JSE, et al. Pregnancy outcome of non-anonymous oocyte donation: A case-control study. European Journal of Obstetrics Gynecology and Reproductive Biology 2014;182:107-112.

Van Tilborg TC, Broekmans FJ, Dolleman M, et al. Individualised FSH dosing and IVF outcome in agonist downregulated cycles: a systematic review. Acta Obstet Gynecol Scand 2016. PMID: 27687487.

van Tilborg TC, Eijkemans MJ, Laven JS, et al. The OPTIMIST study: optimisation of cost effectiveness through individualised FSH stimulation dosages for IVF treatment. A randomised controlled trial. BMC Womens Health 2012;12:29. PMID: 22989359.

van Wely M, Kwan I, Burt AL, et al. Recombinant versus urinary gonadotrophin for ovarian stimulation in assisted reproductive technology cycles. Cochrane Database Syst Rev 2011;(2):Cd005354. PMID: 21328276.

Vasario E, Borgarello V, Bossotti C, et al. IVF twins have similar obstetric and neonatal outcome as spontaneously conceived twins: a prospective follow-up study. Reprod Biomed Online 2010;21(3):422-8. PMID: 20638334.

Vega MG, Gleicher N, Darmon SK, et al. IVF outcomes in average- and poor-prognosis infertile women according to the number of embryos transferred. Reproductive BioMedicine Online 2016;33(3):370-375.

Veleva Z, Orava M, Nuojua-Huttunen S, et al. Factors affecting the outcome of frozen-thawed embryo transfer. Hum Reprod 2013;28(9):2425-31. PMID: 23756705. 
Verhaak CM, Lintsen AM, Evers AW, et al. Who is at risk of emotional problems and how do you know? Screening of women going for IVF treatment. Hum Reprod 2010;25(5):1234-40.

PMID: 20228392.

Verpoest W, Staessen C, Bossuyt PM, et al. Preimplantation genetic testing for aneuploidy by microarray analysis of polar bodies in advanced maternal age: a randomized clinical trial. Hum Reprod 2018;33(9):1767-1776. PMID: 30085138.

Vidal M, Vellve K, Gonzalez-Comadran M, et al. Perinatal outcomes in children born after fresh or frozen embryo transfer: a Catalan cohort study based on 14,262 newborns. Fertil Steril 2017;107(4):940-947. PMID: 28292612.

Vikstrom J, Hammar M, Josefsson A, et al. Birth characteristics in a clinical sample of women seeking infertility treatment: a case-control study. BMJ Open 2014;4(3):e004197. PMID: 24613821.

Vikstrom J, Josefsson A, Bladh M, et al. Mental health in women 20-23 years after IVF treatment: a Swedish cross-sectional study. BMJ Open 2015;5(10):e009426. PMID: 26510732.

Vikstrom J, Josefsson A, Hammar M, et al. Risk of postpartum psychosis after IVF treatment: a nationwide case-control study. Hum Reprod 2017;32(1):139-146. PMID: 27927846.

Vikstrom J, Sydsjo G, Hammar M, et al. Risk of postnatal depression or suicide after in vitro fertilisation treatment: a nationwide case-control study. Bjog 2015. PMID: 26663705.

Visschers BA, Bots RS, Peeters MF, et al. Removal of cervical mucus: effect on pregnancy rates in IVF/ICSI. Reprod Biomed Online 2007;15(3):310-5. PMID: 17854530.

Vithoulkas A, Levanduski M, Goudas VT, et al. Co-culture of human embryos with autologous cumulus cell clusters and its beneficial impact of secreted growth factors on preimplantation development as compared to standard embryo culture in assisted reproductive technologies (ART). Middle East Fertility Society Journal 2017;22(4):317-322.

Vitthala S, Gelbaya TA, Hunter H, et al. Stimulated intrauterine insemination (SIUI) and donor insemination (DI) as first line management for a selected subfertile population: the Manchester experience. J Assist Reprod Genet 2008;25(9-10):431-6. PMID: 18830693.

Volgsten $\mathrm{H}$ and Schmidt L. Live birth outcome, spontaneous pregnancy and adoption up to five years after undergoing assisted reproductive technology treatment. Acta Obstet Gynecol Scand 2017;96(8):954-959. PMID: 28374421.

Vuong LN, Dang VQ, Ho TM, et al. IVF Transfer of Fresh or Frozen Embryos in Women without Polycystic Ovaries. N Engl J Med 2018;378(2):137-147. PMID: 29320655.

Vuong TN, Phung HT and Ho MT. Recombinant follicle-stimulating hormone and recombinant luteinizing hormone versus recombinant follicle-stimulating hormone alone during $\mathrm{GnRH}$ antagonist ovarian stimulation in patients aged $>/=35$ years: a randomized controlled trial. Hum Reprod 2015;30(5):1188-95. PMID: 25740882. 
Wainstock T, Walfisch A, Shoham-Vardi I, et al. Fertility treatments and pediatric neoplasms of the offspring: results of a population-based cohort with a median follow-up of 10 years. Am J Obstet Gynecol 2017;216(3):314.e1-314.e14. PMID: 28153657.

Wan CY, Song C, Diao LH, et al. Laser-assisted hatching improves clinical outcomes of vitrified-warmed blastocysts developed from low-grade cleavage-stage embryos: a prospective randomized study. Reprod Biomed Online 2014;28(5):582-9. PMID: 24631166.

Wang AC, Wang Y, Wu FX, et al. Assessing predictors for the success of GnRH antagonist protocol in reproductive women in IVF/ICSI - in fresh cycles. Biomedical Reports 2017;7(5):482-486.

Wang AY, Chughtai AA, Lui K, et al. Morbidity and mortality among very preterm singletons following fertility treatment in Australia and New Zealand, a population cohort study. BMC Pregnancy Childbirth 2017;17(1):50. PMID: 28148237.

Wang ET, Ozimek JA, Greene N, et al. Impact of fertility treatment on severe maternal morbidity. Fertil Steril 2016. PMID: 27063600.

Wang ET, Ramos L, Vyas N, et al. Maternal and neonatal outcomes associated with infertility. J Matern Fetal Neonatal Med 2018:1-4. PMID: 29510646.

Wang HL, Lai HH, Chuang TH, et al. A patient friendly corifollitropin alfa protocol without routine pituitary suppression in normal responders. PLoS ONE 2016;11(4).

Wang YA, Nikravan R, Smith HC, et al. Higher prevalence of gestational diabetes mellitus following assisted reproduction technology treatment. Hum Reprod 2013;28(9):2554-61. PMID: 23814097.

Waylen AL, Metwally M, Jones GL, et al. Effects of cigarette smoking upon clinical outcomes of assisted reproduction: a meta-analysis. Hum Reprod Update 2009;15(1):31-44. PMID: 18927070.

Wechowski J, Connolly M, McEwan P, et al. An economic evaluation of highly purified HMG and recombinant FSH based on a large randomized trial. Reprod Biomed Online 2007;15(5):5006. PMID: 18028739.

Weiss A, Beck-Fruchter R, Lavee M, et al. A randomized trial comparing time intervals from HCG trigger to intrauterine insemination for cycles utilizing GnRH antagonists. Syst Biol Reprod Med 2015;61(1):44-9. PMID: 25133644.

Weissman A, Horowitz E, Ravhon A, et al. Spontaneous ovulation versus HCG triggering for timing natural-cycle frozen-thawed embryo transfer: a randomized study. Reprod Biomed Online 2011;23(4):484-9. PMID: 21840758.

Weller A, Daniel S, Koren G, et al. The fetal safety of clomiphene citrate: a population-based retrospective cohort study. BJOG: An International Journal of Obstetrics and Gynaecology 2017;124(11):1664-1670. 
Wen SW, Leader A, White RR, et al. A comprehensive assessment of outcomes in pregnancies conceived by in vitro fertilization/intracytoplasmic sperm injection. Eur J Obstet Gynecol Reprod Biol 2010;150(2):160-5. PMID: 20207067.

Wennberg AL, Opdahl S, Bergh C, et al. Effect of maternal age on maternal and neonatal outcomes after assisted reproductive technology. Fertil Steril 2016;106(5):1142-1149.e14. PMID: 27399261.

Wennerholm UB, Henningsen AK, Romundstad LB, et al. Perinatal outcomes of children born after frozen-thawed embryo transfer: a Nordic cohort study from the CoNARTaS group. Hum Reprod 2013;28(9):2545-53. PMID: 23832793.

Westerlund E, Brandt L, Hovatta O, et al. Incidence of hypertension, stroke, coronary heart disease, and diabetes in women who have delivered after in vitro fertilization: a population-based cohort study from Sweden. Fertil Steril 2014;102(4):1096-102. PMID: 25064407.

Whitelaw N, Bhattacharya S, Hoad G, et al. Epigenetic status in the offspring of spontaneous and assisted conception. Hum Reprod 2014;29(7):1452-8. PMID: 24812310.

Wijers CH, van Rooij IA, Rassouli R, et al. Parental subfertility, fertility treatment, and the risk of congenital anorectal malformations. Epidemiology 2015;26(2):169-76. PMID: 25563433.

Winger EE, Reed JL, Ashoush S, et al. Birth defect rates in women using Adalimumab (Humira((R)) ) to treat immunologic-based infertility in IVF patients. Am J Reprod Immunol 2011;66(3):237-41. PMID: 21501282.

Wirleitner B, Schuff M, Vanderzwalmen P, et al. Intrauterine administration of human chorionic gonadotropin does not improve pregnancy and life birth rates independently of blastocyst quality: a randomised prospective study. Reprod Biol Endocrinol 2015;13:70. PMID: 26141379.

Wisborg K, Ingerslev HJ and Henriksen TB. In vitro fertilization and preterm delivery, low birth weight, and admission to the neonatal intensive care unit: a prospective follow-up study. Fertil Steril 2010;94(6):2102-6. PMID: 20188361.

Wisborg K, Ingerslev HJ and Henriksen TB. IVF and stillbirth: a prospective follow-up study. Hum Reprod 2010;25(5):1312-6. PMID: 20179321.

Witteveen T, Van Den Akker T, Zwart JJ, et al. Severe acute maternal morbidity in multiple pregnancies: A nationwide cohort study. American Journal of Obstetrics and Gynecology 2016;214(5):641.e1-641.e10.

Wu AK, Elliott P, Katz PP, et al. Time costs of fertility care: the hidden hardship of building a family. Fertil Steril 2013;99(7):2025-30. PMID: 23454007.

Wu AK, Odisho AY, Washington SL, 3rd, et al. Out-of-pocket fertility patient expense: data from a multicenter prospective infertility cohort. J Urol 2014;191(2):427-32. PMID: 24018235. 
Yakin K, Ata B, Ercelen N, et al. The effect of preimplantation genetic screening on the probability of live birth in young women with recurrent implantation failure; a nonrandomized parallel group trial. Eur J Obstet Gynecol Reprod Biol 2008;140(2):224-9. PMID: 18603347.

Yang M, Fan XB, Wu JN, et al. Association of assisted reproductive technology and multiple pregnancies with the risks of birth defects and stillbirth: A retrospective cohort study. Sci Rep 2018;8(1):8296. PMID: 29844441.

Yavas Y. Curvilinear relationship between age and assisted reproduction technique success: retrospective analyses of US National ART Surveillance System data from 2010-2014. Reprod Biomed Online 2017;35(6):657-668. PMID: 28865756.

Yazbeck C, Ben Jamaa N, Hazout A, et al. Advantages of the two-step embryo transfer strategy in human IVF/ICSI cycles. Zygote 2013;21(1):77-83. PMID: 22008412.

Ye H, Huang G, Pei L, et al. Outcome of in vitro fertilization following stimulation with highly purified hMG or recombinant FSH in downregulated women of advanced reproductive age: a prospective, randomized and controlled trial. Gynecol Endocrinol 2012;28(7):540-4. PMID: 22390186.

Ye H, Huang GN, Zeng PH, et al. IVF/ICSI outcomes between cycles with luteal estradiol (E2) pre-treatment before GnRH antagonist protocol and standard long GnRH agonist protocol: a prospective and randomized study. J Assist Reprod Genet 2009;26(2-3):105-11. PMID: 19225876.

Yeh JS, Steward RG, Dude AM, et al. Pregnancy rates in donor oocyte cycles compared to similar autologous in vitro fertilization cycles: an analysis of 26,457 fresh cycles from the Society for Assisted Reproductive Technology. Fertil Steril 2014;102(2):399-404. PMID: 24842672.

Yeung EH, Sundaram R, Bell EM, et al. Examining Infertility Treatment and Early Childhood Development in the Upstate KIDS Study. JAMA Pediatr 2016;170(3):251-8. PMID: 26746435.

Yeung EH, Sundaram R, Bell EM, et al. Infertility treatment and children's longitudinal growth between birth and 3 years of age. Hum Reprod 2016. PMID: 27165624.

Yeung TW, Chai J, Li RH, et al. The effect of endometrial injury on ongoing pregnancy rate in unselected subfertile women undergoing in vitro fertilization: a randomized controlled trial. Hum Reprod 2014;29(11):2474-81. PMID: 25205759.

Yildiz GA, Sukur YE, Ates C, et al. The addition of gonadotrophin releasing hormone agonist to routine luteal phase support in intracytoplasmic sperm injection and embryo transfer cycles: a randomized clinical trial. Eur J Obstet Gynecol Reprod Biol 2014;182:66-70. PMID: 25238659.

Yilmaz N, Engin-Ustun Y, Inal H, et al. The impact of single embryo transfer policy on pregnancy outcomes after legislative change. Gynecol Endocrinol 2013;29(6):600-2. PMID: 23656390. 
Yilmaz N, Uygur D, Ozgu E, et al. Does coasting, a procedure to avoid ovarian hyperstimulation syndrome, affect assisted reproduction cycle outcome? Fertil Steril 2010;94(1):189-93. PMID: 19376515.

Yılmaz NK, Sargın A, Erkılınç S, et al. Does ovulation induction and intrauterine insemination affect perinatal outcomes in singletons?. Journal of Maternal-Fetal and Neonatal Medicine 2018;31(1):14-17.

Yli-Kuha AN, Gissler M, Klemetti R, et al. Cancer morbidity in a cohort of 9175 Finnish women treated for infertility. Hum Reprod 2012;27(4):1149-55. PMID: 22343550.

Youssef MA, Al-Inany HG, Aboulghar M, et al. Recombinant versus urinary human chorionic gonadotrophin for final oocyte maturation triggering in IVF and ICSI cycles. Cochrane Database Syst Rev 2011;(4):Cd003719. PMID: 21491386.

Youssef MA, Al-Inany HG, Evers JL, et al. Intra-venous fluids for the prevention of severe ovarian hyperstimulation syndrome. Cochrane Database Syst Rev 2011;(2):Cd001302. PMID: 21328249.

Youssef MA, Van der Veen F, Al-Inany HG, et al. Gonadotropin-releasing hormone agonist versus HCG for oocyte triggering in antagonist-assisted reproductive technology. Cochrane Database Syst Rev 2014;10:Cd008046. PMID: 25358904.

Yu B, Mumford S, Royster GDt, et al. Cost-effectiveness analysis comparing continuation of assisted reproductive technology with conversion to intrauterine insemination in patients with low follicle numbers. Fertil Steril 2014;102(2):435-9. PMID: 24951366.

Yu HT, Wang CJ, Lee CL, et al. The role of diagnostic hysteroscopy before the first in vitro fertilization/intracytoplasmic sperm injection cycle. Arch Gynecol Obstet 2012;286(5):1323-8. PMID: 22791384.

Yun BH, Chon SJ, Park JH, et al. Minimal stimulation using gonadotropin combined with clomiphene citrate or letrozole for intrauterine insemination. Yonsei Med J 2015;56(2):490-6. PMID: 25684000.

Zahiri Sorouri Z, Rashid Shomali R and Pourmarzi D. Single versus Double Intrauterine Insemination in Controlled Ovarian Hyperstimulation Cycles: A Randomized Trial. Arch Iran Med 2016;19(7):465-9. PMID: 27362239.

Zainul Rashid MR, Lim JF, Nawawi NH, et al. A pilot study to determine whether progestogen supplementation using dydrogesterone during the first trimester will reduce the incidence of gestational hypertension in primigravidae. Gynecol Endocrinol 2014;30(3):217-20. PMID: 24552449.

Zarei A, Parsanezhad ME, Younesi M, et al. Intrauterine administration of recombinant human chorionic gonadotropin before embryo transfer on outcome of in vitro fertilization/ intracytoplasmic sperm injection: A randomized clinical trial. Iran J Reprod Med 2014;12(1):1-6. PMID: 24799855. 
Zargar M, Nikbakht R, Pourmatroud E, et al. Comparison of the clinical efficacy of two different cabergoline regimens on prevention of ovarian hyperstimulation syndrome (OHSS). Research Journal of Obstetrics and Gynecology 2011;4(2):51-58.

Zargar M, Saadati N and Ejtahed MS. Comparison the effectiveness of oral dydrogesterone, vaginal progesterone suppository and progesterone ampule for luteal phase support on pregnancy rate during ART cycles. International Journal of Pharmaceutical Research and Allied Sciences 2016;5(3):229-236.

Zegers-Hochschild F, Crosby J, Musri C, et al. Assisted reproduction techniques in Latin America: the Latin American Registry, 2014. Reproductive BioMedicine Online 2017;35(3):287-295.

Zegers-Hochschild F, Schwarze JE, Crosby J, et al. Assisted reproductive technologies in Latin America: The Latin American registry, 2010. Jornal Brasileiro de Reproducao Assistida 2012;16(6):320-328.

Zegers-Hochschild F, Schwarze JE, Crosby JA, et al. Assisted reproductive techniques in Latin America: The Latin American registry, 2014. Jornal Brasileiro de Reproducao Assistida 2017;21(3):164-175.

Zhang D, Zhu Y, Gao H, et al. Overweight and obesity negatively affect the outcomes of ovarian stimulation and in vitro fertilisation: a cohort study of 2628 Chinese women. Gynecol Endocrinol 2010;26(5):325-32. PMID: 20192898.

Zhang JJ, Merhi Z, Yang M, et al. Minimal stimulation IVF vs conventional IVF: a randomized controlled trial. Am J Obstet Gynecol 2016;214(1):96.e1-8. PMID: 26259908.

Zhao J, Xu B, Xie S, et al. Whether G-CSF administration has beneficial effect on the outcome after assisted reproductive technology? A systematic review and meta-analysis. Reproductive Biology and Endocrinology 2016;14(1).

Zhao P, Li M, Lian Y, et al. The clinical outcomes of day 3 4-cell embryos after extended in vitro culture. J Assist Reprod Genet 2015;32(1):55-60. PMID: 25308253.

Zheng HY, Shi XY, Wang LL, et al. Study of DNA methylation patterns of imprinted genes in children born after assisted reproductive technologies reveals no imprinting errors: A pilot study. Experimental and Therapeutic Medicine 2011;2(4):751-755.

Zhu HY, Xue YM, Yang LY, et al. Slow freezing should not be totally substituted by vitrification when applied to day 3 embryo cryopreservation: an analysis of 5613 frozen cycles. J Assist Reprod Genet 2015. PMID: 26238389.

Zhu JL, Basso O, Obel C, et al. Infertility, infertility treatment and psychomotor development: the Danish National Birth Cohort. Paediatr Perinat Epidemiol 2009;23(2):98-106. PMID: 19159396. 
Zhu JL, Basso O, Obel C, et al. Parental infertility and sexual maturation in children. Hum Reprod 2009;24(2):445-50. PMID: 18840889.

Zhu JL, Hvidtjorn D, Basso O, et al. Parental infertility and cerebral palsy in children. Hum Reprod 2010;25(12):3142-5. PMID: 21045245.

Zhu JL, Obel C, Basso O, et al. Infertility, infertility treatment, and mixed-handedness in children. Early Hum Dev 2009;85(12):745-9. PMID: 19875254.

Zhu JL, Obel C, Basso O, et al. Parental infertility and developmental coordination disorder in children. Hum Reprod 2010;25(4):908-13. PMID: 20139428.

Zhu JL, Obel C, Hammer Bech B, et al. Infertility, infertility treatment, and fetal growth restriction. Obstet Gynecol 2007;110(6):1326-34. PMID: 18055728.

Zhu X, Ye H and Fu Y. Duphaston and human menopausal gonadotropin protocol in normally ovulatory women undergoing controlled ovarian hyperstimulation during in vitro fertilization/intracytoplasmic sperm injection treatments in combination with embryo cryopreservation. Fertil Steril 2017;108(3):505-512.e2. PMID: 28697910.

Zhu X, Ye J, Fu Y, et al. The effect of human chorionic gonadotrophin contained in human menopausal gonadotropin on the clinical outcomes during progestin-primed ovarian stimulation. Oncotarget 2017;8(50):87340-87352.

Zwink N, Jenetzky E, Hirsch K, et al. Assisted reproductive techniques and risk of exstrophyepispadias complex: a German case-control study. J Urol 2013;189(4):1524-9. PMID: 23201374.

Zwink N, Jenetzky E, Schmiedeke E, et al. Assisted reproductive techniques and the risk of anorectal malformations: a German case-control study. Orphanet J Rare Dis 2012;7:65. PMID: 22978793.

\section{Does not meet study design criteria by outcome type:}

Abdel Raheem A, Rushwan N, Garaffa G, et al. Factors influencing intracytoplasmic sperm injection (ICSI) outcome in men with azoospermia. BJU Int 2013;112(2):258-64. PMID: 23356885.

Abu Hashim H, Foda O and El Rakhawy M. Unilateral or bilateral laparoscopic ovarian drilling in polycystic ovary syndrome: a meta-analysis of randomized trials. Arch Gynecol Obstet 2018;297(4):859-870. PMID: 29374790.

Aflatoonian A, Karimzadeh Maybodi MA, Aflatoonian N, et al. Perinatal outcome in fresh versus frozen embryo transfer in ART cycles. International Journal of Reproductive BioMedicine 2016;14(3):167-172.

Al-Inany HG, Youssef MA, Aboulghar M, et al. Gonadotrophin-releasing hormone antagonists for assisted reproductive technology. Cochrane Database Syst Rev 2011;(5):Cd001750. PMID: 21563131. 
Al-Inany HG, Youssef MA, Ayeleke RO, et al. Gonadotrophin-releasing hormone antagonists for assisted reproductive technology. Cochrane Database Syst Rev 2016;4:Cd001750. PMID: 27126581.

Ali SB, Jeelall Y, Pennell CE, et al. The role of immunological testing and intervention in reproductive medicine: A fertile collaboration?. Am J Reprod Immunol 2018;79(3). PMID: 29154465.

Aljawoan FY, Hunt LP and Gordon UD. Prediction of ovarian hyperstimulation syndrome in coasted patients in an IVF/ICSI program. J Hum Reprod Sci 2012;5(1):32-6. PMID: 22870012.

Amer SA, Shamy TTE, James C, et al. The impact of laparoscopic ovarian drilling on AMH and ovarian reserve: a meta-analysis. Reproduction 2017;154(1):R13-r21. PMID: 28420801.

Anaya Y, Mata DA, Letourneau J, et al. A novel oocyte maturation trigger using 1500 IU of human chorionic gonadotropin plus $450 \mathrm{IU}$ of follicle-stimulating hormone may decrease ovarian hyperstimulation syndrome across all in vitro fertilization stimulation protocols. Journal of Assisted Reproduction and Genetics 2018;35(2):297-307.

Anonymous. Fertility drugs and cancer: a guideline. Fertil Steril 2016;106(7):1617-1626. PMID: 27573989.

Anonymous. Performing the embryo transfer: a guideline. Fertil Steril 2017;107(4):882-896. PMID: 28366416.

Anonymous. Role of metformin for ovulation induction in infertile patients with polycystic ovary syndrome (PCOS): a guideline. Fertil Steril 2017;108(3):426-441. PMID: 28865539.

Anonymous. Guidelines for the number of embryos to transfer following in vitro fertilization No. 182, September 2006. Int J Gynaecol Obstet 2008;102(2):203-16. PMID: 18773532.

Arab-Zozani M and Nastri CO. Single versus double intrauterine insemination (IUI) for pregnancy: A systematic review and meta-analysis. Eur J Obstet Gynecol Reprod Biol 2017;215:75-84. PMID: 28605667.

Arefi S, Hoseini A, Farifteh F, et al. Modified natural cycle frozen-thawed embryo transfer in patients with repeated implantation failure: An observational study. International Journal of Reproductive BioMedicine 2016;14(7):465-470.

Arora P, Arora RS and Cahill D. Essure ${ }^{\circledR}$ for management of hydrosalpinx prior to in vitro fertilisation-a systematic review and pooled analysis. Bjog 2014;121(5):527-36. PMID: 24393165.

Ashrafi M, Bahmanabadi A, Akhond MR, et al. Predictive factors of early moderate/severe ovarian hyperstimulation syndrome in non-polycystic ovarian syndrome patients: a statistical model. Arch Gynecol Obstet 2015. PMID: 25920524. 
Ashrafi M, Madani T, Movahedi M, et al. Increasing The Number of Embryos Transferred from Two to Three, Does not Increase Pregnancy Rates in Good Prognosis Patients. Int J Fertil Steril 2015;9(3):292-9. PMID: 26644851.

Attia AM, Abou-Setta AM and Al-Inany HG. Gonadotrophins for idiopathic male factor subfertility. Cochrane Database Syst Rev 2013;8:Cd005071. PMID: 23970458.

Balen AH, Morley LC, Misso M, et al. The management of anovulatory infertility in women with polycystic ovary syndrome: an analysis of the evidence to support the development of global WHO guidance. Hum Reprod Update 2016;22(6):687-708. PMID: 27511809.

Bang JK, Lyu SW, Choi J, et al. Does infertility treatment increase male reproductive tract disorder? Urology 2013;81(3):644-8. PMID: 23452810.

Barbosa MA, Teixeira DM, Navarro PA, et al. Impact of endometriosis and its staging on assisted reproduction outcome: systematic review and meta-analysis. Ultrasound Obstet Gynecol 2014;44(3):261-78. PMID: 24639087.

Barbosa MW, Silva LR, Navarro PA, et al. Dydrogesterone versus progesterone for luteal-phase support: systematic review and meta-analysis of randomized controlled trials. Ultrasound Obstet Gynecol 2015. PMID: 26577241.

Barbosa MW, Sotiriadis A, Papatheodorou SI, et al. High miscarriage rate in women treated with Essure(R) for hydrosalpinx before embryo transfer: a systematic review and meta-analysis. Ultrasound Obstet Gynecol 2016;48(5):556-565. PMID: 27854386.

Barua S, Hng TM, Smith H, et al. Ovulatory disorders are an independent risk factor for pregnancy complications in women receiving assisted reproduction treatments. Aust N Z J Obstet Gynaecol 2016. PMID: 27396715.

Bechtejew TN, Nadai MN, Nastri CO, et al. Clomiphene citrate and letrozole to reduce folliclestimulating hormone consumption during ovarian stimulation: systematic review and metaanalysis. Ultrasound Obstet Gynecol 2017;50(3):315-323. PMID: 28236310.

Begueria R, Garcia D, Obradors A, et al. Paternal age and assisted reproductive outcomes in ICSI donor oocytes: is there an effect of older fathers? Hum Reprod 2014;29(10):2114-22. PMID: 25073975.

Benaglia L, Bermejo A, Somigliana E, et al. Pregnancy outcome in women with endometriomas achieving pregnancy through IVF. Hum Reprod 2012;27(6):1663-7. PMID: 22447627.

Bensdorp A, Cohlen BJ, Heineman MJ, et al. Intra-uterine insemination for male subfertility. Cochrane Database of Systematic Reviews 2007;(1).

Bensdorp A, Cohlen BJ, Heineman MJ, et al. Intra-uterine insemination for male subfertility. Cochrane Database Syst Rev 2007;(4):Cd000360. PMID: 17943739. 
Bensdorp AJ, Cohlen BJ, Heineman MJ, et al. Intra-uterine insemination for male subfertility. Cochrane Database Syst Rev 2007;(3):Cd000360. PMID: 17636632.

Besselink DE, Farquhar C, Kremer JA, et al. Cervical insemination versus intra-uterine insemination of donor sperm for subfertility. Cochrane Database Syst Rev 2008;(2):Cd000317. PMID: 18425862.

Bhattacharya S, Johnson N, Tijani HA, et al. Female infertility. BMJ Clin Evid 2010;2010. PMID: 21406133.

Bjelica A, Bjelanovic J, Milic N, et al. ALGORITHM OF OVULATION INDUCTION IN PATIENTS WITH POLYCISTIC OVARY SYNDROME. Med Pregl 2016;69(1-2):25-30. PMID: 27498530.

Bongioanni F, Revelli A, Gennarelli G, et al. Ovarian endometriomas and IVF: a retrospective case-control study. Reprod Biol Endocrinol 2011;9:81. PMID: 21679474.

Boomsma CM, Keay SD and Macklon NS. Peri-implantation glucocorticoid administration for assisted reproductive technology cycles. Cochrane Database Syst Rev 2012;(6):Cd005996. PMID: 22696356.

Bordewijk EM, Nahuis M, Costello MF, et al. Metformin during ovulation induction with gonadotrophins followed by timed intercourse or intrauterine insemination for subfertility associated with polycystic ovary syndrome. Cochrane Database Syst Rev 2017;1:Cd009090. PMID: 28118681.

Bosteels J, Weyers S, D'Hooghe TM, et al. Anti-adhesion therapy following operative hysteroscopy for treatment of female subfertility. Cochrane Database Syst Rev 2017;11:Cd011110. PMID: 29178172.

Bosteels J, Weyers S, Mathieu C, et al. The effectiveness of reproductive surgery in the treatment of female infertility: facts, views and vision. Facts Views Vis Obgyn 2010;2(4):23252. PMID: 25009712.

Boujenah J, Hugues JN, Sifer C, et al. Second live birth after undergoing assisted reproductive technology in women operated on for endometriosis. Fertil Steril 2016;105(1):129-33. PMID: 26493118.

Brink Laursen J, Schroll JB, Macklon KT, et al. Surgery versus conservative management of endometriomas in subfertile women. A systematic review. Acta Obstet Gynecol Scand 2017;96(6):727-735. PMID: 28421599.

Bromage SJ, Douglas J, Falconer DA, et al. Factors affecting successful outcome from ICSI in men following previous vasectomy. World J Urol 2007;25(5):519-24. PMID: 17609963.

Brosens IA, De Sutter P, Hamerlynck T, et al. Endometriosis is associated with a decreased risk of pre-eclampsia. Hum Reprod 2007;22(6):1725-9. PMID: 17452394. 
Brown J and Farquhar C. Clomiphene and other antioestrogens for ovulation induction in polycystic ovarian syndrome. Cochrane Database Syst Rev 2016;12:Cd002249. PMID: 27976369.

Brown J, Buckingham K, Buckett W, et al. Ultrasound versus 'clinical touch' for catheter guidance during embryo transfer in women. Cochrane Database of Systematic Reviews 2016;2016(3).

Brown J, Farquhar C, Beck J, et al. Clomiphene and anti-oestrogens for ovulation induction in PCOS. Cochrane Database Syst Rev 2009;(4):Cd002249. PMID: 19821295.

Buckett WM, Chian RC, Dean NL, et al. Pregnancy loss in pregnancies conceived after in vitro oocyte maturation, conventional in vitro fertilization, and intracytoplasmic sperm injection. Fertil Steril 2008;90(3):546-50. PMID: 17904128.

Campbell JM, Lane M, Owens JA, et al. Paternal obesity negatively affects male fertility and assisted reproduction outcomes: a systematic review and meta-analysis. Reprod Biomed Online 2015;31(5):593-604. PMID: 26380863.

Catford SR, McLachlan RI, O'Bryan MK, et al. Long-term follow-up of intra-cytoplasmic sperm injection-conceived offspring compared with in vitro fertilization-conceived offspring: a systematic review of health outcomes beyond the neonatal period. Andrology 2017;5(4):610621. PMID: 28632930.

Chambers GM, Wand H, Macaldowie A, et al. Population trends and live birth rates associated with common ART treatment strategies. Hum Reprod 2016. PMID: 27664207.

Check JH, Bollendorf A, Summers-Chase D, et al. Conventional oocyte insemination may result in a better pregnancy outcome than intracytoplasmic sperm injection (ICSI) for unexplained infertility. Clin Exp Obstet Gynecol 2009;36(3):150-1. PMID: 19860354.

Christopoulos G, Vlismas A, Carby A, et al. GnRH agonist trigger with intensive luteal phase support vs. human chorionic gonadotropin trigger in high responders: an observational study reporting pregnancy outcomes and incidence of ovarian hyperstimulation syndrome. Human Fertility 2016;19(3):199-206.

Chu K, Zhang Q, Han H, et al. A systematic review and meta-analysis of nonpharmacological adjuvant interventions for patients undergoing assisted reproductive technology treatment. Int $\mathrm{J}$ Gynaecol Obstet 2017;139(3):268-277. PMID: 28837219.

Chua SJ, Akande VA and Mol BW. Surgery for tubal infertility. Cochrane Database Syst Rev 2017;1:Cd006415. PMID: 28112384.

Cissen M, Bensdorp A, Cohlen BJ, et al. Assisted reproductive technologies for male subfertility. Cochrane Database Syst Rev 2016;2:Cd000360. PMID: 26915339.

Cobo A, Serra V, Garrido N, et al. Obstetric and perinatal outcome of babies born from vitrified oocytes. Fertil Steril 2014;102(4):1006-1015.e4. PMID: 25064408. 
Cohlen B, Bijkerk A, Van der Poel S, et al. IUI: review and systematic assessment of the evidence that supports global recommendations. Hum Reprod Update 2018. PMID: 29452361.

Cooper AR, O'Neill KE, Allsworth JE, et al. Smaller fetal size in singletons after infertility therapies: the influence of technology and the underlying infertility. Fertil Steril 2011;96(5):1100-6. PMID: 21944928.

Craciunas L, Tsampras N, Coomarasamy A, et al. Intrauterine administration of human chorionic gonadotropin (hCG) for subfertile women undergoing assisted reproduction. Cochrane Database Syst Rev 2016;5:Cd011537. PMID: 27195724.

Crowe M, Lee S, Seow CH, et al. The impact of surgical therapies for inflammatory bowel disease on female fertility. Cochrane Database of Systematic Reviews 2017;2017(7).

Cui N, Wang H, Wang W, et al. Impact of Body Mass Index on Outcomes of In Vitro Fertilization/Intracytoplasmic Sperm Injection Among Polycystic Ovarian Syndrome Patients. Cellular Physiology and Biochemistry 2016:1723-1734.

Dalal RJ, Pai H and Palshetkar N. Effectiveness of HP-hMG vs r-FSH in patients undergoing IVF/ICSI cycles with moderate male factor infertility. International Journal of Infertility and Fetal Medicine 2012;3(2):51-56.

Das M, Son WY, Buckett W, et al. In-vitro maturation versus IVF with GnRH antagonist for women with polycystic ovary syndrome: treatment outcome and rates of ovarian hyperstimulation syndrome. Reprod Biomed Online 2014;29(5):545-51. PMID: 25262236.

Davies MJ, Rumbold AR, Marino JL, et al. Maternal factors and the risk of birth defects after IVF and ICSI: a whole of population cohort study. Bjog 2016. PMID: 27748040.

Dayan N, Pilote L, Opatrny L, et al. Combined impact of high body mass index and in vitro fertilization on preeclampsia risk: a hospital-based cohort study. Obesity (Silver Spring) 2015;23(1):200-6. PMID: 25293810.

Decanter C, Robin G, Thomas P, et al. First intention IVF protocol for polycystic ovaries: does oral contraceptive pill pretreatment influence $\mathrm{COH}$ outcome? Reprod Biol Endocrinol 2013;11:54. PMID: 23782540.

De Silva PM, Chu JJ, Gallos ID, et al. Fallopian tube catheterization in the treatment of proximal tubal obstruction: a systematic review and meta-analysis. Hum Reprod 2017;32(4):836-852.

PMID: 28184438.

Dieamant FC, Petersen CG, Mauri AL, et al. Fresh embryos versus freeze-all embryos - transfer strategies: Nuances of a meta-analysis. Jornal Brasileiro de Reproducao Assistida 2017;21(3):260-272.

Dinelli L, Courbiere B, Achard V, et al. Prognosis factors of pregnancy after intrauterine insemination with the husband's sperm: conclusions of an analysis of 2,019 cycles. Fertil Steril 2014;101(4):994-1000. PMID: 24534285. 
Di Spiezio Sardo A, Di Carlo C, Minozzi S, et al. Efficacy of hysteroscopy in improving reproductive outcomes of infertile couples: a systematic review and meta-analysis. Hum Reprod Update 2016. PMID: 27008893.

Ding N, Chang J, Jian Q, et al. Luteal phase clomiphene citrate for ovulation induction in women with polycystic ovary syndrome: a systematic review and meta-analysis. Gynecol Endocrinol 2016;32(11):866-871. PMID: 27425581.

Donoso P, Tournaye H and Devroey P. Which is the best sperm retrieval technique for nonobstructive azoospermia? A systematic review. Hum Reprod Update 2007;13(6):539-49. PMID: 17895238.

Duffy JM, Arambage K, Correa FJ, et al. Laparoscopic surgery for endometriosis. Cochrane Database Syst Rev 2014;4:Cd011031. PMID: 24696265.

Duffy JM, Johnson N, Ahmad G, et al. Postoperative procedures for improving fertility following pelvic reproductive surgery. Cochrane Database Syst Rev 2009;(2):Cd001897. PMID: 19370571.

Dunietz GL, Holzman C, McKane P, et al. Assisted reproductive technology and the risk of preterm birth among primiparas. Fertil Steril 2015;103(4):974-979.e1. PMID: 25707336.

El Khattabi L, Dupont C, Sermondade N, et al. Is intracytoplasmic morphologically selected sperm injection effective in patients with infertility related to teratozoospermia or repeated implantation failure? Fertil Steril 2013;100(1):62-8. PMID: 23548938.

El Meliegy A, Motawi A and El Salam MAA. Systematic review of hormone replacement therapy in the infertile man. Arab Journal of Urology 2018;16(1):140-147.

Elassar A, Mann JS, Engmann L, et al. Luteal phase estradiol versus luteal phase estradiol and antagonist protocol for controlled ovarian stimulation before in vitro fertilization in poor responders. Fertil Steril 2011;95(1):324-6. PMID: 20797708.

Elgafor El Sharkwy IA. Metformin versus laparoscopic unilateral ovarian drilling in clomiphene resistant women with polycystic ovary syndrome. Middle East Fertility Society Journal 2013;18(3):202-207.

Erdem A, Erdem M, Atmaca S, et al. Factors affecting live birth rate in intrauterine insemination cycles with recombinant gonadotrophin stimulation. Reprod Biomed Online 2008;17(2):199-206. PMID: 18681993.

Esteves SC, Miyaoka R, Roque M, et al. Outcome of varicocele repair in men with nonobstructive azoospermia: systematic review and meta-analysis. Asian J Androl 2016;18(2):246-53. PMID: 26680033.

Esteves SC, Oliveira FV and Bertolla RP. Clinical outcome of intracytoplasmic sperm injection in infertile men with treated and untreated clinical varicocele. J Urol 2010;184(4):1442-6. PMID: 20727535. 
Esteves SC, Prudencio C, Seol B, et al. Comparison of sperm retrieval and reproductive outcome in azoospermic men with testicular failure and obstructive azoospermia treated for infertility. Asian J Androl 2014;16(4):602-6. PMID: 24759580.

Esteves SC, Roque M and Agarwal A. Outcome of assisted reproductive technology in men with treated and untreated varicocele: systematic review and meta-analysis. Asian J Androl 2016;18(2):254-8. PMID: 26510504.

Esteves SC, Roque M, Bradley CK, et al. Reproductive outcomes of testicular versus ejaculated sperm for intracytoplasmic sperm injection among men with high levels of DNA fragmentation in semen: systematic review and meta-analysis. Fertil Steril 2017;108(3):456-467.e1. PMID: 28865546.

Fang C, Huang R, Wei LN, et al. Frozen-thawed day 5 blastocyst transfer is associated with a lower risk of ectopic pregnancy than day 3 transfer and fresh transfer. Fertility and Sterility 2015;103(3):655-661.e3.

Farag S, Padilla PF, Smith KA, et al. Management, Prevention, and Sequelae of Adhesions in Women Undergoing Laparoscopic Gynecologic Surgery: A Systematic Review. Journal of Minimally Invasive Gynecology 2018.

Farquhar C, Brown J and Marjoribanks J. Laparoscopic drilling by diathermy or laser for ovulation induction in anovulatory polycystic ovary syndrome. Cochrane Database Syst Rev 2012;6:Cd001122. PMID: 22696324.

Farquhar C, Lilford RJ, Marjoribanks J, et al. Laparoscopic 'drilling' by diathermy or laser for ovulation induction in anovulatory polycystic ovary syndrome. Cochrane Database Syst Rev 2007;(3):Cd001122. PMID: 17636653.

Farquhar C, Marjoribanks J, Brown J, et al. Management of ovarian stimulation for IVF: narrative review of evidence provided for World Health Organization guidance. Reprod Biomed Online 2017;35(1):3-16. PMID: 28501428.

Farquhar C, Rishworth JR, Brown J, et al. Assisted reproductive technology: an overview of Cochrane Reviews. Cochrane Database Syst Rev 2015;7:Cd010537. PMID: 26174592.

Farquhar C, Rombauts L, Kremer JA, et al. Oral contraceptive pill, progestogen or oestrogen pretreatment for ovarian stimulation protocols for women undergoing assisted reproductive techniques. Cochrane Database Syst Rev 2017;5:Cd006109. PMID: 28540977.

Fedder J, Loft A, Parner ET, et al. Neonatal outcome and congenital malformations in children born after ICSI with testicular or epididymal sperm: a controlled national cohort study. Hum Reprod 2013;28(1):230-40. PMID: 23154066.

Fernando S, Breheny S, Jaques AM, et al. Preterm birth, ovarian endometriomata, and assisted reproduction technologies. Fertil Steril 2009;91(2):325-30. PMID: 18384780. 
Franik S, Kremer JA, Nelen WL, et al. Aromatase inhibitors for subfertile women with polycystic ovary syndrome. Cochrane Database Syst Rev 2014;2:Cd010287. PMID: 24563180.

Franik S, Kremer JA, Nelen WL, et al. Aromatase inhibitors for subfertile women with polycystic ovary syndrome: summary of a Cochrane review. Fertil Steril 2015;103(2):353-5. PMID: 25455536.

Fukuda J, Abe T, Okuno T, et al. Administering human chorionic gonadotropin injections for triggering follicle maturation could impact fertility during the subsequent menstrual cycle. Int $\mathrm{J}$ Gynaecol Obstet 2016;132(3):309-13. PMID: 26643301.

Gadalla MA, Huang S, Wang R, et al. Effect of clomiphene citrate on endometrial thickness, ovulation, pregnancy and live birth in anovulatory women: systematic review and meta-analysis. Ultrasound Obstet Gynecol 2018;51(1):64-76. PMID: 29055102.

Gallos ID, Eapen A, Price MJ, et al. Controlled ovarian stimulation protocols for assisted reproduction: A network meta-analysis. Cochrane Database of Systematic Reviews $2017 ; 2017(3)$.

Gameiro S and Finnigan A. Long-term adjustment to unmet parenthood goals following ART: a systematic review and meta-analysis. Hum Reprod Update 2017;23(3):322-337. PMID: 28164236.

Garrido N, Bellver J, Remohi J, et al. Cumulative live-birth rates per total number of embryos needed to reach newborn in consecutive in vitro fertilization (IVF) cycles: a new approach to measuring the likelihood of IVF success. Fertil Steril 2011;96(1):40-6. PMID: 21621770.

Gasparri ML, Nirgianakis K, Taghavi K, et al. Placenta previa and placental abruption after assisted reproductive technology in patients with endometriosis: a systematic review and metaanalysis. Arch Gynecol Obstet 2018. PMID: 29602980.

Gatimel N, Parinaud J and Leandri RD. Intracytoplasmic morphologically selected sperm injection (IMSI) does not improve outcome in patients with two successive IVF-ICSI failures. Journal of Assisted Reproduction and Genetics 2016;33(3):349-355.

Glavind MT, Forman A, Arendt LH, et al. Endometriosis and pregnancy complications: a Danish cohort study. Fertil Steril 2017;107(1):160-166. PMID: 27743699.

Gnoth C, Markhinin V, Maxrath B, et al. Impact of sperm cell source on the results of intracytoplasmic sperm injection. Arch Gynecol Obstet 2015;291(3):663-9. PMID: 25193430.

Gong F, Li X, Zhang S, et al. A modified ultra-long pituitary downregulation protocol improved endometrial receptivity and clinical outcome for infertile patients with polycystic ovarian syndrome. Experimental and Therapeutic Medicine 2015;10(5):1865-1870.

Goudarzi ZM, Fallahzadeh H, Aflatoonian A, et al. Laparoscopic ovarian electrocautery versus gonadotropin therapy in infertile women with clomiphene citrate-resistant polycystic ovary 
syndrome: A systematic review and meta-analysis. Iran J Reprod Med 2014;12(8):531-8. PMID: 25408702.

Green KA, Zolton JR, Schermerhorn SM, et al. Progesterone luteal support after ovulation induction and intrauterine insemination: an updated systematic review and meta-analysis. Fertil Steril 2017;107(4):924-933.e5. PMID: 28238492.

Gremeau AS, Andreadis N, Fatum M, et al. In vitro maturation or in vitro fertilization for women with polycystic ovaries? A case-control study of 194 treatment cycles. Fertil Steril 2012;98(2):355-60. PMID: 22658347.

Gressier F, Letranchant A, Cazas O, et al. Post-partum depressive symptoms and medically assisted conception: A systematic review and meta-analysis. Human Reproduction 2015;30(11):2575-2586.

Guan Y, Fan H, Styer AK, et al. A modified natural cycle results in higher live birth rate in vitrified-thawed embryo transfer for women with regular menstruation. Systems Biology in Reproductive Medicine 2016;62(5):335-342.

Gül Ü and Turunç T. The effect of human chorionic gonadotropin treatment before testicular sperm extraction in non-obstructive azoospermia. Journal of Clinical and Analytical Medicine 2016;7(1):55-59.

Gunn DD and Bates GW. Evidence-based approach to unexplained infertility: a systematic review. Fertil Steril 2016. PMID: 26902860.

Güven D, Batığlu A, Sari S, et al. The impact of progesterone supplementation on pregnancy rates after intrauterine insemination in patients developing a single follicle. Human Fertility 2016;19(2):111-113.

Hakimi O and Cameron LC. Effect of Exercise on Ovulation: A Systematic Review. Sports Med 2017;47(8):1555-1567. PMID: 28035585.

Hamdan M, Dunselman G, Li TC, et al. The impact of endometrioma on IVF/ICSI outcomes: a systematic review and meta-analysis. Hum Reprod Update 2015. PMID: 26168799.

Hamdan M, Omar SZ, Dunselman G, et al. Influence of endometriosis on assisted reproductive technology outcomes: a systematic review and meta-analysis. Obstet Gynecol. 2015;125(1):7988. PMID: 25560108.

Han AR, Kim HO, Cha SW, et al. Adverse pregnancy outcomes with assisted reproductive technology in non-obese women with polycystic ovary syndrome: a case-control study. Clin Exp Reprod Med 2011;38(2):103-8. PMID: 22384427.

Harb HM, Gallos ID, Chu J, et al. The effect of endometriosis on in vitro fertilisation outcome: a systematic review and meta-analysis. Bjog 2013;120(11):1308-20. PMID: 23834505. 
Harris A and Tsaltas J. Endometriosis and infertility: A systematic review. Journal of Endometriosis and Pelvic Pain Disorders 2017;9(3):139-149.

Hart Roger J, Hickey M, Maouris P, et al. Excisional surgery versus ablative surgery for ovarian endometriomata. 2008;(2):CD004992.

Healy DL, Breheny S, Halliday J, et al. Prevalence and risk factors for obstetric haemorrhage in 6730 singleton births after assisted reproductive technology in Victoria Australia. Hum Reprod 2010;25(1):265-74. PMID: 19897853.

Hibi H, Sumitomo M, Fukunaga N, et al. Superior clinical pregnancy rates after microsurgical epididymal sperm aspiration. Reproductive Medicine and Biology 2018;17(1):59-63.

Hill MJ, McWilliams GD, Miller KA, et al. A luteal estradiol protocol for anticipated poorresponder patients may improve delivery rates. Fertil Steril 2009;91(3):739-43. PMID: 18314123.

Hirsch M, Duffy JM, Kusznir JO, et al. Variation in outcome reporting in endometriosis trials: a systematic review. Am J Obstet Gynecol 2016;214(4):452-64. PMID: 26778385.

Hirst WM, Vail A, Brison DR, et al. Prognostic factors influencing fresh and frozen IVF outcomes: An analysis of the UK national database. Reproductive BioMedicine Online 2011;22(5):437-448.

Hoorsan H, Mirmiran P, Chaichian S, et al. Congenital malformations in infants of mothers undergoing assisted reproductive technologies: A systematic review and meta-analysis study. Journal of Preventive Medicine and Public Health 2017;50(6):347-360.

Hu Q, Hong L, Nie M, et al. The effect of dehydroepiandrosterone supplementation on ovarian response is associated with androgen receptor in diminished ovarian reserve women. J Ovarian Res 2017;10(1):32. PMID: 28472976.

Huang X, Wang P, Tal R, et al. A systematic review and meta-analysis of metformin among patients with polycystic ovary syndrome undergoing assisted reproductive technology procedures. International Journal of Gynecology and Obstetrics 2015;131(2):111-116.

Hughes E, Brown J, Collins JJ, et al. Clomiphene citrate for unexplained subfertility in women. Cochrane Database Syst Rev 2010;(1):Cd000057. PMID: 20091498.

Hughes E, Brown J, Collins John J, et al. Ovulation suppression for endometriosis for women with subfertility. 2007;(3):CD000155.

Hughes E, Brown J, Tiffin G, et al. Danazol for unexplained subfertility. Cochrane Database Syst Rev 2007;(1):Cd000069. PMID: 17253444.

Humphries LA, Chang O, Humm K, et al. Influence of race and ethnicity on in vitro fertilization outcomes: systematic review. Am J Obstet Gynecol 2016;214(2):212.e1-212.e17. PMID: 26363483. 
Jefferys A, Siassakos D and Wardle P. The management of retrograde ejaculation: a systematic review and update. Fertil Steril 2012;97(2):306-12. PMID: 22177462.

Jeve YB and Bhandari HM. Effective treatment protocol for poor ovarian response: A systematic review and meta-analysis. Journal of Human Reproductive Sciences 2016;9(2):70-81.

Jin X and Ruiz Beguerie J. Laparoscopic surgery for subfertility related to endometriosis: a meta-analysis. Taiwan J Obstet Gynecol 2014;53(3):303-8. PMID: 25286781.

Johnson N, van Voorst S, Sowter MC, et al. Surgical treatment for tubal disease in women due to undergo in vitro fertilisation. Cochrane Database Syst Rev 2010;(1):Cd002125. PMID: 20091531.

Johnson N. Metformin is a reasonable first-line treatment option for non-obese women with infertility related to anovulatory polycystic ovary syndrome--a meta-analysis of randomised trials. Aust N Z J Obstet Gynaecol 2011;51(2):125-9. PMID: 21466513.

Junk SM and Yeap D. Improved implantation and ongoing pregnancy rates after single-embryo transfer with an optimized protocol for in vitro oocyte maturation in women with polycystic ovaries and polycystic ovary syndrome. Fertil Steril 2012;98(4):888-92. PMID: 22835445.

Kakourou G, Kahraman S, Ekmekci GC, et al. The clinical utility of PGD with HLA matching: A collaborative multi-centre ESHRE study. Human Reproduction 2018;33(3):520-530.

Kalsi J, Thum MY, Muneer A, et al. Analysis of the outcome of intracytoplasmic sperm injection using fresh or frozen sperm. BJU Int 2011;107(7):1124-8. PMID: 21156015.

Kalu E, Thum MY and Abdalla H. Intrauterine insemination in natural cycle may give better results in older women. J Assist Reprod Genet 2007;24(2-3):83-6. PMID: 17226077.

Kamath MS, Bhave PTK, Aleyamma TK, et al. Predictive factors for pregnancy after intrauterine insemination: A prospective study of factors affecting outcome. Journal of Human Reproductive Sciences 2010;3(3):129-134.

Karacan M, Alwaeely F, Erkan S, et al. Outcome of intracytoplasmic sperm injection cycles with fresh testicular spermatozoa obtained on the day of or the day before oocyte collection and with cryopreserved testicular sperm in patients with azoospermia. Fertil Steril 2013;100(4):975-80. PMID: 23891272.

Kathiresan AS, Ibrahim E, Aballa TC, et al. Comparison of in vitro fertilization/intracytoplasmic sperm injection outcomes in male factor infertility patients with and without spinal cord injuries. Fertil Steril 2011;96(3):562-6. PMID: 21807365.

Kereszturi A, Kozinszky Z, Daru J, et al. Pregnancy Rate after Controlled Ovarian Hyperstimulation and Intrauterine Insemination for the Treatment of Endometriosis following Surgery. Biomed Res Int 2015;2015:282301. PMID: 26247014. 
Khairy M, Dhillon RK, Chu J, et al. The effect of peri-implantation administration of uterine relaxing agents in assisted reproduction treatment cycles: A systematic review and meta-analysis. Reproductive BioMedicine Online 2016;32(4):362-376.

Khalili MA, Halvaei I, Ghazali S, et al. Performing ICSI with commercial microinjection pipettes enhanced pregnancy rates. Turk J Med Sci 2017;47(3):801-805. PMID: 28618725.

Kim HH, Bundorf MK, Behr B, et al. Use and outcomes of intracytoplasmic sperm injection for non-male factor infertility. Fertil Steril 2007;88(3):622-8. PMID: 17445809.

Kim HO, Sung N and Song IO. Predictors of live birth and pregnancy success after in vitro fertilization in infertile women aged 40 and over. Clin Exp Reprod Med 2017;44(2):111-117. PMID: 28795051.

Kirby EW, Wiener LE, Rajanahally S, et al. Undergoing varicocele repair before assisted reproduction improves pregnancy rate and live birth rate in azoospermic and oligospermic men with a varicocele: a systematic review and meta-analysis. Fertil Steril 2016;106(6):1338-1343. PMID: 27526630.

Klement AH, Koren-Morag N, Itsykson P, et al. Intracytoplasmic morphologically selected sperm injection versus intracytoplasmic sperm injection: a step toward a clinical algorithm. Fertil Steril 2013;99(5):1290-3. PMID: 23357455.

Kolanska K, Cohen J, Bendifallah S, et al. Pregnancy outcomes after controlled ovarian hyperstimulation in women with endometriosis-associated infertility: GnRH-agonist versus GnRH-antagonist. J Gynecol Obstet Hum Reprod 2017;46(9):681-686. PMID: 28970135.

Kresowik JD, Sparks AE and Van Voorhis BJ. Clinical factors associated with live birth after single embryo transfer. Fertil Steril 2012;98(5):1152-6. PMID: 22959461.

Kuivasaari-Pirinen P, Hippelainen M, Hakkarainen H, et al. Cumulative baby take-home rate among women with PCOS treated by IVF. Gynecol Endocrinol 2010;26(8):582-9. PMID: 20175706.

Kuivasaari-Pirinen P, Raatikainen K, Hippelainen M, et al. Adverse Outcomes of IVF/ICSI Pregnancies Vary Depending on Aetiology of Infertility. ISRN Obstet Gynecol 2012;2012:451915. PMID: 22570795.

Kumbak B and Kahraman S. Efficacy of metformin supplementation during ovarian stimulation of lean PCOS patients undergoing in vitro fertilization. Acta Obstet Gynecol Scand 2009;88(5):563-8. PMID: 19306135.

Kushnir VA, Safdie M, Darmon SK, et al. Age-Specific IVF Outcomes in Infertile Women With Baseline FSH Levels >/=20 mIU/mL. Reprod Sci 2017:1933719117697130. PMID: 28264624.

L RH, Khan S, Dai J, et al. Low-Dose Urinary Human Chorionic Gonadotropin Is Effective for Oocyte Maturation in In Vitro Fertilization/ Intracytoplasmic Sperm Injection Cycles Independent of Body Mass Index. Int J Fertil Steril 2017;11(1):7-14. PMID: 28367299. 
Lacamara C, Ortega C, Villa S, et al. Are children born from singleton pregnancies conceived by ICSI at increased risk for congenital malformations when compared to children conceived naturally? A systematic review and meta-analysis. JBRA Assist Reprod 2017;21(3):251-259. PMID: 28837036.

Lainas TG, Sfontouris IA, Venetis CA, et al. Live birth rates after modified natural cycle compared with high-dose FSH stimulation using GnRH antagonists in poor responders. Hum Reprod 2015. PMID: 26307091.

Lan L, Harrison CL, Misso M, et al. Systematic review and meta-analysis of the impact of preconception lifestyle interventions on fertility, obstetric, fetal, anthropometric and metabolic outcomes in men and women. Hum Reprod 2017;32(9):1925-1940. PMID: 28854715.

Lannon BM, Choi B, Hacker MR, et al. Predicting personalized multiple birth risks after in vitro fertilization-double embryo transfer. Fertil Steril 2012;98(1):69-76. PMID: 22673597.

Le QV, Abhari S, Abuzeid OM, et al. Modified natural cycle for embryo transfer using frozenthawed blastocysts: A satisfactory option. Eur J Obstet Gynecol Reprod Biol 2017;213:58-63. PMID: 28437630.

Ledee N, Prat-Ellenberg L, Chevrier L, et al. Uterine immune profiling for increasing live birth rate: A one-to-one matched cohort study. J Reprod Immunol 2017;119:23-30. PMID: 27915039.

Legro RS. Effects of obesity treatment on female reproduction: results do not match expectations. Fertil Steril 2017;107(4):860-867. PMID: 28366412.

Lee E, Chambers GM, Hale L, et al. Assisted reproductive technology (ART) cumulative live birth rates following preimplantation genetic diagnosis for aneuploidy (PGD-A) or morphological assessment of embryos: A cohort analysis. Australian and New Zealand Journal of Obstetrics and Gynaecology 2017.

Lee JW, Cha JH, Shin SH, et al. Efficacy of embryo transfer on day 2 versus day 3 according to maternal age in patients with normal ovarian response. Clin Exp Reprod Med 2017;44(3):141145. PMID: 29026720.

Lee YJ, Kim CH, Kim DY, et al. Human chorionic gonadotropin-administered natural cycle versus spontaneous ovulatory cycle in patients undergoing two pronuclear zygote frozen-thawed embryo transfer. Obstet Gynecol Sci 2018;61(2):247-252. PMID: 29564316.

Legro RS, Dodson WC, Kunselman AR, et al. Benefit of Delayed Fertility Therapy with Preconception Weight Loss over Immediate Therapy in Obese Women with PCOS. J Clin Endocrinol Metab 2016:jc20161659. PMID: 27172435.

Lensen SF, Manders M, Nastri CO, et al. Endometrial injury for pregnancy following sexual intercourse or intrauterine insemination. Cochrane Database Syst Rev 2016(6):Cd011424. PMID: 27296541. 
Leone Roberti Maggiore U, Ferrero S, Candiani M, et al. Bladder Endometriosis: A Systematic Review of Pathogenesis, Diagnosis, Treatment, Impact on Fertility, and Risk of Malignant Transformation. Eur Urol 2017;71(5):790-807. PMID: 28040358.

Leone Roberti Maggiore U, Scala C, Tafi E, et al. Spontaneous fertility after expectant or surgical management of rectovaginal endometriosis in women with or without ovarian endometrioma: a retrospective analysis. Fertil Steril 2017;107(4):969-976.e5. PMID: 28366418.

Levron J, Madgar I, Shefi S, et al. IVF outcome with cryopreserved testicular sperm. Andrologia 2011;43(1):48-51. PMID: 21219382.

Li HW, Lee VC, Lau EY, et al. Cumulative live-birth rate in women with polycystic ovary syndrome or isolated polycystic ovaries undergoing in-vitro fertilisation treatment. J Assist Reprod Genet 2014;31(2):205-11. PMID: 24337962.

Li J, Chen Y, Liu C, et al. Intravenous immunoglobulin treatment for repeated IVF/ICSI failure and unexplained infertility: a systematic review and a meta-analysis. Am J Reprod Immunol 2013;70(6):434-47. PMID: 24238107.

Li J, Chen Y, Wang A, et al. A meta-analysis of atosiban supplementation among patients undergoing assisted reproduction. Arch Gynecol Obstet 2017;296(4):623-634. PMID: 28695395.

Li RS, Hwu YM, Lee RK, et al. Day 4 good morula embryo transfer provided compatible live birth rate with day 5 blastocyst embryo in fresh IVF/ET cycles. Taiwan J Obstet Gynecol 2018;57(1):52-57. PMID: 29458903.

Li Z, Sullivan EA, Chapman M, et al. Risk of ectopic pregnancy lowest with transfer of single frozen blastocyst. Hum Reprod 2015;30(9):2048-54. PMID: 26202917.

Li Y, Luo K, Tang Y, et al. Progesterone/estradiol ratio $<0.25$ on the day of human chorionic gonadotropin administration is associated with adverse pregnancy outcomes in prolonged protocols for in vitro fertilization/intracytoplasmic sperm injection. Taiwan J Obstet Gynecol 2017;56(1):27-31. PMID: 28254221.

Liao CC, Lee RKK, Lin SY, et al. Outcomes of anti-Müllerian hormone-tailored ovarian stimulation protocols in in vitro fertilization/intracytoplasmic sperm injection cycles in women of advanced age. Taiwanese Journal of Obstetrics and Gynecology 2016;55(2):239-243.

Lin $\mathrm{H}, \mathrm{Li} \mathrm{Y,} \mathrm{Li} \mathrm{L,} \mathrm{et} \mathrm{al.} \mathrm{Is} \mathrm{a} \mathrm{GnRH} \mathrm{antagonist} \mathrm{protocol} \mathrm{better} \mathrm{in} \mathrm{PCOS} \mathrm{patients?} \mathrm{A} \mathrm{meta-analysis}$ of RCTs. PLoS One 2014;9(3):e91796. PMID: 24642641.

Lin J, Dang Y, Guo G, et al. The influence of dehydroepiandrosterone (DHEA) supplementation for in vitro fertilization in women with diminished ovarian reserve: A meta-analysis of randomized controlled trials. International Journal of Clinical and Experimental Medicine 2017;10(12):15878-15885. 
Lin J, Wang P, Zhao J, et al. Outcomes of in vitro fertilization cycles among patients with polycystic ovary syndrome following ovarian puncture for in vitro maturation. Int J Gynaecol Obstet 2016;135(3):319-323. PMID: 27836468.

Lin YJ, Ou YC, Huang FJ, et al. Ovarian response to gonadotropins in patients with tubal factor infertility: salpingectomy versus nonsalpingectomy. J Minim Invasive Gynecol 2013;20(5):63741. PMID: 23706676.

Liu A, Zheng C, Lang J, et al. Letrozole versus clomiphene citrate for unexplained infertility: a systematic review and meta-analysis. J Obstet Gynaecol Res 2014;40(5):1205-16. PMID: 24754848.

Liu KE and Case A. No. 346-Advanced Reproductive Age and Fertility. J Obstet Gynaecol Can 2017;39(8):685-695. PMID: 28549563.

Liu L, Cai J, Li P, et al. Clinical outcome of cycles with oocyte degeneration after intracytoplasmic sperm injection. Syst Biol Reprod Med 2017;63(2):113-119. PMID: 28151020.

Liu M, Su Y and Wang WH. Assessment of clinical application of preimplantation genetic screening on cryopreserved human blastocysts. Reproductive Biology and Endocrinology 2016;14(1).

Lludgar MFU, Sedo CA, Fiszbajn G, et al. Newborn outcome after assisted reproductive technologies with annexin V (MACS): First report. Jornal Brasileiro de Reproducao Assistida 2013;17(3):183-188.

Lu HF, Peng FS, Chen SU, et al. The outcomes of intracytoplasmic sperm injection and laser assisted hatching in women undergoing in vitro fertilization are affected by the cause of infertility. Int J Fertil Steril 2015;9(1):33-40. PMID: 25918590.

Lu S, Cui Y, Li X, et al. Association of cystic fibrosis transmembrane-conductance regulator gene mutation with negative outcome of intracytoplasmic sperm injection pregnancy in cases of congenital bilateral absence of vas deferens. Fertil Steril 2014;101(5):1255-60. PMID: 24559724.

Lu YH, Gao HJ, Li BJ, et al. Different sperm sources and parameters can influence intracytoplasmic sperm injection outcomes before embryo implantation. J Zhejiang Univ Sci B 2012;13(1):1-10. PMID: 22205614.

Luke B, Brown MB, Wantman E, et al. A prediction model for live birth and multiple births within the first three cycles of assisted reproductive technology. Fertil Steril 2014;102(3):744-52. PMID: 24934487.

Luke B, Brown MB, Wantman E, et al. Application of a validated prediction model for in vitro fertilization: comparison of live birth rates and multiple birth rates with 1 embryo transferred over 2 cycles vs 2 embryos in 1 cycle. Am J Obstet Gynecol 2015;212(5):676.e1-7. PMID: 25683965. 
Ma QW and Tan Y. Effectiveness of co-treatment with traditional Chinese medicine and letrozole for polycystic ovary syndrome: a meta-analysis. J Integr Med 2017;15(2):95-101. PMID: 28285614.

Mackens S, Santos-Ribeiro S, van de Vijver A, et al. Frozen embryo transfer: a review on the optimal endometrial preparation and timing. Hum Reprod 2017;32(11):2234-2242. PMID: 29025055.

Madero S, Rodriguez A, Vassena R, et al. Endometrial preparation: Effect of estrogen dose and administration route on reproductive outcomes in oocyte donation cycles with fresh embryo transfer. Human Reproduction 2016;31(8):1755-1764.

Majzoub A and Agarwal A. Systematic review of antioxidant types and doses in male infertility: Benefits on semen parameters, advanced sperm function, assisted reproduction and live-birth rate. Arab Journal of Urology 2018;16(1):113-124.

Makinen S, Soderstrom-Anttila V, Vainio J, et al. Does long in vitro culture promote large for gestational age babies? Hum Reprod 2013;28(3):828-34. PMID: 23232355.

Malacova E, Kemp A, Hart R, et al. Effectiveness of in vitro fertilization in women with previous tubal sterilization. Contraception 2015;91(3):240-4. PMID: 25499586.

Marci R, Murisier F, Lo Monte G, et al. Clinical outcome after IMSI procedure in an unselected infertile population: a pilot study. Reprod Health 2013;10:16. PMID: 23521828.

Matsuzaki T, Tungalagsuvd A, Iwasa T, et al. Clinical outcome of various metformin treatments for women with polycystic ovary syndrome. 2017;16(2):179-187.

McLernon DJ, Maheshwari A, Lee AJ, et al. Cumulative live birth rates after one or more complete cycles of IVF: A population-based study of linked cycle data from 178898 women. Human Reproduction 2016;31(3):572-581.

Melnick AP, Setton R, Stone LD, et al. Replacing single frozen-thawed euploid embryos in a natural cycle in ovulatory women may increase live birth rates compared to medicated cycles in anovulatory women. Journal of Assisted Reproduction and Genetics 2017;34(10):1325-1331.

Messerlian C, Platt RW, Ata B, et al. Do the causes of infertility play a direct role in the aetiology of preterm birth? Paediatr Perinat Epidemiol 2015;29(2):101-12. PMID: 25644431.

Mignini Renzini M, Brigante C, Coticchio G, et al. Retrospective analysis of treatments with recombinant FSH and recombinant LH versus human menopausal gonadotropin in women with reduced ovarian reserve. Journal of Assisted Reproduction and Genetics 2017;34(12):1645-1651.

Misso ML, Costello MF, Garrubba M, et al. Metformin versus clomiphene citrate for infertility in non-obese women with polycystic ovary syndrome: a systematic review and meta-analysis. Hum Reprod Update 2013;19(1):2-11. PMID: 22956412. 
Misso ML, Wong JL, Teede HJ, et al. Aromatase inhibitors for PCOS: a systematic review and meta-analysis. Hum Reprod Update 2012;18(3):301-12. PMID: 22431566.

Mochtar MH, Danhof NA, Ayeleke RO, et al. Recombinant luteinizing hormone (rLH) and recombinant follicle stimulating hormone (rFSH) for ovarian stimulation in IVF/ICSI cycles. 2017(5). PMID: CD005070.

Live birth rate in fresh and frozen embryo transfer cycles in women with endometriosis. Eur $\mathrm{J}$ Obstet Gynecol Reprod Biol 2011;156(2):177-80. PMID: 21353737.

Moll E, van der Veen F and van Wely M. The role of metformin in polycystic ovary syndrome: a systematic review. Hum Reprod Update 2007;13(6):527-37. PMID: 17767003.

Monraisin O, Chansel-Debordeaux L, Chiron A, et al. Evaluation of intrauterine insemination practices: a 1-year prospective study in seven French assisted reproduction technology centers. Fertil Steril 2016. PMID: 26916177.

Moolenaar LM, Cissen M, de Bruin JP, et al. Cost-effectiveness of assisted conception for male subfertility. Reprod Biomed Online 2015;30(6):659-66. PMID: 25900905.

Moolenaar LM, Mohiuddin S, Munro Davie M, et al. High live birth rate in the subsequent IVF cycle after first-cycle poor response among women with mean age 35 and normal FSH. Reprod Biomed Online 2013;27(4):362-6. PMID: 23953583.

Morley LC, Tang T, Yasmin E, et al. Insulin-sensitising drugs (metformin, rosiglitazone, pioglitazone, D-chiro-inositol) for women with polycystic ovary syndrome, oligo amenorrhoea and subfertility. Cochrane Database Syst Rev 2017;11:Cd003053. PMID: 29183107.

Mouhayar Y, Yin O, Mumford SL, et al. Hysteroscopic polypectomy prior to infertility treatment: A cost analysis and systematic review. Eur J Obstet Gynecol Reprod Biol 2017;213:107-115. PMID: 28445799.

Mourad S, Brown J and Farquhar C. Interventions for the prevention of OHSS in ART cycles: An overview of Cochrane reviews. Cochrane Database of Systematic Reviews 2016;2016(2).

Mourad S, Brown J and Farquhar C. Interventions for the prevention of OHSS in ART cycles: an overview of Cochrane reviews. Cochrane Database Syst Rev 2017;1:Cd012103. PMID: 28111738.

Munch EM, Sparks AE, Zimmerman MB, et al. High FSH dosing is associated with reduced live birth rate in fresh but not subsequent frozen embryo transfers. Human Reproduction 2017;32(7):1402-1409.

Murugesu S, Saso S, Jones BP, et al. Does the use of calcium ionophore during artificial oocyte activation demonstrate an effect on pregnancy rate? A meta-analysis. Fertil Steril 2017;108(3):468-482.e3. PMID: 28865547. 
Mushtaq R, Pundir J, Achilli C, et al. Effect of male body mass index on assisted reproduction treatment outcome: An updated systematic review and meta-analysis. Reproductive BioMedicine Online 2018

Myers ER, McCrory DC, Mills AA, et al. Effectiveness of assisted reproductive technology (ART). Evid Rep Technol Assess (Full Rep) 2008;(167):1-195. PMID: 18620469.

Nagels HE, Rishworth JR, Siristatidis CS, et al. Androgens (dehydroepiandrosterone or testosterone) for women undergoing assisted reproduction. Cochrane Database Syst Rev 2015;11:Cd009749. PMID: 26608695.

Nair R, Nikolopoulos KI and Claydon LS. Clinical outcomes in women undergoing laparoscopic hysteropexy: A systematic review. Eur J Obstet Gynecol Reprod Biol 2017;208:71-80. PMID: 27894032.

Nawaz FH, Khalid R, Naru T, et al. Does continuous use of metformin throughout pregnancy improve pregnancy outcomes in women with polycystic ovarian syndrome? J Obstet Gynaecol Res 2008;34(5):832-7. PMID: 18834342.

Negri L, Patrizio P, Albani E, et al. ICSI outcome is significantly better with testicular spermatozoa in patients with necrozoospermia: a retrospective study. Gynecol Endocrinol 2014;30(1):48-52. PMID: 24147853.

Nejad EST, Rashidi BH, Larti A, et al. The outcome of in vitro fertilization/intracytoplasmic sperm injection in endometriosis associated and tubal factor infertility. Iranian Journal of Reproductive Medicine 2009;7(1):1-5.

Nelson SM and Lawlor DA. Predicting live birth, preterm delivery, and low birth weight in infants born from in vitro fertilisation: a prospective study of 144,018 treatment cycles. PLoS Med 2011;8(1):e1000386. PMID: 21245905.

Nickkho-Amiry M, Savant R, Majumder K, et al. The effect of surgical management of endometrioma on the IVF/ICSI outcomes when compared with no treatment? A systematic review and meta-analysis. Arch Gynecol Obstet 2018;297(4):1043-1057. PMID: 29344847.

Noble G, Harvey C, Napier C, et al. Preimplantation genetic screening in advanced maternal age: A systematic review. Expert Review of Obstetrics and Gynecology 2010;5(6):707-716.

Noventa M, Gizzo S, Saccardi C, et al. Salpingectomy before assisted reproductive technologies: a systematic literature review. J Ovarian Res 2016;9(1):74. PMID: 27809927.

Okun N and Sierra S. Pregnancy outcomes after assisted human reproduction. J Obstet Gynaecol Can 2014;36(1):64-83. PMID: 24444289.

Oldereid NB, Hanevik HI, Bakkevig I, et al. Pregnancy outcome according to male diagnosis after ICSI with non-ejaculated sperm compared with ejaculated sperm controls. Reprod Biomed Online 2014;29(4):417-23. PMID: 25131554. 
Oliveira JB, Cavagna M, Petersen CG, et al. Pregnancy outcomes in women with repeated implantation failures after intracytoplasmic morphologically selected sperm injection (IMSI). Reprod Biol Endocrinol 2011;9:99. PMID: 21781299.

O’Neill KE, Senapati S, Maina I, et al. GnRH agonist with low-dose hCG (dual trigger) is associated with higher risk of severe ovarian hyperstimulation syndrome compared to $\mathrm{GnRH}$ agonist alone. Journal of Assisted Reproduction and Genetics 2016;33(9):1175-1184.

Onalan G, Tohma YA and Zeyneloglu HB. Effect of Etanercept on the Success of Assisted Reproductive Technology in Patients with Endometrioma. Gynecol Obstet Invest 2017. PMID: 29208847.

Opoien HK, Fedorcsak P, Byholm T, et al. Complete surgical removal of minimal and mild endometriosis improves outcome of subsequent IVF/ICSI treatment. Reprod Biomed Online 2011;23(3):389-95. PMID: 21764382.

Opoien HK, Fedorcsak P, Omland AK, et al. In vitro fertilization is a successful treatment in endometriosis-associated infertility. Fertil Steril 2012;97(4):912-8. PMID: 22341637.

Oron G, Fisch B, Sapir O, et al. Pregnancy outcome after ICSI with thawed testicular sperm from men with non-obstructive azoospermia compared to ICSI with ejaculated sperm from men with severe oligoasthenoteratozoospermia and IVF with normal ejaculated sperm. Gynecol Endocrinol 2014;30(2):103-6. PMID: 24303883.

Oron G, Sokal-Arnon T, Son WY, et al. Extended embryo culture is not associated with increased adverse obstetric or perinatal outcome. Am J Obstet Gynecol 2014;211(2):165.e1-7. PMID: 24631436.

Osman A, Alsomait H, Seshadri S, et al. The effect of sperm DNA fragmentation on live birth rate after IVF or ICSI: a systematic review and meta-analysis. Reprod Biomed Online 2015;30(2):120-7. PMID: 25530036.

Ozgur K, Bulut H, Berkkanoglu M, et al. Artificial cryopreserved embryo transfer cycle success depends on blastocyst developmental rate and progesterone timing. Reproductive BioMedicine Online 2018;36(3):269-276.

Ozgur K, Bulut H, Berkkanoglu M, et al. ICSI pregnancy outcomes following hysteroscopic placement of Essure devices for hydrosalpinx in laparoscopic contraindicated patients. Reprod Biomed Online 2014;29(1):113-8. PMID: 24813749.

Ozmen B, Sukur YE, Seval MM, et al. Dual suppression with oral contraceptive pills in GnRH antagonist cycles for patients with polycystic ovary syndrome undergoing intracytoplasmic sperm injection. Eur J Obstet Gynecol Reprod Biol 2014;183:137-40. PMID: 25461367.

Palomba S, Falbo A and La Sala GB. Effects of metformin in women with polycystic ovary syndrome treated with gonadotrophins for in vitro fertilisation and intracytoplasmic sperm injection cycles: a systematic review and meta-analysis of randomised controlled trials. Bjog 2013;120(3):267-76. PMID: 23194199. 
Palomba S, Falbo A and La Sala GB. Metformin and gonadotropins for ovulation induction in patients with polycystic ovary syndrome: a systematic review with meta-analysis of randomized controlled trials. Reprod Biol Endocrinol 2014;12:3. PMID: 24387273.

Palomba S, Homburg R, Santagni S, et al. Risk of adverse pregnancy and perinatal outcomes after high technology infertility treatment: a comprehensive systematic review. Reprod Biol Endocrinol 2016;14(1):76. PMID: 27814762.

Palomba S, Pasquali R, Orio F, Jr., et al. Clomiphene citrate, metformin or both as first-step approach in treating anovulatory infertility in patients with polycystic ovary syndrome (PCOS): a systematic review of head-to-head randomized controlled studies and meta-analysis. Clin Endocrinol (Oxf) 2009;70(2):311-21. PMID: 18691273.

Palomba S, Santagni S, Gibbins K, et al. Pregnancy complications in spontaneous and assisted conceptions of women with infertility and subfertility factors. A comprehensive review. Reprod Biomed Online 2016;33(5):612-628. PMID: 27591135.

Panagiotopoulou N, Karavolos S and Choudhary M. Endometrial injury prior to assisted reproductive techniques for recurrent implantation failure: A systematic literature review. European Journal of Obstetrics Gynecology and Reproductive Biology 2015;193:27-33.

Pandian Z, Akande VA, Harrild K, et al. Surgery for tubal infertility. Cochrane Database Syst Rev 2008;(3):Cd006415. PMID: 18646152.

Pandian Z, Gibreel A and Bhattacharya S. In vitro fertilisation for unexplained subfertility. Cochrane Database Syst Rev 2012;4:Cd003357. PMID: 22513911.

Pandian Z, Gibreel A and Bhattacharya S. In vitro fertilisation for unexplained subfertility. Cochrane Database Syst Rev 2015;11:Cd003357. PMID: 26583517.

Pandian Z, McTavish AR, Aucott L, et al. Interventions for 'poor responders' to controlled ovarian hyper stimulation (COH) in in-vitro fertilisation (IVF). Cochrane Database Syst Rev. 2010(1):Cd004379. PMID: 20091563.

Park H, Kim CH, Kim EY, et al. Effect of second-line surgery on in vitro fertilization outcome in infertile women with ovarian endometrioma recurrence after primary conservative surgery for moderate to severe endometriosis. Obstet Gynecol Sci 2015;58(6):481-6. PMID: 26623412.

Parsanezhad ME, Zarei A, Sayadi M, et al. Surgical ovulation induction in women with polycystic ovary syndrome: A systematic review. Iranian Journal of Medical Sciences 2010;35(2):225-241.

Peng J, Zhang Z, Yuan Y, et al. Pregnancy and live birth rates after microsurgical vasoepididymostomy for azoospermic patients with epididymal obstruction. Hum Reprod 2017;32(2):284-289. PMID: 28057874. 
Pereira N, Friedman C, Hutchinson AP, et al. Increased odds of live birth in fresh in vitro fertilization cycles with shorter ovarian stimulation. Fertil Steril 2017;107(1):104-109.e2. PMID: 27793370.

Pereira N, O'Neill C, Lu V, et al. The safety of intracytoplasmic sperm injection and long-term outcomes. Reproduction 2017;154(6):F61-f70. PMID: 28710292.

Pinheiro LMA, Cândido PS, Moreto TC, et al. Estradiol use in the luteal phase and its effects on pregnancy rates in IVF cycles with GnRH antagonist: A systematic review. Jornal Brasileiro de Reproducao Assistida 2017;21(3):247-250.

Polyzos NP, Tsappi M, Mauri D, et al. Aromatase inhibitors for infertility in polycystic ovary syndrome. The beginning or the end of a new era? Fertil Steril 2008;89(2):278-80. PMID: 18275882.

Polyzos NP, Tzioras S, Mauri D, et al. Treatment of unexplained infertility with aromatase inhibitors or clomiphene citrate: a systematic review and meta-analysis. Obstet Gynecol Surv 2008;63(7):472-9. PMID: 18559123.

Pouwer AW, Farquhar C, Kremer JAM, et al. Long-acting follicle-stimulating hormone versus daily follicle-stimulating hormone for women undergoing assisted reproduction. Fertility and Sterility 2016;105(6):1454-1456.

Pundir J, Psaroudakis D, Savnur P, et al. Inositol treatment of anovulation in women with polycystic ovary syndrome: a meta-analysis of randomised trials. Bjog 2018;125(3):299-308. PMID: 28544572.

Qin J, Liu X, Sheng X, et al. Assisted reproductive technology and the risk of pregnancy-related complications and adverse pregnancy outcomes in singleton pregnancies: a meta-analysis of cohort studies. Fertil Steril 2016;105(1):73-85.e1-6. PMID: 26453266.

Qin J, Sheng X, Wang H, et al. Assisted reproductive technology and risk of congenital malformations: a meta-analysis based on cohort studies. Archives of Gynecology and Obstetrics 2015;292(4):777-798.

Rao M, Zeng Z and Tang L. Maternal physical activity before IVF/ICSI cycles improves clinical pregnancy rate and live birth rate: a systematic review and meta-analysis. Reprod Biol Endocrinol 2018;16(1):11. PMID: 29415732.

Ratts VS, Pauls RN, Pinto AB, et al. Risk of multiple gestation after ovulation induction in polycystic ovary syndrome. J Reprod Med 2007;52(10):896-900. PMID: 17977162.

Reavey J, Vincent K, Child T, et al. Human chorionic gonadotrophin priming for fertility treatment with in vitro maturation. Cochrane Database Syst Rev 2016;11:Cd008720. PMID: 27852101. 
Reichman DE, Chung P, Meyer L, et al. Consecutive gonadotropin-releasing hormone-antagonist in vitro fertilization cycles: does the elapsed time interval between successive treatments affect outcomes? Fertil Steril 2013;99(5):1277-82. PMID: 23290740.

Reifsnyder JE, Ramasamy R, Husseini J, et al. Role of optimizing testosterone before microdissection testicular sperm extraction in men with nonobstructive azoospermia. J Urol 2012;188(2):532-6. PMID: 22704105.

Ren J, Sha A, Han D, et al. Does prolonged pituitary down-regulation with gonadotropinreleasing hormone agonist improve the live-birth rate in in vitro fertilization treatment? Fertil Steril 2014;102(1):75-81. PMID: 24746740.

Roesner S, Dietrich JE, Weigert J, et al. Time-lapse imaging reveals differences in growth dynamics of embryos after in vitro maturation compared with conventional stimulation. Fertil Steril 2017;107(3):606-612.e3. PMID: 28139236.

Rolland L, Perrin J, Villes V, et al. IVF oocyte retrieval: prospective evaluation of the type of anesthesia on live birth rate, pain, and patient satisfaction. Journal of Assisted Reproduction and Genetics 2017;34(11):1523-1528.

Roustan A, Perrin J, Debals-Gonthier M, et al. Surgical diminished ovarian reserve after endometrioma cystectomy versus idiopathic DOR: comparison of in vitro fertilization outcome. Hum Reprod 2015;30(4):840-7. PMID: 25740883.

Rossi AC and Prefumo F. The effects of surgery for endometriosis on pregnancy outcomes following in vitro fertilization and embryo transfer: a systematic review and meta-analysis. Archives of Gynecology and Obstetrics 2016;294(3):647-655.

Roux P, Perrin J, Mancini J, et al. Factors associated with a poor prognosis for the IVF-ICSI live birth rate in women with rAFS stage III and IV endometriosis. J Assist Reprod Genet 2017;34(7):921-928. PMID: 28523409.

Rozdarz KM, Flatley CJ and Kumar S. Intrapartum and neonatal outcomes in singleton pregnancies following conception by assisted reproduction techniques. Australian and New Zealand Journal of Obstetrics and Gynaecology 2017;57(6):588-592.

Rumbold AR, Moore VM, Whitrow MJ, et al. The impact of specific fertility treatments on cognitive development in childhood and adolescence: a systematic review. Hum Reprod 2017;32(7):1489-1507. PMID: 28472417.

Sacha CR, Dimitriadis I, Christou G, et al. The effect of day 2 versus day 3 embryo transfer on early pregnancy outcomes in women with a low yield of fertilized oocytes. Journal of Assisted Reproduction and Genetics 2018:1-6.

Salamun V, Verdenik I, Lagana AS, et al. Should we consider integrated approach for endometriosis-associated infertility as gold standard management? Rationale and results from a large cohort analysis. Arch Gynecol Obstet 2018;297(3):613-621. PMID: 29274003. 
Salem WH, Abdullah A, Abuzeid O, et al. Decreased live births among women of Middle Eastern/North African ethnicity compared to Caucasian women. J Assist Reprod Genet 2017;34(5):581-586. PMID: 28337714.

Sallem A, Santulli P, Barraud-Lange V, et al. Extended culture of poor-quality supernumerary embryos improves ART outcomes. Journal of Assisted Reproduction and Genetics 2018;35(2):311-319.

Samplaski MK, Lo KC, Grober ED, et al. Varicocelectomy to "upgrade" semen quality to allow couples to use less invasive forms of assisted reproductive technology. Fertil Steril 2017;108(4):609-612. PMID: 28911932.

Sampo AV, Palena C, Ganzer L, et al. The adverse effect of overweight in assisted reproduction treatment outcomes. Jornal Brasileiro de Reproducao Assistida 2017;21(3):212-216.

Santos-Ribeiro S, Tournaye H and Polyzos NP. Trends in ectopic pregnancy rates following assisted reproductive technologies in the UK: a 12-year nationwide analysis including 160000 pregnancies. Hum Reprod 2016;31(2):393-402. PMID: 26724796.

Sarais V, Pagliardini L, Rebonato G, et al. A comprehensive analysis of body mass index effect on in vitro fertilization outcomes. Nutrients 2016;8(3).

Sarhan A, Beydoun H, Jones HW, Jr., et al. Gonadotrophin ovulation induction and enhancement outcomes: analysis of more than 1400 cycles. Reprod Biomed Online 2011;23(2):220-6. PMID: 21665547.

Schachter-Safrai N, Karavani G, Levitas E, et al. Does cryopreservation of sperm affect fertilization in nonobstructive azoospermia or cryptozoospermia?. Fertil Steril 2017;107(5):1148-1152. PMID: 28392002.

Schippert C, Soergel P, Staboulidou I, et al. The risk of ectopic pregnancy following tubal reconstructive microsurgery and assisted reproductive technology procedures. Arch Gynecol Obstet 2012;285(3):863-71. PMID: 21947340.

Scholten I, van Zijl M, Custers IM, et al. The effectiveness of intrauterine insemination: A matched cohort study. Eur J Obstet Gynecol Reprod Biol 2017;212:91-95. PMID: 28349891.

Schwarze JE, Valdebenito P, Ortega C, et al. Do women offered assisted reproduction technologies have a higher incidence of gynecologic cancer? A systematic review and metaanalysis. JBRA Assist Reprod 2017;21(2):115-119. PMID: 28609278.

Selçuk S, Küçükbaş M, Yenidede İ, et al. The outcomes of controlled ovarian hyperstimulation/ intrauterine insemination in patients with unilateral tubal occlusion on hysterosalpingograph. Turk Jinekoloji ve Obstetrik Dernegi Dergisi 2016;13(1):7-10.

Selvaraj P, Selvaraj K, Kalaichelvi S, et al. Semen preparation techniques in intrauterine insemination: A comparison of non-temperature and temperature controlled centrifugation in cases of unexplained infertility. J Hum Reprod Sci 2013;6(4):241-4. PMID: 24672162. 
Shah JS, Nasab SH, Chappell N, et al. Neonatal outcomes among twins stratified by method of conception: secondary analysis of maternal fetal medicine (MFMU) network database. Journal of Assisted Reproduction and Genetics 2018:1-7.

Shalom-Paz E, Holzer H, Son W, et al. PCOS patients can benefit from in vitro maturation (IVM) of oocytes. Eur J Obstet Gynecol Reprod Biol 2012;165(1):53-6. PMID: 22819571.

Shanbhag S, Aucott L, Bhattacharya S, et al. Interventions for 'poor responders' to controlled ovarian hyperstimulation (COH) in in-vitro fertilisation (IVF). Cochrane Database Syst Rev 2007(1):Cd004379. PMID: 17253503.

Shavit T, Ellenbogen A, Michaeli M, et al. In-vitro maturation of oocytes vs in-vitro fertilization with a gonadotropin-releasing hormone antagonist for women with polycystic ovarian syndrome: can superiority be defined? Eur J Obstet Gynecol Reprod Biol 2014;179:46-50. PMID: 24965979.

Shavit T, Shalom-Paz E, Samara N, et al. Comparison between stimulation with highly purified hMG or recombinant FSH in patients undergoing IVF with GnRH antagonist protocol. Gynecol Endocrinol 2016:1-5. PMID: 26939574.

Shebl O, Sifferlinger I, Habelsberger A, et al. Oocyte competence in in vitro fertilization and intracytoplasmic sperm injection patients suffering from endometriosis and its possible association with subsequent treatment outcome: a matched case-control study. Acta Obstet Gynecol Scand 2017;96(6):736-744. PMID: 27317364.

Shiotani M, Matsumoto Y, Okamoto E, et al. Is human chorionic gonadotropin supplementation beneficial for frozen and thawed embryo transfer in estrogen/progesterone replacement cycles?: A randomized clinical trial. 2017;16(2):166-169.

Shirlow R, Healey M, Volovsky M, et al. The effects of adjuvant therapies on embryo transfer success. Journal of Reproduction and Infertility 2017;18(4):368-378.

Showell MG, Brown J, Yazdani A, et al. Antioxidants for male subfertility. Cochrane Database Syst Rev 2011;(1):Cd007411. PMID: 21249690.

Showell MG, Mackenzie-Proctor R, Brown J, et al. Antioxidants for male subfertility. Cochrane Database Syst Rev 2014;12:Cd007411. PMID: 25504418.

Siebert TI, Viola MI, Steyn DW, et al. Is metformin indicated as primary ovulation induction agent in women with PCOS? A systematic review and meta-analysis. Gynecol Obstet Invest 2012;73(4):304-13. PMID: 22516925.

Sifer C, Herbemont C, Adda-Herzog E, et al. Clinical predictive criteria associated with live birth following elective single embryo transfer. Eur J Obstet Gynecol Reprod Biol 2014;181:229-32. PMID: 25171268.

Singh N, Naha M, Malhotra N, et al. Comparison of gonadotropin-releasing hormone agonist with GnRH antagonist in polycystic ovary syndrome patients undergoing in vitro fertilization 
cycle: Retrospective analysis from a tertiary center and review of literature. J Hum Reprod Sci 2014;7(1):52-7. PMID: 24829532.

Siristatidis CS, Gibreel A, Basios G, et al. Gonadotrophin-releasing hormone agonist protocols for pituitary suppression in assisted reproduction. Cochrane Database Syst Rev 2015;11:Cd006919. PMID: 26558801.

Siristatidis C, Sergentanis TN, Vogiatzi P, et al. In Vitro Maturation in Women with vs. without Polycystic Ovarian Syndrome: A Systematic Review and Meta-Analysis. PLoS One 2015;10(8):e0134696. PMID: 26241855.

Skalkidou A, Sergentanis TN, Gialamas SP, et al. Risk of endometrial cancer in women treated with ovary-stimulating drugs for subfertility. Cochrane Database Syst Rev 2017;3:Cd010931. PMID: 28349511.

Smith NK, Madeira J and Millard HR. Sexual function and fertility quality of life in women using in vitro fertilization. J Sex Med 2015;12(4):985-93. PMID: 25639162.

Stern JE, Luke B, Hornstein MD, et al. The effect of father's age in fertile, subfertile, and assisted reproductive technology pregnancies: a population based cohort study. J Assist Reprod Genet 2014;31(11):1437-44. PMID: 25193289.

Sun X, Zhang D and Zhang W. Effect of metformin on ovulation and reproductive outcomes in women with polycystic ovary syndrome: a meta-analysis of randomized controlled trials. Arch Gynecol Obstet 2013;288(2):423-30. PMID: 23430028.

Sundhararaj U, Madne M, Biliangady R, et al. Single blastocyst transfer: The key to reduce multiple pregnancy rates without compromising the live birth rate. Journal of Human Reproductive Sciences 2017;10(3):201-207.

Sunkara SK, Antonisamy B, Selliah HY, et al. Perinatal outcomes after gestational surrogacy versus autologous IVF: analysis of national data. Reproductive BioMedicine Online 2017;35(6):708-714.

Swanton A, Storey L, McVeigh E, et al. IVF outcome in women with PCOS, PCO and normal ovarian morphology. Eur J Obstet Gynecol Reprod Biol 2010;149(1):68-71. PMID: 20022685.

Tabbalat AM, Pereira N, Klauck D, et al. Arabian Peninsula ethnicity is associated with lower ovarian reserve and ovarian response in women undergoing fresh ICSI cycles. J Assist Reprod Genet 2018;35(2):331-337. PMID: 29063502.

Takashima A, Takeshita N and Kinoshita T. Pregnancy outcomes after assisted reproductive procedures with embryos that had been derived from affected and unaffected ovaries among women with small unilateral endometriomas. Reprod Med Biol 2017;16(2):152-156. PMID: 29259463. 
Takemura Y, Osuga Y, Fujimoto A, et al. Increased risk of placenta previa is associated with endometriosis and tubal factor infertility in assisted reproductive technology pregnancy. Gynecol Endocrinol 2013;29(2):113-5. PMID: 22835092.

Tan J, Moriarty S, Taskin O, et al. Reproductive Outcomes after Fertility-Sparing Surgery for Focal and Diffuse Adenomyosis: a Systematic Review. J Minim Invasive Gynecol 2018. PMID: 29305234.

Tan TY, Lau SK, Loh SF, et al. Female ageing and reproductive outcome in assisted reproduction cycles. Singapore Med J 2014;55(6):305-9. PMID: 25017405.

Tang T, Lord JM, Norman RJ, et al. Insulin-sensitising drugs (metformin, rosiglitazone, pioglitazone, D-chiro-inositol) for women with polycystic ovary syndrome, oligo amenorrhoea and subfertility. Cochrane Database Syst Rev 2009;(4):Cd003053. PMID: 19821299.

Tang T, Lord JM, Norman RJ, et al. Insulin-sensitising drugs (metformin, rosiglitazone, pioglitazone, D-chiro-inositol) for women with polycystic ovary syndrome, oligo amenorrhoea and subfertility. Cochrane Database Syst Rev 2010;(1):Cd003053. PMID: 20091537.

Tang T, Lord JM, Norman RJ, et al. Insulin-sensitising drugs (metformin, rosiglitazone, pioglitazone, D-chiro-inositol) for women with polycystic ovary syndrome, oligo amenorrhoea and subfertility. Cochrane Database Syst Rev 2012;5:Cd003053. PMID: 22592687.

Tannus S, Hatirnaz S, Tan J, et al. Predictive factors for live birth after in vitro maturation of oocytes in women with polycystic ovary syndrome. Arch Gynecol Obstet 2018;297(1):199-204. PMID: 28993961.

Tannus S, Son WY, Gilman A, et al. The role of intracytoplasmic sperm injection in non-male factor infertility in advanced maternal age. Hum Reprod 2016. PMID: 27852688.

Tannus S, Tan J, Son WY, et al. Prevalence, clinical characteristics, and reproductive outcomes of polycystic ovary syndrome in older women referred for tertiary fertility care. Arch Gynecol Obstet 2018;297(4):1037-1042. PMID: 29289990.

Tannus S, Turki R, Cohen Y, et al. Reproductive outcomes after a single dose of gonadotropinreleasing hormone agonist compared with human chorionic gonadotropin for the induction of final oocyte maturation in hyper-responder women aged 35-40 years. Fertil Steril 2017;107(6):1323-1328.e2. PMID: 28501366.

Tao X, Chen L, Ge S, et al. Weigh the pros and cons to ovarian reserve before stripping ovarian endometriomas prior to IVF/ICSI: A meta-analysis. PLoS One 2017;12(6):e0177426. PMID: 28574993.

Tarasconi B, Tadros T, Ayoubi JM, et al. Serum antimullerian hormone levels are independently related to miscarriage rates after in vitro fertilization-embryo transfer. Fertil Steril 2017;108(3):518-524. PMID: 28865551. 
Tatsumi T, Jwa SC, Kuwahara A, et al. Pregnancy and neonatal outcomes following letrozole use in frozen-thawed single embryo transfer cycles. Hum Reprod 2017;32(6):1244-1248. PMID: 28398491.

Teixeira DM, Barbosa MA, Ferriani RA, et al. Regular (ICSI) versus ultra-high magnification (IMSI) sperm selection for assisted reproduction. Cochrane Database Syst Rev 2013;7:Cd010167. PMID: 23884963.

Thomopoulos C, Salamalekis G, Kintis K, et al. Risk of hypertensive disorders in pregnancy following assisted reproductive technology: overview and meta-analysis. J Clin Hypertens (Greenwich) 2017;19(2):173-183. PMID: 28071857.

Thomopoulos C, Tsioufis C, Michalopoulou H, et al. Assisted reproductive technology and pregnancy-related hypertensive complications: a systematic review. J Hum Hypertens 2013;27(3):148-57. PMID: 22495105.

Tiseo BC, Esteves SC and Cocuzza MS. Summary evidence on the effects of varicocele treatment to improve natural fertility in subfertile men. Asian J Androl 2016;18(2):239-45. PMID: 26806080.

Toshimitsu M, Nagamatsu T, Nagasaka T, et al. Increased risk of pregnancy-induced hypertension and operative delivery after conception induced by in vitro fertilization/intracytoplasmic sperm injection in women aged 40 years and older. Fertil Steril 2014;102(4):1065-1070.e1. PMID: 25123639.

Tranoulis A, Laios A, Pampanos A, et al. Efficacy and safety of pulsatile gonadotropin-releasing hormone therapy among patients with idiopathic and functional hypothalamic amenorrhea: a systematic review of the literature and a meta-analysis. Fertil Steril 2018. PMID: 29605411.

Tsiami A, Chaimani A, Mavridis D, et al. Surgical treatment for hydrosalpinx prior to in-vitro fertilization embryo transfer: a network meta-analysis. Ultrasound Obstet Gynecol 2016;48(4):434-445. PMID: 26922863.

Tso LO, Costello MF, Albuquerque LE, et al. Metformin treatment before and during IVF or ICSI in women with polycystic ovary syndrome. Cochrane Database Syst Rev 2009;(2):Cd006105. PMID: 19370625.

Tso LO, Costello MF, Albuquerque LE, et al. Metformin treatment before and during IVF or ICSI in women with polycystic ovary syndrome. Cochrane Database Syst Rev 2014;11:Cd006105. PMID: 25406011.

Tu J, Lin G, Lu C, et al. A novel modified ultra-long agonist protocol improves the outcome of high body mass index women with polycystic ovary syndrome undergoing IVF/ICSI. Gynecol Endocrinol 2014;30(3):209-12. PMID: 24351071.

Tuncay G, Karaer A, Inci Coskun E, et al. The impact of thyroid-stimulating hormone levels in euthyroid women on intrauterine insemination outcome. BMC Womens Health 2018;18(1):51. PMID: 29558997. 
Turhan N, Pekel A, Ayrim A, et al. Effectiveness of HP-hMG versus r-FSH in patients undergoing IVF/ICSI cycles with moderate male-factor infertility. Turkish Journal of Medical Sciences 2013;43(1):144-149.

Turkgeldi E, Yagmur H, Seyhan A, et al. Short and long term outcomes of children conceived with assisted reproductive technology. Eur J Obstet Gynecol Reprod Biol 2016;207:129-136. PMID: 27846448.

Ubaldi FM, Cimadomo D, Capalbo A, et al. Preimplantation genetic diagnosis for aneuploidy testing in women older than 44 years: a multicenter experience. Fertil Steril 2017;107(5):11731180. PMID: 28433373.

Ulkumen B, Silfeler D, Sofuoglu K, et al. The incidence of preeclampsia in ICSI pregnancies. Pak J Med Sci 2014;30(1):101-5. PMID: 24639840.

Unuane D, Velkeniers B, Bravenboer B, et al. Impact of thyroid autoimmunity in euthyroid women on live birth rate after IUI. Hum Reprod 2017;32(4):915-922. PMID: 28333271.

Vaegter KK, Lakic TG, Olovsson M, et al. Which factors are most predictive for live birth after in vitro fertilization and intracytoplasmic sperm injection (IVF/ICSI) treatments? Analysis of 100 prospectively recorded variables in 8,400 IVF/ICSI single-embryo transfers. Fertil Steril 2017;107(3):641-648.e2. PMID: 28108009.

van Kessel M, Tros R, Oosterhuis J, et al. The prognostic capacity of transvaginal hydrolaparoscopy to predict non-IVF conception. Reprod Biomed Online 2018. PMID: 29456102.

van Seeters JAH, Chua SJ, Mol BWJ, et al. Tubal anastomosis after previous sterilization: a systematic review. Hum Reprod Update 2017;23(3):358-370. PMID: 28333337.

van Wely M, Barbey N, Meissner A, et al. Live birth rates after MESA or TESE in men with obstructive azoospermia: is there a difference? Hum Reprod 2015;30(4):761-6. PMID: 25740877.

Vaughan DA, Leung A, Resetkova N, et al. How many oocytes are optimal to achieve multiple live births with one stimulation cycle? The one-and-done approach. Fertil Steril 2017;107(2):397-404.e3. PMID: 27916206.

Veltman-Verhulst SM, Cohlen BJ, Hughes E, et al. Intra-uterine insemination for unexplained subfertility. Cochrane Database Syst Rev 2012;9:Cd001838. PMID: 22972053.

Veltman-Verhulst SM, Fauser BC and Eijkemans MJ. High singleton live birth rate confirmed after ovulation induction in women with anovulatory polycystic ovary syndrome: validation of a prediction model for clinical practice. Fertil Steril 2012;98(3):761-768.e1. PMID: 22633255.

Veltman-Verhulst SM, Hughes E, Ayeleke RO, et al. Intra-uterine insemination for unexplained subfertility. Cochrane Database Syst Rev 2016;2:Cd001838. PMID: 26892070. 
Vitagliano A, Noventa M, Saccone G, et al. Endometrial scratch injury before intrauterine insemination: is it time to re-evaluate its value? Evidence from a systematic review and metaanalysis of randomized controlled trials. Fertil Steril 2018;109(1):84-96.e4. PMID: 29175068.

Vloeberghs V, Verheyen G, Haentjens P, et al. How successful is TESE-ICSI in couples with non-obstructive azoospermia? Hum Reprod 2015;30(8):1790-6. PMID: 26082482.

Wahd SA, Alalaf SK, Al-Shawaf T, et al. Ovarian reserve markers and assisted reproductive technique (ART) outcomes in women with advanced endometriosis. Reprod Biol Endocrinol 2014;12:120. PMID: 25442239.

Wang N, Wang Y, Chen Q, et al. Luteal-phase ovarian stimulation vs conventional ovarian stimulation in patients with normal ovarian reserve treated for IVF: a large retrospective cohort study. Clin Endocrinol (Oxf) 2016;84(5):720-8. PMID: 26603821.

Wang R, Kim BV, van Wely M, et al. Treatment strategies for women with WHO group II anovulation: systematic review and network meta-analysis. Bmj 2017;356:j138. PMID: 28143834.

Wang X, Hao J, Zhang F, et al. Effects of female and male body mass indices on the treatment outcomes and neonatal birth weights associated with in vitro fertilization/intracytoplasmic sperm injection treatment in China. Fertility and Sterility 2016;106(2):460-466.

Wang YA, Healy D, Black D, et al. Age-specific success rate for women undertaking their first assisted reproduction technology treatment using their own oocytes in Australia, 2002-2005. Hum Reprod 2008;23(7):1633-8. PMID: 18441345.

Wei Z, Cheng X, Li H, et al. Effects of prolonging administration gonadotropin on unexpectedly poor ovarian responders undergoing in vitro fertilization. Reprod Biol Endocrinol 2010;8:26. PMID: 20236519.

Weiss Nienke S, Nahuis M, Bayram N, et al. Gonadotrophins for ovulation induction in women with polycystic ovarian syndrome. 2015;(9):CD010290.

Weiss NS, Braam S, Konig TE, et al. How long should we continue clomiphene citrate in anovulatory women? Hum Reprod 2014;29(11):2482-6. PMID: 25164024.

Wilkinson J, Roberts SA, Showell M, et al. No common denominator: a review of outcome measures in IVF RCTs. Hum Reprod 2016;31(12):2714-2722. PMID: 27664214.

Woldringh GH, Besselink DE, Tillema AH, et al. Karyotyping, congenital anomalies and followup of children after intracytoplasmic sperm injection with non-ejaculated sperm: a systematic review. Hum Reprod Update 2010;16(1):12-9. PMID: 19700489.

$\mathrm{Wu} \mathrm{CH}$, Lee $\mathrm{TH}$, Chen $\mathrm{HH}$, et al. The influence of female age on the cumulative live-birth rate of fresh cycles and subsequent frozen cycles using vitrified blastocysts in hyper-responders. Taiwan J Obstet Gynecol 2015;54(5):567-71. PMID: 26522112. 
Xing W, Lin H, Li Y, et al. GnRH antagonist protocol is it an optimal protocol for policytic ovary syndrome (PCOS) patients undergoing in vitro fertilisation (IVF)/intracytoplasmic sperm injection (ICSI)?. Medical Science Technology 2017;58(1):10-14.

Xiong F, Hu L, Zhang Y, et al. Correlation of hypertensive disorders in pregnancy with procedures of in vitro fertilization and pregnancy outcomes. Experimental and Therapeutic Medicine 2017;14(6):5405-5410.

Xu B, Zhang Q, Zhao J, et al. Pregnancy outcome of in vitro fertilization after Essure and laparoscopic management of hydrosalpinx: a systematic review and meta-analysis. Fertil Steril 2017;108(1):84-95.e5. PMID: 28579408.

$\mathrm{Xu} \mathrm{Y,} \mathrm{Li} \mathrm{J,} \mathrm{Zhou} \mathrm{G,} \mathrm{et} \mathrm{al.} \mathrm{Clinical} \mathrm{outcomes} \mathrm{for} \mathrm{various} \mathrm{causes} \mathrm{of} \mathrm{infertility} \mathrm{with} \mathrm{natural-cycle} \mathrm{in}$ vitro fertilization combined with in vitro maturation of immature oocytes. Fertil Steril 2010;94(2):777-80. PMID: 19909949.

Xue T, Li SW and Wang Y. Effectiveness of bromocriptine monotherapy or combination treatment with clomiphene for infertility in women with galactorrhea and normal prolactin: A systematic review and meta-analysis. Curr Ther Res Clin Exp 2010;71(4):199-210. PMID: 24688144.

Yang S, Peng HY, Li Y, et al. Intrauterine Insemination Treatment Strategy for Women over 35 Years Old: Based on a Large Sample Multi-center Retrospective Analysis. Chin Med J (Engl) 2016;129(23):2873-2875. PMID: 27901004.

Yarali H, Polat M, Mumusoglu S, et al. Preparation of endometrium for frozen embryo replacement cycles: a systematic review and meta-analysis. Journal of Assisted Reproduction and Genetics 2016;33(10):1287-1304.

Yerebasmaz N, Demir B, Kahyaoglu I, et al. Does male factor infertility affect intracytoplasmic sperm injection pregnancy results?. Crescent Journal of Medical and Biological Sciences 2017;4(2):85-89.

Youssef MA and Mourad S. Volume expanders for the prevention of ovarian hyperstimulation syndrome. Cochrane Database Syst Rev 2016(8):Cd001302. PMID: 27577848.

Youssef MA, Abou-Setta AM and Lam WS. Recombinant versus urinary human chorionic gonadotrophin for final oocyte maturation triggering in IVF and ICSI cycles. Cochrane Database Syst Rev 2016;4:Cd003719. PMID: 27106604.

Youssef MA, van Wely M, Mochtar M, et al. Low dosing of gonadotropins in in vitro fertilization cycles for women with poor ovarian reserve: systematic review and meta-analysis. Fertil Steril 2018;109(2):289-301. PMID: 29317127.

Youssef MM, Mantikou E, van Wely M, et al. Culture media for human pre-implantation embryos in assisted reproductive technology cycles. Cochrane Database Syst Rev 2015;11:Cd007876. PMID: 26585317. 
Zakhari A, Ates S, Shaulov T, et al. Does ovarian reserve affect outcomes in single ideal blastocyst transfers in women less than 40 years of age?. Arch Gynecol Obstet 2018;297(1):233239. PMID: 29082421.

Zhang J, Wang Y, Mao X, et al. Dual trigger of final oocyte maturation in poor ovarian responders undergoing IVF/ICSI cycles. Reproductive BioMedicine Online 2017;35(6):701-707.

Zhang JJ, Yang M and Merhi Z. Reproductive potential of mature oocytes after conventional ovarian hyperstimulation for in vitro fertilization. International Journal of Gynecology and Obstetrics 2016;133(2):230-233.

Zhang X, Ma C, Wu Z, et al. Frozen-Thawed Embryo Transfer Cycles Have a Lower Incidence of Ectopic Pregnancy Compared With Fresh Embryo Transfer Cycles. Reprod Sci 2017:1933719117746759. PMID: 29254433.

Zhang Z, Su D, Zhu P, et al. Effect of different luteal support schemes on clinical outcome in frozen-Thawed embryos transfer cycles. Clinical and Experimental Obstetrics and Gynecology 2016;43(4):486-489.

Zhao JZ, Lin JJ, Yang HY, et al. Effects of oral contraceptives and metformin on the outcome of in vitro maturation in infertile women with polycystic ovary syndrome. J Womens Health (Larchmt) 2010;19(2):261-5. PMID: 20113146.

Zhao W, Hao J, Wang S, et al. Clinical outcomes comparison of single fresh and frozen-thawed superior blastocyst transfer. International Journal of Clinical and Experimental Medicine 2017;10(8):12605-12608.

Zheng YM, Li L, Zhou LM, et al. Alterations in the frequency of trinucleotide repeat dynamic mutations in offspring conceived through assisted reproductive technology. Hum Reprod 2013;28(9):2570-80. PMID: 23861482.

Zhou K, Zhang J, Xu L, et al. Chinese herbal medicine for subfertile women with polycystic ovarian syndrome. Cochrane Database Syst Rev 2016;10:Cd007535. PMID: 27731904.

Zhu J, Lin S, Li M, et al. Effect of in vitro culture period on birthweight of singleton newborns. Hum Reprod 2014;29(3):448-54. PMID: 24408317.

Zorn B, Virant-Klun I, Drobni S, et al. Male and female factors that influence ICSI outcome in azoospermia or aspermia. Reprod Biomed Online 2009;18(2):168-76. PMID: 19192334. 


\section{Appendix E. Characteristics of Included Studies}

Table E-1 shows the study characteristics for the included studies. For full study citations, please refer to the report's main reference list.

\section{Table E-1. Characteristics of included studies}

\begin{tabular}{|c|c|c|c|c|c|}
\hline $\begin{array}{c}\text { Study } \\
\text { ACRONYM } \\
\text { KQs } \\
\text { Companion Studies }\end{array}$ & $\begin{array}{l}\text { Study Design } \\
\text { Geographic } \\
\text { Location }\end{array}$ & $\begin{array}{l}\text { N Enrolled } \\
\text { N Completed } \\
\text { Underlying } \\
\text { Diagnosis }\end{array}$ & Interventions & $\begin{array}{c}\text { Outcomes } \\
\text { (Subgroups analyzed) }\end{array}$ & Quality \\
\hline $\begin{array}{l}\text { Abdellah, } 2011^{156} \\
\text { KQ } 1\end{array}$ & $\begin{array}{l}\mathrm{RCT} \\
\text { Africa }\end{array}$ & $\begin{array}{l}147 \\
140 \\
\text { PCOS }\end{array}$ & $\begin{array}{l}\text { Letrozole } 5 \mathrm{mg} / \mathrm{d} \text { for } 5 \text { days, maximum treatment } \\
\text { duration } 6 \text { consecutive cycles. } \\
\text { vs. } \\
\text { Laparoscopic ovarian drilling (LOD) }\end{array}$ & $\begin{array}{l}\text { Live birth } \\
\text { Miscarriage } \\
\text { Multiple births }\end{array}$ & Good \\
\hline $\begin{array}{l}\text { Aboulghar, } 2010^{162} \\
\text { KQ } 1\end{array}$ & $\begin{array}{l}\mathrm{RCT} \\
\text { Africa }\end{array}$ & $\begin{array}{l}84 \\
\text { NR } \\
\text { PCOS }\end{array}$ & $\begin{array}{l}\text { Routine IVF/ICSI using highly purified uFSH } \\
\text { (Fostimon) } \\
\text { vs. } \\
\text { rFSH (Gonal F) }\end{array}$ & OHSS & Good \\
\hline $\begin{array}{l}\text { Abu Hashim, } 2010^{168} \\
\text { KQ } 1\end{array}$ & $\begin{array}{l}\mathrm{RCT} \\
\text { Africa }\end{array}$ & $\begin{array}{l}260 \\
260 \\
\text { PCOS }\end{array}$ & $\begin{array}{l}\text { Letrozole, } 2.5 \mathrm{mg} / \mathrm{d} \text { for } 5 \text { days, maximum } \\
\text { treatment duration } 6 \text { cycles } \\
\text { vs. } \\
\text { Laparoscopic ovarian diathermy (LOD) }\end{array}$ & $\begin{array}{l}\text { Live birth } \\
\text { Miscarriage } \\
\text { Multiple births } \\
\text { OHSS }\end{array}$ & Good \\
\hline $\begin{array}{l}\text { Abu Hashim, } 2011^{154} \\
\text { KQ } 1\end{array}$ & $\begin{array}{l}\mathrm{RCT} \\
\text { Africa }\end{array}$ & $\begin{array}{l}176 \\
165 \\
\text { PCOS }\end{array}$ & $\begin{array}{l}\text { Laparoscopic ovarian diathermy (LOD) } \\
\text { performed at least } 8 \text { weeks after the last CC } \\
\text { dosage } \\
\text { vs. } \\
\text { CC } 50-150 \mathrm{mg} / \mathrm{d} \text { for } 5 \text { days, maximum treatment } \\
\text { duration } 6 \text { cycles }\end{array}$ & $\begin{array}{l}\text { Live birth } \\
\text { Miscarriage } \\
\text { OHSS }\end{array}$ & Fair \\
\hline $\begin{array}{l}\text { Abu Hashim, } 2011^{157} \\
\text { KQ } 1\end{array}$ & $\begin{array}{l}\text { RCT } \\
\text { Middle East }\end{array}$ & $\begin{array}{l}188 \\
188 \\
\text { PCOS }\end{array}$ & $\begin{array}{l}\text { CC } 50 \text { - } 150 \mathrm{mg} / \mathrm{d} \text {, maximum treatment duration } \\
3 \text { cycles followed by IUI } \\
\text { vs. } \\
\text { Timed intercourse }\end{array}$ & $\begin{array}{l}\text { Live birth } \\
\text { Ectopic pregnancy } \\
\text { Miscarriage } \\
\text { Multiple births } \\
\text { OHSS }\end{array}$ & Good \\
\hline $\begin{array}{l}\text { Abu Hashim, } 2012^{204} \\
\text { KQ } 2\end{array}$ & $\begin{array}{l}\mathrm{RCT} \\
\text { Africa }\end{array}$ & $\begin{array}{l}136 \\
125 \\
\text { Endometriosis }\end{array}$ & $\begin{array}{l}\text { IUI following hCG injection using Letrozole } 5 \\
\mathrm{mg} / \mathrm{d} \\
\mathrm{vs} . \\
\text { CC } 100 \mathrm{mg} / \mathrm{d} \text { on cycle days } 3-9\end{array}$ & $\begin{array}{l}\text { Live birth } \\
\text { Miscarriage }\end{array}$ & Good \\
\hline
\end{tabular}




\begin{tabular}{|c|c|c|c|c|c|}
\hline $\begin{array}{c}\text { Study } \\
\text { ACRONYM } \\
\text { KQs } \\
\text { Companion Studies }\end{array}$ & $\begin{array}{l}\text { Study Design } \\
\text { Geographic } \\
\text { Location }\end{array}$ & $\begin{array}{l}\text { N Enrolled } \\
\text { N Completed } \\
\text { Underlying } \\
\text { Diagnosis }\end{array}$ & Interventions & $\begin{array}{c}\text { Outcomes } \\
\text { (Subgroups analyzed) }\end{array}$ & Quality \\
\hline $\begin{array}{l}\text { Aghahosseini, } 2017^{178} \\
\text { KQ } 1\end{array}$ & $\begin{array}{l}\text { RCT } \\
\text { Middle East }\end{array}$ & $\begin{array}{l}100 \\
80 \\
\text { PCOS }\end{array}$ & $\begin{array}{l}\text { Low dose hCG } 35 \text { hours after GnRH agonist } \\
\text { Vs } \\
\text { Low dose hCG simultaneously with GnRH } \\
\text { agonist } \\
\text { in PCOS patients undergoing IVF }\end{array}$ & OHSS & Good \\
\hline $\begin{array}{l}\text { Amer, } 2009^{165} \\
\text { KQ } 1\end{array}$ & $\begin{array}{l}\text { RCT } \\
\text { UK/Europe }\end{array}$ & $\begin{array}{l}72 \\
65 \\
\text { PCOS }\end{array}$ & $\begin{array}{l}\text { Laparoscopic ovarian diathermy for } \\
\text { endometriosis, periadnexal adhesions, and } \\
\text { adhesions } \\
\text { vs. } \\
\text { CC } 50-150 \mathrm{mg} / \mathrm{d} \text { on cycle days } 2-6\end{array}$ & $\begin{array}{l}\text { Live birth } \\
\text { Miscarriage } \\
\text { Multiple births } \\
\text { OHSS } \\
\text { Surgical complications }\end{array}$ & Fair \\
\hline $\begin{array}{l}\text { Amer, } 2017^{179} \\
\text { KQ } 1\end{array}$ & $\begin{array}{l}\text { RCT } \\
\text { UK/Europe }\end{array}$ & $\begin{array}{l}159 \\
149 \\
\text { PCOS }\end{array}$ & $\begin{array}{l}\text { Clomiphene } \\
\text { vs. } \\
\text { Letrozole }\end{array}$ & Live birth & Good \\
\hline $\begin{array}{l}\text { An, } 2014^{146} \\
\text { KQ } 1\end{array}$ & $\begin{array}{l}\mathrm{RCT} \\
\text { Asia }\end{array}$ & $\begin{array}{l}150 \\
109 \\
\text { PCOS }\end{array}$ & $\begin{array}{l}\text { Berberine was administered at a dosage of } 3 \times \\
500 \mathrm{mg} \text { daily for greater than or equal to } 12 \\
\text { weeks before controlled ovarian stimulation. } \\
\text { vs. } \\
\text { Metformin was administered at a dosage of } 3 \times \\
500 \mathrm{mg} \text { daily for greater than or equal to } 12 \\
\text { weeks before controlled ovarian stimulation. } \\
\text { vs. } \\
\text { Placebo was administered as one tablet three } \\
\text { times daily for greater than or equal to } 12 \text { weeks } \\
\text { before controlled ovarian stimulation. }\end{array}$ & Live birth & Fair \\
\hline $\begin{array}{l}\text { Badawy, 2008 } \\
\text { KQ } 1 \\
\end{array}$ & $\begin{array}{l}\text { RCT } \\
\text { Middle East }\end{array}$ & $\begin{array}{l}318 \\
318 \\
\text { PCOS }\end{array}$ & $\begin{array}{l}\text { Clomiphene citrate } \\
\text { vs. } \\
\text { Gonadotrophin }\end{array}$ & Miscarriage & Fair \\
\hline $\begin{array}{l}\text { Badawy, 2009222 } \\
\text { KQ } 3\end{array}$ & $\begin{array}{l}\text { RCT } \\
\text { Middle East }\end{array}$ & $\begin{array}{l}996 \\
996 \\
\text { Unknown }\end{array}$ & $\begin{array}{l}\text { Letrozole } 5 \mathrm{mg} / \mathrm{d} \text { for } 5 \text { days } \\
\text { vs. } \\
\text { Anastrozole } 1 \mathrm{mg} / \mathrm{d} \text { for } 5 \text { days } \\
\text { vs. } \\
\text { CC } 100 \mathrm{mg} / \mathrm{d} \text { for } 5 \text { days. } \\
\text { vs. } \\
\text { Spontaneous pregnancy }\end{array}$ & $\begin{array}{l}\text { Miscarriage } \\
\text { Ectopic pregnancy } \\
\text { Birthweight } \\
\text { Neonatal death }\end{array}$ & Fair \\
\hline
\end{tabular}




\begin{tabular}{|c|c|c|c|c|c|}
\hline $\begin{array}{c}\text { Study } \\
\text { ACRONYM } \\
\text { KQs } \\
\text { Companion Studies } \\
\end{array}$ & $\begin{array}{l}\text { Study Design } \\
\text { Geographic } \\
\text { Location }\end{array}$ & $\begin{array}{l}\text { N Enrolled } \\
\text { N Completed } \\
\text { Underlying } \\
\text { Diagnosis }\end{array}$ & Interventions & $\begin{array}{c}\text { Outcomes } \\
\text { (Subgroups analyzed) }\end{array}$ & Quality \\
\hline $\begin{array}{l}\text { Bagis, } 2010^{221} \\
\text { KQ 3, } 5\end{array}$ & $\begin{array}{l}\text { RCT } \\
\text { UK/Europe }\end{array}$ & $\begin{array}{l}228 \\
226 \\
\text { Unknown, Male }\end{array}$ & $\begin{array}{l}\text { IUI performed } 36 \text { hours after hCG injection } \\
\text { vs. } \\
\text { IUI performed } 18 \text { hours after hCG injection } \\
\text { followed by second IUI performed } 40 \text { hours after } \\
\text { hCG }\end{array}$ & $\begin{array}{l}\text { Live birth (male) } \\
\text { Miscarriage }\end{array}$ & Fair \\
\hline $\begin{array}{l}\text { Balaban, } 2011^{258} \\
\text { KQ } 5\end{array}$ & $\begin{array}{l}\text { RCT } \\
\text { Middle East }\end{array}$ & $\begin{array}{l}77 \text { cycles } \\
77 \text { cycles } \\
\text { Male }\end{array}$ & $\begin{array}{l}\text { ICSI } \\
\text { vS. } \\
\text { IMSI }\end{array}$ & Live birth & Fair \\
\hline $\begin{array}{l}\text { Barad, } 2017^{280} \\
\text { Across All KQs }\end{array}$ & $\begin{array}{l}\text { Observational } \\
\text { US }\end{array}$ & $\begin{array}{l}33,756 \\
21,008 \\
\text { All }\end{array}$ & $\begin{array}{l}\text { PGD } \\
\text { vs. } \\
\text { Non-PGD in donor oocyte cycles }\end{array}$ & $\begin{array}{l}\text { Live birth } \\
\text { Miscarriage }\end{array}$ & Fair \\
\hline $\begin{array}{l}\text { Belva, } 2011^{259} \\
\text { KQ } 5\end{array}$ & $\begin{array}{l}\text { Observational } \\
\text { UK/Europe }\end{array}$ & $\begin{array}{l}120 \\
120 \\
\text { Male }\end{array}$ & $\begin{array}{l}\text { Male offspring born to parents who underwent } \\
\text { ICSI } \\
\text { vs. } \\
\text { Male offspring born to parents who conceived } \\
\text { spontaneously }\end{array}$ & Birthweight & Poor \\
\hline $\begin{array}{l}\text { Bhattacharya, } 2008^{135} \\
\text { SUIT } \\
\text { KQ } 3\end{array}$ & $\begin{array}{l}\text { RCT } \\
\text { UK/Europe }\end{array}$ & $\begin{array}{l}580 \\
576 \\
\text { Unknown }\end{array}$ & $\begin{array}{l}\text { Expectant Management } \\
\text { vs. } \\
\text { CC } 50 \text { mg days } 2-6 \text { of cycle } \\
\text { vS. } \\
\text { IUI }\end{array}$ & $\begin{array}{l}\text { Live birth (diagnostic } \\
\text { criteria) } \\
\text { Time to pregnancy } \\
\text { Ectopic pregnancy } \\
\text { Miscarriage } \\
\text { Patient costs } \\
\end{array}$ & Good \\
\hline $\begin{array}{l}\text { Bodri, } 2008^{268} \\
\text { KQ } 6\end{array}$ & $\begin{array}{l}\text { Observational } \\
\text { UK/Europe }\end{array}$ & $\begin{array}{l}2,653 \\
2,653 \\
\text { Donor }\end{array}$ & $\begin{array}{l}\text { Ovarian stimulation with } \mathrm{GnRH} \text { agonist } \\
\text { vs. } \\
\text { Ovarian stimulation with } \mathrm{GnRH} \text { antagonist/hCG } \\
\text { vs. } \\
\text { Ovarian stimulation with GnRH antagonist/GnRH } \\
\text { agonist } \\
\text { vs. } \\
\text { A control group was created by taking into } \\
\text { account all IVF cycles reaching oocyte retrieval } \\
\text { performed during the same period }\end{array}$ & OHSS & Fair \\
\hline $\begin{array}{l}\text { Bodri, } 2009^{269} \\
\text { KQ } 6\end{array}$ & $\begin{array}{l}\text { Observational } \\
\text { UK/Europe }\end{array}$ & $\begin{array}{l}1,171 \\
1,171 \\
\text { Donor }\end{array}$ & $\begin{array}{l}\text { Triggering with recombinant hCG } \\
\text { vs. } \\
\text { Triggering with } \mathrm{GnRH}\end{array}$ & OHSS & Fair \\
\hline
\end{tabular}




\begin{tabular}{|c|c|c|c|c|c|}
\hline $\begin{array}{c}\text { Study } \\
\text { ACRONYM } \\
\text { KQs } \\
\text { Companion Studies }\end{array}$ & $\begin{array}{c}\text { Study Design } \\
\text { Geographic } \\
\text { Location }\end{array}$ & $\begin{array}{l}\text { N Enrolled } \\
\text { N Completed } \\
\text { Underlying } \\
\text { Diagnosis }\end{array}$ & Interventions & $\begin{array}{c}\text { Outcomes } \\
\text { (Subgroups analyzed) }\end{array}$ & Quality \\
\hline $\begin{array}{l}\text { Boulet, } 2015^{254} \\
\text { NASS } \\
\text { KQ } 5\end{array}$ & $\begin{array}{l}\text { Observational } \\
\text { US }\end{array}$ & $\begin{array}{l}\text { 499,135 cycles } \\
\text { NA } \\
\text { Male }\end{array}$ & $\begin{array}{l}\text { Conventional IVF } \\
\text { vs. } \\
\text { ICSI }\end{array}$ & $\begin{array}{l}\text { Live birth } \\
\text { Miscarriage } \\
\text { Multiple pregnancies } \\
\text { Birthweight }\end{array}$ & Fair \\
\hline $\begin{array}{l}\text { Boulet, } 2016^{277} \\
\text { Across All KQs }\end{array}$ & $\begin{array}{l}\text { Observational } \\
\text { US }\end{array}$ & $\begin{array}{l}4,618,076 \\
4,618,076 \\
\text { All }\end{array}$ & $\begin{array}{l}\text { No intervention (spontaneous conception) } \\
\text { vs. } \\
\text { Conventional IVF } \\
\text { vs. } \\
\text { IVF + ICSI }\end{array}$ & Congenital anomalies & Good \\
\hline $\begin{array}{l}\text { Brinton, 2015 } \\
\text { Across All KQs } \\
\text { Companions: } \\
\text { Brinton, 2013'38; } \\
\text { Brinton, 2014 } \\
\text { Trabert, } 2013^{26} \\
\end{array}$ & $\begin{array}{l}\text { Observational } \\
\text { US }\end{array}$ & $\begin{array}{l}9,892 \\
9,892 \\
\text { All }\end{array}$ & $\begin{array}{l}\text { Control } \\
\text { vs. } \\
\text { CC } \\
\text { vs. } \\
\text { Gonadotropins }\end{array}$ & Maternal cancer & Good \\
\hline $\begin{array}{l}\text { Butts, } 2014^{122} \\
\text { NASS } \\
\text { KQ } 3\end{array}$ & $\begin{array}{l}\text { Observational } \\
\text { US }\end{array}$ & $\begin{array}{l}38,926 \\
38,926 \\
\text { Unknown }\end{array}$ & $\begin{array}{l}\text { ICSI } \\
\text { vs. } \\
\text { IVF } \\
\text { vs. } \\
\text { Assisted hatching } \\
\text { vs. } \\
\text { No assisted hatching }\end{array}$ & Live birth (ovarian reserve) & Fair \\
\hline $\begin{array}{l}\text { Chang, } 2016^{276} \\
\text { Across All KQs }\end{array}$ & $\begin{array}{l}\text { Observational } \\
\text { US }\end{array}$ & $\begin{array}{l}106,902 \text { cycles } \\
106,902 \text { cycles } \\
\text { All }\end{array}$ & $\begin{array}{l}\text { PGD } \\
\text { vs. } \\
\text { PGD done for concern of aneuploidy } \\
\text { vs. } \\
\text { PGD done for other concern outside of } \\
\text { genetics/aneuploidy } \\
\text { vs. } \\
\text { No PGD } \\
\end{array}$ & $\begin{array}{l}\text { Live birth } \\
\text { Multiple births } \\
\text { Miscarriage } \\
\text { Birthweight }\end{array}$ & Good \\
\hline
\end{tabular}




\begin{tabular}{|c|c|c|c|c|c|}
\hline $\begin{array}{c}\text { Study } \\
\text { ACRONYM } \\
\text { KQs } \\
\text { Companion Studies } \\
\end{array}$ & $\begin{array}{c}\text { Study Design } \\
\text { Geographic } \\
\text { Location }\end{array}$ & $\begin{array}{l}\text { N Enrolled } \\
\text { N Completed } \\
\text { Underlying } \\
\text { Diagnosis } \\
\end{array}$ & Interventions & $\begin{array}{c}\text { Outcomes } \\
\text { (Subgroups analyzed) }\end{array}$ & Quality \\
\hline $\begin{array}{l}\text { Chen, } 2016^{174} \\
\text { KQ } 1\end{array}$ & $\begin{array}{l}\text { RCT } \\
\text { Asia }\end{array}$ & $\begin{array}{l}1,508 \\
1,508 \\
\text { PCOS }\end{array}$ & $\begin{array}{l}\text { Frozen embryo transfer } \\
\text { vs. } \\
\text { Fresh embryo transfer }\end{array}$ & $\begin{array}{l}\text { Live birth } \\
\text { Miscarriage } \\
\text { Multiple births } \\
\text { Birthweight } \\
\text { OHSS } \\
\text { Ectopic pregnancy } \\
\text { Neonatal death } \\
\text { Congenital anomalies }\end{array}$ & Good \\
\hline $\begin{array}{l}\text { Choi, 2012 }{ }^{147} \\
\text { KQ } 1\end{array}$ & $\begin{array}{l}\text { RCT } \\
\text { Asia }\end{array}$ & $\begin{array}{l}61 \\
61 \\
\text { PCOS }\end{array}$ & $\begin{array}{l}\text { IVM/IVF with FSH and hCG priming protocol } \\
\text { vs. } \\
\text { GnRH agonist long protocol group } \\
\text { vs. } \\
\text { GnRH antagonist multi-dose flexible protocol }\end{array}$ & Live birth & Poor \\
\hline $\begin{array}{l}\text { Crawford, } 2017^{281} \\
\text { Across All KQs }\end{array}$ & $\begin{array}{l}\text { Observational } \\
\text { US }\end{array}$ & $\begin{array}{l}105,517 \text { cycles } \\
105,517 \text { cycles } \\
\text { All }\end{array}$ & $\begin{array}{l}\text { Autologous cycles: } \\
\text { Cryopreserved oocyte } \\
\text { vs. } \\
\text { Fresh oocyte } \\
\text { Donor cycles: } \\
\text { Cryopreserved oocyte } \\
\text { vs. } \\
\text { Fresh oocyte }\end{array}$ & $\begin{array}{l}\text { Live birth } \\
\text { Miscarriage }\end{array}$ & Good \\
\hline $\begin{array}{l}\text { Custers, } 2012^{139} \\
\text { KQ } 3\end{array}$ & $\begin{array}{l}\text { RCT } \\
\text { UK/Europe }\end{array}$ & $\begin{array}{l}253 \\
253 \\
\text { Unknown }\end{array}$ & $\begin{array}{l}\text { Expectant Management for } 6 \text { months, followed } \\
\text { by six cycles of IUI-COS, followed by } 3 \text { cycles of } \\
\text { IVF } \\
\text { vs. } \\
\text { IUI with controlled ovarian stimulation (IUI-COS) } \\
\text { for } 6 \text { months followed by } 3 \text { cycles of IVF }\end{array}$ & $\begin{array}{l}\text { Miscarriage } \\
\text { Ectopic pregnancy } \\
\text { Multiple birth } \\
\text { Health system costs }\end{array}$ & Good \\
\hline $\begin{array}{l}\text { Danhof, } 2018^{244} \\
\text { KQ } 3\end{array}$ & $\begin{array}{l}\text { RCT } \\
\text { UK/Europe }\end{array}$ & $\begin{array}{l}738 \\
738 \\
\text { Unknown }\end{array}$ & $\begin{array}{l}\text { CC with IUI } \\
\text { vs. } \\
\text { FSH with IUI }\end{array}$ & $\begin{array}{l}\text { Live birth } \\
\text { Ectopic pregnancy } \\
\text { Miscarriage } \\
\text { Multiple births } \\
\text { OHSS }\end{array}$ & Fair \\
\hline
\end{tabular}




\begin{tabular}{|c|c|c|c|c|c|}
\hline $\begin{array}{c}\text { Study } \\
\text { ACRONYM } \\
\text { KQs } \\
\text { Companion Studies }\end{array}$ & $\begin{array}{l}\text { Study Design } \\
\text { Geographic } \\
\text { Location }\end{array}$ & $\begin{array}{l}\text { N Enrolled } \\
\text { N Completed } \\
\text { Underlying } \\
\text { Diagnosis }\end{array}$ & Interventions & $\begin{array}{c}\text { Outcomes } \\
\text { (Subgroups analyzed) }\end{array}$ & Quality \\
\hline $\begin{array}{l}\text { de Wilde, } 2017^{180} \\
\text { KQ } 1\end{array}$ & $\begin{array}{l}\text { Observational } \\
\text { UK/Europe }\end{array}$ & $\begin{array}{l}3,077 \\
3,077 \\
\text { PCOS }\end{array}$ & $\begin{array}{l}\text { Natural conception } \\
\text { vs. } \\
\text { Ovulation Induction } \\
\text { vs. } \\
\text { IVF-ICSI }\end{array}$ & $\begin{array}{l}\text { Birthweight } \\
\text { Neonatal death }\end{array}$ & Good \\
\hline $\begin{array}{l}\text { Demirol, } 2007^{230} \\
\text { KQ } 3\end{array}$ & $\begin{array}{l}\text { RCT } \\
\text { UK/Europe }\end{array}$ & $\begin{array}{l}241 \\
241 \\
\text { Unknown }\end{array}$ & $\begin{array}{l}\text { IUl following : } \\
\text { Group I (Gonal-F, Serono, Turkey), } 81 \text { Follitropin } \\
\text { alpha } \\
\text { vs. } \\
\text { Group II (Metrodin-HP, Serono), highly-purified } \\
\text { uFSH } \\
\text { vs. } \\
\text { Group III (Pergonal, Serono), hMG }\end{array}$ & $\begin{array}{l}\text { Miscarriage } \\
\text { OHSS }\end{array}$ & Good \\
\hline $\begin{array}{l}\text { Dhalwani, } 2016^{236} \\
\text { KQ } 3 \text { and } 5\end{array}$ & $\begin{array}{l}\text { Observational } \\
\text { US }\end{array}$ & $\begin{array}{l}3,896,242 \text { births } \\
3,896,242 \text { births } \\
\text { Unknown, Male }\end{array}$ & $\begin{array}{l}\text { ART } \\
\text { vs. } \\
\text { No intervention (spontaneous conception) }\end{array}$ & Birthweight & Good \\
\hline $\begin{array}{l}\text { Diamond, } 2015^{233} \\
\text { KQ } 3\end{array}$ & $\begin{array}{l}\text { RCT } \\
\text { US }\end{array}$ & $\begin{array}{l}900 \\
746 \\
\text { Unknown }\end{array}$ & $\begin{array}{l}\text { Gonadotropin } \\
\text { vs. } \\
\text { Clomiphene } \\
\text { vs. } \\
\text { Letrozole }\end{array}$ & $\begin{array}{l}\text { Live birth } \\
\text { Multiple births } \\
\text { Miscarriage } \\
\text { Ectopic pregnancy } \\
\text { Birthweight } \\
\text { Neonatal death } \\
\text { Congenital anomalies }\end{array}$ & Good \\
\hline $\begin{array}{l}\text { Dreyer, } 2016^{250} \\
\text { KQ } 4\end{array}$ & $\begin{array}{l}\text { RCT } \\
\text { UK/Europe }\end{array}$ & $\begin{array}{l}85 \\
85 \text { (for ITT } \\
\text { analysis) } \\
\text { Tubal }\end{array}$ & $\begin{array}{l}\text { Hysteroscopic proximal occlusion by intratubal } \\
\text { device placement (Essure Device) } \\
\text { vs. } \\
\text { Laparoscopic salpingectomy }\end{array}$ & $\begin{array}{l}\text { Live birth } \\
\text { Miscarriage } \\
\text { Ectopic pregnancy } \\
\text { Time to pregnancy }\end{array}$ & Good \\
\hline $\begin{array}{l}\text { Ebrahimi, } 2010^{229} \\
\text { KQ } 3\end{array}$ & $\begin{array}{l}\text { RCT } \\
\text { Middle East }\end{array}$ & $\begin{array}{l}200 \\
179 \\
\text { Unknown }\end{array}$ & $\begin{array}{l}\text { CC } 50 \mathrm{mg} \text { BID on cycle days } 3-7 \text { followed by } 75 \\
\text { IU hMG on cycle days } 7-9 \text { and adjusted } \\
\text { thereafter. IUl performed following triggered } \\
\text { ovulation. } \\
\text { Cyclogest vaginal pessaries } 400 \mathrm{mg} / \text { daily } \\
\text { through the tenth week of pregnancy } \\
\text { vs. } \\
\text { No luteal phase support }\end{array}$ & $\begin{array}{l}\text { Live birth } \\
\text { Multiple births }\end{array}$ & Good \\
\hline
\end{tabular}




\begin{tabular}{|c|c|c|c|c|c|}
\hline $\begin{array}{c}\text { Study } \\
\text { ACRONYM } \\
\text { KQs } \\
\text { Companion Studies } \\
\end{array}$ & $\begin{array}{l}\text { Study Design } \\
\text { Geographic } \\
\text { Location }\end{array}$ & $\begin{array}{l}\text { N Enrolled } \\
\text { N Completed } \\
\text { Underlying } \\
\text { Diagnosis } \\
\end{array}$ & Interventions & $\begin{array}{c}\text { Outcomes } \\
\text { (Subgroups analyzed) }\end{array}$ & Quality \\
\hline $\begin{array}{l}\text { Einarsson, } 2017^{181} \\
\text { KQ } 1\end{array}$ & $\begin{array}{l}\text { RCT } \\
\text { UK/Europe }\end{array}$ & $\begin{array}{l}962 \\
317 \\
\text { PCOS }\end{array}$ & $\begin{array}{l}\text { Lifestyle change (weight reduction) +IVF } \\
\text { vs. } \\
\text { IVF only }\end{array}$ & $\begin{array}{l}\text { Live birth } \\
\text { Multiple births } \\
\text { Miscarriage } \\
\text { Ectopic pregnancy } \\
\text { OHSS }\end{array}$ & Good \\
\hline $\begin{array}{l}\text { Elsedeek, 2014 } \\
\text { KQ } 1\end{array}$ & $\begin{array}{l}\text { RCT } \\
\text { Middle East }\end{array}$ & $\begin{array}{l}220 \text { cycles } \\
220 \text { cycles } \\
\text { Pcos }\end{array}$ & $\begin{array}{l}\text { Clomiphene citrate } 200 \mathrm{mg} / \text { day over } 5 \text { days } \\
\text { vs. } \\
\text { Clomiphene citrate } 100 \mathrm{mg} / \text { day over } 10 \text { days }\end{array}$ & Live birth & Fair \\
\hline $\begin{array}{l}\text { Emekci, } 2017^{182} \\
\text { KQ } 1\end{array}$ & $\begin{array}{l}\text { RCT } \\
\text { Middle East }\end{array}$ & $\begin{array}{l}196 \\
196 \\
\text { PCOS }\end{array}$ & $\begin{array}{l}4 \mathrm{~g} \text { Myo-Inositol (MYO) plus } 400 \mathrm{mg} \text { folic acid } \\
\text { vs. } \\
\text { Recombinant FSH and no MYO administration }\end{array}$ & Miscarriage & Good \\
\hline $\begin{array}{l}\text { Erdem, } 2009^{225} \\
\text { KQ } 3\end{array}$ & $\begin{array}{l}\text { RCT } \\
\text { UK/Europe }\end{array}$ & $\begin{array}{l}214 \\
214 \\
\text { Unknown }\end{array}$ & $\begin{array}{l}\text { Gonadotropin IUI followed by luteal support with } \\
\text { crinone once a day beginning } 2 \text { days after } \\
\text { insemination until pregnancy testing. } \\
\text { vs. } \\
\text { Gonadotropin IUI without luteal support. }\end{array}$ & Live birth & Fair \\
\hline $\begin{array}{l}\text { Erdem, } 2015^{208} \\
\text { KQ } 3,5\end{array}$ & $\begin{array}{l}\text { RCT } \\
\text { Middle East }\end{array}$ & $\begin{array}{l}219 \\
174 \\
\text { Unknown, Male }\end{array}$ & $\begin{array}{l}\text { rFSH followed by triggered ovulation } \\
\text { vs. } \\
\text { CC } 100 \mathrm{mg} / \mathrm{d} \text { on days 3-7 of cycle followed by } \\
\text { triggered ovulation }\end{array}$ & Live birth & Good \\
\hline $\begin{array}{l}\text { Farquhar, } 2018^{238} \\
\text { KQ } 3\end{array}$ & $\begin{array}{l}\text { RCT } \\
\text { Australia/N.Z. }\end{array}$ & $\begin{array}{l}473 \\
201 \\
\text { Unknown }\end{array}$ & $\begin{array}{l}\text { IUI } \\
\text { vs. } \\
\text { Expectant Management }\end{array}$ & $\begin{array}{l}\text { Live birth } \\
\text { Multiple births } \\
\text { Miscarriage } \\
\text { Ectopic pregnancy }\end{array}$ & Good \\
\hline $\begin{array}{l}\mathrm{Ge}, 2008^{167} \\
\mathrm{KQ} 1\end{array}$ & $\begin{array}{l}\text { RCT } \\
\text { Asia }\end{array}$ & $\begin{array}{l}62 \\
62 \\
\text { PCos }\end{array}$ & $\begin{array}{l}\text { IVF using oocytes cultured in media containing } \\
\text { hCG, rFSH, and rhCG } \\
\text { vs. } \\
\text { hCG-free media with rFSH for the first } 10 \text { hours, } \\
\text { then were transferred to the same medium the } \\
\text { group above } \\
\text { vs. } \\
\text { hCG-free media only }\end{array}$ & $\begin{array}{l}\text { Live birth } \\
\text { Miscarriage }\end{array}$ & Good \\
\hline
\end{tabular}




\begin{tabular}{|c|c|c|c|c|c|}
\hline $\begin{array}{c}\text { Study } \\
\text { ACRONYM } \\
\text { KQs } \\
\text { Companion Studies }\end{array}$ & $\begin{array}{l}\text { Study Design } \\
\text { Geographic } \\
\text { Location }\end{array}$ & $\begin{array}{l}\text { N Enrolled } \\
\text { N Completed } \\
\text { Underlying } \\
\text { Diagnosis }\end{array}$ & Interventions & $\begin{array}{c}\text { Outcomes } \\
\text { (Subgroups analyzed) }\end{array}$ & Quality \\
\hline $\begin{array}{l}\text { Ghahiri, } 2016^{170} \\
\text { KQ } 1\end{array}$ & $\begin{array}{l}\text { RCT } \\
\text { Middle East }\end{array}$ & $\begin{array}{l}101 \\
101 \\
\text { PCOS }\end{array}$ & $\begin{array}{l}\text { Clomiphene } 100 \mathrm{mg} \\
\text { vs. } \\
\text { Letrozole } 5 \mathrm{mg}\end{array}$ & $\begin{array}{l}\text { Multiple births } \\
\text { Miscarriage } \\
\text { Ectopic pregnancy } \\
\text { OHSS } \\
\text { Time to pregnancy }\end{array}$ & Fair \\
\hline $\begin{array}{l}\text { Ghanem, } 2013^{145} \\
\text { KQ } 1\end{array}$ & $\begin{array}{l}\text { RCT } \\
\text { Middle East }\end{array}$ & $\begin{array}{l}174 \\
159 \\
\text { PCOS }\end{array}$ & $\begin{array}{l}\text { CC } 100 \mathrm{mg} / \mathrm{d} \text { for } 5 \text { days plus uFSH } 37.5 \mathrm{IU} / \mathrm{d} \text {. } \\
\text { vs. } \\
\text { uFSH } 37.5 \mathrm{IU} / \mathrm{d} \text { only }\end{array}$ & Live birth & Good \\
\hline $\begin{array}{l}\text { Gibreel, } 2013^{228} \\
\text { KQ } 3\end{array}$ & $\begin{array}{l}\mathrm{RCT} \\
\text { Africa }\end{array}$ & $\begin{array}{l}105 \\
90 \\
\text { Unknown }\end{array}$ & $\begin{array}{l}\text { Endometrial scratching using a pipelle biopsy } \\
\text { catheter with biopsies obtained } \\
\text { vs. } \\
\text { Sham procedure using uterine sound only }\end{array}$ & $\begin{array}{l}\text { Miscarriage } \\
\text { Multiple births }\end{array}$ & Good \\
\hline $\begin{array}{l}\text { Goldman, } 2014^{210} \\
\text { FORT-T } \\
\text { KQ } 3\end{array}$ & $\begin{array}{l}\text { RCT } \\
\text { US }\end{array}$ & $\begin{array}{l}154 \\
115 \\
\text { Unknown }\end{array}$ & $\begin{array}{l}\text { CC } 100 \mathrm{mg} / \mathrm{d} \text { for } 5 \text { days followed by IUI, } \\
\text { maximum } 2 \text { cycles after which patients } \\
\text { proceeded to IVF up to } 6 \text { cycles } \\
\text { vs. } \\
\text { rFSH followed by IUI. maximum } 2 \text { cycles after } \\
\text { which patients proceeded to IVF up to } 6 \text { cycles } \\
\text { vs. } \\
\text { Immediate IVF up to } 6 \text { cycles. }\end{array}$ & $\begin{array}{l}\text { Live birth } \\
\text { Time to pregnancy } \\
\text { Ectopic pregnancy } \\
\text { Miscarriage } \\
\text { Multiple births } \\
\text { Birthweight } \\
\text { Neonatal death } \\
\text { OHSS }\end{array}$ & Good \\
\hline $\begin{array}{l}\text { Gregoriou, } 2008^{226} \\
\text { KQ } 3\end{array}$ & $\begin{array}{l}\text { RCT } \\
\text { UK/Europe }\end{array}$ & $\begin{array}{l}50 \\
50 \\
\text { Unknown }\end{array}$ & $\begin{array}{l}\text { rFSH beginning on cycle day } 3 \\
\text { vs. } \\
\text { Letrozole } 5 \mathrm{mg} / \mathrm{d} \text { on cycle day } 3\end{array}$ & Live birth (prior treatments) & Good \\
\hline $\begin{array}{l}\text { Grimstad, } 2016^{251} \\
\text { KQ } 4\end{array}$ & $\begin{array}{l}\text { Observational } \\
\text { US }\end{array}$ & $\begin{array}{l}7,145 \\
7,145 \\
\text { Tubal }\end{array}$ & $\begin{array}{l}\text { IVF } \\
\text { vS. } \\
\text { ICSI }\end{array}$ & $\begin{array}{l}\text { Live birth } \\
\text { Multiple births } \\
\text { Miscarriage } \\
\text { Birthweight }\end{array}$ & Good \\
\hline $\begin{array}{l}\text { Hajizadeh, } 2017^{261} \\
\text { KQ } 5\end{array}$ & $\begin{array}{l}\text { RCT } \\
\text { Middle East }\end{array}$ & $\begin{array}{l}419 \\
386 \\
\text { Male }\end{array}$ & $\begin{array}{l}\text { Structured aerobic exercise for } 12 \text { weeks } \\
\text { vs. } \\
\text { No exercise }\end{array}$ & Live birth & Good \\
\hline $\begin{array}{l}\text { Harira, } 2018^{246} \\
\text { KQ } 3\end{array}$ & $\begin{array}{l}\text { RCT } \\
\text { Middle East }\end{array}$ & $\begin{array}{l}172 \\
172 \\
\text { Unknown }\end{array}$ & $\begin{array}{l}\text { CC + estradiol } \\
\text { vs. } \\
\text { Letrozole }\end{array}$ & $\begin{array}{l}\text { Ectopic pregnancy } \\
\text { Miscarriage } \\
\text { OHSS }\end{array}$ & Good \\
\hline $\begin{array}{l}\text { Hassan, } 2017^{183} \\
\text { KQ } 1\end{array}$ & $\begin{array}{l}\text { RCT } \\
\text { Middle East }\end{array}$ & $\begin{array}{l}182 \\
140 \\
\text { PCOS }\end{array}$ & $\begin{array}{l}\text { Letrozole } \\
\text { vs. } \\
\text { FSH }\end{array}$ & $\begin{array}{l}\text { Miscarriage } \\
\text { Adverse events } \\
\text { Cost effectiveness }\end{array}$ & Good \\
\hline
\end{tabular}




\begin{tabular}{|c|c|c|c|c|c|}
\hline $\begin{array}{c}\text { Study } \\
\text { ACRONYM } \\
\text { KQs } \\
\text { Companion Studies } \\
\end{array}$ & $\begin{array}{l}\text { Study Design } \\
\text { Geographic } \\
\text { Location }\end{array}$ & $\begin{array}{l}\text { N Enrolled } \\
\text { N Completed } \\
\text { Underlying } \\
\text { Diagnosis }\end{array}$ & Interventions & $\begin{array}{c}\text { Outcomes } \\
\text { (Subgroups analyzed) }\end{array}$ & Quality \\
\hline $\begin{array}{l}\text { Hershko-Klement, } \\
2016^{260} \\
\text { KQ } 5\end{array}$ & $\begin{array}{l}\text { Observational } \\
\text { Middle East }\end{array}$ & $\begin{array}{l}2,406 \\
1,981 \\
\text { Male }\end{array}$ & $\begin{array}{l}\text { ICSI } \\
\text { VS. } \\
\text { IMSI }\end{array}$ & Congenital anomalies & Fair \\
\hline $\begin{array}{l}\text { Homburg, } 2012^{152} \\
\text { KQ } 1\end{array}$ & $\begin{array}{l}\text { RCT } \\
\text { UK/Europe \& } \\
\text { Latin America }\end{array}$ & $\begin{array}{l}302 \\
255 \\
\text { PCOS }\end{array}$ & $\begin{array}{l}\text { CC } 50 \text { - } 150 \mathrm{mg} / \mathrm{d} \text { for } 5 \text { days, triggered ovulation } \\
\text { vs. } \\
\text { rhFSH, triggered ovulation }\end{array}$ & $\begin{array}{l}\text { Live birth } \\
\text { Ectopic pregnancy } \\
\text { Miscarriage } \\
\text { Multiple births }\end{array}$ & Good \\
\hline $\begin{array}{l}\text { Hosseini, } 2010^{158} \\
\text { KQ } 1\end{array}$ & $\begin{array}{l}\text { RCT } \\
\text { Middle East }\end{array}$ & $\begin{array}{l}\text { NR } \\
112 \\
\text { PCOS }\end{array}$ & $\begin{array}{l}\text { Long-term desensitization protocol using GnRH } \\
\text { agonist buserelin } 500 \mathrm{mcg} \text { SQ. Gonal F started } \\
\text { on day } 3 \text {, replaced by hMG after } 7 \text { th day of } \\
\text { stimulation. } \\
\text { vs. } \\
\text { Gonal F for ovarian stimulation. Cetrorelix } \\
\text { (GnRH antagonist) } 0.35 \mathrm{mg} / \mathrm{d} \text { injected SQ for } 3 \\
\text { days. hMG prescribed after } 7 \text { th day of } \\
\text { stimulation. Fertilization via ICSI. } 3 \text { good quality } \\
\text { embryos transferred } 3 \text { days later. }\end{array}$ & $\begin{array}{l}\text { Miscarriage } \\
\text { OHSS }\end{array}$ & Fair \\
\hline $\begin{array}{l}\text { Hossein-Rashidi, } \\
2016^{176} \\
\text { KQ } 1\end{array}$ & $\begin{array}{l}\text { RCT } \\
\text { Middle East }\end{array}$ & $\begin{array}{l}104 \\
96 \\
\text { PCOS }\end{array}$ & $\begin{array}{l}\text { Clomiphene } \\
\text { vs. } \\
\text { Recombinant human fSH }\end{array}$ & $\begin{array}{l}\text { Live birth } \\
\text { Miscarriage } \\
\text { OHSS }\end{array}$ & Fair \\
\hline $\begin{array}{l}\text { Ibrahim, } 2017^{189} \\
\text { KQ } 1\end{array}$ & $\begin{array}{l}\text { RCT } \\
\text { Middle East }\end{array}$ & $\begin{array}{l}80 \\
80 \\
\text { PCOS }\end{array}$ & $\begin{array}{l}\text { Laparoscopic Ovarian Drilling } \\
\text { vs. } \\
\text { Letrozole }\end{array}$ & Miscarriage & Fair \\
\hline $\begin{array}{l}\text { Jacob, } 2016^{177} \\
\text { KQ } 1\end{array}$ & $\begin{array}{l}\text { RCT } \\
\text { UK/Europe }\end{array}$ & $\begin{array}{l}153 \\
153 \\
\text { PCOS }\end{array}$ & $\begin{array}{l}\text { Placebo } \\
\text { vs. } \\
\text { Metformin }\end{array}$ & $\begin{array}{l}\text { Live birth } \\
\text { OHSS }\end{array}$ & Good \\
\hline $\begin{array}{l}\text { Jahromi, } 2017^{243} \\
\text { KQ } 3\end{array}$ & $\begin{array}{l}\text { RCT } \\
\text { Middle East }\end{array}$ & $\begin{array}{l}80 \\
66 \\
\text { Unknown }\end{array}$ & $\begin{array}{l}\text { Melatonin along with ART } \\
\text { vs. } \\
\text { Placebo with ART }\end{array}$ & Miscarriage & Fair \\
\hline
\end{tabular}




\begin{tabular}{|c|c|c|c|c|c|}
\hline $\begin{array}{c}\text { Study } \\
\text { ACRONYM } \\
\text { KQs } \\
\text { Companion Studies }\end{array}$ & $\begin{array}{l}\text { Study Design } \\
\text { Geographic } \\
\text { Location }\end{array}$ & $\begin{array}{l}\text { N Enrolled } \\
\text { N Completed } \\
\text { Underlying } \\
\text { Diagnosis }\end{array}$ & Interventions & $\begin{array}{c}\text { Outcomes } \\
\text { (Subgroups analyzed) }\end{array}$ & Quality \\
\hline $\begin{array}{l}\text { Johnson, } 2010^{160} \\
\text { KQ } 1\end{array}$ & $\begin{array}{l}\text { RCT } \\
\text { Australia/NZ }\end{array}$ & $\begin{array}{l}171 \\
168 \\
\text { PCOS }\end{array}$ & $\begin{array}{l}\text { Placebo, BMl>32. Standard care. } \\
\text { vs. } \\
\text { Metformin, BMl>32. } \\
\text { vs. } \\
\text { CC, BMl<=32 } \\
\text { vs. } \\
\text { Metformin, BMl<=32 } \\
\text { vs. } \\
\text { Both, BMl<=32 }\end{array}$ & $\begin{array}{l}\text { Live birth (obesity/BMI) } \\
\text { Miscarriage (obesity/BMI) } \\
\text { Multiple births (obesity/BMI) }\end{array}$ & Good \\
\hline $\begin{array}{l}\text { Kansal Kalra, } 2008^{223} \\
\text { KQ } 3\end{array}$ & $\begin{array}{l}\text { RCT } \\
\text { US }\end{array}$ & $\begin{array}{l}18 \\
18 \\
\text { Unknown }\end{array}$ & $\begin{array}{l}\text { Follicular arm - rFSH on cycle day } 1 \text { or } 2 \text { of the } \\
\text { oocyte retrieval cycle. } \\
\text { vs. } \\
\text { Luteal phase arm - rFSH } 9 \text { days after } \\
\text { spontaneous LH surge of the menstrual cycle } \\
\text { preceding oocyte retrieval }\end{array}$ & Live birth (ovarian reserve) & Fair \\
\hline $\begin{array}{l}\text { Kar, } 2015^{173} \\
\text { KQ } 1\end{array}$ & $\begin{array}{l}\text { RCT } \\
\text { Asia }\end{array}$ & $\begin{array}{l}105 \\
80 \\
\text { PCOS }\end{array}$ & $\begin{array}{l}\text { Clomiphene } \\
\text { vs. } \\
\text { Metformin } \\
\text { vs. } \\
\text { Clomiphene + Metformin }\end{array}$ & $\begin{array}{l}\text { Live birth } \\
\text { Miscarriage }\end{array}$ & Fair \\
\hline $\begin{array}{l}\text { Kettner, } 2016^{184} \\
\text { KQ 1, 4, } 5\end{array}$ & $\begin{array}{l}\text { Observational } \\
\text { UK/Europe }\end{array}$ & $\begin{array}{l}565,116 \\
\text { pregnancies } \\
565,116 \\
\text { pregnancies } \\
\text { PCOS, Tubal, } \\
\text { Male }\end{array}$ & $\begin{array}{l}\text { No fertility treatment } \\
\text { vs. } \\
\text { IUI or OI } \\
\text { vs. } \\
\text { IVF or ICSI }\end{array}$ & Type 1 diabetes in children & Good \\
\hline $\begin{array}{l}\text { Keyhan, } 2018^{185} \\
\text { KQ 1, 2, 3, 4, } 5\end{array}$ & $\begin{array}{l}\text { Observational } \\
\text { US }\end{array}$ & $\begin{array}{l}\text { 90,401 cycles } \\
90,401 \text { cycles } \\
\text { PCOS, } \\
\text { Endometriosis, } \\
\text { Unknown, Tubal, } \\
\text { Male }\end{array}$ & $\begin{array}{l}\text { ICSI } \\
\text { VS. } \\
\text { IVF }\end{array}$ & $\begin{array}{l}\text { Birthweight } \\
\text { (male infertility, age) }\end{array}$ & Fair \\
\hline $\begin{array}{l}\text { Khosravi, } 2015^{232} \\
\text { KQ } 3\end{array}$ & $\begin{array}{l}\mathrm{RCT} \\
\text { Middle East }\end{array}$ & $\begin{array}{l}180 \\
150 \\
\text { Unknown }\end{array}$ & $\begin{array}{l}\text { Oral dydrogesterone } \\
\text { vs. } \\
\text { Vaginal cyclogest }\end{array}$ & Miscarriage & Fair \\
\hline
\end{tabular}




\begin{tabular}{|c|c|c|c|c|c|}
\hline $\begin{array}{c}\text { Study } \\
\text { ACRONYM } \\
\text { KQs } \\
\text { Companion Studies }\end{array}$ & $\begin{array}{l}\text { Study Design } \\
\text { Geographic } \\
\text { Location }\end{array}$ & $\begin{array}{l}\text { N Enrolled } \\
\text { N Completed } \\
\text { Underlying } \\
\text { Diagnosis }\end{array}$ & Interventions & $\begin{array}{c}\text { Outcomes } \\
\text { (Subgroups analyzed) }\end{array}$ & Quality \\
\hline $\begin{array}{l}\text { Kim, 2011 } \\
\text { KQ } 3\end{array}$ & $\begin{array}{l}\text { RCT } \\
\text { Asia }\end{array}$ & $\begin{array}{l}110 \\
110 \\
\text { Unknown }\end{array}$ & $\begin{array}{l}\text { IVF/ICSI with testosterone gel pretreatment (12.5 } \\
\text { mg/d) starting on cycle day } 6 \text { of the estrogen- } \\
\text { progesterone pretreatment. } \\
\text { vs. } \\
21 \text { days pretreatment with estradiol valerate and } \\
\text { norethindrone }\end{array}$ & $\begin{array}{l}\text { Live birth (ovarian reserve) } \\
\text { Miscarriage (ovarian } \\
\text { reserve) }\end{array}$ & Fair \\
\hline $\begin{array}{l}\text { Kim, } 2012^{150} \\
\text { KQ } 1\end{array}$ & $\begin{array}{l}\text { RCT } \\
\text { Asia }\end{array}$ & $\begin{array}{l}211 \\
208 \\
\text { PCOS }\end{array}$ & $\begin{array}{l}\text { Ovarian stimulation using } 50 \text { - } 150 \text { IU of rhFSH } \\
\text { after establishing ovarian and uterine quiescence } \\
\text { using vaginal ultrasound. GnRH antagonist, } \\
\text { cetrorelix (Cetrotide) } 0.125 \mathrm{mg} / \mathrm{d} \text { was } \\
\text { administered in the morning of stimulation day } 1 \\
\text { and } 2 \text {. When the mean diameter of lead follicle } \\
\text { reached } 13 \mathrm{~mm} \text {, cetrorelix at a dose of } 0.25 \mathrm{mg} / \mathrm{d} \\
\text { was started again and continued daily up to the } \\
\text { day of rhCG (injection). } \\
\text { vs. } \\
\text { GnRH agonist, triptorelin (Decapeptyl) at a dose } \\
\text { of } 0.1 \mathrm{mg} / \mathrm{d} \text { was initiated from day } 18 \text { of oral } \\
\text { contraceptive pretreatment cycle. All patients } \\
\text { had withdrawal bleeding after discontinuation of } \\
\text { oral contraceptive. When pituitary desensitization } \\
\text { was achieved, ovarian stimulation was started } \\
\text { and the dose of triptorelin was reduced to } 0.05 \\
\text { mg daily and continues up to day of rhCG } \\
\text { administration. Ovarian stimulation was } \\
\text { performed in the same manner. }\end{array}$ & Live birth & Fair \\
\hline $\begin{array}{l}\text { Kissin, } 2015^{272} \\
\text { Across All KQs }\end{array}$ & $\begin{array}{l}\text { Observational } \\
\text { US }\end{array}$ & $\begin{array}{l}42,383 \\
42,383 \\
\text { All }\end{array}$ & $\begin{array}{l}\text { Singleton ICSI } \\
\text { vs. } \\
\text { Singleton Conventional IVF (without ICSI) } \\
\text { vs. } \\
\text { Multiples ICSI } \\
\text { vs. } \\
\text { Multiples Conventional IVF (without ICSI) }\end{array}$ & $\begin{array}{l}\text { Neurodevelopment-autism } \\
\text { diagnosis (male) }\end{array}$ & Good \\
\hline
\end{tabular}




\begin{tabular}{|c|c|c|c|c|c|}
\hline $\begin{array}{c}\text { Study } \\
\text { ACRONYM } \\
\text { KQs } \\
\text { Companion Studies }\end{array}$ & $\begin{array}{l}\text { Study Design } \\
\text { Geographic } \\
\text { Location }\end{array}$ & $\begin{array}{l}\text { N Enrolled } \\
\text { N Completed } \\
\text { Underlying } \\
\text { Diagnosis } \\
\end{array}$ & Interventions & $\begin{array}{c}\text { Outcomes } \\
\text { (Subgroups analyzed) }\end{array}$ & Quality \\
\hline $\begin{array}{l}\text { Kjotrod, 2011 } \\
\text { KQ } 1\end{array}$ & $\begin{array}{l}\text { RCT } \\
\text { UK/Europe }\end{array}$ & $\begin{array}{l}150 \\
149 \\
\text { PCOS }\end{array}$ & $\begin{array}{l}\text { Metformin prior to, and during, AFT } \\
\text { vs. } \\
\text { Placebo prior to, and during, AFT }\end{array}$ & Live birth & $\begin{array}{l}\text { Fair } \\
\text { (ITT } \\
\text { results) } \\
\text { Poor } \\
\text { (non- } \\
\text { ITT } \\
\text { results) }\end{array}$ \\
\hline $\begin{array}{l}\text { Knudtson, } 2017^{282} \\
\text { Across All KQs }\end{array}$ & $\begin{array}{l}\text { Observational } \\
\text { US }\end{array}$ & $\begin{array}{l}151,533 \text { first- } \\
\text { cycle } \\
151,533 \text { first- } \\
\text { cycle } \\
\text { All }\end{array}$ & $\begin{array}{l}\text { Frozen embryo without assisted hatching } \\
\text { vs. } \\
\text { Frozen embryo with assisted hatching }\end{array}$ & $\begin{array}{l}\text { Live birth } \\
\text { (race, age, etiology of } \\
\text { infertility) }\end{array}$ & Good \\
\hline $\begin{array}{l}\text { Kramer, } 2009^{271} \\
\text { KQ } 6\end{array}$ & $\begin{array}{l}\text { Observational } \\
\text { US }\end{array}$ & $\begin{array}{l}287 \\
155 \\
\text { Donor }\end{array}$ & $\begin{array}{l}\text { All respondents were egg donors } \\
\text { vs. } \\
\text { No comparator }\end{array}$ & $\begin{array}{l}\text { Adverse effects of } \\
\text { treatments } \\
\text { OHSS } \\
\text { Downstream fertility }\end{array}$ & Poor \\
\hline $\begin{array}{l}\text { Kurzawa, 2008 } \\
\text { KQ } 1\end{array}$ & $\begin{array}{l}\text { RCT } \\
\text { UK/Europe }\end{array}$ & $\begin{array}{l}74 \\
70 \\
\text { Pcos }\end{array}$ & $\begin{array}{l}\text { All patients received oral contraceptives pills } \times 1 \\
\text { month before starting controlled ovarian } \\
\text { hyperstimulation. None of the patients used oral } \\
\text { antidiabetic medications (biguanides or } \\
\text { thiazolidinediones). } \\
\text { rhFSH started on cycle day } 2 \text { at } 150 \mathrm{IU} / \mathrm{d} \text { and } \\
\text { adjusted depending on an ovarian response. A } \\
\text { GnRH antagonist - cetrorelix } 0.25 \mathrm{mg} \\
\text { subcutaneous injections were given until the } \\
\text { criteria for recombinant hCG administration were } \\
\text { met } \\
\text { vs. } \\
\text { During oral contraception on days } 16-18 \text { of the } \\
\text { preceding cycle, after transvaginal } \\
\text { ultrasonographic screening of ovaries, an } \\
\text { intramuscular injection of GnRH agonist } \\
\text { triptorelin (Diphereline SR } 3.75 ; \text { Boufor Ibsen } \\
\text { Pharma, France) was given. After confirmation of } \\
\text { pituitary desensitization (LH <2 mIU/mL and } \\
\text { estradiol <40 pg/mL) the administration of FSH } \\
\text { was commenced. rFSH and hCG administered } \\
\text { as above }\end{array}$ & $\begin{array}{l}\text { Live birth } \\
\text { Miscarriage } \\
\text { Multiple births } \\
\text { OHSS }\end{array}$ & Fair \\
\hline
\end{tabular}




\begin{tabular}{|c|c|c|c|c|c|}
\hline $\begin{array}{c}\text { Study } \\
\text { ACRONYM } \\
\text { KQs } \\
\text { Companion Studies } \\
\end{array}$ & $\begin{array}{l}\text { Study Design } \\
\text { Geographic } \\
\text { Location }\end{array}$ & $\begin{array}{l}\text { N Enrolled } \\
\text { N Completed } \\
\text { Underlying } \\
\text { Diagnosis }\end{array}$ & Interventions & $\begin{array}{c}\text { Outcomes } \\
\text { (Subgroups analyzed) }\end{array}$ & Quality \\
\hline $\begin{array}{l}\text { Kuzmin, } 2014^{248} \\
\text { KQ } 4\end{array}$ & $\begin{array}{l}\text { RCT } \\
\text { Asia }\end{array}$ & $\begin{array}{l}468 \\
468 \\
\text { Tubal Factor }\end{array}$ & $\begin{array}{l}\text { Laparoscopy, salpingolysis, salpingostomy, and } \\
\text { transcervical falloposcopy tubal dilatation (TFTD) } \\
\text { vs. } \\
\text { Laparoscopy, salpingolysis, salpingostomy }\end{array}$ & Ectopic pregnancy & Poor \\
\hline $\begin{array}{l}\text { La Sala, } 2015^{253} \\
\text { KQ } 5\end{array}$ & $\begin{array}{l}\text { RCT } \\
\text { UK/Europe }\end{array}$ & $\begin{array}{l}242 \\
242 \\
\text { Male }\end{array}$ & $\begin{array}{l}\text { IMSI } \\
\text { vS. } \\
\text { ICSI }\end{array}$ & $\begin{array}{l}\text { Live birth } \\
\text { Miscarriage } \\
\text { Multiple births } \\
\text { Birthweight } \\
\text { Congenital anomalies }\end{array}$ & Poor \\
\hline $\begin{array}{l}\text { Leandri, } 2013^{256} \\
\text { KQ } 5\end{array}$ & $\begin{array}{l}\text { RCT } \\
\text { UK/Europe }\end{array}$ & $\begin{array}{l}255 \\
255 \\
\text { Male } \\
\end{array}$ & $\begin{array}{l}\text { Conventional ICSI } \\
\text { vs. } \\
\text { IMSI }\end{array}$ & Live birth & Fair \\
\hline $\begin{array}{l}\text { Legro, } 2007^{128} \\
\text { PPCOS } \\
\text { KQ } 1 \\
\text { Companion: } \\
\text { Rausch, 2009129 }\end{array}$ & $\begin{array}{l}\text { RCT } \\
\text { US }\end{array}$ & $\begin{array}{l}626 \\
450 \\
\text { PCOS }\end{array}$ & $\begin{array}{l}\text { CC initial dose of } 50 \mathrm{mg} \text { of CC on days } 3-7 \text { of } \\
\text { each treatment cycle on-study } \\
\text { vs. } \\
\text { Metformin } 500 \mathrm{mg} / \mathrm{d} \text {, increased to } 2000 \mathrm{mg} / \mathrm{d} \\
\text { Vs. } \\
\text { Combination of CC and metformin }\end{array}$ & $\begin{array}{l}\text { Live birth (obesity/BMI) } \\
\text { Ectopic pregnancy } \\
\text { Miscarriage } \\
\text { Multiple births } \\
\text { Congenital anomalies }\end{array}$ & Good \\
\hline $\begin{array}{l}\text { Legro, 2014 }{ }^{131} \\
\text { PPCOS } 2 \\
\text { KQ } 1 \\
\text { Companion: } \\
\text { Polotsky, 2015 }\end{array}$ & $\begin{array}{l}\text { RCT } \\
\text { US }\end{array}$ & $\begin{array}{l}750 \\
750 \\
\text { PCOS }\end{array}$ & $\begin{array}{l}\text { CC } 50 \mathrm{mg} \text { daily starting on cycle day } 3 \text { for } 5 \text { days } \\
\text { vs. } \\
\text { Letrozole } 2.5 \mathrm{mg} \text { daily starting on cycle day } 3 \text { for } \\
5 \text { days } \\
\text { Maximum treatment duration up to } 5 \text { cycles }\end{array}$ & $\begin{array}{l}\text { Live birth } \\
\text { Time to pregnancy } \\
\text { Ectopic pregnancy } \\
\text { Miscarriage } \\
\text { Birthweight } \\
\text { Congenital anomalies } \\
\text { Neonatal death }\end{array}$ & Good \\
\hline $\begin{array}{l}\text { Legro, } 2015^{172} \\
\text { KQ } 1\end{array}$ & $\begin{array}{l}\text { RCT } \\
\text { US }\end{array}$ & $\begin{array}{l}149 \\
132 \\
\text { PCOS }\end{array}$ & $\begin{array}{l}\text { Continuous OCP } \\
\text { vs. } \\
\text { Lifestyle changes (caloric restriction, physical } \\
\text { activity, weight loss medication) } \\
\text { vs. } \\
\text { Combined OCP and lifestyle changes }\end{array}$ & $\begin{array}{l}\text { Live birth } \\
\text { Birthweight } \\
\text { Ectopic pregnancy } \\
\text { Miscarriage }\end{array}$ & Good \\
\hline
\end{tabular}




\begin{tabular}{|c|c|c|c|c|c|}
\hline $\begin{array}{c}\text { Study } \\
\text { ACRONYM } \\
\text { KQs } \\
\text { Companion Studies }\end{array}$ & $\begin{array}{l}\text { Study Design } \\
\text { Geographic } \\
\text { Location }\end{array}$ & $\begin{array}{l}\text { N Enrolled } \\
\text { N Completed } \\
\text { Underlying } \\
\text { Diagnosis }\end{array}$ & Interventions & $\begin{array}{c}\text { Outcomes } \\
\text { (Subgroups analyzed) }\end{array}$ & Quality \\
\hline $\begin{array}{l}\text { Levi Dunietz, } 2017^{239} \\
\text { KQ } 3\end{array}$ & $\begin{array}{l}\text { Observational } \\
\text { US }\end{array}$ & $\begin{array}{l}4,292,779 \\
4,292,779 \\
\text { Unknown }\end{array}$ & $\begin{array}{l}\text { Non-ART } \\
\text { vs. } \\
\text { Fresh embryos } \\
\text { vs. } \\
\text { Cryopreserved embryos }\end{array}$ & Birthweight & Good \\
\hline $\begin{array}{l}\text { Litzky, } 2018^{284} \\
\text { Across All KQs }\end{array}$ & $\begin{array}{l}\text { Observational } \\
\text { US }\end{array}$ & $\begin{array}{l}1,008,393 \\
180,184 \\
\text { All }\end{array}$ & $\begin{array}{l}\text { Fresh embryo transfers } \\
\text { vs. } \\
\text { Frozen/thawed embryo transfers }\end{array}$ & Birthweight & Fair \\
\hline $\begin{array}{l}\text { Litzky, } 2018^{290} \\
\text { Across All KQs }\end{array}$ & $\begin{array}{l}\text { Observational } \\
\text { US }\end{array}$ & $\begin{array}{l}124,154 \\
124,154 \\
\text { All }\end{array}$ & $\begin{array}{l}\text { Blastocyst stage transfer } \\
\text { vs. } \\
\text { Cleavage stage transfer }\end{array}$ & Birthweight & Good \\
\hline $\begin{array}{l}\text { Londra, } 2016^{278} \\
\text { Across All KQs }\end{array}$ & $\begin{array}{l}\text { Observational } \\
\text { US }\end{array}$ & $\begin{array}{l}136,605 \text { cycles } \\
\text { that resulted in a } \\
\text { pregnancy } \\
136,605 \\
\text { All }\end{array}$ & $\begin{array}{l}\text { Luteal GnRH agonist cycles } \\
\text { vs. } \\
\text { GnRH antagonist } \\
\text { vs. } \\
\text { GnRH agonist flare cycles }\end{array}$ & Ectopic pregnancy & Good \\
\hline $\begin{array}{l}\text { Luke, } 2010^{123} \\
\text { NASS } \\
\text { KQ 2, 3, 4, } 5\end{array}$ & $\begin{array}{l}\text { Observational } \\
\text { US }\end{array}$ & $\begin{array}{l}69,028 \text { cycles } \\
69,028 \text { cycles } \\
\text { Endometriosis, } \\
\text { Unknown, Tubal } \\
\text { Factor, Male }\end{array}$ & $\begin{array}{l}\text { Elective single embryo transfer (eSET)--1 } \\
\text { embryo } \\
\text { vs. } \\
\text { eSET--2 embryos } \\
\text { vS. } \\
\text { eSET--3 embryos } \\
\text { vs. } \\
\text { eSET--4 or more embryos }\end{array}$ & Live birth & Fair \\
\hline $\begin{array}{l}\text { Luke, } 2016^{274} \\
\text { Across All KQs }\end{array}$ & $\begin{array}{l}\text { Observational } \\
\text { US }\end{array}$ & $\begin{array}{l}53,859 \\
53,859 \\
\text { All }\end{array}$ & $\begin{array}{l}\text { ART } \\
\text { vS. } \\
\text { ART }\end{array}$ & $\begin{array}{l}\text { Birthweight } \\
\text { Congenital anomalies }\end{array}$ & Good \\
\hline
\end{tabular}




\begin{tabular}{|c|c|c|c|c|c|}
\hline $\begin{array}{c}\text { Study } \\
\text { ACRONYM } \\
\text { KQs } \\
\text { Companion Studies }\end{array}$ & $\begin{array}{l}\text { Study Design } \\
\text { Geographic } \\
\text { Location }\end{array}$ & $\begin{array}{l}\text { N Enrolled } \\
\text { N Completed } \\
\text { Underlying } \\
\text { Diagnosis }\end{array}$ & Interventions & $\begin{array}{c}\text { Outcomes } \\
\text { (Subgroups analyzed) }\end{array}$ & Quality \\
\hline $\begin{array}{l}\text { Magnusson, } 2018^{287} \\
\text { Across All KQs }\end{array}$ & $\begin{array}{l}\text { Observational } \\
\text { UK/Europe }\end{array}$ & $\begin{array}{l}44,369 \\
27,359 \\
\text { All }\end{array}$ & $\begin{array}{l}\text { Number of oocytes retrieved: } \\
<10 \\
\text { vs. } \\
10-14 \\
\text { vs. } \\
15-19 \\
\text { vs. } \\
\geq 20\end{array}$ & $\begin{array}{l}\text { Birthweight } \\
\text { Congenital anomalies } \\
\text { Neonatal death }\end{array}$ & Good \\
\hline $\begin{array}{l}\text { Maher, } 2018^{245} \\
\text { KQ } 3\end{array}$ & $\begin{array}{l}\text { RCT } \\
\text { Middle East }\end{array}$ & $\begin{array}{l}714 \\
714 \\
\text { Unknown }\end{array}$ & $\begin{array}{l}\text { Unstimulated IUI: } \\
\text { Cervical mucus removal (internal and external) } \\
\text { vs. } \\
\text { No mucus removal }\end{array}$ & $\begin{array}{l}\text { Live birth } \\
\text { Miscarriage } \\
\text { Multiple births }\end{array}$ & Fair \\
\hline $\begin{array}{l}\text { Majumdar, } 2013^{214} \\
\text { KQ } 3\end{array}$ & $\begin{array}{l}\text { RCT } \\
\text { Asia }\end{array}$ & $\begin{array}{l}156 \\
151 \\
\text { Unknown }\end{array}$ & $\begin{array}{l}\text { ICSI with sperm selection based on visual } \\
\text { assessment } \\
\text { vs. } \\
\text { ICSI with sperm selection based on ability to bind } \\
\text { hyaluronic acid }\end{array}$ & $\begin{array}{l}\text { Live birth } \\
\text { Miscarriage }\end{array}$ & Fair \\
\hline $\begin{array}{l}\text { Malchau, } 2017^{186} \\
\text { KQ 1, 2, 3, } 5\end{array}$ & $\begin{array}{l}\text { Observational } \\
\text { UK/Europe }\end{array}$ & $\begin{array}{l}19,884 \\
19,884 \\
\text { PCOS, } \\
\text { Endometriosis, } \\
\text { Unknown, Male }\end{array}$ & $\begin{array}{l}\text { IUI } \\
\text { VS. } \\
\text { ART } \\
\text { vs. } \\
\text { No treatment }\end{array}$ & $\begin{array}{l}\text { Live birth } \\
\text { Time to birth }\end{array}$ & Good \\
\hline $\begin{array}{l}\text { Mancuso, } 2016^{283} \\
\text { Across All KQs }\end{array}$ & $\begin{array}{l}\text { Observational } \\
\text { US }\end{array}$ & $\begin{array}{l}914 \\
914 \\
\text { All }\end{array}$ & $\begin{array}{l}\text { Elective single embryo transfer (eSET) } \\
\text { vs. } \\
\text { Double embryo transfer (DET) }\end{array}$ & $\begin{array}{l}\text { Live birth } \\
\text { Multiple birth }\end{array}$ & Good \\
\hline $\begin{array}{l}\text { Maxwell, } 2008^{270} \\
\text { KQ } 6\end{array}$ & $\begin{array}{l}\text { Observational } \\
\text { US }\end{array}$ & $\begin{array}{l}587 \\
587 \\
\text { Donor }\end{array}$ & $\begin{array}{l}\text { Oocyte Donors } \\
\text { vs. } \\
\text { No comparison }\end{array}$ & $\begin{array}{l}\text { Adverse effects of } \\
\text { treatments } \\
\text { OHSS }\end{array}$ & Fair \\
\hline $\begin{array}{l}\text { Mehrabian, } 2012^{148} \\
\text { KQ } 1\end{array}$ & $\begin{array}{l}\text { RCT } \\
\text { Middle East }\end{array}$ & $\begin{array}{l}104 \\
\text { Unclear } \\
\text { PCOS }\end{array}$ & $\begin{array}{l}\text { hMG followed by triggered ovulation } \\
\text { vs. } \\
\text { Laparoscopic ovarian drilling } \\
\text { If after } 3 \text { cycles anovulation persisted, CC was } \\
\text { prescribed and gonadotropin was administered if } \\
\text { anovulation persisted after CC. }\end{array}$ & OHSS & Fair \\
\hline
\end{tabular}




\begin{tabular}{|c|c|c|c|c|c|}
\hline $\begin{array}{c}\text { Study } \\
\text { ACRONYM } \\
\text { KQs } \\
\text { Companion Studies }\end{array}$ & $\begin{array}{l}\text { Study Design } \\
\text { Geographic } \\
\text { Location }\end{array}$ & $\begin{array}{l}\text { N Enrolled } \\
\text { N Completed } \\
\text { Underlying } \\
\text { Diagnosis }\end{array}$ & Interventions & $\begin{array}{l}\text { Outcomes } \\
\text { (Subgroups analyzed) }\end{array}$ & Quality \\
\hline $\begin{array}{l}\text { Mohammadi, 2018 } \\
\text { KQ } 1\end{array}$ & $\begin{array}{l}\text { RCT } \\
\text { Middle East }\end{array}$ & $\begin{array}{l}219 \\
184 \\
\text { PCOS }\end{array}$ & $\begin{array}{l}\text { Methylprednisolone } \\
\text { vs. } \\
\text { No treatment }\end{array}$ & OHSS & Fair \\
\hline $\begin{array}{l}\text { Morad, 2012227 } \\
\text { KQ } 3\end{array}$ & $\begin{array}{l}\mathrm{RCT} \\
\text { Africa }\end{array}$ & $\begin{array}{l}234 \\
231 \\
\text { Unknown }\end{array}$ & $\begin{array}{l}\text { Hydrotubation performed one day before IUI } \\
\text { using: } \\
20 \mathrm{~mL} \text { of saline } \\
\text { vs. } \\
20 \mathrm{~mL} \text { of } 0.1 \mathrm{mg} \text { Lidocaine } / \mathrm{mL} \text { saline mixed with } \\
19.9 \mathrm{cc} \text { of saline) }\end{array}$ & $\begin{array}{l}\text { Ectopic pregnancy } \\
\text { Miscarriage } \\
\text { Multiple births } \\
\text { OHSS }\end{array}$ & Good \\
\hline $\begin{array}{l}\text { Morin-Papunen, } \\
2012^{151} \\
\text { KQ } 1\end{array}$ & $\begin{array}{l}\text { RCT } \\
\text { UK/Europe }\end{array}$ & $\begin{array}{l}320 \\
259 \\
\text { Pcos }\end{array}$ & $\begin{array}{l}\text { Metformin ( } 500 \mathrm{mg} \text { ) was initiated at a dose of } \\
\text { one tablet once a day for the first week and } \\
\text { increased thereafter by one tablet daily in weekly } \\
\text { steps up to three tablets (one + two daily) in } \\
\text { nonobese women and to four tablets (two + two } \\
\text { daily) in obese women and was continued up to } \\
\text { a maximum of } 9 \text { months. } \\
\text { If pregnancy occurred, metformin was continued } \\
\text { up to the } 12 \text { th week. } \\
\text { The women used metformin or placebo alone for } \\
\text { at least } 3 \text { months. If pregnancy did not occur, } \\
\text { ovulation induction was commenced: if the } \\
\text { woman ovulated after CC, she continued } \\
\text { metformin/placebo with the same dose of } \\
\text { clomiphene for four to six cycles or until the } 12 \text { th } \\
\text { week of pregnancy. After four to six unsuccessful } \\
\text { cycles with metformin/placebo and CC, either } \\
\text { gonadotrophins or aromatase inhibitors were } \\
\text { used. } \\
\text { vs. } \\
\text { Placebo with all other management the same as } \\
\text { above. }\end{array}$ & $\begin{array}{l}\text { Live birth (obesity/BMI) } \\
\text { Time to pregnancy }\end{array}$ & Good \\
\hline
\end{tabular}




\begin{tabular}{|c|c|c|c|c|c|}
\hline $\begin{array}{c}\text { Study } \\
\text { ACRONYM } \\
\text { KQs } \\
\text { Companion Studies }\end{array}$ & $\begin{array}{l}\text { Study Design } \\
\text { Geographic } \\
\text { Location }\end{array}$ & $\begin{array}{l}\text { N Enrolled } \\
\text { N Completed } \\
\text { Underlying } \\
\text { Diagnosis }\end{array}$ & Interventions & $\begin{array}{c}\text { Outcomes } \\
\text { (Subgroups analyzed) }\end{array}$ & Quality \\
\hline $\begin{array}{l}\text { Muller, } 2017^{205} \\
\text { KQ } 2\end{array}$ & $\begin{array}{l}\text { RCT } \\
\text { UK/Europe }\end{array}$ & $\begin{array}{l}144 \\
144 \\
\text { Endometriosis }\end{array}$ & $\begin{array}{l}\text { Dienogest } \\
\text { vs. } \\
\text { a-GnRH } \\
\text { vs. } \\
\text { No treatment }\end{array}$ & Live birth & Fair \\
\hline $\begin{array}{l}\text { Mutsaerts, } 2016^{119} \\
\text { KQ 1, 3, } 5 \\
\text { Companions: } \\
\text { van Oers, 2016 } \\
\text { van Oers, 2018 }\end{array}$ & $\begin{array}{l}\text { RCT } \\
\text { UK/Europe }\end{array}$ & $\begin{array}{l}577 \\
564 \\
\text { PCOS, } \\
\text { Unknown, Male }\end{array}$ & $\begin{array}{l}\text { 6-month lifestyle intervention preceding } \\
\text { treatment for infertility } \\
\text { vs. } \\
\text { Prompt treatment for infertility }\end{array}$ & $\begin{array}{l}\text { Live birth } \\
\text { Ectopic pregnancy } \\
\text { Miscarriage } \\
\text { Time to pregnancy } \\
\text { Neonatal death } \\
\text { Birthweight } \\
\text { Congenital anomalies }\end{array}$ & Good \\
\hline $\begin{array}{l}\text { Nada, } 2016^{234} \\
\text { KQ } 3\end{array}$ & $\begin{array}{l}\mathrm{RCT} \\
\text { Africa }\end{array}$ & $\begin{array}{l}622 \\
595 \\
\text { Unknown }\end{array}$ & $\begin{array}{l}\text { Pts given human menopausal gonadotropins } \\
\text { form Day } 2 \text { to reach DF of } 18-22 \mathrm{~mm} \text {. Then } \\
\text { Ganirelix acetate } 0.25 \mathrm{mg} \text { SQ started from Day } 6 \\
\text { or } 7 \text {. Then IUI of } 0.5 \mathrm{~mL} \text {. } \\
\text { vs. } \\
\text { Clomiphene citrate } 100 \mathrm{mg} \text { from Day } 2 \text { to } 6 \text {. } \\
\text { Monitor ovulation., the hCG given at dose of } \\
10,000 \text { IU IM. Then, IUI of } 0.5 \mathrm{~mL}\end{array}$ & $\begin{array}{l}\text { OHSS } \\
\text { Cost }\end{array}$ & Good \\
\hline $\begin{array}{l}\text { Nahuis, 2011 } \\
\text { KQ } 1 \\
\text { Companion: } \\
\text { Nahuis, 2012 }\end{array}$ & $\begin{array}{l}\text { RCT } \\
\text { UK/Europe }\end{array}$ & $\begin{array}{l}168 \\
168 \\
\text { PCOS }\end{array}$ & $\begin{array}{l}\text { Laparoscopic electrocautery (LEC). LEC } \\
\text { followed by clomiphene citrate and then rFSH if } \\
\text { still anovulatory. } \\
\text { vs. } \\
\text { rFSH }\end{array}$ & $\begin{array}{l}\text { Live birth } \\
\text { Patient costs }\end{array}$ & Fair \\
\hline $\begin{array}{l}\text { Nandi, } 2017^{240} \\
\text { KQ } 3\end{array}$ & $\begin{array}{l}\text { RCT } \\
\text { UK/Europe }\end{array}$ & $\begin{array}{l}207 \\
207 \\
\text { Unknown }\end{array}$ & $\begin{array}{l}\text { Three cycles of IUI+COH } \\
\text { vs. } \\
\text { One cycle of IVF }\end{array}$ & $\begin{array}{l}\text { Live birth } \\
\text { Multiple birth } \\
\text { Miscarriage } \\
\text { Ectopic pregnancy } \\
\text { OHSS }\end{array}$ & Fair \\
\hline $\begin{array}{l}\text { Nangia, } 2011^{124} \\
\text { NASS } \\
\text { KQ } 5\end{array}$ & $\begin{array}{l}\text { Observational } \\
\text { US }\end{array}$ & $\begin{array}{l}77,432 \text { cycles } \\
77,432 \text { cycles } \\
\text { Male }\end{array}$ & $\begin{array}{l}\text { IVF w/o ICSI, Male only } \\
\text { vs. } \\
\text { ICSI, Male only }\end{array}$ & $\begin{array}{l}\text { Live birth } \\
\text { Birthweight }\end{array}$ & Fair \\
\hline
\end{tabular}




\begin{tabular}{|c|c|c|c|c|c|}
\hline $\begin{array}{c}\text { Study } \\
\text { ACRONYM } \\
\text { KQs } \\
\text { Companion Studies }\end{array}$ & $\begin{array}{c}\text { Study Design } \\
\text { Geographic } \\
\text { Location }\end{array}$ & $\begin{array}{l}\mathrm{N} \text { Enrolled } \\
\mathrm{N} \text { Completed } \\
\text { Underlying } \\
\text { Diagnosis }\end{array}$ & Interventions & $\begin{array}{c}\text { Outcomes } \\
\text { (Subgroups analyzed) }\end{array}$ & Quality \\
\hline $\begin{array}{l}\text { Oyesanya } 2009^{224} \\
\text { KQ } 3\end{array}$ & $\begin{array}{l}\text { RCT } \\
\text { UK/Europe }\end{array}$ & $\begin{array}{l}353 \\
351 \\
\text { Unknown }\end{array}$ & $\begin{array}{l}\text { Provided that } 6 \text { or more oocytes were retrieved } \\
\text { from the prospective donor, half were given to } \\
\text { the recipient and half were given to another } \\
\text { recipient. } \\
\text { vs. } \\
\text { Recipients received all retrieved oocytes from } \\
\text { their altruistic donor. }\end{array}$ & $\begin{array}{l}\text { Ectopic pregnancy } \\
\text { (diagnostic criteria) }\end{array}$ & Fair \\
\hline $\begin{array}{l}\text { Palomba, } 2010^{163} \\
\text { KQ } 1\end{array}$ & $\begin{array}{l}\text { RCT } \\
\text { UK/Europe }\end{array}$ & $\begin{array}{l}50 \\
47 \\
\text { PCOS }\end{array}$ & $\begin{array}{l}\text { Laparoscopic ovarian diathermy. No drugs to } \\
\text { trigger ovulation. } \\
\text { vs. } \\
\text { CC for up to } 6 \text { cycles plus metformin } 500 \mathrm{mg} \\
\text { tapered upwards. No drugs to trigger ovulation. }\end{array}$ & $\begin{array}{l}\text { Live birth } \\
\text { Surgical complications }\end{array}$ & Good \\
\hline $\begin{array}{l}\text { Palombia, 2011 } \\
\text { KQ } 1\end{array}$ & $\begin{array}{l}\text { RCT } \\
\text { UK/Europe }\end{array}$ & $\begin{array}{l}120 \\
120 \\
\text { PCOS }\end{array}$ & $\begin{array}{l}\text { Metformin } 500 \mathrm{mg} \text { three times daily } \\
\text { vs. } \\
\text { Placebo } \\
\text { Metformin and placebo treatments started on the } \\
\text { same day of GnRH-a administration } \\
\text { (pretreatment) and were continued during the } \\
\text { gonadotropin ovarian stimulation (cotreatment), } \\
\text { treatment that started at least } 14 \text { days later. Both } \\
\text { active drug and placebo were stopped when a } \\
\text { positive pregnancy test or menstrual bleeding } \\
\text { appeared. }\end{array}$ & $\begin{array}{l}\text { Live birth } \\
\text { OHSS }\end{array}$ & Good \\
\hline $\begin{array}{l}\text { Peeraer, } 2015^{288} \\
\text { Across All KQs }\end{array}$ & $\begin{array}{l}\text { RCT } \\
\text { UK/Europe }\end{array}$ & $\begin{array}{l}579 \text { cycles } \\
434 \text { cycles } \\
\text { All }\end{array}$ & $\begin{array}{l}\text { Frozen/thawed embryo transfer in women with a } \\
\text { natural cycle } \\
\text { vs. } \\
\text { Frozen/thawed embryo transfer in women } \\
\text { injected with hMG }\end{array}$ & Live birth & Good \\
\hline
\end{tabular}




\begin{tabular}{|c|c|c|c|c|c|}
\hline $\begin{array}{c}\text { Study } \\
\text { ACRONYM } \\
\text { KQs } \\
\text { Companion Studies }\end{array}$ & $\begin{array}{l}\text { Study Design } \\
\text { Geographic } \\
\text { Location }\end{array}$ & $\begin{array}{l}\text { N Enrolled } \\
\text { N Completed } \\
\text { Underlying } \\
\text { Diagnosis }\end{array}$ & Interventions & $\begin{array}{c}\text { Outcomes } \\
\text { (Subgroups analyzed) }\end{array}$ & Quality \\
\hline $\begin{array}{l}\text { Pourali, } 2017^{241} \\
\text { KQ } 3\end{array}$ & $\begin{array}{l}\text { RCT } \\
\text { Middle East }\end{array}$ & $\begin{array}{l}180 \\
170 \\
\text { Unknown }\end{array}$ & $\begin{array}{l}5 \mathrm{mg} / \text { day letrozole on day 3-7 of menstrual cycle } \\
\text { vs. } \\
100 \mathrm{mg} / \text { day clomiphene on day 3-7 of menstrual } \\
\text { cycle } \\
\text { In both groups, human menopausal } \\
\text { gonadotropin was administered every day } \\
\text { starting on day between 6-8 of cycle. Ovulation } \\
\text { was triggered with urinary Human Chorionic } \\
\text { Gonadotropin ( } 5000 \text { IU) when have two follicles } \\
\text { of } \geq 16 \mathrm{~mm} \text {. IUI was performed } 36 \text { hr later }\end{array}$ & $\begin{array}{l}\text { Miscarriage } \\
\text { OHSS } \\
\text { Cost }\end{array}$ & Fair \\
\hline $\begin{array}{l}\text { Provost, } 2016^{279} \\
\text { Across All KQs }\end{array}$ & $\begin{array}{l}\text { Observational } \\
\text { US }\end{array}$ & $\begin{array}{l}173.968 \text { cycles } \\
173,968 \text { cycles } \\
\text { All }\end{array}$ & $\begin{array}{l}\text { Treatment received in US states that mandated } \\
\text { coverage for IVF } \\
\text { vs. } \\
\text { Treatment received in US states that did not } \\
\text { mandate coverage for IVF }\end{array}$ & $\begin{array}{l}\text { Live birth } \\
\text { Multiple births } \\
\text { Birthweight }\end{array}$ & Good \\
\hline $\begin{array}{l}\text { Qu, } 2017^{252} \\
\text { KQ } 4\end{array}$ & $\begin{array}{l}\text { RCT } \\
\text { Asia }\end{array}$ & $\begin{array}{l}481 \\
442 \\
\text { Tubal }\end{array}$ & $\begin{array}{l}\text { IVF + TEAS-2Hz } \\
\text { Vs. } \\
\text { IVF + TEAS-100 Hz } \\
\text { Vs. } \\
\text { IVF + TEAS-2/100Hz } \\
\text { Vs. } \\
\text { IVF }\end{array}$ & Live birth & Fair \\
\hline $\begin{array}{l}\text { Ragni, } 2012^{218} \\
\text { KQ } 3\end{array}$ & $\begin{array}{l}\text { RCT } \\
\text { UK/Europe }\end{array}$ & $\begin{array}{l}304 \\
249 \\
\text { Unknown }\end{array}$ & $\begin{array}{l}\text { CC oral tablets at dose of } 150 \mathrm{mg} / \mathrm{d} \text { from day } 3 \\
\text { to day } 7 \\
\text { vs. } \\
\text { Daily SQ injections of triptoreline started on day } \\
\text { one or two of the menstrual cycle and } 450 \text { IU of } \\
\text { SQ rFSH from day } 3 \text { of the cycle. }\end{array}$ & $\begin{array}{l}\text { Live birth (ovarian reserve) } \\
\text { Patient costs }\end{array}$ & Fair \\
\hline
\end{tabular}




\begin{tabular}{|c|c|c|c|c|c|}
\hline $\begin{array}{c}\text { Study } \\
\text { ACRONYM } \\
\text { KQs } \\
\text { Companion Studies }\end{array}$ & $\begin{array}{l}\text { Study Design } \\
\text { Geographic } \\
\text { Location }\end{array}$ & $\begin{array}{l}\text { N Enrolled } \\
\text { N Completed } \\
\text { Underlying } \\
\text { Diagnosis }\end{array}$ & Interventions & $\begin{array}{c}\text { Outcomes } \\
\text { (Subgroups analyzed) }\end{array}$ & Quality \\
\hline $\begin{array}{l}\text { Rahman, } 2017^{264} \\
\text { KQ } 5\end{array}$ & $\begin{array}{l}\text { RCT } \\
\text { UK/Europe }\end{array}$ & $\begin{array}{l}66 \\
61 \\
\text { Male }\end{array}$ & $\begin{array}{l}\text { Recombinant FSH (r-FSH) supplemented by r- } \\
\text { LH in the late follicular phase starting the same } \\
\text { day of GnRH-antagonist (GnRH-ant) } \\
\text { administration } \\
\text { vs. } \\
\text { r-FSH alone } \\
\text { All patients were stimulated with a GnRH-ant } \\
\text { flexible protocol starting r-FSH on day } 2 \text { of a } \\
\text { spontaneous or induced cycle. hCG (10000 IU) } \\
\text { was administered by intramuscular route when at } \\
\text { least } 2 \text { follicles reached } 18 \mathrm{~mm} \text { in diameter }\end{array}$ & Miscarriage & Fair \\
\hline $\begin{array}{l}\text { Rashidi, } 2015^{143} \\
\text { KQ } 1\end{array}$ & $\begin{array}{l}\text { RCT } \\
\text { Middle East }\end{array}$ & $\begin{array}{l}276 \\
276 \\
\text { PCOS }\end{array}$ & $\begin{array}{l}\text { Ovulation induction with CC day 3-7 followed by: } \\
75 \text { IU of rFSH day 7-9 and IUI } \\
\text { vs. } \\
75 \text { IU of hMG day 7-9 and IUI }\end{array}$ & $\begin{array}{l}\text { Live birth } \\
\text { Miscarriage } \\
\text { Multiple births } \\
\text { OHSS }\end{array}$ & Fair \\
\hline $\begin{array}{l}\text { Rashidi,2013216 } \\
\text { KQ } 3\end{array}$ & $\begin{array}{l}\text { RCT } \\
\text { Middle East }\end{array}$ & $\begin{array}{l}280 \\
259 \\
\text { Unknown }\end{array}$ & $\begin{array}{l}\text { Induction of ovulation with CC } 100 \mathrm{mg} / \mathrm{d} \text { on cycle } \\
\text { days } 3-7 \text {, followed by } 75 \mathrm{IU} / \mathrm{d} \text { hMG on cycle } \\
\text { days } 7-9 \text {, followed by triggered ovulation and IUI } \\
\text { vs. } \\
\text { Same protocol except hMG replaced by } 75 \mathrm{IU} \\
\text { rFSH. }\end{array}$ & $\begin{array}{l}\text { Live birth } \\
\text { Miscarriage } \\
\text { OHSS }\end{array}$ & Fair \\
\hline $\begin{array}{l}\text { Razi, } 2013^{255} \\
\text { KQ } 5\end{array}$ & $\begin{array}{l}\text { RCT } \\
\text { Middle East }\end{array}$ & $\begin{array}{l}182 \\
182 \\
\text { Male }\end{array}$ & $\begin{array}{l}\text { Laser Assisted Hatching (LAH) following ICSI } \\
\text { vs. } \\
\text { Control group. Intact transferred embryos without } \\
\text { LAH }\end{array}$ & $\begin{array}{l}\text { Live birth } \\
\text { Multiple births } \\
\text { Congenital anomalies }\end{array}$ & Poor \\
\hline $\begin{array}{l}\text { Reindollar, } 2010^{52} \\
\text { FASTT } \\
\text { KQ } 3\end{array}$ & $\begin{array}{l}\text { RCT } \\
\text { US }\end{array}$ & $\begin{array}{l}503 \\
503 \\
\text { Unknown }\end{array}$ & $\begin{array}{l}\text { CC/IUI } \times 3 \text { cycles followed by up to } 3 \text { cycles } \\
\text { gonadotropin/IUI followed by } 6 \text { cycles of IVF (of } \\
\text { which } 2 \text { could be frozen cycles) } \\
\text { vs. } \\
\text { CC/IUI } \times 3 \text { cycles followed by } 6 \text { cycles of IVF (of } \\
\text { which } 2 \text { could be frozen cycles) }\end{array}$ & $\begin{array}{l}\text { Live birth } \\
\text { Ectopic pregnancy } \\
\text { Miscarriage } \\
\text { Multiple births } \\
\text { Time to pregnancy } \\
\text { OHSS } \\
\text { Birthweight } \\
\text { Neonatal death } \\
\text { Health system costs }\end{array}$ & Good \\
\hline
\end{tabular}




\begin{tabular}{|c|c|c|c|c|c|}
\hline $\begin{array}{c}\text { Study } \\
\text { ACRONYM } \\
\text { KQs } \\
\text { Companion Studies }\end{array}$ & $\begin{array}{l}\text { Study Design } \\
\text { Geographic } \\
\text { Location }\end{array}$ & $\begin{array}{l}\text { N Enrolled } \\
\text { N Completed } \\
\text { Underlying } \\
\text { Diagnosis }\end{array}$ & Interventions & $\begin{array}{c}\text { Outcomes } \\
\text { (Subgroups analyzed) }\end{array}$ & Quality \\
\hline $\begin{array}{l}\text { Rubio, } 2013^{217} \\
\text { KQ } 3\end{array}$ & $\begin{array}{l}\text { RCT } \\
\text { UK/Europe }\end{array}$ & $\begin{array}{l}274 \\
274 \\
\text { Unknown }\end{array}$ & $\begin{array}{l}\text { IVF with day- } 5 \text { blastocyst transfer (no PGD) } \\
\text { vs. } \\
\text { IVF, embryo biopsy and FISH for } 9 \\
\text { chromosomes on day } 3 \text {, transfer on day } 5\end{array}$ & $\begin{array}{l}\text { Live birth } \\
\text { Miscarriage } \\
\text { Multiple births } \\
\text { (RIF and AMA) }\end{array}$ & Fair \\
\hline $\begin{array}{l}\text { Schendelaar, } 2011^{273} \\
\text { Across All KQs }\end{array}$ & $\begin{array}{l}\text { Observational } \\
\text { UK/Europe }\end{array}$ & $\begin{array}{l}\text { NR } \\
310\end{array}$ & $\begin{array}{l}\text { Children who were not born to sub-fertile parents } \\
\text { vs. } \\
\text { Children born to sub-fertile parents who received } \\
\text { IVF (either COH-IVF or MNC-IVF) }\end{array}$ & Neurodevelopmental issues & Fair \\
\hline $\begin{array}{l}\text { Seckin, } 2014^{209} \\
\text { KQ } 3\end{array}$ & $\begin{array}{l}\text { RCT } \\
\text { UK/Europe }\end{array}$ & $\begin{array}{l}149 \\
139 \\
\text { Unknown }\end{array}$ & $\begin{array}{l}\text { Luteal phase support with } 90 \mathrm{mg} / \mathrm{d} \text { vaginal } 8 \% \\
\text { progesterone gel starting on the day of IUI until } \\
\text { pregnancy testing } \\
\text { vs. } \\
\text { No drug for luteal phase support }\end{array}$ & Live birth & Good \\
\hline $\begin{array}{l}\text { Selman, } 2016^{237} \\
\text { KQ } 3\end{array}$ & $\begin{array}{l}\text { RCT } \\
\text { UK/Europe }\end{array}$ & $\begin{array}{l}85 \\
76 \\
\text { Unknown }\end{array}$ & $\begin{array}{l}\text { Clomiphene citrate and corifollitropin alfa for the } \\
\text { first } 7 \text { days of stimulation followed by } \\
\text { recombinant follicle stimulating hormone (rFSH) } \\
\text { in a gonadotropin-releasing hormone antagonist } \\
\text { protocol } \\
\text { vs. } \\
\text { Clomiphene citrate and a daily injection of rFSH } \\
\text { in a gonadotropin-releasing hormone antagonist } \\
\text { protocol }\end{array}$ & $\begin{array}{l}\text { Live birth } \\
\text { Miscarriage }\end{array}$ & Good \\
\hline $\begin{array}{l}\text { Shi, } 2018^{262} \\
\text { KQ } 4 \text { and } 5\end{array}$ & $\begin{array}{l}\text { RCT } \\
\text { Asia }\end{array}$ & $\begin{array}{l}2,157 \\
1,782 \\
\text { Tubal, Male }\end{array}$ & $\begin{array}{l}\text { Fresh-embryo transfer } \\
\text { vs. } \\
\text { Embryo cryopreservation followed by frozen- } \\
\text { embryo transfer }\end{array}$ & $\begin{array}{l}\text { Live birth } \\
\text { Multiple births } \\
\text { Miscarriage } \\
\text { OHSS } \\
\text { Birthweight }\end{array}$ & Good \\
\hline $\begin{array}{l}\text { Sismanoglu, } 2009^{267} \\
\text { KQ } 6\end{array}$ & $\begin{array}{l}\text { RCT } \\
\text { UK/Europe }\end{array}$ & $\begin{array}{l}50 \\
44 \\
\text { Donor }\end{array}$ & $\begin{array}{l}\text { Donor triggering with } \mathrm{hCG} \\
\text { vs. } \\
\text { Donor triggering with } \mathrm{GnRH} \text { agonist }\end{array}$ & OHSS & Fair \\
\hline
\end{tabular}




\begin{tabular}{|c|c|c|c|c|c|}
\hline $\begin{array}{c}\text { Study } \\
\text { ACRONYM } \\
\text { KQs } \\
\text { Companion Studies }\end{array}$ & $\begin{array}{l}\text { Study Design } \\
\text { Geographic } \\
\text { Location }\end{array}$ & $\begin{array}{l}\text { N Enrolled } \\
\mathrm{N} \text { Completed } \\
\text { Underlying } \\
\text { Diagnosis }\end{array}$ & Interventions & $\begin{array}{c}\text { Outcomes } \\
\text { (Subgroups analyzed) }\end{array}$ & Quality \\
\hline $\begin{array}{l}\text { Spaan, 2015 } \\
\text { OMEGA } \\
\text { Across All KQs } \\
\text { Companions: } \\
\text { Spaan, 2016 } \\
\text { van Leeuwen, } 2011^{126}\end{array}$ & $\begin{array}{l}\text { Observational } \\
\text { UK/Europe }\end{array}$ & $\begin{array}{l}25,108 \\
25,108 \\
\text { All }\end{array}$ & $\begin{array}{l}\text { Received at least one IVF cycle with ovarian } \\
\text { stimulation } \\
\text { vs. } \\
\text { Subfertile women with other treatments including } \\
\text { tubal surgery, IUI, or hormonal treatment OR } \\
\text { withdrew from IVF waiting list }\end{array}$ & Maternal cancer & Good \\
\hline $\begin{array}{l}\text { Stadtmauer, 2011'159 } \\
\text { KQ } 1\end{array}$ & $\begin{array}{l}\text { RCT } \\
\text { US }\end{array}$ & $\begin{array}{l}98 \\
\text { NR } \\
\text { PCOS }\end{array}$ & $\begin{array}{l}\text { rFSH Follistim starting on cycle day } 3 \text { until the } \\
\text { appropriate follicle size was reached. } \\
\text { vs. } \\
\text { Ganirelix } 0.25 \mathrm{mg} \text { SQ/d added to rFSH in a } \\
\text { flexible protocol when the leading follicle } \\
\text { diameter reached R13 mm. } \\
\text { vs. } \\
\text { rFSH and Ganirelix }\end{array}$ & $\begin{array}{l}\text { Live birth } \\
\text { Miscarriage } \\
\text { Multiple births }\end{array}$ & Fair \\
\hline $\begin{array}{l}\text { Stewart, } 2013^{203} \\
\text { KQ } 2\end{array}$ & $\begin{array}{l}\text { Observational } \\
\text { Australia/NZ }\end{array}$ & $\begin{array}{l}22,045 \\
21,646 \\
\text { Endometriosis }\end{array}$ & $\begin{array}{l}\text { Women receiving infertility treatment but not IVF } \\
\text { vs. } \\
\text { Women receiving IVF }\end{array}$ & Maternal cancer (parity) & Fair \\
\hline $\begin{array}{l}\text { Tartagni, } 2015^{207} \\
\text { KQ } 3\end{array}$ & $\begin{array}{l}\text { RCT } \\
\text { UK/Europe }\end{array}$ & $\begin{array}{l}109 \\
109 \\
\text { Unknown }\end{array}$ & $\begin{array}{l}\text { IVF with DHEA } 75 \mathrm{mg} / \mathrm{d} \text { for } 8 \text { weeks pre- } \\
\text { treatment } \\
\text { vs. } \\
\text { Placebo }\end{array}$ & $\begin{array}{l}\text { Live birth } \\
\text { Miscarriage }\end{array}$ & Fair \\
\hline $\begin{array}{l}\text { Tehraninejad, } 2010^{161} \\
\text { KQ } 1\end{array}$ & $\begin{array}{l}\text { RCT } \\
\text { Middle East }\end{array}$ & $\begin{array}{l}95 \\
90 \\
\text { Pcos }\end{array}$ & $\begin{array}{l}\text { Pretreatment with OCP for } 21 \text { days, controlled } \\
\text { ovarian stim started on day } 2-3 \text {. Cetrolix } \\
\text { (antagonist) } 0.25 \mathrm{mg} \text { SQ started when follicles } \\
12-14 \mathrm{~mm} \text {. } \\
\text { vs. } \\
\text { Control group. Pretreatment with OCP for } 21 \\
\text { days, along with buserelin (agonist) } 500 \mathrm{mcg} / \mathrm{d} \\
\text { SQ. Buserelin then reduced to } 250 \mathrm{mcg} / \mathrm{d} \text {. } \\
\text { Controlled ovarian stimulation with } \mathrm{hMG} \text {. }\end{array}$ & OHSS & Fair \\
\hline
\end{tabular}




\begin{tabular}{|c|c|c|c|c|c|}
\hline $\begin{array}{c}\text { Study } \\
\text { ACRONYM } \\
\text { KQs } \\
\text { Companion Studies }\end{array}$ & $\begin{array}{l}\text { Study Design } \\
\text { Geographic } \\
\text { Location }\end{array}$ & $\begin{array}{l}\text { N Enrolled } \\
\text { N Completed } \\
\text { Underlying } \\
\text { Diagnosis }\end{array}$ & Interventions & $\begin{array}{c}\text { Outcomes } \\
\text { (Subgroups analyzed) }\end{array}$ & Quality \\
\hline $\begin{array}{l}\text { Toftager, } 2016^{275} \\
\text { Across All KQs }\end{array}$ & $\begin{array}{l}\text { RCT } \\
\text { UK/Europe }\end{array}$ & $\begin{array}{l}1,099 \\
1,023 \\
\text { All }\end{array}$ & $\begin{array}{l}\text { GnRH antagonist protocol: Women with regular } \\
\text { cycles ( } \leq 35 \text { days) received daily injections with } \\
\text { recombinant human FSH. Controlled ovarian } \\
\text { stimulation (COS) was initiated on cycle day } 2 \text { or } \\
3 \text { and continued until the day of ovulation } \\
\text { induction. A fixed dose of rFSH was used for the } \\
\text { first } 6 \text { days, either } 150 \text { or } 225 \text { IU according to } \\
\text { age } \leq 36 \text { years or age }>36 \text { years. After } 6 \text { days of } \\
\text { stimulation the rFSH doses were adjusted } \\
\text { according to ovarian response evaluated using } \\
\text { transvaginal ultrasonography. A daily GnRH } \\
\text { antagonist (Ganirelix) dose of } 0.25 \text { mg was used } \\
\text { starting on stimulation day } 6 \text { and was } \\
\text { administered until the day of ovulation induction. } \\
\text { vs. } \\
\text { GnRH agonist protocol: Women with regular } \\
\text { cycles ( } \leq 35 \text { days) started daily nasal } \\
\text { administration of } 200 \text { mg three times daily of } \\
\text { GnRH agonist (Nafarelin-Synarelaw; Pfizer, } \\
\text { Ballerup, Denmark) on Day } 21 \text { of the preceding } \\
\text { cycle to initiate pituitary down-regulation if luteal } \\
\text { phase characteristics were verified by } \\
\text { transvaginal ultrasound. After } 14 \text { days of GnRH } \\
\text { agonist administration (cycle day } 35 \text { ) } \\
\text { gonadotrophin stimulation was initiated if ovarian } \\
\text { follicles } \leq 10 \text { mm diameter and endometrial } \\
\text { thickness } \leq 6 \text { mm was confirmed by transvaginal } \\
\text { ultrasound. A fixed dose of rFSH (Puregon) was } \\
\text { used for the first } 6 \text { days, either } 150 \text { or } 225 \text { IU } \\
\text { according to age } \leq 36 \text { or . } 36 \text { years. After } 6 \text { days } \\
\text { of stimulation the rFSH doses were adjusted } \\
\text { according to ovarian response evaluated using } \\
\text { transvaginal ultrasonography. Nasal } \\
\text { administration of } 200 \text { mg two times daily of } \\
\text { GnRH agonist was continued during rFSH } \\
\text { stimulation and until ovulation induction. }\end{array}$ & $\begin{array}{l}\text { Live birth } \\
\text { OHSS }\end{array}$ & Good \\
\hline $\begin{array}{l}\text { Toftager, } 2017^{285} \\
\text { Across All KQs }\end{array}$ & $\begin{array}{l}\text { RCT } \\
\text { UK/Europe }\end{array}$ & $\begin{array}{l}1,050 \\
1,023 \\
\text { All }\end{array}$ & $\begin{array}{l}\text { Short GnRH-antagonist protocol } \\
\text { vs. } \\
\text { Long GnRH-agonist protocol }\end{array}$ & $\begin{array}{l}\text { Live birth } \\
\text { Miscarriage } \\
\text { Time to pregnancy }\end{array}$ & Good \\
\hline
\end{tabular}




\begin{tabular}{|c|c|c|c|c|c|}
\hline $\begin{array}{c}\text { Study } \\
\text { ACRONYM } \\
\text { KQs } \\
\text { Companion Studies }\end{array}$ & $\begin{array}{l}\text { Study Design } \\
\text { Geographic } \\
\text { Location }\end{array}$ & $\begin{array}{l}\text { N Enrolled } \\
\text { N Completed } \\
\text { Underlying } \\
\text { Diagnosis }\end{array}$ & Interventions & $\begin{array}{c}\text { Outcomes } \\
\text { (Subgroups analyzed) }\end{array}$ & Quality \\
\hline $\begin{array}{l}\text { Topcu, } 2017^{191} \\
\text { KQ } 1\end{array}$ & $\begin{array}{l}\text { RCT } \\
\text { Middle East }\end{array}$ & $\begin{array}{l}101 \\
88 \\
\text { PCOS }\end{array}$ & $\begin{array}{l}\text { Tamoxifen } 20 \mathrm{mg} / \text { daily for } 5 \text { consecutive days } \\
\text { vs. } \\
\text { Clomiphene citrate } 50 \mathrm{mg} / \text { daily for } 5 \text { consecutive } \\
\text { days } \\
\text { Both medications were started on day } 5 \text { of the } \\
\text { menstrual cycle }\end{array}$ & $\begin{array}{l}\text { Live birth } \\
\text { Miscarriage } \\
\text { Multiple births } \\
\text { OHSS }\end{array}$ & Fair \\
\hline $\begin{array}{l}\text { Tsai, } 2011^{257} \\
\text { KQ } 5\end{array}$ & $\begin{array}{l}\text { Observational } \\
\text { Asia }\end{array}$ & $\begin{array}{l}\text { NA } \\
191 \text { cycles } \\
\text { Male }\end{array}$ & $\begin{array}{l}\text { Testicular sperm extraction (TESE) via biopsy in } \\
\text { men w/ azoospermia } \\
\text { vs. } \\
\text { Fresh ejaculated sperm from men with extreme } \\
\text { severe oligo-astheno-teratozoospermia (OAT) } \\
\text { sperm }\end{array}$ & $\begin{array}{l}\text { Live birth } \\
\text { Miscarriage } \\
\text { Birthweight } \\
\text { Congenital anomalies }\end{array}$ & Poor \\
\hline $\begin{array}{l}\text { van Rijswijk, } 2017^{242} \\
\text { KQ } 3\end{array}$ & $\begin{array}{l}\text { RCT } \\
\text { UK/Europe }\end{array}$ & $\begin{array}{l}498 \\
481 \\
\text { Unknown }\end{array}$ & $\begin{array}{l}15 \text { min of supine immobilization following IUI for } \\
\text { a maximum of six cycles } \\
\text { vs. } \\
\text { Immediate mobilization following IUI }\end{array}$ & $\begin{array}{l}\text { Live birth } \\
\text { Multiple births } \\
\text { Miscarriage } \\
\text { Time to pregnancy }\end{array}$ & Fair \\
\hline $\begin{array}{l}\text { van Rumste, } 2014^{212} \\
\text { KQ } 3\end{array}$ & $\begin{array}{l}\text { RCT } \\
\text { UK/Europe }\end{array}$ & $\begin{array}{l}116 \\
116 \\
\text { Unknown } \\
\end{array}$ & $\begin{array}{l}\text { IVF elective single embryo transfer } \\
\text { vs. } \\
\mathrm{COH} / \mathrm{IUI} \text {, maximum treatment duration } 3 \text { cycles }\end{array}$ & $\begin{array}{l}\text { Live birth } \\
\text { Health system costs }\end{array}$ & Fair \\
\hline $\begin{array}{l}\text { Verhoeve, } 2013^{249} \\
\text { KQ } 4\end{array}$ & $\begin{array}{l}\text { Observational } \\
\text { UK/Europe }\end{array}$ & $\begin{array}{l}5000 \\
5000 \\
\text { Tubal Factor }\end{array}$ & $\begin{array}{l}\text { No diagnostics and no treatment } \\
\text { vs. } \\
\text { No diagnostics and immediate treatment (up to } 3 \\
\text { IVF treatments) } \\
\text { vs. } \\
\text { No diagnostics and delayed treatment (no } \\
\text { treatment for } 1 \text { yr, then up to } 3 \text { IVF treatments) } \\
\text { vs. } \\
\text { Hysterosalpingogram followed by tailored } \\
\text { treatment (delayed or immediate IVF) }\end{array}$ & $\begin{array}{l}\text { Live birth } \\
\text { Patient costs }\end{array}$ & Good \\
\hline $\begin{array}{l}\text { Vitek, } 2013^{215} \\
\text { KQ } 3\end{array}$ & $\begin{array}{l}\text { Observational } \\
\text { US }\end{array}$ & $\begin{array}{l}154 \\
154 \\
\text { Unknown }\end{array}$ & $\begin{array}{l}\text { Split IVF/ICSI } \\
\text { vS. } \\
\text { Computer simulated- all IVF } \\
\text { vs. } \\
\text { Computer simulated- all ICSI }\end{array}$ & Patient costs & Good \\
\hline
\end{tabular}




\begin{tabular}{|c|c|c|c|c|c|}
\hline $\begin{array}{c}\text { Study } \\
\text { ACRONYM } \\
\text { KQs } \\
\text { Companion Studies }\end{array}$ & $\begin{array}{l}\text { Study Design } \\
\text { Geographic } \\
\text { Location }\end{array}$ & $\begin{array}{l}\text { N Enrolled } \\
\text { N Completed } \\
\text { Underlying } \\
\text { Diagnosis }\end{array}$ & Interventions & $\begin{array}{c}\text { Outcomes } \\
\text { (Subgroups analyzed) }\end{array}$ & Quality \\
\hline $\begin{array}{l}\text { Wang, } 2016^{171} \\
\text { KQ } 1\end{array}$ & $\begin{array}{l}\text { RCT } \\
\text { Asia }\end{array}$ & $\begin{array}{l}120 \\
108 \\
\text { PCOS }\end{array}$ & $\begin{array}{l}\text { hMG } 150-225 I \text { Iday and MPA 10mg/day from } \\
\text { day } 3 \text { of menstruation. Both cotriggered with } \\
\text { GnRHa and hCG and underwent IVF/ICSI. } \\
\text { vs. } \\
\text { Patients received GnRHa (decapeptyl) } 0.1 \mathrm{mg} \\
\text { beginning of day } 2 \text { of menstruation and adding } \\
\text { hMG on day } 3 \text { of menstruation. Both cotriggered } \\
\text { with GnRHa and hCG and underwent IVF/ICSI. }\end{array}$ & $\begin{array}{l}\text { Multiple births } \\
\text { Miscarriage } \\
\text { Ectopic pregnancy } \\
\text { OHSS }\end{array}$ & Fair \\
\hline $\begin{array}{l}\text { Wang, } 2017^{286} \\
\text { Across All KQs }\end{array}$ & $\begin{array}{l}\text { Observational } \\
\text { US }\end{array}$ & $\begin{array}{l}509,938 \text { cycles } \\
509,938 \text { cycles } \\
\text { All }\end{array}$ & $\begin{array}{l}\text { Fresh blastocyst } \\
\text { vs. } \\
\text { Fresh non-blastocyst } \\
\text { vs. } \\
\text { Frozen blastocyst } \\
\text { vs. } \\
\text { Frozen non-blastocyst }\end{array}$ & $\begin{array}{l}\text { Live birth } \\
\text { Miscarriage } \\
\text { Ectopic pregnancy }\end{array}$ & Good \\
\hline $\begin{array}{l}\text { Weiss, } 2018^{187} \\
\text { KQ } 1 \text { and } 3\end{array}$ & $\begin{array}{l}\text { RCT } \\
\text { UK/Europe }\end{array}$ & $\begin{array}{l}666 \\
661 \\
\text { PCOS, Unknown }\end{array}$ & $\begin{array}{l}\text { Six cycles with gonadotrophins plus intrauterine } \\
\text { insemination } \\
\text { vs. } \\
\text { Six cycles with gonadotrophins plus intercourse } \\
\text { vs. } \\
\text { Six cycles with clomifene citrate plus intrauterine } \\
\text { insemination } \\
\text { vs. } \\
\text { Six cycles with clomifene citrate plus intercourse } \\
\text { Clomifene citrate dosages varied from } 50 \text { to } 150 \\
\text { mg daily orally and gonadotrophin starting dose } \\
\text { was } 50 \text { or } 75 \text { IU daily subcutaneously }\end{array}$ & $\begin{array}{l}\text { Live birth } \\
\text { Multiple births } \\
\text { Miscarriage } \\
\text { Ectopic pregnancy } \\
\text { Time to pregnancy } \\
\text { Birthweight }\end{array}$ & Good \\
\hline $\begin{array}{l}\text { Williams, } 2013^{213} \\
\text { KQ 3, } 5\end{array}$ & $\begin{array}{l}\text { Observational } \\
\text { UK/Europe }\end{array}$ & $\begin{array}{l}106,013 \\
\text { NA } \\
\text { Unknown, Male }\end{array}$ & $\begin{array}{l}\text { Assisted Reproduction } \\
\text { vs. } \\
\text { General Population }\end{array}$ & Child cancer & Fair \\
\hline $\begin{array}{l}\text { Williams, } 2018^{289} \\
\text { Across All KQs }\end{array}$ & $\begin{array}{l}\text { Observational } \\
\text { UK/Europe }\end{array}$ & $\begin{array}{l}255,786 \\
255,786 \\
\text { All }\end{array}$ & $\begin{array}{l}\text { Assisted Reproduction } \\
\text { vs. } \\
\text { General Population }\end{array}$ & Maternal cancer & Good \\
\hline
\end{tabular}




\begin{tabular}{|c|c|c|c|c|c|}
\hline $\begin{array}{c}\text { Study } \\
\text { ACRONYM } \\
\text { KQs } \\
\text { Companion Studies }\end{array}$ & $\begin{array}{l}\text { Study Design } \\
\text { Geographic } \\
\text { Location }\end{array}$ & $\begin{array}{l}\text { N Enrolled } \\
\text { N Completed } \\
\text { Underlying } \\
\text { Diagnosis }\end{array}$ & Interventions & $\begin{array}{c}\text { Outcomes } \\
\text { (Subgroups analyzed) }\end{array}$ & Quality \\
\hline $\begin{array}{l}\text { Wiser, } 2010^{220} \\
\text { KQ } 3\end{array}$ & $\begin{array}{l}\text { RCT } \\
\text { Middle East }\end{array}$ & $\begin{array}{l}33 \\
33 \\
\text { Unknown }\end{array}$ & $\begin{array}{l}\text { Long-stimulation protocol IVF with } 75 \text { mg DHEA } \\
\text { orally, once a day, at least } 6 \text { weeks before } \\
\text { starting the first cycle of ovulation induction. } \\
\text { Patients who did not conceive and continued to } \\
\text { the second cycle took DHEA for at least 16-18 } \\
\text { weeks. } \\
\text { vs. } \\
\text { Standard long-stimulation protocol IVF }\end{array}$ & Live birth (ovarian reserve) & Fair \\
\hline $\begin{array}{l}\text { Wu, } 2016^{175} \\
\text { KQ } 1\end{array}$ & $\begin{array}{l}\text { RCT } \\
\text { Asia }\end{array}$ & $\begin{array}{l}644 \\
644 \text { in analysis } \\
\text { PCOS }\end{array}$ & $\begin{array}{l}\text { Letrozole } 2.5 \mathrm{mg} \text { plus placebo for Berberine, } \\
\text { increased to } 5.0 \mathrm{mg} \text { on days } 3-7 \text { of last } 3 \\
\text { treatment cycles } \\
\text { vs. } \\
\text { Berberine } 1.5 \mathrm{mg} \text { plus placebo for Letrozole } \\
\text { vs. } \\
\text { Combination letrozole and berberine }\end{array}$ & $\begin{array}{l}\text { Live birth } \\
\text { Multiple births } \\
\text { Miscarriage } \\
\text { Ectopic pregnancy } \\
\text { Birthweight } \\
\text { Neonatal death }\end{array}$ & Good \\
\hline $\begin{array}{l}\text { Wu, } 2017^{188} \\
\text { KQ } 1\end{array}$ & $\begin{array}{l}\text { RCT } \\
\text { Asia }\end{array}$ & $\begin{array}{l}1,000 \\
926 \\
\text { PCOS }\end{array}$ & $\begin{array}{l}\text { Active acupuncture administered twice a week } \\
\text { plus clomiphene administered for } 5 \text { days per } \\
\text { cycle, for up to } 4 \text { cycles } \\
\text { vs. } \\
\text { Active acupuncture administered twice a week } \\
\text { plus placebo for clomiphene administered for } 5 \\
\text { days per cycle, for up to } 4 \text { cycles } \\
\text { vs. } \\
\text { Control acupuncture administered twice a week } \\
\text { plus clomiphene administered for } 5 \text { days per } \\
\text { cycle, for up to } 4 \text { cycles } \\
\text { vs. } \\
\text { Control acupuncture administered twice a week } \\
\text { plus placebo for clomiphene administered for } 5 \\
\text { days per cycle, for up to } 4 \text { cycles }\end{array}$ & $\begin{array}{l}\text { Live birth } \\
\text { Multiple births } \\
\text { Miscarriage } \\
\text { Ectopic pregnancy } \\
\text { Neonatal death }\end{array}$ & Good \\
\hline $\begin{array}{l}\text { Xiong, } 2017^{263} \\
\text { KQ } 5\end{array}$ & $\begin{array}{l}\text { Observational } \\
\text { US }\end{array}$ & $\begin{array}{l}141,030 \\
141,030 \\
\text { Male }\end{array}$ & $\begin{array}{l}\text { IVF } \\
\text { VS. } \\
\text { ICSI }\end{array}$ & $\begin{array}{l}\text { Neonatal death } \\
\text { Preterm birth } \\
\text { Congenital anomaly }\end{array}$ & Fair \\
\hline
\end{tabular}




\begin{tabular}{|c|c|c|c|c|c|}
\hline $\begin{array}{c}\text { Study } \\
\text { ACRONYM } \\
\text { KQs } \\
\text { Companion Studies }\end{array}$ & $\begin{array}{l}\text { Study Design } \\
\text { Geographic } \\
\text { Location }\end{array}$ & $\begin{array}{l}\text { N Enrolled } \\
\text { N Completed } \\
\text { Underlying } \\
\text { Diagnosis }\end{array}$ & Interventions & $\begin{array}{c}\text { Outcomes } \\
\text { (Subgroups analyzed) }\end{array}$ & Quality \\
\hline $\begin{array}{l}\text { Yapca, } 2015^{206} \\
\text { KQ } 3\end{array}$ & $\begin{array}{l}\text { RCT } \\
\text { UK/Europe }\end{array}$ & $\begin{array}{l}80 \\
80 \\
\text { Unknown }\end{array}$ & $\begin{array}{l}\text { CC } 100 \mathrm{mg} / \mathrm{d} \text { on cycle days } 3-7 \text { followed by } \\
\text { time-limited hydrotubation performed after } \\
\text { detection of the dominant follicle and then timed } \\
\text { intercourse } \\
\text { vs. } \\
\text { No hydrotubation }\end{array}$ & $\begin{array}{l}\text { Live birth } \\
\text { Miscarriage }\end{array}$ & Fair \\
\hline $\begin{array}{l}\text { Yazici, } 2014^{144} \\
\text { KQ } 1\end{array}$ & $\begin{array}{l}\text { RCT } \\
\text { UK/Europe }\end{array}$ & $\begin{array}{l}110 \\
56 \\
\text { PCOS }\end{array}$ & $\begin{array}{l}\text { Ovarian stimulation using rFSH followed by IUI } \\
\text { and luteal support with vaginal micronized } \\
\text { progesterone } 300 \mathrm{mg} / \mathrm{d} \\
\text { vs. } \\
\text { No luteal support }\end{array}$ & Live birth & Poor \\
\hline $\begin{array}{l}\text { Yildiz, } 2014^{211} \\
\text { KQ } 3\end{array}$ & $\begin{array}{l}\text { RCT } \\
\text { UK/Europe }\end{array}$ & $\begin{array}{l}180 \\
180 \\
\text { Unknown }\end{array}$ & $\begin{array}{l}\text { Follitropin alpha, follitropin beta, uhMG and } \\
\text { urofolitropin were used for ovarian stimulation. } \\
\text { Ovulation induction was started between } 2-5 \\
\text { days of menstruation on patients who had no } \\
\text { residual cysts larger than } 15 \text { mm as visualized } \\
\text { with basal transvaginal USG (ultrasound). All } \\
\text { patients had } 75-150 \text { IU/d drug as an initial dose. } \\
\text { On cycle day 5-6, stimulated follicles were } \\
\text { measured ultrasonographically. Induction doses } \\
\text { were increased or decreased between } 37.5-75 \\
\text { IU/d according to follicle size. When } 1-2 \text { follicles } \\
\text { reached a mean diameter of } 17 \text { mm, } 250 \text { mcg of } \\
\text { rhCG was administered to trigger ovulation. } \\
\text { Uterine washing was accomplished by } \\
\text { introducing a silicone catheter through the } \\
\text { internal cervical os, after which } 20 \text { cc saline and } \\
1 \text { cc jetocain were slowly injected. The speculum } \\
\text { was removed and the procedure completed after } \\
\text { the injection. At } 35-36 \text { hours after the hCG } \\
\text { injection, IUI was performed. } \\
\text { vs. } \\
\text { Same procedures except no uterine washing } \\
\text { performed }\end{array}$ & $\begin{array}{l}\text { Live birth } \\
\text { Miscarriage }\end{array}$ & Poor \\
\hline
\end{tabular}




\begin{tabular}{|c|c|c|c|c|c|}
\hline $\begin{array}{c}\text { Study } \\
\text { ACRONYM } \\
\text { KQs } \\
\text { Companion Studies } \\
\end{array}$ & $\begin{array}{l}\text { Study Design } \\
\text { Geographic } \\
\text { Location }\end{array}$ & $\begin{array}{l}\text { N Enrolled } \\
\text { N Completed } \\
\text { Underlying } \\
\text { Diagnosis }\end{array}$ & Interventions & $\begin{array}{l}\text { Outcomes } \\
\text { (Subgroups analyzed) }\end{array}$ & Quality \\
\hline $\begin{array}{l}\text { Youssef, 2016 } \\
\text { KQ } 3\end{array}$ & $\begin{array}{l}\text { RCT } \\
\text { Africa }\end{array}$ & $\begin{array}{l}394 \\
394 \\
\text { Unknown }\end{array}$ & 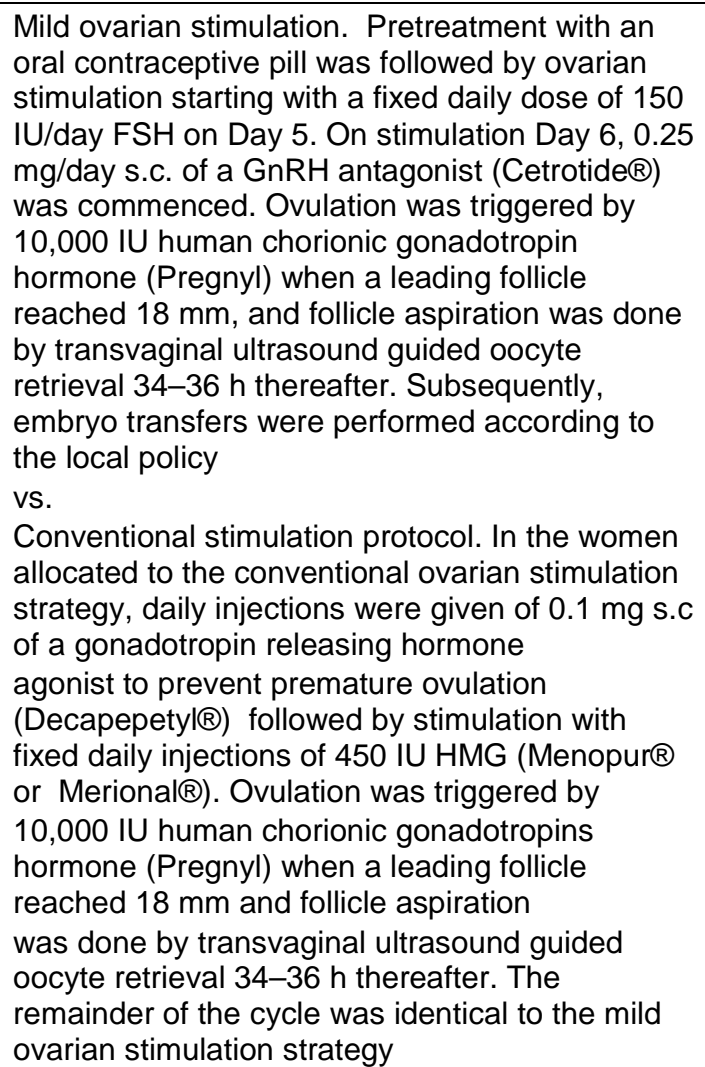 & Miscarriage & Good \\
\hline $\begin{array}{l}\text { Yu, 2018 } \\
\text { KQ } 3\end{array}$ & $\begin{array}{l}\text { RCT } \\
\text { Asia }\end{array}$ & $\begin{array}{l}116 \\
116 \\
\text { Unknown }\end{array}$ & $\begin{array}{l}\text { Modified GnRH agonist (triptorelin) } \\
\text { vs. } \\
\text { Mild stimulation protocol with letrozole } \\
\text { vs. } \\
\text { Antagonist protocol with triptorelin }\end{array}$ & Live birth & Fair \\
\hline $\begin{array}{l}\text { Zahran, } 2018^{192} \\
\text { KQ } 1\end{array}$ & $\begin{array}{l}\text { RCT } \\
\text { Middle East }\end{array}$ & $\begin{array}{l}130 \\
120 \\
\text { PCOS }\end{array}$ & $\begin{array}{l}\text { CC + Cabergoline } \\
\text { vs. } \\
\text { CC alone }\end{array}$ & $\begin{array}{l}\text { Miscarriage } \\
\text { OHSS }\end{array}$ & Fair \\
\hline
\end{tabular}




\begin{tabular}{|c|c|c|c|c|c|}
\hline $\begin{array}{c}\text { Study } \\
\text { ACRONYM } \\
\text { KQs } \\
\text { Companion Studies }\end{array}$ & $\begin{array}{l}\text { Study Design } \\
\text { Geographic } \\
\text { Location }\end{array}$ & $\begin{array}{l}\text { N Enrolled } \\
\text { N Completed } \\
\text { Underlying } \\
\text { Diagnosis }\end{array}$ & Interventions & $\begin{array}{c}\text { Outcomes } \\
\text { (Subgroups analyzed) }\end{array}$ & Quality \\
\hline $\begin{array}{l}\text { Zain, 2009166 } \\
\text { KQ } 1\end{array}$ & $\begin{array}{l}\text { RCT } \\
\text { Asia }\end{array}$ & $\begin{array}{l}124 \\
115 \\
\text { Pcos }\end{array}$ & $\begin{array}{l}\text { Metformin tablets at the initial dose of } 500 \mathrm{mg} \\
\text { and increased in a step- wise fashion during the } \\
\text { first } 3 \text { weeks to a total dose of } 1,500 \mathrm{mg} / \mathrm{d} \text {. The } \\
\text { patients were then asked to make a telephone } \\
\text { call once they had a menstrual period and a } \\
\text { transvaginal ultrasound (TVS) and follicular } \\
\text { tracking was done to document evidence of } \\
\text { follicular growth and ovulation on days } 2,8,12 \text {, } \\
\text { and } 16 \text {. A menstrual calendar chart recorded } \\
\text { menses cycles monthly. } \\
\text { vs. } \\
\text { CC at a dose of } 50 \text { mg on days } 2-6 \text {. The TVS } \\
\text { and follicular tracking were done to document } \\
\text { follicular growth and ovulation on days } 2,8,12 \text {, } \\
\text { and } 16 \text {. If there was absence of ovulation, the } \\
\text { CC dose was increased stepwise on a treatment } \\
\text { cycle basis after a P withdrawal bleed to a } \\
\text { maximum of } 200 \text { mg. If there was evidence of } \\
\text { ovulation but the patient did not get pregnant, the } \\
\text { same dosage was continued for a maximum of } \\
\text { six cycles. } \\
\text { vs. } \\
\text { Metformin was given in a similar manner to the } \\
\text { metformin only group. CC was given at a dose of } \\
50 \text { mg on days } 2-6 \text {. The TVS and follicular } \\
\text { tracking were done to document evidence of } \\
\text { follicular growth and ovulation on days } 2,8,12 \text {, } \\
\text { and } 16 \text {. If there was absence of ovulation, the } \\
\text { CC dose was increased step-wise on a treatment } \\
\text { cycle basis after a P withdrawal bleed to a } \\
\text { maximum of } 200 \text { mg. If there was evidence of } \\
\text { ovulation but patient did not get pregnant, a } \\
\text { similar dosage was continued for a maximum of } \\
\text { six cycles. }\end{array}$ & $\begin{array}{l}\text { Live birth } \\
\text { Ectopic pregnancy } \\
\text { Miscarriage } \\
\text { Multiple births }\end{array}$ & Fair \\
\hline
\end{tabular}




\begin{tabular}{|c|c|c|c|c|c|}
\hline $\begin{array}{c}\text { Study } \\
\text { ACRONYM } \\
\text { KQs } \\
\text { Companion Studies }\end{array}$ & $\begin{array}{l}\text { Study Design } \\
\text { Geographic } \\
\text { Location }\end{array}$ & $\begin{array}{l}\text { N Enrolled } \\
\text { N Completed } \\
\text { Underlying } \\
\text { Diagnosis }\end{array}$ & Interventions & $\begin{array}{l}\text { Outcomes } \\
\text { (Subgroups analyzed) }\end{array}$ & Quality \\
\hline $\begin{array}{l}\text { Zakherah, 2010169 } \\
\text { KQ } 1\end{array}$ & $\begin{array}{l}\text { RCT } \\
\text { Africa }\end{array}$ & $\begin{array}{l}150 \\
150 \\
\text { Pcos }\end{array}$ & $\begin{array}{l}\text { CC } 150 \mathrm{mg}+\text { Tamoxifen } 40 \mathrm{mg} \text { from cycle days } \\
3 \text { to } 7 \text {, maximum treatment duration } 6 \text { cycles. } \\
\text { vs. } \\
\text { Laparoscopic ovarian drilling (LOD) performed } \\
\text { through triple-puncture laparoscopy followed by } \\
\text { timed intercourse. }\end{array}$ & $\begin{array}{l}\text { Live birth } \\
\text { Miscarriage }\end{array}$ & Good \\
\hline $\begin{array}{l}\text { Zarei, 2016 } \\
\text { KQ } 3\end{array}$ & $\begin{array}{l}\text { RCT } \\
\text { Middle East }\end{array}$ & $\begin{array}{l}260 \\
260 \\
\text { Unknown }\end{array}$ & $\begin{array}{l}\text { Clomid days 5-9, recombinant FSH days } 8+\text {, } \\
\text { hCG trigger, IUI x up to } 3 \text { cycles. Piroxicam days } \\
4-6 \text { after IUI. } \\
\text { vs. } \\
\text { Clomid days } 5-9 \text {, recombinant FSH days } 8+\text {, } \\
\text { hCG trigger, IUI } x \text { up to } 3 \text { cycles. }\end{array}$ & Miscarriage & Fair \\
\hline $\begin{array}{l}\text { Zheng, } 2012^{149} \\
\text { KQ } 1\end{array}$ & $\begin{array}{l}\text { RCT } \\
\text { Asia }\end{array}$ & $\begin{array}{l}82 \\
74 \\
\text { PCOS }\end{array}$ & $\begin{array}{l}\text { Primed with } 10,000 \text { IU hCG after progesterone } \\
\text { induced withdrawal bleeding. } \\
\text { Immature oocytes were collected } 36-38 \text { hours } \\
\text { after hCG priming. IVM and ICSI were done. } \\
\text { vs. } \\
\text { No priming after progesterone induced } \\
\text { withdrawal bleeding. } \\
\text { Immature oocytes were collected directly after } \\
\text { allocation to non-priming group. IVM and ICSI } \\
\text { were done. }\end{array}$ & Live birth & Fair \\
\hline $\begin{array}{l}\text { Zhu, 2014 } 202 \\
\text { KQ } 2\end{array}$ & $\begin{array}{l}\text { RCT } \\
\text { Asia }\end{array}$ & $\begin{array}{l}163 \\
156 \\
\text { Endometriosis }\end{array}$ & $\begin{array}{l}\text { Oral contraceptive for } 63 \text { days, every } 3 \text { month } \\
\text { visits for } 14 \text { months, if no pregnancy within } 12 \\
\text { months of stopping OCP, advised to undergo IVF } \\
\text { vs. } \\
\text { Oral contraceptive for } 33 \text { days, followed by a } \\
\text { combination of oral contraceptive and } 30 \mathrm{~g} / \mathrm{d} \\
\text { Dan'e mixture for } 30 \text { days; every } 3 \text { month visits } \\
\text { for } 14 \text { months, if no pregnancy within } 12 \text { months } \\
\text { of stopping OCP, advised to undergo IVF } \\
\text { vs. } \\
\text { No treatment; q3month visits for } 12 \text { months, if no } \\
\text { pregnancy within } 12 \text { months, advised to undergo } \\
\text { IVF }\end{array}$ & $\begin{array}{l}\text { Live birth } \\
\text { Ectopic pregnancy } \\
\text { Miscarriage } \\
\text { Neonatal death }\end{array}$ & Fair \\
\hline
\end{tabular}


Abbreviations: ART=assisted reproductive technology; BID=two times per day; BMI=body mass index; CARE Consortium=Centres for Assisted Reproduction; $\mathrm{CC}=$ clomiphene citrate; $\mathrm{COH}=$ controlled ovarian hyperstimulation; $\mathrm{COS}=$ controlled ovarian stimulation; DHEA=dehydroepiandrosterone; FASTT=Fast Track and Standard Treatment Trial; FISH=fluorescence in situ hybridization; FORT-T=Forty and Over Treatment Trial; FSH=follicle stimulating hormone;

GnRH=gonadotropin-releasing hormone; hCG=human chorionic gonadotropin; hMG=human menopausal gonadotropin; ICSI=intracytoplasmic sperm injection; IMSI=Intrycytoplasmic morphologically selected sperm injection; ITT=intention-to-treat; IU=international units; IUI=intrauterine insemination; IVF=in vitro

fertilization; IVM=in vitro maturation; KQ=key question; mcg=microgram; MNC=modified natural cycle; MOSART=Massachusetts Outcomes Study of Assisted Reproductive Technologies; NA=not applicable; NASS=National Artificial Reproductive Technology Surveillance System; NR=Not Reported;

$\mathrm{OCP}=$ oral contraceptive pill; OHSS=Ovarian Hyperstimulation Syndrome; PCOS=Polycystic Ovary Syndrome; PGD=preimplantation genetic diagnosis; PPCOS=Pregnancy in Polycystic Ovary Syndrome; RCT=Randomized Controlled Trial; rFSH=recombinant follicle stimulating hormone; rhFSH=recombinant human follicle stimulating hormone; SQ=subcutaneous; SUIT=Scottish Unexplained Infertility Trial; TVS=transvaginal ultrasound; uFSH=urinary follicle stimulating hormone; uhMG=urinary human menopausal gonadotropin 


\section{Appendix F. AMSTAR Quality Assessment for Systematic Reviews}

Table F-1 shows the AMSTAR (A Measurement Tool to Assess the Methodological Quality of Systematic Reviews) quality assessment for the included systematic reviews. For full study citations, please refer to the report's main reference list.

Table F-1. AMSTAR assessment for included systematic reviews

\begin{tabular}{|c|c|c|c|c|c|c|c|c|c|c|c|}
\hline Study & 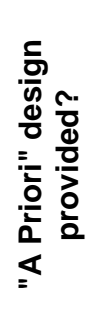 & 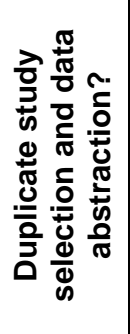 & 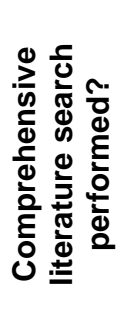 & 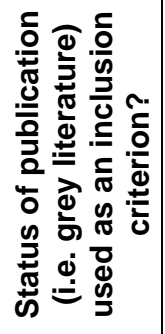 & 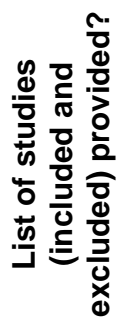 & 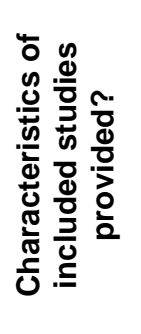 & 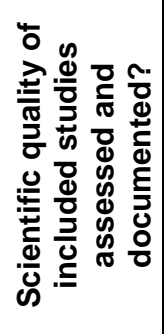 & 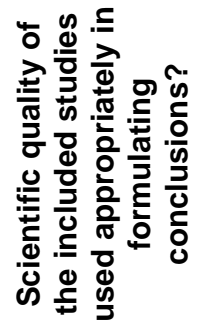 & 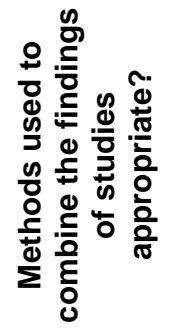 & 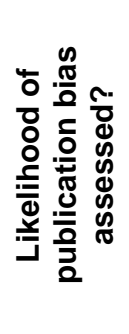 & 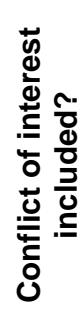 \\
\hline Al-Inany, $2016^{320}$ & $\mathrm{Y}$ & $\mathrm{Y}$ & $\mathrm{Y}$ & $\mathrm{Y}$ & $\mathrm{Y}$ & $\mathrm{Y}$ & $\mathrm{Y}$ & $\mathrm{Y}$ & $\mathrm{Y}$ & $\mathrm{Y}$ & $\mathrm{Y}$ \\
\hline Attia, $2013^{73}$ & $\mathrm{Y}$ & $Y$ & $\mathrm{Y}$ & $\mathrm{Y}$ & $\mathrm{Y}$ & $\bar{Y}$ & $\bar{Y}$ & $\bar{Y}$ & $\mathrm{Y}$ & $\mathrm{Y}$ & $\bar{Y}$ \\
\hline Barbosa, $2014^{64}$ & $\bar{Y}$ & $\mathrm{Y}$ & $\mathrm{Y}$ & $\mathrm{Y}$ & $\mathrm{Y}$ & $\bar{Y}$ & $\bar{Y}$ & $\bar{Y}$ & $\bar{Y}$ & $\bar{Y}$ & $\bar{Y}$ \\
\hline Bensdorp, $2007^{265}$ & $\mathrm{Y}$ & $\mathrm{Y}$ & $\mathrm{Y}$ & $\mathrm{Y}$ & $\mathrm{Y}$ & $\mathrm{Y}$ & $\mathrm{Y}$ & $\mathrm{Y}$ & $\mathrm{Y}$ & $\mathrm{Y}$ & $\mathrm{Y}$ \\
\hline Bhattacharya, $2010^{90}$ & $\mathrm{Y}$ & $\mathrm{C}$ & $\mathrm{Y}$ & $\mathrm{N}$ & $\mathrm{Y}$ & $\mathrm{Y}$ & $\mathrm{Y}$ & $\mathrm{Y}$ & $\mathrm{Y}$ & $\mathrm{N}$ & $\mathrm{Y}$ \\
\hline Bordewijk, $2017^{197}$ & $\mathrm{Y}$ & $\mathrm{Y}$ & $\mathrm{Y}$ & $\mathrm{Y}$ & $\mathrm{Y}$ & $\bar{Y}$ & $\mathrm{Y}$ & $\mathrm{Y}$ & $\mathrm{Y}$ & $\mathrm{Y}$ & $\bar{Y}$ \\
\hline Brown, $2016^{198}$ & $\bar{Y}$ & $\mathrm{Y}$ & $\mathrm{Y}$ & $\mathrm{Y}$ & $\mathrm{Y}$ & $\bar{Y}$ & $\bar{Y}$ & $\mathrm{Y}$ & $\bar{Y}$ & $\bar{Y}$ & $\mathrm{Y}$ \\
\hline Brown, $2016^{321}$ & $\mathrm{Y}$ & $\mathrm{Y}$ & $\mathrm{Y}$ & $\mathrm{Y}$ & $\mathrm{Y}$ & $\mathrm{Y}$ & $\mathrm{Y}$ & $\mathrm{Y}$ & $\mathrm{Y}$ & $\mathrm{Y}$ & $\mathrm{Y}$ \\
\hline Brown, $2016^{322}$ & $\bar{Y}$ & $\mathrm{Y}$ & $\mathrm{Y}$ & $\mathrm{Y}$ & $\mathrm{Y}$ & $\bar{Y}$ & $\bar{Y}$ & $\bar{Y}$ & $\mathrm{Y}$ & $\bar{Y}$ & $\bar{Y}$ \\
\hline Chua, $2017^{323}$ & $\mathrm{Y}$ & $\mathrm{Y}$ & $\mathrm{Y}$ & $\mathrm{Y}$ & $\mathrm{Y}$ & $\mathrm{Y}$ & $\mathrm{Y}$ & $\mathrm{Y}$ & $\mathrm{Y}$ & $\mathrm{Y}$ & $\mathrm{Y}$ \\
\hline Cissen, $2016^{266}$ & $\mathrm{Y}$ & $\mathrm{Y}$ & $\mathrm{Y}$ & $\mathrm{Y}$ & $\mathrm{Y}$ & $\mathrm{Y}$ & $\mathrm{Y}$ & $\mathrm{Y}$ & $\mathrm{Y}$ & $\mathrm{Y}$ & $Y$ \\
\hline Duffy, $2014^{65}$ & $\mathrm{Y}$ & $\mathrm{Y}$ & $\mathrm{Y}$ & $\mathrm{Y}$ & $\mathrm{Y}$ & $\mathrm{Y}$ & $\mathrm{Y}$ & $\mathrm{Y}$ & $\mathrm{Y}$ & $\mathrm{Y}$ & $\mathrm{Y}$ \\
\hline Farquhar, $2018^{324}$ & $\bar{Y}$ & $\mathrm{Y}$ & $\mathrm{Y}$ & $\mathrm{Y}$ & $\mathrm{Y}$ & $\bar{Y}$ & $\bar{Y}$ & $\bar{Y}$ & $\bar{Y}$ & $\bar{Y}$ & $\bar{Y}$ \\
\hline Farquhar, $2012^{81}$ & $\mathrm{Y}$ & $\mathrm{Y}$ & $\mathrm{Y}$ & $\mathrm{Y}$ & $\mathrm{Y}$ & $\mathrm{Y}$ & $\mathrm{Y}$ & $\mathrm{Y}$ & $\mathrm{Y}$ & $\mathrm{Y}$ & $\mathrm{Y}$ \\
\hline Farquhar, $2017^{325}$ & $\bar{Y}$ & $\mathrm{Y}$ & $\mathrm{Y}$ & $\mathrm{Y}$ & $\mathrm{Y}$ & $\bar{Y}$ & $\bar{Y}$ & $\bar{Y}$ & $\mathrm{Y}$ & $\bar{Y}$ & $\bar{Y}$ \\
\hline Franik, $2018^{326}$ & $\mathrm{Y}$ & $\mathrm{Y}$ & $\mathrm{Y}$ & $\mathrm{Y}$ & $\mathrm{Y}$ & $\bar{Y}$ & $\bar{Y}$ & $\bar{Y}$ & $\mathrm{Y}$ & $\bar{Y}$ & $\bar{Y}$ \\
\hline Franik, $2015^{327}$ & $\mathrm{Y}$ & $\mathrm{Y}$ & $\mathrm{Y}$ & $\mathrm{Y}$ & $\mathrm{Y}$ & $Y$ & $Y$ & $Y$ & $\mathrm{Y}$ & $\mathrm{Y}$ & $Y$ \\
\hline Franik, 2014 66 & $\mathrm{Y}$ & $\mathrm{Y}$ & $\mathrm{Y}$ & $\mathrm{Y}$ & $\mathrm{Y}$ & $\mathrm{Y}$ & $\mathrm{Y}$ & $\mathrm{Y}$ & $\mathrm{Y}$ & $\mathrm{Y}$ & $\mathrm{Y}$ \\
\hline Ghobara, $2017^{328}$ & $\bar{Y}$ & $Y$ & $\mathrm{Y}$ & $Y$ & $\mathrm{Y}$ & $\bar{Y}$ & $\bar{Y}$ & $\bar{Y}$ & $Y$ & $\mathrm{Y}$ & $\bar{Y}$ \\
\hline Hamdan, $2015^{60}$ & $\mathrm{Y}$ & $\mathrm{Y}$ & $\mathrm{Y}$ & $\mathrm{Y}$ & $\mathrm{N}$ & $\bar{Y}$ & $\bar{Y}$ & $\bar{Y}$ & $\mathrm{Y}$ & $\mathrm{N}$ & $\bar{Y}$ \\
\hline Hamdan, $2015^{329}$ & $\mathrm{Y}$ & $\mathrm{Y}$ & $\mathrm{Y}$ & $\mathrm{N}$ & $\mathrm{N}$ & $\mathrm{Y}$ & $\mathrm{Y}$ & $\mathrm{Y}$ & $\mathrm{Y}$ & $\mathrm{N}$ & $\mathrm{Y}$ \\
\hline $\mathrm{Hu}, 2018^{330}$ & $\mathrm{Y}$ & $\mathrm{Y}$ & $\mathrm{Y}$ & $\mathrm{Y}$ & $\mathrm{Y}$ & $\mathrm{Y}$ & $\mathrm{Y}$ & $\mathrm{Y}$ & $\mathrm{Y}$ & $\mathrm{Y}$ & $\mathrm{Y}$ \\
\hline Hughes, $2010^{92}$ & $\mathrm{Y}$ & $\mathrm{Y}$ & $\mathrm{Y}$ & $\mathrm{Y}$ & $\mathrm{Y}$ & $\mathrm{Y}$ & $\mathrm{Y}$ & $\mathrm{Y}$ & $\mathrm{Y}$ & $\mathrm{Y}$ & $Y$ \\
\hline
\end{tabular}




\begin{tabular}{|c|c|c|c|c|c|c|c|c|c|c|c|}
\hline Study & 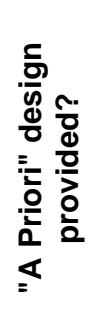 & 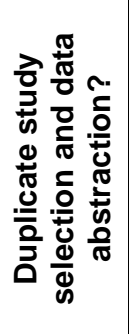 & 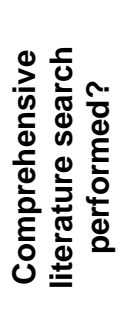 & 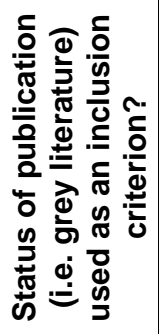 & 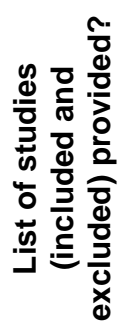 & 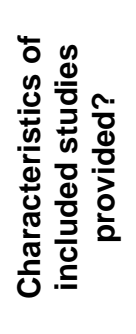 & 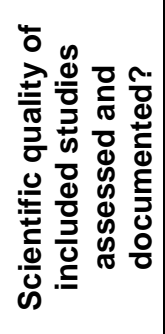 & 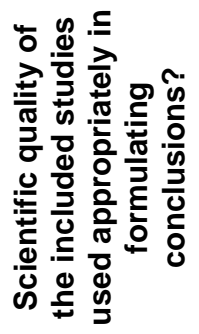 & 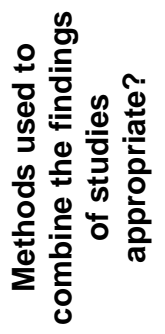 & 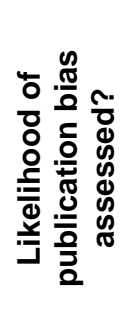 & 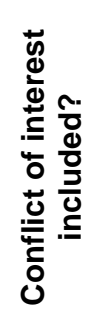 \\
\hline Jie, $2018^{331}$ & $\mathrm{Y}$ & $\mathrm{Y}$ & $\mathrm{Y}$ & $\mathrm{Y}$ & $\mathrm{Y}$ & $\mathrm{Y}$ & $\mathrm{Y}$ & $\mathrm{Y}$ & $\mathrm{Y}$ & $\mathrm{Y}$ & $\mathrm{Y}$ \\
\hline Johnson, $2010^{93}$ & $\mathrm{Y}$ & $\mathrm{Y}$ & $\mathrm{Y}$ & $\mathrm{Y}$ & $\mathrm{Y}$ & $Y$ & $\mathrm{Y}$ & $\mathrm{Y}$ & $\mathrm{Y}$ & $\mathrm{Y}$ & $\mathrm{Y}$ \\
\hline Lensen, $2016^{332}$ & $\mathrm{Y}$ & $\mathrm{Y}$ & $\mathrm{Y}$ & $\mathrm{Y}$ & $\mathrm{Y}$ & $\mathrm{Y}$ & $\mathrm{Y}$ & $\mathrm{Y}$ & $\mathrm{Y}$ & $\mathrm{Y}$ & $\mathrm{Y}$ \\
\hline Lim, $2016^{195}$ & $\mathrm{Y}$ & $\mathrm{Y}$ & $\mathrm{Y}$ & $\mathrm{Y}$ & $\mathrm{Y}$ & $\mathrm{Y}$ & $\mathrm{Y}$ & $Y$ & $Y$ & $\mathrm{Y}$ & $Y$ \\
\hline Liu, $2014^{70}$ & $\mathrm{Y}$ & $\mathrm{Y}$ & $\mathrm{Y}$ & $\mathrm{Y}$ & $\mathrm{N}$ & $\mathrm{Y}$ & $\mathrm{Y}$ & $\mathrm{Y}$ & $\mathrm{Y}$ & $\mathrm{Y}$ & $\mathrm{Y}$ \\
\hline Morley, $2017^{199}$ & $\mathrm{Y}$ & $\mathrm{Y}$ & $\mathrm{Y}$ & $\mathrm{Y}$ & $\mathrm{Y}$ & $\mathrm{Y}$ & $\mathrm{Y}$ & $\mathrm{Y}$ & $\mathrm{Y}$ & $\mathrm{Y}$ & $\mathrm{Y}$ \\
\hline Mourad, $2017^{333}$ & $\mathrm{Y}$ & $\mathrm{Y}$ & $\mathrm{Y}$ & $\mathrm{Y}$ & $\mathrm{Y}$ & $\mathrm{Y}$ & $\mathrm{Y}$ & $\mathrm{Y}$ & $\mathrm{Y}$ & $\mathrm{Y}$ & $\mathrm{Y}$ \\
\hline Nagels, $2015^{334}$ & $\mathrm{Y}$ & $\mathrm{Y}$ & $\mathrm{Y}$ & $\mathrm{Y}$ & $\mathrm{Y}$ & $\mathrm{Y}$ & $\mathrm{Y}$ & $\mathrm{Y}$ & $\mathrm{Y}$ & $\mathrm{Y}$ & $\mathrm{Y}$ \\
\hline Noble, $2010^{94}$ & $\mathrm{Y}$ & $\mathrm{Y}$ & $\mathrm{Y}$ & $\mathrm{C}$ & $\mathrm{Y}$ & $\mathrm{Y}$ & $\mathrm{N}$ & $\mathrm{N}$ & $\mathrm{Y}$ & $\mathrm{N}$ & $\mathrm{Y}$ \\
\hline Osman, $2015^{61}$ & $\mathrm{Y}$ & $\mathrm{Y}$ & $\mathrm{Y}$ & $Y$ & $\mathrm{~N}$ & $\mathrm{Y}$ & $\mathrm{N}$ & $\mathrm{N}$ & $\mathrm{Y}$ & $\mathrm{N}$ & $\mathrm{Y}$ \\
\hline Pandian, $2012^{84}$ & $\mathrm{Y}$ & $\mathrm{Y}$ & $\mathrm{Y}$ & $\mathrm{Y}$ & $\mathrm{Y}$ & $\mathrm{Y}$ & $\mathrm{Y}$ & $\mathrm{Y}$ & $\mathrm{Y}$ & $\mathrm{Y}$ & $\mathrm{Y}$ \\
\hline Pandian, $2015^{335}$ & $\mathrm{Y}$ & $\mathrm{Y}$ & $\mathrm{Y}$ & $\mathrm{Y}$ & $\mathrm{Y}$ & $\mathrm{Y}$ & $\mathrm{Y}$ & $\mathrm{Y}$ & $\mathrm{Y}$ & $\mathrm{Y}$ & $\mathrm{Y}$ \\
\hline Pandian, $2010^{95}$ & $\mathrm{Y}$ & $\mathrm{Y}$ & $\mathrm{Y}$ & $\mathrm{Y}$ & $\mathrm{Y}$ & $\mathrm{Y}$ & $\mathrm{Y}$ & $\mathrm{Y}$ & $Y$ & $\mathrm{Y}$ & $\mathrm{Y}$ \\
\hline Reavey, $2016^{196}$ & $\mathrm{Y}$ & $\mathrm{Y}$ & $\mathrm{Y}$ & $\mathrm{Y}$ & $\mathrm{Y}$ & $\mathrm{Y}$ & $\mathrm{Y}$ & $\mathrm{Y}$ & $\mathrm{Y}$ & $\mathrm{Y}$ & $\mathrm{Y}$ \\
\hline Showell, $2011^{336}$ & $Y$ & $Y$ & $Y$ & $Y$ & $Y$ & $Y$ & $Y$ & $\mathrm{Y}$ & $Y$ & $Y$ & $Y$ \\
\hline Siristatidis, $2016^{337}$ & $\mathrm{Y}$ & $\mathrm{Y}$ & $\mathrm{Y}$ & $\mathrm{Y}$ & $\mathrm{Y}$ & $\mathrm{Y}$ & $\mathrm{Y}$ & $\mathrm{Y}$ & $\mathrm{Y}$ & $\mathrm{Y}$ & $\mathrm{Y}$ \\
\hline Siristatidis, $2015^{338}$ & $\mathrm{Y}$ & $\mathrm{Y}$ & $\mathrm{Y}$ & $\mathrm{Y}$ & $\mathrm{Y}$ & $\mathrm{Y}$ & $\mathrm{Y}$ & $\mathrm{Y}$ & $\mathrm{Y}$ & $\mathrm{Y}$ & $\mathrm{Y}$ \\
\hline Sun, $2013^{78}$ & $\mathrm{Y}$ & $\mathrm{Y}$ & $\mathrm{Y}$ & $\mathrm{C}$ & $\mathrm{N}$ & $\mathrm{Y}$ & $\mathrm{N}$ & $\mathrm{N}$ & $\mathrm{Y}$ & $\mathrm{N}$ & $\mathrm{Y}$ \\
\hline Tang, $2016^{339}$ & $\mathrm{Y}$ & $\mathrm{Y}$ & $\mathrm{Y}$ & $\mathrm{Y}$ & $\mathrm{Y}$ & $\mathrm{Y}$ & $\mathrm{Y}$ & $\mathrm{Y}$ & $\mathrm{Y}$ & $\mathrm{Y}$ & $\mathrm{Y}$ \\
\hline Teixeira, $2013^{79}$ & $\mathrm{Y}$ & $\mathrm{Y}$ & $\mathrm{Y}$ & $\mathrm{Y}$ & $\mathrm{Y}$ & $\mathrm{Y}$ & $\mathrm{Y}$ & $\mathrm{Y}$ & $\mathrm{Y}$ & $\mathrm{Y}$ & $\mathrm{Y}$ \\
\hline Veltman-Verhulst, $2012^{87}$ & $\mathrm{Y}$ & $\mathrm{Y}$ & $\mathrm{Y}$ & $\mathrm{Y}$ & $\mathrm{Y}$ & $\mathrm{Y}$ & $\mathrm{Y}$ & $\mathrm{Y}$ & $\mathrm{Y}$ & $\mathrm{Y}$ & $\mathrm{Y}$ \\
\hline Veltman-Verhulst, $2016^{340}$ & $\mathrm{Y}$ & $\mathrm{Y}$ & $\mathrm{Y}$ & $\mathrm{Y}$ & $\mathrm{Y}$ & $\mathrm{Y}$ & $\mathrm{Y}$ & $\mathrm{Y}$ & $\mathrm{Y}$ & $\mathrm{Y}$ & $\mathrm{Y}$ \\
\hline Vermey, $2018^{341}$ & $\mathrm{Y}$ & $\mathrm{Y}$ & $\mathrm{Y}$ & $\mathrm{Y}$ & $\mathrm{Y}$ & $\mathrm{Y}$ & $\mathrm{Y}$ & $\mathrm{Y}$ & $\mathrm{Y}$ & $\mathrm{Y}$ & $\mathrm{Y}$ \\
\hline Vitaglia, $2018^{342}$ & $\mathrm{Y}$ & $\mathrm{Y}$ & $\mathrm{Y}$ & $\mathrm{Y}$ & $\mathrm{Y}$ & $\mathrm{Y}$ & $\mathrm{Y}$ & $\mathrm{Y}$ & $\mathrm{Y}$ & $\mathrm{Y}$ & $\mathrm{Y}$ \\
\hline Youssef, $2016^{343}$ & $\mathrm{Y}$ & $\mathrm{Y}$ & $\mathrm{Y}$ & $\mathrm{Y}$ & $\mathrm{Y}$ & $\mathrm{Y}$ & $\mathrm{Y}$ & $\mathrm{Y}$ & $\mathrm{Y}$ & $\mathrm{Y}$ & $\mathrm{Y}$ \\
\hline Youssef, $2015^{344}$ & $\mathrm{Y}$ & $\mathrm{Y}$ & $\mathrm{Y}$ & $\mathrm{Y}$ & $\mathrm{Y}$ & $\mathrm{Y}$ & $\mathrm{Y}$ & $\mathrm{Y}$ & $\mathrm{Y}$ & $\mathrm{Y}$ & $\bar{Y}$ \\
\hline Yu, $2018^{345}$ & $Y$ & $\mathrm{Y}$ & $\mathrm{Y}$ & $\mathrm{N}$ & $\mathrm{Y}$ & $\mathrm{Y}$ & $\mathrm{N}$ & $\mathrm{N}$ & $\mathrm{Y}$ & $\mathrm{N}$ & $Y$ \\
\hline Zhou, $2016^{346}$ & $\mathrm{Y}$ & $\mathrm{Y}$ & $\mathrm{Y}$ & $\mathrm{Y}$ & $\mathrm{Y}$ & $\mathrm{Y}$ & $Y$ & $\mathrm{Y}$ & $\mathrm{Y}$ & $\mathrm{Y}$ & $\mathrm{Y}$ \\
\hline
\end{tabular}

Abbreviations: $\mathrm{C}=$ Can't answer; $\mathrm{N}=\mathrm{No}$; $\mathrm{Y}=\mathrm{Yes}$ 
Figure F-1. Summary of AMSTAR quality assessment for systematic reviews

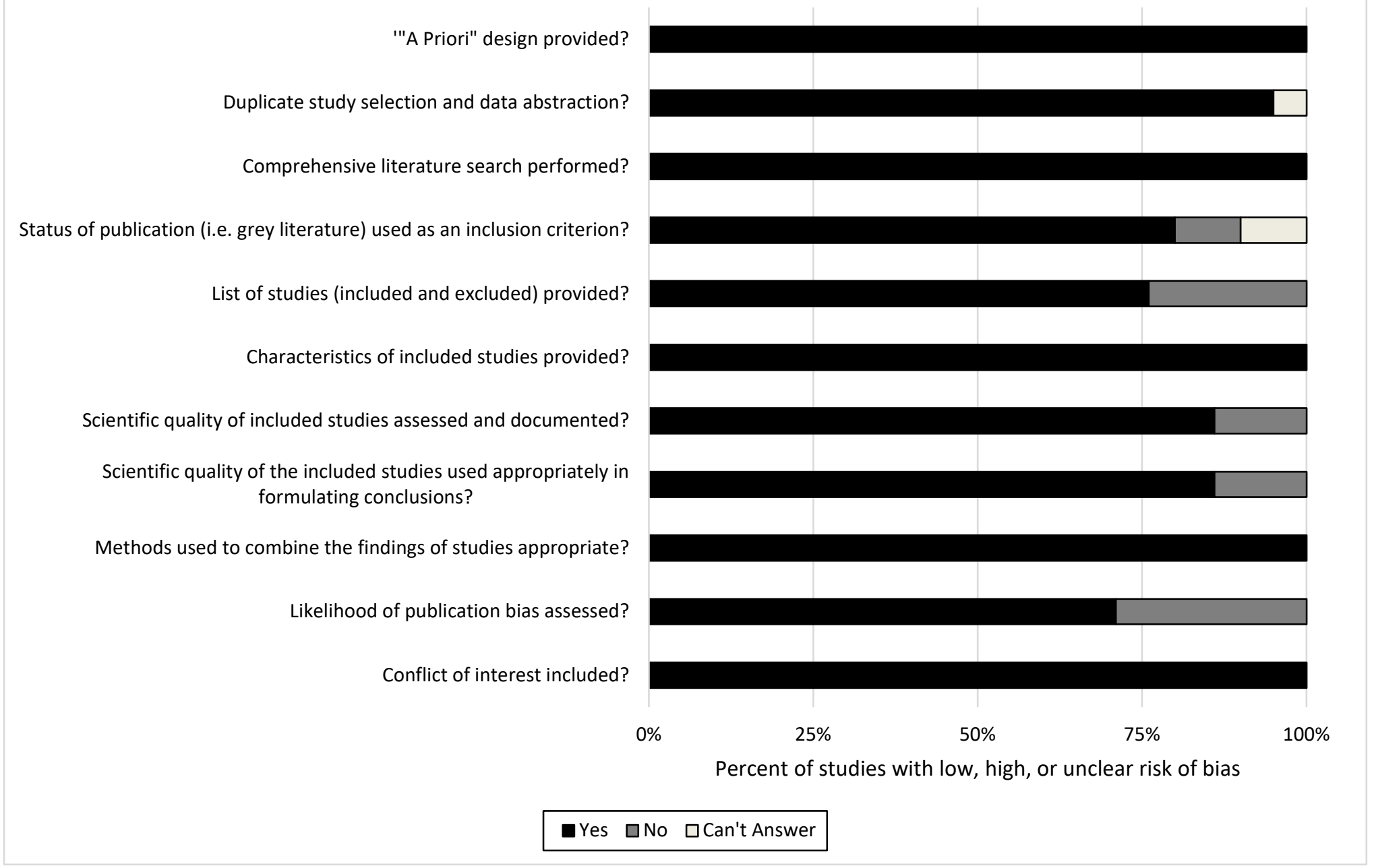




\section{Appendix G. Risk of Bias Assessment for Included Studies}

Table G-1 shows the risk of bias quality assessment for the included cohort studies. For full study citations, please refer to the report's main reference list.

Table G-1. Risk of bias assessment for included cohort studies

\begin{tabular}{|c|c|c|c|c|c|c|c|c|c|c|c|c|c|}
\hline Study & 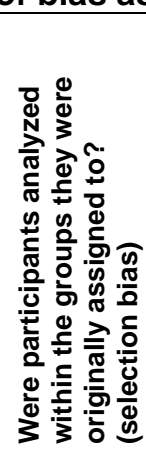 & 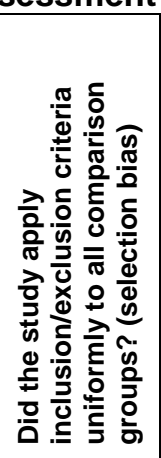 & 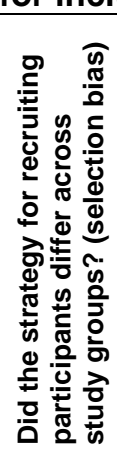 & 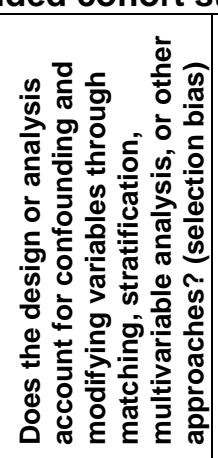 & 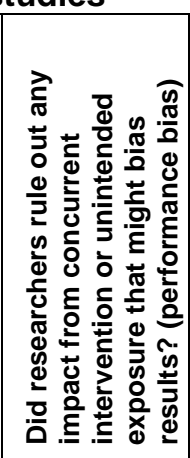 & 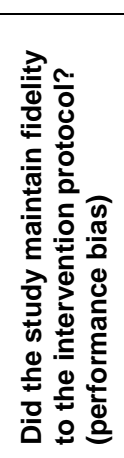 & 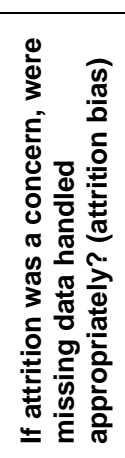 & 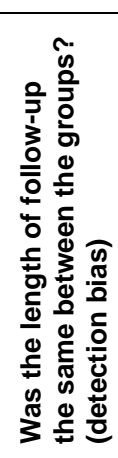 & 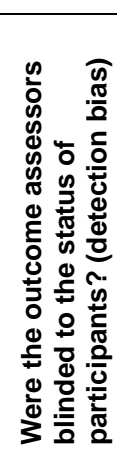 & 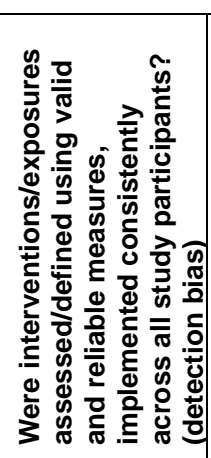 & 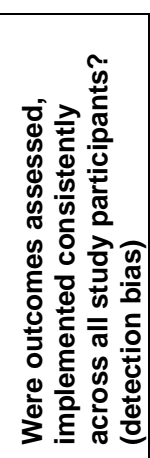 & 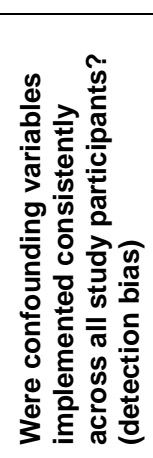 & 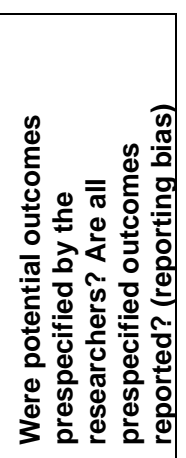 \\
\hline Belva, 2011259 & $\mathrm{N}$ & $\mathrm{N}$ & $U$ & $\mathrm{~N}$ & $\mathrm{~N}$ & $\mathrm{~N}$ & $\mathrm{~N}$ & $\mathrm{~N}$ & $\bar{Y}$ & $\bar{U}$ & $\bar{Y}$ & $\mathrm{~N}$ & $\mathrm{~N}$ \\
\hline Bodri, 2008 268 & $\mathrm{~N}$ & $\mathrm{Y}$ & $U$ & $\mathrm{~N}$ & $\mathrm{~N}$ & $U$ & $U$ & $\mathrm{Y}$ & $\mathrm{N}$ & $\bar{Y}$ & $\mathrm{U}$ & $\mathrm{Y}$ & $\mathrm{Y}$ \\
\hline Bodri, 2009 & $\mathrm{Y}$ & $\mathrm{Y}$ & $U$ & $\mathrm{~N}$ & $\mathrm{~N}$ & $U$ & $U$ & $\mathrm{Y}$ & $\mathrm{N}$ & $\mathrm{Y}$ & $\mathrm{Y}$ & $\mathrm{N}$ & $\mathrm{Y}$ \\
\hline Boulet, 2015 $5^{254}$ & $\mathrm{~N}$ & $\mathrm{~N}$ & $U$ & $\mathrm{Y}$ & $\mathrm{Y}$ & $\mathrm{N}$ & $\mathrm{Y}$ & $\mathrm{N}$ & $U$ & $U$ & $U$ & $\mathrm{Y}$ & $\mathrm{Y}$ \\
\hline Boulet, $2016^{277}$ & $\mathrm{Y}$ & $\mathrm{Y}$ & $\mathrm{N}$ & $\mathrm{Y}$ & $\mathrm{Y}$ & $\mathrm{Y}$ & $\mathrm{Y}$ & $\bar{U}$ & $\mathrm{U}$ & $\mathrm{Y}$ & $\mathrm{Y}$ & $\mathrm{Y}$ & $\mathrm{Y}$ \\
\hline Brinton, 2015 $5^{136}$ & $\mathrm{Y}$ & $\mathrm{Y}$ & $\mathrm{N}$ & $\mathrm{Y}$ & $U$ & $\mathrm{Y}$ & $\mathrm{Y}$ & $\mathrm{Y}$ & $U$ & $\mathrm{Y}$ & $\mathrm{Y}$ & $\mathrm{Y}$ & $U$ \\
\hline Butts, 2014 ${ }^{122}$ & $\mathrm{Y}$ & $\mathrm{Y}$ & $\mathrm{N}$ & $\mathrm{Y}$ & $U$ & $\mathrm{Y}$ & $\mathrm{Y}$ & $\mathrm{Y}$ & $\mathrm{N}$ & $\mathrm{Y}$ & $\mathrm{Y}$ & $\mathrm{Y}$ & $\mathrm{Y}$ \\
\hline Chang, 2016276 & $\mathrm{Y}$ & $\mathrm{Y}$ & $\mathrm{N}$ & $\mathrm{Y}$ & $\mathrm{Y}$ & $\mathrm{Y}$ & U & U & $\mathrm{U}$ & $\mathrm{Y}$ & $\mathrm{Y}$ & $\bar{Y}$ & $\mathrm{Y}$ \\
\hline Crawford, $2017^{281}$ & $\mathrm{Y}$ & $\mathrm{Y}$ & $\mathrm{N}$ & $\mathrm{Y}$ & $\mathrm{Y}$ & $\mathrm{Y}$ & $\mathrm{Y}$ & $\mathrm{Y}$ & $\mathrm{Y}$ & $\mathrm{Y}$ & $\mathrm{Y}$ & $\mathrm{Y}$ & $\mathrm{Y}$ \\
\hline de Wilde, $2017^{180}$ & $\mathrm{Y}$ & $\mathrm{Y}$ & $\mathrm{N}$ & $\mathrm{Y}$ & $\mathrm{Y}$ & $\mathrm{Y}$ & $U$ & $\mathrm{Y}$ & $\mathrm{Y}$ & $\mathrm{Y}$ & $\mathrm{Y}$ & $\bar{Y}$ & $\mathrm{Y}$ \\
\hline Dhalwani, $2016^{236}$ & $\mathrm{Y}$ & $\mathrm{Y}$ & $\mathrm{N}$ & $\mathrm{Y}$ & $\mathrm{Y}$ & $\mathrm{Y}$ & $\mathrm{Y}$ & $\mathrm{Y}$ & $\mathrm{N}$ & $\mathrm{Y}$ & $\mathrm{Y}$ & $\mathrm{Y}$ & $\mathrm{Y}$ \\
\hline Hershko-Klement, 2016 $6^{260}$ & $\mathrm{U}$ & $\mathrm{Y}$ & $\mathrm{U}$ & $\mathrm{N}$ & $\mathrm{U}$ & $\mathrm{U}$ & $\mathrm{U}$ & $\mathrm{Y}$ & $\mathrm{U}$ & $\mathrm{U}$ & $\mathrm{U}$ & $\mathrm{U}$ & U \\
\hline Kettner, 2016 ${ }^{184}$ & $\mathrm{Y}$ & $\mathrm{Y}$ & $\mathrm{N}$ & $\mathrm{Y}$ & $\mathrm{Y}$ & $\mathrm{Y}$ & $\mathrm{Y}$ & $\mathrm{Y}$ & $\mathrm{N}$ & $\mathrm{Y}$ & $\mathrm{Y}$ & $\mathrm{Y}$ & $\mathrm{Y}$ \\
\hline Keyhan, $2018^{185}$ & $\mathrm{Y}$ & $\mathrm{Y}$ & $\mathrm{N}$ & $\mathrm{Y}$ & $U$ & $U$ & $\mathrm{Y}$ & $\mathrm{Y}$ & $\mathrm{N}$ & $\mathrm{N}$ & $\mathrm{Y}$ & $\mathrm{N}$ & $\mathrm{Y}$ \\
\hline Kissin, $2015^{272}$ & $\mathrm{Y}$ & $\bar{Y}$ & $\mathrm{Y}$ & $\mathrm{Y}$ & $\mathrm{Y}$ & $\mathrm{Y}$ & $\mathrm{Y}$ & $\bar{Y}$ & $\mathrm{Y}$ & $\mathrm{Y}$ & $\bar{Y}$ & $\mathrm{Y}$ & $\bar{Y}$ \\
\hline Knudtson, $2017^{282}$ & $\mathrm{Y}$ & $\mathrm{Y}$ & $\mathrm{N}$ & $\mathrm{Y}$ & $\mathrm{Y}$ & $\mathrm{Y}$ & $\mathrm{Y}$ & $\mathrm{Y}$ & $\mathrm{N}$ & $\mathrm{Y}$ & $\mathrm{Y}$ & $\mathrm{Y}$ & $\mathrm{Y}$ \\
\hline Levi Dunietz, $2017^{239}$ & $\mathrm{Y}$ & $\mathrm{Y}$ & $\mathrm{N}$ & $\mathrm{Y}$ & $\mathrm{Y}$ & $\mathrm{Y}$ & $\mathrm{Y}$ & $\bar{Y}$ & $\mathrm{Y}$ & $\bar{Y}$ & $\mathrm{Y}$ & $\bar{Y}$ & $\bar{Y}$ \\
\hline Litzky, $2018^{84}$ & $\mathrm{Y}$ & $\mathrm{Y}$ & $\mathrm{N}$ & $\mathrm{Y}$ & $u$ & $\mathrm{Y}$ & $\mathrm{Y}$ & $\mathrm{Y}$ & $\mathrm{N}$ & $\mathrm{Y}$ & $\mathrm{Y}$ & $\mathrm{Y}$ & $\mathrm{Y}$ \\
\hline Litzky, $2018^{290}$ & $\mathrm{Y}$ & $\mathrm{Y}$ & $\mathrm{N}$ & $\mathrm{Y}$ & $U$ & $\mathrm{Y}$ & $\mathrm{Y}$ & $\mathrm{Y}$ & $\mathrm{U}$ & $\mathrm{Y}$ & $\mathrm{Y}$ & $\mathrm{Y}$ & $\mathrm{Y}$ \\
\hline Luke, $2010^{123}$ & $\mathrm{~N}$ & $\mathrm{Y}$ & $U$ & $\mathrm{~N}$ & $\mathrm{~N}$ & $\mathrm{~N}$ & $\mathrm{~N}$ & $\mathrm{~N}$ & $\mathrm{U}$ & $\bar{Y}$ & $\mathrm{Y}$ & $\mathrm{U}$ & $\mathrm{Y}$ \\
\hline Magnusson, $2018^{287}$ & $\mathrm{Y}$ & $\mathrm{Y}$ & $\mathrm{N}$ & $\mathrm{Y}$ & $U$ & $\mathrm{Y}$ & $\mathrm{Y}$ & $\mathrm{Y}$ & $\mathrm{U}$ & $\mathrm{Y}$ & $\mathrm{Y}$ & $\bar{Y}$ & $\mathrm{Y}$ \\
\hline Malchau, $2017^{186}$ & $\mathrm{Y}$ & $\mathrm{Y}$ & $\mathrm{N}$ & $\mathrm{Y}$ & $U$ & $\mathrm{Y}$ & $\mathrm{Y}$ & $\mathrm{Y}$ & $\mathrm{U}$ & $\mathrm{Y}$ & $\mathrm{Y}$ & $\mathrm{Y}$ & $\mathrm{Y}$ \\
\hline Mancuso, $2016^{283}$ & $\mathrm{Y}$ & $\mathrm{Y}$ & $\mathrm{N}$ & $\mathrm{Y}$ & $\mathrm{u}$ & $\mathrm{Y}$ & $\mathrm{Y}$ & $\mathrm{u}$ & $\mathrm{U}$ & $\mathrm{Y}$ & $\mathrm{Y}$ & $\mathrm{Y}$ & $\mathrm{Y}$ \\
\hline Maxwell, $2008^{270}$ & $\mathrm{~N}$ & $\mathrm{Y}$ & $\mathrm{N}$ & $\mathrm{N}$ & $\mathrm{N}$ & $U$ & $\mathrm{Y}$ & $\mathrm{N}$ & $\mathrm{N}$ & $\mathrm{Y}$ & $\mathrm{N}$ & $\mathrm{N}$ & $\mathrm{N}$ \\
\hline Muller, $2017^{205}$ & $\mathrm{Y}$ & $\mathrm{Y}$ & $\mathrm{N}$ & $\mathrm{N}$ & $U$ & 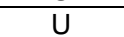 & $U$ & $\mathrm{Y}$ & $U$ & $\mathrm{Y}$ & $\mathrm{Y}$ & $\mathrm{N}$ & $\mathrm{Y}$ \\
\hline
\end{tabular}

G-1 


\begin{tabular}{|c|c|c|c|c|c|c|c|c|c|c|c|c|c|}
\hline Study & 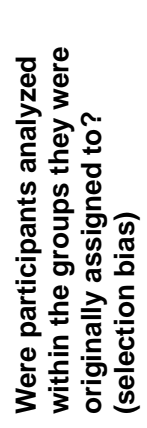 & 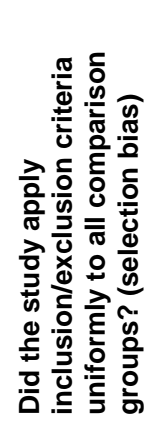 & 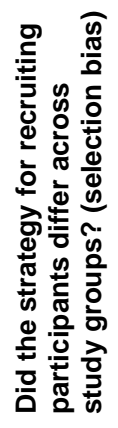 & 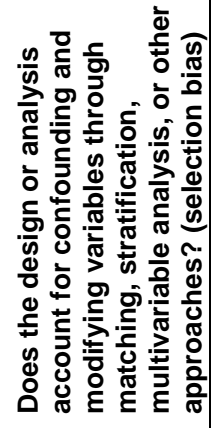 & 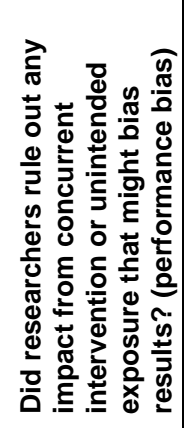 & 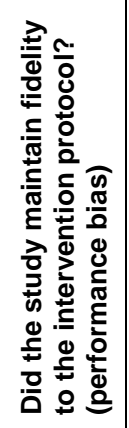 & 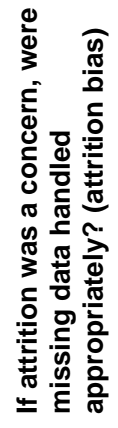 & 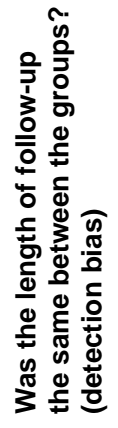 & 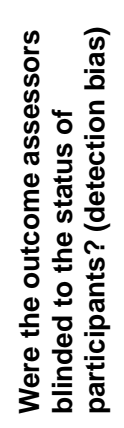 & 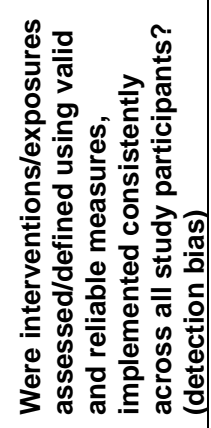 & 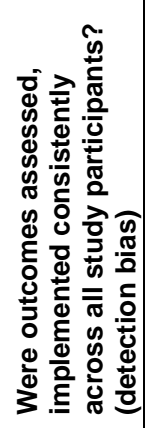 & 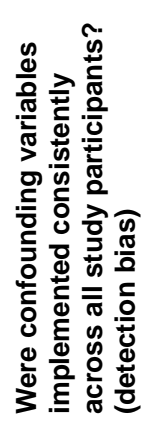 & 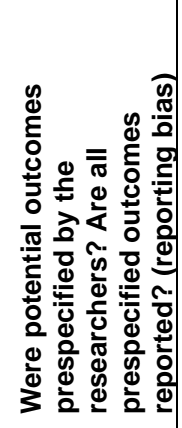 \\
\hline Nangia, 2011 124 & $\mathrm{U}$ & $\mathrm{Y}$ & $\mathrm{U}$ & $\mathrm{Y}$ & $\mathrm{N}$ & $\mathrm{U}$ & $\mathrm{U}$ & $\mathrm{N}$ & $\mathrm{U}$ & $\mathrm{Y}$ & $\mathrm{Y}$ & $\mathrm{Y}$ & $\mathrm{Y}$ \\
\hline Oyesanya, $2009^{224}$ & $\mathrm{Y}$ & $\mathrm{Y}$ & $\mathrm{N}$ & $\mathrm{N}$ & $\mathrm{N}$ & $\mathrm{Y}$ & $\mathrm{Y}$ & $\mathrm{Y}$ & $\mathrm{N}$ & $\mathrm{Y}$ & $\mathrm{Y}$ & $\mathrm{N}$ & $\mathrm{U}$ \\
\hline Schendelaar, $2011^{273}$ & $\mathrm{Y}$ & $\mathrm{Y}$ & $\mathrm{Y}$ & $\mathrm{Y}$ & $\mathrm{U}$ & $U$ & $\mathrm{Y}$ & $\mathrm{Y}$ & $\mathrm{N}$ & $\mathrm{Y}$ & $\mathrm{Y}$ & $U$ & $\mathrm{U}$ \\
\hline Spaan, $2015^{125}$ & $\mathrm{~N}$ & $\mathrm{Y}$ & $\mathrm{N}$ & $\mathrm{Y}$ & $\mathrm{Y}$ & $\mathrm{Y}$ & $U$ & $\mathrm{Y}$ & $U$ & $\mathrm{Y}$ & $\mathrm{Y}$ & $\mathrm{Y}$ & $\mathrm{U}$ \\
\hline Stewart, $2013^{203}$ & $\mathrm{Y}$ & $\mathrm{Y}$ & $\mathrm{N}$ & $\mathrm{N}$ & $\mathrm{U}$ & $\mathrm{Y}$ & $\mathrm{Y}$ & $\mathrm{Y}$ & $U$ & $\mathrm{Y}$ & $\mathrm{Y}$ & $\mathrm{N}$ & $\mathrm{Y}$ \\
\hline Tsai, $2011^{257}$ & $\mathrm{~N}$ & $\mathrm{~N}$ & $\mathrm{Y}$ & $\mathrm{N}$ & $\mathrm{N}$ & $U$ & $\mathrm{~N}$ & $\mathrm{~N}$ & $\mathrm{~N}$ & $\mathrm{Y}$ & $\mathrm{Y}$ & $\mathrm{N}$ & $\mathrm{Y}$ \\
\hline Verhoeve, $2013^{249}$ & $\mathrm{U}$ & $\mathrm{U}$ & $\mathrm{U}$ & $\mathrm{U}$ & $\mathrm{U}$ & $U$ & $\mathrm{U}$ & $\mathrm{U}$ & $\mathrm{U}$ & $\mathrm{U}$ & $\mathrm{U}$ & $\mathrm{U}$ & $\mathrm{U}$ \\
\hline Vitek, $2013^{215}$ & $\mathrm{Y}$ & $\mathrm{Y}$ & $\mathrm{N}$ & $\mathrm{Y}$ & $\mathrm{Y}$ & $\mathrm{Y}$ & $\mathrm{Y}$ & $\mathrm{Y}$ & $\mathrm{U}$ & $\mathrm{Y}$ & $\mathrm{Y}$ & $\mathrm{Y}$ & $\mathrm{U}$ \\
\hline Wang, $2017^{286}$ & $\mathrm{Y}$ & $\mathrm{Y}$ & $\mathrm{N}$ & $\mathrm{Y}$ & $\mathrm{U}$ & $\mathrm{Y}$ & $\mathrm{Y}$ & $\mathrm{Y}$ & $\mathrm{U}$ & $\mathrm{Y}$ & $\mathrm{Y}$ & $\mathrm{Y}$ & $\mathrm{Y}$ \\
\hline Williams, $2013^{213}$ & $\mathrm{Y}$ & $\mathrm{Y}$ & $\mathrm{N}$ & $\mathrm{N}$ & $\mathrm{U}$ & $\mathrm{Y}$ & $\mathrm{Y}$ & $\mathrm{Y}$ & $\mathrm{U}$ & $\mathrm{Y}$ & $\mathrm{Y}$ & $U$ & $\mathrm{Y}$ \\
\hline Williams, $2018^{289}$ & $\mathrm{Y}$ & $\mathrm{Y}$ & $\mathrm{N}$ & $\mathrm{Y}$ & $\mathrm{U}$ & $\mathrm{Y}$ & $\mathrm{Y}$ & $\mathrm{N}$ & $\mathrm{U}$ & $\mathrm{Y}$ & $\mathrm{Y}$ & $\mathrm{Y}$ & $\mathrm{Y}$ \\
\hline Xiong, $2017^{263}$ & $\mathrm{Y}$ & $\mathrm{Y}$ & $\mathrm{U}$ & $\mathrm{Y}$ & $\mathrm{N}$ & $U$ & $U$ & $\mathrm{Y}$ & $U$ & $\mathrm{Y}$ & $\mathrm{Y}$ & $\mathrm{Y}$ & $\mathrm{Y}$ \\
\hline
\end{tabular}




\section{Figure G-1. Summary of risk of bias assessment for included cohort studies}

Were participants analyzed within the groups they were originally assigned to? (selection bias)

Did the study apply inclusion/exclusion criteria uniformly to all comparison groups? (selection bias)

Did the strategy for recruiting participants into the study differ across study groups? (selection bias)

Does the design or analysis control account for important confounding and modifying variables through matching, stratification, multivariable analysis, or..

Did researchers rule out any impact from a concurrent intervention or an unintended exposure that might bias results? (performance bias)

Did the study maintain fidelity to the intervention protocol? (performance bias)

If attrition was a concern, were missing data handled appropriately? (attrition bias)

In prospective studies, was the length of follow-up the same between the groups, or in case-control studies, was the time period between the...

Were the outcome assessors blinded to the intervention or exposure status of participants? (detection bias)

Were interventions/exposures assessed/defined using valid and reliable measures, implemented consistently across all study participants? (detection bias)

Were outcomes assessed/defined using valid and reliable measures, implemented consistently across all study participants? (detection bias)

Were confounding variables assessed using valid and reliable measures, implemented consistently across all study participants? (detection bias)

Were the potential outcomes prespecified by the researchers? Are all prespecified outcomes reported? (reporting bias)
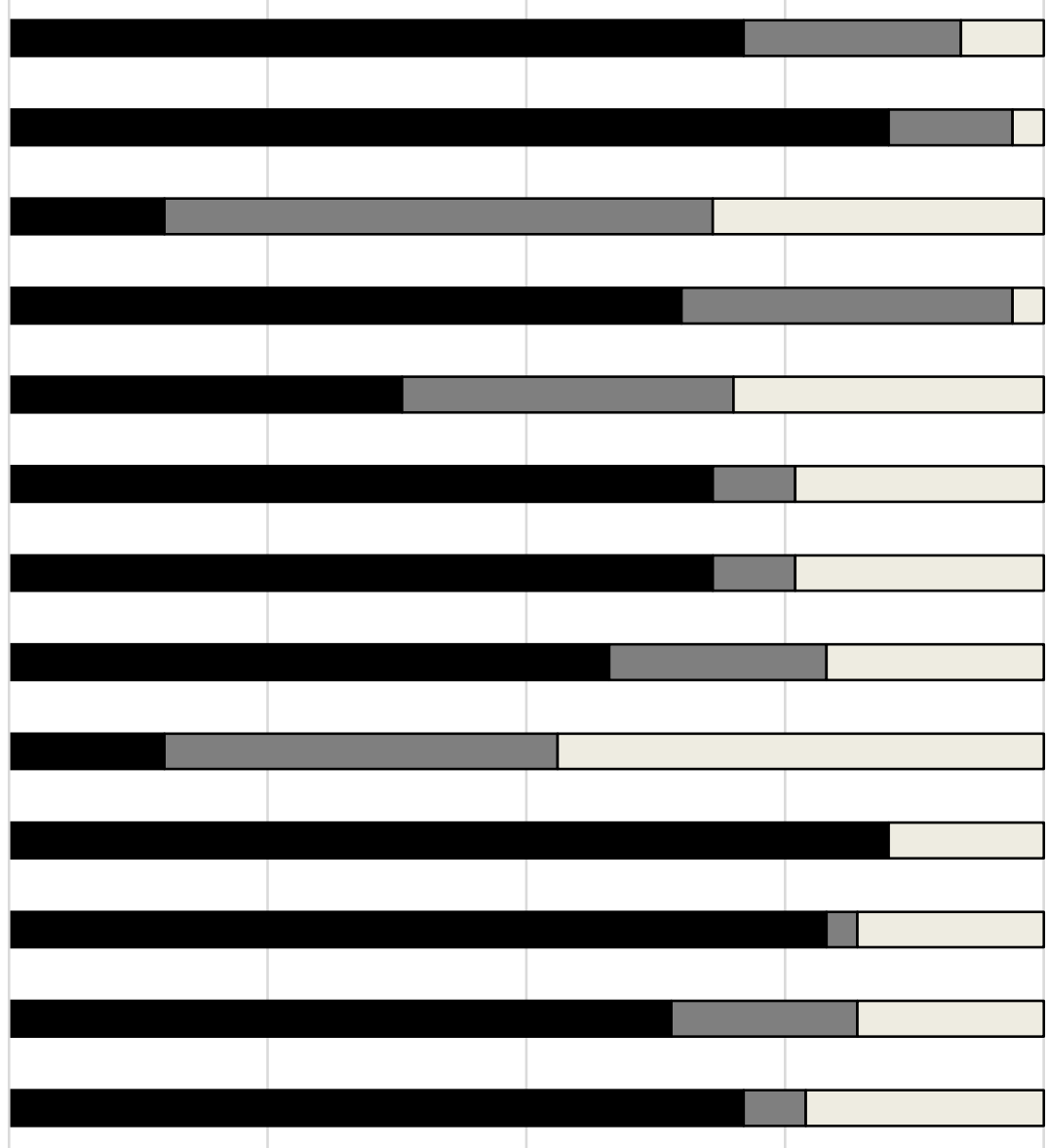

$0 \%$

$25 \%$

$50 \%$

$75 \%$

Percent of studies with low, high, or unclear risk of bias

- Yes aNo aUnclear 
Table G-2 shows the risk of bias quality assessment for the included cross-sectional studies. For full study citations, please refer to the report's main reference list.

\section{Table G-2. Risk of bias assessment for included cross-sectional studies}

\begin{tabular}{|c|c|c|c|c|c|c|c|c|c|}
\hline Study & 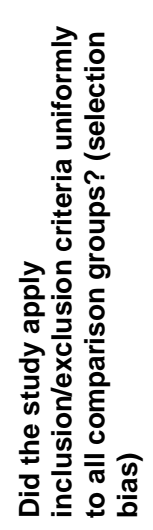 & 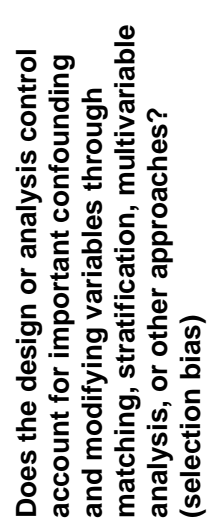 & 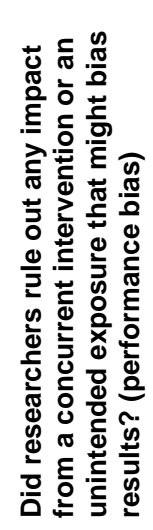 & 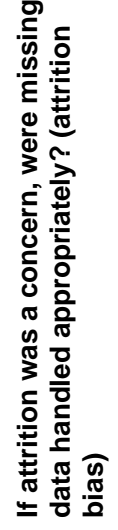 & 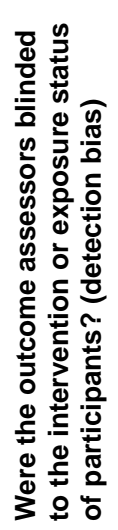 & 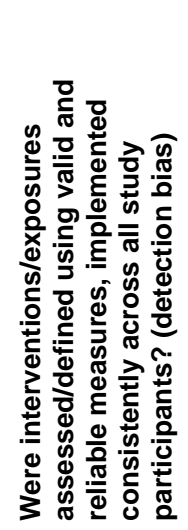 & 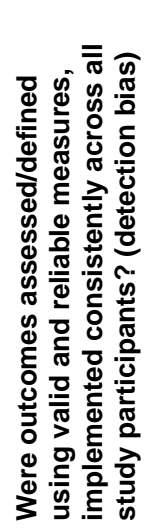 & 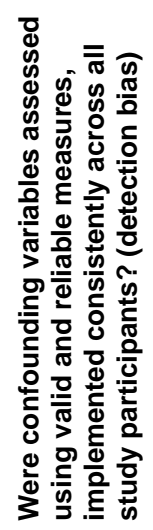 & 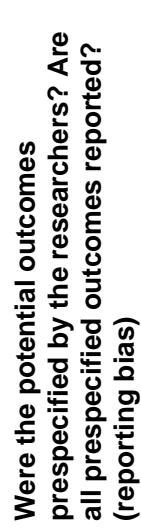 \\
\hline Barad, $2017^{280}$ & $\mathrm{Y}$ & $\mathrm{Y}$ & $\mathrm{U}$ & $\mathrm{N}$ & $\mathrm{Y}$ & $\mathrm{Y}$ & $\mathrm{Y}$ & $\mathrm{Y}$ & $\bar{Y}$ \\
\hline Grimstad, $2016^{251}$ & $\mathrm{Y}$ & $\mathrm{Y}$ & $\mathrm{Y}$ & $\mathrm{Y}$ & $U$ & $\mathrm{Y}$ & $\bar{Y}$ & $\mathrm{Y}$ & $\bar{Y}$ \\
\hline Kramer, 2009 ${ }^{271}$ & $\mathrm{Y}$ & $\mathrm{N}$ & $\mathrm{N}$ & $\mathrm{N}$ & $U$ & $\mathrm{~N}$ & $\mathrm{Y}$ & $\mathrm{Y}$ & $\mathrm{Y}$ \\
\hline Londra, $2016^{278}$ & $\mathrm{Y}$ & $\mathrm{Y}$ & $\mathrm{Y}$ & $\mathrm{Y}$ & $U$ & $\mathrm{Y}$ & $Y$ & $\mathrm{Y}$ & $\mathrm{Y}$ \\
\hline Luke, $2016^{274}$ & $\mathrm{Y}$ & $\mathrm{Y}$ & $\mathrm{Y}$ & $\mathrm{Y}$ & $U$ & $\mathrm{Y}$ & $\mathrm{Y}$ & $\mathrm{Y}$ & $\mathrm{Y}$ \\
\hline Provost, $2016^{279}$ & $\mathrm{Y}$ & $\mathrm{Y}$ & $\mathrm{Y}$ & $\mathrm{Y}$ & $U$ & $\mathrm{Y}$ & $\mathrm{Y}$ & $\mathrm{Y}$ & $\mathrm{Y}$ \\
\hline
\end{tabular}

Abbreviations: $\mathrm{N}=\mathrm{No}$; $\mathrm{U}=$ Unclear; $\mathrm{Y}=\mathrm{Yes}$ 


\section{Figure G-2. Summary of risk of bias assessment for included cross-sectional studies}

Did the study apply inclusion/exclusion criteria uniformly to all comparison groups? (selection bias)

Does the design or analysis control account for important confounding and modifying variables through matching, stratification, multivariable analysis, or other approaches? (selection bias)

Did researchers rule out any impact from a concurrent intervention or an unintended exposure that might bias results? (performance bias)

If attrition was a concern, were missing data handled appropriately? (attrition bias)

Were the outcome assessors blinded to the intervention or exposure status of participants? (detection bias)

Were interventions/exposures assessed/defined using valid and reliable measures, implemented consistently across all study participants? (detection bias)

Were outcomes assessed/defined using valid and reliable measures, implemented consistently across all study participants? (detection bias)

Were confounding variables assessed using valid and reliable measures, implemented consistently across all study participants? (detection bias)

Were the potential outcomes prespecified by the researchers? Are all prespecified outcomes reported? (reporting bias)

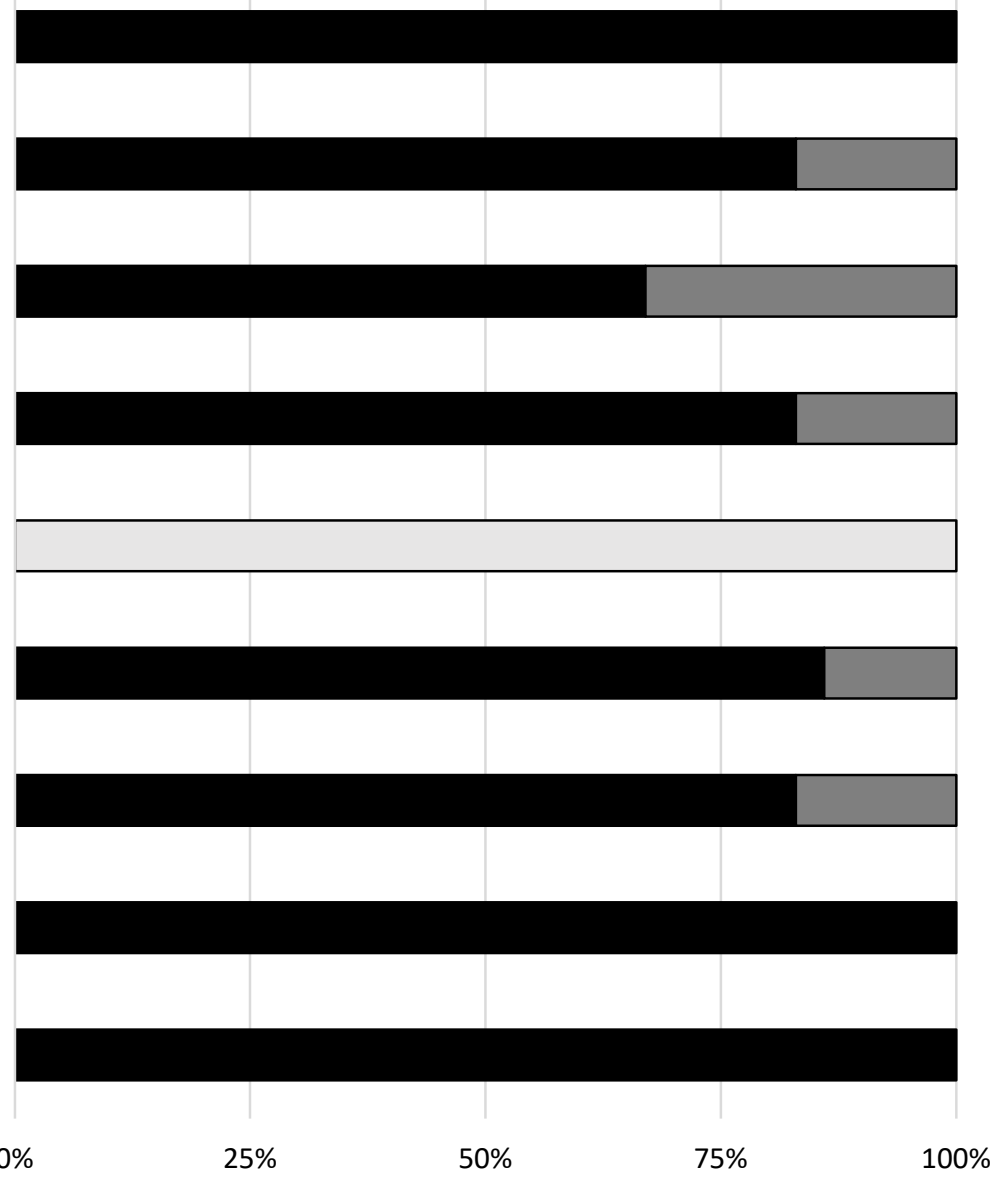

Percent of studies with low, high, or unclear risk of bias

-Yes $\square$ No $\square$ Unclear 
Table G-3 shows the risk of bias quality assessment for the included RCTs. For full study citations, please refer to the report's main reference list.

Table G-3. Risk of bias assessment for included RCTs

\begin{tabular}{|c|c|c|c|c|c|c|c|c|c|c|c|c|}
\hline Study & 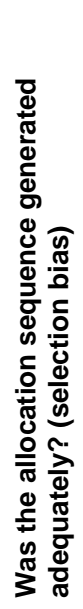 & 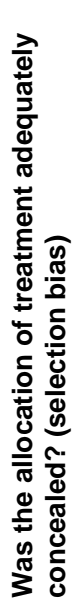 & 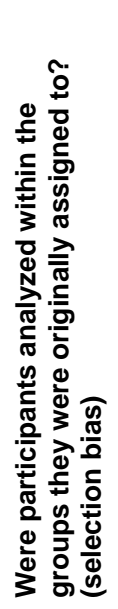 & 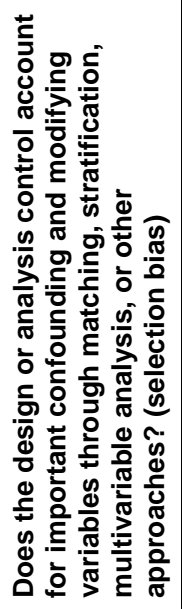 & 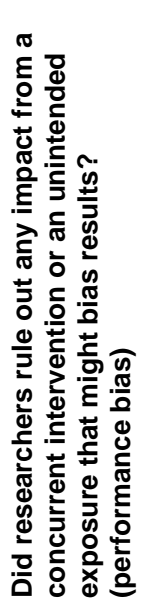 & 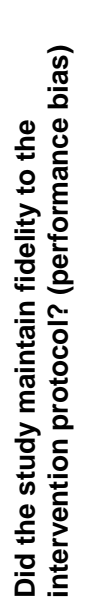 & 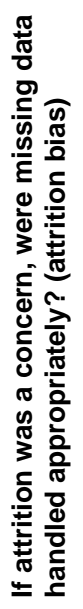 & 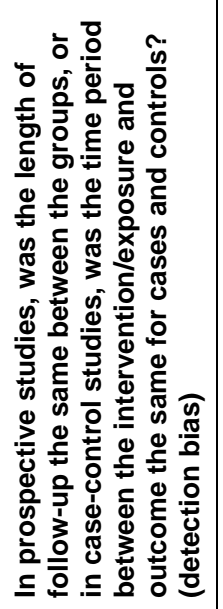 & 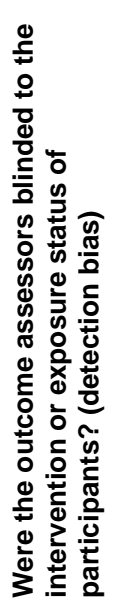 & 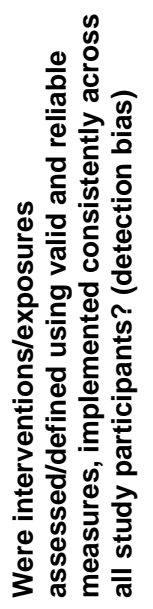 & 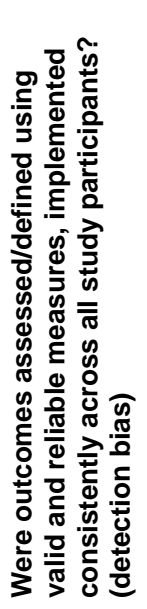 & 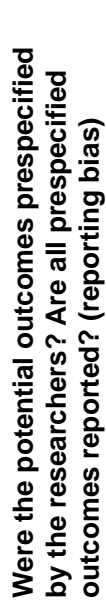 \\
\hline Abdellah, $2011^{156}$ & $\mathrm{Y}$ & $\mathrm{Y}$ & $\mathrm{Y}$ & $\mathrm{Y}$ & $\mathrm{N}$ & $\mathrm{Y}$ & $\mathrm{N}$ & $\mathrm{Y}$ & $\mathrm{Y}$ & $\mathrm{Y}$ & $\mathrm{Y}$ & $\mathrm{Y}$ \\
\hline Aboulghar, $2010^{162}$ & $\mathrm{Y}$ & $\mathrm{Y}$ & $\bar{Y}$ & $\mathrm{~N}$ & $\mathrm{Y}$ & $\mathrm{Y}$ & $\mathrm{Y}$ & $\mathrm{Y}$ & $\mathrm{N}$ & $\mathrm{Y}$ & $\mathrm{Y}$ & $\mathrm{Y}$ \\
\hline Abu Hashim, 2012 ${ }^{204}$ & $\mathrm{Y}$ & $\mathrm{Y}$ & $\mathrm{Y}$ & $\bar{Y}$ & $\mathrm{Y}$ & $\mathrm{Y}$ & $\bar{Y}$ & $\mathrm{Y}$ & $\mathrm{Y}$ & $\mathrm{Y}$ & $\bar{Y}$ & $\bar{Y}$ \\
\hline Abu Hashim, $2011^{154}$ & $\mathrm{Y}$ & $\mathrm{Y}$ & $\mathrm{Y}$ & $\mathrm{Y}$ & $\mathrm{N}$ & $\mathrm{Y}$ & $\mathrm{Y}$ & $\mathrm{Y}$ & $\mathrm{N}$ & $\mathrm{Y}$ & $\mathrm{Y}$ & $\mathrm{Y}$ \\
\hline Abu Hashim, 2010 & $\mathrm{Y}$ & $\mathrm{Y}$ & $\mathrm{Y}$ & $\mathrm{N}$ & $\mathrm{N}$ & $U$ & $\mathrm{Y}$ & $\mathrm{Y}$ & $\mathrm{Y}$ & $\mathrm{Y}$ & $\mathrm{Y}$ & $\mathrm{Y}$ \\
\hline Abu Hashim, $2011^{157}$ & $\mathrm{Y}$ & $\mathrm{Y}$ & $\mathrm{Y}$ & $\mathrm{Y}$ & $\mathrm{N}$ & $\mathrm{Y}$ & 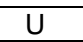 & $\mathrm{Y}$ & U & $\mathrm{Y}$ & $\mathrm{Y}$ & $\mathrm{Y}$ \\
\hline Aghahosseini, $2017^{178}$ & $\mathrm{Y}$ & $U$ & $\bar{Y}$ & $\bar{Y}$ & $\bar{Y}$ & $\mathrm{Y}$ & $\mathrm{Y}$ & $\bar{Y}$ & $U$ & $\mathrm{Y}$ & $\bar{Y}$ & $\bar{Y}$ \\
\hline Amer, $2017^{179}$ & $\mathrm{Y}$ & $\mathrm{Y}$ & $\mathrm{Y}$ & $\mathrm{Y}$ & $\mathrm{Y}$ & $\mathrm{Y}$ & $\mathrm{Y}$ & $\mathrm{Y}$ & $\mathrm{Y}$ & $\mathrm{Y}$ & $\mathrm{Y}$ & $\mathrm{Y}$ \\
\hline Amer, 2009 165 & $\bar{Y}$ & $\mathrm{Y}$ & $\mathrm{N}$ & $\mathrm{Y}$ & $\mathrm{N}$ & $\mathrm{N}$ & $\mathrm{N}$ & $\mathrm{Y}$ & $U$ & $\mathrm{Y}$ & $\mathrm{Y}$ & $\mathrm{N}$ \\
\hline An, $2014^{146}$ & $\mathrm{Y}$ & $\mathrm{Y}$ & $\mathrm{Y}$ & $\mathrm{Y}$ & $\mathrm{Y}$ & $\mathrm{Y}$ & $\mathrm{Y}$ & $\mathrm{Y}$ & $\mathrm{Y}$ & $\mathrm{Y}$ & $\mathrm{Y}$ & $\mathrm{Y}$ \\
\hline Badawy, 2008 ${ }^{193}$ & $\mathrm{Y}$ & $U$ & $\mathrm{Y}$ & $\mathrm{N}$ & $\mathrm{U}$ & $U$ & $\mathrm{Y}$ & $\mathrm{Y}$ & $U$ & $\mathrm{Y}$ & $\bar{Y}$ & $\bar{Y}$ \\
\hline Badawy, 2009 222 & $\mathrm{~N}$ & $\mathrm{~N}$ & $\mathrm{Y}$ & $\mathrm{N}$ & $\mathrm{U}$ & $\mathrm{Y}$ & $\mathrm{U}$ & $\mathrm{N}$ & $\mathrm{N}$ & $\mathrm{Y}$ & $\mathrm{U}$ & $\mathrm{U}$ \\
\hline Bagis, $2010^{221}$ & $\mathrm{Y}$ & $\mathrm{Y}$ & $\mathrm{Y}$ & $\mathrm{Y}$ & $\mathrm{Y}$ & $\mathrm{Y}$ & $\mathrm{Y}$ & $\mathrm{Y}$ & $\mathrm{U}$ & $\mathrm{Y}$ & $\mathrm{Y}$ & $\mathrm{Y}$ \\
\hline Balaban, 2011258 & $U$ & $U$ & $\mathrm{Y}$ & $\mathrm{N}$ & $\mathrm{N}$ & $U$ & $U$ & $\mathrm{Y}$ & $\mathrm{Y}$ & $\mathrm{Y}$ & $\mathrm{Y}$ & $\mathrm{Y}$ \\
\hline Bhattacharya, $2008^{135}$ & $\mathrm{Y}$ & $\mathrm{U}$ & $\mathrm{Y}$ & $\mathrm{Y}$ & $\mathrm{Y}$ & $\mathrm{Y}$ & $\mathrm{Y}$ & $\mathrm{Y}$ & $\mathrm{N}$ & $\mathrm{Y}$ & $\bar{Y}$ & $\mathrm{Y}$ \\
\hline Chen, $2016^{174}$ & $\mathrm{Y}$ & $\mathrm{Y}$ & $\mathrm{Y}$ & $\mathrm{Y}$ & $\mathrm{Y}$ & $\mathrm{Y}$ & $\mathrm{Y}$ & $\mathrm{Y}$ & $\mathrm{Y}$ & $\mathrm{Y}$ & $\mathrm{Y}$ & $\mathrm{Y}$ \\
\hline Choi , 2012 ${ }^{147}$ & $\mathrm{U}$ & $\mathrm{U}$ & $\mathrm{Y}$ & $\mathrm{U}$ & $\mathrm{U}$ & $\mathrm{U}$ & $\mathrm{U}$ & $\bar{U}$ & $\mathrm{U}$ & $\mathrm{Y}$ & $\mathrm{Y}$ & $\mathrm{Y}$ \\
\hline Custers, $2012^{139}$ & $\mathrm{Y}$ & $\mathrm{Y}$ & $\mathrm{Y}$ & $\mathrm{Y}$ & $\mathrm{Y}$ & $\mathrm{Y}$ & $\mathrm{Y}$ & $\mathrm{Y}$ & $\mathrm{U}$ & $\mathrm{Y}$ & $\mathrm{Y}$ & $\mathrm{Y}$ \\
\hline Danhof,, $2018^{244}$ & $\mathrm{~N}$ & $\mathrm{~N}$ & $\mathrm{Y}$ & $\mathrm{N}$ & $U$ & $U$ & $U$ & $\mathrm{Y}$ & $\mathrm{N}$ & $\mathrm{Y}$ & $\mathrm{Y}$ & $U$ \\
\hline Demirol, $2007^{230}$ & $\mathrm{Y}$ & $\mathrm{Y}$ & $\mathrm{Y}$ & $\mathrm{Y}$ & $\mathrm{Y}$ & $\mathrm{Y}$ & $\mathrm{Y}$ & $\mathrm{Y}$ & $\mathrm{N}$ & $\mathrm{Y}$ & $\mathrm{Y}$ & $\mathrm{Y}$ \\
\hline Diamond, $2015^{233}$ & $\bar{Y}$ & $\bar{Y}$ & $\bar{Y}$ & $\bar{Y}$ & $\bar{Y}$ & $\bar{Y}$ & $\bar{Y}$ & $\mathrm{Y}$ & $\bar{Y}$ & $\bar{Y}$ & $\bar{Y}$ & $\bar{Y}$ \\
\hline Dreyer, $2016^{250}$ & $\mathrm{Y}$ & $\mathrm{Y}$ & $\mathrm{N}$ & $\mathrm{Y}$ & $\mathrm{Y}$ & $\mathrm{Y}$ & $\mathrm{Y}$ & $\mathrm{Y}$ & U & $\mathrm{Y}$ & $\bar{Y}$ & $\bar{Y}$ \\
\hline Ebrahimi, $2010^{229}$ & $\mathrm{Y}$ & $\mathrm{Y}$ & $\mathrm{Y}$ & $\mathrm{Y}$ & $\mathrm{Y}$ & $\mathrm{Y}$ & $\mathrm{Y}$ & $\mathrm{Y}$ & $\mathrm{N}$ & $\mathrm{Y}$ & $\mathrm{Y}$ & $\mathrm{Y}$ \\
\hline Einarsson, $2017^{181}$ & $\mathrm{Y}$ & $U$ & $\mathrm{Y}$ & $\mathrm{Y}$ & $\mathrm{Y}$ & $\mathrm{Y}$ & $\mathrm{Y}$ & $\mathrm{N}$ & $\mathrm{N}$ & $\mathrm{Y}$ & $\mathrm{Y}$ & $\mathrm{Y}$ \\
\hline
\end{tabular}




\begin{tabular}{|c|c|c|c|c|c|c|c|c|c|c|c|c|}
\hline Study & 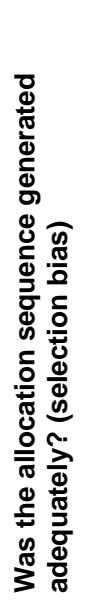 & 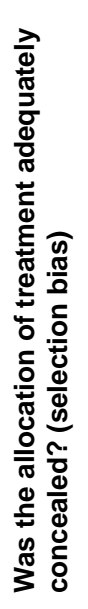 & 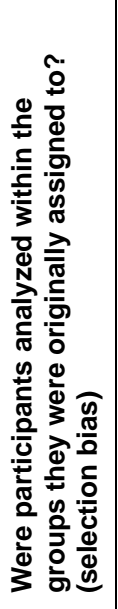 & 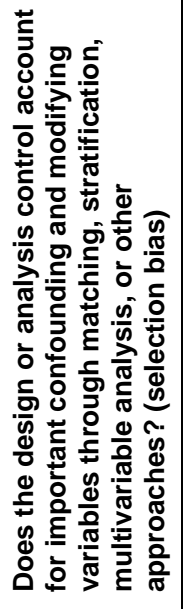 & 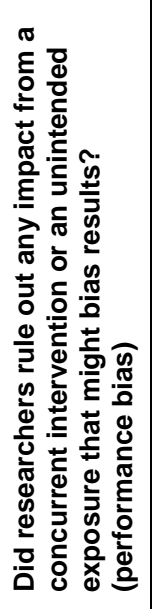 & 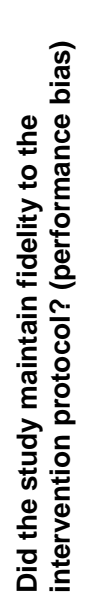 & 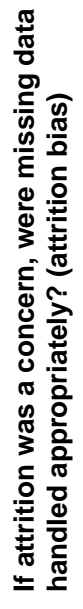 & 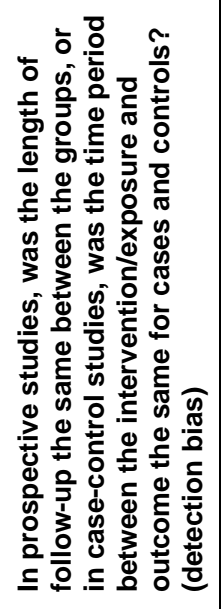 & 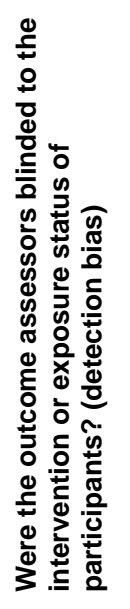 & 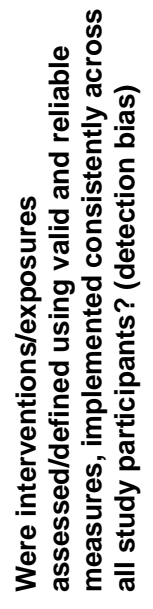 & 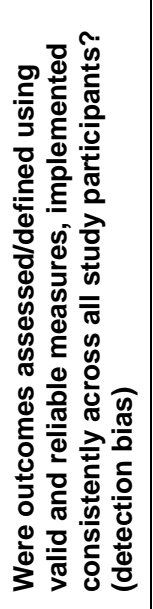 & 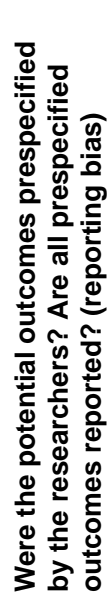 \\
\hline Elsedeek, 2014 ${ }^{194}$ & $\bar{Y}$ & $\mathrm{Y}$ & $\bar{U}$ & $\mathrm{Y}$ & $\mathrm{Y}$ & $\bar{Y}$ & $\mathrm{U}$ & $\mathrm{Y}$ & $\mathrm{Y}$ & $\mathrm{Y}$ & $\bar{Y}$ & $\mathrm{Y}$ \\
\hline Emekci Ozay, $2017^{182}$ & $\mathrm{Y}$ & $\mathrm{Y}$ & $\mathrm{Y}$ & $\mathrm{U}$ & $\mathrm{Y}$ & $\mathrm{Y}$ & $\mathrm{U}$ & $\mathrm{Y}$ & $\mathrm{Y}$ & $\mathrm{Y}$ & $\mathrm{Y}$ & $\mathrm{Y}$ \\
\hline Erdem, $2009^{225}$ & $\mathrm{Y}$ & $\mathrm{N}$ & $\mathrm{Y}$ & $\mathrm{N}$ & $U$ & $\mathrm{Y}$ & $\mathrm{Y}$ & $\mathrm{Y}$ & $\mathrm{N}$ & $\mathrm{Y}$ & $\mathrm{Y}$ & $\mathrm{Y}$ \\
\hline Erdem, $2015^{208}$ & $\bar{Y}$ & $\mathrm{Y}$ & $\bar{Y}$ & $\mathrm{Y}$ & $\bar{Y}$ & $\bar{Y}$ & $\bar{Y}$ & $\bar{Y}$ & $\mathrm{U}$ & $\bar{Y}$ & $\mathrm{Y}$ & $\mathrm{Y}$ \\
\hline Farquhar, $2018^{238}$ & $\overline{\mathrm{Y}}$ & $\mathrm{Y}$ & $\bar{Y}$ & $\bar{Y}$ & $\bar{Y}$ & $\bar{Y}$ & $\bar{Y}$ & $\bar{Y}$ & $\mathrm{~N}$ & $\bar{Y}$ & $\bar{Y}$ & $\bar{Y}$ \\
\hline $\mathrm{Ge}, 2008^{167}$ & $\mathrm{Y}$ & $\mathrm{Y}$ & $\mathrm{Y}$ & $\mathrm{Y}$ & $\mathrm{Y}$ & $\bar{Y}$ & $\mathrm{Y}$ & $\mathrm{Y}$ & $\mathrm{U}$ & $\mathrm{Y}$ & $\mathrm{Y}$ & $\mathrm{Y}$ \\
\hline Ghahiri, $2016^{170}$ & $\bar{Y}$ & $\mathrm{Y}$ & $\bar{Y}$ & $\mathrm{Y}$ & $\bar{Y}$ & $\bar{Y}$ & $\mathrm{~N}$ & $U$ & $\mathrm{U}$ & $\mathrm{Y}$ & $\mathrm{U}$ & $\mathrm{N}$ \\
\hline Ghanem, $2013^{145}$ & $\mathrm{Y}$ & $\mathrm{N}$ & $\mathrm{Y}$ & $\mathrm{Y}$ & $\mathrm{Y}$ & $\mathrm{Y}$ & $\mathrm{Y}$ & $\mathrm{U}$ & $\mathrm{N}$ & $\mathrm{Y}$ & $\mathrm{Y}$ & $\mathrm{Y}$ \\
\hline Gibreel, $2013^{228}$ & $\mathrm{Y}$ & $\mathrm{Y}$ & $\mathrm{Y}$ & $\mathrm{Y}$ & $\bar{Y}$ & $\bar{Y}$ & $\bar{Y}$ & $\bar{Y}$ & $\mathrm{~N}$ & $\mathrm{Y}$ & $\mathrm{Y}$ & $\mathrm{Y}$ \\
\hline Goldman, $2014^{210}$ & $\mathrm{Y}$ & $\mathrm{Y}$ & $\mathrm{Y}$ & $\mathrm{Y}$ & $U$ & $\mathrm{Y}$ & $\mathrm{Y}$ & $\mathrm{Y}$ & $\mathrm{Y}$ & $\mathrm{Y}$ & $\mathrm{Y}$ & $\mathrm{Y}$ \\
\hline Gregoriou, $2008^{226}$ & $\mathrm{Y}$ & $\mathrm{Y}$ & $\mathrm{Y}$ & $\mathrm{Y}$ & $\mathrm{Y}$ & $\mathrm{Y}$ & $\mathrm{Y}$ & $\mathrm{Y}$ & $\mathrm{U}$ & $\mathrm{Y}$ & $\mathrm{Y}$ & $\mathrm{Y}$ \\
\hline Hajizadeh, $2017^{261}$ & $\mathrm{Y}$ & $\mathrm{Y}$ & $\mathrm{Y}$ & $\mathrm{Y}$ & $\mathrm{Y}$ & $\mathrm{Y}$ & $\mathrm{Y}$ & $\mathrm{Y}$ & $\mathrm{N}$ & $\mathrm{Y}$ & $\mathrm{Y}$ & $\mathrm{Y}$ \\
\hline Harira, $2018^{246}$ & $\mathrm{Y}$ & $\bar{Y}$ & $\mathrm{Y}$ & $\mathrm{U}$ & $\bar{U}$ & $\bar{Y}$ & $\bar{Y}$ & $\bar{Y}$ & $\mathrm{Y}$ & $\bar{Y}$ & $\bar{Y}$ & $\mathrm{U}$ \\
\hline Hassan, $2017^{183}$ & $\mathrm{Y}$ & $\mathrm{Y}$ & $\mathrm{Y}$ & $\mathrm{Y}$ & $\mathrm{Y}$ & $\mathrm{Y}$ & $\mathrm{Y}$ & $\mathrm{Y}$ & $U$ & $\mathrm{Y}$ & $\mathrm{Y}$ & $\mathrm{Y}$ \\
\hline Homburg, $2012^{152}$ & $\mathrm{Y}$ & $\mathrm{Y}$ & $\mathrm{Y}$ & $\mathrm{Y}$ & $\mathrm{Y}$ & $\mathrm{Y}$ & $\mathrm{Y}$ & $\mathrm{Y}$ & $\mathrm{U}$ & $\mathrm{Y}$ & $\mathrm{Y}$ & $\mathrm{Y}$ \\
\hline Hosseini, $2010^{158}$ & $\mathrm{U}$ & $\mathrm{U}$ & $\mathrm{U}$ & $\mathrm{N}$ & $\mathrm{N}$ & $\mathrm{U}$ & $\mathrm{U}$ & $\mathrm{Y}$ & $\mathrm{Y}$ & $\mathrm{Y}$ & $\mathrm{Y}$ & $\mathrm{Y}$ \\
\hline Hossein-Rashidi, $2016^{176}$ & $\mathrm{Y}$ & $\mathrm{U}$ & $\mathrm{U}$ & $\mathrm{Y}$ & $\mathrm{Y}$ & $\mathrm{Y}$ & $\mathrm{N}$ & $\mathrm{Y}$ & $U$ & $\mathrm{Y}$ & $\mathrm{Y}$ & $\mathrm{Y}$ \\
\hline Ibrahim, $2017^{189}$ & $\mathrm{Y}$ & $\mathrm{U}$ & $\mathrm{Y}$ & $\mathrm{N}$ & $\mathrm{N}$ & $\mathrm{Y}$ & $\mathrm{U}$ & $\mathrm{Y}$ & $\mathrm{Y}$ & $\mathrm{Y}$ & $\mathrm{Y}$ & $\mathrm{U}$ \\
\hline Jacob, $2016^{177}$ & $\mathrm{Y}$ & $\mathrm{Y}$ & $\mathrm{Y}$ & $\mathrm{Y}$ & $\mathrm{Y}$ & $\mathrm{Y}$ & $\mathrm{Y}$ & $\mathrm{Y}$ & $\mathrm{Y}$ & $\mathrm{Y}$ & $\mathrm{Y}$ & $\mathrm{Y}$ \\
\hline Jahromi, $2017^{243}$ & $\mathrm{Y}$ & $\mathrm{Y}$ & $\mathrm{Y}$ & $\mathrm{N}$ & $\mathrm{U}$ & $\mathrm{Y}$ & $\mathrm{U}$ & $\mathrm{Y}$ & $\mathrm{Y}$ & $\mathrm{Y}$ & $\mathrm{Y}$ & $\mathrm{U}$ \\
\hline Johnson, $2010^{160}$ & $\mathrm{Y}$ & $\mathrm{Y}$ & $\mathrm{Y}$ & $\bar{Y}$ & $\mathrm{~N}$ & $\bar{Y}$ & $\mathrm{Y}$ & $\mathrm{Y}$ & $\mathrm{Y}$ & $\bar{U}$ & $\mathrm{Y}$ & $\mathrm{Y}$ \\
\hline Kansal Kalra, 2008 223 & $\mathrm{Y}$ & $\mathrm{Y}$ & $\mathrm{Y}$ & $\mathrm{N}$ & $\mathrm{U}$ & $\bar{Y}$ & $\bar{Y}$ & $\mathrm{Y}$ & $\mathrm{N}$ & $\mathrm{Y}$ & $\mathrm{Y}$ & $\mathrm{Y}$ \\
\hline Kar, $2015^{173}$ & $\mathrm{~N}$ & $\mathrm{Y}$ & $\mathrm{Y}$ & $\mathrm{Y}$ & $\mathrm{Y}$ & $\mathrm{Y}$ & $\mathrm{N}$ & $\mathrm{Y}$ & $\mathrm{Y}$ & $\mathrm{Y}$ & $\mathrm{N}$ & $\mathrm{Y}$ \\
\hline Khosravi , 2015 32 & $\mathrm{Y}$ & $\mathrm{Y}$ & $\mathrm{Y}$ & $\mathrm{Y}$ & $\mathrm{Y}$ & $U$ & $\mathrm{U}$ & $\mathrm{Y}$ & $\mathrm{Y}$ & $\mathrm{Y}$ & $\mathrm{Y}$ & $\mathrm{Y}$ \\
\hline Kim, $2011^{219}$ & $\mathrm{U}$ & $\mathrm{U}$ & $\mathrm{Y}$ & $\mathrm{N}$ & $\mathrm{U}$ & $\mathrm{Y}$ & $\mathrm{U}$ & $\mathrm{U}$ & $\mathrm{U}$ & $\mathrm{Y}$ & $\mathrm{Y}$ & $\mathrm{U}$ \\
\hline Kim, $2012^{150}$ & $\mathrm{Y}$ & $\mathrm{Y}$ & $\mathrm{Y}$ & $\mathrm{Y}$ & $\mathrm{Y}$ & $\mathrm{Y}$ & $\mathrm{Y}$ & $\mathrm{U}$ & $\mathrm{N}$ & $\mathrm{Y}$ & $\mathrm{Y}$ & $\mathrm{Y}$ \\
\hline Kjotrod, $2011^{155}$ & $\mathrm{Y}$ & $\mathrm{Y}$ & $Y$ & $\mathrm{Y}$ & $\mathrm{N}$ & $\mathrm{Y}$ & $\mathrm{Y}$ & $\mathrm{Y}$ & $\mathrm{Y}$ & $\mathrm{Y}$ & $\mathrm{Y}$ & $\mathrm{Y}$ \\
\hline Kurzawa, $2008^{164}$ & $\mathrm{Y}$ & $\mathrm{N}$ & $\mathrm{Y}$ & $\mathrm{Y}$ & $\mathrm{Y}$ & $\mathrm{Y}$ & $\mathrm{U}$ & $\mathrm{Y}$ & $\mathrm{N}$ & $\mathrm{Y}$ & $\mathrm{U}$ & $\mathrm{Y}$ \\
\hline Kuzmin, $2014^{248}$ & $\mathrm{U}$ & $\mathrm{U}$ & $\mathrm{U}$ & $\mathrm{U}$ & $\mathrm{N}$ & $\mathrm{U}$ & $\mathrm{U}$ & $U$ & $\mathrm{~N}$ & $\mathrm{U}$ & $\mathrm{Y}$ & $\mathrm{Y}$ \\
\hline
\end{tabular}

G-7 


\begin{tabular}{|c|c|c|c|c|c|c|c|c|c|c|c|c|}
\hline Study & 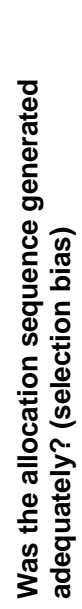 & 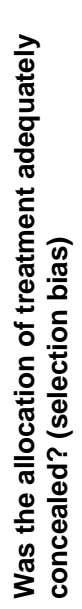 & 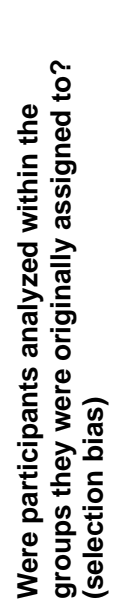 & 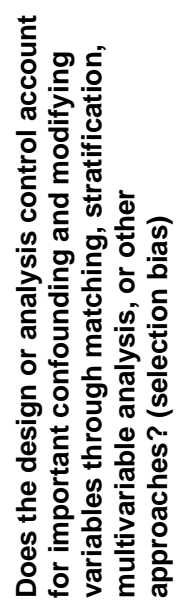 & 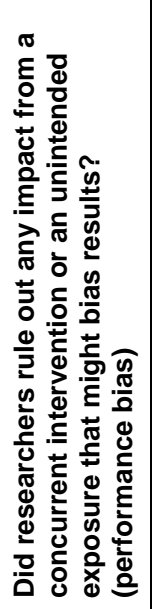 & 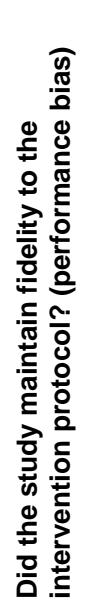 & 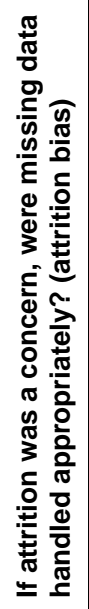 & 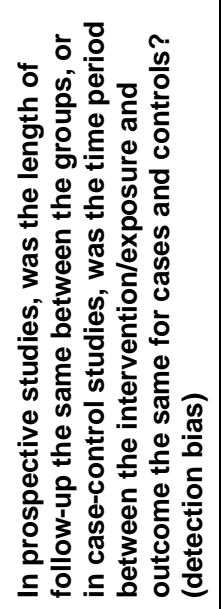 & 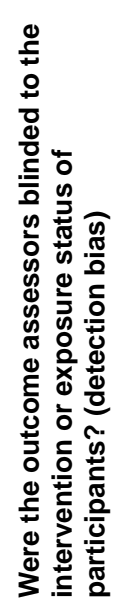 & 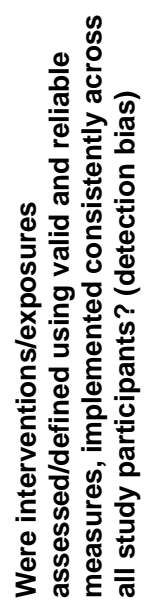 & 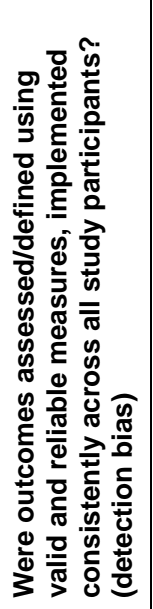 & 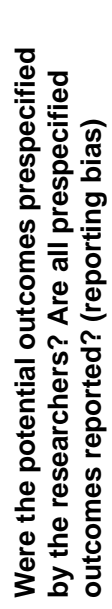 \\
\hline La Sala, $2015^{253}$ & $\mathrm{~N}$ & $\mathrm{~N}$ & $\mathrm{U}$ & $\mathrm{Y}$ & $\mathrm{N}$ & $\mathrm{U}$ & $\mathrm{Y}$ & $\mathrm{Y}$ & $\mathrm{Y}$ & $\mathrm{Y}$ & $\mathrm{Y}$ & $\mathrm{Y}$ \\
\hline Leandri, $2013^{256}$ & $\mathrm{Y}$ & $\mathrm{Y}$ & $\mathrm{U}$ & $\mathrm{Y}$ & $\mathrm{Y}$ & $\mathrm{U}$ & $U$ & $\mathrm{Y}$ & $\mathrm{Y}$ & $\mathrm{Y}$ & $\mathrm{Y}$ & $\mathrm{Y}$ \\
\hline Legro, $2007^{128}$ & $\mathrm{Y}$ & $\mathrm{Y}$ & $\mathrm{Y}$ & $\mathrm{Y}$ & $\mathrm{Y}$ & $\mathrm{Y}$ & $\mathrm{Y}$ & $\mathrm{Y}$ & $\mathrm{Y}$ & $\mathrm{Y}$ & $\mathrm{Y}$ & $\mathrm{N}$ \\
\hline Legro, $2014^{131}$ & $\mathrm{Y}$ & $\mathrm{Y}$ & $\mathrm{Y}$ & $\mathrm{Y}$ & $\mathrm{Y}$ & $\mathrm{Y}$ & $\mathrm{Y}$ & $\mathrm{Y}$ & $\mathrm{Y}$ & $\mathrm{Y}$ & $\mathrm{Y}$ & $\mathrm{Y}$ \\
\hline Legro, $2015^{172}$ & $\mathrm{Y}$ & $\bar{Y}$ & $Y$ & $\mathrm{Y}$ & $Y$ & $\bar{Y}$ & $\bar{Y}$ & $Y$ & $\mathrm{Y}$ & $\bar{Y}$ & $Y$ & $\mathrm{Y}$ \\
\hline Maher, $2018^{245}$ & $\mathrm{Y}$ & $\mathrm{Y}$ & $\bar{Y}$ & $\mathrm{~N}$ & $U$ & $\bar{Y}$ & $\bar{Y}$ & $\bar{Y}$ & $\mathrm{~N}$ & $\bar{Y}$ & $\mathrm{Y}$ & $\mathrm{U}$ \\
\hline Majumdar, $2013^{214}$ & $\mathrm{Y}$ & $\mathrm{U}$ & $\mathrm{N}$ & $\mathrm{N}$ & $\mathrm{U}$ & $\mathrm{U}$ & $\mathrm{N}$ & $\mathrm{Y}$ & $\mathrm{U}$ & $\mathrm{Y}$ & $\mathrm{Y}$ & $\mathrm{U}$ \\
\hline Mehrabian, $2012^{148}$ & $\mathrm{Y}$ & $U$ & $\mathrm{Y}$ & $\mathrm{Y}$ & $\mathrm{U}$ & $\mathrm{Y}$ & $\mathrm{U}$ & U & $\mathrm{U}$ & $\mathrm{Y}$ & $\mathrm{Y}$ & $\mathrm{Y}$ \\
\hline Mohammadi Yeganeh, $2018^{190}$ & $\bar{Y}$ & $U$ & $\mathrm{Y}$ & $\mathrm{N}$ & $\mathrm{U}$ & $\bar{Y}$ & $\mathrm{~N}$ & $\mathrm{Y}$ & $\mathrm{U}$ & $\mathrm{Y}$ & $\bar{Y}$ & $\bar{Y}$ \\
\hline Morad, $2012^{227}$ & $\mathrm{Y}$ & $\bar{Y}$ & $\bar{Y}$ & $\mathrm{Y}$ & $\mathrm{Y}$ & $\mathrm{Y}$ & $Y$ & $\mathrm{Y}$ & $\mathrm{N}$ & $\mathrm{Y}$ & $\mathrm{Y}$ & $\mathrm{Y}$ \\
\hline Morin-Papunen, $2012^{151}$ & $\mathrm{Y}$ & $\mathrm{Y}$ & $\mathrm{Y}$ & $\mathrm{Y}$ & $\mathrm{Y}$ & $\mathrm{Y}$ & $\mathrm{Y}$ & $U$ & $\mathrm{Y}$ & $\mathrm{Y}$ & $\mathrm{Y}$ & $\mathrm{Y}$ \\
\hline Mutsaerts, $2016^{119}$ & $\mathrm{Y}$ & $\mathrm{Y}$ & $\mathrm{Y}$ & $\mathrm{Y}$ & $\mathrm{Y}$ & $\mathrm{Y}$ & $\mathrm{Y}$ & $\mathrm{Y}$ & $\mathrm{N}$ & $\mathrm{Y}$ & $\mathrm{Y}$ & $\mathrm{Y}$ \\
\hline Nada, $2016^{234}$ & $\mathrm{Y}$ & $\bar{Y}$ & $\mathrm{Y}$ & $\mathrm{Y}$ & $\bar{Y}$ & $\mathrm{Y}$ & $\bar{Y}$ & $\mathrm{Y}$ & $\mathrm{U}$ & $\mathrm{Y}$ & $\mathrm{Y}$ & $\mathrm{Y}$ \\
\hline Nahuis, $2011^{141}$ & $\mathrm{U}$ & $\mathrm{U}$ & $\mathrm{Y}$ & $\mathrm{Y}$ & $\mathrm{Y}$ & $\mathrm{Y}$ & $\mathrm{Y}$ & $\mathrm{Y}$ & $\mathrm{U}$ & $\mathrm{Y}$ & $\mathrm{Y}$ & $\mathrm{U}$ \\
\hline Nandi, $2017^{240}$ & $\mathrm{U}$ & $\mathrm{Y}$ & $\bar{Y}$ & $\mathrm{~N}$ & $U$ & $\mathrm{U}$ & $\bar{Y}$ & $\mathrm{Y}$ & $\mathrm{N}$ & $\bar{Y}$ & $\mathrm{Y}$ & $\bar{Y}$ \\
\hline Palomba, $2010^{163}$ & $\mathrm{Y}$ & $\mathrm{Y}$ & $\mathrm{Y}$ & $\mathrm{N}$ & $\mathrm{N}$ & $\mathrm{Y}$ & $\mathrm{Y}$ & $\mathrm{Y}$ & $\mathrm{Y}$ & $\mathrm{Y}$ & $\mathrm{Y}$ & $\mathrm{Y}$ \\
\hline Palomba, 2011 ${ }^{153}$ & $\mathrm{Y}$ & $\mathrm{Y}$ & $\mathrm{Y}$ & $\mathrm{Y}$ & $Y$ & $\mathrm{Y}$ & $\mathrm{Y}$ & $U$ & $\mathrm{Y}$ & $\mathrm{Y}$ & $\mathrm{Y}$ & $\mathrm{Y}$ \\
\hline Peeraer, $2015^{288}$ & $\mathrm{Y}$ & $\mathrm{Y}$ & $\mathrm{Y}$ & $\mathrm{Y}$ & $\mathrm{Y}$ & $\mathrm{Y}$ & $\mathrm{Y}$ & $\mathrm{Y}$ & $U$ & $\mathrm{Y}$ & $\mathrm{Y}$ & $\mathrm{Y}$ \\
\hline Pourali, $2017^{241}$ & $\mathrm{U}$ & $\mathrm{Y}$ & $Y$ & $\mathrm{~N}$ & $\mathrm{U}$ & $\mathrm{Y}$ & $U$ & $Y$ & $\mathrm{Y}$ & $\mathrm{Y}$ & $Y$ & $\mathrm{Y}$ \\
\hline $\mathrm{Qu}, 2017^{252}$ & $Y$ & $\bar{Y}$ & $\mathrm{Y}$ & $\mathrm{N}$ & $\mathrm{N}$ & $\bar{Y}$ & $\mathrm{~N}$ & $\mathrm{Y}$ & $\mathrm{Y}$ & $U$ & $\mathrm{Y}$ & $\mathrm{Y}$ \\
\hline Ragni, $2012^{218}$ & $\mathrm{Y}$ & $\mathrm{Y}$ & $\mathrm{Y}$ & $\mathrm{N}$ & $\mathrm{U}$ & $\mathrm{Y}$ & $\mathrm{N}$ & $\mathrm{Y}$ & $\mathrm{U}$ & $\mathrm{Y}$ & $\mathrm{Y}$ & $\bar{Y}$ \\
\hline Rahman, $2017^{264}$ & $\mathrm{Y}$ & $U$ & $\mathrm{Y}$ & $\mathrm{N}$ & $\mathrm{U}$ & $\mathrm{Y}$ & $\mathrm{N}$ & $U$ & $\mathrm{U}$ & $\mathrm{Y}$ & $\bar{Y}$ & $\bar{Y}$ \\
\hline Rashidi, $2013^{216}$ & $\mathrm{U}$ & $\mathrm{U}$ & $\mathrm{Y}$ & $\mathrm{N}$ & $\mathrm{U}$ & $\mathrm{Y}$ & $\mathrm{N}$ & $\mathrm{Y}$ & $\mathrm{U}$ & $\mathrm{Y}$ & $U$ & $U$ \\
\hline Rashidi, $2015^{143}$ & $\mathrm{U}$ & $\mathrm{U}$ & $\mathrm{Y}$ & $\mathrm{U}$ & $\mathrm{U}$ & $\mathrm{Y}$ & $\mathrm{Y}$ & $\mathrm{Y}$ & $\mathrm{U}$ & $\mathrm{Y}$ & $\mathrm{Y}$ & $\mathrm{Y}$ \\
\hline Razi, $2013^{255}$ & $\mathrm{~N}$ & $\mathrm{~N}$ & 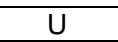 & $\mathrm{N}$ & $\mathrm{N}$ & U & $\mathrm{Y}$ & $\mathrm{Y}$ & $\mathrm{U}$ & $\mathrm{Y}$ & $\mathrm{Y}$ & $\mathrm{U}$ \\
\hline Reindollar, $2010^{52}$ & $\mathrm{Y}$ & $\mathrm{Y}$ & $\mathrm{Y}$ & $\mathrm{Y}$ & $\mathrm{Y}$ & $\mathrm{Y}$ & $\mathrm{Y}$ & $\mathrm{Y}$ & $\mathrm{Y}$ & $\mathrm{Y}$ & $\bar{Y}$ & $\bar{Y}$ \\
\hline Rubio, $2013^{217}$ & $\mathrm{Y}$ & $\mathrm{N}$ & $\mathrm{Y}$ & $\mathrm{N}$ & $U$ & $\mathrm{Y}$ & $U$ & $\mathrm{Y}$ & $\mathrm{N}$ & $\mathrm{Y}$ & $\mathrm{N}$ & $U$ \\
\hline Seckin, 2014 ${ }^{209}$ & $\mathrm{Y}$ & $\mathrm{U}$ & $\mathrm{Y}$ & $\mathrm{Y}$ & $\mathrm{Y}$ & $\mathrm{Y}$ & $\mathrm{Y}$ & $U$ & $\mathrm{~N}$ & $\mathrm{Y}$ & $\mathrm{Y}$ & $\mathrm{Y}$ \\
\hline Selman, $2016^{237}$ & $\mathrm{Y}$ & $\mathrm{Y}$ & $\mathrm{Y}$ & $\bar{Y}$ & $\mathrm{Y}$ & $\mathrm{Y}$ & $\bar{Y}$ & $\mathrm{Y}$ & $\mathrm{U}$ & $\bar{Y}$ & $\bar{Y}$ & $\mathrm{Y}$ \\
\hline
\end{tabular}

G-8 


\begin{tabular}{|c|c|c|c|c|c|c|c|c|c|c|c|c|}
\hline Study & 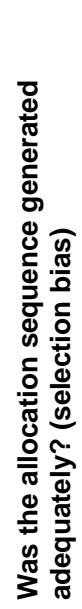 & 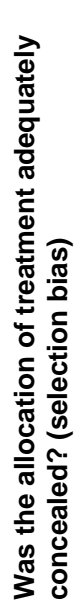 & 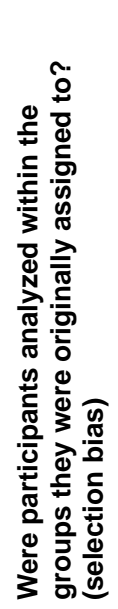 & 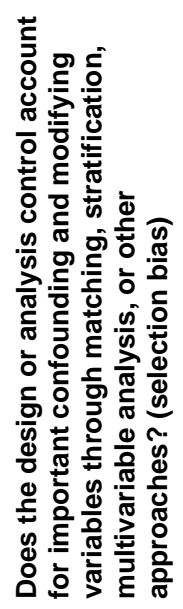 & 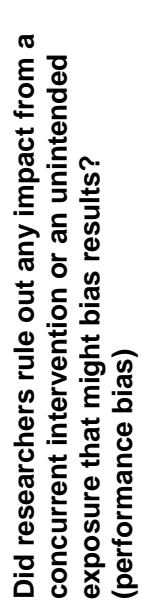 & 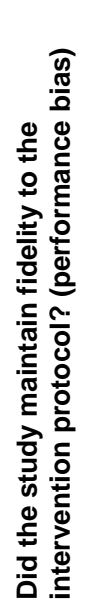 & 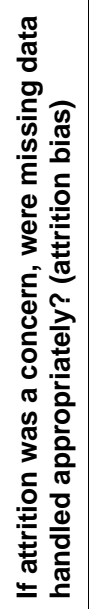 & 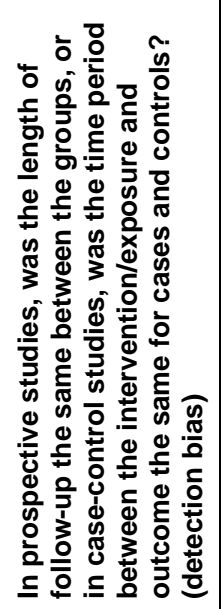 & 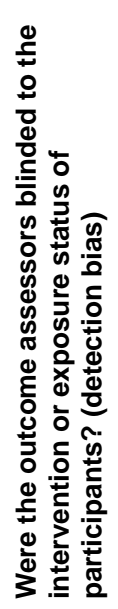 & 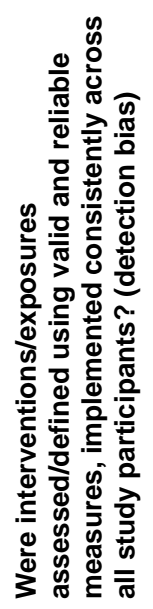 & 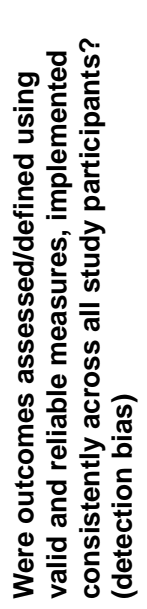 & 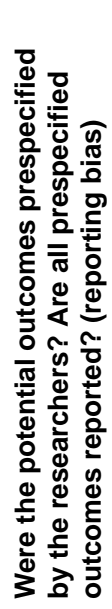 \\
\hline Shi, $2018^{262}$ & $\mathrm{Y}$ & $\mathrm{Y}$ & $\mathrm{Y}$ & $\mathrm{U}$ & $\mathrm{N}$ & $U$ & $\mathrm{Y}$ & $\mathrm{Y}$ & $U$ & $\mathrm{Y}$ & $\mathrm{Y}$ & $\mathrm{Y}$ \\
\hline Sismanoglu, $2009^{267}$ & $\mathrm{Y}$ & $\mathrm{N}$ & $\mathrm{N}$ & $\mathrm{Y}$ & $\mathrm{N}$ & $\mathrm{Y}$ & $\mathrm{Y}$ & $\mathrm{Y}$ & $\mathrm{Y}$ & $\mathrm{Y}$ & U & $\mathrm{Y}$ \\
\hline Stadtmauer, $2011^{159}$ & $\mathrm{Y}$ & $\mathrm{Y}$ & $\mathrm{Y}$ & $\mathrm{Y}$ & $\mathrm{N}$ & U & $\mathrm{Y}$ & $\mathrm{Y}$ & $\mathrm{N}$ & $\mathrm{Y}$ & $\mathrm{Y}$ & $\mathrm{Y}$ \\
\hline Tartagni, $2015^{207}$ & $\bar{Y}$ & $\mathrm{U}$ & $\bar{Y}$ & $\mathrm{~N}$ & $U$ & $Y$ & $U$ & $\mathrm{Y}$ & $\mathrm{Y}$ & $U$ & $\mathrm{Y}$ & $\mathrm{Y}$ \\
\hline Tehraninejad, $2010^{161}$ & $\mathrm{Y}$ & $\mathrm{Y}$ & $\mathrm{Y}$ & $\mathrm{Y}$ & $\mathrm{N}$ & $\mathrm{Y}$ & $\mathrm{N}$ & $\mathrm{Y}$ & $\mathrm{N}$ & $\mathrm{Y}$ & $\mathrm{Y}$ & $\mathrm{Y}$ \\
\hline Toftager, $2016^{275}$ & $\mathrm{Y}$ & $\mathrm{Y}$ & $\mathrm{Y}$ & $\mathrm{Y}$ & $\mathrm{Y}$ & $\mathrm{Y}$ & $\mathrm{Y}$ & $\mathrm{Y}$ & $\mathrm{U}$ & $\mathrm{Y}$ & $\mathrm{Y}$ & $\mathrm{Y}$ \\
\hline Toftager, $2017^{285}$ & $\mathrm{Y}$ & $\mathrm{Y}$ & $\mathrm{Y}$ & $\mathrm{Y}$ & $\mathrm{Y}$ & $\mathrm{U}$ & $\mathrm{Y}$ & $\mathrm{Y}$ & $\mathrm{N}$ & $\mathrm{Y}$ & $\mathrm{Y}$ & $\mathrm{Y}$ \\
\hline Topçu, $2017^{191}$ & $\mathrm{Y}$ & $\mathrm{N}$ & $\mathrm{Y}$ & $\mathrm{Y}$ & $\mathrm{Y}$ & $\mathrm{Y}$ & $\mathrm{Y}$ & $\mathrm{Y}$ & $\mathrm{U}$ & $\mathrm{Y}$ & $\mathrm{Y}$ & $\mathrm{Y}$ \\
\hline van Rijswijk, $2017^{242}$ & $\mathrm{Y}$ & $\mathrm{Y}$ & $\mathrm{Y}$ & $\mathrm{Y}$ & $\bar{Y}$ & U & $\bar{Y}$ & $\mathrm{Y}$ & $\mathrm{U}$ & $U$ & $\mathrm{Y}$ & $\mathrm{Y}$ \\
\hline van Rumste, $2014^{212}$ & $\mathrm{U}$ & $\mathrm{U}$ & $\mathrm{U}$ & $\mathrm{U}$ & $\mathrm{U}$ & $\mathrm{Y}$ & $\mathrm{Y}$ & $\mathrm{Y}$ & $\mathrm{U}$ & $\mathrm{Y}$ & $\mathrm{Y}$ & $\mathrm{U}$ \\
\hline Wang, $2016^{171}$ & $\mathrm{~N}$ & $\mathrm{Y}$ & $\mathrm{Y}$ & $\mathrm{Y}$ & $\mathrm{Y}$ & $\mathrm{Y}$ & $\mathrm{N}$ & $Y$ & $\mathrm{Y}$ & $\mathrm{Y}$ & $\mathrm{Y}$ & $\mathrm{N}$ \\
\hline Weiss, $2018^{187}$ & $\mathrm{Y}$ & $\mathrm{U}$ & $\mathrm{Y}$ & $U$ & $\mathrm{Y}$ & $\mathrm{Y}$ & $\mathrm{Y}$ & $\mathrm{Y}$ & $\mathrm{N}$ & $\mathrm{Y}$ & $\mathrm{Y}$ & $\mathrm{Y}$ \\
\hline Wiser, $2010^{220}$ & $\mathrm{Y}$ & $\bar{Y}$ & $\mathrm{Y}$ & $\mathrm{N}$ & $U$ & $\mathrm{Y}$ & U & $\mathrm{Y}$ & $U$ & $\mathrm{~N}$ & $\mathrm{Y}$ & $U$ \\
\hline $\mathrm{Wu}, 2016^{175}$ & $\mathrm{Y}$ & $\mathrm{Y}$ & $\mathrm{Y}$ & $\mathrm{Y}$ & $U$ & $U$ & $\mathrm{Y}$ & $\mathrm{Y}$ & $\mathrm{U}$ & $\mathrm{Y}$ & $\mathrm{Y}$ & $\mathrm{Y}$ \\
\hline $\mathrm{Wu}, 2017^{188}$ & $\mathrm{Y}$ & $\mathrm{Y}$ & $\mathrm{N}$ & $\mathrm{U}$ & $\mathrm{Y}$ & $\mathrm{Y}$ & $\mathrm{N}$ & $\mathrm{Y}$ & $\mathrm{Y}$ & $\mathrm{Y}$ & $\mathrm{Y}$ & $\mathrm{Y}$ \\
\hline Yapca, $2015^{206}$ & $\mathrm{~N}$ & $\mathrm{Y}$ & $\mathrm{Y}$ & $\mathrm{N}$ & $\mathrm{U}$ & $\mathrm{Y}$ & $\mathrm{U}$ & $\mathrm{Y}$ & $\mathrm{N}$ & $\mathrm{Y}$ & $\mathrm{U}$ & $\mathrm{U}$ \\
\hline Yazici, $2014^{144}$ & $\mathrm{Y}$ & $\mathrm{N}$ & $\mathrm{U}$ & $\mathrm{Y}$ & $U$ & $\mathrm{Y}$ & $\mathrm{U}$ & $U$ & $\mathrm{~N}$ & $\mathrm{Y}$ & $\mathrm{Y}$ & $\mathrm{Y}$ \\
\hline Yildiz, $2014^{211}$ & $\mathrm{~N}$ & $\mathrm{~N}$ & $\mathrm{Y}$ & $\mathrm{N}$ & $U$ & $\mathrm{Y}$ & $\mathrm{U}$ & $\mathrm{Y}$ & $\mathrm{U}$ & $\mathrm{Y}$ & U & $\mathrm{U}$ \\
\hline Youssef, $2016^{235}$ & $\mathrm{Y}$ & $\mathrm{Y}$ & $\mathrm{Y}$ & $\mathrm{Y}$ & $\mathrm{Y}$ & $\mathrm{Y}$ & $\mathrm{Y}$ & $\mathrm{Y}$ & $\mathrm{U}$ & $\mathrm{Y}$ & $\mathrm{Y}$ & $\mathrm{Y}$ \\
\hline $\mathrm{Yu}, 2018^{247}$ & $\mathrm{Y}$ & $\mathrm{U}$ & $Y$ & $\mathrm{U}$ & $\mathrm{U}$ & U & $U$ & $Y$ & $\mathrm{U}$ & $\bar{Y}$ & $\mathrm{Y}$ & $\mathrm{U}$ \\
\hline Zahran, $2018^{192}$ & $\mathrm{Y}$ & $\mathrm{Y}$ & $\mathrm{N}$ & $\mathrm{Y}$ & $\mathrm{Y}$ & $\mathrm{Y}$ & $\mathrm{N}$ & $\mathrm{Y}$ & $\mathrm{Y}$ & $\bar{Y}$ & $\mathrm{Y}$ & $\mathrm{Y}$ \\
\hline Zain, $2009^{166}$ & $\mathrm{Y}$ & $\mathrm{N}$ & $\mathrm{Y}$ & $\mathrm{U}$ & $\mathrm{N}$ & $\mathrm{Y}$ & $\mathrm{N}$ & $Y$ & $\mathrm{~N}$ & $\mathrm{Y}$ & $\mathrm{Y}$ & $\mathrm{Y}$ \\
\hline Zakherah, $2010^{169}$ & $\mathrm{Y}$ & $\mathrm{Y}$ & $\mathrm{Y}$ & $\mathrm{N}$ & $\mathrm{N}$ & $\mathrm{Y}$ & $\mathrm{Y}$ & $\mathrm{Y}$ & $\mathrm{Y}$ & $\mathrm{Y}$ & $\mathrm{Y}$ & $\mathrm{Y}$ \\
\hline Zarei, $2016^{231}$ & $\mathrm{Y}$ & $\mathrm{U}$ & $\mathrm{Y}$ & $\mathrm{Y}$ & $\mathrm{U}$ & $\mathrm{Y}$ & $\mathrm{Y}$ & $Y$ & $\mathrm{U}$ & $\mathrm{Y}$ & $\mathrm{U}$ & $\mathrm{Y}$ \\
\hline Zheng, $2012^{149}$ & $\mathrm{Y}$ & $\mathrm{U}$ & $\mathrm{Y}$ & $\mathrm{Y}$ & $U$ & $\mathrm{Y}$ & $U$ & $U$ & $\mathrm{Y}$ & $\mathrm{Y}$ & $\bar{Y}$ & $\bar{Y}$ \\
\hline Zhu, $2014^{202}$ & $\mathrm{Y}$ & $\mathrm{Y}$ & $\mathrm{Y}$ & $\mathrm{Y}$ & $\mathrm{N}$ & $\mathrm{Y}$ & $\mathrm{Y}$ & $\mathrm{Y}$ & $U$ & $\mathrm{Y}$ & $Y$ & $Y$ \\
\hline
\end{tabular}




\section{Figure G-3. Summary of risk of bias assessment for included RCTs}

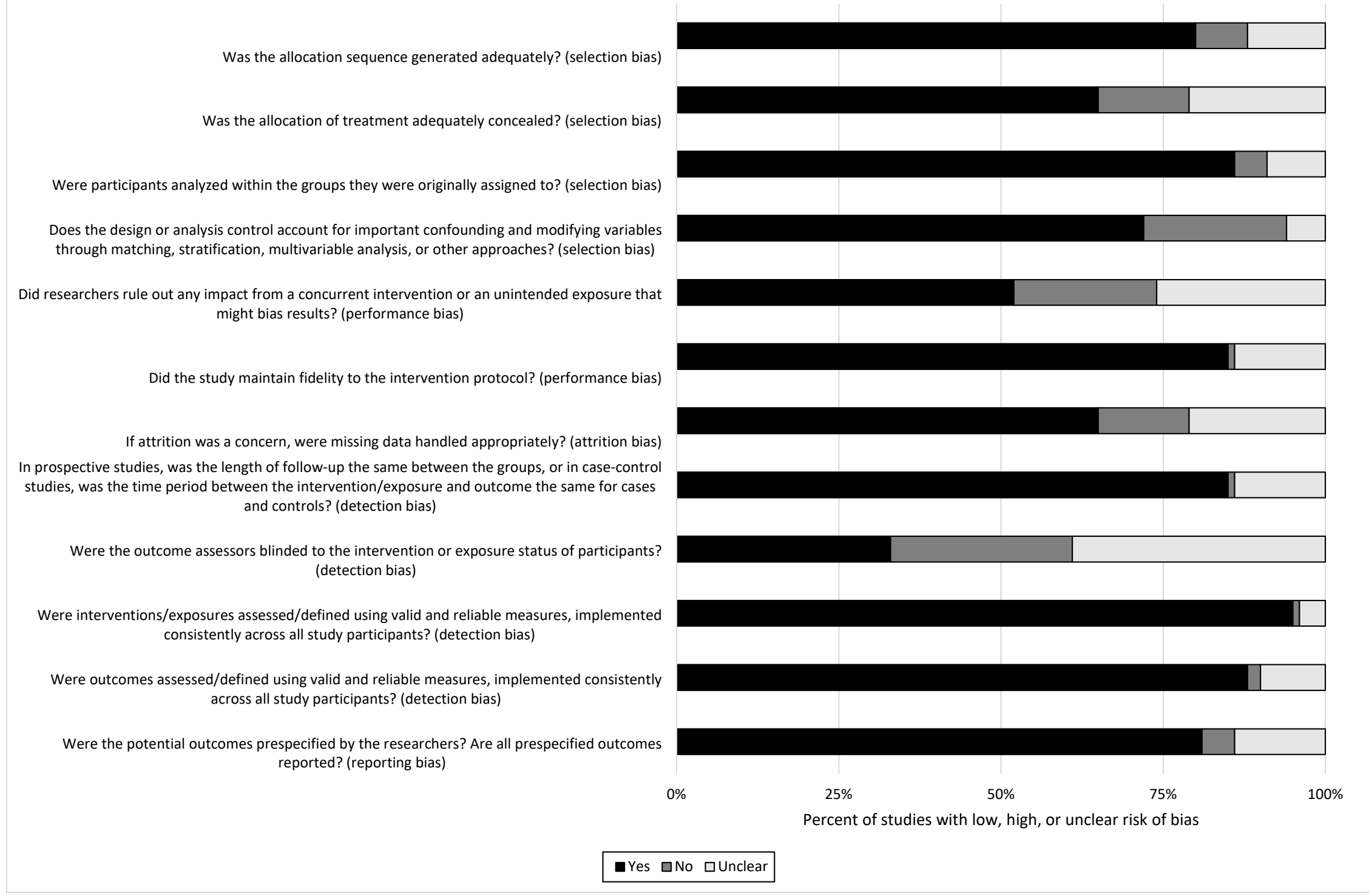




\section{Appendix H. Supplemental Project To Assess the Transparency of Reporting for Trials Evaluating Treatment for Infertility}

Authors: Williams JW Jr., Eaton JL, Gierisch JM, Masilamani V, von Isenburg M, Chobot MM ${ }^{\mathrm{a}}$

\section{Background and Objectives}

Selective reporting can bias estimates of effect, yet methods to detect such biases are limited. ${ }^{1,2}$ Statistical methods for detecting publication bias (e.g., funnel plots, Beggs rank correlation) are underpowered. ${ }^{3}$ Comparing outcomes listed under Methods versus those reported under Results in published manuscripts is an expedient but crude method for detecting reporting bias. ${ }^{4}$ Another method is to search ClinicalTrials.gov (CT.gov) and (a) compare studies identified there to published studies (to detect publication bias) and (b) compare planned analyses and outcomes reported in CT.gov to those reported in the final publication (to detect reporting bias). ${ }^{4,5}$ The EPC guidance recommends this approach. ${ }^{6}$ While conceptually sound, this approach may be labor-intensive, and its utility uncertain.

The overall goal of this project was to evaluate the utility of CT.gov for detecting selective reporting, and to determine the impact of selective reporting on the estimates of treatment effect. A secondary goal was to estimate the person-hours required to complete these analyses.

To accomplish these goals, we used an ongoing review, Management of Infertility, to explore differences between information from published sources included in the review and CT.gov.

\section{Methods}

\section{Scope and General Approach}

We adopted a pragmatic approach, using methods that could be readily incorporated into future systematic reviews. To maintain feasibility while still applying our methods to a range of interventions, we included KQ 1, KQ 2, and KQ 4 from the Management of Infertility review in this analysis. The KQs are listed below:

KQ 1: What are the comparative safety and effectiveness of available treatment strategies for women with polycystic ovary syndrome (PCOS) who are subfertile/infertile and who wish to become pregnant?

KQ 2: What are the comparative safety and effectiveness of available treatment strategies for women with endometriosis who are subfertile/infertile and who wish to become pregnant?

KQ 4: What are the comparative safety and effectiveness of available treatments for women with tubal or peritoneal factors (e.g., pelvic adhesions) who are subfertile/infertile and who wish to become pregnant?

\footnotetext{
${ }^{\text {a }}$ Williams JW Jr., Eaton JL, Gierisch JM, Masilamani V, von Isenburg M, Chobot M. Supplemental Project To Assess the Transparency of Reporting for Trials Evaluating Treatment for Infertility. Methods Research Report. AHRQ Publication No. 17EHC022-EF. Rockville, MD: Agency for Healthcare Research and Quality. June 2017.
} 


\section{Searching CT.gov}

We searched CT.gov for trials potentially applicable to the KQs with the assistance of our search librarian. Because CT.gov does not use MeSH-based search terms, we adapted the search strategies developed for the Management of Infertility review to language appropriate for CT.gov. We conducted two searches, a broad search using the basic interface and a more specific search using the advanced interface in CT.gov. For the broad search, we searched for synonyms for infertility (infertility OR infertile OR subfertility OR subfertile OR sub-fertility OR subfertile) in the conditions field and limited our results to interventional studies. For the narrow search, we searched for the same synonyms for infertility in the broader search terms field and combined this with multiple, separate searches for each of the conditions of interest. This narrower search was also limited to interventional studies. Exact search strings used in both searches are given in Appendix A.

Results of the two searches were imported into Excel.

\section{Matching Studies}

We matched randomized controlled trials (RCTs) identified in CT.gov with those identified for the Management of Infertility review at several levels.

First, we determined whether RCTs reporting a live birth outcome that were included in the Management of Infertility review had a matching record in CT.gov. Matching was performed initially using the NCT identifier (NCTID). Our intention was to conduct this matching using a semi-automated process within EndNote. This approach proved infeasible due to inconsistent assignment of NCTIDs to EndNote fields. Thus, all matching was accomplished by manual review. For unmatched studies, we conducted a secondary match using other trial registration numbers and then trial characteristics, including: condition, intervention, sample size, and author/investigator. Matching was performed initially for the broad CT.gov search. We then determined the proportion of matched studies that were not identified by the narrow CT.gov search.

Second, for matched studies (i.e., studies included in the Management of Infertility review with a CT.gov record), we abstracted selected variables from the CT.gov record to determine whether key study design variables and reported outcomes matched information in the published manuscript. Variables abstracted were:

- Date of completion

- Number of study arms

- Intervention description

- Study design

- Outcomes measures and results prioritized in the Management of Infertility review

- Analysis approach

- Subgroup analyses

Data from CT.gov were compared to published data. For each variable, the result was classified as: matching, discrepant, or possibly discrepant. Discrepant data were defined as cases where information was absent in one source but reported in another, or when the information given in the two sources was contradictory. Discrepancies were summarized narratively.

Third, we screened the unmatched CT.gov citations for potentially eligible completed trials. Eligibility criteria for each KQ are given in Table 1 of the Methods chapter of the main 
Management of Infertility review. For potentially eligible studies identified from CT.gov, we used author names and intervention terms to search for a matching publication in PubMed. We classified studies into two groups: (1) potentially eligible completed study without a published manuscript; and (2) potentially eligible completed study with a matching published manuscript that was not identified in the systematic review search.

All matching was limited to studies published since the 2005 International Committee of Medical Journal Editors (ICMJE) policy requiring trial registration. Matching was performed initially by a research assistant, and reviewed by a study investigator. Team members involved in matching piloted the data collection forms and procedures to refine them before full use.

\section{Estimate of Person-Hours Required To Complete the Project}

EPC staff routinely log the time spent working on projects using project-specific codes. Coinvestigators do not log project time routinely. Therefore, our project coordinator sent regular queries to co-investigators asking for estimates of time spent (to nearest 15 minutes) completing project-specific tasks. These estimates were tracked in an Excel spreadsheet. We used the staff logs and co-investigator reports to estimate the total staff time and co-investigator time dedicated to completing project-related activities.

\section{Impact on Systematic Review Conclusions}

Study conclusions will flow from the strength of evidence (SOE). We used the GRADE framework for evaluating SOE, a framework that includes assessment of risk of bias, consistency, precision, directness, and publication bias. The EPC risk of bias tool explicitly considers reporting bias. Therefore, risk of bias and publication bias are the domains most likely to be affected by supplemental data from CT.gov. In collaboration with authors of the Management of Infertility review, we reviewed the SOE table to determine qualitatively whether study conclusions would change.

\section{Results}

Results are presented in five sections: (1) concordance between RCTs included in the Management of Infertility review and in CT.gov; (2) studies identified from CT.gov as potentially eligible but not included in the Management of Infertility review; (3) concordance between data from CT.gov and published studies for studies present in both sources; (4) effects of CT.gov results on SOE and review conclusions; and (5) person-hours required to generate these results.

\section{Concordance Between RCTs Included in the Management of Infertility Review and in CT.gov}

Twenty-four unique RCTs reported live birth as an outcome and were included for KQs 1, 2, and 4 in the Management of Infertility review. The majority of these trials $(\mathrm{n}=22)$ were applicable to KQ 1 . Of the 24 trials:

- 8 were matched to a CT.gov record by NCTID

- 3 were matched by other trial ID number

- 1 was matched by other criteria (i.e., study characteristics)

- 12 were not matched 
All matched studies were confirmed by an investigator. Three preliminary matches based on "other criteria" were not confirmed by study investigators and are included in the 12 unmatched studies above.

Only one-third of the included trials were matched to a CT.gov record using the NCTID, the most reliable and readily applied matching variable. When using all available data, 50\% (95\% CI, 30 to 50\%) of the eligible studies were matched to a CT.gov record.

\section{Studies Identified From CT.gov as Potentially Eligible but Not Included in the Management of Infertility Review}

Using broad search criteria, we searched CT.gov for potentially eligible studies. The search yielded 858 registered studies. Of those, 376 were classified as “completed.” The 355 studies published from 2005 forward were reviewed by two study staff, and 94 were flagged as potentially eligible for the Management of Infertility review, with relevance to KQs as follows: $\mathrm{KQ} 1=14$, KQ $2=1$, KQ $3=69$, KQ $4=1$, KQ $5=3$, KQ $6=1$, and multiple KQs $=5$.

Of the 16 studies potentially relevant to KQs 1, 2, or 4, 11 had been identified in the Management of Infertility search and included in the review. The other 5 studies were reviewed by an investigator; details are reported in the Table $\mathrm{H}-1$.

Table H-1. Potentially eligible studies not included in the review

\begin{tabular}{|l|l|l|l|}
\hline NCTID & $\begin{array}{l}\text { Search } \\
\text { Strategy } \\
\text { Identifying Trial }\end{array}$ & $\begin{array}{l}\text { CT.gov } \\
\text { Completion } \\
\text { Date }\end{array}$ & Classification \\
\hline NCT01675843 & Both & March 2012 & Potentially eligible; no citation in PubMed \\
\hline NCT01679574 & Both & January 2012 & Potentially eligible; no citation in PubMed \\
\hline NCT01894074 & Broad & July 2015 & Potentially eligible; no citation in PubMed \\
\hline NCT00220545 & Both & March 2006 & $\begin{array}{l}\text { Identified in original review search but excluded } \\
\text { at title-and-abstract screening stage. Full text } \\
\text { reviewed and study included in Management of } \\
\text { Infertility review }\end{array}$ \\
\hline NCT01581359 & Both & May 2015 & Potentially eligible; no citation in PubMed \\
\hline
\end{tabular}

Only 5 potentially eligible studies were identified across the 3 KQs. Of these, 2 are recently completed trials (2015) and no journal publication was expected. Two trials with a combined sample size of 340 patients were completed more than 3 years ago, indicating potential publication bias. Both of these trials were applicable to KQ 1 . One trial was excluded at the titleand-abstract screening phase of the review; upon review of the full text, the study was reclassified as eligible and included in the review.

\section{Concordance Between Data From CT.gov and Published Studies for Studies Present in Both Sources}

Study investigators participating in the transparency project abstracted data independently from CT.gov for the 8 studies matched by the NCTID. These data were compared to data abstracted from published data by the Management of Infertility investigators.

Overall, there were no important differences in the study characteristic descriptions between the two sources. Details are described below: 
- The KQ classification matched for all 8 studies.

- The study design and number of study arms matched for all 8 studies.

- Of 5 studies reporting the enrolled "n," 4 were exact matches and 1 had a discrepancy in the estimated enrollment (326) vs. the number enrolled (320). Three studies did not report the sample size in CT.gov and thus were classified as discrepant.

- Intervention descriptions were substantially concordant for all 8 studies and thus were classified as matching.

- The analytic approach and any plans for subgroup analyses were not addressed in CT.gov for any of the studies. However, subgroup analyses were not reported in the published manuscripts for any of these trials.

- The funding sources was classified as matched for 6 studies. Two studies were classified as discrepant: 1 of these was classified as non-government/non-industry from CT.gov and as "not reported" from manuscript, and 1 was classified as non-government/non-industry from CT.gov and as government from the published manuscript.

Outcomes were compared at 2 levels: the outcomes planned from CT.gov to those reported in published manuscripts, and the results reported in CT.gov to those reported in published manuscripts.

- Planned outcomes: 11 outcomes were reported in both sources and classified as matched. Three outcomes reported as planned in CT.gov were not abstracted from manuscripts: quality of life, ${ }^{7}$ miscarriage, ${ }^{8}$ and live birth. ${ }^{9}$ In 4 studies, outcomes reported in published manuscripts were not described in CT.gov: live birth, ${ }^{8,10}$ miscarriage, ${ }^{11}$ multiple births, ${ }^{11,12}$ and surgical complications. ${ }^{12}$

- Only $1^{7}$ of the 8 trials reported results in CT.gov, and these results matched those reported in the manuscript for the single outcome present in both sources.

\section{Effects of CT.gov Results on Strength of Evidence}

Overall, data from CT.gov had little impact on the SOE ratings. Using a threshold of 3 years since reported completion, only 2 completed trials were identified from CT.gov that did not have a matching journal publication. Both trials were applicable to KQ 1 and had a combined sample size of 340 patients. Thirty trials (10,718 patients) were included in the SOE rating for KQ 1 , and thus these 2 "missing" trials are unlikely to have had a meaningful impact on study results. Similarly, there was little evidence of reporting bias, with only single mismatches for 3 different outcomes between planned outcomes in CT.gov and reported outcomes in published manuscripts. 


\section{Person-Hours Required for Data Collection and Analysis}

Overall, the project team devoted an estimated 74.5 hours to planning and conducting this study. Data by investigator vs. staff are given in Table H-2.

Table H-2. Person-hours required, investigators vs. staff

\begin{tabular}{|l|c|c|c|c|c|}
\hline Name & $\begin{array}{c}\text { Administrative } \\
\text { (meetings, etc) }\end{array}$ & $\begin{array}{c}\text { Planningl } \\
\text { designing }\end{array}$ & $\begin{array}{c}\text { Running searches/ } \\
\text { abstracting data }\end{array}$ & $\begin{array}{c}\text { Synthesizing } \\
\text { data/writing }\end{array}$ & Total \\
\hline Investigator & 7 & 9 & 10.75 & 7.75 & 34.5 \\
\hline EPC Staff & 23 & 0 & 14 & 3 & 40 \\
\hline Totals & 30 & 9 & 24.75 & 10.75 & 74.5 \\
\hline
\end{tabular}

\section{Discussion}

This substudy found that CT.gov has important limitations for identifying selective reporting. Only one-third of the studies included in the Management of Infertility review were matched to a CT.gov record based on NCTID, and only 1 of those studies reported results in CT.gov. In addition, there were few discrepancies between planned outcomes reported in CT.gov and those reported in published manuscripts. A careful search and inspection of CT.gov for potentially eligible studies not identified by the review team yielded only 2 studies without a publication and 1 study incorrectly excluded at the title-and-abstract screening stage. These data had no impact on the SOE ratings or study conclusions, but required substantial person-hours to generate.

It is possible that CT.gov will mature into a more useful resource for the purpose of identifying selective reporting. Using data from CT.gov for the dates of trial registration compared to conduct of the study, it is clear that some studies were registered retrospectively. Prospective registration may yield more complete records and more informative data. However, it is likely that changes to CT.gov will be required for this database to serve as a useful source for identifying selective reporting.

At present, these results do not support the routine use of CT.gov to evaluate selective reporting. However, our study examined a small set of interventions for a single condition (infertility) and included a relatively small set of trials. Additional studies are needed before definitive conclusions can be drawn about the utility of CT.gov for detecting selective reporting. If changes to CT.gov were made to facilitate its use for this purpose, other resources could improve efficiency, including: a customized EndNote filter for importing CT.gov results, a standard methodology to guide investigators, and additional data on the activities that can be reliably completed by study staff versus investigators.

\section{References to Appendix H}

1. Borenstein M, Hedges LV, Higgins JPT, et al. Publication Bias. Introduction to MetaAnalysis: John Wiley \& Sons, Ltd; 2009:277-92.

2. Song F, Parekh S, Hooper L, et al. Dissemination and publication of research findings: an updated review of related biases. Health Technology Assessment. 2010;14(8):iii, ix-xi, 1-193. PMID: 20181324.
3. Sterne JA, Sutton AJ, Ioannidis JP, et al. Recommendations for examining and interpreting funnel plot asymmetry in metaanalyses of randomised controlled trials. BMJ. 2011;343:d4002. PMID: 21784880. 
4. Dwan K, Altman DG, Clarke M, et al. Evidence for the selective reporting of analyses and discrepancies in clinical trials: a systematic review of cohort studies of clinical trials. PLoS Med. 2014;11(6):e1001666. PMID: 24959719.

5. Hartung DM, Zarin DA, Guise JM, et al. Reporting discrepancies between the ClinicalTrials.gov results database and peerreviewed publications. Ann Intern Med. 2014;160(7):477-83. PMID: 24687070.

6. Agency for Healthcare Research and Quality (AHRQ). Methods Guide for Effectiveness and Comparative Effectiveness Reviews. AHRQ Publication No. 10(14)-EHC063-EF. Rockville, MD: Agency for Healthcare Research and Quality. February 2015. Available at: https://www.effectivehealthcare.ahrq.gov/to pics/cer-methods-guide/overview. Accessed April 1, 2016.

7. Legro RS, Brzyski RG, Diamond MP, et al. Letrozole versus clomiphene for infertility in the polycystic ovary syndrome. $\mathrm{N}$ Engl J Med. 2014;371(2):119-29. PMID: 25006718.

8. Morin-Papunen L, Rantala AS, UnkilaKallio L, et al. Metformin improves pregnancy and live-birth rates in women with polycystic ovary syndrome (PCOS): a multicenter, double-blind, placebocontrolled randomized trial. J Clin Endocrinol Metab. 2012;97(5):1492-500. PMID: 22419702.
9. Palomba S, Falbo A, Carrillo L, et al. Metformin reduces risk of ovarian hyperstimulation syndrome in patients with polycystic ovary syndrome during gonadotropin-stimulated in vitro fertilization cycles: a randomized, controlled trial. Fertil Steril. 2011;96(6):1384-90.e4. PMID: 21982727.

10. Abu Hashim H, El Rakhawy M, Abd Elaal I. Randomized comparison of superovulation with letrozole vs. clomiphene citrate in an IUI program for women with recently surgically treated minimal to mild endometriosis. Acta Obstet Gynecol Scand. 2012;91(3):338-45. PMID: 22181973.

11. Johnson NP, Stewart AW, Falkiner J, et al. PCOSMIC: a multi-centre randomized trial in women with PolyCystic Ovary Syndrome evaluating Metformin for Infertility with Clomiphene. Hum Reprod. 2010;25(7):1675-83. PMID: 20435692.

12. Palomba S, Falbo A, Battista L, et al. Laparoscopic ovarian diathermy vs clomiphene citrate plus metformin as second-line strategy for infertile anovulatory patients with polycystic ovary syndrome: a randomized controlled trial. Am J Obstet Gynecol. 2010;202(6):577.e1-8. PMID: 20096821. 